\title{
Kinks, Sistemas Integrables y Geodésicas: Solitones en el Modelo Sigma $O(3)$ Lineal
}

\author{
Departamento de Matemática Aplicada \\ Universidad de Salamanca
}

Memoria presentada por Miguel Ángel González León
para optar al Grado de Doctor en Matemáticas

Salamanca, Enero 2001 


\section{Índice}

$\begin{array}{ll}\text { Introducción } & 1\end{array}$

1 De Kinks y Sistemas Integrables $\quad 11$

1.1 Solitones y Kinks . . . . . . . . . . . . . . . . . . . . . . . . . . . 11

1.1.1 Orígenes de la Teoría de Solitones . . . . . . . . . . . . . 12

1.1.2 Aplicaciones de la Teoría de Solitones . . . . . . . . . . . . 17

1.1.3 Solitones en Teoría de Campos . . . . . . . . . . . . . 21

1.1.4 Ondas Solitarias y Defectos de tipo pared . . . . . . . . 23

1.2 El problema de la búsqueda de Kinks como un Sistema Dinámico . 25

1.3 Sistemas Dinámicos Integrables y Separables Hamilton-Jacobi . . . 29

1.3.1 Sistemas Integrables . . . . . . . . . . . . . . . 30

1.3.2 El Método de Hamilton-Jacobi . . . . . . . . . . . . . 37

1.3.3 Simetrías ocultas y Deformaciones Integrables . . . . . . . . 40

2 De las Ondas Solitarias en el Modelo Sigma $O(N)$ Lineal Defor$\begin{array}{ll}\text { mado } & 43\end{array}$

2.1 Modelo Sigma $O(N)$ Lineal y sus deformaciones . . . . . . . . . . . 44

2.2 Modelo Sigma Lineal Deformado y búsqueda de Kinks . . . . . . . 48

2.3 Coordenadas Elípticas . . . . . . . . . . . . . . . . . . 54

2.4 Separabilidad de la Ecuación de Hamilton-Jacobi . . . . . . . . . . 65

3 De la variedad de Kinks en el Modelo Sigma $O(3)$ Lineal Deformado

3.1 Modelo Sigma $O(2)$ Lineal Deformado . . . . . . . . . . . . . 77

3.2 Modelo Sigma $O(3)$ Deformado . . . . . . . . . . . . . . . 86

3.2.1 Solución General de la Ecuación de Hamilton-Jacobi . . . . 87

3.2.2 Tipos de Kinks . . . . . . . . . . . . . . . . . . . . . . . . . . 92

3.2.3 Límites Especiales y Reglas de Suma . . . . . . . . . . . . 111 
3.2.4 Espacio "modular" de Kinks . . . . . . . . . . . . . . . . 129

4 Mecánica Analítica en Variedades Riemannianas 141

4.1 Cálculo Variacional en Sistemas Dinámicos . . . . . . . . . . . . . . 142

4.2 Métrica de Jacobi . . . . . . . . . . . . . . . . . . . . . . . . . . . . 146

4.2 .1 Teorema de Jacobi . . . . . . . . . . . . . . . . . . . . 147

4.2.2 Hessiano Dinámico y Hessianos Geodésicos . . . . . . . . . . 154

4.2.3 Operador de Desviación Geodésica y Diagonalización de la Curvatura seccional . . . . . . . . . . . . . . . 163

4.3 Sistemas Pre-Supersimétricos . . . . . . . . . . . . . . . . 165

4.3.1 N=2 Pre-Supersimetría . . . . . . . . . . . . . . . . . . . . 169

5 De la Estabilidad de los Kinks del Modelo Sigma Lineal Defor$\begin{array}{lr}\text { mado } & 175\end{array}$

5.1 Geodésicas de tipo Kink en la Métrica de Jacobi . . . . . . . . . . . 176

5.1 .1 Modelo $O(2)$ deformado . . . . . . . . . . . . . 176

5.1 .2 Modelos Sigma $O(3)$ y $O(N)$ Deformados . . . . . . . . . . 196

5.2 Funcional Segunda Variación. Modelo $O(2)$. . . . . . . . . . . . . . 198

5.2.1 Funcional Hessiano Dinámico: TK1 . . . . . . . . . . . . . . 199

5.2.2 Funcionales Hessianos Geométricos: TK1 . . . . . . . . . . . 208

5.2 .3 Funcional Hessiano Dinámico: TK2 . . . . . . . . . . . . . . 218

5.2.4 Funcionales Hessianos Geométricos: TK2 . . . . . . . . . . . 225

5.3 Funcional Segunda Variación. Modelo $O(3)$. . . . . . . . . . . . . 230

5.3.1 Funcionales Hessianos para el kink TK1 . . . . . . . . . . . 230

5.3.2 Funcional Hessiano Dinámico: TK2 $\sigma_{2}$ y TK2 $\sigma_{3} \quad \ldots . . .232$

5.3.3 Funcional Hessiano Geométrico: TK2 $\sigma_{2}$ y TK2 $2 \sigma_{3} \ldots \ldots$. . . 236

6 Teoría de Morse de Kinks $\quad 241$

6.1 El Superpotencial y las Ecuaciones de primer orden . . . . . . . . . 242

6.1.1 Cálculo del Superpotencial . . . . . . . . . . . . . . . . . . . 245

6.1 .2 Ecuaciones de primer orden . . . . . . . . . . . . 248

6.2 Solución general de las ecuaciones de primer orden . . . . . . . . . . 252

6.3 Teoría de Morse de Kinks . . . . . . . . . . . . . . . . . . . . . . . 271

6.3.1 La serie de Morse de $\mathcal{C}$. . . . . . . . . . . . . . . . . 273

6.3.2 Análisis de la estabilidad por el Teorema del Índice de Morse 277 
$\begin{array}{ll}\text { A Modelo Elíptico-Cilíndrico } & 283\end{array}$

A.1 Planteamiento del Modelo Elíptico-Cilíndrico . . . . . . . . . . . . . 283

A.2 Tipos de Soluciones . . . . . . . . . . . . . . . . . . . . 292

B Superpotencial del Modelo $O(3)$ en Coordenadas Cartesianas 301

B.1 Cambio de coordenadas inverso . . . . . . . . . . . . . . 301

B.2 Superpotencial en coordenadas cartesianas . . . . . . . . . . . . . 304

C Recopilación Breve de Geometría Riemanniana 307

C.1 Conexiones y derivadas covariantes . . . . . . . . . . . . . . 307

C.2 Tensor de Curvatura . . . . . . . . . . . . . . . . . . . 314

$\begin{array}{ll}\text { D Cálculo Variacional Aplicado a Geodésicas } & 317\end{array}$

D.1 Conceptos Básicos del Cálculo Variacional . . . . . . . . . . . . . . 317

D.2 Variaciones y Teorema Fundamental . . . . . . . . . . . . . . . . 318

D.3 Cálculo Variacional aplicado a Geodésicas . . . . . . . . . . . . . 320

D.4 Desviación Geodésica . . . . . . . . . . . . . . . . . . . . . 322

D.5 Teoría de Morse . . . . . . . . . . . . . . . . . . . . 323

E Correcciones cuánticas a la masa de Kinks 327

E.1 Kinks cuánticos en el Modelo Sigma $O(3)$ Lineal Deformado . . . . 330

E.1.1 El Kink TK1 . . . . . . . . . . . . . . . . . . 330

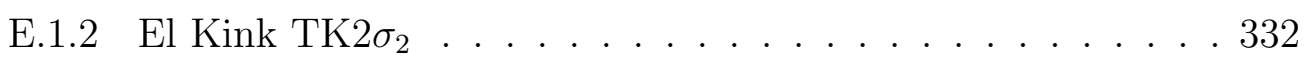

$\begin{array}{ll}\text { Bibliografía } & 339\end{array}$ 


\section{Introducción}

La finalidad de esta Memoria es el estudio de los defectos topológicos de tipo kink que existen en teorías de campos relativistas con varias componentes escalares definidas sobre un universo bidimensional.

La literatura existente sobre este tema es abundantísima en lo que respecta a teorías con un solo campo escalar real (los ejemplos más famosos de este tipo de objetos extensos son el kink de la teoría $\phi^{4}$ y el solitón del modelo de Seno-Gordon [22]). Sin embargo, son muy escasas las referencias que atañen a sistemas con varias componentes escalares. Se han obtenido algunos resultados parciales, como por ejemplo los trabajos citados en las referencias [11] y [18].

El estudio que se ha realizado en este trabajo tiene, como pretendemos demostrar, gran importancia tanto desde el punto de vista físico como desde el matemático. Nuestro interés, sin embargo, se centrará en éste último. El objeto del trabajo, por tanto, será el desarrollo de técnicas matemáticas que nos permitirán calcular explícitamente todas las soluciones de tipo onda solitaria, o kinks, de la deformación natural del conocido Modelo Sigma $O(N)$ Lineal, así como analizar el espacio funcional que dichas soluciones constituyen y su estabilidad.

\section{Importancia de los kinks en Teorías Físicas}

Las soluciones de tipo solitón, o kink, en una Teoría de Campos, son importantes por varias razones:

a) Forman parte del espacio de estados (desde la perspectiva cuántica), o del espacio de soluciones (desde la clásica) de la teoría y, en consecuencia, es necesario su cálculo para disponer del conjunto completo de soluciones.

b) Este tipo de soluciones describen estados extensos con propiedades muy distintas a las que presentan los cuantos habituales, y con muchas implicaciones en Física Fundamental, Cosmología y Física de la Materia Condensada. 
c) El tipo de kinks que vamos a estudiar en este trabajo, muy poco conocido en la literatura científica hasta este momento, aparece en modelos tan importantes, en el contexto expuesto en b), como los que enumeraremos a continuación:

- El sector de Higgs de la teoría electrodébil de interacción entre partículas elementales se describe en términos de una acción (ver [106]) susceptible de admitir defectos de este tipo, si bien en forma de "pared" por ser vistos en una perspectiva $(3+1)$-dimensional. Lo mismo puede afirmarse del sector de Higgs de los modelos de gran unificación.

- El Modelo Sigma $O(N)$ Lineal propuesto por Gell-Mann y Lèvy en los años 60, ver [43] y [102], para explicar la fenomenología hadrónica a baja energía, es también un sistema con $N$ campos escalares.

- Importantes avances en el estudio de la Gluodinámica N=1 supersimétrica, [29], y el modelo de Wess-Zumino, [44], se han conseguido mediante el estudio de una configuración de membrana en una teoría de cuerdas débilmente acoplada cuyo límite de baja energía da lugar a las teorías de campos aludidas, [109]. Los defectos de tipo "pared" que dan lugar a las membranas bidimensionales aparecen así en modelos con sector escalar que incluye más de un campo.

- Como último ámbito en el que sistemas del tipo que estudiamos en esta Memoria juegan un papel importante citaremos la física de la materia condensada: para configuraciones estáticas la energía de los modelos a considerar puede interpretarse como la energía libre de la teoría de Ginzburg, [48], de las transiciones de fase y sus generalizaciones.

\section{Interés matemático del cálculo de soluciones de tipo kink}

Es bien conocido, desde los años 60, que la búsqueda de soluciones estáticas a las ecuaciones de evolución de los campos en un espacio-tiempo bidimensional equivale a la resolución de un sistema mecánico en dimensión $N$, donde $N$ es el número de campos escalares de la teoría. Por tanto el estudio de los defectos de tipo kink con $N$ componentes escalares reales se traduce, por medio de esta equivalencia o "analogía mecánica", en el análisis de sistemas dinámicos con $N$ grados de libertad. En el caso de teorías con un único campo escalar $(N=1)$, el sistema mecánico 
análogo será unidimensional y, en consecuencia, completamente integrable. Muy pronto se reconoció el salto en dificultad de resolución del problema si el número de campos escalares presentes en la teoría es superior a uno $(N>1)$, como prueba la cita de la referencia clásica sobre este tema, el libro de R. Rajaraman [87]:

"Unlike the $N=1$ motion equation which could be integrated by quadrature, there is no simple way of integrating the $N$ coupled differential equations ruling the evolution of the $N$-dimensional mechanical system. Here the analogy to mechanics does not help. Given a general potential and end-point boundary conditions, it is not easy to integrate Newton's law of motion in two or more dimensions. This may seem surprising in view of the long history of mechanics and the several classical text on orbit theory".

El problema, por tanto, reside en el hecho de que para $N>1$ no está asegurada la integrabilidad del sistema dinámico análogo y, de esta forma, no es posible en general encontrar las soluciones kink de la teoría. La estrategia que utilizaremos en este trabajo es la de centrarnos en el estudio detallado de los sistemas completamente integrables y de su integración efectiva, de cara a la obtención de soluciones de tipo kink en teorías con varios campos reales.

El pesimismo de Rajaraman ha sido superado en los últimos años por varios resultados importantes. Un ejemplo de ellos es el Modelo MSTB (de MontonenTrullinger-Sarker y Bishop), con $N=2$ campos escalares: en [71] y [91] este modelo se propuso en el contexto de la búsqueda de solitones no topológicos cuya estabilidad proviene de una simetría interna respecto del grupo $U(1)$. En [66], el modelo MSTB se consideró como la aproximación continua clásica a un cristal unidimensional con un parámetro de orden de dos componentes. Más importante aún que la diversidad de aplicaciones del mismo es el hecho de que en [66] se reconoció que el sistema mecánico análogo a resolver en la búsqueda de kinks es un sistema completamente integrable. H. Ito, a continuación, [52], encontró todos los kinks del sistema usando el hecho de que la ecuación de Hamilton-Jacobi es separable. Realmente el sistema dinámico no es otro que el sistema de Garnier, [42], cuya completa integrabilidad y, de hecho, su integración explícita, había sido demostrada por R. Garnier en 1919 en otro contexto diferente al de la búsqueda de kinks.

Un segundo aspecto en el que la presencia de más de un campo escalar produce un problema cualitativamente mucho más difícil que en el caso $N=1$ es el estudio de la estabilidad de los diferentes tipos de kinks de un modelo determinado. El 
estudio del espectro de matrices $N \times N$ de operadores diferenciales no es tarea fácil en absoluto. Por esta razón hay incluso errores flagrantes en la literatura sobre esta materia. El método correcto de análisis de la estabilidad en el Modelo MSTB fue sugerido en la referencia [53] y desarrollado completamente en la serie de artículos [67], [68] y [69]. La idea fundamental se basa en la analogía existente con el estudio de la estabilidad de geodésicas tal como se enmarca en la Teoría de Morse, [70]. Ello requiere plantear el problema dinámico como un problema geodésico y la prescripción para conseguirlo es el principio de Maupertuis-Jacobi: las trayectorias de una partícula en un potencial son las geodésicas en una variedad riemanniana dotada de la métrica de Jacobi asociada al mismo, [65].

\section{Objetivos de este trabajo}

El propósito fundamental de este trabajo es extender el procedimiento de resolución del Modelo MSTB citado anteriormente al Modelo Sigma $O(N)$ Lineal Deformado que generaliza el modelo MSTB al caso de $N$ campos escalares. El sistema dinámico a resolver es entonces el modelo de Garnier en dimensión N, [42]. Las dificultades analíticas aumentan notablemente pero emerge una estructura en la variedad de kinks del sistema de gran interés: de modo natural constituye un espacio modular, como los espacios de monopolos BPS, [10], o instantones, [36], si bien el grupo por el que se ha de tomar cociente es discreto.

En particular, nos centraremos en describir la riquísima estructura del espacio modular de kinks en el caso $N=3$, que da la pauta para los sistemas con $N>3$. El caso $N=2$ es bien conocido, sin embargo la amplísima oferta de modelos de Liouville, con sistemas dinámicos asociados completamente integrables (ver [80]), que presentan soluciones de tipo kink, sugiere que en teoría de campos escalares complejos hay multitud de sistemas donde existen defectos tipo kink que se pueden describir analíticamente con gran precisión. En la tesina de Grado de A. Alonso y en la referencia [4] se ha explotado esta idea y se han encontrado variedades de kinks con propiedades muy ricas. La posible limitación a modelos con sistema dinámico asociado integrable se puede evitar parcialmente estudiando deformaciones realistas de los sistemas en que se conoce la variedad de kinks y analizando cuáles de ellos sobreviven a la deformación. El estudio general del caso $N=2$ es el objetivo de la Memoria titulada: "Kinks, Sistemas Integrables y Supersimetría" elaborada por A. Alonso y que puede considerarse, en buena medida, paralela a la que aquí presentamos. 
En lo que respecta a la estabilidad de las soluciones, en la Memoria se establecen con precisión, y desde un punto de vista general, las diferencias existentes, al segundo orden en el cálculo de variaciones, entre el criterio de estabilidad proporcionado por las variaciones segundas del funcional de acción (estabilidad dinámica) y las correspondientes a los funcionales de energía y longitud en la métrica de Jacobi asociada (estabilidad geométrica).

El análisis general anterior permite completar el estudio del Modelo Sigma $O(3)$ Lineal Deformado al particularizarlo a sus soluciones kink concretas. La teoría de Morse del sistema de Garnier queda demostrada así de modo riguroso y con ello identificadas las propiedades de estabilidad de los distintos tipos de kink.

Por último, en la Memoria se ha demostrado que el Modelo Sigma $O(N)$ Lineal Deformado es un modelo pre-supersimétrico, es decir: admite una extensión supersimétrica. Se profundiza asimismo en la relación no trivial que existe entre la completa integrabilidad de un sistema y su carácter pre-supersimétrico.

\section{Estructura de la Memoria}

En el Capítulo 1 de la Memoria se presentan, a modo de introducción general, las ideas fundamentales que se manejan en este trabajo. Se trata por tanto de una recopilación de resultados y teorías muy tratadas en la literatura científica, y en la que en ningún caso pretendemos ser originales. Más bien lo contrario, hemos procurado seleccionar las que a nuestro modo de ver son las referencias más significativas sobre el tema tratado y hemos reproducido de ellas los resultados y comentarios principales.

Este capítulo está dividido en tres secciones. La primera de ellas da cuenta de aspectos históricos acerca de los solitones y sus aplicaciones. Se resalta particularmente la importancia de los solitones en las teorías de campos y se presentan las diferentes definiciones posibles de los conceptos de solitón y onda solitaria. En la segunda se presenta la ya citada "analogía mecánica" que permite calcular los kinks de una teoría de campos escalares 1+1-dimensionales por medio de la resolución de un sistema dinámico en Mecánica Clásica. Aunque dicho resultado está descrito en varias referencias bibliográficas citadas en la Memoria, es de resaltar que la presentación que aquí realizamos es más completa que las que pueden encontrarse habitualmente en la literatura. Finalmente, la tercera sección de este capítulo está dedicada a exponer los aspectos más relevantes de la Teoría de Sistemas Integrables, y particularmente los separables Hamilton-Jacobi, que van a ser utilizados en el resto de la Memoria. De nuevo se ha optado por acudir a las 
referencias clásicas sobre el tema para la elaboración de dicha sección.

En el Capítulo 2 se resuelve completamente, por el método de separación de variables, la ecuación de Hamilton-Jacobi del sistema de Garnier, asociado vía la analogía mecánica al Modelo Sigma $O(N)$ Lineal Deformado.

En una primera sección se define el Modelo Sigma $O(N)$ Lineal y se plantean las diferentes deformaciones del mismo que pueden realizarse. Se describe también cómo, desde el punto de vista de las aplicaciones físicas del modelo, dichas deformaciones son inevitables. Se establece, en la segunda sección, la deformación concreta que se estudiará fundamentalmente en esta Memoria, que no es otra que la generalización natural del Modelo MSTB al caso de $N$ campos escalares, y se analiza el sistema dinámico asociado que, como ya hemos dicho, es el sistema de Garnier $N$-dimensional. Tras una sección dedicada a la definición y presentación detallada de las propiedades de las coordenadas elípticas de Jacobi, se demuestra la separabilidad, en dichas coordenadas, de la ecuación de Hamilton-Jacobi del sistema, procediéndose al planteamiento de las cuadraturas que resuelven completamente la misma. El planteamiento realizado en esta sección es muy novedoso y constituye el punto de partida de las varias aportaciones completamente originales que contiene esta Memoria.

El Capítulo tercero trata la particularización de los resultados anteriores al caso $N=3$, así como el análisis pormenorizado de los diferentes tipos de soluciones kink del modelo. Para favorecer la claridad expositiva se han recordado, en una primera sección, los resultados conocidos acerca del Modelo MSTB, presentados ahora en las coordenadas elípticas antes citadas, lo cual resulta novedoso frente a la literatura existente.

La rica estructura del espacio de soluciones del Modelo $O(3)$ es tratada de modo exhaustivo y con precisión. Se demuestran con todo detalle aspectos no triviales de la misma como son las reglas de suma de las energías de los diferentes tipos de kink o los límites asintóticos de los mismos, que permiten plantear finalmente diferentes compactificaciones del espacio modular de soluciones. Los resultados citados son absolutamente originales y permiten una comprensión completa del Modelo $O(3)$.

Hemos dedicado el cuarto capítulo al planteamiento variacional de la Mecánica Analítica sobre variedades Riemannianas. Se ha dedicado una primera sección a exponer brevemente los aspectos fundamentales, o más conocidos, del mismo. Posteriormente nos hemos centrado en el Teorema de Jacobi (o Principio de MaupertuisJacobi) y sus implicaciones en el segundo orden del Cálculo de Variaciones. Fi- 
nalmente, de una manera muy novedosa, se relaciona la métrica de Jacobi de un sistema con su carácter pre-supersimétrico. Podemos resumir, por tanto, en cuatro puntos concretos los objetivos abordados en este capítulo:

- En primer lugar se trata de formular con precisión el Teorema de Jacobi acerca de la equivalencia entre la resolución de un sistema dinámico y el estudio de las geodésicas en una variedad Riemanniana asociada (Principio de MaupertuisJacobi).

- En segundo lugar, y relacionado con el anterior, hemos demostrado la relación que existe entre la "estabilidad dinámica", determinada por el espectro del funcional de segunda variación de la acción del sistema, y la "estabilidad geométrica" asociada al espectro del operador de desviación geodésica en la variedad asociada. Mediante dos Teoremas, se establece la no-equivalencia estricta entre ambos conceptos, si bien se desvela la relación existente entre los dos espectros citados. Los resultados aquí demostrados no están incluidos en ninguna de las referencias que conocemos sobre el tema.

- Se plantea, en el apartado 4.2.3, un método de diagonalización de la ecuación de desviación geodésica que generaliza al planteado habitualmente en la literatura (ver por ejemplo [70]) para el caso de variedades localmente simétricas. Bajo ciertas condiciones se demuestra que dicha ecuación puede diagonalizarse aún en el caso de variedades no localmente simétricas. Se extiende por tanto, de manera novedosa, un resultado particular a un caso mucho más general.

- Finalmente se estudian en este capítulo los sistemas pre-supersimétricos y las consecuencias que dicho carácter tiene sobre la métrica de Jacobi correspondiente. Se presenta asimismo un elegante y original Teorema que caracteriza los sistemas con $\mathrm{N}=2$ pre-supersimetría en términos de la curvatura Gaussiana de la variedad riemanniana asociada vía la métrica de Jacobi.

El Capítulo 5 está dedicado al análisis de la estabilidad de los kinks del Modelo Sigma $O(3)$ Lineal Deformado. Se aplican, evidentemente, los resultados generales obtenidos en el capítulo anterior. Para ello se comienza resolviendo las ecuaciones de las geodésicas de la métrica de Jacobi asociada y, posteriormente, se calculan los espectros de los funcionales hessianos correspondientes.

Se consigue así demostrar completamente, y de forma analítica, la estabilidad e inestabilidad de los diferentes tipos de kinks, algo que, como se ha dicho antes, no estaba logrado previamente.

En el Capítulo 6 se aborda el estudio de la Teoría de Morse del sistema tratado. 
En una primera sección se demuestra que el modelo es pre-supersimétrico, algo que no había sido probado hasta ahora. Para ello se integra la ecuación en derivadas parciales que determina el superpotencial de la teoría. La obtención del superpotencial permite el planteamiento de ecuaciones de primer orden para el sistema, y, en consecuencia, la clasificación de los kinks en dos tipos: kinks BPS y kinks no-BPS. Esta clasificación establece a su vez un criterio acerca de la estabilidad, cuya aplicación al Modelo Sigma O(3) Lineal Deformado proporciona, obviamente, idénticos resultados a los determinados en el capítulo anterior.

En una segunda sección se resuelven las ecuaciones de primer orden del sistema. Los resultados evidentemente coinciden con los obtenidos por medio de la integración de la ecuación de Hamilton-Jacobi en el Capítulo 3. La nueva resolución aporta, no obstante, la obtención de las energías de los kinks como cotas de Bogomol'nyi de muy diferentes formas para cada uno de los tipos de kink, según las características de los mismos.

Finalmente se estudia la Teoría de Morse del modelo. La aplicación del Teorema del Índice de Morse permite establecer la estabilidad o inestabilidad de cada solución de acuerdo con el número de puntos conjugados por los que dicha solución pase. Se determinan así cuáles son dichos puntos conjugados y cuál es el índice correspondiente a cada uno de ellos, para aplicar posteriormente el Teorema de Morse.

La Memoria se completa con cinco apéndices de muy diferente carácter:

- Dedicamos el Apéndice A al estudio de un modelo con tres campos escalares que engloba al Modelo Sigma $O(1)$ Lineal y al Modelo MSTB. Hemos denominado a dicho sistema Modelo Elíptico-Cilíndrico. Este modelo constituye, en cierto sentido, un paso intermedio entre el Modelo MSTB y el que hemos denominado Modelo Sigma $O(3)$ Lineal Deformado. A pesar de ello, presenta aspectos bien diferenciados de los correspondientes a los distintos modelos del caso general, como por ejemplo el poseer cuatro puntos de vacío; por esta razón lo incluimos dentro de un apéndice y no en un capítulo de la Memoria, a pesar de tratarse de resultados originales de este trabajo.

- El Apéndice B recoge los largos cálculos que conducen a la obtención, en coordenadas cartesianas, del superpotencial del Modelo Sigma $O(3)$ Lineal Deformado que se ha presentado en el Capítulo 6 (en coordenadas elípticas). Con el fin de no entorpecer el discurso lógico de los razonamientos incluidos en dicho capítulo, nos ha parecido oportuno dedicar un apéndice a las expresiones y cálculos citados. 
- Los Apéndices C y D son de una índole muy distinta. Se trata de dos breves recopilaciones de materias a las que la Memoria se remite con frecuencia y que habitualmente vienen presentadas en la literatura de formas y notaciones muy diversas. El primero de ellos está dedicado a la Geometría Riemanniana y con el mismo se pretende fijar con precisión la notación escogida y las definiciones adoptadas. El segundo, por su parte, trata de presentar someramente el Cálculo de Variaciones aplicado a Geodésicas, incluyendo la ecuación de desviación geodésica y una pequeña descripción de los aspectos fundamentales de la Teoría de Morse.

- En el Apéndice E presentamos, de una manera concisa, los resultados que hemos obtenido acerca de la cuantificación de las ondas solitarias que se han calculado en la Memoria. Si bien el proceso de cuantificación de los kinks escapa, en principio, a los objetivos perseguidos por este trabajo, razón por la cual se incorporan dichos cálculos en forma de Apéndice, nos parece totalmente adecuado incluir estos resultados, que complementan los objetivos alcanzados y, finalmente, cierran el estudio completo del Modelo Sigma $O(3)$ Deformado. No está de menos resaltar, además, que la cuantificación realizada representa una aportación completamente novedosa a la literatura sobre el tema.

Por último, se ha incluido una lista abundante de referencias bibliográficas. Algunas de ellas, evidentemente, han representado un papel fundamental en la elaboración de esta Memoria, otras, por contra, han sido consultadas únicamente para completar algún aspecto puntual de los conceptos citados en la misma. Las citas que se realizan a lo largo del trabajo a ellas determinan uno u otro carácter. Pedimos disculpas por aquellas referencias importantes que hayamos podido olvidar.

Creemos adecuado resaltar que los aspectos más originales del trabajo que aquí presentamos están recogidos en los Capítulos tercero, quinto y sexto, y en las secciones 2.4 y 4.3 de los Capítulos segundo y cuarto, respectivamente. 


\section{Capítulo 1}

\section{De Kinks y Sistemas Integrables}

Dividiremos este primer capítulo en tres secciones, en una primera se presentan los conceptos y teorías fundamentales acerca de los solitones y su importancia en las teorías de campos, en segundo lugar se planteará la búsqueda de soluciones de tipo solitónico (kinks) en una teoría de campos, sobre un espacio-tiempo bidimensional, por medio del análisis de un sistema dinámico en Mecánica Clásica, y finalizaremos con una sección dedicada a revisar la teoría de los Sistemas Dinámicos Integrables y de los Sistemas separables Hamilton-Jacobi.

\subsection{Solitones y Kinks}

Existen en la literatura física y matemática muchas definiciones del concepto de solitón, dependiendo del marco de estudio o de la aplicación concreta que se esté analizando. Intentaremos en esta sección aclarar en buena medida los diferentes conceptos y definiciones acerca de los objetos solitónicos que aparecen en el ámbito de la Matemática Aplicada y de la Física Matemática. Nuestro análisis será más detallado en lo que respecta a la importancia de la Teoría de Solitones en las Teorías Cuánticas de Campos, marco en el que pueden encuadrarse algunos de los objetivos principales de este trabajo.

De una manera general se suele denominar solitón a toda solución de tipo onda solitaria de una ecuación no lineal. Se trata, por tanto, de una onda viajera $u(x, t)=f(x-v t)$ tal que $f$ es una función diferenciable que tiende a cero rápidamente en el infinito. La presencia, para algunos tipos de ecuaciones, de peculiares propiedades de scattering de las soluciones (fenómenos de superposición no lineal) puede refinar la definición general, y establece habitualmente en la lite- 
ratura una clasificación entre solitones propiamente dichos (cuando se requiere la verificación de las propiedades de superposición) y ondas solitarias o kinks ${ }^{1}$ (lumps en el lenguaje de Coleman [22]). Aclararemos todos estos conceptos a continuación, utilizando fundamentalmente el siguiente soporte bibliográfico: el artículo de R.S. Palais [79] y los textos de T.D. Lee [63], R. Rajaraman [87], P.G. Drazin y D.S. Johnson [28], y M.J. Ablowitz y P.A. Clarkson [1].

\subsubsection{Orígenes de la Teoría de Solitones}

La Historia de la Teoría de Solitones se remonta al mes de Agosto del año 1834, cuando el naturalista e ingeniero naval John Scott Russell observó, montado en su caballo, una "Onda de Traslación" viajando en un canal cercano a Edimburgo ("Union Canal"). Reproduciendo sus propias palabras, del famoso "Report on Waves" de 1844 [90]:

" I believe I shall best introduce this phænomenon by describing the circumstances of my own first acquaintance with it. I was observing the motion of a boat which was rapidly drawn along a narrow channel by a pair of horses, when the boat suddenly stopped-not so the mass of water in the channel which it had put in motion; it accumulated round the prow of the vessel in a state of violent agitation, then suddenly leaving it behind, rolled forward with great velocity, assuming the form of a large solitary elevation, a rounded, smooth and well-defined heap of water, which continued its course along the channel apparently without change of form or diminution of speed. I followed it on horseback, and overtook it still rolling on at a rate of some eight or nine miles an hour, preserving its original figure some thirty feet long and a foot to a foot and a half in height. Its height gradually diminished, and after a chase of one or two miles I lost it in the windings of the channel. Such, in the month of August 1834, was my first chance interview with that singular and beautiful phænomenon which I have called the Wave of Translation, a name which it now very generally bears; which I have since found to be an important element in almost every case of fluid resistance, and ascertained to be the type of that great moving elevation of the sea,

\footnotetext{
${ }^{1}$ Es conveniente precisar que la palabra kink no siempre se utiliza en la literatura desde el punto de vista general que hemos adoptado en este trabajo, y que se especificará en las secciones siguientes. En varios contextos diferentes es posible encontrar la denominación kink usada para describir un tipo de onda solitaria muy concreto.
} 
which, with the regularity of a planet, ascends our rivers and rolls along our shores".

J.S. Russell quedó fascinado con este fenómeno y realizó numerosos experimentos con este tipo de ondas en un tanque de ondas de su propio diseño. Obtuvo la fórmula que relaciona la velocidad y la altura de estas ondas:

$$
c^{2}=g(h+\eta)
$$

(donde $c$ es la velocidad, $g$ la aceleración de la gravedad, $h$ la profundidad del canal y $\eta$ la amplitud de la onda), y propuso que el objeto solitario que había encontrado realmente representaba un tipo general de soluciones de la Hidrodinámica, que primeramente denominó "ondas de traslación", y más tarde "ondas solitarias". A diferencia de la onda de choque, singular en el frente de choque, la "onda solitaria" es regular en todas partes, no presenta singularidades. La onda solitaria es no dispersiva y estable, por tanto es diferente de cualquier paquete de ondas compuesto por las habituales soluciones en ondas planas.

El éxito de Russell fue mucho menor en lo que respecta a convencer a los científicos de su tiempo de estos hechos. Airy y Stokes, las mayores autoridades en la materia de la época, aparentemente "demostraron" que una onda de ese tipo era necesariamente inestable. Los trabajos posteriores de Boussinesq (1871) [16], Lord Rayleigh (1876) [88] y finalmente D.J. Korteweg y G. de Vries (1895) [60] señalaron los errores de Airy y Stokes y reivindicaron las conclusiones de Russell.

En el artículo citado [60] "On the change of form of long waves advancing in a rectangular canal, and on a new type of long stationary waves", Korteweg y de Vries dieron una explicación analítica completa de lo que actualmente se denomina "solución solitón" de la ecuación hidrodinámica no lineal (la llamada ecuación de Korteweg-de Vries), ecuación que gobierna la evolución de ondas propagándose en un canal de aguas poco profundas, unidimensionales y de pequeña amplitud [1]:

$$
\frac{\partial \eta}{\partial \tau}=\frac{3}{2} \sqrt{\frac{g}{h}} \frac{\partial}{\partial \xi}\left(\frac{1}{2} \eta^{2}+\frac{2}{3} \alpha \eta+\frac{1}{3} \sigma \frac{\partial^{2} \eta}{\partial \xi^{2}}\right), \quad \sigma=\frac{1}{3} h^{3}-\frac{T h}{\rho g}
$$

donde $\eta$ es la elevación de la onda sobre el nivel de equilibrio $h, \alpha$ es un parámetro constante relativo al movimiento uniforme del líquido, $g$ es la aceleración de la gravedad, $T$ es la tensión superficial y $\rho$ la densidad. La ecuación puede pasarse a la forma no-dimensional: por medio de los cambios

$$
t=\frac{1}{2} \sqrt{g /(h \sigma)} \tau, \quad x=-\frac{1}{\sqrt{\sigma}} \xi, \quad u=\frac{1}{2} \eta+\frac{1}{3} \alpha
$$


se obtiene

$$
u_{t}+6 u u_{x}+u_{x x x}=0
$$

donde el subíndice significa obviamente diferenciación. ${ }^{2}$

La solución "onda solitaria" de Korteweg y de Vries es:

$$
u(x, t)=2 \kappa^{2} \operatorname{sech}^{2}\left\{\kappa\left(x-4 \kappa^{2} t-x_{0}\right)\right\}
$$

con $\kappa$ y $x_{0}$ constantes.

Podemos ya definir, de una manera precisa, qué se entiende exactamente por onda solitaria actualmente:

Definición 1. ([1]) Una solución "onda solitaria" de una ecuación en derivadas parciales:

$$
\Phi(x, t, u)=0
$$

donde $x, t \in \mathbb{R}$ (variables espacial y temporal respectivamente) y $u: \mathbb{R}^{2} \rightarrow \mathbb{R}$ es la variable dependiente, es una solución de onda viajera de la forma:

$$
u(x, t)=\phi(x-\gamma t)=\phi(z)
$$

para alguna velocidad $\gamma$, y cuya transición ocurre desde un estado asintótico constante, cuando $z \rightarrow-\infty$, hasta otro estado asintótico constante cuando $z \rightarrow+\infty$.

Una cuestión quedó sin resolver en tiempos de Korteweg y de Vries: cuándo tales soluciones estables, no singulares y no disipativas pueden ocurrir en otras ramas de la física, independientemente de la Hidrodinámica.

El estudio de los solitones permaneció en un "estado estacionario" hasta sesenta años después. Entre 1954 y 1955 se construyó en Los Álamos una nueva computadora, MANIAC I, para la realización de los cálculos necesarios en el diseño de la primera bomba de hidrógeno. Enrico Fermi y Stanislaw Ulam propusieron un problema-prueba para calibrar las posibilidades de la nueva máquina; buscaron un problema sencillo de establecer tal que su resolución requiriera una cantidad de cálculos tan grande que no pudiera ser resuelto "a mano" ni utilizando las computadoras mecánicas existentes. Naturalmente, debía de tratarse de un problema con solución ya conocida. La propuesta que realizaron fue el estudio de una cuerda elástica, con extremos fijos, sujeta no sólo a la fuerza elástica usual, de intensidad proporcional al estiramiento, sino también a un término corrector no lineal. La

\footnotetext{
${ }^{2}$ Es importante resaltar que cualquiera de los tres sumandos de la ecuación puede ser modificado por una constante arbitraria sin más que re-escalar adecuadamente las variables independientes y dependiente.
} 
cuestión a estudiar era cómo el movimiento global termalizaría al cabo del tiempo. Se trataba, por tanto, de verificar un hecho que constituía un artículo de fe en mecánica estadística: la creencia de que cuando un sistema mecánico, con varios grados de libertad y cercano a una posición de equilibrio estable, es sometido a una interacción no lineal genérica, ésta termalizará su energía total, es decir la energía estará equidistribuida entre los modos normales del correspondiente sistema linealizado. Fermi creía haber demostrado este hecho, [34].

El experimento fue llevado a cabo por Fermi, Ulam y John Pasta, que programó el MANIAC I. El resultado del hoy conocido como experimento de Fermi-PastaUlam fue completamente diferente a lo que se esperaba, de hecho no encontraron termalización en absoluto. Los detalles del planteamiento y de los cálculos realizados pueden verse en [79], o en la referencia original [35]; recordaremos simplemente que el experimento se realizó aproximando (discretizando) la cuerda (continua, unidimensional, con extremos fijos y sujeta a fuerzas elásticas no lineales) por un número finito de puntos, de tal manera que las ecuaciones en derivadas parciales que describen el movimiento de la cuerda pasan a ser un número finito de ecuaciones diferenciales ordinarias (en el experimento FPU, exactamente 64).

$$
m \frac{\partial^{2} y_{n}}{\partial t^{2}}=K\left(y_{n+1}-2 y_{n}+y_{n-1}\right)\left[1+\alpha\left(y_{n+1}-y_{n-1}\right)\right]
$$

El resultado del experimento fue que la energía, inicialmente concentrada en el primer modo, no se distribuyó por igual entre todos los modos sino que fue pasando sucesivamente casi en su totalidad de modo en modo. De esta manera, al cabo de un cierto tiempo había retornado al primer modo con una exactitud del $99 \%$ y el proceso se reinició.

La explicación del experimento FPU no fue completada hasta diez años después en el famoso artículo de M. Kruskal y N. Zabusky [62]. Kruskal y Zabusky demostraron que, en un límite continuo, ciertas soluciones de las ecuaciones FPU podían ser descritas en términos de las soluciones de la ecuación de Korteweg-de Vries; probaron además, utilizando cálculo numérico para el problema de valor inicial en la ecuación KdV, que estas soluciones "pulsos de onda solitaria" o "solitones" (Kruskal y Zabusky fueron los primeros en utilizar esta denominación, por analogía con el comportamiento de las partículas), tras interaccionar nolinealmente, permanecían inalteradas, es decir, y utilizando las palabras de Kruskal y Zabusky: los solitones se "atraviesan" unos a otros sin perder su identidad tras interaccionar. ${ }^{3}$

\footnotetext{
${ }^{3}$ Realmente este hecho fue descubierto también por Russell, ver [28].
} 
Es el momento de precisar, por tanto, qué es un solitón, al menos en la versión de sus "descubridores":

Definición 2. ([1]) Un solitón es una onda solitaria que preserva asintóticamente su forma y su velocidad bajo interacciones no lineales con otras ondas solitarias, o de manera más general, con otra perturbación localizada arbitraria.

Mucho se ha investigado con relación a los solitones desde la época de Kruskal y Zabusky. Por otra parte, se ha diversificado extraordinariamente el campo de aplicación de dichas investigaciones. En las próximas secciones reseñaremos algunas de las aplicaciones más remarcables.

Para finalizar esta breve exposición histórica, resaltaremos dos resultados muy importantes y relacionados entre sí. El primero de ellos fue el descubrimiento de que la ecuación KdV tenía un número infinito de leyes de conservación independientes asociadas, y, por tanto, un número infinito de cantidades conservadas. Sin ánimo de entrar en detalles técnicos, diremos que la importancia de esta presencia de infinitas simetrías fue crucial, por un lado para la comprensión de las propiedades de las soluciones solitónicas y, por otro, para la obtención del segundo de los resultados, el método del scattering inverso.

En el año 1967, C. Gardner, J. Greene, M. Kruskal y R. Miura, en uno de los artículos más citados en la historia de la Matemáticas, [40], introdujeron el Método de la Transformación de Scattering Inverso para resolver la ecuación KdV. En los años siguientes este método cambió la Matemática Aplicada como ningún otro desde la Transformada de Fourier (con el que está intimamente relacionado), y pronto quedó claro que era la llave para la comprensión de las propiedades de las ecuaciones de los solitones.

La idea fundamental del Método del Scattering Inverso fue asociar a la ecuación KdV una ecuación de Schrödinger independiente del tiempo para un proceso de scattering en la que el potencial no era otro que la solución de KdV. Este paso surgió de manera natural en la transformación de Miura (que servía para transformar soluciones de la llamada ecuación KdV modificada en soluciones de KdV, y ulteriormente probar que ambas ecuaciones tenían asociadas un número infinito de leyes de conservación, como ya hemos citado). En definitiva, el método consta de tres pasos:

- 1. Problema directo: Dadas las soluciones a tiempo $t=0$ (condiciones iniciales ${ }^{4}$ para la ecuación KdV), resolver el problema de Scattering asociado,

\footnotetext{
${ }^{4} \mathrm{El}$ Método es efectivo para condiciones iniciales de la forma $u(x, 0)=f(x)$ tales que $f(x)$ tiende a cero lo suficientemente rápido cuando $|x| \rightarrow \infty$, como ocurre en las ecuaciones con
} 
es decir, calcular el espectro de la ecuación de Schrödinger:

$$
v_{x x}+u(x, 0) v=\lambda v
$$

- 2. Evolución temporal: Obtenido el espectro (datos de scattering) a tiempo cero, su evolución temporal viene determinada por la ecuación:

$$
v_{t}=\left(\gamma+u_{x}\right) v-(4 \lambda+2 u) v_{x}
$$

donde $\gamma$ es una constante arbitraria. Es posible, por tanto, obtener los datos de scattering a tiempo $t$.

- 3. Problema Inverso: Reconstruir el potencial de la ecuación de Schrödinger a tiempo $t$ (es decir, las soluciones $u(x, t)$ ) a partir de los datos de scattering a tiempo $t$. El procedimiento es altamente no trivial, requiere la construcción y resolución de una ecuación integral lineal (ecuación de Gel'fand-LevitanMarchenko).

La analogía con el Método de Fourier es clara, los datos de scattering juegan el papel de transformación de Fourier y el problema inverso el de la transformación inversa de Fourier.

Existen multitud de textos y publicaciones donde el método de Scattering Inverso está explicado y detallado desde diferentes puntos de vista, por ejemplo: [77], [33], [1], [28], etc.

\subsubsection{Aplicaciones de la Teoría de Solitones}

Hemos limitado la sección anterior a la que posiblemente es la ecuación de solitones más conocida, la de Korteweg-de Vries, existen sin embargo muchas otras ecuaciones con soluciones solitónicas o de tipo solitónico $u(x, t)$, las más famosas, y las que más relevancia han tenido por su amplio rango de aplicación son sin duda la citada KdV, la ecuación Seno-Gordon (SG) y la ecuación de Schrödinger no lineal (SNL).

Además de la primera aplicación de $\mathrm{KdV}$, ya mencionada en el apartado anterior, en 1960 Gardner y Morikawa [41] "redescubrieron" la ecuación KdV en el estudio de ondas hidromagnéticas. Otras aplicaciones han sido desarrolladas

soluciones solitónicas. 
en otros campos de la Física, como ondas internas estratificadas, ondas iónicoacústicas, física de plasmas, dinámica de redes, etc. Existen, además, otras ecuaciones hidrodinámicas relacionadas en mayor o menor medida con la ecuación $\mathrm{KdV}$, como la ecuación de Benjamin-Ono o la ecuación de ondas largas intermedia. Una ecuación muy relacionada con $\mathrm{KdV}$, y que en cierto modo la generaliza para dos variables independientes espaciales y una temporal, es la conocida ecuación de Kadomtsev-Petviashvili. Por otro lado, muchos son los experimentos y observaciones que se han realizado sobre la presencia de fenómenos solitónicos hidrodinámicos en la naturaleza; podemos citar como más espectaculares los solitones oceánicos, vistos por primera vez en el Mar de Andaman, por Osborne y Burch (para detalles, ver [1] y las referencias allí indicadas).

La ecuación Seno-Gordon apareció por primera vez, a mediados del siglo XIX, en el estudio de las superficies de curvatura Gaussiana constante negativa, en $\mathbb{R}^{3}$, es decir las superficies pseudoesféricas o "pseudoesferas" ${ }^{5}$. Las ecuaciones de Codazzi-Mainardi, para este tipo de superficies, se reducen a la ecuación del Seno-Gordon ${ }^{6}$ :

$$
u_{x t}=\operatorname{sen} u
$$

de tal forma que existe una correspondencia biyectiva entre las clases de isometría de las inmersiones isométricas del plano hiperbólico en $\mathbb{R}^{3}$ y las soluciones de la ecuación $\mathrm{SG}^{7}$. En este contexto, $u$ no es más que el ángulo entre dos direcciones asintóticas $(x, t)$ en la superficie a lo largo de la cual la segunda forma de curvatura es nula, [78].

Esta teoría dió lugar al concepto de transformaciones de Bäcklund (descubiertas por Bianchi), un hecho verdaderamente notable. Sin ánimo de extendernos demasiado (ver por ejemplo [100] para detalles), recordaremos simplemente que dichas

\footnotetext{
${ }^{5}$ Bianchi denominó a esta ecuación "l'equazione fondamentale di tutta la teoria delle superficie pseudosferiche".

${ }^{6}$ La denominación Seno-Gordon (Sine-Gordon en inglés) procede de una aliteración con el nombre de la ecuación de Klein-Gordon, apareció por primera vez en un artículo de Rubinstein, y parece que hay cierta disputa, en cuanto al origen de la broma, entre Finkelstein y Kruskal (ver [87], pág. 35); el "mérito", en cualquier caso, es discutible (Coleman califica este nombre como "tonto" [22], pág. 262).

${ }^{7}$ Siendo rigurosos, es necesario precisar que esta correspondencia biyectiva existe si se relaja la condición de inmersión para admitir singularidades cuspidales a lo largo de curvas. De hecho, y a pesar de que la ecuación SG tiene soluciones definidas globalmente en $\mathbb{R}^{2}$, las superficies correspondientes siempre tienen singularidades. Hilbert demostró que no existe ninguna superficie completa inmersa en $\mathbb{R}^{3}$ con curvatura seccional constante negativa.
} 
transformaciones permiten, dada una solución de la ecuación SG, la creación de una familia biparamétrica de nuevas soluciones. Una pequeña complicación es que ello requiere la resolución de una nueva ecuación diferencial ordinaria. La "fórmula de permutación de Bianchi" permite sin embargo componer fácilmente transformaciones de Bäcklund de manera que una vez se ha encontrado el primer conjunto de nuevas soluciones, la aplicación de una transformación a una de ellas produce una segunda familia que se escribe, explícitamente, en términos de funciones algebraicas de la primera, sin resolver ninguna nueva ecuación diferencial. Se puede así conseguir una serie infinita de familias de soluciones de la ecuación SG cada vez más complicadas. Si se comienza este procedimiento con la solución trivial, idénticamente nula, en el primer paso encontraremos la llamada solución kink de la ecuación SG, cuya familia correspondiente de superficies pseudoesféricas es la familia de Dini (que incluye a la pseudoesfera de Beltrami). Usando la fórmula de Bianchi obtendremos la solución doble-solitón de SG y correspondientemente la famosa superficie de Küen, y así sucesivamente. Como vemos, los geómetras de la segunda mitad del pasado siglo conocían muy bien el sector solitónico del conjunto de soluciones de la ecuación SG. La explicación de porqué no descubrieron realmente los solitones no es difícil de encontrar, por un lado sólo conocían las soluciones solitónicas, y no sabían nada acerca de la existencia de otro sector de soluciones, dispersivas y no-solitónicas; no tenían manera alguna, además, de descubrir la transformación de Scattering Inversa, crucial para comprender completamente el espacio de todas las soluciones. Por otro lado, y quizás la razón más importante, nunca se plantearon la ecuación SG como una ecuación de evolución para una onda unidimensional, con lo cual no percibieron las extrañas propiedades de scattering que sus soluciones tenían.

Sin embargo, el trabajo de estos matemáticos no fue en absoluto en vano, el método de las transformaciones de Bäcklund es transportable a la ecuación de Korteweg-de Vries y, de hecho, todas las ecuaciones de solitones tienen sus transformaciones de Bäcklund asociadas.

Para finalizar con esta presentación de la ecuación SG citaremos únicamente que aparece en muy diversos dominios, como por ejemplo en la modelización de la propagación de dislocaciones en una red cristalina, o en el estudio del efecto túnel Josephson en superconductores (ver [27], pág. 164 y siguientes), o como modelo simplificado de una teoría de partículas elementales que describe bosones y fermiones simultáneamente (ver [22], pág. 262, ó [87], pág. 214) mediante la identificación de la ecuación SG con un sector del Modelo de Thirring masivo. 
La ecuación de Schrödinger no lineal (SNL),

$$
u_{t}=\frac{i}{2}\left(u_{x x}+2|u|^{2} u\right)
$$

tiene también una historia muy interesante. Fue descubierta "disfrazada" a principios de este siglo por el matemático italiano Da Rios, [25], en su tesis doctoral bajo la dirección de Levi-Civita. La ecuación de Da Rios ${ }^{8}$ modelizaba el comportamiento de un filamento vórtice fino en un fluido viscoso de la siguiente forma: si la curva $\vec{\gamma}(x, t)$ representa al filamento en $\mathbb{R}^{3}$, entonces la ecuación que describe su movimiento será: $\vec{\gamma}_{t}=\vec{\gamma}_{x} \times \vec{\gamma}_{x x}$. Usando las ecuaciones de Frenet: $\vec{\gamma}_{x} \times \vec{\gamma}_{x x}=\kappa \vec{B}$. Es decir, la curva evoluciona en el tiempo en la dirección de su vector binormal y a una velocidad igual a su curvatura. En 1971, Hasimoto, [49], encontró una transformación de tipo gauge que transforma la ecuación de Da Rios en la ecuación SNL.

La ecuación SNL ha sido "redescubierta" varias veces más en el presente siglo. Su actualidad, en cualquier caso, radica en su uso como ecuación envolvente: si una onda unidimensional de alta frecuencia y amplitud modulada se mueve en un medio no lineal altamente dispersivo, entonces es una buena aproximación suponer que la onda envolvente, en un sistema de coordenadas moviéndose a la velocidad de grupo, evoluciona de acuerdo con la ecuación SNL. Este hecho significa en la práctica que el estudio de la ecuación SNL tiene una gran importancia en la transmisión de datos por fibra óptica, de hecho se están realizando actualmente serios intentos, tanto teórica como técnicamente, para utilizar las soluciones solitónicas en la transmisión de datos por Internet (ver [79], pág. 383).

Muchas otras ecuaciones con soluciones solitónicas han sido estudiadas y aplicadas en los últimos años; algunas, como la ecuación de Fisher, simultáneamente en áreas tan dispares como la selección genética, las teorías de combustión o la Cinética Química. Desde un punto de vista experimental, cabe resaltar los resultados obtenidos en Óptica, fundamentalmente en la última década, con rayos de luz que exhiben comportamientos solitónicos al propagarse en medios no lineales (ver [93] y referencias allí recogidas).

En otros casos, como en el ejemplo citado de la ecuación SG, las ecuaciones solitónicas aparecen en muy diversas áreas de las propias matemáticas. Citando a C.L. Terng y K. Uhlenbeck: "Soliton theory is an enticingtly elegant part of modern mathematics. It has a multitude of interpretations in geometry, analysis

\footnotetext{
${ }^{8}$ Frecuentemente llamada ecuación del anillo de humo.
} 
and algebra" [99]. Algunas referencias importantes a este respecto son [78], [98], [100] y el libro [99].

Un apartado especial merece la aplicación de los solitones a la Física de la Materia Condensada y a los Defectos de Tipo pared en Cosmología, lo que se ha venido en llamar Teoría de Defectos Topológicos o Defectos en Teorías de Campos. Incluiremos estos resultados en el próximo apartado, no obstante citaremos como principal referencia de esos temas el libro de A. Vilenkin y E. Shellard [104] (ver también el trabajo de Tesis Doctoral de W. García Fuertes [39]).

\subsubsection{Solitones en Teoría de Campos}

Dedicaremos esta sección a presentar la parte de la Teoría de Solitones en la que las aportaciones fundamentales de esta memoria se concentran.

Durante los años 70 fue desarrollada, por un amplio abanico de científicos de la Física Matemática, la técnica de evaluar el impacto que en el espacio de estados de ciertas Teorías Cuánticas de Campos tiene la existencia de soluciones clásicas de las ecuaciones correspondientes. Existen varias referencias que recogen con detalle la génesis y el desarrollo de estas ideas, como por ejemplo: [22], [32], [55] y [87], a las que nos remitimos.

De una forma general, por tanto, se trata de estudiar las soluciones de una Teoría Cuántica de Campos, en una primera aproximación, analizando las ecuaciones desde un punto de vista clásico, es decir, como ecuaciones que gobiernan el comportamiento de configuraciones de campos clásicos y no como operadores cuánticos. La posterior cuantización de dichas soluciones permitirá "volver" a la teoría cuántica original.

En este contexto, T.D. Lee [63] define solitones como "soluciones localizadas espacialmente y no dispersivas de una teoría clásica de campos". Restringiéndonos a teorías clásicas de campos locales y relativistas (de cara a una cuantización ulterior), consideraremos soluciones de las ecuaciones (en la métrica de Minkowski) tales que su energía esté localizada espacialmente y tales que preserven su forma, en definitiva ondas solitarias. En este punto es necesario precisar que en la literatura al respecto se han denominado habitualmente solitones a estas ondas solitarias, aunque no necesariamente presenten fenómenos de superposición no lineal, como veremos. La explicación de esta aparente confusión terminológica es, por otro lado, evidente: los solitones en esta perspectiva son paquetes localizados no dispersivos de energía moviéndose uniformemente, es decir "se parecen" a las partículas elementales reales. Por otro lado, la cuantificación de las soluciones puede entenderse 
como asociar a cada solución clásica un estado cuántico de partícula-solitón fundamental, más una serie de estados excitados relacionados y obtenidos al cuantizar las fluctuaciones alrededor de dicho estado fundamental. Existe toda una técnica sistemática, basada en la expansión semiclásica, para obtener todas las propiedades cuánticas del solitón cuántico a partir de las correspondientes propiedades del solitón clásico.

El carácter no dispersivo de las soluciones que consideramos obliga a que estén presentes en la teoría acoplamientos no lineales, pues en caso contrario todas las soluciones serán ondas planas y, en definitiva, soluciones dispersivas. Es de resaltar entonces que el acoplamiento no lineal puede ser de cualquier magnitud y por tanto no necesariamente fuerte. Este hecho tiene una gran importancia desde el punto de vista cuántico, no olvidemos que esta forma de obtener soluciones de una Teoría Cuántica de Campos es no-perturbativa, de manera que determinados fenómenos, no explicables perturbativamente, son ahora susceptibles de una explicación completa.

En el caso de que se estudien las ecuaciones euclídeas de una teoría clásica de campos, que no trataremos en este trabajo, las soluciones de tipo onda solitaria reciben el nombre de instantones, y son configuraciones clásicas localizadas de acción finita de la versión euclídea de las ecuaciones de cualquier modelo considerado. A nivel clásico, los instantones no son muy diferentes de las soluciones estáticas solitónicas de las ecuaciones independientes del tiempo, de hecho los instantones de un modelo $d$-dimensional son esencialmente los solitones estáticos del mismo modelo $d+1$-dimensional. Su traducción cuántica, sin embargo, es muy diferente, mientras que los solitones conducen, como hemos dicho, a estados extensos de partícula, los instantones son los responsables de efectos túnel entre los diferentes estados de vacío del sistema, que pueden conducir, como en el modelo de Higgs dos dimensional abeliano, a fenómenos de confinamiento de la carga.

La cuantización de los solitones se puede realizar por varios métodos: Existen métodos semiclásicos como los del tipo Wentzel-Kramers-Brillouin (WKB), desarrollados por Dashen, Hasslacher y Neveu [26], métodos basados en el formalismo de integral funcional (Faddeev y Korepin [32]), o métodos variacionales (similares al método de Born-Oppenheimer) debidos a Goldstone y Jackiw [46].

De manera general tendremos que las soluciones clásicas pueden ser de diversos tipos: soluciones constantes (independientes del tiempo y del espacio), soluciones estáticas (dependientes únicamente del espacio), soluciones dependientes del tiempo y del espacio y, por último, las citadas soluciones euclídeas que conducen 
a soluciones de tipo instantón y que no trataremos.

El significado cuántico de las soluciones constantes es claro: se trata de la primera aproximación a los valores esperados en el vacío de los campos cuánticos, y frecuentemente señalan el fenómeno de Goldstone de ruptura espontánea de la simetría. Las soluciones estáticas, y las dependientes del tiempo y del espacio, señalan, por su parte, la presencia de estados de partículas que no aparecen, como ya hemos comentado, desde el punto de vista perturbativo.

\subsubsection{Ondas Solitarias y Defectos de tipo pared}

Tal y como hemos comentado y siguiendo a Rajaraman [87], definiremos el concepto de solitón, en este contexto, utilizando la densidad de energía de la solución, más que la solución misma ${ }^{9}$. De esta manera consideraremos un sistema con $n$ campos $\phi_{1}(\vec{x}, t), \ldots, \phi_{n}(\vec{x}, t)$, con una densidad de energía $\varepsilon(\vec{x}, t)$, función de los campos, y tal que su integral a todo el espacio, $E\left[\phi_{i}\right]$, será la energía total, es decir una cantidad conservada. Dada la arbitrariedad en la definición de la energía podemos, sin pérdida de generalidad, suponer que el valor mínimo que alcanza $E$ es cero. En este contexto, llamaremos localizada a toda configuración de los campos, solución de las ecuaciones del sistema y tales que la densidad de energía a ellos asociada sea una función localizada en el espacio para cualquier tiempo finito $t$, es decir, que sea finita en una región espacial concreta y que tienda a cero en el infinito espacial lo suficientemente deprisa para que su integral converja. Una vez definida la localización en este sentido, podemos definir:

Definición 3. ([87]) Llamaremos solitón a toda solución no singular y localizada de una teoría de campos no lineal, tal que su densidad de energía sea localizada y con una dependencia espacio-temporal de la forma:

$$
\varepsilon(\vec{x}, t)=\varepsilon(\vec{x}-\vec{v} t)
$$

para algún vector velocidad $\vec{v}$.

Dicho con otras palabras, se trata de que la densidad de energía se mueva, sin distorsionarse, con una velocidad constante.

Esta definición sugiere interesantes comentarios, en primer lugar toda solución estática (independiente del tiempo) y localizada, desde este punto de vista, es un solitón; en segundo lugar, y dado que estamos interesados en soluciones de las

\footnotetext{
${ }^{9}$ Rajaraman distingue entre onda solitaria y solitón, de manera análoga a como nosotros lo hemos hecho en las definiciones 1 y 2, si bien desde el punto de vista de la densidad de energía. Nosotros llamaremos de manera genérica solitón a ambos, como es común en la literatura.
} 
ecuaciones en el espacio de Minkowski, es decir, en el estudio de teorías relativistas, tendremos que la invarianza relativista nos conduce a que toda solución estática que verifique la definición 3 puede ser transformada, mediante una transformación de Lorentz, en una dependiente del tiempo y del espacio, y recíprocamente. Concluimos, por tanto, que, a nuestros efectos, nos bastará con estudiar las soluciones independientes del tiempo para analizar la totalidad de las soluciones de carácter solitónico.

La definición anterior engloba a los solitones de cualquier dimensión espaciotemporal. Sin embargo, nos limitaremos en este trabajo a teorías en $1+1$ dimensiones, cuyas soluciones solitónicas denominaremos kinks. Las razones por las cuales nos restringimos al estudio de los kinks son varias y de diferente índole. Tal y como puede verse con detalle en la referencia ya citada [39], los llamados defectos topológicos en teorías de campos, soluciones solitónicas como a las que nos estamos refiriendo, se denominan kinks en el caso $1+1$ dimensional, vórtices en el $2+1$ y monopolos en el $3+1$. El estudio de los kinks es relevante por varios motivos, por un lado existe una larga tradición en Física de utilizar modelos $1+1$ dimensionales, que permiten un cálculo matemático simplificado frente a dimensiones mayores, sin que se pierdan por ello todos los aspectos significativos de un modelo concreto; en definitiva, se obtiene información de un caso simplificado, que permitirá después "saltar" a modelos más realistas en dimensiones mayores. No hay que olvidar, por otra parte, que el conocido teorema de Derrick, [87] y [63], no permite la existencia de soluciones solitónicas estáticas en teorías de campos escalares de dimensiones espaciales iguales o superiores a dos. Para esos casos, por tanto, es necesario considerar campos más complejos, no sólo campos escalares ${ }^{10}$. Se obtienen soluciones solitónicas con $D>1$ considerando, por ejemplo, campos de espín no nulo o soluciones dependientes del tiempo aunque no dispersivas. El primero de estos casos nos lleva a solitones gauge topológicos ${ }^{11}$ (como los vórtices de Nielsen-Olesen [76] en dos dimensiones espaciales o los monopolos magnéticos de t'Hooft [97] y Polyakov [83] en dimensión espacial 3). El segundo de los casos conduce a solitones no-topológicos multidimensionales (ver [63] y [38]).

Existe otra razón que motiva el estudio específico de las teorías en $1+1$ dimensiones y no es otro que la abundancia de problemas que son modelizables

\footnotetext{
${ }^{10}$ El Teorema de Derrick establece que para $D>1$ ( $D$ es la dimensión espacial) las únicas soluciones independientes del tiempo de energía finita son los estados fundamentales, es decir $\phi_{a}(\vec{x}, t)=$ Cte., siendo $\phi_{a}$ un cero (y mínimo) de la densidad de energía potencial considerada en el modelo (ver [63] para la demostración y detalles).

${ }^{11}$ La distinción entre solitones topológicos y no-topológicos se hará en la próxima sección 1.2.
} 
realmente en este contexto. Así por ejemplo, en Física de la materia condensada, problemas tales como las cadenas de polímeros lineales, o cristales tridimensionales pero fuertemente anisótropos de tal manera que los electrones se mueven predominantemente sobre uno de los ejes cristalinos, o las uniones Josephson en superconductores, ya citadas anteriormente al referirnos a las aplicaciones de la ecuación SG, son algunos ejemplos de problemas físicos estudiables en $1+1$ dimensiones. En algunos de estos casos se han obtenido resultados que podríamos calificar de espectaculares, como por ejemplo la cadena lineal del poliacetileno, donde se ha observado un proceso de fraccionización de la carga eléctrica, algo que Jackiw y Rebbi habían encontrado de manera teórica al tratar con un modelo de interacción fermión-solitón sobre la recta, donde aparecían números fermiónicos semienteros, ver [56].

Otro ejemplo remarcable y que ha generado mucha literatura en los últimos años son las paredes de dominio o "domain walls" en Cosmología. En este caso, se estudian defectos topológicos en $3+1$ dimensiones pero tales que son triviales en dos de las variables espaciales, es decir, se reduce el problema real a $1+1$ dimensiones. Las soluciones kink estáticas, unidimensionales, lógicamente son en realidad "muros" tridimensionales (espacialmente) al considerar todos los valores posibles de las dos variables trivializadas. Aparecen incluso fenómenos de defectos dentro de defectos (por ejemplo "domain ribbons" dentro de "domain walls", [72]), al calcularse, para los modelos en los que es posible, kinks en otra variable, es decir, kinks dentro de kinks. De las muchas referencias existentes en este campo, destacaremos las de Vilenkin, [103] y [104].

\subsection{El problema de la búsqueda de Kinks como un Sistema Dinámico}

Siguiendo la notación y las definiciones adoptadas en el apartado 1.1.4, consideraremos una teoría relativista de campos escalares en $1+1$ dimensiones determinada por la acción

$$
S=\int_{-\infty}^{+\infty} d x^{0} \int_{-\infty}^{+\infty} d x^{1}\left\{\frac{1}{2} \partial_{\mu} \vec{\phi} \cdot \partial^{\mu} \vec{\phi}-V(\vec{\phi})\right\}
$$


donde $\mu=0,1$, se utiliza el convenio de sumación de índices repetidos de Einstein, la métrica de Minkowski $1+1$ dimensional se ha tomado de la forma:

$$
g_{\mu \nu}=\left(\begin{array}{cc}
1 & 0 \\
0 & -1
\end{array}\right)
$$

y $\vec{\phi} \equiv\left(\phi_{1}, \ldots, \phi_{N}\right)$ es un campo vectorial de $N$ componentes escalares, es decir una aplicación del espacio tiempo $\mathbb{R}^{1,1}$ de Minkowski en la representación irreducible fundamental de $O(N), \mathbb{R}^{N}$. En este "espacio interno" $\mathbb{R}^{N}$ de la teoría consideramos el producto escalar euclídeo estándar.

Sin pérdida de generalidad podemos considerar que el potencial $V(\vec{\phi})$ es semidefinido positivo, de tal forma que se anule en sus valores mínimos.

La energía derivada de la acción (1.6) es:

$$
E=\int_{-\infty}^{+\infty} d x^{1}\left\{\frac{1}{2} \frac{\partial \vec{\phi}}{\partial x^{0}} \cdot \frac{\partial \vec{\phi}}{\partial x^{0}}+\frac{1}{2} \frac{\partial \vec{\phi}}{\partial x^{1}} \cdot \frac{\partial \vec{\phi}}{\partial x^{1}}+V(\vec{\phi})\right\}
$$

de manera que la condición de energía finita se traduce en unas condiciones asintóticas concretas: las configuraciones solitónicas tienen que ser tales que tiendan a uno de los ceros de $V(\vec{\phi})$ cuando $\left|x^{1}\right| \rightarrow \infty$.

Consideraremos en general la existencia de $n$ mínimos (y por tanto ceros) no degenerados de la función $V(\vec{\phi})$, de manera que el conjunto de todos ellos lo denominamos:

$$
\mathcal{V}=\left\{\vec{v}_{1}, \vec{v}_{2}, \ldots, \vec{v}_{n}\right\}
$$

variedad de vacío del modelo. El significado cuántico de las configuraciones $\vec{v}_{i}$ es muy claro y justifica el nombre de variedad de vacío. $\vec{v}_{i}$ son las soluciones constantes de la teoría clásica con energía finita, y se corresponden por tanto con los valores esperados del operador de campo cuántico sobre los estados fundamentales o de vacío en la teoría cuántica correspondiente.

Tal y como se explicó en la sección anterior, dada la invarianza relativista, nos basta con buscar las soluciones estáticas para obtener todos los kinks, puesto que un boost aplicado a un kink estático nos proporciona directamente la correspondiente solución solitónica de velocidad arbitraria.

Para las configuraciones estáticas $\phi_{a}\left(x^{0}, x^{1}\right)=q_{a}\left(x^{1}\right)$, el funcional de energía se reduce a:

$$
E[\vec{q}]=\int_{-\infty}^{+\infty} d x^{1}\left\{\frac{1}{2} \frac{d \vec{q}}{d x^{1}} \cdot \frac{d \vec{q}}{d x^{1}}+V(\vec{q})\right\}
$$


donde $\vec{q}=\left(q_{1}, q_{2}, \ldots, q_{N}\right)$. El espacio de configuración de la teoría de campos es, en definitiva, el espacio de funciones $\vec{q}: \mathbb{R} \rightarrow \mathbb{R}^{N}$, tales que $E[\vec{q}]<\infty$ :

$$
\mathcal{C}=\left\{\vec{q}\left(x^{1}\right) / E[\vec{q}]<\infty\right\}
$$

y, por tanto, las condiciones asintóticas:

$$
\lim _{x^{1} \rightarrow \pm \infty} \frac{d \vec{q}}{d x^{1}}=\overrightarrow{0} ; \quad \lim _{x^{1} \rightarrow \pm \infty} \vec{q}=\vec{v}_{i}
$$

para algún vector $\vec{v}_{i}=\left(v_{i}^{1}, \ldots, v_{i}^{N}\right)$ de $\mathcal{V}$, deben ser satisfechas. El estudio de la estructura de $\mathcal{C}$ en diversos modelos de interés físico será uno de los objetivos que se abordarán en este trabajo.

La presencia de más de un elemento en $\mathcal{V}$, esto es, la degeneración del estado fundamental de la teoría cuántica, conduce a la identificación dentro de $\mathcal{C}$ de diversos sectores, diferenciados por los vacíos que se tomen en las condiciones (1.9) cuando $x^{1} \rightarrow-\infty$ y cuando $x^{1} \rightarrow+\infty$. Dos configuraciones correspondientes a valores asintóticos diferentes están separadas, además, por una barrera infinita de potencial, de modo que hay que descartar que la evolución temporal, ya sea clásica o cuántica, transforme la una en la otra (ver [39] y referencias allí recogidas para detalles). $\mathcal{C}$ se separa, de esta forma, en sectores topológicos determinados por las aplicaciones de $\partial \mathbb{R}=\{-\infty,+\infty\}$ en $\mathcal{V}$. Dado que $\operatorname{card}(\mathcal{V})=n$, tendremos $2^{n}$ sectores topológicos disconexos:

$$
\mathcal{C}=\bigsqcup_{i, j=1}^{n} \mathcal{C}^{(i, j)}
$$

donde los superíndices denotan el vacío alcanzado en $-\infty$ y en $+\infty$ respectivamente.

Es usual en la literatura asociar a cada campo una "carga topológica", dependiente de los puntos de vacío alcanzados en $x^{1} \rightarrow-\infty$ y en $x^{1} \rightarrow+\infty$, de la forma:

$$
Q_{a}^{T}=\frac{1}{2}\left(\hat{q}_{a}(-\infty)-\hat{q}_{a}(+\infty)\right), \quad \forall a=1, \ldots, N
$$

donde $\hat{q}_{a}=q_{a} /\left|v^{a}\right|$ y siendo $v^{a}$ la componente $a$-ésima del vacío correspondiente alcanzado $\vec{v}$. Tenemos, de esta forma, en un modelo con $N$ campos, un vector de cargas topológicas $\vec{Q}^{T}(\vec{q})$, que toma idéntico valor para configuraciones en un mismo sector de $\mathcal{C}$. Llamaremos solitones topológicos, desde esta perspectiva, a aquéllos pertenecientes a sectores con carga topológica diferente de cero (del vector nulo), mientras que serán no-topológicos los que "salen" (en $-\infty)$ y "llegan" (en $+\infty)$ al mismo punto de vacío, y por tanto tienen carga nula. 
Lógicamente, para que existan los solitones topológicos es necesaria la condición impuesta de que el vacío del modelo esté degenerado, para los no-topológicos esta restricción no es obligada.

Las ecuaciones de Euler-Lagrange de la acción (1.6) son:

$$
\square \phi_{a}=-\frac{\partial V}{\partial \phi_{a}}
$$

que son verificadas trivialmente por las configuraciones constantes citadas

$$
\vec{\phi}_{0}\left(x^{0}, x^{1}\right)=\vec{v}_{i}, \quad i=1, \ldots, n ; \quad \vec{v}_{i} \in \mathcal{V}
$$

dado que los puntos de vacío $v_{i}$ son los mínimos del potencial.

Las pequeñas deformaciones en torno a la solución estable $\vec{\phi}_{0}$ que siguen siendo soluciones de las ecuaciones (1.11) se corresponderán con los cuantos fundamentales de la teoría cuántica. La función

$$
\vec{\Phi}_{0}^{\vec{v}}\left(\vec{\kappa} ; x^{0}, x^{1}\right)=\vec{v}+\sum_{\kappa} \vec{a}(\kappa) e^{i \vec{\kappa} \cdot \vec{x}} \quad \vec{\kappa}=\left(\kappa^{0}, \kappa^{1}\right), \vec{x}=\left(x^{0}, x^{1}\right)
$$

es una solución en ondas planas de (1.11) si se verifica la relación de dispersión:

$$
\delta_{a b}\left(\kappa^{0}\right)^{2}=\delta_{a b}\left(\kappa^{1}\right)^{2}+M_{a b}^{2}(\vec{v}), \quad M_{a b}^{2}(\vec{v})=\frac{\partial^{2} V}{\partial \phi_{a} \partial \phi_{b}}(\vec{v})
$$

$M_{a b}^{2}(\vec{v})$ es de esta manera la matriz de masas en el punto crítico escogido. Dado que los puntos $\vec{v}$ que consideramos son todos mínimos de $V$, no tendremos autovalores negativos de $M_{a b}^{2}(\vec{v})$ y así las soluciones de pequeñas deformaciones (1.12) alrededor del mínimo constante $\vec{v}$ dependen del tiempo de la forma $e^{i \kappa^{0} x^{0}}\left(\right.$ con $\left.\kappa^{0} \in \mathbb{R}\right)$, es decir, no divergen con el tiempo.

Las ecuaciones (1.11), para las configuraciones estáticas, $\vec{\phi}=\vec{q}$, se reducen a la forma:

$$
\frac{d^{2} q_{a}}{d\left(x^{1}\right)^{2}}=\frac{\partial V}{\partial q_{a}}
$$

que pueden reinterpretarse, mediante lo que llamaremos Método de la Analogía Mecánica, como las ecuaciones de Newton para un sistema dinámico en Mecánica Clásica donde $U=-V$ es el potencial al que se ve sometida una partícula en el espacio $\mathbb{R}^{N}$, cuya posición a "tiempo" $t=x^{1}$ está determinada por el vector $\vec{q}(t)=\left(q_{1}(t), \ldots, q_{N}(t)\right)$.

$$
\frac{d^{2} q_{a}}{d t^{2}}=-\frac{\partial U}{\partial q_{a}}
$$


La acción de dicho sistema mecánico será:

$$
J[\vec{q}]=\int_{-\infty}^{+\infty} d t\left\{\frac{1}{2} \frac{d \vec{q}}{d t} \cdot \frac{d \vec{q}}{d t}-U(\vec{q})\right\}=\int_{-\infty}^{+\infty} d t\left\{\frac{1}{2} \frac{d \vec{q}}{d t} \cdot \frac{d \vec{q}}{d t}+V(\vec{q})\right\}=E[\vec{q}]
$$

es decir, coincide exactamente con la energía total de la configuración estática en la teoría clásica de campos.

Las condiciones asintóticas (1.9) se trasladan al sistema dinámico y así las trayectorias de acción $J$ finita están en correspondencia biunívoca con los kinks, de energía $E=J$. Como veremos en los próximos capítulos dichas condiciones determinarán que las trayectorias del sistema que corresponden a soluciones kink de la teoría de campos son exactamente las soluciones separatrices entre el movimiento acotado y el no acotado del sistema.

Se puede concluir, por tanto, con que la búsqueda de kinks en un modelo de este tipo se traduce, mediante la analogía mecánica, en la resolución de un Sistema Dinámico. Dedicaremos la sección siguiente a recordar conceptos fundamentales de la Teoría de Sistemas Dinámicos de cara a abordar, en las siguientes, la integración del sistema mecánico asociado al Modelo Sigma Lineal Deformado, objeto principal de este trabajo.

\subsection{Sistemas Dinámicos Integrables y Separables Hamilton-Jacobi}

Una vez establecida la necesidad de estudiar sistemas dinámicos clásicos de cara a encontrar las soluciones de tipo onda estacionaria o kink de las teorías de campos bajo consideración en este trabajo, presentaremos ahora una breve exposición de los principios, definiciones y propiedades fundamentales de los primeros, con el único objetivo de fijar la notación y los métodos principales que van a ser utilizados a lo largo de la memoria. No pretendemos en ningún momento ser exhaustivos, ni detallaremos en ningún caso las demostraciones de los teoremas expuestos. Posteriormente, en el Capítulo 4, desarrollaremos algunos de los aspectos que aquí citamos y nos extenderemos en el análisis de los aspectos variacionales de la Mecánica Analítica en variedades riemannianas, con el objetivo final de estudiar la estabilidad de las soluciones de un sistema dinámico.

La exposición que presentaremos a continuación está basada fundamentalmente en el soporte bibliográfico proporcionado por el texto de V.I. Arnold [9] y las referencias complementarias [12], [61], [64] y [80]. 
Plantearemos esta sección utilizando una notación rigurosa desde el punto de vista geométrico, pero sin olvidar que trataremos en este trabajo con sistemas mecánicos clásicos estándar; no profundizaremos, de esta manera, en las muchas generalizaciones matemáticas que estos conceptos admiten y que están muy tratadas en la literatura.

\subsubsection{Sistemas Integrables}

Comenzaremos este resumen de los conceptos fundamentales acerca de los Sistemas Dinámicos desde un punto de vista Lagrangiano. Un sistema dinámico estará determinado, por tanto, por un funcional de acción:

$$
S[\sigma]=\int_{t_{0}}^{t_{1}} \mathcal{L}(\vec{q}(t), \dot{\vec{q}}(t)) d t
$$

donde $\sigma:\left[t_{0}, t_{1}\right] \rightarrow M$ es una curva diferenciable en una variedad riemanniana $M$, $(\vec{q}, \dot{\vec{q}})=\left(q_{1}, \ldots, q_{n} ; \dot{q}_{1}, \ldots, \dot{q}_{n}\right)$ son un sistema de coordenadas canónico ${ }^{12}$ en $T M$, fibrado tangente de la variedad $M$, y $\mathcal{L}$, lagrangiana del sistema, es la función real en $T M^{13}$ :

$$
\mathcal{L}(\vec{q}, \dot{\vec{q}})=T-U=\frac{1}{2} \sum_{i, j}^{n} g_{i j} \dot{q}_{i} \dot{q}_{j}-U(\vec{q})
$$

siendo $g_{i j}$ la métrica riemanniana de $M$ y $U(\vec{q})=U(\pi(\vec{q}, \dot{\vec{q}}))$ una función real en $M=\pi(T M)$, energía potencial del sistema y característica del mismo.

Dada la curva citada $\sigma:\left[t_{0}, t_{1}\right] \rightarrow M$, su vector velocidad vendrá determinado por $\sigma^{\prime}:\left[t_{0}, t_{1}\right] \rightarrow T M$, y así, si llamamos $x_{i}(t)=x_{i}(\sigma(t))$ a las componentes de $\sigma$ y $q_{i}(t)=q_{i}\left(\sigma^{\prime}(t)\right)=x_{i}(t)$, tendremos que $\dot{q}_{i}(t)=\dot{q}_{i}\left(\sigma^{\prime}(t)\right)=d x_{i}\left(\sigma^{\prime}(t)\right)=\frac{d x_{i}(t)}{d t}=$ $\frac{d q_{i}(t)}{d t}$. De esta manera:

$$
S[\sigma]=\int_{t_{0}}^{t_{1}}\left(\frac{1}{2} \sum_{i, j=1}^{n} g_{i j}(\vec{x}(t)) \frac{d x_{i}}{d t} \frac{d x_{j}}{d t}-U(\vec{x}(t))\right) d t
$$

El principio de acción de Hamilton nos dice que las trayectorias solución del sistema se corresponden con aquéllas que hacen extremal el funcional de acción,

\footnotetext{
${ }^{12} \mathrm{Se}$ llama sistema de coordenadas canónico al construido de la siguiente forma: Sea $\left(x_{1}, \ldots, x_{n}\right)$ un sistema de coordenadas locales en un abierto $V$ de $M$, entonces el sistema de coordenadas canónico asociado a él en $\pi^{-1}(V) \subset T M,(\vec{q}, \dot{\vec{q}})=\left(q_{1}, \ldots, q_{n} ; \dot{q}_{1}, \ldots, \dot{q}_{n}\right)$ se define: $q_{i}=x_{i} \circ \pi$ y $\dot{q}_{i}=d x_{i}$.

${ }^{13}$ Nos restringiremos, como ya hemos dicho, a funciones lagrangianas estándar, es decir de la forma $\mathcal{L}=T-U$ siendo $T$ la energía cinética (forma cuadrática no degenerada) y $U$ la energía potencial, llamadas frecuentemente en la literatura lagrangianas naturales.
} 
es fácil comprobar que la condición $\delta S=0$ conduce a las ecuaciones de EulerLagrange (ver Apéndice D):

$$
\frac{\partial \mathcal{L}}{\partial q_{i}}-\frac{d}{d t} \frac{\partial \mathcal{L}}{\partial \dot{q}_{i}}=0, \quad \forall i=1, \ldots, n
$$

Si sustituimos en las ecuaciones de Euler-Lagrange (1.18) la expresión del lagrangiano (1.16) ó (1.17), y recordamos la definición de los símbolos de Christoffel asociados a la métrica riemanniana $g,(1.18)$ se escribirán ${ }^{14}$ :

$$
\frac{d^{2} x_{i}}{d t^{2}}=-\Gamma_{j k}^{i}(x) \frac{d x_{j}}{d t} \frac{d x_{k}}{d t}-g^{i k} \frac{\partial U}{\partial x_{k}}
$$

que no son más que las ecuaciones de Newton del sistema generalizadas para una métrica riemanniana cualquiera ${ }^{15}$. El gradiente de la función energía potencial, cambiado de signo, es así la fuerza generalizada. Evidentemente, si consideramos el caso de energía potencial nula, las ecuaciones (1.19) se reducen a las ecuaciones de las geodésicas sobre la variedad $M$.

No es difícil demostrar, por otro lado, que si se define la energía total o función hamiltoniana del sistema como la función real en $T M: H=2 T-\mathcal{L}=T+U$, entonces $H$ es constante sobre las trayectorias solución del sistema, es decir, es una constante del movimiento.

La presencia de más integrales primeras del sistema, que nos sirvan para simplificar la resolución de las ecuaciones (1.18), está a menudo asociada con la existencia de simetrías en el sistema.

Llamaremos simetría del sistema, desde el punto de vista lagrangiano que estamos tratando, a todo difeomorfismo $\psi$ de $M$ que deje invariante a la función lagrangiana $\mathcal{L}^{16}$. Lógicamente, $\mathcal{L}$ es una función en $T M$, de manera que $\psi$ actúa sobre $\mathcal{L}$ por medio del difeomorfismo inducido $\psi_{*}$ de $\psi$ en $T M$, así $\psi$ es una simetría del sistema si $\mathcal{L}\left(\psi_{*}(\mathbf{v})\right)=\mathcal{L}(\mathbf{v}), \forall \mathbf{v} \in T M$. Un campo de vectores $X$ se denomina simetría infinitesimal del sistema si genera un grupo uniparamétrico de simetrías.

Sea $X$ una simetría infinitesimal y sea $\psi_{s}$ el grupo uniparamétrico de difeomorfismos de $M$ por él generado, asociamos entonces a $X$ una función $\tilde{X}$ en $T M$, que llamaremos su función momento conjugado, de la forma $\tilde{X}(\mathbf{v})=\left\langle\mathbf{v}, X_{\pi(\mathbf{v})}\right\rangle$ (donde

\footnotetext{
${ }^{14}$ En el Capítulo 4 y en el Apéndice C están detalladas estas ideas.

${ }^{15}$ En la expresión (1.19) se ha tomado el convenio de Einstein de suma sobre índices repetidos. $g^{i j}$ son las componentes de $g^{-1}$.

${ }^{16}$ Para lagrangianas naturales, las simetrías del sistema no serán más que las isometrías de $M$ que preserven la energía potencial $U$.
} 
el paréntesis denota el producto escalar en $T M$ y $\pi$ es la proyección natural de $T M$ en $M)$. Tenemos entonces el conocido Teorema de E. Noether:

Teorema de Noether. El momento conjugado de una simetría infinitesimal es una constante del movimiento.

Es decir, la función $\tilde{X}$ es constante sobre las trayectorias solución. En las coordenadas canónicas antes citadas tendremos:

$$
\tilde{X}(\vec{q}, \dot{\vec{q}})=\left.\frac{\partial \mathcal{L}}{\partial \dot{\vec{q}}} \frac{d \psi_{s}(\vec{q})}{d s}\right|_{s=0}
$$

Los momentos conjugados a los campos $\frac{\partial}{\partial q_{i}}$ serán denotados por $P_{i}=\sum_{j=1}^{n} g_{i j} \dot{q}_{j}$, que para los lagrangianos naturales, a los que nos estamos restringiendo, serán: $P_{i}=\frac{\partial \mathcal{L}}{\partial \dot{q}_{i}}$, los momentos generalizados. De hecho, $\left(q_{1}, \ldots, q_{n} ; P_{1}, \ldots, P_{n}\right)$ es un sistema de coordenadas locales en $T M$. Es posible escribir así las ecuaciones de Euler-Lagrange (1.18), o las equivalentes ecuaciones de Newton (1.19), en función de estas coordenadas:

$$
\frac{d q_{i}}{d t}=\dot{q}_{i} ; \quad \frac{d P_{i}}{d t}=\frac{\partial \mathcal{L}}{\partial q_{i}}
$$

Por otro lado, y teniendo en cuenta que la función hamiltoniana se puede escribir $H=\sum_{i} P_{i} \dot{q}_{i}-\mathcal{L}$, tras un breve cálculo se obtienen las ecuaciones, equivalentes a las anteriores:

$$
\frac{d q_{i}}{d t}=\frac{\partial H}{\partial P_{i}} ; \quad \frac{d P_{i}}{d t}=-\frac{\partial H}{\partial q_{i}}
$$

ecuaciones de Hamilton del sistema considerado ${ }^{17}$.

Desde un punto de vista general, un sistema mecánico como el que hemos descrito se dice integrable si las ecuaciones que determinan su dinámica, (1.18), (1.19) ó (1.20) son resolubles, es decir son integrables por cuadraturas. Sin embargo, esta definición aparentemente sencilla no lo es tanto a la hora de llevarla a la práctica. Sin ánimo de extendernos demasiado en las diferentes definiciones de integrabilidad que pueden darse, recordaremos simplemente dos frases oportunas sobre este concepto, una de ellas debida a Birkhoff [12]: "When, however, one attempts to formulate a precise definition of integrability, many possibilities appear, each with a certain intrinsic theoretic interest", y una segunda, citada en [12], de Poincaré: "a system of differential equations is only more or less integrable".

\footnotetext{
${ }^{17}$ Nótese que estas ecuaciones de Hamilton están deducidas sin abandonar el punto de vista lagrangiano. A pesar de que los abusos de notación son habituales en estos contextos, y de que nosotros hemos incurrido en ellos, hemos denotado a los momentos con mayúsculas, reservando las minúsculas para el formalismo hamiltoniano propiamente dicho.
} 
Utilizaremos en este trabajo el concepto de integrabilidad de un sistema dinámico asociándolo al Teorema de Arnold-Liouville que expondremos más tarde.

Plantearemos a continuación el Formalismo Hamiltoniano, es decir, el estudio de la dinámica del sistema en el fibrado cotangente (espacio de fases) de la variedad $M$ (espacio de configuración).

El mecanismo de paso del Formalismo Lagrangiano al Hamiltoniano es la Transformación de Legendre. Para los lagrangianos naturales, a los que nos hemos restringido, esta transformación es un isomorfismo de fibrados, de manera que ambas formulaciones son absolutamente equivalentes. No nos extenderemos, por tanto, en detalles sobre las transformaciones de Legendre, pertinentes cuando uno estudia el caso general ${ }^{18}$.

En el fibrado cotangente $T^{*} M$, tenemos definida de manera natural una forma simpléctica $\omega$ (dos-forma cerrada, no degenerada e independiente de la métrica que pueda estar definida en $M$ ).

De manera análoga al fibrado tangente, en $T^{*} M$ podemos dar unas coordenadas locales naturales $\left(q_{1}, \ldots, q_{n} ; p_{1}, \ldots, p_{n}\right)$, de tal forma que la transformación de Legendre $L: T M \rightarrow T^{*} M$ se comporta de la forma $q_{i} \circ L=q_{i}, p_{i} \circ L=P_{i}$, siendo $P_{i}$ el momento generalizado antes definido.

Con el abuso de notación habitual en la literatura, llamaremos de nuevo función lagrangiana $\mathcal{L}$ y función hamiltoniana $H$ a las transformadas de las definidas en el formalismo lagrangiano: $\mathcal{L}=\mathcal{L} \circ L^{-1}, H=H \circ L^{-1}$.

La forma simpléctica citada anteriormente viene dada, en las coordenadas naturales $(\vec{q}, \vec{p})$, por la expresión

$$
\omega=\sum_{i=1}^{n} d p_{i} \wedge d q_{i}
$$

de hecho se denominan coordenadas canónicas a cualesquiera coordenadas locales $\left(q_{1}, \ldots, q_{n} ; p_{1}, \ldots, p_{n}\right)$ del fibrado $T^{*} M$ en las que $\omega$ se escriba de esa forma. En el caso que nos ocupa son las coordenadas naturales, pero su existencia está garantizada, localmente, en toda variedad simpléctica general $(\Omega, \omega)$, por el conocido Teorema de Darboux. Por este motivo las coordenadas canónicas se llaman a menudo coordenadas de Darboux.

La estructura simpléctica nos permite definir, asociado a cada función diferenciable $f$ en $T^{*} M$ (o en $\Omega$ para el caso general), un campo $X_{f}$ en $T^{*} M$, que

\footnotetext{
${ }^{18}$ Para los lagrangianos singulares la transformación de Legendre no es un isomorfismo y ambas formulaciones no son equivalentes.
} 
llamaremos campo hamiltoniano, por medio de la contracción interior con $\omega$, de la forma: $i X \omega=d f$. La función $f$ se llama función hamiltoniana de $X_{f}$.

Toda variedad simpléctica tiene una estructura asociada de variedad de Poisson. El paréntesis de Poisson asociado a $\omega$ se define de la siguiente forma: $\forall f, g$ funciones diferenciables en $T^{*} M$, se define

$$
\{f, g\}=\omega\left(X_{f}, X_{g}\right)
$$

Es trivial comprobar entonces que en un sistema de coordenadas canónico, el paréntesis de Poisson tiene la siguiente expresión:

$$
\{f, g\}=\sum_{i=1}^{n}\left(\frac{\partial f}{\partial p_{i}} \frac{\partial g}{\partial q_{i}}-\frac{\partial f}{\partial q_{i}} \frac{\partial g}{\partial p_{i}}\right)
$$

Es necesario comentar, aun siendo reiterativos, que estamos restringiendo nuestro análisis al caso de lagrangianas naturales e independientes del tiempo. Es posible generalizar totalmente este análisis desde varios puntos de vista. En particular, es habitual en la literatura (ver por ejemplo [80]) plantear un sistema dinámico simplemente como una variedad de Poisson con una función hamiltoniana (energía) determinada ${ }^{19}$.

Las ecuaciones de Hamilton, ahora ya en las coordenadas naturales canónicas $(\vec{q}, \vec{p})$, se escriben en la forma:

$$
\frac{d q_{i}}{d t}=\frac{\partial H}{\partial p_{i}}=\left\{H, q_{i}\right\} ; \quad \frac{d p_{i}}{d t}=-\frac{\partial H}{\partial q_{i}}=\left\{H, p_{i}\right\}
$$

En general, por tanto, si $f$ es una función diferenciable en $T^{*} M$, tendremos:

$$
\dot{f}=\{H, f\}
$$

de manera que toda función $f$ tal que $\{H, f\}=0$ es una constante del movimiento, una integral primera del sistema.

Existe un análogo evidente al teorema de Noether en versión hamiltoniana, donde se construyen los invariantes por medio de la aplicación momento. Puede verse con detalle en muchas de referencias, resaltaremos [19] y [80].

Independientemente del origen de las integrales primeras, es fácil demostrar que el paréntesis de Poisson de dos de ellas es otra integral primera, de tal manera que las integrales primeras de un sistema dinámico, con el paréntesis de Poisson,

\footnotetext{
${ }^{19}$ La estructura de variedad de Poisson es más general que la de variedad simpléctica, toda variedad simpléctica es de Poisson, pero el recíproco no es cierto.
} 
constituyen un álgebra de Lie. Se dice, por otro lado, que dos integrales primeras están en involución si su paréntesis de Poisson es nulo. Como ya hemos comentado anteriormente, la existencia de integrales primeras en un sistema está vinculada a la integrabilidad del mismo, en el sentido ya citado de reducir la integración de las ecuaciones a la evaluación de cuadraturas, es decir, integrales indefinidas de funciones conocidas. El Teorema siguiente, formulado inicialmente por Jacobi en 1842, demostrado independientemente por Bour y Liouville en 1855, y reformulado por Arnold en los años cincuenta, establece con precisión esta vinculación ${ }^{20}$ :

Teorema de Arnold-Liouville. ([80]) Sea $T^{*} M \approx \mathbb{R}^{2 n} \equiv(\vec{q}, \vec{p})$ el espacio de fases de un sistema hamiltoniano con el paréntesis de Poisson estándar y hamiltoniano $H(\vec{p}, \vec{q}, t)$. Supongamos que el sistema tiene $n$ integrales primeras en involución $F_{1}, \ldots, F_{n}$, es decir:

$$
\frac{\partial F_{j}}{\partial t}+\left\{F_{j}, H\right\}=0, \quad\left\{F_{j}, F_{k}\right\}=0
$$

Si las funciones $F_{1}, \ldots, F_{n}$ son funcionalmente independientes en el conjunto:

$$
M_{\vec{a}}=\left\{(\vec{p}, \vec{q}, t) \in \mathbb{R}^{2 n} \times \mathbb{R}: F_{j}(\vec{p}, \vec{q}, t)=a_{j}, \quad j=1, \ldots, n\right\}
$$

entonces las soluciones de las ecuaciones de Hamilton:

$$
\dot{p}_{j}=-\frac{\partial H}{\partial q_{j}}, \quad \dot{q}_{j}=\frac{\partial H}{\partial p_{j}}
$$

pertenecientes a $M_{\vec{a}}$ pueden obtenerse por cuadraturas.

Evidentemente, para los sistemas que no dependen explícitamente del tiempo, como los que estamos considerando, la condición (1.22) se reduce a que:

$$
\left\{F_{j}, H\right\}=\left\{F_{j}, F_{k}\right\}=0, \quad \forall j, k=1, \ldots, n
$$

Existen varias generalizaciones de este teorema (en [80] pueden encontrarse seis), para una exposición detallada, así como las demostraciones correspondientes nos remitiremos al texto de Arnold [9]. Citaremos la primera generalización, a variedades cualesquiera (no necesariamente tales que $T^{*} M \approx \mathbb{R}^{2 n}$ globalmente):

Teorema de Arnold-Liouville II. Sean $F_{1}, \ldots, F_{n}$ funciones diferenciables en involución en una variedad simpléctica $2 n$ dimensional $\Omega=T^{*} M$ :

$$
\left\{F_{i}, F_{j}\right\}=0, \quad i, j=1, \ldots, n
$$

Si se cumple:

\footnotetext{
${ }^{20}$ Es habitual denominar a este teorema como Teorema de Arnold-Liouville, a pesar de los demás autores citados.
} 
- 1. $F_{1}, \ldots, F_{n}$ son independientes en cada $M_{\vec{a}}$ (definida en (1.23)), es decir sus diferenciales son linealmente independientes en cada punto de $M_{\vec{a}}$.

- 2. Los campos hamiltonianos $X_{j}=X_{F_{j}}$ son completos en $M_{\vec{a}}$.

entonces se verifica:

- a) $M_{\vec{a}}$ es una variedad diferenciable invariante bajo el flujo de fase inducido por las funciones hamiltonianas $H=F_{j}, \forall j=1, \ldots, n$.

- b) cada componente conexa de $M_{\vec{a}}$ es difeomorfa al producto de un toro $k$ dimensional y un espacio vectorial euclídeo $(n-k)$-dimensional: $M_{\vec{a}} \approx T^{k} \times$ $\mathbb{R}^{n-k}$. Si $M_{\vec{a}}$ es compacta, entonces: $M_{\vec{a}} \approx T^{n}$.

- c) existe una elección de coordenadas $\varphi_{1}, \ldots, \varphi_{k}, y_{1}, \ldots, y_{n-k}$ en $M_{\vec{a}}$ tal que las ecuaciones de Hamilton para la función hamiltoniana $F_{j}$ toman la forma:

$$
\dot{\varphi}_{m}=\omega_{m j}, \quad \dot{y}_{s}=c_{s j} \quad(\omega=\omega(\vec{a}), \quad c=\text { cte. })
$$

- d) $S i M_{\vec{a}}$ es compacta, entonces existe un entorno de $M_{\vec{a}}$ en $\Omega$ que es difeomorfo simplécticamente a varias copias de $T^{n} \times D^{n}$, siendo $D^{n}$ la bola $n$ dimensional en $\mathbb{R}^{n}$ y siendo la estructura simpléctica de $T^{n} \times D^{n}$ la del entorno de $T^{n} \times\{0\}$ en el fibrado cotangente $T^{*} T^{n}=T^{n} \times \mathbb{R}^{n}$.

Es habitual en la literatura denominar sistemas completamente integrables bien a los que verifican las hipótesis de este teorema, bien a los que verifican únicamente la primera de ellas (la segunda es difícil de establecer en muchos sistemas). Nos quedaremos con la segunda opción.

La propiedad c) establecida en el teorema II nos permite introducir las variables de acción-ángulo. En el caso $M_{\vec{a}}$ compacto, las variables $F_{j}, \varphi_{k}, j, k=1, \ldots n$, forman un sistema de coordenadas en $T^{*} M$, no necesariamente canónico, pero no es difícil demostrar que existen $n$ variables $I_{1}, \ldots, I_{n}$, dependientes únicamente de las funciones $F_{j}$, y tales que la forma simpléctica, en coordenadas $I_{1}, \ldots, I_{n}, \varphi_{1}, \ldots, \varphi_{n}$, se escribe:

$$
\omega=\sum_{i=1}^{n} d I_{i} \wedge d \varphi_{i}
$$

es decir, coordenadas canónicas en $T^{*} M$. Fijando así las constantes de movimiento $F_{j}$, y por tanto, las $I_{j}$, se determina el toro invariante que describe la dinámica, las variables $\varphi_{j}$ determinarán la posición de un punto en dicho toro y variarán linealmente con el tiempo (ver [9] para los detalles). 


\subsubsection{El Método de Hamilton-Jacobi}

Una vez establecido con claridad el concepto de Sistema Completamente Integrable, dos son los problemas fundamentales que permanecen: el primero, obtener el número necesario de integrales primeras (si es que existe) para saber si el sistema es completamente integrable y el segundo, y en general nada fácil, integrar realmente el sistema de ecuaciones resultante. Tal y como afirma Arnold [9], de todos los métodos existentes para integrar las ecuaciones diferenciales de la Dinámica el más efectivo sin duda es el llamado Método de Hamilton-Jacobi, desarrollado por Jacobi para integrar las ecuaciones de Hamilton, de ahí su nombre. La técnica de Hamilton-Jacobi consiste en calcular funciones generadoras de transformaciones canónicas, conceptos que definiremos a continuación, y en definitiva, se reduce a integrar una ecuación en derivadas parciales, la ecuación de Hamilton-Jacobi. Una vez más, no dispondremos de un método general para resolver esta ecuación. La técnica más efectiva para hacerlo, y la que mayores éxitos ha conseguido en la historia de la Mecánica, es el método de separación de variables. Este método requiere encontrar un sistema de coordenadas en el cual la ecuación de Hamilton-Jacobi sea separable, lo cual en principio no es trivial en absoluto. No conviene olvidar, sin embargo, una célebre frase de Jacobi ([54], recogida en [9] y [61]): "La principal dificultad para integrar una ecuación diferencial dada estriba en la introducción de variables adecuadas, para cuyo hallazgo no existen reglas. Por lo tanto, debemos recorrer el camino inverso y después de encontrar alguna sustitución evidente, buscar problemas para los cuales se puede aplicar satisfactoriamente" ${ }^{21}$.

Por otra parte, un teorema de Jacobi asegura que si un sistema es completamente separable en unas determinadas variables, entonces una solución general (dependiente por tanto de $n$-parámetros independientes) de la ecuación de Hamilton-Jacobi se encuentra por cuadraturas, pero también entonces el sistema correspondiente de ecuaciones canónicas podrá ser integrado por cuadraturas.

Otro teorema, éste de Stäckel, nos relacionará la separabilidad de la ecuación de Hamilton-Jacobi con la existencia de un determinado tipo de integrales primeras, como veremos también en esta sección.

La idea del método de Hamilton-Jacobi consiste en lo siguiente: bajo transformaciones canónicas de coordenadas en el espacio de fases (cambios de coordenadas que preservan la forma simpléctica, es decir, cambios de coordenadas canónicas a

\footnotetext{
${ }^{21}$ No está de más precisar, con respecto a esta frase, que Jacobi llamaba sustitución "evidente" a situaciones del estilo al hallazgo de las coordenadas elípticas, que veremos en un apartado posterior.
} 
nuevas coordenadas canónicas) se conservan las ecuaciones de Hamilton, como se puede probar fácilmente. De esta manera, si se consigue encontrar una transformación canónica que convierta la función hamiltoniana en una función tal que sea posible integrar las ecuaciones de Hamilton, entonces las ecuaciones originales también serán integrables.

Sin ánimo de extendernos demasiado, y remitiéndonos de nuevo al texto de Arnold [9], diremos que este proceso, es decir, la obtención de la transformación canónica correcta para estos fines, requiere integrar la ecuación:

$$
H\left(\frac{\partial S(\vec{Q}, \vec{q})}{\partial \vec{q}}, \vec{q}, t\right)=K(\vec{Q})
$$

caso especial de la llamada ecuación de Hamilton-Jacobi:

$$
\frac{\partial S}{\partial t}+H\left(\frac{\partial S}{\partial \vec{q}}, \vec{q}, t\right)=0
$$

donde se está considerando el cambio de coordenadas canónicas $(\vec{q}, \vec{p})$ a nuevas coordenadas canónicas $(\vec{Q}, \vec{P})$ de tal forma que la función hamiltoniana $H(\vec{p}, \vec{q})$ se convierta en $K(\vec{Q})$. S es la función generadora de dicha transformación canónica, es decir una función dependiente de $(\vec{Q})$ y de $(\vec{q})$ y tal que verifica:

$$
\frac{\partial S(\vec{Q}, \vec{q})}{\partial \vec{q}}=\vec{p}, \quad \frac{\partial S(\vec{Q}, \vec{q})}{\partial \vec{Q}}=\vec{P}
$$

Las ecuaciones canónicas, en variables $(\vec{Q}, \vec{P})$ tomarán la forma:

$$
\dot{Q}_{i}=0, \quad \dot{P}_{i}=-\frac{\partial K}{\partial Q_{i}}
$$

de tal manera que las nuevas coordenadas $Q_{i}$ serán constantes sobre las soluciones del sistema.

Con todos estos ingredientes, podemos plantear el teorema:

Teorema de Jacobi. ([9]) Si se encuentra una solución general (integral completa) $S(\vec{Q}, \vec{q})$ de la ecuación de Hamilton-Jacobi (1.24), dependiente de $n$ parámetros arbitrarios $Q_{i}$, y tal que 22

$$
\operatorname{Det}\left(\frac{\partial^{2} S}{\partial \vec{Q} \partial \vec{q}}\right) \neq 0
$$

\footnotetext{
${ }^{22}$ Esta condición hace que la transformación canónica sea "libre", ver [9].
} 
entonces las ecuaciones canónicas del sistema:

$$
\dot{q}_{i}=\frac{\partial H}{\partial p_{i}}, \quad \dot{p}_{i}=-\frac{\partial H}{\partial q_{i}}
$$

se pueden resolver explícitamente por cuadraturas. Las funciones $\vec{Q} \equiv Q_{i}(\vec{q}, \vec{p})$ determinadas por las ecuaciones

$$
\frac{\partial S(\vec{Q}, \vec{q})}{\partial \vec{q}}=\vec{p}
$$

son integrales primeras de las ecuaciones (1.27).

Un comentario pertinente a este respecto es el de Arnold: "El Teorema de Jacobi reduce la solución de un sistema de ecuaciones diferenciales ordinarias (1.27) al hallazgo de una integral completa de la ecuación en derivadas parciales (1.24). Puede parecer sorprendente que esta 'reducción' desde la simple a la complicada nos proporcione un método efectivo para resolver problemas concretos. Sin embargo, el resultado es que éste es el método más valioso conocido para la integración exacta, y muchos problemas que fueron resueltos por Jacobi no pudieron serlo por otros métodos".

El proceso de separación de variables para la resolución de la ecuación de Hamilton-Jacobi o directamente de las ecuaciones de movimiento ha sido muy investigado, fundamentalmente en el siglo pasado y en los principios del presente. Un análisis muy completo de los casos más importantes puede verse en el texto ya citado de Perelomov [80]. Resaltaremos aquí el caso de los sistemas llamados de Stäckel, así como los teoremas relacionados con ellos, puesto que nos serán de utilidad en los capítulos próximos.

Llamaremos sistema de Stäckel a todo sistema dinámico cuyo hamiltoniano se escriba de la forma (en un adecuado sistema de coordenadas):

$$
H=\sum_{i=1}^{n} a_{i}\left(q_{1}, \ldots, q_{n}\right)\left[\frac{1}{2} p_{i}^{2}+U_{i}\left(q_{i}\right)\right]
$$

Para este tipo de sistemas Stäckel (ver [80] y referencias allí recogidas) demostró el siguiente teorema:

Teorema de Stäckel. Un sistema con hamiltoniano de la forma (1.28) admite separación de variables en su ecuación de Hamilton-Jacobi si y sólo si existe una matriz $B, n \times n$, cuyos elementos $b_{i j}$ dependan únicamente de la coordenada $q_{j}, y$ tales que:

$$
\operatorname{Det} B \neq 0, \quad \sum_{k=1}^{n} b_{j k}\left(q_{k}\right) a_{k}\left(q_{1}, \ldots, q_{n}\right)=\delta_{j 1}
$$


Si se denota por $A \equiv\left(a_{j k}\right)$ a la matriz inversa de $B$, entonces se tiene que los coeficientes $a_{i}$ del hamiltoniano (1.28) serán

$$
a_{i}=a_{i 1}
$$

Un segundo resultado que puede deducirse para los sistemas de Stäckel es que las funciones:

$$
I_{j}=\sum_{i=1}^{n} a_{i j}\left[\frac{1}{2} p_{i}^{2}+U_{i}\left(q_{i}\right)\right], \quad I_{1}=H
$$

forman un conjunto de integrales primeras en involución para el sistema (1.28). Finalmente, el teorema de Stäckel puede reformularse en una versión más fuerte (debida a L.A. Pars, ver [80]):

Teorema de Stäckel II. Para un sistema dinámico con hamiltoniano (1.28), los siguientes enunciados son equivalentes:

- 1) La ecuación de Hamilton-Jacobi es separable.

- 2) Existe una matriz invertible $B$, cuyos elementos $b_{i j}$ dependen únicamente de la coordenada $q_{j}$ y que satisface las ecuaciones (1.29).

- 3) Existen $n$ integrales primeras funcionalmente independientes y en involución, cuadráticas en los momentos y con la forma (1.30).

Como vemos, los sistemas que verifican el teorema de Stäckel tienen asociadas $n$ integrales primeras en involución y además ortogonales (se dice que son ortogonales en el caso en que no contienen términos cruzados en los momentos, es decir términos de la forma $a_{i j} p_{i} p_{j}, i \neq j$ ).

Como ya hemos dicho, un análisis detallado de diversos sistemas separables y algunos resultados generales sobre los mismos puede verse en [80], en particular, se estudian los conocidos sistemas de Liouville, que no son más que un caso particular de los sistemas de Stäckel.

\subsubsection{Simetrías ocultas y Deformaciones Integrables}

En el primer apartado de esta sección recordábamos el teorema de Noether, que nos permite calcular constantes de movimiento de un sistema dinámico cuando conocemos una simetría de dicho sistema. Como resultado directo se obtenía que las 
cantidades conservadas (o invariantes Noether), para funciones lagrangianas naturales, eran funciones lineales y homogéneas de las velocidades $\dot{\vec{q}}$. Una exposición detallada de este hecho puede verse en [85].

Tenemos, por tanto, desde el punto de vista Hamiltoniano, que a cada grupo de transformaciones que conservan la acción Hamiltoniana le corresponde una integral primera de las ecuaciones de movimiento. El enunciado recíproco es también cierto en el siguiente sentido ([61]): El flujo de fase de un sistema Hamiltoniano con una integral primera adicional conocida tomada como Hamiltoniano transforma soluciones de las ecuaciones de movimiento originales en soluciones de las mismas ecuaciones.

Se suele utilizar en la literatura el nombre de simetrías ocultas o generalizadas para denominar la situación antedicha, es decir, la existencia de integrales primeras de un sistema dinámico no explicables por medio de la aplicación del Teorema de Noether a simetrías obvias del mismo. Muchos y variados son los ejemplos de este tipo de situaciones, como el problema de Kepler, las geodésicas de un elipsoide o el caso ya citado de las ecuaciones que dan origen a soluciones solitónicas (KdV, $\mathrm{SG}$, etc), donde aparecen infinitas cantidades conservadas independientes (ver [9], apéndice 13 y referencias allí recogidas).

En algunos casos, y particularmente en el sistema dinámico asociado al Modelo Sigma $O(N)$ Lineal Deformado que estudiamos en este trabajo, la presencia de integrales primeras que no son invariantes Noether correspondientes a la acción de ningún grupo está asociado precisamente al hecho de tratarse de deformaciones de sistemas que sí que admiten dichas simetrías Noether. De esta forma se parte de un sistema completamente integrable gracias a la presencia del suficiente número de integrales primeras en involución, debidas a una simetría del sistema bajo la acción de un grupo de Lie (más la integral obvia de la energía) y se llega a un nuevo sistema, también completamente integrable, donde las integrales primeras han sufrido a su vez la deformación, de tal manera que se corresponden en el modelo deformado con simetrías ocultas. En el caso del sistema citado, que analizaremos más adelante, la deformación del modelo considerada rompe una invarianza bajo la acción del grupo $O(N)$, el nuevo grupo de simetría es discreto, no tiene por tanto invariantes Noether asociados y, sin embargo, el sistema continúa siendo completamente integrable. Llamaremos a este tipo de modificaciones Deformaciones Integrables. 
CAPÍTULO 1 


\section{Capítulo 2}

\section{De las Ondas Solitarias en el Modelo Sigma $O(N)$ Lineal Deformado}

Una vez introducidos, en el Capítulo primero, los principales temas que se estudian en este trabajo, dedicaremos el Capítulo segundo a presentar el que hemos denominado Modelo Sigma $O(N)$ Lineal Deformado y a calcular explícitamente todas las soluciones de tipo kink del mismo.

En una primera sección recopilaremos brevemente las características principales del Modelo Sigma Lineal, así como sus aplicaciones. Especificaremos también las posibles deformaciones que de dicho modelo pueden realizarse al mismo tiempo que analizaremos la necesariedad e interés de las mismas desde diferentes puntos de vista.

En la Sección 2.2 se planteará el sistema mecánico análogo al modelo y sus propiedades. El sistema dinámico resulta ser completamente integrable en el sentido del Teorema de Arnold-Liouville comentado en el Capítulo precedente. De hecho se trata de un sistema ya tratado en la literatura matemática, si bien desde un punto de vista diferente al que aquí nos ocupa, por R. Garnier en 1919 [42].

Tras una sección dedicada al estudio de las coordenadas elípticas, resolveremos finalmente la ecuación de Hamilton-Jacobi del sistema, que resulta ser una ecuación separable en las coordenadas citadas. Demostraremos además, usando razonamientos dinámicos, que las ondas solitarias de este modelo están restringidas a una determinada región en el espacio interno $\mathbb{R}^{N}$ del sistema, una elipse y su interior para el caso $N=2$ (como era ya conocido en la literatura desde los años ochenta, si bien no estaba demostrado), un elipsoide y su interior para $N=3 \mathrm{y}$ 
en general un hiperelipsoide y su interior para $N \geq 3$.

En el Capítulo 6 abordaremos otra resolución de este sistema dinámico a partir de las ecuaciones de primer orden que para el mismo se derivan de su carácter presupersimétrico. Estudiaremos allí la equivalencia, para los sistemas de este tipo, entre ambos planteamientos.

\subsection{Modelo Sigma $O(N)$ Lineal y sus deforma- ciones}

Los orígenes del Modelo Sigma $O(N)$ Lineal se remontan a principios de los años sesenta, cuando Gell-Mann y Lèvy estudiaron la fenomenología hadrónica de baja energía introduciendo una teoría Lagrangiana efectiva de este tipo (ver [43] para detalles). El modelo viene dado por el funcional de acción:

$$
S_{\sigma}[\vec{\chi}]=\int d^{2} x\left\{\frac{1}{2} \partial_{\mu} \vec{\chi} \cdot \partial^{\mu} \vec{\chi}-\frac{\lambda^{2}}{4}\left(\vec{\chi} \cdot \vec{\chi}-\alpha^{2}\right)^{2}\right\}
$$

(donde $\vec{\chi} \equiv\left(\chi_{1}, \ldots, \chi_{N}\right)$ es un campo vectorial que representa a $N$ campos escalares en $1+1$ dimensiones, $\lambda^{2}$ y $\alpha^{2}$ son constantes) y describe sistemas en Teoría Cuántica de Campos con ruptura espontánea de simetría y bosones de Goldstone en el espectro de partículas.

Originariamente las deformaciones del modelo fueron usadas para tratar a los mesones ligeros como pseudo-partículas de Goldstone. Además de convertirse en una parte central del álgebra de corrientes, el Modelo Sigma Lineal pasó a tener una crucial importancia en la Física fundamental como el sector de Higgs para las teorías gauge de las partículas elementales (una exposición detallada de estos aspectos puede verse en [102]). Como ejemplos, podemos citar el modelo sigma $O(4)$, en su versión lineal, que corresponde al sector de Higgs de la teoría electrodébil, o el caso $O(24) \times O(5)$, que proporciona el sector bosónico de la teoría de gran unificación $S U(5)$ mínima. Hoy en día estas teorías se consideran también como límites de baja energía de teorías de cuerdas fundamentales. En esta línea, consideraremos también en esta memoria deformaciones del Modelo Sigma $O(N)$ Lineal similares a las propuestas en [11]; se trata de modelos tales que su renormalización falla (en dimensiones físicas como $2+1$ o $3+1$, no así en $1+1$ dimensiones) pero que describen correctamente los aspectos de baja energía.

Estudiaremos en este trabajo las soluciones de tipo solitónico o kinks de una 
deformación natural del Modelo Sigma Lineal de $N$ campos

$$
S[\chi]=\int d^{2} x\left\{\frac{1}{2} \partial_{\mu} \vec{\chi} \cdot \partial^{\mu} \vec{\chi}-\frac{\lambda^{2}}{4}\left(\vec{\chi} \cdot \vec{\chi}-\alpha^{2}\right)^{2}-\sum_{a=1}^{N} \frac{\beta_{a}^{2}}{4} \chi_{a}^{2}\right\}
$$

donde $\beta_{a}^{2}$ son constantes y se ha supuesto el Convenio de Einstein de suma en los índices griegos.

Los motivos que nos llevan a considerar una deformación y la razón de que tomemos específicamente ésta son varios. Por un lado la estructura de solitones que encontraremos es muy rica, con solitones topológicos y no-topológicos. Desde el punto de vista de la aplicación a la Cosmología, ya citada en el Capítulo anterior, los solitones de esta teoría ("domain walls" desde esta perspectiva), al tener estructura interna, permitirán la existencia de defectos dentro de defectos, situación similar a la propuesta por Morris en [72].

Por otro lado, el teorema de Coleman prohíbe la existencia de bosones de Goldstone en teorías en $1+1$ dimensiones debido a la presencia de divergencias infrarrojas (ver [23]). De esta forma, no es posible una cuantización correcta del Modelo Sigma Lineal en 1+1 dimensiones pues la ruptura espontánea de la simetría de un grupo continuo $(O(N))$ produciría tales bosones de Goldstone. Se hace necesaria, por tanto, una deformación que conduzca a que en lugar de una ruptura espontánea de la simetría de $O(N)$ por una variedad de vacío degenerada $S^{N-1}$, aparezcan términos de masa que rompan explícitamente la simetría $O(N)$ a una discreta. Se trata, en definitiva, de considerar en el modelo original la deformación que se generaría, en cualquier caso, al introducir las correcciones cuánticas. La deformación que trataremos principalmente en este trabajo será, desde este punto de vista, la más sencilla posible y romperá la simetría $O(N)$ explícitamente a un grupo discreto, $\mathbb{Z}_{2}^{\otimes N}$. Ello permitirá la existencia de un vacío degenerado, con ruptura espontánea de la simetría al tomar uno de los puntos de vacío posibles, pero sin la presencia de bosones de Goldstone.

La historia de este modelo, o de la versión deformada del Modelo Sigma $O(N)$ Lineal, se remonta a los trabajos de C. Montonen [71] y S. Sarker, S.E. Trulliger y A.R. Bishop [91] sobre el modelo para dos únicos campos escalares, que es llamado por ese motivo modelo MSTB en la literatura. Los autores citados propusieron el modelo en el contexto de la búsqueda de solitones no topológicos con estabilidad garantizada por una simetría interna $(U(1))$. R. Rajaraman, [86], utilizando la técnica de las órbitas de prueba, encontró algunas soluciones solitónicas para este modelo. Posteriormente E. Magyari y H. Thomas demostraron, en 1984 [66], que 
el sistema dinámico asociado vía la analogía mecánica al modelo MSTB era un sistema hamiltoniano completamente integrable, apuntando además que este modelo podía verse como una aproximación continua clásica a un cristal unidimensional con un parámetro de orden de dos componentes.

H. Ito, en 1985 [52], demostró que el sistema dinámico no sólo era completamente integrable sino además separable Hamilton-Jacobi, y calculó explícitamente la totalidad de las soluciones del problema mecánico y, por tanto, todas las configuraciones solitónicas de la teoría. La resolución de Ito aclaraba completamente, por otra parte, la peculiar regla de suma de las energías de los diferentes tipos de kinks, que constituía hasta entonces un hecho casi empírico.

El mismo Ito y H. Tasaki analizaron la estabilidad de estas soluciones mediante el uso de una generalización del Teorema del Índice de Morse [53]. Finalmente J. Mateos Guilarte, [67] y [68], estudió completamente estos aspectos utilizando Teoría de Morse à la Bott, tras identificar la topología del espacio de configuración como la del espacio de caminos en la esfera $S^{2}$.

En la sección 3.1 del próximo capítulo describiremos el cálculo de los kinks de este modelo y sus principales propiedades.

En el artículo [4] A. Alonso Izquierdo, M.A. González León y J. Mateos Guilarte estudiamos dos sistemas muy similares al modelo MSTB, uno de ellos consistente en una deformación de éste, mientras que el segundo trata con una energía potencial del tipo Chern-Simons-Higgs, que surge en las teorías gauge planas autoduales. En la referencia [3] se extiende dicho estudio a toda una infinidad de modelos con dos campos escalares reales, al realizarse un estudio general de los sistemas dinámicos bidimensionales completamente integrables asociados.

Trataremos en este trabajo, como ya hemos citado, el modelo análogo al MSTB para el caso de $N$ campos escalares. Una primera presentación de los resultados obtenidos puede verse en [5] y en [7].

A la hora de generalizar a $N$ campos la deformación del Modelo Sigma Lineal que presenta el modelo MSTB surgen diferentes opciones, según los diferentes valores que puedan tomar las constantes $\beta_{a}$ introducidas en (2.2). Un punto de vista clarificador es el de considerar todas las posibilidades dentro de una familia de modelos caracterizados por una densidad de energía potencial:

$$
V=\frac{\lambda^{2}}{4}\left(\sum_{a=1}^{N} \epsilon_{a} \chi_{a}^{2}-\alpha^{2}\right)^{2}+\sum_{a=1}^{N} \frac{\beta_{a}^{2}}{4} \chi_{a}^{2}+\sum_{a=1}^{N} \sum_{b=1}^{N} \frac{\gamma_{a b}^{2}}{8} \chi_{a}^{2} \chi_{b}^{2}
$$

donde $\epsilon_{a}, \beta_{a}^{2}$ y $\gamma_{a b}^{2}$ son los parámetros de la familia. Las divergencias ultravioletas en la teoría cuántica asociada están controladas por el orden normal del producto 
de operadores, pero surge la necesidad de introducir un punto de renormalización $\mu^{2}$, y la dependencia de los parámetros renormalizados en $\mu^{2}$ estará determinada por la ecuación del grupo de renormalización.

Una solución especial, con un flujo del grupo de renormalización específico, podría llevar al "punto"

$$
\epsilon_{a}^{R}\left(\mu^{2}\right)=1, \quad \beta_{a}^{R}\left(\mu^{2}\right)=0, \quad \gamma_{a b}^{R}\left(\mu^{2}\right)=0, \quad \forall a, b=1, \ldots, N
$$

en el espacio de teorías cuánticas de campos asociadas a la familia. Este punto es, lógicamente, el Modelo Sigma $O(N)$ Lineal, con su correspondiente grupo de simetría $G=O(N)$. La órbita de vacío, como ya hemos dicho, será $S^{N-1}$, y aparecen, por tanto, $N-1$ partículas sin masa, bosones de Goldstone, como corresponde a una situación con grupo continuo de simetría.

Otro punto distinguido ocurre en el caso:

$$
\epsilon_{a}^{R}\left(\mu^{2}\right)=1, \quad \gamma_{a b}^{R}\left(\mu^{2}\right)=0, \quad \forall a, b=1, \ldots, N
$$

pero $\beta_{1}^{R}\left(\mu^{2}\right)=\cdots=\beta_{r}^{R}\left(\mu^{2}\right)=0$ y $\beta_{r+1}^{R}\left(\mu^{2}\right)<\cdots<\beta_{N}^{R}\left(\mu^{2}\right)$. La órbita de vacío será ahora $S^{r-1}$ y tendremos, en consecuencia, $r-1$ bosones de Goldstone.

Como ya se ha citado anteriormente, el Teorema de Coleman impide la existencia de bosones de Goldstone en teorías 1+1 dimensionales. El comportamiento asintótico infrarrojo de las funciones de Green de dos puntos de un campo escalar cuántico prohíbe los polos en $\kappa^{2}=0$. De esta manera, no es posible buscar el Modelo Sigma Lineal, o la deformación que acabamos de explicar (con grupo $O(r)$ espontáneamente rota a $O(r-1)$ ), en el flujo del grupo de renormalización. Los puntos permitidos más cercanos a éstos serán los de la forma:

$$
\begin{gathered}
\epsilon_{a}^{R}\left(\mu^{2}\right)=1, \quad \gamma_{a b}^{R}\left(\mu^{2}\right)=0, \quad \forall a, b=1, \ldots, N \\
\beta_{1}^{R}\left(\mu^{2}\right)=0<\beta_{2}^{R}\left(\mu^{2}\right)=\beta_{2} \leq \cdots \leq \beta_{N}^{R}\left(\mu^{2}\right)=\beta_{N}
\end{gathered}
$$

Nos centraremos en el caso de máxima simetría explícitamente rota, es decir, cuando tenemos las desigualdades estrictas y, por tanto, nuestro modelo a tratar será el de la ecuación (2.2) con constantes de deformación que producen máxima anisotropía en los términos armónicos:

$$
\beta_{1}=0<\beta_{2}<\cdots<\beta_{N}
$$

Los demás casos permitidos corresponden a situaciones del tipo:

$$
\beta_{1}=0<\beta_{2}=\cdots=\beta_{r_{1}}<\beta_{r_{1}+1}=\cdots=\beta_{r_{2}}<\cdots<\beta_{r_{k}+1}=\cdots=\beta_{N}
$$


que presentan un espectro de partículas degenerado pero no bosones de Goldstone. Los generadores de subgrupo de simetría $O\left(r_{1}-1\right) \times O\left(r_{2}-r_{1}\right) \times \cdots \times O\left(N-r_{k}\right)$ estarán todos en el grupo pequeño del vacío. El grupo de simetría es $G=\mathbb{Z}_{2} \times$ $O\left(r_{1}-1\right) \times O\left(r_{2}-r_{1}\right) \times \cdots \times O\left(N-r_{k}\right)$, el grupo pequeño o grupo de simetría remanente tras escoger uno de los vacíos es $H=O\left(r_{1}-1\right) \times O\left(r_{2}-r_{1}\right) \times \cdots \times$ $O\left(N-r_{k}\right)$, y, en definitiva, la variedad de vacío $\mathcal{V}$ puede entenderse como la órbita de uno de sus elementos por la acción del grupo: $\mathcal{V}=G / H=\mathbb{Z}_{2}$.

Existe una gran analogía entre los razonamientos expuestos y el teorema $c$ de Zamolodchikov [112]: las deformaciones en el espacio de teorías de campos $(1+1)$ dimensionales que conducen de sistemas conformes a sistemas integrables son las más interesantes. En este caso la situación es análoga pero finito-dimensional, el papel del grupo conforme lo representa el grupo $O(N)$ y la integrabilidad de sistemas con infinitos grados de libertad es sustituida por la integrabilidad de un sistema mecánico $N$-dimensional.

Trataremos a continuación, como ya hemos citado, la deformación maximalmente asimétrica. Por otro lado, analizaremos más adelante las consecuencias que tiene sobre la estructura de la variedad de soluciones kink los diferentes valores relativos que puedan tomar las constantes del modelo, avanzaremos simplemente que el caso más rico se corresponde con el rango $\beta_{a}^{2}<\alpha^{2} \lambda^{2}, \forall a=1, \ldots, N$, al que nos restringiremos por ahora.

\subsection{Modelo Sigma Lineal Deformado y búsqueda de Kinks}

Tal y como hemos comentado en la sección anterior, estudiaremos una teoría de campos $(1+1)$-dimensional con $N$ campos escalares reales, que representaremos agrupados en un campo vectorial $N$-dimensional $\vec{\chi} \equiv\left(\chi_{1}, \chi_{2}, \ldots, \chi_{N}\right)$, cuya dinámica viene determinada por el funcional de acción ${ }^{1}$ :

$$
S[\vec{\chi}]=\int d^{2} y\left\{\frac{1}{2} \partial_{\mu} \vec{\chi} \cdot \partial^{\mu} \vec{\chi}-\bar{V}\left(\chi_{1}, \ldots, \chi_{N}\right)\right\}
$$

donde $\mu=0,1$, se está considerando la métrica $g_{\mu \nu}$ del espacio de Minkowski $1+1$ dimensional en la forma

$$
g_{\mu \nu}=\left(\begin{array}{rr}
1 & 0 \\
0 & -1
\end{array}\right)
$$

\footnotetext{
${ }^{1}$ Como ya se ha especificado, se utiliza el Convenio de Einstein de suma en índices repetidos únicamente en los índices denotados con letras griegas.
} 
y el producto escalar es el euclídeo estándar en $\mathbb{R}^{N}$.

La densidad de energía potencial de la deformación del Modelo Sigma Lineal que consideramos es:

$$
\bar{V}\left(\chi_{1}, \ldots, \chi_{N}\right)=\frac{\lambda^{2}}{4}\left(\vec{\chi} \cdot \vec{\chi}-\frac{m^{2}}{\lambda^{2}}\right)^{2}+\sum_{a=1}^{N} \frac{\beta_{a}^{2}}{4} \chi_{a}^{2}
$$

Como ya hemos comentado, nos restringiremos al caso en el que los parámetros verifican $\beta_{a} \neq \beta_{b}, \forall a \neq b$, y dentro del rango determinado por las inecuaciones ${ }^{2}$ : $\beta_{a}^{2}<m^{2}$.

Introduciendo variables sin dimensiones, de cara a obtener expresiones más sencillas para la acción ${ }^{3}: \vec{\chi} \rightarrow \frac{m}{\lambda} \vec{\Phi} ; y_{\mu} \rightarrow \frac{\sqrt{2}}{m} x_{\mu} ; \frac{\beta_{a}^{2}}{m^{2}} \rightarrow \sigma_{a}^{2}$, tendremos:

$$
\begin{gathered}
S[\vec{\Phi}]=\frac{m^{2}}{\lambda^{2}} \int_{-\infty}^{+\infty} d x^{0} \int_{-\infty}^{+\infty} d x^{1}\left\{\frac{1}{2} \partial_{\mu} \vec{\Phi} \cdot \partial^{\mu} \vec{\Phi}-V\left(\phi_{1}, \ldots, \phi_{N}\right)\right\} \\
V\left(\phi_{1}, \ldots, \phi_{N}\right)=\frac{1}{2}(\vec{\Phi} \cdot \vec{\Phi}-1)^{2}+\frac{1}{2} \sum_{a=1}^{N} \sigma_{a}^{2} \phi_{a}^{2}
\end{gathered}
$$

mientras que la condición (2.4), junto con los rangos de parámetros tomados, se traducen en la elección

$$
\sigma_{1}=0<\sigma_{2}<\cdots<\sigma_{N}<1
$$

de las constantes de la deformación.

La búsqueda de ondas solitarias o kinks de la teoría de campos nos lleva a centrar nuestra atención en las configuraciones estáticas, es decir: $\vec{\Phi}\left(x^{0}, x^{1}\right)=$ $\vec{q}\left(x^{1}\right)$. Para ellas, el funcional de energía se reduce a la expresión:

$$
E[\vec{q}]=\frac{m^{3}}{\sqrt{2} \lambda^{2}} \int_{-\infty}^{+\infty} d x^{1}\left\{\frac{1}{2} \frac{d \vec{q}}{d x^{1}} \cdot \frac{d \vec{q}}{d x^{1}}+V\left(q_{1}, \ldots, q_{N}\right)\right\}
$$

El espacio de configuración que consideraremos es por tanto el conjunto de aplicaciones $\vec{q}: \mathbb{R} \rightarrow \mathbb{R}^{N}$ tales que la energía $E[\vec{q}]$ sea finita:

$$
\mathcal{C}=\left\{\vec{q}\left(x^{1}\right) / E[\vec{q}]<+\infty\right\}
$$

\footnotetext{
${ }^{2}$ Nótese que se ha escogido la constante $\alpha^{2}$ de la forma: $\alpha^{2}=\frac{m^{2}}{\lambda^{2}}$, así $\beta_{a}^{2}<\alpha^{2} \lambda^{2}$ equivale a $\beta_{a}^{2}<m^{2}$.

${ }^{3}$ Es necesario precisar, en este punto, que en la expresión (2.5) se han utilizado unidades naturales.
} 
de tal forma que pertenecerán a $\mathcal{C}$ aquellas configuraciones $\vec{q}$ continuas que satisfagan las condiciones asintóticas

$$
\lim _{x^{1} \rightarrow \pm \infty} \frac{d \vec{q}}{d x^{1}}=\overrightarrow{0}, \quad \lim _{x^{1} \rightarrow \pm \infty} \vec{q}\left(x^{1}\right)=\vec{v}
$$

donde $\vec{v}$ es un vector constante de $\mathcal{V}$, la variedad de vacío del modelo, es decir, los mínimos, y simultáneamente ceros, de la densidad de energía potencial tomada. Obviamente, para este caso, será un conjunto de dos elementos:

$$
\mathcal{V}=\left\{\vec{v}^{ \pm}=( \pm 1,0, \ldots, 0)\right\}
$$

y, por tanto, la variedad de vacío, a pesar de estar degenerada, es un conjunto discreto.

Las simetrías del sistema serán, además de la invarianza natural bajo la acción del grupo de Poincaré bidimensional, una invarianza o simetría interna bajo la acción del grupo discreto $G=\mathbb{Z}_{2} \times \cdots \times \mathbb{Z}_{2}$, generado por $q_{a} \rightarrow(-1)^{\delta_{a b}} q_{a}, \forall b=$ $1, \ldots, N$. La variedad de vacío puede verse así como la órbita de un elemento bajo la acción del grupo:

$$
\mathcal{V}=G / H_{\vec{v}^{ \pm}}=\mathbb{Z}_{2}
$$

donde $H_{\vec{v}^{ \pm}}$es el grupo pequeño o grupo de isotropía del vacío $\vec{v}^{ \pm}$, al que rompe $G$ al elegir el vacío concreto, es decir, el grupo de simetría restante al cuantizar alrededor de $\vec{v}^{ \pm}$.

Los ceros de $V$ son al mismo tiempo soluciones constantes de las ecuaciones de los campos (2.13): por ser mínimos del potencial verifican

$$
\left.\frac{\partial V}{\partial q_{a}}\right|_{\vec{q}=\vec{v}}=0 \quad \forall a=1, \ldots, N
$$

y, por tanto, cumplen las ecuaciones

$$
\square q_{a}=-\frac{\partial V}{\partial q_{a}}
$$

de manera trivial.

Por otro lado, el espectro de partículas, $M_{a b}^{2}\left(\vec{v}^{ \pm}\right)$, matriz de masa en cada punto crítico tomado, será

$$
M^{2}\left(\vec{v}^{ \pm}\right)=\left(\begin{array}{cccc}
4 & 0 & \cdots & 0 \\
0 & \sigma_{2}^{2} & \cdots & 0 \\
\vdots & \vdots & \ddots & \vdots \\
0 & 0 & \cdots & \sigma_{N}^{2}
\end{array}\right) \cdot \frac{m^{2}}{2}
$$


cuya simetría es exactamente el grupo pequeño del vacío considerado $H_{\vec{v}^{ \pm}}$. Al ser los puntos $\vec{v}$ mínimos de $V$, los autovalores $M^{2}\left(\vec{v}^{ \pm}\right)$son todos positivos y así, tal y como se explicó en la Sección 1.2, las soluciones de pequeñas deformaciones alrededor del mínimo constante $\vec{v}^{ \pm}$no serán divergentes con el tiempo. Si entendemos el modelo como una aproximación continua de un cristal unidimensional con un parámetro de orden con $N$ componentes (de manera similar a lo apuntado por Magyari y Thomas, [66], para el modelo MSTB), encontramos que el espectro de partículas está dado por $N$ ramas de fonones en una única fase.

La existencia de dos vectores de vacío, $\vec{v}^{+}$y $\vec{v}^{-}$, en $\mathcal{V}$, hace que el espacio de configuración del modelo sea la unión, en este caso, de 4 sectores topológicamente disconexos

$$
\mathcal{C}=\mathcal{C}^{(+,+)} \sqcup \mathcal{C}^{(+,-)} \sqcup \mathcal{C}^{(-,+)} \sqcup \mathcal{C}^{(-,-)}
$$

Esta estructura topológica es la misma que la del modelo MSTB, profundamente analizada en las referencias $[67,68]$. Utilizando la identidad $\pi_{0}(\mathcal{C})=\pi_{0}(\mathcal{V})$ entre los grupos de homotopía de orden cero de $\mathcal{C}$ y $\mathcal{V}$ y las condiciones asintóticas (2.11) encontraremos que $\pi_{0}(\mathcal{C})=\mathbb{Z}_{2}$ (ver [17] para detalles).

Teniendo en cuenta que los dos vacíos $\vec{v}^{ \pm}$de nuestro modelo tienen todas las componentes nulas salvo la primera, es posible definir la carga topológica de cada configuración como un escalar dependiente únicamente del primero de los campos, $q_{1}$. De esta forma:

$$
Q^{T}(\vec{q})=\frac{1}{2}\left(\hat{q}_{1}(\infty)-\hat{q}_{1}(-\infty)\right)
$$

donde $\hat{q}_{1}=\frac{q_{1}}{\left|v_{1}\right|}$. Podemos utilizar $Q^{T}$ para etiquetar las clases de homotopía y así la carga topológica será nula para las configuraciones de los sectores $\mathcal{C}^{(+,+)}$y $\mathcal{C}^{(-,-)}$, kinks no-topológicos, mientras que los sectores $\mathcal{C}^{(+,-)}$y $\mathcal{C}^{(-,+)}$contienen kinks topológicos, con cargas +1 y -1 respectivamente, y que denominaremos en adelante kinks para el sector $\mathcal{C}^{(-,+)}$, y antikinks para el $\mathcal{C}^{(+,-)}$.

El sistema de ecuaciones (2.13) se reduce a un sistema de ecuaciones diferenciales ordinarias para estas soluciones estacionarias:

$$
\frac{d^{2} q_{a}}{d\left(x^{1}\right)^{2}}=\frac{\partial V}{\partial q_{a}}
$$

junto con las condiciones asintóticas (2.11).

La resolución de este sistema es equivalente, por la analogía mecánica explicada en la Sección 1.2, a encontrar las soluciones de un sistema dinámico Lagrangiano

\footnotetext{
${ }^{4}$ Mantenemos la definición similar a la (1.10) si bien en este caso $\left|v_{1}\right|=1$ independientemente del vacío alcanzado, con lo que se podría obviar esta "normalización".
} 
en el cual la coordenada $x^{1}=t$ representa al "tiempo" mecánico, mientras que $\vec{q}(t)$ nos determina la posición de la "partícula" descrita por dicho sistema Lagrangiano en el espacio $\mathbb{R}^{N}$. Esta interpretación cobra sentido si tomamos como energía potencial de la "partícula" a $U(\vec{q})=-V(\vec{q})$. Las ecuaciones $(2.15)$ se escriben, desde esta perspectiva:

$$
\frac{d^{2} q_{a}}{d t^{2}}=-\frac{\partial U}{\partial q_{a}}
$$

La acción del sistema mecánico no es más que la energía de las configuraciones estáticas (salvo las constantes multiplicativas):

$$
\begin{gathered}
J[\vec{q}]=\int_{-\infty}^{+\infty} d t\left\{\frac{1}{2} \frac{d \vec{q}}{d t} \cdot \frac{d \vec{q}}{d t}-U(\vec{q})\right\}=\frac{\sqrt{2} \lambda^{2}}{m^{3}} E[\vec{q}] \\
U(\vec{q})=-\frac{1}{2}(\vec{q} \cdot \vec{q}-1)^{2}-\frac{1}{2} \sum_{a=1}^{N} \sigma_{a}^{2} q_{a}^{2}
\end{gathered}
$$

y así las trayectorias $\vec{q}(t)$ de acción $J$ finita están en correspondencia biunívoca con los kinks, de energía $E=\frac{m^{3}}{\sqrt{2} \lambda^{2}} J$.

Como ya hemos comentado, el método de la analogía mecánica fue utilizado exitosamente en el modelo MSTB por Magyari y Thomas [66], demostrando que el sistema mecánico asociado era completamente integrable en el sentido de ArnoldLiouville. Ito, [52], demostró que además el sistema era separable Hamilton-Jacobi, encontrando de forma explícita todas las trayectorias. Demostraremos a continuación que este modelo con $N$ campos mantiene estas propiedades dinámicas y calcularemos todas su soluciones.

Las ecuaciones de movimiento de la partícula (2.16), escritas de forma explícita, serán:

$$
\frac{d^{2} q_{a}}{d t^{2}}=2 q_{a}(\vec{q} \cdot \vec{q}-1)+\sigma_{a}^{2} q_{a}
$$

donde $a=1, \ldots, N$.

Las condiciones asintóticas (2.11) se traducen en:

$$
\lim _{t \rightarrow \pm \infty} \frac{d q_{a}}{d t}=0, \quad \lim _{t \rightarrow \pm \infty} q_{a}(t)= \pm \delta_{a 1}
$$

Utilizaremos el Formalismo Hamiltoniano para integrar el sistema mecánico. El momento canónico asociado a la variable $q_{a}$ será, lógicamente:

$$
p_{a}(t)=\frac{\delta J}{\delta \frac{d q_{a}}{d t}}(t)=\frac{d q_{a}}{d t}(t)
$$


De esta forma $\left(q_{a}, p_{a}\right)$ constituirán un sistema de coordenadas locales del espacio de fases del sistema. La función de Hamilton se calcula fácilmente:

$$
I_{1}=\frac{1}{2} \sum_{a=1}^{N} p_{a}^{2}-\frac{1}{2}\left(\sum_{a=1}^{N} q_{a}^{2}-1\right)^{2}-\frac{1}{2} \sum_{a=1}^{N} \sigma_{a}^{2} q_{a}^{2}
$$

que nos conduce a las ecuaciones canónicas, equivalentes a (2.19):

$$
\frac{d q_{a}}{d t}=\left\{I_{1}, q_{a}\right\}, \quad \frac{d p_{a}}{d t}=\left\{I_{1}, p_{a}\right\}
$$

donde se utiliza el paréntesis de Poisson estándar definido de manera natural en el espacio de fases:

$$
\{F, G\}=\sum_{a=1}^{N}\left(\frac{\partial F}{\partial p_{a}} \cdot \frac{\partial G}{\partial f_{a}}-\frac{\partial F}{\partial f_{a}} \cdot \frac{\partial G}{\partial p_{a}}\right)
$$

R. Garnier, en su artículo de 1919 titulado: "Sur une classe de Systèmes différentiels Abéliens deduits de la Théorie des équations linéaires", [42], integró las ecuaciones (2.19) en términos de las funciones theta. El sistema mecánico dado por (2.17), asociado al sistema de ecuaciones (2.19), ha sido frecuentemente llamado Sistema de Garnier por esta razón.

La técnica empleada por Garnier se basaba en la separabilidad de las ecuaciones en coordenadas elípticas, si bien Garnier no las empleaba explícitamente. En el artículo citado se demuestra la integrabilidad de un tipo general de sistemas de ecuaciones diferenciales de los cuales (2.19) no es más que un caso particular. Este hecho tal vez sea el causante de que durante años se hayan publicado diferentes artículos exponiendo resultados parciales acerca de la integración del sistema (2.19) sin conocer sus autores que dicho problema estaba resuelto.

El Sistema de Garnier ha sido utilizado en el campo de la Geometría Algebraica para estudiar espacios de moduli de ciertas variedades abelianas. Desde este punto de vista el Sistema de Garnier constituye un ejemplo de sistema completamente integrable algebraico (ver el Capítulo VII de la referencia [101] para detalles).

En 1980, Grosse demostró que las funciones:

$$
K_{a}=\sum_{b=1, b \neq a}^{N} \frac{1}{\sigma_{b}^{2}-\sigma_{a}^{2}} l_{a b}^{2}+p_{a}^{2}+\left(2-\sigma_{a}^{2}\right) q_{a}^{2}-q_{a}^{2} \sum_{b=1}^{N} q_{b}^{2}
$$

con $a=1, \ldots, N$ y donde

$$
l_{a b}=p_{a} q_{b}-p_{b} q_{a}
$$


son integrales primeras del sistema dinámico y que, además, están en involución:

$$
\left\{I_{1}, K_{a}\right\}=0 ; \quad\left\{K_{a}, K_{b}\right\}=0, \quad \forall a, b
$$

Las funciones $K_{a}$ constituyen un conjunto completo ${ }^{5}$ de integrales independientes. Aparentemente tenemos un conjunto, por tanto, de $N+1$ integrales primeras en involución $\left\{I_{1}, K_{1}, \ldots, K_{N}\right\}$. No se trata, sin embargo, de un sistema sobreintegrable o superintegrable puesto que es fácil comprobar que $K_{1}+K_{2}+$ $\cdots+K_{N}=2 I_{1}+1$, es decir sólo $N$ de las integrales son independientes.

De acuerdo con el Teorema de Arnold-Liouville, el sistema es completamente integrable.

La verificación de las condiciones asintóticas (2.20), por otra parte, determina que las trayectorias del sistema que constituyen las soluciones de tipo onda solitaria de la teoría de campos subyacente son aquéllas para las cuales las constantes de movimiento toman los valores siguientes:

$$
I_{1}=K_{2}=K_{3}=\cdots=K_{N}=0, \quad K_{1}=1
$$

como puede comprobarse fácilmente.

Dado que las integrales primeras son cuadráticas en los momentos, pero no ortogonales (contienen términos de la forma $p_{i} p_{j}$, con $i \neq j$ ), no es posible aplicar directamente el Teorema de Stäckel. Sin embargo, el sistema resulta ser no sólo completamente integrable, sino separable. Es decir existe algún sistema de coordenadas en el que las ecuaciones reducen a un conjunto de ecuaciones diferenciales ordinarias no acopladas, o, alternativamente, si se utiliza el Formalismo de Hamilton-Jacobi, como nosotros haremos, la ecuación de Hamilton-Jacobi es separable e integrable por cuadraturas. Evidentemente, en este sistema de coordenadas sí que se verificarán todas las hipótesis del Teorema de Stäckel citado. El sistema de coordenadas adecuado a nuestro modelo no es otro que las coordenadas elípticas de Jacobi, a las que dedicaremos la próxima sección.

\subsection{Coordenadas Elípticas}

Las coordenadas elípticas fueron descubiertas por G. Lamé en 1837 e independientemente por C.G.J. Jacobi [54] en 1838.

\footnotetext{
${ }^{5}$ En el sentido del Teorema de Arnold-Liouville.
} 
Jacobi encontró el sistema de coordenadas ortogonales que permitía separar las ecuaciones de las geodésicas sobre el elipsoide triaxial y escribir las cuadraturas que determinaban dichas geodésicas: las coordenadas elípticas. Sin embargo, Jacobi no pudo calcular explícitamente dichas cuadraturas, pues se necesitaban las funciones theta de género dos, absolutamente desconocidas en su época. Fue K. Weierstrass, en 1861, quien resolvió definitivamente el problema. Un estudio cualitativo del comportamiento de dichas geodésicas puede verse en el texto de V.I. Arnold [9], mientras que una exposición detallada del proceso de integración de las cuadraturas con funciones theta, así como de las principales propiedades de las coordenadas elípticas puede encontrarse en [81]. Otra referencia importante sobre las coordenadas elípticas y el proceso de separación de variables que veremos en la próxima sección es [111].

Lamé estudiaba los problemas de la transmisión del calor en un elipsoide, es decir la ecuación de Laplace tomando el elipsoide como superficie equipotencial. Históricamente, sin embargo, se ha conocido a estas coordenadas como Coordenadas Elípticas de Jacobi. Estos resultados históricos se refieren al caso bidimensional, no es difícil, sin embargo, generalizar la definición y las propiedades de estas coordenadas al caso $N$-dimensional. Las coordenadas elípticas han sido usadas, además, para la resolución del problema de la fuerza elástica sobre el elipsoide triaxial (Jacobi [54]) o, más recientemente, para el problema de los billares elipsoídicos (Chang y Friedberg [24]).

Dados $N$ números reales positivos y diferentes $0<a_{1}<a_{2}<\ldots<a_{N}$, podemos construir la ecuación:

$$
Q_{\lambda}(\vec{x})=\sum_{j=1}^{N} \frac{x_{j}^{2}}{a_{j}-\lambda}=1
$$

donde $Q_{\lambda}(\vec{x})$ puede verse alternativamente como una función de $\mathbb{R}^{N}$, es decir de las variables $\left(x_{1}, \ldots, x_{N}\right) \equiv \vec{x} \in \mathbb{R}^{N}$, o como una función de la variable compleja $\lambda$, a $\vec{x}$ fijo.

Es posible escribir esta ecuación en la forma:

$$
1-Q_{\lambda}(\vec{x})=1+\sum_{j=1}^{N} \frac{x_{j}^{2}}{\lambda-a_{j}}=\frac{\prod_{j=1}^{N}\left(\lambda-\lambda_{j}\right)}{\prod_{j=1}^{N}\left(\lambda-a_{j}\right)}=0
$$

donde se ha tomado por un lado común denominador, con lo que éste será un 
polinomio de grado $N$ en $\lambda$ y, por otro, dado que el numerador también es un polinomio de grado $N$, se ha escrito factorizado en términos de sus raíces $\left(\lambda_{1}, \ldots, \lambda_{N}\right)$. El hecho crucial es que dichas raíces, del numerador y por tanto de la ecuación, son números reales y además separados por las constantes tomadas $\left(a_{1}, \ldots, a_{N}\right)$ de la forma:

$$
\lambda_{1}<a_{1}<\lambda_{2}<a_{2}<\ldots<\lambda_{N}<a_{N}
$$

(ver Figura 2.1 $)^{6}$.

Se llaman coordenadas elípticas del punto $\vec{x}=\left(x_{1}, \ldots, x_{N}\right) \in \mathbb{R}^{N}$ a las raíces $\left(\lambda_{1}, \ldots, \lambda_{N}\right)$ de dicha ecuación $(2.24)$.

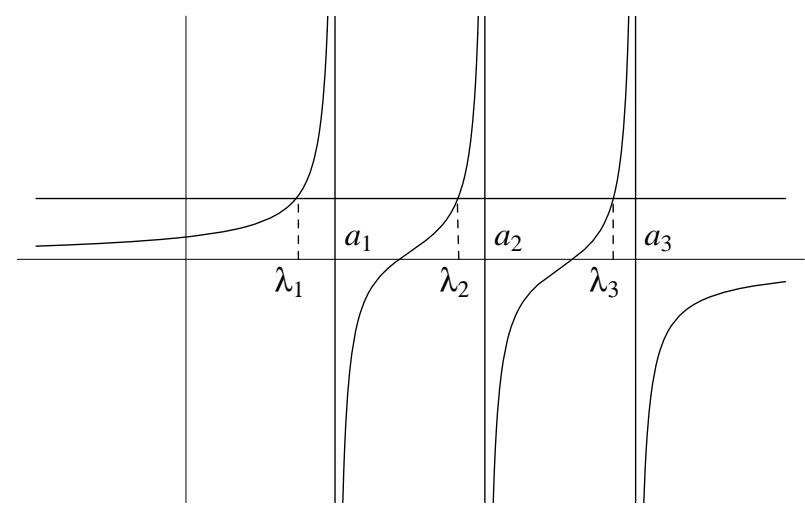

Figura 2.1: Gráfica de la función $Q_{\lambda}(\vec{x})$ sobre el eje real $(\lambda)$ para el caso $0<a_{1}<a_{2}<a_{3}$ y de la recta $y=1$. Los puntos de corte definen las coordenadas elípticas $\lambda_{1}, \lambda_{2}$ y $\lambda_{3}$.

Desde un punto de vista geométrico, la ecuación (2.24) con $\lambda=\lambda_{i}$ y vista por tanto en las incógnitas $\left(x_{1}, \ldots, x_{N}\right)$, representa una familia de cuádricas en $\mathbb{R}^{N}$, de forma que el hiperplano "elíptico" $\lambda_{1}=$ cte. nos proporciona la ecuación de un hiperelipsoide, mientras que $\lambda_{i}=$ cte, $\forall i=2, \ldots, N$ se corresponden con los hiperhiperboloides de todas las signaturas posibles en $\mathbb{R}^{N}$.

Por simplicidad en la notación, llamaremos a los dos productos que aparecen

\footnotetext{
${ }^{6}$ Es fácil demostrar que las raíces son reales: el dominio real de $Q_{\lambda}(\vec{x})$ (como función de $\lambda$ ) es $\mathbb{R}-\left\{a_{1}, \ldots, a_{N}\right\}=\left(-\infty, a_{1}\right) \cup\left(a_{1}, a_{2}\right) \cup \ldots \cup\left(a_{N}, \infty\right)$, que coincide con su dominio de continuidad y de derivabilidad. La derivada de $\left(1-Q_{\lambda}(\vec{x})\right)$ es diferente de cero para todo $\lambda$ del dominio, de esta forma tendrá a lo más una raíz en cada parte conexa del mismo. Como además en todos los intervalos del dominio excepto en el último $\left(a_{N}, \infty\right)$, la función $1-Q_{\lambda}(\vec{x})$ cambia de signo, por el Teorema de Bolzano queda probada la existencia de $N$ raíces reales.
} 
en la ecuación (2.25) de la siguiente manera:

$$
\Lambda(\lambda)=\prod_{j=1}^{N}\left(\lambda-\lambda_{j}\right) ; \quad A(\lambda)=\prod_{j=1}^{N}\left(\lambda-a_{j}\right)
$$

de manera que (2.25) se reescribe:

$$
1+\sum_{j=1}^{N} \frac{x_{j}^{2}}{\lambda-a_{k}}=\frac{\prod_{j=1}^{N}\left(\lambda-\lambda_{j}\right)}{\prod_{j=1}^{N}\left(\lambda-a_{j}\right)} \equiv \frac{\Lambda(\lambda)}{A(\lambda)}
$$

Para calcular la expresión de las coordenadas cartesianas $\left(x_{1}, \ldots, x_{N}\right)$ en términos de las coordenadas elípticas $\left(\lambda_{1}, \ldots, \lambda_{N}\right)$ podemos seguir varios caminos equivalentes en sus resultados:

- Una primera posibilidad es plantear la ecuación (2.24) para todos los valores de las coordenadas elípticas $\lambda=\lambda_{i}$, obtendremos así un sistema de $N$ ecuaciones lineales en las incógnitas $x_{j}^{2}$ :

$$
\begin{array}{ccc}
\frac{x_{1}^{2}}{a_{1}-\lambda_{1}}+\frac{x_{2}^{2}}{a_{2}-\lambda_{1}}+\cdots+\frac{x_{n}^{2}}{a_{n}-\lambda_{1}} & =1 \\
\frac{x_{1}^{2}}{a_{1}-\lambda_{2}}+\frac{x_{2}^{2}}{a_{2}-\lambda_{2}}+\cdots+\frac{x_{n}^{2}}{a_{n}-\lambda_{2}} & =1 \\
\vdots & \vdots & \ddots \\
\vdots & =\vdots \\
\frac{x_{1}^{2}}{a_{1}-\lambda_{n}}+\frac{x_{2}^{2}}{a_{2}-\lambda_{n}}+\cdots+\frac{x_{n}^{2}}{a_{n}-\lambda_{n}} & =1
\end{array}
$$

La matriz asociada a dicho sistema es conocida en la literatura como la "matriz de Cauchy":

$$
A=\left(\begin{array}{ccc}
\frac{1}{a_{1}-\lambda_{1}} & \cdots & \frac{1}{a_{n}-\lambda_{1}} \\
\vdots & \ddots & \vdots \\
\frac{1}{a_{1}-\lambda_{n}} & \cdots & \frac{1}{a_{n}-\lambda_{n}}
\end{array}\right)
$$

No es difícil pero tampoco trivial demostrar que su determinante vale

$$
\Delta=\frac{\prod_{j<k}^{n}\left(a_{j}-a_{k}\right) \prod_{j<k}^{n}\left(\lambda_{j}-\lambda_{k}\right)}{\prod_{j, k}^{n}\left(a_{j}-\lambda_{k}\right)}
$$

y construir sus soluciones directamente, por la Regla de Cramer. 
- Alternativamente, podemos ver la ecuación (2.26) como una ecuación en la variable compleja $\lambda$ y calcular los residuos de ambos miembros en cada polo $\lambda=a_{i}$. Para el primer miembro tendremos, trivialmente:

$$
\left.\operatorname{Res}\left(1-Q_{\lambda}(x)\right)\right|_{\lambda=a_{i}}=x_{i}^{2}
$$

mientras que en el segundo aplicamos la propiedad que nos da el residuo de un cociente de funciones analíticas:

$$
\left.\operatorname{Res} \frac{\Lambda(\lambda)}{A(\lambda)}\right|_{\lambda=a_{i}}=\frac{\Lambda\left(a_{i}\right)}{A^{\prime}\left(a_{i}\right)}
$$

de manera que

$$
x_{i}^{2}=\frac{\Lambda\left(a_{i}\right)}{A^{\prime}\left(a_{i}\right)}=\frac{\prod_{j=1}^{N}\left(a_{i}-\lambda_{j}\right)}{\prod_{j=1, j \neq i}^{N}\left(a_{i}-a_{j}\right)}, \quad i=1,2, \ldots, N
$$

donde, obviamente, $A^{\prime}\left(a_{i}\right)=\left.\frac{d A(\lambda)}{d \lambda}\right|_{\lambda=a_{i}}$. Escrito de forma explícita tendremos:

$$
x_{i}^{2}=\frac{\left(a_{i}-\lambda_{1}\right)\left(a_{i}-\lambda_{2}\right) \ldots\left(a_{i}-\lambda_{N}\right)}{\left(a_{i}-a_{1}\right)\left(a_{i}-a_{2}\right) \ldots{ }^{(i)} \ldots\left(a_{i}-a_{N}\right)}
$$

donde la notación escogida refleja evidentemente que falta el factor $\left(a_{i}-a_{i}\right)$ en el denominador.

- Incluiremos una tercera deducción alternativa de estas expresiones. El motivo de hacerlo es que, a pesar de ser menos elegante que las anteriores, nos proporcionará al mismo tiempo varias identidades que relacionan expresiones algebraicas en coordenadas elípticas y en cartesianas, que nos resultarán más adelante de crucial utilidad. Si escribimos el primer miembro de (2.26) en la forma:

$$
\frac{\prod_{j=1}^{N}\left(\lambda-a_{j}\right)+\sum_{j=1}^{N} x_{j}^{2} \prod_{j=1, j \neq i}^{N}\left(\lambda-a_{j}\right)}{\prod_{j=1}^{N}\left(\lambda-a_{j}\right)}
$$

el denominador es exactamente lo que hemos denominado $A(\lambda)$, mientras que el numerador es igual al polinomio $\Lambda(\lambda)$ de grado $N$ en $\lambda$. Desarrollamos el 
numerador en potencias de $\lambda$ e igualamos al desarrollo correspondiente de $\Lambda(\lambda)$, para obtener la igualdad polinómica:

$$
\begin{gathered}
\prod_{j=1}^{N}\left(\lambda-a_{j}\right)+\sum_{j=1}^{N} x_{j}^{2} \prod_{k=1, k \neq j}^{N}\left(\lambda-a_{k}\right)=\Lambda(\lambda) \\
\lambda^{N}-\left(\sum_{j=1}^{N} a_{j}\right) \lambda^{N-1}+\left(\sum_{i<j}^{N} a_{i} a_{j}\right) \lambda^{N-2}+\ldots+(-1)^{N} \prod_{j=1}^{N} a_{j}+ \\
+\sum_{j=1}^{N} x_{j}^{2}\left(\lambda^{N-1}-\lambda^{N-2}\left(\sum_{i=1, i \neq j}^{N} a_{i}\right)+\ldots+(-1)^{N-1} \prod_{i=1, i \neq j}^{N} a_{i}\right)= \\
=\lambda^{N}+\lambda^{N-1}\left(-\sum_{j=1}^{N} a_{j}+\sum_{j=1}^{N} x_{j}^{2}\right)+ \\
+\lambda^{N-2}\left(\sum_{i<j}^{N} \sum_{i} a_{j}-\sum_{j=1}^{N} x_{j}^{2} \sum_{i=1, i \neq j}^{N} a_{i}\right)+\ldots= \\
=\lambda^{N}-\left(\sum_{j=1}^{N} \lambda_{j}\right) \lambda^{N-1}+\left(\sum_{i<j}^{N} \lambda_{i} \lambda_{j}\right) \lambda^{N-2}+\ldots+(-1)^{N} \prod_{j=1}^{N} \lambda_{j}
\end{gathered}
$$

Si particularizamos la ecuación (2.28) para $\lambda=a_{i}$, obtenemos de forma inmediata la expresión (2.27) del cambio de coordenadas, como puede comprobarse fácilmente.

Por otro lado, igualando término a término los dos miembros de la ecuación (2.29) tenemos $N$ identidades no triviales. Resaltaremos las dos primeras, correspondientes a los términos en $\lambda^{N-1}$ y en $\lambda^{N-2}$ :

$$
\begin{gathered}
\sum_{j=1}^{N} \lambda_{j}=\sum_{j=1}^{N} a_{j}-\sum_{j=1}^{N} x_{j}^{2} \\
\sum \sum_{i<j}^{N} \lambda_{i} \lambda_{j}=\sum \sum_{i<j}^{N} a_{i} a_{j}-\sum_{j=1}^{N} x_{j}^{2} \sum_{i=1, i \neq j}^{N} a_{i}
\end{gathered}
$$

Manipulando (2.31) y utilizando (2.30), podemos reescribir (2.31) de la forma:

$$
\sum \sum_{i<j}^{N} \lambda_{i} \lambda_{j}=\sum_{j=1}^{N} a_{j} x_{j}^{2}-\sum_{j=1}^{N} x_{j}^{2} \sum_{i=1}^{N} a_{i}+\sum \sum_{i<j}^{N} a_{i} a_{j}
$$


Una vez deducidas las expresiones (2.27), es trivial observar que el cambio de coordenadas no es biunívoco, la transformación $\left(x_{1}, \ldots, x_{N}\right) \rightarrow\left(\lambda_{1}, \ldots, \lambda_{N}\right)$ es $2^{N}$ a uno al estar las coordenadas cartesianas elevadas al cuadrado en las ecuaciones que determinan dicho cambio.

En general el cambio inverso es imposible de obtener en términos analíticos. Para el caso bidimensional no es difícil pues requiere resolver ecuaciones cuadráticas. Para 3 y 4 dimensiones, las ecuaciones (2.27) son cúbicas y cuárticas respectivamente, con lo que cabe aplicar las fórmulas de Cardano-Tartaglia, si bien el resultado es extraordinariamente complicado. Para dimensiones mayores evidentemente las ecuaciones ya no son resolubles por radicales de manera general. Por este motivo, las identidades que se deducen de (2.29) cobran una gran importancia, aunque no conozcamos el cambio inverso de coordenas, estas identidades permiten al menos expresar en términos de las coordenadas cartesianas relaciones algebraicas sencillas de las coordenadas elípticas ${ }^{7}$.

Existe una importante propiedad de las coordenadas elípticas que no podemos olvidar, y que será también de gran utilidad en el proceso de separación de variables en la ecuación de Hamilton-Jacobi que realizaremos en la próxima sección:

Lema (Jacobi). La expresión

$$
\sum_{k=1}^{N} \frac{\alpha_{k}^{s}}{\left(\alpha_{k}-\alpha_{1}\right)\left(\alpha_{k}-\alpha_{2}\right) \ldots{ }^{(k)} \ldots\left(\alpha_{k}-\alpha_{N}\right)}
$$

donde $\left(\alpha_{1}, \ldots, \alpha_{N}\right)$ son cualesquiera números reales que verifiquen $\alpha_{1}<\alpha_{2}<\cdots<$ $\alpha_{N}$ y $s$ es un número entero no negativo, es exactamente igual a 0 si $s \leq N-2$ y a 1 si $s=N-1$.

Demostración: La manera más fácil de demostrar el Lema, y de obtener además nuevas identidades de las coordenadas elípticas, es considerar la función:

$$
f_{s}(z)=\frac{z^{s}}{\prod_{j=1}^{N}\left(z-\alpha_{j}\right)}
$$

\footnotetext{
${ }^{7}$ Un ejemplo claro de este hecho son las identidades que se deducen para el caso tridimensional:

$$
\begin{aligned}
& \lambda_{1}+\lambda_{2}+\lambda_{3}=a-\left(x^{2}+y^{2}+z^{2}\right) \\
& \lambda_{1} \lambda_{2}+\lambda_{1} \lambda_{3}+\lambda_{2} \lambda_{3}=b-a\left(x^{2}+y^{2}+z^{2}\right)+a_{1} x^{2}+a_{2} y^{2}+a_{3} z^{2} \\
& \lambda_{1} \lambda_{2} \lambda_{3}=a_{1} a_{2} a_{3}-a_{2} a_{3} x^{2}-a_{1} a_{3} y^{2}-a_{1} a_{2} z^{2}
\end{aligned}
$$

donde $a=a_{1}+a_{2}+a_{3}$ y $b=a_{2} a_{3}+a_{1} a_{3}+a_{1} a_{2}$.
} 
con $N$ polos en el plano complejo (en cada $z=\alpha_{i}$ ) más un posible polo en el infinito, dependiendo del valor de $s$. Si integramos $f_{s}(z)$

$$
\frac{1}{2 \pi i} \int_{\gamma} f_{s}(z) d z
$$

en una curva cerrada $\gamma$ en el plano complejo tal que todos los polos finitos de la función pertenezcan a la región interior a la curva, obtendremos, por aplicación directa del teorema de los residuos de Cauchy, el resultado:

$$
\sum_{k=1}^{N} \operatorname{Res}\left(f_{s}\right)\left(\alpha_{k}\right)=-\operatorname{Res}\left(f_{s}\right)(\infty)
$$

El primer miembro de (2.34) es obvio:

$$
\sum_{k=1}^{N} \operatorname{Res}\left(f_{s}\right)\left(\alpha_{k}\right)=\sum_{k=1}^{N} \frac{\alpha_{k}^{s}}{\left(\alpha_{k}-\alpha_{1}\right)\left(\alpha_{k}-\alpha_{2}\right) \ldots{ }^{(k)} \ldots\left(\alpha_{k}-\alpha_{N}\right)}
$$

mientras que el residuo de $f_{s}(z)$ en el infinito se calcula con relativa facilidad teniendo en cuenta que su denominador,

$$
\prod_{j=1}^{N}\left(z-\alpha_{j}\right)
$$

es un polinomio de grado $N$. Podemos así escribir la función en la forma:

$$
\begin{gathered}
f_{s}(z)=z^{s-N} \frac{1}{1-\left(\sum_{j} \alpha_{j}\right) \frac{1}{z}+\left(\sum \sum_{i<j} \alpha_{i} \alpha_{j}\right) \frac{1}{z^{2}}-\ldots}= \\
=z^{s-N}\left(1+\sum_{j=1}^{N} \alpha_{j} \frac{1}{z}+\left(\left(\sum_{j=1}^{N} \alpha_{j}\right)^{2}-\sum \sum_{i<j}^{N} \alpha_{i} \alpha_{j}\right) \frac{1}{z^{2}}+\ldots\right)
\end{gathered}
$$

El residuo $\operatorname{Res}\left(f_{s}\right)(\infty)$ será menos el coeficiente de $\frac{1}{z}$, de manera que, para los diferentes valores de $s$, obtendremos:

$$
\begin{aligned}
& \sum_{k=1}^{N} \frac{\alpha_{k}^{s}}{\left(\alpha_{k}-\alpha_{1}\right)\left(\alpha_{k}-\alpha_{2}\right) \ldots{ }^{(k)} \ldots\left(\alpha_{k}-\alpha_{N}\right)}=0, \quad \forall s<N-1 \\
& \sum_{k=1}^{N} \frac{\alpha_{k}^{N-1}}{\left(\alpha_{k}-\alpha_{1}\right)\left(\alpha_{k}-\alpha_{2}\right) \ldots{ }^{(k)} \ldots\left(\alpha_{k}-\alpha_{N}\right)}=1
\end{aligned}
$$

Q.E.D. 
Si consideramos el Lema para el caso $\alpha_{j}=\lambda_{j}, \forall j=1, \ldots, N$, las expresiones anteriores se traducen en:

$$
\begin{aligned}
& \sum_{j=1}^{N} \frac{\lambda_{j}^{s}}{\Lambda^{\prime}\left(\lambda_{j}\right)}=0, \quad \forall s<N-1 \\
& \sum_{j=1}^{N} \frac{\lambda_{j}^{N-1}}{\Lambda^{\prime}\left(\lambda_{j}\right)}=1
\end{aligned}
$$

puesto que el denominador en este caso no es más que

$$
\Lambda^{\prime}\left(\lambda_{j}\right)=\left(\lambda_{j}-\lambda_{1}\right)\left(\lambda_{j}-\lambda_{2}\right) \ldots{ }^{(j)} \ldots\left(\lambda_{j}-\lambda_{N}\right)
$$

Mientras que si $\alpha_{j}=a_{j}$ :

$$
\begin{aligned}
& \sum_{j=1}^{N} \frac{a_{j}^{m}}{A^{\prime}\left(a_{j}\right)}=0, \quad \forall s<N-1 \\
& \sum_{j=1}^{N} \frac{a_{j}^{N-1}}{A^{\prime}\left(a_{j}\right)}=1
\end{aligned}
$$

Por otro lado, la demostración que hemos hecho implica más resultados de los que hemos resaltado, es posible calcular fácilmente los residuos para valores del exponente $s$ mayores o iguales que $N$. Así por ejemplo, para $s=N$ y $\alpha_{j}=\lambda_{j}$ tendremos:

$$
\sum_{j=1}^{N} \frac{\lambda_{j}^{N}}{\Lambda^{\prime}\left(\lambda_{j}\right)}=\sum_{j=1}^{N} \lambda_{j}
$$

mientras que para $s=N+1$ :

$$
\sum_{j=1}^{N} \frac{\lambda_{j}^{N+1}}{\Lambda^{\prime}\left(\lambda_{j}\right)}=\left(\sum_{j=1}^{N} \lambda_{j}\right)^{2}-\sum \sum_{i<j}^{N} \lambda_{i} \lambda_{j}
$$

y así sucesivamente ${ }^{8}$.

${ }^{8}$ Nótese que estas expresiones son fácilmente combinables con las anteriormente comentadas que se derivaban de la ecuación (2.29). Por ejemplo y de nuevo para el caso tridimensional:

$$
\begin{aligned}
& \frac{C}{\Lambda^{\prime}\left(\lambda_{1}\right)}+\frac{C}{\Lambda^{\prime}\left(\lambda_{2}\right)}+\frac{C}{\Lambda^{\prime}\left(\lambda_{3}\right)}=0, \quad \forall C=\text { cte. } \\
& \frac{\lambda_{1}}{\Lambda^{\prime}\left(\lambda_{1}\right)}+\frac{\lambda_{2}}{\Lambda^{\prime}\left(\lambda_{2}\right)}+\frac{\lambda_{3}}{\Lambda^{\prime}\left(\lambda_{3}\right)}=0
\end{aligned}
$$


Las consecuencias del Lema de Jacobi no terminan aquí. No es difícil demostrar, por ejemplo, que fijados los índices $i$ y $k($ con $i \neq k)$ se verifica:

$$
\sum_{j=1}^{N} \frac{x_{j}^{2}}{\left(a_{j}-\lambda_{i}\right)\left(a_{j}-\lambda_{k}\right)}=0
$$

Efectivamente:

$$
\begin{aligned}
& \sum_{j=1}^{N} \frac{x_{j}^{2}}{\left(a_{j}-\lambda_{i}\right)\left(a_{j}-\lambda_{k}\right)}=\sum_{j=1}^{N} \frac{\frac{\Lambda\left(a_{j}\right)}{A^{\prime}\left(a_{j}\right)}}{\left(a_{j}-\lambda_{i}\right)\left(a_{j}-\lambda_{k}\right)}= \\
& =\sum_{j=1}^{N} \frac{\prod_{l=1}^{N}\left(a_{j}-\lambda_{l}\right)}{A^{\prime}\left(a_{j}\right)\left(a_{j}-\lambda_{i}\right)\left(a_{j}-\lambda_{k}\right)}=\sum_{j=1}^{N} \frac{\prod_{l=1, l \neq i, l \neq k}\left(a_{j}-\lambda_{l}\right)}{A^{\prime}\left(a_{j}\right)}= \\
& =\sum_{j=1}^{N} \frac{a_{j}^{N-2}-a_{j}^{N-3} \sum_{l=1, l \neq i, l \neq k} \lambda_{l}+\ldots+(-1)^{N-2} \prod_{l=1, l \neq i, l \neq k} \lambda_{l}}{A^{\prime}\left(a_{j}\right)}=0
\end{aligned}
$$

por aplicaciones sucesivas del Lema.

Si recordamos que la ecuación inicial (2.24)

$$
Q_{\lambda}(\vec{x})=\sum_{j=1}^{N} \frac{x_{j}^{2}}{a_{j}-\lambda} ; \quad Q_{\lambda}(\vec{x})=1
$$

era la ecuación de la familia de cuádricas citada para los diferentes valores de $\lambda$ (elipsoide si $\lambda<a_{1}$, e hiperboloides en los demás casos). Entonces

$$
\vec{n}(\lambda) \equiv\left(n_{j}(\lambda)\right)=\left(\frac{x_{j}}{a_{j}-\lambda}\right)
$$

puede verse como el vector normal a la cuádrica en el punto $\vec{x} \equiv\left(x_{j}\right)$ y así la expresión

$$
\begin{gathered}
\sum_{j=1}^{N} \frac{x_{j}^{2}}{\left(a_{j}-\lambda_{i}\right)\left(a_{j}-\lambda_{k}\right)}=0 \\
\frac{\lambda_{1}^{2}}{\Lambda^{\prime}\left(\lambda_{1}\right)}+\frac{\lambda_{2}^{2}}{\Lambda^{\prime}\left(\lambda_{2}\right)}+\frac{\lambda_{3}^{2}}{\Lambda^{\prime}\left(\lambda_{3}\right)}=1 \\
\frac{\lambda_{1}^{3}}{\Lambda^{\prime}\left(\lambda_{1}\right)}+\frac{\lambda_{2}^{3}}{\Lambda^{\prime}\left(\lambda_{2}\right)}+\frac{\lambda_{3}^{3}}{\Lambda^{\prime}\left(\lambda_{3}\right)}=\lambda_{1}+\lambda_{2}+\lambda_{3} \\
\frac{\lambda_{1}^{4}}{\Lambda^{\prime}\left(\lambda_{1}\right)}+\frac{\lambda_{2}^{4}}{\Lambda^{\prime}\left(\lambda_{2}\right)}+\frac{\lambda_{3}^{4}}{\Lambda^{\prime}\left(\lambda_{3}\right)}=\left(\lambda_{1}+\lambda_{2}+\lambda_{3}\right)^{2}-\left(\lambda_{1} \lambda_{2}+\lambda_{1} \lambda_{3}+\lambda_{2} \lambda_{3}\right)
\end{gathered}
$$

y así sucesivamente. 
deducida anteriormente, no es más que el producto escalar de los vectores normales a dos de las cuádricas $\left(\lambda=\lambda_{i}\right.$ y $\left.\lambda=\lambda_{k}\right)$ en el mismo punto

$$
\sum_{j=1}^{n} n_{j}\left(\lambda_{i}\right) n_{j}\left(\lambda_{k}\right)=0
$$

Concluimos por tanto que todas las cuádricas son ortogonales entre sí y, por tanto, las coordenadas elípticas constituyen un sistema ortogonal.

Calcularemos, por último, el elemento de línea en este sistema de coordenadas. La métrica euclídea estándar en cartesianas,

$$
d s^{2}=\sum_{j=1}^{N} d x_{j}^{2}
$$

puede expresarse en coordenadas elípticas de la forma:

$$
d s^{2}=\sum \sum_{i, j=1}^{N} g_{i j} d \lambda_{i} d \lambda_{j}
$$

Diferenciando en (2.27) tendremos

$$
\frac{2 d x_{j}}{x_{j}}=(-1)^{N} \sum_{k=1}^{n} \frac{d \lambda_{k}}{a_{j}-\lambda_{k}}
$$

y, usando el Lema de Jacobi,

$$
4 d x_{j}^{2}=x_{j}^{2} \sum_{k=1}^{N} \frac{d \lambda_{k}^{2}}{a_{j}-\lambda_{k}}
$$

que, tras sustituir (2.27), nos proporciona la expresión:

$$
d s^{2}=\frac{1}{4} \sum_{k=1}^{n} M_{j}(\lambda)\left(d \lambda_{j}\right)^{2}
$$

donde:

$$
M_{j}(\lambda)=\sum_{k=1}^{N} \frac{x_{k}^{2}}{\left(a_{k}-\lambda_{j}\right)^{2}}=-\frac{\prod_{k \neq j, k=1}^{N}\left(\lambda_{j}-\lambda_{k}\right)}{\prod_{k=1}^{N}\left(\lambda_{j}-a_{k}\right)}
$$

que evidencia que la métrica es definida positiva a pesar del signo del último miembro. 
En definitiva:

$$
g_{j j}=-\frac{1}{4} \frac{\Lambda^{\prime}\left(\lambda_{j}\right)}{A\left(\lambda_{j}\right)}, g_{i j}=0, \forall i \neq j
$$

es decir comprobamos de nuevo que se trata de un sistema ortogonal de coordenadas en $N$ dimensiones.

La energía cinética es ahora fácilmente calculable:

$$
T=\frac{1}{2} \sum_{k=1}^{N} \dot{x}_{k}^{2}=\frac{1}{2} \sum_{j=1}^{N} g_{j j} \dot{\lambda}_{j}^{2}
$$

que, si definimos $\pi_{j}$, momento asociado a la coordenada $\lambda_{j}$ :

$$
\pi_{j}=\frac{\partial T}{\partial \dot{\lambda}_{j}}
$$

y, abusando de la notación, volvemos a llamar $T$ a la energía cinética en el formalismo Hamiltoniano:

$$
T=\sum_{j=1}^{N} \pi_{j} \dot{\lambda}_{j}-T=\frac{1}{2} \sum_{j=1}^{N} \frac{1}{g_{j j}} \pi_{j}^{2}=-2 \sum_{j=1}^{N} \frac{A\left(\lambda_{j}\right)}{\Lambda^{\prime}\left(\lambda_{j}\right)} \pi_{j}^{2}
$$

\subsection{Separabilidad de la Ecuación de Hamilton- Jacobi}

Tal y como se apuntaba en la primera sección de este capítulo, el sistema dinámico asociado al Modelo Sigma $O(N)$ Lineal Deformado que estamos considerando no sólo es completamente integrable, sino que además es Hamilton-Jacobi separable si se utilizan las coordenadas elípticas de Jacobi que hemos introducido en la sección anterior.

Definimos coordenadas elípticas en $\mathbb{R}^{N}$ visto como espacio de configuración del sistema dinámico. Pasamos por tanto de nuestras coordenadas canónicas $\vec{q}=$ $\left(q_{1}, \ldots, q_{N}\right)$ a $\vec{\lambda}=\left(\lambda_{1}, \ldots, \lambda_{N}\right)$ definidas por las ecuaciones $(2.27)$ en las que son $q_{a}$ quienes juegan el papel de coordenadas cartesianas $x_{j}$ y donde se ha tomado una familia de constantes adecuada al sistema, de la siguiente forma:

$$
a_{N-i+1}=\bar{\sigma}_{i}^{2}=1-\sigma_{i}^{2}, \quad \forall i=1, \ldots, N
$$

de manera que la condición (2.9) se traduce ahora en que

$$
\bar{\sigma}_{N}^{2}<\bar{\sigma}_{N-1}^{2}<\cdots<\bar{\sigma}_{1}^{2}=1
$$


La ordenación en los índices de las constantes $\bar{\sigma}_{a}^{2}$, inversa a la presentada para las constantes que definen el cambio de coordenadas en la sección anterior, nos conduce a una identificación de las coordenadas concretas $q_{a}$ con las genéricas de la Sección $2.2, x_{i}$, de la forma:

$$
x_{i} \equiv q_{N-i+1} ; \quad i=1,2, \ldots, N
$$

De esta forma la ecuación del cambio de coordenadas (2.27) se mantiene formalmente en nuestro caso:

$$
q_{a}^{2}=\frac{\prod_{b=1}^{N}\left(\bar{\sigma}_{a}^{2}-\lambda_{b}\right)}{\prod_{b=1, b \neq a}^{N}\left(\bar{\sigma}_{a}^{2}-\bar{\sigma}_{b}^{2}\right)}=\frac{\Lambda\left(\bar{\sigma}_{a}^{2}\right)}{A^{\prime}\left(\bar{\sigma}_{a}^{2}\right)}, \quad \forall a=1, \ldots, N
$$

Las variables elípticas estarán separadas en la forma:

$$
-\infty<\lambda_{1}<\bar{\sigma}_{N}^{2}<\lambda_{2}<\bar{\sigma}_{N-1}^{2}<\cdots<\bar{\sigma}_{2}^{2}<\lambda_{N}<1
$$

El producto escalar estándar euclídeo que teníamos en las variables $q_{a}$ se transforma como hemos visto en el apartado anterior:

$$
d s^{2}=\sum_{a=1}^{N}\left(d q_{a}\right)^{2}=\sum_{b=1}^{N} g_{b b}\left(d \lambda_{b}\right)^{2}, \quad g_{b b}=\frac{-1}{4} \frac{\Lambda^{\prime}\left(\lambda_{b}\right)}{A\left(\lambda_{b}\right)}, g_{a b}=0, \forall a \neq b
$$

Y, en definitiva, el Lagrangiano pasa a ser

$$
L=\frac{1}{2} \sum_{a=1}^{N}\left(\frac{d q_{a}}{d t}\right)^{2}-U\left(q_{1}, \ldots, q_{N}\right)=\frac{1}{2} \sum_{b=1}^{N} g_{b b}(\lambda)\left(\frac{d \lambda_{b}}{d t}\right)^{2}-U_{\lambda}\left(\lambda_{1}, \ldots, \lambda_{N}\right)
$$

El cálculo de la expresión de $U_{\lambda}\left(\lambda_{1}, \ldots, \lambda_{N}\right)$ está lejos de ser trivial y requiere la utilización de las identidades que hemos obtenido en el apartado anterior (2.29, 2.35, 2.36, 2.37, 2.38). Si pasamos al formalismo Hamiltoniano, la función Hamiltoniana será:

$$
H=\frac{1}{2} \sum_{b=1}^{N} \frac{-4 A\left(\lambda_{b}\right)}{f_{b}(\vec{\lambda})} \pi_{b}^{2}-\sum_{b=1}^{N} \frac{1}{2} \frac{\lambda_{b}^{N+1}-(\alpha-1) \lambda_{b}^{N}+(1-\alpha+\beta) \lambda_{b}^{N-1}}{f_{b}(\vec{\lambda})}
$$

donde las constantes $\alpha$ y $\beta$ se escriben en términos de las $\bar{\sigma}_{a}^{2}$ de la forma:

$$
\alpha=\sum_{b=1}^{N} \bar{\sigma}_{b}^{2}, \quad \beta=\frac{1}{2}\left[\alpha^{2}-\sum_{b=1}^{N} \bar{\sigma}_{b}^{4}\right]=\sum \sum_{a<b}^{N} \bar{\sigma}_{a}^{2} \bar{\sigma}_{b}^{2}
$$


$\pi_{a}$ es el momento canónico asociado a la variable $\lambda_{a}$,

$$
\pi_{a}=\sum_{b=1}^{N} g_{a b} \frac{d \lambda_{b}}{d t}=g_{a a} \frac{d \lambda_{a}}{d t}
$$

y donde se ha denotado $f_{b}(\vec{\lambda})=\Lambda^{\prime}\left(\lambda_{b}\right)^{9}$.

El Hamiltoniano puede escribirse como una suma, en forma de sistema de Stäckel (ver [80]):

$$
H=\sum_{b=1}^{N} \frac{H_{b}}{f_{b}(\vec{\lambda})}
$$

con

$$
H_{b}=-2 A\left(\lambda_{b}\right) \pi_{b}^{2}-\frac{1}{2}\left(\lambda_{b}^{N+1}-(\alpha-1) \lambda_{b}^{N}+(1-\alpha+\beta) \lambda_{b}^{N-1}\right)
$$

de tal manera que la ecuación de Hamilton-Jacobi

$$
\frac{\partial \mathcal{S}}{\partial t}+H\left(\frac{\partial \mathcal{S}}{\partial \lambda_{1}}, \ldots, \frac{\partial \mathcal{S}}{\partial \lambda_{N}}, \lambda_{1}, \ldots, \lambda_{N}\right)=0
$$

es completamente separable, si bien de una manera no trivial. Demostraremos a continuación este hecho.

Si escribimos $\mathcal{S}$ de la forma:

$$
\mathcal{S}=-I_{1} t+\sum_{b=1}^{N} \mathcal{S}_{b}\left(\lambda_{b}\right)
$$

donde $I_{1}=H$, integral primera evidente del sistema, entonces (2.46) se reduce a:

$$
I_{1}=H\left(\frac{d \mathcal{S}_{1}}{d \lambda_{1}}, \ldots, \frac{d \mathcal{S}_{2}}{d \lambda_{N}} ; \lambda_{1}, \ldots, \lambda_{N}\right)=\sum_{a=1}^{N} \frac{H_{a}}{f_{a}(\vec{\lambda})}
$$

La ecuación (2.46) es entonces equivalente a un sistema de ecuaciones diferenciales ordinarias desacopladas:

$$
H_{b}\left(\frac{d \mathcal{S}_{b}}{d \lambda_{b}}, \lambda_{b}\right)=\eta_{1} \lambda_{b}^{N-1}+\eta_{2} \lambda_{b}^{N-2}+\ldots+\eta_{N-1} \lambda_{b}+\eta_{N}
$$

para cada $b=1, \ldots, N$, y donde:

$$
H_{b}\left(\frac{d \mathcal{S}_{b}}{d \lambda_{b}}, \lambda_{b}\right)=-2 A\left(\lambda_{b}\right)\left(\frac{d \mathcal{S}_{b}}{d \lambda_{b}}\right)^{2}-\frac{1}{2}\left(\lambda_{b}^{N+1}-(\alpha-1) \lambda_{b}^{N}+(1-\alpha+\beta) \lambda_{b}^{N-1}\right)
$$

\footnotetext{
${ }^{9}$ La notación utilizada habitualmente en la literatura es $\Lambda^{\prime}\left(\lambda_{b}\right)=\prod_{a=1, a \neq b}^{N}\left(\lambda_{a}-\lambda_{b}\right)$. Se ha elegido aquí la notación $f_{b}(\vec{\lambda})=\Lambda^{\prime}\left(\lambda_{b}\right)$, al igual que en la referencia [7], para enfatizar el hecho de que esta función depende de todas las componentes de $\vec{\lambda}$ y no sólo de $\lambda_{b}$.
} 
Esta equivalencia es fácilmente demostrable si se tiene en cuenta la identidad

$$
\sum_{b=1}^{N} \frac{H_{b}}{\Lambda^{\prime}\left(\lambda_{b}\right)}=I_{1}=\eta_{1} \sum_{b=1}^{N} \frac{\lambda_{b}^{N-1}}{\Lambda^{\prime}\left(\lambda_{b}\right)}+\eta_{2} \sum_{b=1}^{N} \frac{\lambda_{b}^{N-2}}{\Lambda^{\prime}\left(\lambda_{b}\right)}+\ldots+\eta_{N} \sum_{b=1}^{N} \frac{1}{\Lambda^{\prime}\left(\lambda_{b}\right)}
$$

que se deriva de las identidades deducidas en la Sección anterior a partir del Lema de Jacobi:

$$
\begin{aligned}
& \sum_{b=1}^{N} \frac{\lambda_{b}^{N-1}}{\Lambda^{\prime}\left(\lambda_{b}\right)}=1 \\
& \sum_{b=1}^{N} \frac{\lambda_{b}^{N-i}}{\Lambda^{\prime}\left(\lambda_{b}\right)}=0, \quad \forall i>1
\end{aligned}
$$

Las constantes $\eta_{a}, a=1, \ldots, N$ son, evidentemente, arbitrarias.

Hemos demostrado, por tanto, que las soluciones del sistema de ecuaciones (2.49), sustituidas en (2.47), conducen a una $\mathcal{S}$ solución de la Ecuación de HamiltonJacobi (2.46).

Es posible demostrar la proposición recíproca, es decir: las ecuaciones (2.49) son deducibles directamente de (2.48) y, por tanto, las soluciones que resulten de (2.49) son todas las soluciones de (2.46) de la forma (2.47). Veamos: si escribimos (2.48) de forma explícita:

$$
\begin{aligned}
& \frac{H_{1}\left(\lambda_{1}\right)}{\left(\lambda_{1}-\lambda_{2}\right)\left(\lambda_{1}-\lambda_{3}\right) \cdots\left(\lambda_{1}-\lambda_{N}\right)}+\frac{H_{2}\left(\lambda_{2}\right)}{\left(\lambda_{2}-\lambda_{1}\right)\left(\lambda_{2}-\lambda_{3}\right) \cdots\left(\lambda_{2}-\lambda_{N}\right)}+\cdots \\
& +\frac{H_{N}\left(\lambda_{N}\right)}{\left(\lambda_{N}-\lambda_{1}\right)\left(\lambda_{N}-\lambda_{2}\right) \cdots\left(\lambda_{N}-\lambda_{N-1}\right)}=I_{1}
\end{aligned}
$$

entonces cada uno de las funciones $H_{a}\left(\lambda_{a}\right)$ es de la forma (2.50) debido a la suposición (2.47). Multiplicando (2.53) por $\left(\lambda_{1}-\lambda_{2}\right)$ y tomando $\lambda_{1}=\lambda_{2}$ se obtiene que $H_{1}(\lambda)=H_{2}(\lambda)$ y, por simetría, todos $\operatorname{los} H_{a}(\lambda), a=1, \ldots, N$, serán idénticos. De esta manera es suficiente con calcular uno de ellos, por ejemplo $H_{1}(\lambda)$. Si multiplicamos $(2.53)$ por $f_{1}(\vec{\lambda})$ obtendremos:

$H_{1}\left(\lambda_{1}\right)+P_{2}(\vec{\lambda}) H_{2}\left(\lambda_{2}\right)+\cdots+P_{N}(\vec{\lambda}) H_{N}\left(\lambda_{N}\right)=I_{1}\left(\lambda_{1}-\lambda_{2}\right)\left(\lambda_{1}-\lambda_{3}\right) \cdots\left(\lambda_{1}-\lambda_{N}\right)$,

donde cada $P_{a}(\vec{\lambda}), a=2,3, \cdots, N$, es un polinomio de grado $N-1$ en $\lambda_{1}$. Derivando (2.54) $N$ veces con respecto a $\lambda_{1}$ tendremos

$$
\frac{d^{N} H_{1}\left(\lambda_{1}\right)}{d \lambda_{1}^{N}}=N ! I_{1}
$$


y así $H_{1}(\lambda)$ es necesariamente un polinomio de grado $N$ en $\lambda$ con $I_{1}$ como coeficiente del término de mayor grado, lo que concuerda con (2.49).

El conjunto de constantes de separación $\eta_{i}, i=1, \ldots, N$, es otro conjunto completo de integrales primeras. Su expresión en términos de las coordenadas elípticas del espacio de fases, $\lambda_{b}$ y $\pi_{b}=\frac{d \mathcal{S}_{b}}{d \lambda_{b}}$, se obtiene fácilmente resolviendo el sistema de ecuaciones (2.49), lineales en las incógnitas $\eta_{i}$, pues resulta ser un sistema de tipo Vandermonde.

Tenemos además otro conjunto de $N$ invariantes en involución: se trata de las $N-1$ raíces del polinomio

$$
\lambda_{a}^{N-1}+\frac{\eta_{a}}{I_{1}} \lambda_{a}^{N-2}+\ldots+\frac{\eta_{N-1}}{I_{1}} \lambda_{a}+\frac{\eta_{N}}{I_{1}}=\left(\lambda_{a}-F_{2}\right)\left(\lambda_{a}-F_{3}\right) \cdots\left(\lambda_{a}-F_{N}\right)
$$

más la energía $F_{1}=I_{1}$. Los dos conjuntos están relacionados por las expresiones: $\eta_{1}=I_{1} \mathrm{y}$

$$
\eta_{a}=(-1)^{a} I_{1} \sum_{i_{1}<i_{2}<\cdots<i_{a}} F_{i_{1}} F_{i_{2}} \ldots F_{i_{a}} ; \quad a=1,2, \ldots, N, \quad i_{\alpha}=2,3, \ldots, N
$$

Se concluye entonces que todas las constantes $\eta_{a}$ son proporcionales a $I_{1}$. De esta manera, si tenemos en cuenta las condiciones asintóticas (2.20), que era necesario imponer al sistema dinámico de cara a obtener soluciones de energía (de los campos, es decir acción del sistema mecánico) finita, tendremos $\left.I_{1}\right|_{t= \pm \infty}=0, \mathrm{y}$ al ser una constante del movimiento, $I_{1}=0, \forall t \in(-\infty,+\infty)$. Por tanto $\eta_{a}=0$, $\forall a=1, \ldots, N$.

El imponer $\eta_{a}=0, \forall a=1, \ldots, N$, convierte a las ecuaciones (2.49) en las que describen las trayectorias separatrices entre el movimiento acotado y el no acotado del sistema. Son estas separatrices las que están en correspondencia biunívoca (módulo la identificación $\mathbb{Z}^{\otimes N}$ ) con los kinks u ondas solitarias de la teoría de campos original.

Definiremos el polinomio $B\left(\lambda_{b}\right)$ de la forma

$$
B\left(\lambda_{b}\right)=\lambda_{b}^{N+1}-(\alpha-1) \lambda_{b}^{N}+\left(1-\alpha+\beta+2 \eta_{1}\right) \lambda_{b}^{N-1}+2 \eta_{2} \lambda_{b}^{N-2}+\cdots+2 \eta_{N}
$$

con el fin de escribir una expresión sencilla para $\mathcal{S}_{b}$ :

$$
\mathcal{S}_{b}\left(\lambda_{b}\right)=\frac{1}{2} \operatorname{sign}\left(\frac{d \mathcal{S}_{b}}{d \lambda_{b}}\right) \int \sqrt{\left|\frac{B\left(\lambda_{b}\right)}{A\left(\lambda_{b}\right)}\right|} d \lambda_{b}
$$

y así la solución general de la Ecuación de Hamilton-Jacobi será:

$$
\mathcal{S}=-\eta_{1} t+\sum_{b=1}^{N} \mathcal{S}_{b}=-\eta_{1} t+\sum_{b=1}^{N} \frac{1}{2} \operatorname{sign}\left(\frac{d \mathcal{S}_{b}}{d \lambda_{b}}\right) \int \sqrt{\left|\frac{B\left(\lambda_{b}\right)}{A\left(\lambda_{b}\right)}\right|} d \lambda_{b}
$$


La integración completa de estas cuadraturas, para cualesquiera valores de las constantes de separación, es un problema complejo y sólo resoluble utilizando funciones theta (de géneros dependientes del $N$ considerado, lógicamente). Sin embargo, los valores de las constantes que imponen las condiciones asintóticas simplifican las integrales de tal manera que se consigue sin dificultad la integración efectiva, como veremos en el próximo capítulo para el caso tridimensional. De hecho, la consideración del límite tendiendo a cero de las constantes de separación conduce a que alguno de los periodos de los que dependen las funciones theta tienda a infinito, con lo que las mismas son expresables en términos de arcotangentes hiperbólicos o, alternativamente, de combinaciones de logaritmos neperianos.

La acción restringida a las trayectorias correspondientes a los valores $\eta_{a}=0$, $\forall a=1, \ldots, N$, es finita, incluso en tiempo infinito ${ }^{10}$

$$
\tilde{\mathcal{S}}=\sum_{b=1}^{N} \tilde{\mathcal{S}}_{b}=\sum_{b=1}^{N} \frac{1}{2} \operatorname{sign}\left(\frac{d \tilde{\mathcal{S}}_{b}}{d \lambda_{b}}\right) \int_{\lambda_{b}(-\infty)}^{\lambda_{b}(+\infty)} \sqrt{\left|\frac{\tilde{B}\left(\lambda_{b}\right)}{A\left(\lambda_{b}\right)}\right|} d \lambda_{b}
$$

y su valor, como ya hemos demostrado en anteriores apartados, no es más que la energía del correspondiente solitón (salvo constantes multiplicativas).

La Teoría general de Hamilton-Jacobi nos determina la forma explícita de las trayectorias solución, así como de la dependencia temporal de las mismas, por medio de las ecuaciones:

$$
\gamma_{a}=\frac{\partial \mathcal{S}}{\partial \eta_{a}}, \quad a=1, \ldots, N
$$

donde $\gamma_{a}$ son constantes.

Sobre la hipersuperficie del espacio de fases determinada por $\eta_{1}=\cdots=\eta_{N}=0$, la primera ecuación del sistema,

$$
\gamma_{1}=-t+\sum_{a=1}^{N} \frac{1}{2} \operatorname{sign}\left(\pi_{a}\right) \int \frac{\lambda_{a}^{N-1} d \lambda_{a}}{\left|A\left(\lambda_{a}\right)\right|} \sqrt{\left|\frac{A\left(\lambda_{a}\right)}{\lambda_{a}^{N+1}-(\alpha-1) \lambda_{a}^{N}+(1-\alpha+\beta) \lambda_{a}^{N-1}}\right|}
$$

nos indica la dependencia temporal de la partícula en su movimiento a lo largo de su órbita, mientras que las otras $N-1$ ecuaciones,

$$
\gamma_{i}=\sum_{a=1}^{N} \frac{1}{2} \operatorname{sign}\left(\pi_{a}\right) \int \frac{\lambda_{a}^{N-i}}{\left|A\left(\lambda_{a}\right)\right|} d \lambda_{a} \cdot \sqrt{\left|\frac{A\left(\lambda_{a}\right)}{\lambda_{a}^{N+1}-(\alpha-1) \lambda_{a}^{N}+(1-\alpha+\beta) \lambda_{a}^{N-1}}\right|}
$$

\footnotetext{
${ }^{10} \mathrm{La}$ tilde sobre una letra significa que la función correspondiente debe ser evaluada considerando $\eta_{a}=0, \forall a=1, \ldots, N$.
} 


$$
i=2, \ldots, N
$$

determinan dicha órbita, la trayectoria de la partícula como intersección de $N-1$ hipersuperficies en el espacio de configuración.

Es interesante, por último, escribir las ecuaciones (2.58) y (2.59) en forma diferencial:

$$
\begin{aligned}
d t & =\sum_{a=1}^{N} \frac{\operatorname{sign}\left(\pi_{a}\right) \lambda_{a}^{N-1} d \lambda_{a}}{2 \sqrt{\left|A\left(\lambda_{a}\right)\left(\lambda_{a}^{N+1}-(\alpha-1) \lambda_{a}^{N}+(1-\alpha+\beta) \lambda_{a}^{N-1}\right)\right|}} \\
0 & =\sum_{a=1}^{N} \frac{\operatorname{sign}\left(\pi_{a}\right) \lambda_{a}^{N-i} d \lambda_{a}}{2 \sqrt{\left|A\left(\lambda_{a}\right)\left(\lambda_{a}^{N+1}-(\alpha-1) \lambda_{a}^{N}+(1-\alpha+\beta) \lambda_{a}^{N-1}\right)\right|}}
\end{aligned}
$$

para $i=2, \ldots, N$. Volveremos a analizar estas ecuaciones en el Capítulo 6 donde se deducirán por medio de la descomposición de Bogomolny que, como veremos, el sistema mecánico admite.

Antes de pasar al Capítulo tercero en el que estudiamos con detalle las soluciones $(2.58,2.59)$ para los casos $N=2$ y $N=3$, demostraremos que los valores de las constantes de movimiento deducidos de las condiciones asintóticas del sistema obligan a que las órbitas (2.59) del sistema dinámico estén restringidas necesariamente a una región finita del espacio $\mathbb{R}^{N}$ o, alternativamente, a una región finita del espacio "elíptico".

Para demostrar este hecho recordemos el hamiltoniano (2.45):

$$
H=\sum_{b=1}^{N} \frac{H_{b}}{f_{b}(\vec{\lambda})}
$$

con

$$
H_{b}=-2 A\left(\lambda_{b}\right) \pi_{b}^{2}-\frac{1}{2}\left(\lambda_{b}^{N+1}-(\alpha-1) \lambda_{b}^{N}+(1-\alpha+\beta) \lambda_{b}^{N-1}\right)
$$

Utilizando las identidades (2.35) no es difícil obtener una expresión de $H_{b}$ diferente a la anterior pero que sigue verificando la ecuación (2.45). Efectivamente, es posible escribir para $H$ la expresión:

$$
H=\sum_{b=1}^{N} \frac{\bar{H}_{b}}{f_{b}(\vec{\lambda})}
$$

donde ahora:

$$
\bar{H}_{b}=-2 A\left(\lambda_{b}\right) \pi_{b}^{2}-\frac{1}{2} A\left(\lambda_{b}\right) \frac{\lambda_{b}^{2}}{\lambda_{b}-1}
$$


Esta aparente arbitrariedad en la forma de elegir el potencial del modelo queda aclarada completamente, como hemos citado, si se consideran las identidades $(2.35)$ :

$$
\sum_{a=1}^{N} \frac{\lambda_{a}^{s}}{f_{a}(\vec{\lambda})}=0
$$

$\forall s<N-1$. Dado que la diferencia entre $H_{b}$ y $\bar{H}_{b}$ se reduce a un polinomio de grado $N-2$, la contribución de dicha diferencia al hamiltoniano $H$ es nula.

Si tomamos entonces en la ecuación (2.49) las funciones $\bar{H}_{b}$ e imponemos que las constantes $\eta_{b}$ se anulen para todo $b$, como prescriben las condiciones asintóticas, tendremos que $\bar{H}_{b}=0, \forall b=1, \ldots, N$, necesariamente para las soluciones del sistema en las que estamos interesados (soluciones de acción finita).

Por otro lado, las coordenadas elípticas $\vec{\lambda} \equiv\left(\lambda_{1}, \ldots, \lambda_{N}\right)$ varían en la región $P_{N}(\infty)$ determinada por las inecuaciones:

$$
-\infty<\lambda_{1}<\bar{\sigma}_{N}^{2}<\lambda_{2}<\bar{\sigma}_{N-1}^{2}<\cdots<\bar{\sigma}_{2}^{2}<\lambda_{N}<1
$$

Es decir, las coordenadas $\lambda_{2}, \ldots, \lambda_{N}$ están acotadas por definición. Fijemos nuestra atención por tanto en la coordenada $\lambda_{1}$. Según lo expuesto tendremos:

$$
\bar{H}_{1}=0 \Rightarrow \frac{1}{2} \pi^{2}+\frac{1}{8} \frac{\lambda_{1}^{2}}{\lambda_{1}-1}=0
$$

y esta ecuación, (2.63), puede interpretarse como la que describe la dinámica de una partícula con energía cero que se mueve bajo la influencia de un potencial de la forma:

$$
\begin{aligned}
V\left(\lambda_{1}\right)= & =\frac{1}{8} \frac{\lambda_{1}^{2}}{\lambda_{1}-1} & & -\infty<\lambda_{1}<\bar{\sigma}_{N}^{2} \\
& =\infty & & \bar{\sigma}_{N}^{2}<\lambda_{1}<\infty
\end{aligned}
$$

Como puede verse en la Figura 2.2, el potencial tiene un máximo en $\lambda_{1}=0$ y tiende a $-\infty$ cuando $\lambda_{1}$ tiende a $-\infty$; por tanto el movimiento acotado ocurre únicamente en el intervalo $\lambda_{1} \in\left[0, \bar{\sigma}_{N}^{2}\right]$.

Este razonamiento nos conduce a que las trayectorias kink del sistema están restringidas a la región finita $P_{N}(0) \subset P_{N}(\infty)$ dada por:

$$
0 \leq \lambda_{1}<\bar{\sigma}_{N}^{2}<\lambda_{2}<\bar{\sigma}_{N-1}^{2}<\cdots<\bar{\sigma}_{2}^{2}<\lambda_{N}<1
$$

El hiperplano $\lambda_{1}=0$ que "cierra" la región $P_{N}(0)$ se corresponde en coordenadas cartesianas con el hiperelipsoide:

$$
q_{1}^{2}+\frac{q_{2}^{2}}{\bar{\sigma}_{2}^{2}}+\frac{q_{3}^{2}}{\bar{\sigma}_{3}^{2}}+\cdots+\frac{q_{N}^{2}}{\bar{\sigma}_{N}^{2}}=1
$$




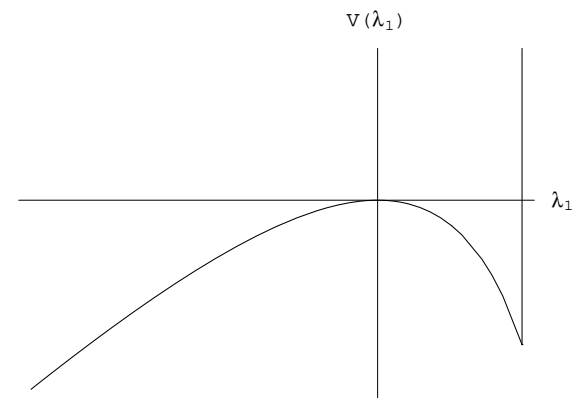

Figura 2.2: Gráfica del potencial $V\left(\lambda_{1}\right)$. 


\section{Capítulo 3}

\section{De la variedad de Kinks en el Modelo Sigma $O(3)$ Lineal Deformado}

En el capítulo anterior hemos resuelto de forma general la ecuación de HamiltonJacobi correspondiente al modelo que hemos denominado Modelo Sigma $O(N)$ Lineal Deformado. Dedicaremos este capítulo a particularizar dichos resultados a casos concretos. En primer lugar detallaremos la resolución del Modelo Sigma $O(2)$ Lineal Deformado (modelo MSTB) como paso previo al estudio del modelo $O(3)$. Como ya se ha comentado, el Modelo MSTB está muy estudiado en la literatura, la inclusión en esta memoria de los aspectos más relevantes del mismo, así como del análisis de su variedad de soluciones, está plenamente justificada si se tiene en cuenta que este modelo está contenido como submodelo dentro del caso $N=3$ y de una manera no trivial, como veremos.

Realizaremos un análisis detallado del Modelo $O(3)$ que revelará el hecho de que las soluciones recogidas en las ecuaciones (2.58) y (2.59), para valores reales de las constantes arbitrarias en ellas involucradas, no incluyen a la totalidad de las soluciones del sistema. Las razones de este comportamiento son, por un lado, la singularidad de las coordenadas elípticas sobre determinadas regiones del espacio y, por otro, la peculiar estructura del espacio de soluciones, que nos llevará a clasificar los kinks de cada modelo es tres categorías: embebidos, envolventes y genéricos. Demostraremos que todas las soluciones pueden ser descritas de una manera conjunta si se amplía el espacio de parámetros de que dependen incluyendo casos singulares que dan cuenta de las soluciones no genéricas.

Antes de abordar el estudio del Modelo $O(2)$ es recomendable, de alguna ma- 
nera, "empezar por el principio", es decir, comentar, aunque sea brevemente, el caso $N=1$. El Modelo Sigma $O(1)$ Lineal $^{1}$ no es otro que el conocido Modelo $\phi^{4}$ (ver [87] y [22]). El sistema mecánico análogo es evidentemente unidimensional y en consecuencia integrable.

El funcional de acción correspondiente al modelo será:

$$
S[\chi]=\int d^{2} y\left\{\frac{1}{2} \partial_{\mu} \chi \partial^{\mu} \chi-\frac{\lambda^{2}}{4}\left(\chi^{2}-\frac{m^{2}}{\lambda^{2}}\right)^{2}\right\}
$$

mientras que la acción del sistema mecánico análogo (escrita ya en variables sin dimensiones y obviando la constante multiplicativa) será:

$$
J[q]=\int d t\left\{\frac{1}{2}\left(\frac{d q}{d t}\right)^{2}+\frac{1}{2}\left(q^{2}-1\right)^{2}\right\}
$$

La única integral primera del sistema, su energía $I_{1}$, se escribe entonces en la forma:

$$
I_{1}=\frac{1}{2}\left(\frac{d q}{d t}\right)^{2}+\frac{1}{2}\left(q^{2}-1\right)^{2}
$$

y las condiciones asintóticas (2.20) nos imponen evidentemente que $I_{1}$ sea nula para las trayectorias de tipo kink.

$I_{1}=0$ conduce directamente a las cuadraturas:

$$
\int \frac{d q}{q^{2}-1}= \pm \int d t
$$

y, en consecuencia, a las soluciones:

$$
q(t)= \pm \tanh \left(t-t_{0}\right)
$$

Es habitual denominar solución TK1 (kink topológico de una componente) al kink $q(t)=\tanh \left(t-t_{0}\right)$, perteneciente al sector topológico $\mathcal{C}^{(-,+)}$, y ATK1 (antikink topológico de una componente) a $q(t)=-\tanh \left(t-t_{0}\right)$, de $\mathcal{C}^{(+,-)}$.

Comentaremos más detalles acerca de este tipo de kinks en las secciones siguientes.

\footnotetext{
${ }^{1}$ Nótese que para el Modelo $O(1)$ la distinción entre el modelo deformado y no deformado es innecesaria, ambos son equivalentes (siempre y cuando $\sigma^{2}$ sea menor que 2, puesto que en ese rango la deformación puede ser "absorbida" por una adecuada redefinición del campo $\chi$ ).
} 


\subsection{Modelo Sigma $O(2)$ Lineal Deformado}

Como ya hemos comentado, el Modelo Sigma $O(2)$ Lineal Deformado (o Modelo MSTB) está muy estudiado en la literatura. Ito, en [52], demostró la separabilidad de la ecuación de Hamilton-Jacobi del sistema mecánico asociado al modelo, utilizando para ello coordenadas elípticas bidimensionales (en su versión trigonométrica). En la referencia [4] se plantea dicha resolución en coordenadas elípticas bidimensionales de Euler y ahora presentaremos el planteamiento en coordenadas elípticas de Jacobi.

El interés de incluir esta sección dedicada al Modelo MSTB reside en que, por un lado, se trata de un buen primer acercamiento, en orden de complejidad, al caso $N=3$ que veremos posteriormente; se presentarán, además, pautas de comportamiento de las soluciones que se repetirán en modelos con mayor número de campos. Por otro lado, el Modelo MSTB está contenido dos veces en el Modelo $O(3)$ y, en consecuencia, está justificada su presencia en sí misma de cara a un análisis detallado de las soluciones de éste último.

La acción (2.2), para el caso $N=2$, se escribe:

$$
\begin{aligned}
S[\vec{\chi}]= & \int d^{2} y\left\{\frac{1}{2}\left(\partial_{\mu} \chi_{1} \cdot \partial^{\mu} \chi_{1}+\partial_{\mu} \chi_{2} \cdot \partial^{\mu} \chi_{2}\right)\right. \\
& \left.-\frac{\lambda^{2}}{4}\left(\chi_{1}^{2}+\chi_{2}^{2}-\frac{m^{2}}{\lambda^{2}}\right)^{2}-\frac{\beta_{1}^{2}}{4} \chi_{1}^{2}-\frac{\beta_{2}^{2}}{4} \chi_{2}^{2}\right\}
\end{aligned}
$$

que, en variables sin dimensiones (definidas en el capítulo anterior de forma general), será:

$$
\begin{gathered}
S[\vec{\Phi}]=\frac{m^{2}}{\lambda^{2}} \int_{-\infty}^{+\infty} d x^{0} \int_{-\infty}^{+\infty} d x^{1}\left\{\frac{1}{2} \partial_{\mu} \vec{\Phi} \cdot \partial^{\mu} \vec{\Phi}-V\left(\phi_{1}, \phi_{2}\right)\right\} \\
V\left(\phi_{1}, \phi_{2}\right)=\frac{1}{2}\left(\phi_{1}^{2}+\phi_{2}^{2}-1\right)^{2}+\frac{\sigma_{1}^{2}}{2} \phi_{1}^{2}+\frac{\sigma_{2}^{2}}{2} \phi_{2}^{2}
\end{gathered}
$$

De acuerdo con lo expuesto en el capítulo anterior podemos tomar sin pérdida de generalidad:

$$
\sigma_{1}=0<\sigma_{2}=\sigma
$$

y, además, $\sigma<1$ de cara a considerar el caso en el que el espacio de soluciones es más interesante ${ }^{2}$.

\footnotetext{
${ }^{2}$ Es fácil comprobar que para el caso $\sigma \geq 1$ las únicas soluciones de tipo onda solitaria que presenta el modelo son el kink y el antikink del Modelo $O(1)$ comentado anteriormente.
} 
Para las configuraciones estáticas, $\vec{\Phi}\left(x^{0}, x^{1}\right)=\vec{q}\left(x^{1}\right) \equiv\left(q_{1}\left(x^{1}\right), q_{2}\left(x^{1}\right)\right)$, el funcional de energía se escribe:

$$
\begin{gathered}
E[\vec{q}]=\frac{m^{3}}{\sqrt{2} \lambda^{2}} \int_{-\infty}^{+\infty} d x^{1}\left\{\frac{1}{2}\left(\left(\frac{d q_{1}}{d x^{1}}\right)^{2}+\left(\frac{d q_{2}}{d x^{1}}\right)^{2}\right)+V\left(q_{1}, q_{2}\right)\right\} \\
V\left(q_{1}, q_{2}\right)=\frac{1}{2}\left(q_{1}^{2}+q_{2}^{2}-1\right)^{2}+\frac{\sigma^{2}}{2} q_{2}^{2}
\end{gathered}
$$

de manera que el sistema mecánico análogo vendrá determinado por el funcional de acción:

$$
\begin{gathered}
J[\vec{q}]=\int_{-\infty}^{\infty} \mathcal{L}\left(\vec{q}, \frac{d \vec{q}}{d t}\right) d t \\
\mathcal{L}=\frac{1}{2}\left(\left(\frac{d q_{1}}{d t}\right)^{2}+\left(\frac{d q_{2}}{d t}\right)^{2}\right)-U\left(q_{1}, q_{2}\right)
\end{gathered}
$$

siendo $t \equiv x^{1}$ y $U\left(q_{1}, q_{2}\right)$ la función (ver Figura 3.1):

$$
U\left(q_{1}, q_{2}\right)=-\frac{1}{2}\left(q_{1}^{2}+q_{2}^{2}-1\right)^{2}-\frac{\sigma^{2}}{2} q_{2}^{2}
$$

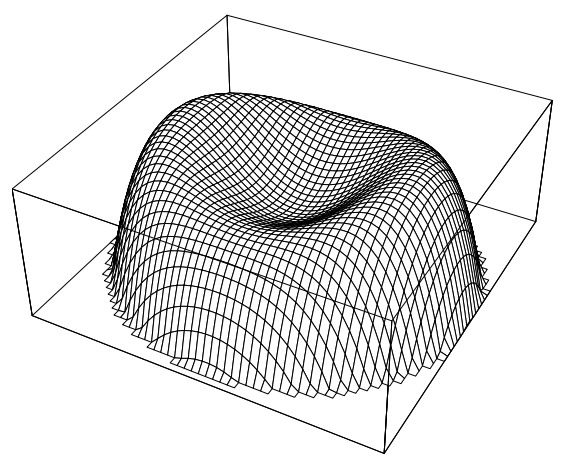

Figura 3.1: Representación gráfica del potencial "mecánico" $U\left(q_{1}, q_{2}\right)$.

El cambio de coordenadas (2.42) se escribirá, para este caso:

$$
q_{1}^{2}=\frac{1}{\sigma^{2}}\left(1-\mu_{1}\right)\left(1-\mu_{2}\right) ; \quad q_{2}^{2}=\frac{1}{\sigma^{2}}\left(\bar{\sigma}^{2}-\mu_{1}\right)\left(\bar{\sigma}^{2}-\mu_{2}\right)
$$

donde $\bar{\sigma}^{2}=1-\sigma^{2}$ y se han denotado $\left(\mu_{1}, \mu_{2}\right)$ a las coordenadas elípticas de Jacobi bidimensionales ${ }^{3}$.

\footnotetext{
${ }^{3}$ Para evitar confusiones reservaremos la notación $\lambda$ para el caso tridimensional.
} 
Evidentemente se tiene:

$$
-\infty<\mu_{1}<\bar{\sigma}^{2}<\mu_{2}<1
$$

y se trata de un cambio de coordenadas en el que se identifica el plano $\mathbb{R}^{2}$ con el paralelogramo infinito $P_{2}(\infty)$. El cambio no es biyectivo, como ya se ha comentado, la invarianza $\mathbb{Z}_{2} \times \mathbb{Z}_{2}$ conduce a que se trate de una aplicación $2^{2}$ a 1 . En la Figura 3.2 puede verse representado dicho cambio de coordenadas.
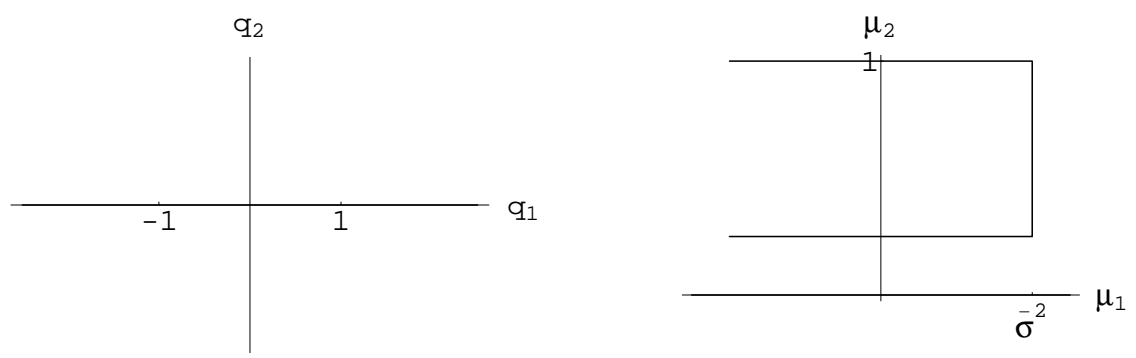

Figura 3.2: La aplicación de $\mathbb{R}^{2}$ en $P_{2}(\infty)$ debida al cambio de coordenadas (3.9).

Los razonamientos expuestos en el Capítulo 2 para el caso general pueden repetirse ahora para demostrar en que las soluciones de tipo kink del modelo se restringen en $P_{2}(\infty)$ a la región $P_{2}(0)$ determinada por las desigualdades:

$$
0 \leq \mu_{1}<\bar{\sigma}^{2}<\mu_{2}<1
$$

o, equivalentemente en el plano $\mathbb{R}^{2}$, a la elipse:

$$
q_{1}^{2}+\frac{q_{2}^{2}}{\bar{\sigma}^{2}}=1
$$

y su interior.

Tal y como se ha indicado en la Figura 3.3, hemos denotado por la letra D a los puntos de vacío (en elípticas es un único punto, mientras que en cartesianas: $\mathrm{D}_{1} \equiv \vec{v}^{-}, \mathrm{D}_{2} \equiv \vec{v}^{+}$), por F a los focos de la elipse (3.10) y por B a los puntos de la elipse determinados por su semieje menor (en la recta $q_{1}=0$ ).

Realizaremos la presentación de los diferentes tipos de kinks en este modelo (tanto para el caso que nos ocupa, con $N=2$, como para el caso general), de acuerdo con una clasificación natural, en las siguientes categorías:

- Kinks Embebidos: Llamaremos de manera general kinks embebidos a las ondas solitarias de un modelo que provienen de la inclusión en el mismo 

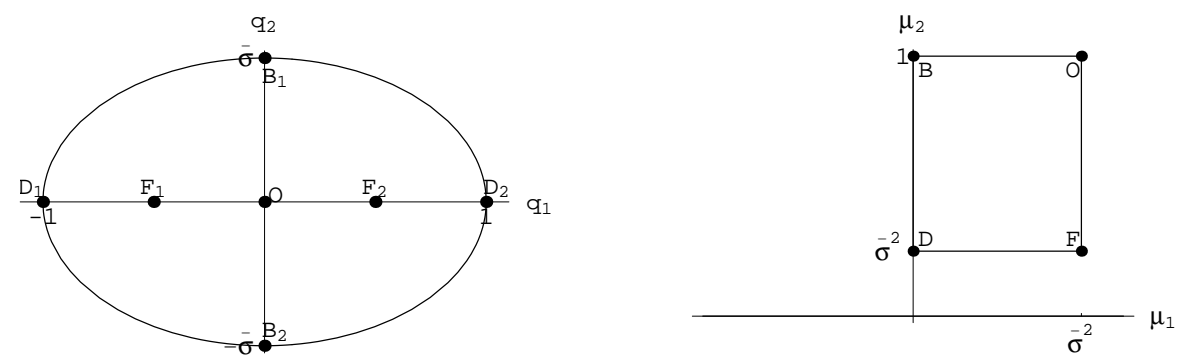

Figura 3.3: Elipse (3.10) en $\mathbb{R}^{2}$ y paralelogramo "elíptico" $P_{2}(0)$.

de otro modelo con un menor número de campos. Como veremos en las secciones siguientes, esta situación es común a los modelos procedentes de deformaciones del Modelo Sigma $O(N)$ Lineal.

- Kinks Envolventes: Hemos adoptado la denominación envolventes para denotar a aquellas ondas solitarias que aparecen sobre la curva (en general hipersuperficie) $\lambda_{1}=0$ que, como vimos, limitaba el "espacio elíptico" $P_{N}(\infty)$ a la región finita $P_{N}(0)$, en la que se desarrollaba la dinámica de las soluciones acotadas en los sistemas mecánicos asociados al modelo para los diferentes valores de $N$.

- Kinks Genéricos: Denominaremos kinks genéricos a las soluciones de la ecuación de Hamilton-Jacobi regulares, asociadas a valores finitos de las constantes de integración.

Una vez establecida esta clasificación, describamos la situación presente en el Modelo $O(2)$ :

\section{Kinks Embebidos}

Los kinks del caso $N=1$ están presentes en el modelo correspondiente a $N=2$ de una manera trivial. Si restringimos el sistema a la recta determinada por la ecuación $q_{2}=0$, tenemos entonces que el sistema dinámico se reduce al característico del caso $N=1$. De esta manera encontramos las soluciones TK1 y ATK1 (kink y antikink topológicos de una componente "cartesiana" no nula):

$$
\vec{q}^{\mathrm{TK} 1}(t)=\left(\begin{array}{c}
\tanh t \\
0
\end{array}\right) ; \quad \vec{q}^{\mathrm{ATK} 1}(t)=\left(\begin{array}{c}
-\tanh t \\
0
\end{array}\right)
$$


En coordenadas elípticas ${ }^{4}$, el kink TK1 recorre dos tramos de la frontera de $P_{2}(0)$ :

I. Arista DF:

$$
\vec{\mu}_{\mathrm{I}}^{\mathrm{TK} 1}(t)=\left(\begin{array}{c}
1-\tanh ^{2}( \pm t) \\
\bar{\sigma}^{2}
\end{array}\right)
$$

recorrida dos veces, de acuerdo con los rangos de variación de $t$ siguientes:

$$
t \in(-\infty, \operatorname{arctanh}(\mp \sigma)] \sqcup[\operatorname{arctanh}( \pm \sigma), \infty)
$$

II. Arista FO:

$$
\vec{\mu}_{\mathrm{II}}^{\mathrm{TK} 1}(t)=\left(\begin{array}{c}
\bar{\sigma}^{2} \\
1-\tanh ^{2}(\mp t)
\end{array}\right)
$$

De nuevo se recorre dos veces la arista, para los valores de $t$ pertenecientes al intervalo:

$$
t \in[\operatorname{arctanh}(\mp \sigma), \operatorname{arctanh}( \pm \sigma)]
$$

(ver Figura 3.4). En las expresiones (3.11), (3.12) y (3.13) se ha escogido, por simplicidad, que la constante $t_{0}$ sea nula. Mantendremos esta consideración en lo sucesivo, a menos que se especifique lo contrario.
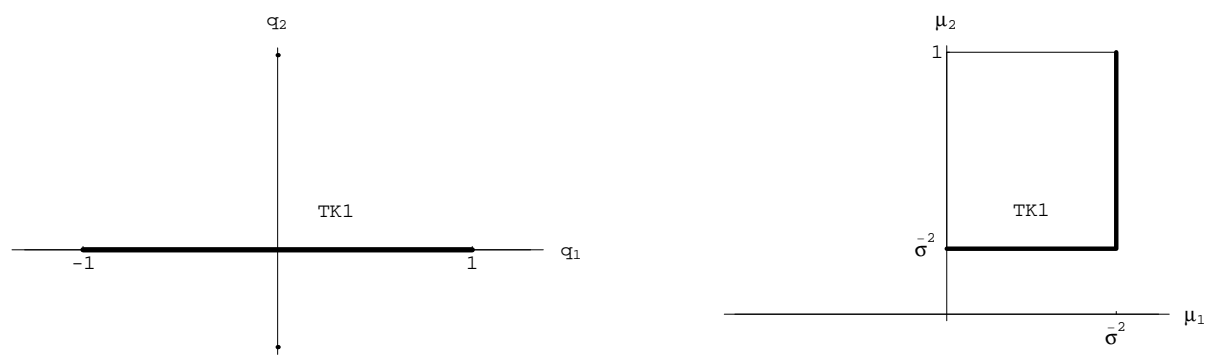

Figura 3.4: Trayectoria del kink TK1 representada en coordenadas cartesianas y elípticas.

\footnotetext{
${ }^{4}$ En coordenadas elípticas, ambos kinks, TK1 y ATK1, son indistinguibles debido a la simetría. Los denominaremos conjuntamente TK1 y mantendremos este criterio en los kinks sucesivos de este modelo y del correspondiente a $N=3$, a pesar del evidente abuso de notación en el que incurrimos.
} 


\section{Kinks Envolventes}

Los primeros kinks genuinos del modelo $O(2)$ aparecen sobre otra de las aristas del borde de $P_{2}(0)$, DB. Si consideramos ahora $\mu_{1}=0$ como órbita, es decir la elipse (3.10), tendremos que:

$$
\vec{q}^{\mathrm{TK} 2}(t)=\left(\begin{array}{c}
q_{1}^{\mathrm{TK} 2}(t) \\
q_{2}^{\mathrm{TK} 2}(t)
\end{array}\right)=\left(\begin{array}{c}
\tanh ( \pm \sigma t) \\
\pm \bar{\sigma} \operatorname{sech}( \pm \sigma t)
\end{array}\right)
$$

son kinks topológicos de dos componentes no nulas. Teniendo en cuenta las diferentes combinaciones de signos que pueden darse, encontramos en total cuatro kinks con denominación genérica TK2. Siguiendo la notación habitual en la literatura, podemos particularizar los kinks del tipo TK2 en la siguiente forma: llamaremos kink TK2 propiamente dicho al correspondiente al sector topológico $\mathcal{C}^{(-,+)}$y en el que se considera la segunda componente positiva, es decir sobre la semielipse $q_{2}=+\bar{\sigma} \sqrt{1-q_{1}^{2}}$. Análogamente, llamaremos TK2* al kink perteneciente al mismo sector pero definido sobre $q_{2}=-\bar{\sigma} \sqrt{1-q_{1}^{2}}$. Los antikinks asociados, en $\mathcal{C}^{(+,-)}$, recibirán los nombres de ATK2 y ATK2*, respectivamente.

En coordenadas elípticas, todos ellos (debido a la invarianza $\mathbb{Z}_{2} \times \mathbb{Z}_{2}$ ) se representan como un único tramo:

$$
\vec{\mu}^{\mathrm{TK} 2}(t)=\left(\begin{array}{c}
0 \\
1-\sigma^{2} \tanh ^{2} \sigma t
\end{array}\right)
$$

recorrido dos veces (la trayectoria completa se obtiene al considerar la sucesión de aristas DB+BD). Ver Figura 3.5.
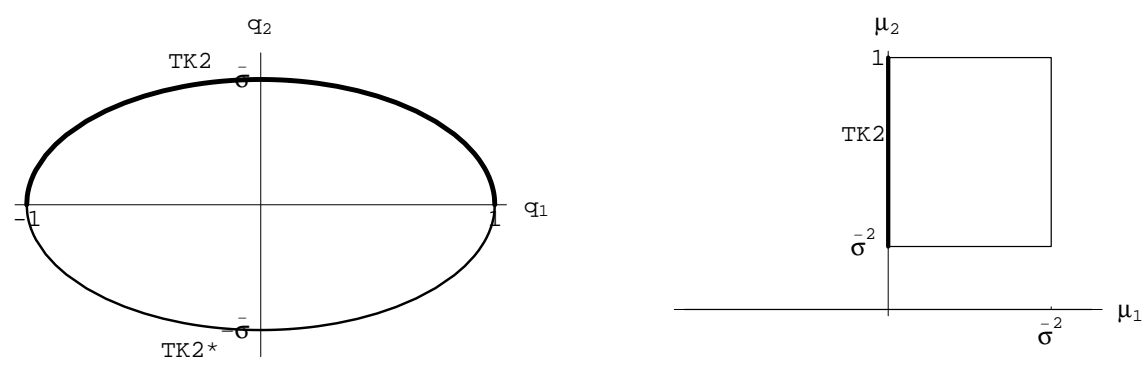

Figura 3.5: Trayectorias de los kinks del tipo TK2 en coordenadas cartesianas y en elípticas. 


\section{Kinks Genéricos}

Los kinks genéricos del modelo $N=2$ se obtienen integrando la ecuación de Hamilton-Jacobi para este caso. El Hamiltoniano se escribe ahora de la forma

$$
H=\frac{1}{2} \frac{-4\left(\mu_{1}-1\right)\left(\mu_{1}-\bar{\sigma}^{2}\right) \pi_{1}^{2}-4\left(1-\mu_{2}\right)\left(\mu_{2}-\bar{\sigma}^{2}\right) \pi_{2}^{2}-\left(\mu_{1}^{3}-\bar{\sigma}^{2} \mu_{1}^{2}\right)+\left(\mu_{2}^{3}-\bar{\sigma}^{2} \mu_{2}^{2}\right)}{\mu_{1}-\mu_{2}}
$$

y la integración de (2.59) conduce, tras simplicaciones evidentes, a la ecuación de las trayectorias

$$
\begin{aligned}
& \left(\left|\frac{\sqrt{1-\mu_{1}}-\sigma}{\sqrt{1-\mu_{1}}+\sigma}\right| \cdot\left|\frac{\sqrt{1-\mu_{1}}+1}{\sqrt{1-\mu_{1}}-1}\right|^{\sigma}\right)^{\operatorname{sign}\left(\pi_{1}\right)} \cdot \\
& \left(\left|\frac{\sqrt{1-\mu_{2}}-\sigma}{\sqrt{1-\mu_{2}}+\sigma}\right| \cdot\left|\frac{\sqrt{1-\mu_{2}}+1}{\sqrt{1-\mu_{2}}-1}\right|^{\sigma}\right)^{\operatorname{sign}\left(\pi_{2}\right)}=e^{2 \sigma \bar{\sigma}^{2} \gamma_{2}}
\end{aligned}
$$

parametrizadas por la constante de integración real $\gamma_{2}$.
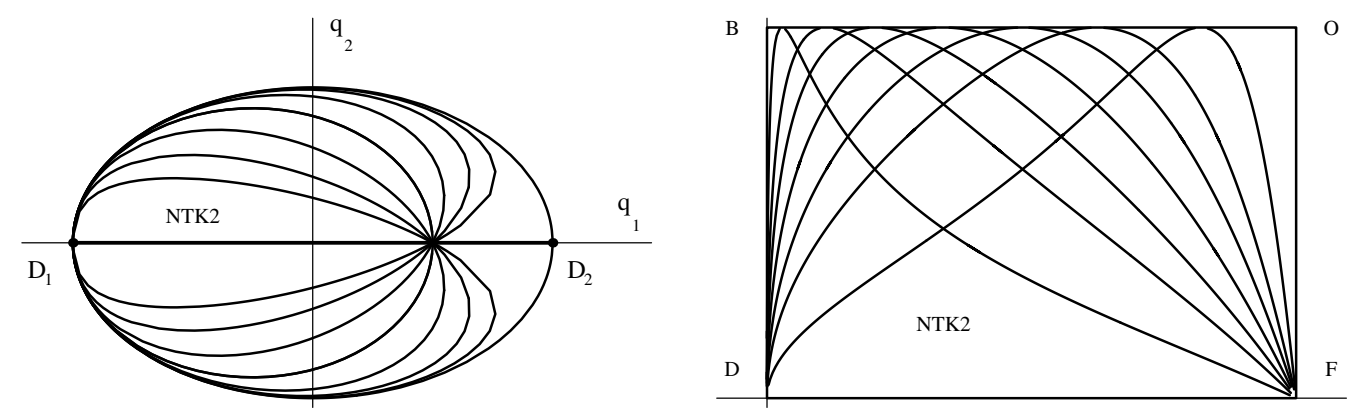

Figura 3.6: Varias trayectorias kink del tipo NTK2 en coordenadas cartesianas y en elípticas.

La dependencia temporal viene dada por la integración de (2.58), que proporciona la expresión:

$$
\left|\frac{\sqrt{1-\mu_{1}}-\sigma}{\sqrt{1-\mu_{1}}+\sigma}\right|^{\bar{\sigma}^{2} \operatorname{sign}\left(\pi_{1}\right)} \cdot\left|\frac{\sqrt{1-\mu_{2}}-\sigma}{\sqrt{1-\mu_{2}}+\sigma}\right|^{\bar{\sigma}^{2} \operatorname{sign}\left(\pi_{2}\right)}=e^{2\left(\gamma_{1}+t\right) \sigma}
$$

La constante $\gamma_{1}$ equivale, evidentemente, a la denominada $t_{0}$ en la descripción del Modelo $O(1)$ y su presencia en la ecuación (3.17) representa la invarianza de las soluciones bajo la acción del grupo de traslaciones temporales.

Los kinks descritos por las ecuaciones (3.16) y (3.17) en coordenadas elípticas no son fácilmente expresables en coordenadas cartesianas, dada la complejidad de 
las mismas. Es posible, a pesar de ello, analizar su comportamiento en el plano "cartesiano" $\mathbb{R}^{2}$.

Evidentemente la distinción entre kinks topológicos y no-topológicos carece de sentido desde el punto de vista de las coordenadas elípticas. Se han representado algunas de las trayectorias descritas por la ecuación (3.16) en la Figura 3.6 (b); como puede observarse en la misma, cada una de las soluciones se compone de cuatro tramos diferenciados por las distintas posibilidades de elección de los signos correspondientes. Su "traducción" a coordenadas cartesianas requiere la imposición de dos requisitos: la trayectoria ha de ser continua y además diferenciable en todo punto de la misma. Si se elige entonces, arbitrariamente, el punto $\mathrm{D}_{1}=\vec{v}^{-}$como punto inicial y el cuadrante $\left(q_{1}<0, q_{2}>0\right)$ como región a la que pertenece el primer tramo (el asociado a los $\left.\operatorname{signos} \operatorname{sign}\left(\pi_{1}\right)=\operatorname{sign}\left(\pi_{2}\right)=+1\right)$, entonces dichos requerimientos obligan a que las trayectorias sean similares a las representadas en la Figura 3.6 (a). Se trata, en consecuencia, de kinks no topológicos de dos componentes cartesianas no nulas y se les denomina, por tanto, NTK2.

Desde el punto de vista de las coordenadas elípticas tenemos que un kink NTK2 genérico parte del punto de vacío D y en un primer tramo (determinado por la elección citada de signos en (3.16)) alcanza la arista OB, para continuar hasta el punto F, foco de la elipse (3.10). Las dos posibilidades de elección de signos restantes reproducen en $P_{2}(0)$ la misma situación, retornando finalmente al punto de vacío original.

La parametrización de la familia NTK2 en términos de la constante de integración $\gamma_{2}$ tiene un significado geométrico claro. En coordenadas cartesianas $\gamma_{2}$ está relacionada con la tangente del ángulo de incidencia de la solución NTK2 tomada sobre el punto focal $\mathrm{F}$ de la forma:

$$
\tan \theta= \pm \frac{2 e^{\sigma \bar{\sigma}^{2} \gamma_{2}}}{1-e^{2 \sigma \bar{\sigma}^{2} \gamma_{2}}}
$$

(ver [3] para detalles). De esta manera, cada valor $\gamma_{2}$ fijado determina una trayectoria y un ángulo de incidencia concreto sobre el punto focal.

Es posible expresar los kinks embebidos y los kinks envolventes como soluciones genéricas si consideramos incluidas en las mismas los límites en los cuales la constante $\gamma_{2}$ de la ecuación (3.16) tiende a sus valores asintóticos $\pm \infty$.

Dada la libertad en la elección de los signos ambos límites son equivalentes. De esta manera consideremos $\gamma_{2} \rightarrow-\infty$. El primer miembro de (3.16) es nulo si se tienen las siguientes situaciones: 
- (a) $\operatorname{sign}\left(\pi_{1}\right)=-1, \mu_{1}=0$, para los dos posibles signos de $\pi_{2}$. Tenemos entonces, en coordenadas cartesianas, los cuatro kinks del tipo TK2.

- (b) $\operatorname{sign}\left(\pi_{1}\right)=+1, \mu_{1}=\bar{\sigma}^{2}$, con las dos elecciones $\operatorname{sign}\left(\pi_{2}\right)= \pm 1$ nos determina el tramo del kink TK1 que yace en la arista FO.

$\operatorname{sign}\left(\pi_{2}\right)=+1, \mu_{2}=\bar{\sigma}^{2}$ dos veces $\left(\operatorname{sign}\left(\pi_{1}\right)= \pm 1\right)$, el otro tramo del kink TK1, sobre DF. En coordenadas cartesianas tenemos por tanto el TK1 completo.

De acuerdo con la interpretación geométrica antedicha de la constante $\gamma_{2}$, los límites asintóticos calculados concuerdan perfectamente con la intuición geométrica del problema: el límite $\gamma \rightarrow \pm \infty$ conduce en la ecuación (3.18) a que $\tan \theta \rightarrow 0$, de manera que el ángulo de incidencia tiende a cero, es decir los kinks NTK2 en el límite tienden, en su primera parte, al kink TK1. Consecuentemente su continuación tiende a un kink TK2 del sector topológico contrario (ver Figura 3.6). Estas consideraciones se plasman completamente si analizamos las energías de los diferentes tipos de kinks del modelo.

Como ya se ha explicado, la energía de cada uno de los kinks equivale (salvo constantes multiplicativas) al valor de la acción del sistema mecánico sobre la solución correspondiente. De esta forma tendremos, teniendo en cuenta el número de veces que cada intervalo de variación de las coordenadas elípticas es recorrido:

$$
\begin{aligned}
E_{\mathrm{NTK} 2} & =\frac{m^{3}}{\lambda^{2} \sqrt{2}}\left(4 \int_{\bar{\sigma}^{2}}^{1} d \mu_{2} \frac{\mu_{2}}{2 \sqrt{1-\mu_{2}}}+2 \int_{0}^{\bar{\sigma}^{2}} d \mu_{1} \frac{\mu_{1}}{2 \sqrt{1-\mu_{1}}}\right)= \\
& =\frac{m^{3}}{\lambda^{2} \sqrt{2}}\left(2 \sigma\left(1-\frac{\sigma^{2}}{3}\right)+\frac{4}{3}\right)
\end{aligned}
$$

mientras que:

$$
\begin{aligned}
& E_{\mathrm{TK} 1}=\frac{m^{3}}{\lambda^{2} \sqrt{2}}\left(2 \int_{0}^{\bar{\sigma}^{2}} d \mu_{1} \frac{\mu_{1}}{2 \sqrt{1-\mu_{1}}}+2 \int_{\bar{\sigma}^{2}}^{1} d \mu_{2} \frac{\mu_{2}}{2 \sqrt{1-\mu_{2}}}\right)=\frac{m^{3}}{\lambda^{2} \sqrt{2}} \frac{4}{3} \\
& E_{\mathrm{TK} 2}=\frac{m^{3}}{\lambda^{2} \sqrt{2}}\left(2 \int_{\bar{\sigma}^{2}}^{1} d \mu_{2} \frac{\mu_{2}}{2 \sqrt{1-\mu_{2}}}\right)=\frac{m^{3}}{\lambda^{2} \sqrt{2}} 2 \sigma\left(1-\frac{\sigma^{2}}{3}\right)
\end{aligned}
$$

Y tenemos por tanto demostrada la regla de suma de las energías:

$$
E_{\mathrm{NTK} 2}=E_{\mathrm{TK} 2}+E_{\mathrm{TK} 1}
$$

ya comentada. 
Queda así probado que la combinación de kinks TK2+TK1 puede interpretarse como un miembro de la familia uniparamétrica NTK2, correspondiente al valor $\gamma_{2} \rightarrow \pm \infty$ del parámetro.

Con el objetivo de sintetizar la presentación de las soluciones del modelo en estudio, definiremos el Espacio Modular de Kinks como el espacio de soluciones de tipo onda solitaria cociente por la acción del grupo de simetría propio del sistema.

Hemos comentado ya que el grupo de simetría del Modelo MSTB es $G=$ $\mathbb{Z}_{2} \times \mathbb{Z}_{2}$. El cambio de coordenadas cartesianas a elípticas, por su parte, lleva aparejada una invarianza bajo la acción de $G$, de tal manera que dicho cambio es un isomorfismo entre $\mathbb{R}^{2} / G$ y $P_{2}(\infty)$.

La conclusión evidente del razonamiento anterior es que si se considera el espacio de soluciones kink desde el punto de vista de las coordenadas elípticas, entonces el cociente bajo la acción de $G$ ya está intrínsecamente tomado y, en definitiva, dicho espacio coincide con el Espacio Modular de Kinks del modelo. Este hecho es trivialmente generalizable a dimensiones superiores dado que, para cualquier $N$ que se considere, el grupo de simetría interna del Modelo Sigma $O(N)$ Lineal Deformado es $G=\mathbb{Z}_{2}^{\times N}$, y exactamente el mismo grupo es el que determina la simetría del cambio de coordenadas $N$-dimensional.

El espacio de soluciones kink del Modelo MSTB, inicialmente isomorfo a la recta real $\mathbb{R}$ dada por los valores de la constante $\gamma_{2}$ puede ser, como hemos visto, completado por el infinito, y donde además se identifican las soluciones que aparecen en $+\infty$ y $-\infty$. El resultado (ver [67] y [68] para detalles) entonces es evidentemente homeomorfo a la esfera $S^{1}$. Concluimos por tanto que el espacio modular de soluciones kink del Modelo Sigma Lineal $O(2)$ Deformado es:

$$
\mathcal{M} \simeq S^{1}
$$

\subsection{Modelo Sigma $O(3)$ Deformado}

Estudiaremos en esta sección el caso $N=3$ del Modelo Sigma $O(N)$ Lineal Deformado que hemos considerado de manera general en el Capítulo 2. Como veremos, la pauta de comportamiento marcada por el Modelo Sigma $O(2)$ Lineal Deformado se repetirá en este caso. De esta manera el Modelo $O(3)$ presentará tres tipos diferentes de soluciones kink: los kinks embebidos (el Modelo $O(2)$ ), los kinks envolventes (en este caso sobre la superficie del elipsoide de ecuación $\lambda_{1}=0$ ) y los kinks genéricos. 
El Modelo $O(2)$ aparecerá embebido dos veces en el $O(3)$, como veremos. Sin entrar en detalle, vislumbraremos más tarde una estructura similar de inmersión del caso $N-1$ en el $N$. Es adecuado, por tanto, analizar detenidamente el caso $N=$ 3 , pues todos los aspectos significativos del caso general quedarán aquí recogidos, si bien con una estructura de soluciones mucho más compleja que la que obtuvimos para el caso $N=2$.

\subsubsection{Solución General de la Ecuación de Hamilton-Jacobi}

La expresión de la función Hamiltoniana (2.21) del sistema, en las coordenadas originales o "cartesianas", será, para el caso $N=3$ :

$$
H=\frac{1}{2}\left(p_{1}^{2}+p_{2}^{2}+p_{3}^{2}\right)-\frac{1}{2}\left(q_{1}^{2}+q_{2}^{2}+q_{3}^{2}-1\right)^{2}-\frac{\sigma_{2}^{2}}{2} q_{2}^{2}-\frac{\sigma_{3}^{2}}{2} q_{3}^{2}
$$

donde ya se ha tomado el valor $\sigma_{1}=0$, de acuerdo con la elección (2.9). En coordenadas elípticas tridimensionales, el Hamiltoniano (3.21) se escribirá en la forma:

$$
H=\sum_{a=1}^{3} \frac{1}{f_{a}(\vec{\lambda})} H_{a}
$$

donde

$$
H_{a}=-2 A\left(\lambda_{a}\right) \pi_{a}^{2}-\frac{1}{2}\left(\lambda_{a}^{2}\left(\lambda_{a}-\bar{\sigma}_{2}^{2}\right)\left(\lambda_{a}-\bar{\sigma}_{3}^{2}\right)\right)
$$

que no es más que la expresión (2.44) para el caso $N=3$, y donde se ha de tener en cuenta que ahora: $\bar{\sigma}_{2}^{2}=1-\sigma_{2}^{2}, \bar{\sigma}_{3}^{2}=1-\sigma_{3}^{2}$, y

$$
\alpha=3-\sigma_{2}^{2}-\sigma_{3}^{2}=1+\bar{\sigma}_{2}^{2}+\bar{\sigma}_{3}^{2}, \quad \beta=\bar{\sigma}_{2}^{2} \bar{\sigma}_{3}^{2}+\bar{\sigma}_{2}^{2}+\bar{\sigma}_{3}^{2}
$$

mientras que las funciones $A\left(\lambda_{a}\right)$ y $f_{a}(\vec{\lambda})=\Lambda^{\prime}\left(\lambda_{a}\right)$ se reducen, en tres dimensiones, a:

$$
\begin{gathered}
A\left(\lambda_{a}\right)=\left(\lambda_{a}-1\right)\left(\lambda_{a}-\bar{\sigma}_{2}^{2}\right)\left(\lambda_{a}-\bar{\sigma}_{3}^{2}\right) \\
f_{1}(\vec{\lambda})=\left(\lambda_{1}-\lambda_{2}\right)\left(\lambda_{1}-\lambda_{3}\right) ; f_{2}(\vec{\lambda})=\left(\lambda_{2}-\lambda_{1}\right)\left(\lambda_{2}-\lambda_{3}\right) ; f_{3}(\vec{\lambda})=\left(\lambda_{3}-\lambda_{1}\right)\left(\lambda_{3}-\lambda_{2}\right)
\end{gathered}
$$

Las dos ecuaciones de las trayectorias, (2.59), serán en este caso:

$$
\begin{aligned}
& \gamma_{2}=\sum_{a=1}^{3} \frac{1}{2} \operatorname{sign}\left(\pi_{a}\right) \int \frac{d \lambda_{a}}{\left|\left(\lambda_{a}-\bar{\sigma}_{2}^{2}\right)\left(\lambda_{a}-\bar{\sigma}_{3}^{2}\right)\right| \sqrt{1-\lambda_{a}}} \\
& \gamma_{3}=\sum_{a=1}^{3} \frac{1}{2} \operatorname{sign}\left(\pi_{a}\right) \int \frac{d \lambda_{a}}{\lambda_{a}\left|\left(\lambda_{a}-\bar{\sigma}_{2}^{2}\right)\left(\lambda_{a}-\bar{\sigma}_{3}^{2}\right)\right| \sqrt{1-\lambda_{a}}}
\end{aligned}
$$


cuya integración y simplificación conduce a las expresiones:

$$
\begin{aligned}
C_{2}= & \left|\frac{\sqrt{1-\lambda_{1}}+\sigma_{2}}{\sqrt{1-\lambda_{1}}-\sigma_{2}}\right|^{\sigma_{3} \operatorname{sign}\left(\pi_{1}\right)} \cdot\left|\frac{\sqrt{1-\lambda_{1}}-\sigma_{3}}{\sqrt{1-\lambda_{1}}+\sigma_{3}}\right|^{\sigma_{2} \operatorname{sign}\left(\pi_{1}\right)} . \\
& \left|\frac{\sqrt{1-\lambda_{2}}+\sigma_{2}}{\sqrt{1-\lambda_{2}}-\sigma_{2}}\right|^{\sigma_{3} \operatorname{sign}\left(\pi_{2}\right)} \cdot\left|\frac{\sqrt{1-\lambda_{2}}-\sigma_{3}}{\sqrt{1-\lambda_{2}}+\sigma_{3}}\right|^{\sigma_{2} \operatorname{sign}\left(\pi_{2}\right)} . \\
& \left|\frac{\sqrt{1-\lambda_{3}}+\sigma_{2}}{\sqrt{1-\lambda_{3}}-\sigma_{2}}\right|^{\sigma_{3} \operatorname{sign}\left(\pi_{3}\right)} \cdot\left|\frac{\sqrt{1-\lambda_{3}}-\sigma_{3}}{\sqrt{1-\lambda_{3}}+\sigma_{3}}\right|^{\sigma_{2} \operatorname{sign}\left(\pi_{3}\right)}
\end{aligned}
$$

donde $C_{2}=\exp \left\{2 \gamma_{2} \sigma_{2} \sigma_{3}\left(\sigma_{2}^{2}-\sigma_{3}^{2}\right)\right\}$ es constante y:

$$
\begin{aligned}
C_{3}= & \left|\frac{\sqrt{1-\lambda_{1}}-1}{\sqrt{1-\lambda_{1}}+1}\right|^{\sigma_{2} \sigma_{3}\left(\sigma_{2}^{2}-\sigma_{3}^{2}\right) \operatorname{sign}\left(\pi_{1}\right)} \cdot\left|\frac{\sqrt{1-\lambda_{1}}+\sigma_{2}}{\sqrt{1-\lambda_{1}}-\sigma_{2}}\right|^{\sigma_{3} \bar{\sigma}_{3}^{2} \operatorname{sign}\left(\pi_{1}\right)} . \\
& \left|\frac{\sqrt{1-\lambda_{1}}-\sigma_{3}}{\sqrt{1-\lambda_{1}}+\sigma_{3}}\right|^{\sigma_{2} \bar{\sigma}_{2}^{2} \operatorname{sign}\left(\pi_{1}\right)} \cdot\left|\frac{\sqrt{1-\lambda_{2}}-1}{\sqrt{1-\lambda_{2}}+1}\right|^{\sigma_{2} \sigma_{3}\left(\sigma_{2}^{2}-\sigma_{3}^{2}\right) \operatorname{sign}\left(\pi_{2}\right)} . \\
& \left|\frac{\sqrt{1-\lambda_{2}}+\sigma_{2}}{\sqrt{1-\lambda_{2}}-\sigma_{2}}\right|^{\sigma_{3} \bar{\sigma}_{3}^{2} \operatorname{sign}\left(\pi_{2}\right)} \cdot\left|\frac{\sqrt{1-\lambda_{2}}-\sigma_{3}}{\sqrt{1-\lambda_{2}}+\sigma_{3}}\right|^{\sigma_{2} \bar{\sigma}_{2}^{2} \operatorname{sign}\left(\pi_{2}\right)} \cdot\left|\frac{\sqrt{1-\lambda_{3}}+\sigma_{2}}{\sqrt{1-\lambda_{3}}-\sigma_{2}}\right|^{\sigma_{3} \bar{\sigma}_{3}^{2} \operatorname{sign}\left(\pi_{3}\right)} . \\
& \left|\frac{\sqrt{1-\lambda_{3}}-1}{\sqrt{1-\lambda_{3}}+1}\right|^{\sigma_{2} \sigma_{3}\left(\sigma_{2}^{2}-\sigma_{3}^{2}\right) \operatorname{sign}\left(\pi_{3}\right)} . \\
& \left|\frac{\sqrt{1-\lambda_{3}}-\sigma_{3}}{\sqrt{1-\lambda_{3}}+\sigma_{3}}\right|^{\sigma_{2} \bar{\sigma}_{2}^{2} \operatorname{sign}\left(\pi_{3}\right)}
\end{aligned}
$$

con $C_{3}=\exp \left\{2 \gamma_{3} \sigma_{2} \sigma_{3} \bar{\sigma}_{2}^{2} \bar{\sigma}_{3}^{2}\left(\sigma_{2}^{2}-\sigma_{3}^{2}\right)\right\}$.

La dependencia temporal de la partícula en cada trayectoria viene marcada por la integración de la cuadratura (2.58) que, en este caso tridimensional, se escribe en la forma:

$$
\gamma_{1}+t=\sum_{a=1}^{3} \frac{1}{2} \operatorname{sign}\left(\pi_{a}\right) \int \frac{\lambda_{a} d \lambda_{a}}{\left|\left(\lambda_{a}-\bar{\sigma}_{2}^{2}\right)\left(\lambda_{a}-\bar{\sigma}_{3}^{2}\right)\right| \sqrt{1-\lambda_{a}}}
$$

y que conduce a la expresión siguiente:

$$
\begin{aligned}
C_{1}(t)= & \left|\frac{\sqrt{1-\lambda_{1}}-\sigma_{2}}{\sqrt{1-\lambda_{1}}+\sigma_{2}}\right|^{\sigma_{3} \bar{\sigma}_{2}^{2} \operatorname{sign}\left(\pi_{1}\right)} \cdot\left|\frac{\sqrt{1-\lambda_{1}}+\sigma_{3}}{\sqrt{1-\lambda_{1}}-\sigma_{3}}\right|^{\sigma_{2} \bar{\sigma}_{3}^{2} \operatorname{sign}\left(\pi_{1}\right)} . \\
& \left|\frac{\sqrt{1-\lambda_{2}}+\sigma_{2}}{\sqrt{1-\lambda_{2}}-\sigma_{2}}\right|^{\sigma_{3} \bar{\sigma}_{2}^{2} \operatorname{sign}\left(\pi_{2}\right)} \cdot\left|\frac{\sqrt{1-\lambda_{2}}-\sigma_{3}}{\sqrt{1-\lambda_{2}}+\sigma_{3}}\right|^{\sigma_{2} \bar{\sigma}_{3}^{2} \operatorname{sign}\left(\pi_{2}\right)} \\
& \left|\frac{\sqrt{1-\lambda_{3}}-\sigma_{2}}{\sqrt{1-\lambda_{3}}+\sigma_{2}}\right|^{\sigma_{3} \bar{\sigma}_{2}^{2} \operatorname{sign}\left(\pi_{3}\right)} \cdot\left|\frac{\sqrt{1-\lambda_{3}}+\sigma_{3}}{\sqrt{1-\lambda_{3}}-\sigma_{3}}\right|^{\sigma_{2} \bar{\sigma}_{3}^{2} \operatorname{sign}\left(\pi_{3}\right)}
\end{aligned}
$$


con $C_{1}(t)=\exp \left\{2\left(\gamma_{1}+t\right)\left(\sigma_{3}^{2}-\sigma_{2}^{2}\right) \sigma_{2} \sigma_{3}\right\}$. Tenemos, en definitiva, que las soluciones están parametrizadas por tres constantes arbitrarias $\gamma_{1}, \gamma_{2}$ y $\gamma_{3}$. El significado de la constante $\gamma_{1}$ es claro: su arbitrariedad refleja la invarianza del sistema mecánico frente a traslaciones temporales, un valor concreto de $\gamma_{1}$ fija el "centro" del kink, el punto donde la densidad de energía alcanza su valor máximo. Obviando por tanto la constante "temporal" $\gamma_{1}$ podemos hablar, sin pérdida de generalidad, de una familia biparamétrica de trayectorias solución en términos de $\gamma_{2}$ y $\gamma_{3}$.

Las ecuaciones $(3.25,3.26)$ representan la totalidad de las trayectorias solución en la región del espacio donde las coordenadas elípticas están bien definidas. Sin embargo, existen varias superficies donde el cambio de coordenadas es singular y, por tanto, el análisis de las posibles trayectorias solución del sistema dinámico debe realizarse con especial cuidado. Es conveniente, por ello, describir los detalles del cambio de coordenadas (2.42) para el caso particular tridimensional. Tendremos:

$$
\begin{aligned}
& q_{1}^{2}=\frac{1}{\sigma_{2}^{2} \sigma_{3}^{2}}\left(1-\lambda_{1}\right)\left(1-\lambda_{2}\right)\left(1-\lambda_{3}\right) \\
& q_{2}^{2}=\frac{-1}{\sigma_{2}^{2}\left(\sigma_{3}^{2}-\sigma_{2}^{2}\right)}\left(\bar{\sigma}_{2}^{2}-\lambda_{1}\right)\left(\bar{\sigma}_{2}^{2}-\lambda_{2}\right)\left(\bar{\sigma}_{2}^{2}-\lambda_{3}\right) \\
& q_{3}^{2}=\frac{-1}{\sigma_{3}^{2}\left(\sigma_{2}^{2}-\sigma_{3}^{2}\right)}\left(\bar{\sigma}_{3}^{2}-\lambda_{1}\right)\left(\bar{\sigma}_{3}^{2}-\lambda_{2}\right)\left(\bar{\sigma}_{3}^{2}-\lambda_{3}\right)
\end{aligned}
$$

de manera que el espacio total $\mathbb{R}^{3}$ se aplica en el paralelepípedo infinito $P_{3}(\infty)$ :

$$
-\infty<\lambda_{1}<\bar{\sigma}_{3}^{2}<\lambda_{2}<\bar{\sigma}_{2}^{2}<\lambda_{3}<1
$$

El cambio de coordenadas es, de esta manera, singular en los planos coordena$\operatorname{dos}\left(q_{3}=0\right),\left(q_{2}=0\right)$ y $\left(q_{1}=0\right)$. Las identificaciones se realizan de la siguiente forma:

- El plano $q_{3}=0$ se corresponde con las caras $\lambda_{2}=\bar{\sigma}_{3}^{2}$ y $\lambda_{1}=\bar{\sigma}_{3}^{2}$ del borde $\partial P_{3}(\infty)$ del paralelepípedo $P_{3}(\infty)$.

- El plano $\mathbb{R}^{2}\left(q_{2}=0\right)$ se aplica en las caras $\lambda_{2}=\bar{\sigma}_{2}^{2}$ y $\lambda_{3}=\bar{\sigma}_{2}^{2}$.

- Por último, la imagen del plano $q_{1}=0$ es una única cara en $\partial P_{3}(\infty), \lambda_{3}=1$.

Dado que las componentes de la métrica en coordenadas elípticas se escribían de la forma:

$$
g_{a a}=-\frac{1}{4} \frac{f_{a}(\vec{\lambda})}{A\left(\lambda_{a}\right)} ; \quad g_{a b}=0, \forall a \neq b
$$


es trivial comprobar que, sobre todas las caras citadas del paralelepípedo $P_{3}(\infty)$, se verifica:

$$
\begin{aligned}
& g_{11}\left(\bar{\sigma}_{3}^{2}, \lambda_{2}, \lambda_{3}\right)=g_{22}\left(\lambda_{1}, \bar{\sigma}_{3}^{2}, \lambda_{3}\right)=g_{22}\left(\lambda_{1}, \bar{\sigma}_{2}^{2}, \lambda_{3}\right)= \\
& =g_{33}\left(\lambda_{1}, \lambda_{2}, \bar{\sigma}_{2}^{2}\right)=g_{33}\left(\lambda_{1}, \lambda_{2}, 1\right)=\infty
\end{aligned}
$$

y, por tanto, el cambio de coordenadas es singular sobre dichas regiones, como se ha afirmado.

Las ecuaciones (3.29) son invariantes bajo la acción del grupo $G=\mathbb{Z}_{2} \times \mathbb{Z}_{2} \times \mathbb{Z}_{2}$, dado por las transformaciones: $q_{a} \rightarrow(-1)^{\delta_{a b}} q_{a}, b=1,2,3$. Este hecho provoca que un punto interior de $P_{3}(\infty)$ se corresponde con $2^{3}=8$ puntos de $\mathbb{R}^{3}$. Las regiones singulares (los planos coordenados citados) lo son, en este sentido, debido a que se trata de las regiones invariantes bajo los diferentes subgrupos no triviales del grupo $G$. Detallaremos dichas invarianzas posteriormente.

Tal y como se demostró de manera general en el Capítulo 2, las trayectorias de acción finita permanecen en la región acotada (paralelepípedo finito) $P_{3}(0)$ determinado por las inecuaciones:

$$
0 \leq \lambda_{1}<\bar{\sigma}_{3}^{2}<\lambda_{2}<\bar{\sigma}_{2}^{2}<\lambda_{3}<1
$$

cuya única cara no-singular (desde el punto de vista del cambio de coordenadas) es $\lambda_{1}=0$, que, en coordenadas cartesianas, no es otro que el elipsoide

$$
q_{1}^{2}+\frac{q_{2}^{2}}{\bar{\sigma}_{2}^{2}}+\frac{q_{3}^{2}}{\bar{\sigma}_{3}^{2}}=1
$$

en $\mathbb{R}^{3}$. Denominaremos $\bar{P}_{3}(0)$ al paralelepípedo $P_{3}(0)$ junto con su borde $\partial P_{3}(0)$, es decir:

$$
\bar{P}_{3}(0)=P_{3}(0) \sqcup \partial P_{3}(0)
$$

En la Figura 3.7 pueden visualizarse $D^{3}$ y $P_{3}(0)$, donde hemos denominado $D^{3}$ a la región cerrada de $\mathbb{R}^{3}$ limitada por el elipsoide (3.30).

Existen elementos distinguidos en el dominio $D^{3}$ y en el paralelepípedo $\bar{P}_{3}(0)$ dignos de ser resaltados por el importante papel que desempeñarán con respecto a las trayectorias solución:

\section{Puntos:}

- El origen O. Se trata del único punto fijo bajo la acción de todo el grupo $G=\mathbb{Z}_{2} \times \mathbb{Z}_{2} \times \mathbb{Z}_{2}$ y así un único punto $\mathrm{O}$ de $D^{3}$ se aplica en el vértice $\mathrm{O}$ de $\bar{P}_{3}(\infty)$ о $\bar{P}_{3}(0)$. 

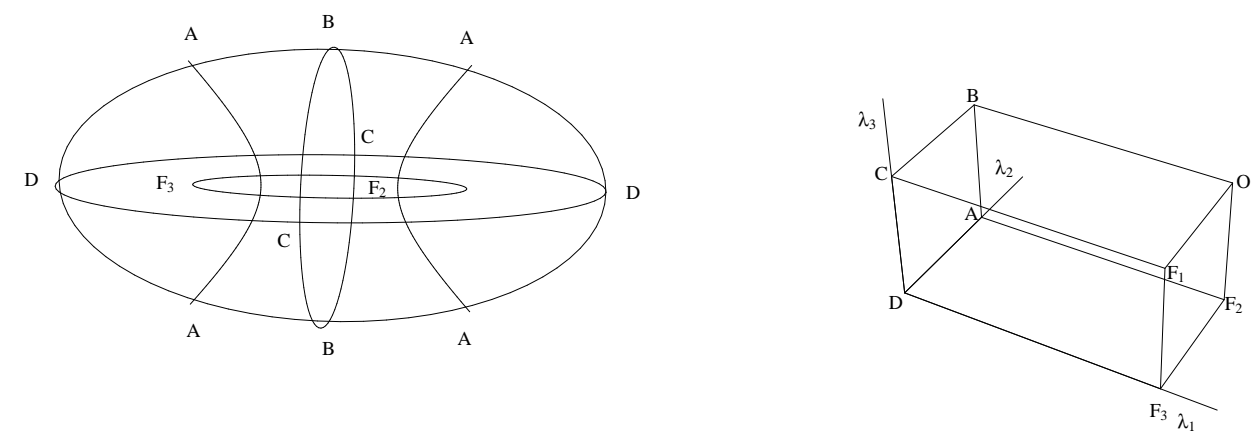

Figura 3.7: Elipsoide (3.30), con sus elementos característicos, y paralelepípedo $P_{3}(0)$.

- Los puntos B, C y D. Se corresponden con los puntos de intersección entre las tres elipses maximales de (3.30), es decir: $q_{1}^{2}+\frac{q_{2}^{2}}{\bar{\sigma}_{2}^{2}}=1, q_{1}^{2}+\frac{q_{3}^{2}}{\bar{\sigma}_{3}^{2}}=1 \mathrm{y}$ $\frac{q_{2}^{2}}{\bar{\sigma}_{2}^{2}}+\frac{q_{3}^{2}}{\bar{\sigma}_{3}^{2}}=1$. Son puntos fijos bajo la acción del subgrupo $\mathbb{Z}_{2} \times \mathbb{Z}_{2}$ de $G$ y, por tanto, dos puntos del borde de $D^{3}$ se aplican en un único punto del borde de $\bar{P}_{3}(0)$. D es evidentemente el punto que se corresponde con los dos vacíos del sistema, $\vec{v}^{-}$y $\vec{v}^{+}$.

- Los focos $\mathrm{F}_{1}, \mathrm{~F}_{2}$ y $\mathrm{F}_{3}$ de las elipses maximales mencionadas. La simetría es idéntica a la de los puntos B, C y D, y, en consecuencia, dos puntos de $D^{3}$ son correlatos de uno del borde de $P_{3}(0), \partial P_{3}(0)$.

- Los puntos umbílicos A del elipsoide (3.30). Se trata ahora de puntos invariantes bajo la acción de un único $\mathbb{Z}_{2}$. Cuatro puntos de $\partial D^{3}$ son aplicados en un vértice de $\bar{P}_{3}(0)$.

\section{Curvas:}

- La elipse, perteneciente al plano $q_{3}=0$, con focos $\mathrm{F}_{2}$ y que pasa por los focos $\mathrm{F}_{3}$ y $\mathrm{F}_{1}$,

$$
\frac{q_{1}^{2}}{\sigma_{3}^{2}}+\frac{q_{2}^{2}}{\sigma_{3}^{2}-\sigma_{2}^{2}}=1
$$

Se trata de la arista $\lambda_{1}=\lambda_{2}=\bar{\sigma}_{3}^{2}$ de $\partial P_{3}(0)$. Cuatro puntos diferentes de (3.31) se corresponden con uno sólo de la arista de $\partial P_{3}(0)$ debido a la invarianza bajo la acción del subgrupo $\mathbb{Z}_{2}$ de $G$. La aplicación de los puntos $\mathrm{F}_{1}$ y $\mathrm{F}_{3}$ es, sin embargo, 2 a 1 por tener un grupo mayor de simetría: $\mathbb{Z}_{2} \times \mathbb{Z}_{2} \subset$ $G$. 
- La hipérbola, del plano $q_{2}=0$, que pasa por $\mathrm{F}_{2}$ y por los puntos umbílicos A, teniendo sus focos en $\mathrm{F}_{3}$,

$$
\frac{q_{1}^{2}}{\sigma_{2}^{2}}-\frac{q_{3}^{2}}{\sigma_{3}^{2}-\sigma_{2}^{2}}=1
$$

que se corresponde (ambas hojas) con la arista $\mathrm{AF}_{2}\left(\lambda_{2}=\lambda_{3}=\bar{\sigma}_{2}^{2}\right)$ de $\partial P_{3}(0)$.

Tras el análisis realizado, es fácil concluir que para obtener todas las soluciones solitónicas de este modelo es necesario estudiar las zonas singulares del cambio de coordenadas independientemente. Como veremos en el siguiente apartado, las superficies singulares contienen una variedad muy rica de soluciones (exceptuando, claro está, el plano $q_{1}=0$, excluido por las condiciones asintóticas al no contener los vacíos del modelo). Demostraremos también cómo estas soluciones "singulares" (desde este punto de vista) se pueden obtener como los casos límite de las soluciones generales (3.25) y (3.26) para los valores asintóticos de las constantes, de una manera altamente no trivial. Todas estas soluciones van a ser, como hemos comentado anteriormente, resultado de la inmersión del modelo $N=2$ (modelo MSTB) en el $N=3$. Sin embargo aparecerán también nuevas soluciones, las que denominaremos envolventes, sobre la superficie del elipsoide (3.30), es decir restringidas a la cara $\lambda_{1}=0$ del paralelepípedo $\bar{P}_{3}(0)$.

\subsubsection{Tipos de Kinks}

\section{Kinks Embebidos}

El estudio de las soluciones del Modelo Sigma $O(3)$ Deformado sobre los planos $q_{2}=0$ y $q_{3}=0$ se reduce a dos casos del Modelo Sigma $O(2)$ Lineal Deformado que hemos estudiado en la sección 3.1 utilizando coordenadas elípticas de Jacobi bidimensionales. Se tratará ahora dicho modelo en coordenadas tridimensionales restringidas a las correspondientes caras de $\partial P_{3}(0)$.

Como ya se comentó en la sección 3.1, encontramos que el Modelo $N=1$ está inmerso en el $N=2$, precisamente ocupando la región singular que queda determinada por el cambio de coordenadas que convierte el sistema dinámico en separable Hamilton-Jacobi. Se repite la situación, como vemos, al analizar la manera en la que el modelo $N=2$ está inmerso en el $N=3$. Por otro lado, los kinks TK2 $2^{5}$ que aparecieron en el modelo $N=2$ como trayectorias singulares

\footnotetext{
${ }^{5}$ Los kinks y antikinks que recorren la elipse (3.10).
} 
desde el punto de vista dinámico, que no geométrico, tendrán ahora su equivalente en las familias de kinks NTK3, trayectorias sobre el elipsoide (3.30), que hemos incluido obviamente en la categoría de Kinks Envolventes.

Es de resaltar, por último, el hecho de que los kinks embebidos del caso $N=2$, es decir los del tipo TK1, aparecen ahora como intersección de los dos modelos $N=2$ embebidos en el caso $N=3$. De esta manera el Modelo $O(3)$ posee un único tipo de kinks unidimensional (es decir con una componente cartesiana no constante).

\section{Modelo $O(1)$ Embebido en el Modelo $O(3)$}

Si consideramos el modelo restringido a la órbita:

$$
M_{1}=\left\{\left(q_{1}, q_{2}, q_{3}\right) \in \mathbb{R}^{3} / q_{2}=q_{3}=0\right\}
$$

tendremos de nuevo el modelo $\phi^{4}$ analizado en la introducción de este capítulo. No es necesario, evidentemente, acudir a la ecuación de Hamilton-Jacobi, las integrales primeras $K_{2}$ y $K_{3}(\operatorname{ver}(2.22))$ son idénticamente nulas, mientras que la ecuación $K_{1}=1$ es la conocida ecuación de Bogomol'nyi:

$$
\frac{d q_{1}}{d t}= \pm\left(q_{1}^{2}-1\right)
$$

cuyas soluciones son:

$$
\vec{q}^{\mathrm{TK} 1}(t)=\left(\begin{array}{c}
\tanh \left(t-t_{0}\right) \\
0 \\
0
\end{array}\right), \quad \vec{q}^{\mathrm{ATK} 1}(t)=\left(\begin{array}{c}
-\tanh \left(t-t_{0}\right) \\
0 \\
0
\end{array}\right)
$$

donde $\vec{q}^{\mathrm{TK} 1}(t)$ denota, una vez más, solución de tipo kink, topológica (pues su carga topológica asociada es 1 , a "tiempo" $t=-\infty$ la "partícula" estará en el vacío $\vec{v}^{-} \equiv \mathrm{D}_{1}$, mientras que a "tiempo" $t=\infty$ alcanza el vacío $\vec{v}^{+} \equiv \mathrm{D}_{2}$ ) y de una sóla componente no nula. La correspondiente $\vec{q}^{\mathrm{ATK} 1}(t)$ será el antikink asociado, es decir la misma trayectoria recorrida en sentido contrario. Desde el punto de vista mecánico, ambas son soluciones heteroclínicas.

En coordenadas elípticas la trayectoria TK1 (y la ATK1) transcurre sobre tres aristas del paralelepípedo $\bar{P}_{3}(0): \mathrm{DF}_{3}, \mathrm{~F}_{3} \mathrm{~F}_{2}$ y $\mathrm{F}_{2} \mathrm{O}$, como puede comprobarse en la Figura 3.8.

Podemos calcular las expresiones del kink TK1 en cada uno de los tramos citados: 

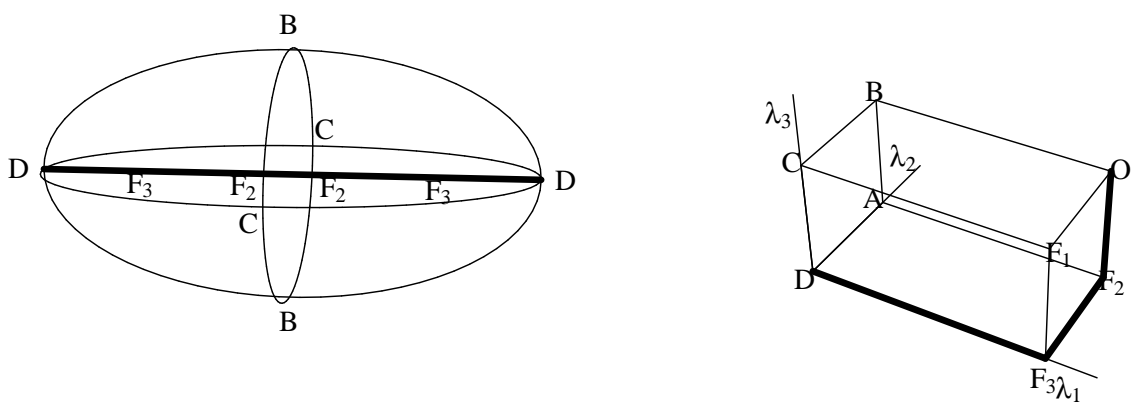

Figura 3.8: Kink TK1 en coordenadas cartesianas (a) y en elípticas (b).

- Primer tramo: Se trata de la arista $\mathrm{DF}_{3}$ determinada por las ecuaciones:

$$
\lambda_{2}=\bar{\sigma}_{3}^{2} ; \quad \lambda_{3}=\bar{\sigma}_{2}^{2}
$$

Si sustituimos dichos valores directamente en la ecuación $I_{1}=0$ tendremos que ésta se reduce a:

$$
\frac{d \lambda_{1}}{d t}= \pm 2 \lambda_{1} \sqrt{1-\lambda_{1}}
$$

de manera que la integración directa nos conduce a la solución:

$$
\vec{\lambda}_{\mathrm{I}}^{\mathrm{TK} 1}=\left(\begin{array}{c}
1-\tanh ^{2}\left(t-t_{0}\right) \\
\bar{\sigma}_{3}^{2} \\
\bar{\sigma}_{2}^{2}
\end{array}\right)
$$

para los valores del parámetro $t$ siguientes:

$$
t \in\left(-\infty, t_{0}+\operatorname{arctanh}\left(-\sigma_{3}\right)\right] \sqcup\left[t_{0}+\operatorname{arctanh}\left(\sigma_{3}\right), \infty\right)
$$

- Segundo tramo: la arista $\mathrm{F}_{3} \mathrm{~F}_{2}$ dada por:

$$
\lambda_{1}=\bar{\sigma}_{3}^{2} ; \quad \lambda_{3}=\bar{\sigma}_{2}^{2}
$$

En este caso la ecuación a integrar será:

$$
\frac{d \lambda_{2}}{d t}= \pm 2 \lambda_{2} \sqrt{1-\lambda_{2}}
$$

y así la solución se escribe de la forma:

$$
\vec{\lambda}_{\mathrm{II}}^{\mathrm{TK} 1}=\left(\begin{array}{c}
\bar{\sigma}_{3}^{2} \\
1-\tanh ^{2}\left(t-t_{0}\right) \\
\bar{\sigma}_{2}^{2}
\end{array}\right)
$$


con:

$t \in\left[t_{0}+\operatorname{arctanh}\left(-\sigma_{3}\right), t_{0}+\operatorname{arctanh}\left(-\sigma_{2}\right)\right] \sqcup\left[t_{0}+\operatorname{arctanh}\left(\sigma_{2}\right), t_{0}+\operatorname{arctanh}\left(\sigma_{3}\right)\right]$

- Finalmente el tercer tramo es la arista $\mathrm{F}_{2} \mathrm{O}$ dada por:

$$
\lambda_{1}=\bar{\sigma}_{3}^{2} ; \quad \lambda_{2}=\bar{\sigma}_{2}^{2}
$$

La ecuación $I_{1}=0$ se reduce ahora a:

$$
\frac{d \lambda_{3}}{d t}= \pm 2 \lambda_{3} \sqrt{1-\lambda_{3}}
$$

con solución:

$$
\vec{\lambda}_{\mathrm{III}}^{\mathrm{TK} 1}=\left(\begin{array}{c}
\bar{\sigma}_{3}^{2} \\
\bar{\sigma}_{2}^{2} \\
1-\tanh ^{2}\left(t-t_{0}\right)
\end{array}\right)
$$

en el rango:

$$
t \in\left[t_{0}+\operatorname{arctanh}\left(-\sigma_{2}\right), t_{0}+\operatorname{arctanh}\left(\sigma_{2}\right)\right]
$$

Los tramos anteriormente presentados describen en coordenadas cartesianas únicamente el trayecto $\mathrm{D}_{1} \rightarrow \mathrm{O}$. La trayectoria completa del kink TK1 (o del antikink ATK1) requiere, evidentemente, que dichos tramos se recorran dos veces.

\section{Modelos $O(2)$ Embebidos en el Modelo $O(3)$}

Existen dos planos del espacio en los que el modelo en estudio se reduce al caso $N=2$, es decir al ya citado modelo MSTB. Son, evidentemente, los determinados por las ecuaciones $q_{2}=0$ y $q_{3}=0$. Se reproducen, por tanto, en este subapartado, los resultados ya expuestos en la sección anterior al tratarse este modelo. En este caso las coordenadas que utilizaremos son las elípticas de Jacobi tridimensionales, restringidas a la región determinada por esas ecuaciones.

Ambos modelos contienen como submodelo (a su vez embebido en ellos) al caso $N=1$ y precisamente en la intersección de ambos. Dado que ya lo hemos detallado en el apartado anterior no repetiremos aquí las expresiones de los correspondientes kinks.

II.A. Modelo MSTB en el plano $q_{2}=0$.

La restricción al plano ${ }^{6}$

$$
M_{2_{\sigma_{3}}}=\left\{\left(q_{1}, q_{2}, q_{3}\right) \in \mathbb{R}^{3} / q_{2}=0\right\}
$$

\footnotetext{
${ }^{6}$ En este apartado y en los siguientes utilizaremos la palabra "planos" en coordenadas cartesianas, no obstante la región interesante desde el punto de vista dinámico está limitada al interior y al borde del elipsoide (3.30).
} 
reduce el sistema tridimensional al caso $N=2$ (modelo MSTB) sobre el plano coordenado por $q_{1} \mathrm{y} q_{3}$.

En coordenadas elípticas, $M_{2_{\sigma_{3}}}$ se traduce en dos caras del paralelepípedo $\bar{P}_{3}(0)$ : $\lambda_{2}=\bar{\sigma}_{2}^{2}$ y $\lambda_{3}=\bar{\sigma}_{2}^{2}$. De esta manera tendremos que:

$$
M_{2_{\sigma_{3}}}=\left\{\left(\lambda_{1}, \lambda_{2}, \lambda_{3}\right) / \lambda_{3}=\bar{\sigma}_{2}^{2}\right\} \sqcup\left\{\left(\lambda_{1}, \lambda_{2}, \lambda_{3}\right) / \lambda_{2}=\bar{\sigma}_{2}^{2}\right\}=M_{2_{\sigma_{3}}}^{1} \sqcup M_{2_{\sigma_{3}}}^{2}
$$

y estudiaremos, en consecuencia, el problema en dos regiones separadamente:

- 1. En $M_{2_{\sigma_{3}}}^{1}$ tomaremos coordenadas restringidas de la forma: $\lambda_{3}=\bar{\sigma}_{2}^{2}$ $\left(q_{2}=0\right) \mathrm{y}$

$$
\begin{aligned}
& q_{1}^{2}=\frac{1}{\sigma_{3}^{2}}\left(1-\lambda_{1}\right)\left(1-\lambda_{2}\right) \\
& q_{3}^{2}=\frac{1}{-\sigma_{3}^{2}}\left(\bar{\sigma}_{3}^{2}-\lambda_{1}\right)\left(\bar{\sigma}_{3}^{2}-\lambda_{2}\right)
\end{aligned}
$$

El rango de variación de estas coordenadas elípticas restringidas será:

$$
0 \leq \lambda_{1}<\bar{\sigma}_{3}^{2}<\lambda_{2}<\bar{\sigma}_{2}^{2}
$$

El Hamiltoniano reducido a $M_{2_{\sigma_{3}}}^{1}$ se escribe de la forma:

$H=\frac{-2 A\left(\lambda_{1}\right) \pi_{1}^{2}-\lambda_{1}^{2}\left(\lambda_{1}-\bar{\sigma}_{2}^{2}\right)\left(\lambda_{1}-\bar{\sigma}_{3}^{2}\right)}{\lambda_{1}-\lambda_{2}}+\frac{-2 A\left(\lambda_{2}\right) \pi_{2}^{2}-\lambda_{2}^{2}\left(\lambda_{2}-\bar{\sigma}_{2}^{2}\right)\left(\lambda_{2}-\bar{\sigma}_{3}^{2}\right)}{\lambda_{2}-\lambda_{1}}$

de forma que la integración de la correspondiente ecuación de HamiltonJacobi nos conduce a la expresión de las trayectorias solución sobre esta cara de $\partial P_{3}(0)$ :

$$
\begin{aligned}
e^{2 \sigma_{3} \bar{\sigma}_{3}^{2} \gamma_{2}=} & \left(\left|\frac{\sqrt{1-\lambda_{1}}-\sigma_{3}}{\sqrt{1-\lambda_{1}}+\sigma_{3}}\right| \cdot\left|\frac{\sqrt{1-\lambda_{1}}+1}{\sqrt{1-\lambda_{1}}-1}\right|^{\sigma_{3}}\right)^{\operatorname{sign}\left(\pi_{1}\right)} . \\
& \left(\left|\frac{\sqrt{1-\lambda_{2}}-\sigma_{3}}{\sqrt{1-\lambda_{2}}+\sigma_{3}}\right| \cdot\left|\frac{\sqrt{1-\lambda_{2}}+1}{\sqrt{1-\lambda_{2}}-1}\right|^{\sigma_{3}}\right)^{\operatorname{sign}\left(\pi_{2}\right)}
\end{aligned}
$$

Esta ecuación describe, por tanto, la porción de las trayectorias que pertenece a la región del plano $q_{2}=0$ limitada por las aristas de $\bar{P}_{3}(0): \mathrm{AD}, \mathrm{AF}_{2}, \mathrm{~F}_{2} \mathrm{~F}_{3}$ y $\mathrm{F}_{3} \mathrm{D}$. Su equivalente en coordenadas cartesianas será la región limitada (en un cuadrante del plano $q_{2}=0$ ) por la elipse

$$
q_{1}^{2}+\frac{q_{3}^{2}}{\bar{\sigma}_{3}^{2}}=1
$$

entre el punto D y el punto umbílico A, el eje $q_{1}$ y la hipérbola característica (3.32). 
- 2. En $M_{2_{\sigma_{3}}}^{2}$, de manera análoga: $\lambda_{2}=\bar{\sigma}_{2}^{2}\left(q_{2}=0\right)$

$$
\begin{aligned}
q_{1}^{2} & =\frac{1}{\sigma_{3}^{2}}\left(1-\lambda_{1}\right)\left(1-\lambda_{3}\right) \\
q_{3}^{2} & =\frac{1}{-\sigma_{3}^{2}}\left(\bar{\sigma}_{3}^{2}-\lambda_{1}\right)\left(\bar{\sigma}_{3}^{2}-\lambda_{3}\right) \\
0 & \leq \lambda_{1}<\bar{\sigma}_{3}^{2} ; \quad \bar{\sigma}_{2}^{2}<\lambda_{3}<1
\end{aligned}
$$

Las ecuaciones de las trayectorias en la superficie determinada por las aristas: $\mathrm{AF}_{2}, \mathrm{~F}_{2} \mathrm{O}$, OB y BA será:

$$
\begin{aligned}
e^{2 \sigma_{3} \bar{\sigma}_{3}^{2} \gamma_{2}=} & \left(\left|\frac{\sqrt{1-\lambda_{1}}-\sigma_{3}}{\sqrt{1-\lambda_{1}}+\sigma_{3}}\right| \cdot\left|\frac{\sqrt{1-\lambda_{1}}+1}{\sqrt{1-\lambda_{1}}-1}\right|^{\sigma_{3}}\right)^{\operatorname{sign}\left(\pi_{1}\right)} . \\
& \left(\left|\frac{\sqrt{1-\lambda_{3}}-\sigma_{3}}{\sqrt{1-\lambda_{3}}+\sigma_{3}}\right| \cdot\left|\frac{\sqrt{1-\lambda_{3}}+1}{\sqrt{1-\lambda_{3}}-1}\right|^{\sigma_{3}}\right)^{\operatorname{sign}\left(\pi_{3}\right)}
\end{aligned}
$$

El método de Hamilton-Jacobi nos proporciona también la dependencia temporal sobre las trayectorias solución indicadas de manera que tendremos la ecuación:

$$
e^{2\left(\gamma_{1}+t\right) \sigma_{3}}=\left|\frac{\sqrt{1-\lambda_{1}}-\sigma_{3}}{\sqrt{1-\lambda_{1}}+\sigma_{3}}\right|^{\bar{\sigma}_{3}^{2} \operatorname{sign}\left(\pi_{1}\right)} \cdot\left|\frac{\sqrt{1-\lambda_{2}}-\sigma_{3}}{\sqrt{1-\lambda_{2}}+\sigma_{3}}\right|^{\bar{\sigma}_{3}^{2} \operatorname{sign}\left(\pi_{2}\right)}
$$

para la parte de la trayectoria que transcurre en $M_{2_{\sigma_{3}}}^{1}$ y que se corresponde con los rangos de variación del "tiempo" $t$ siguientes:

$$
-\infty<t \leq t_{1} ; \quad t_{2} \leq t \leq t_{3} ; \quad t_{4} \leq t<\infty
$$

donde $t_{1}, t_{2}, t_{3}$ y $t_{4}$ son los valores que resultan de resolver el sistema de ecuaciones $(3.34,3.36)$ cuando se alcanzan las aristas del borde de $M_{2_{\sigma_{3}}}^{1}{ }^{7}$.

En la región $M_{2_{\sigma_{3}}}^{2}$ tendremos, análogamente:

$$
e^{2\left(\gamma_{1}+t\right) \sigma_{3}}=\left|\frac{\sqrt{1-\lambda_{1}}-\sigma_{3}}{\sqrt{1-\lambda_{1}}+\sigma_{3}}\right|^{\bar{\sigma}_{3}^{2} \operatorname{sign}\left(\pi_{1}\right)} \cdot\left|\frac{\sqrt{1-\lambda_{3}}-\sigma_{3}}{\sqrt{1-\lambda_{3}}+\sigma_{3}}\right|^{\bar{\sigma}_{3}^{2} \operatorname{sign}\left(\pi_{3}\right)}
$$

para $t$ en los rangos:

$$
t_{1} \leq t \leq t_{2} ; \quad t_{3} \leq t \leq t_{4}
$$

\footnotetext{
${ }^{7}$ Dichos valores, $t_{1}, t_{2}, t_{3}$ y $t_{4}$, dependen evidentemente de $\gamma_{1}, \gamma_{2}, \sigma_{2}$ y $\sigma_{3}$ y determinan los valores del parámetro $t$ correspondientes a la intersección de una trayectoria solución con la arista $\mathrm{AF}_{2}$ de $\partial P_{3}(0)$, ver Figura 3.9.
} 
Para cada valor finito de la constante $\gamma_{2}$ se tiene, en consecuencia, un kink no topológico. Los sectores involucrados son, en definitiva, $\mathcal{C}^{(+,+)}$y $\mathcal{C}^{(-,-)}$, indistinguibles en coordenadas elípticas. La distinción kink-antikink es confusa en este caso, de cualquier manera dos son las soluciones asociadas a cada trayectoria puesto que cada una de ellas puede recorrerse en ambos sentidos. En la Figura 3.9 se representan algunas soluciones de esta familia que denominaremos NTK2 $\sigma_{3}$ (particularmente del sector $\mathcal{C}^{(-,-)}$) en coordenadas cartesianas y elípticas.
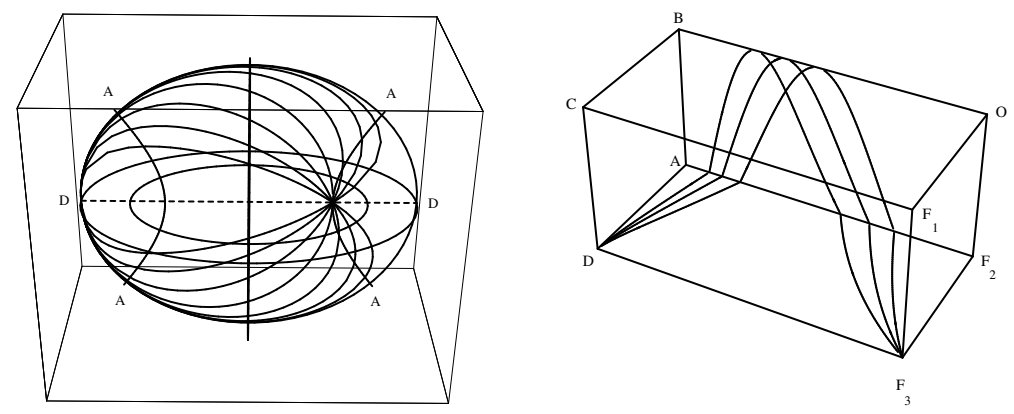

Figura 3.9: Kinks $\mathrm{NTK}_{2} \sigma_{3}$ del sector $\mathcal{C}^{(-,-)}$en coordenadas cartesianas, (a), y en elípticas, (b).

El Modelo MSTB queda completo si se consideran ahora por un lado los kinks del tipo TK1 (kinks embebidos del Modelo $N=1$ en el $N=2$, ya comentados en el apartado anterior) y por otro los kinks del tipo TK2 (kinks envolventes del caso $N=2$ ). Sobre la elipse maximal:

$$
q_{1}^{2}+\frac{q_{3}^{2}}{\bar{\sigma}_{3}^{2}}=1
$$

del elipsoide (3.30), contenida en $M_{2_{\sigma_{3}}}$ y correspondiente en coordenadas elípticas a la unión de las aristas DA y AB, $\left\{\lambda_{1}=0, \lambda_{3}=\bar{\sigma}_{2}^{2}\right\}$ y $\left\{\lambda_{1}=0, \lambda_{2}=\bar{\sigma}_{2}^{2}\right\}$ respectivamente, las soluciones serán:

$$
\begin{aligned}
\vec{q}^{\mathrm{TK} 2 \sigma_{3}} & =\left(\begin{array}{c}
\tanh \sigma_{3}\left(t-t_{0}\right) \\
0 \\
\bar{\sigma}_{3} \operatorname{sech} \sigma_{3}\left(t-t_{0}\right)
\end{array}\right), \vec{q}^{\mathrm{ATK} 2 \sigma_{3}}=\left(\begin{array}{c}
-\tanh \sigma_{3}\left(t-t_{0}\right) \\
0 \\
-\bar{\sigma}_{3} \operatorname{sech} \sigma_{3}\left(t-t_{0}\right)
\end{array}\right) \\
\vec{q}^{\mathrm{TK} 2 \sigma_{3} *} & =\left(\begin{array}{c}
\tanh \sigma_{3}\left(t-t_{0}\right) \\
0 \\
-\bar{\sigma}_{3} \operatorname{sech} \sigma_{3}\left(t-t_{0}\right)
\end{array}\right), \vec{q}^{\mathrm{ATK} 2 \sigma_{3} *}=\left(\begin{array}{c}
-\tanh \sigma_{3}\left(t-t_{0}\right) \\
0 \\
\bar{\sigma}_{3} \operatorname{sech} \sigma_{3}\left(t-t_{0}\right)
\end{array}\right)
\end{aligned}
$$


en coordenadas cartesianas (como se deduce directamente de la sección 3.1). En coordenadas elípticas hemos de analizar, como hemos dicho, dos aristas diferentes de $\partial P_{3}(0)$ :

- Tramo DA: $\left\{\lambda_{1}=0, \lambda_{3}=\bar{\sigma}_{2}^{2}\right\}$. La ecuación $I_{1}=0$ se convierte en este caso en la expresión:

$$
\frac{d \lambda_{2}}{d t}= \pm 2\left(\lambda_{2}-\bar{\sigma}_{3}^{2}\right) \sqrt{1-\lambda_{2}}
$$

de manera que obtenemos la solución ${ }^{8}$ :

$$
\lambda_{1}^{\mathrm{TK} 2 \sigma_{3}(\mathrm{I})}(t)=0 \quad \lambda_{2}^{\mathrm{TK} 2 \sigma_{3}(\mathrm{I})}(t)=1-\sigma_{3}^{2} \tanh ^{2}\left(\sigma_{3} t\right) \quad \lambda_{3}^{\mathrm{TK} 2 \sigma_{3}(\mathrm{I})}(t)=\bar{\sigma}_{2}^{2}
$$

para los valores de $t$ pertenecientes a los intervalos:

$$
t \in\left(-\infty, \frac{-1}{\sigma_{3}} \operatorname{arctanh} \frac{\sigma_{2}}{\sigma_{3}}\right] \sqcup\left[\frac{1}{\sigma_{3}} \operatorname{arctanh} \frac{\sigma_{2}}{\sigma_{3}}, \infty\right)
$$

- Tramo AB: $\left\{\lambda_{1}=0, \lambda_{2}=\bar{\sigma}_{2}^{2}\right\}$. La ecuación a integrar es ahora:

$$
\frac{d \lambda_{3}}{d t}= \pm 2\left(\lambda_{3}-\bar{\sigma}_{3}^{2}\right) \sqrt{1-\lambda_{3}}
$$

que conduce a la solución:

$$
\lambda_{1}^{\mathrm{TK} 2 \sigma_{3}(\mathrm{II})}(t)=0 \quad \lambda_{2}^{\mathrm{TK} 2 \sigma_{3}(\mathrm{II})}(t)=\bar{\sigma}_{2}^{2} \quad \lambda_{3}^{\mathrm{TK} 2 \sigma_{3}(\mathrm{II})}(t)=1-\sigma_{3}^{2} \tanh ^{2}\left(\sigma_{3} t\right)
$$

donde ahora se tiene:

$$
t \in\left[-\frac{1}{\sigma_{3}} \operatorname{arctanh} \frac{\sigma_{2}}{\sigma_{3}}, \frac{1}{\sigma_{3}} \operatorname{arctanh} \frac{\sigma_{2}}{\sigma_{3}}\right]
$$

Evidentemente los tramos anteriormente descritos se recorren dos veces (la trayectoria completa ha de entenderse de la forma: $\mathrm{D} \rightarrow \mathrm{A} \rightarrow \mathrm{B} \rightarrow \mathrm{A} \rightarrow \mathrm{D}$ ).

En la Figura 3.10 está representada la trayectoria de los kinks TK2 $\sigma_{3}$ y $\mathrm{ATK} 2 \sigma_{3}$.

II.B. Modelo MSTB en el plano $q_{3}=0$.

De similares pero no idénticas características es la inmersión en el modelo $N=3$ del modelo $N=2$ a través de la superficie:

$$
M_{2_{\sigma_{2}}}=\left\{\left(q_{1}, q_{2}, q_{3}\right) \in \mathbb{R}^{3} / q_{3}=0\right\}
$$

que, en coordenadas elípticas, se corresponde con:

$$
M_{2_{\sigma_{2}}}=\left\{\left(\lambda_{1}, \lambda_{2}, \lambda_{3}\right) / \lambda_{1}=\bar{\sigma}_{3}^{2}\right\} \sqcup\left\{\left(\lambda_{1}, \lambda_{2}, \lambda_{3}\right) / \lambda_{2}=\bar{\sigma}_{3}^{2}\right\}=M_{2_{\sigma_{2}}}^{1} \sqcup M_{2_{\sigma_{2}}}^{2}
$$

\footnotetext{
${ }^{8}$ Por simplicidad en las expresiones se ha tomado, una vez más, la constante $t_{0}=\gamma_{1}=0$.
} 

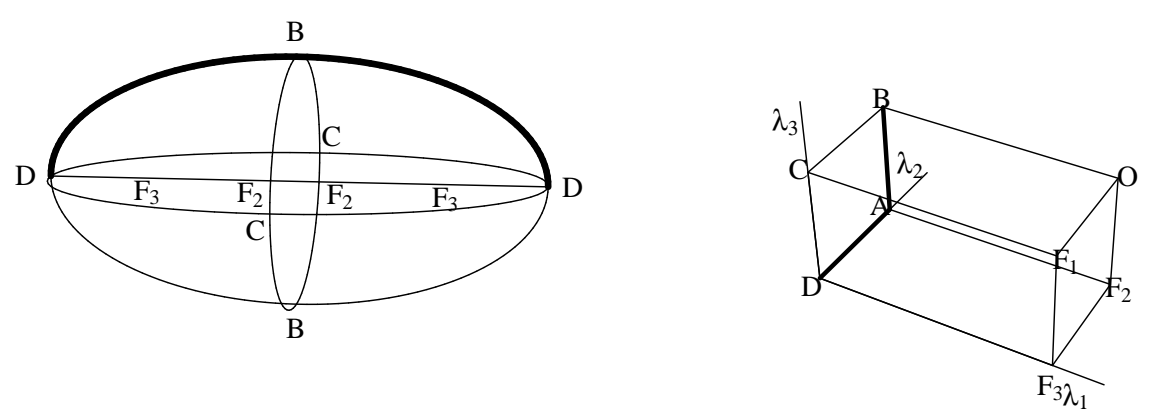

Figura 3.10: Uno de los kinks TK2 del plano $q_{2}=0$ en coordenadas cartesianas, (a), y en elípticas, (b).

- 1. En $M_{2_{\sigma_{2}}}^{1}$ se tiene: $\lambda_{1}=\bar{\sigma}_{3}^{2}$, y así necesariamente: $q_{3}=0$. Las coordenadas elípticas restringidas serán ahora:

$$
\begin{aligned}
& q_{1}^{2}=\frac{1}{\sigma_{2}^{2}}\left(1-\lambda_{2}\right)\left(1-\lambda_{3}\right) \\
& q_{2}^{2}=\frac{1}{-\sigma_{2}^{2}}\left(\bar{\sigma}_{2}^{2}-\lambda_{2}\right)\left(\bar{\sigma}_{2}^{2}-\lambda_{3}\right)
\end{aligned}
$$

dentro de los rangos:

$$
\bar{\sigma}_{3}^{2}<\lambda_{2}<\bar{\sigma}_{2}^{2}<\lambda_{3}<1
$$

Desde el punto de vista de las coordenadas cartesianas esta región no es otra que el interior de la elipse (3.31). La integración de la ecuación de Hamilton-Jacobi para este caso nos proporciona las ecuaciones de las trayectorias solución:

$$
\begin{aligned}
e^{2 \sigma_{2} \bar{\sigma}_{2}^{2} \gamma_{2}=\quad} & \left(\left|\frac{\sqrt{1-\lambda_{2}}-\sigma_{2}}{\sqrt{1-\lambda_{2}}+\sigma_{2}}\right| \cdot\left|\frac{\sqrt{1-\lambda_{2}}+1}{\sqrt{1-\lambda_{2}}-1}\right|^{\sigma_{2}}\right)^{\operatorname{sign}\left(\pi_{2}\right)} . \\
& \left(\left|\frac{\sqrt{1-\lambda_{3}}-\sigma_{2}}{\sqrt{1-\lambda_{3}}+\sigma_{2}}\right| \cdot\left|\frac{\sqrt{1-\lambda_{3}}+1}{\sqrt{1-\lambda_{2}}-1}\right|^{\sigma_{2}}\right)^{\operatorname{sign}\left(\pi_{3}\right)}
\end{aligned}
$$

- 2. En $M_{2_{\sigma_{2}}}^{2}: \lambda_{2}=\bar{\sigma}_{3}^{2}$ es la ecuación que proporciona ahora la restricción $q_{3}=0$. El cambio de coordenadas se escribirá por tanto en la forma:

$$
\begin{aligned}
& q_{1}^{2}=\frac{1}{\sigma_{2}^{2}}\left(1-\lambda_{1}\right)\left(1-\lambda_{3}\right) \\
& q_{2}^{2}=\frac{1}{-\sigma_{2}^{2}}\left(\bar{\sigma}_{2}^{2}-\lambda_{1}\right)\left(\bar{\sigma}_{2}^{2}-\lambda_{3}\right)
\end{aligned}
$$


para el rango

$$
0 \leq \lambda_{1}<\bar{\sigma}_{3}^{2} ; \quad \bar{\sigma}_{2}^{2}<\lambda_{3}<1
$$

de tal manera que las soluciones verifican la siguiente ecuación para las trayectorias:

$$
\begin{aligned}
e^{2 \sigma_{2} \bar{\sigma}_{2}^{2} \gamma_{2}=} & \left(\left|\frac{\sqrt{1-\lambda_{1}}-\sigma_{2}}{\sqrt{1-\lambda_{1}}+\sigma_{2}}\right| \cdot\left|\frac{\sqrt{1-\lambda_{1}}+1}{\sqrt{1-\lambda_{1}}-1}\right|^{\sigma_{2}}\right)^{\operatorname{sign}\left(\pi_{1}\right)} . \\
& \left(\left|\frac{\sqrt{1-\lambda_{3}}-\sigma_{2}}{\sqrt{1-\lambda_{3}}+\sigma_{2}}\right| \cdot\left|\frac{\sqrt{1-\lambda_{3}}+1}{\sqrt{1-\lambda_{2}}-1}\right|^{\sigma_{2}}\right)^{\operatorname{sign}\left(\pi_{3}\right)}
\end{aligned}
$$

Las expresiones anteriores se complementan con las correspondientes ecuaciones que determinan la dependencia temporal. Se tiene así:

$$
e^{2\left(\gamma_{1}+t\right) \sigma_{2}}=\left|\frac{\sqrt{1-\lambda_{2}}-\sigma_{2}}{\sqrt{1-\lambda_{2}}+\sigma_{2}}\right|^{\bar{\sigma}_{2}^{2} \operatorname{sign}\left(\pi_{2}\right)} \cdot\left|\frac{\sqrt{1-\lambda_{3}}-\sigma_{2}}{\sqrt{1-\lambda_{3}}+\sigma_{2}}\right|^{\bar{\sigma}_{2}^{2} \operatorname{sign}\left(\pi_{3}\right)}
$$

para los tramos pertenecientes a $M_{2_{\sigma_{2}}}^{1} \mathrm{y}$ :

$$
e^{2\left(\gamma_{1}+t\right) \sigma_{2}}=\left|\frac{\sqrt{1-\lambda_{1}}-\sigma_{2}}{\sqrt{1-\lambda_{1}}+\sigma_{2}}\right|^{\bar{\sigma}_{2}^{2} \operatorname{sign}\left(\pi_{1}\right)} \cdot\left|\frac{\sqrt{1-\lambda_{3}}-\sigma_{2}}{\sqrt{1-\lambda_{3}}+\sigma_{2}}\right|^{\bar{\sigma}_{2}^{2} \operatorname{sign}\left(\pi_{3}\right)}
$$

para $M_{2_{\sigma_{2}}}^{2}$.
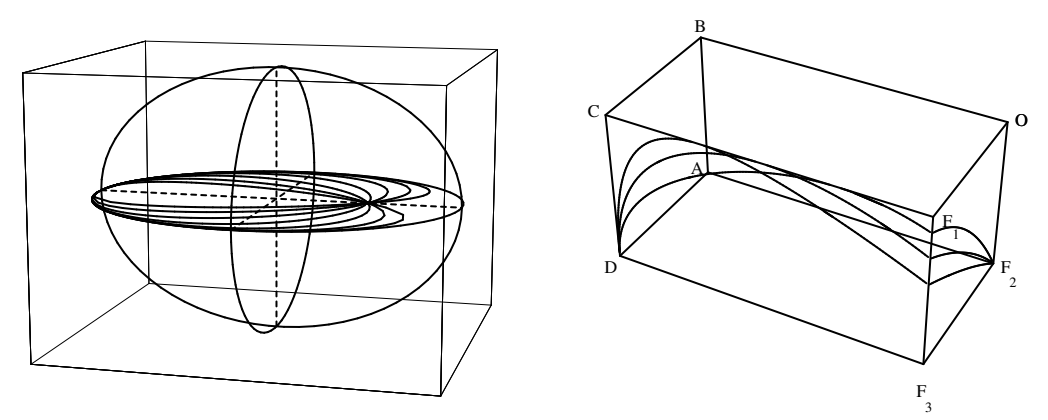

Figura 3.11: Kinks NTK2 $\sigma_{2}$ del sector $\mathcal{C}^{(-,-)}$en coordenadas cartesianas, (a), y en elípticas, (b).

Encontramos de nuevo que para valores finitos de la constante $\gamma_{2}$ se obtiene una familia uniparamétrica de trayectorias kink no topológicas, que denominaremos ahora como NTK $2 \sigma_{2}$, pertenecientes al plano $q_{3}=0$, como puede verse en la Figura 3.11. 
Una vez más el modelo se completa con los kinks del tipo TK1 ya comentados y con los kinks de tipo TK2.

Los kinks envolventes del Modelo MSTB se corresponden en este caso, para $M_{2_{\sigma_{2}}}$, con las soluciones que transcurren sobre la elipse:

$$
q_{1}^{2}+\frac{q_{2}^{2}}{\bar{\sigma}_{2}^{2}}=1
$$

es decir, otra de las elipses maximales contenidas en (3.30). Consecuentemente, tenemos las soluciones:

$$
\begin{aligned}
& \vec{q}^{\mathrm{TK} 2 \sigma_{2}}=\left(\begin{array}{c}
\tanh \sigma_{2}\left(t-t_{0}\right) \\
\bar{\sigma}_{2} \operatorname{sech} \sigma_{2}\left(t-t_{0}\right) \\
0
\end{array}\right), \quad \vec{q}^{\mathrm{ATK} 2 \sigma_{2}}=\left(\begin{array}{c}
-\tanh \sigma_{2}\left(t-t_{0}\right) \\
-\bar{\sigma}_{2} \operatorname{sech} \sigma_{2}\left(t-t_{0}\right) \\
0
\end{array}\right) \\
& \vec{q}^{\mathrm{TK} 2 \sigma_{2} *}=\left(\begin{array}{c}
\tanh \sigma_{2}\left(t-t_{0}\right) \\
-\bar{\sigma}_{2} \operatorname{sech} \sigma_{2}\left(t-t_{0}\right) \\
0
\end{array}\right), \quad \vec{q}^{\mathrm{ATK} 2 \sigma_{2} *}=\left(\begin{array}{c}
-\tanh \sigma_{2}\left(t-t_{0}\right) \\
\bar{\sigma}_{2} \operatorname{sech} \sigma_{2}\left(t-t_{0}\right) \\
0
\end{array}\right)
\end{aligned}
$$

que denotaremos genéricamente por TK2 $\sigma_{2}$.

En coordenadas elípticas tendremos que las trayectorias del tipo TK2 $\sigma_{2}$ parten del punto D, llegan al punto $\mathrm{C}$ y retornan a $\mathrm{D}$, dentro de la elipse (3.43). Se corresponden, por tanto, con una única arista perteneciente a $\partial P_{3}(0)$.

Sobre DC tenemos: $\lambda_{1}=0, \lambda_{2}=\bar{\sigma}_{3}^{2}$. De esta manera las integrales primeras nos proporcionan una única ecuación:

$$
\frac{d \lambda_{3}}{d t}= \pm 2\left(\lambda_{3}-\bar{\sigma}_{2}^{2}\right) \sqrt{1-\lambda_{3}}
$$

y así la expresión de los kinks del tipo TK2 $\sigma_{2}$ se reduce, en elípticas, a la expresión:

$$
\lambda_{1}^{\mathrm{TK} 2 \sigma_{2}}(t)=0 \quad \lambda_{2}^{\mathrm{TK} 2 \sigma_{2}}(t)=\bar{\sigma}_{3}^{2} \quad \lambda_{3}^{\mathrm{TK} 2 \sigma_{2}}(t)=1-\sigma_{2}^{2} \tanh ^{2}\left(\sigma_{2}\left(t-t_{0}\right)\right)
$$

con $t \in(-\infty, \infty)$ y donde evidentemente la arista DC se recorre una vez en cada sentido.

En la Figura 3.12 está representada la trayectoria correspondiente a los kinks $\mathrm{TK} 2 \sigma_{2} *$ y $\mathrm{ATK} 2 \sigma_{2} *$.

\section{Kinks Envolventes}

Como ya se ha comentado anteriormente, la parte del borde de la región $P_{N}(0)$ determinada por la ecuación $\lambda_{1}=0$ constituye la única frontera no singular con 

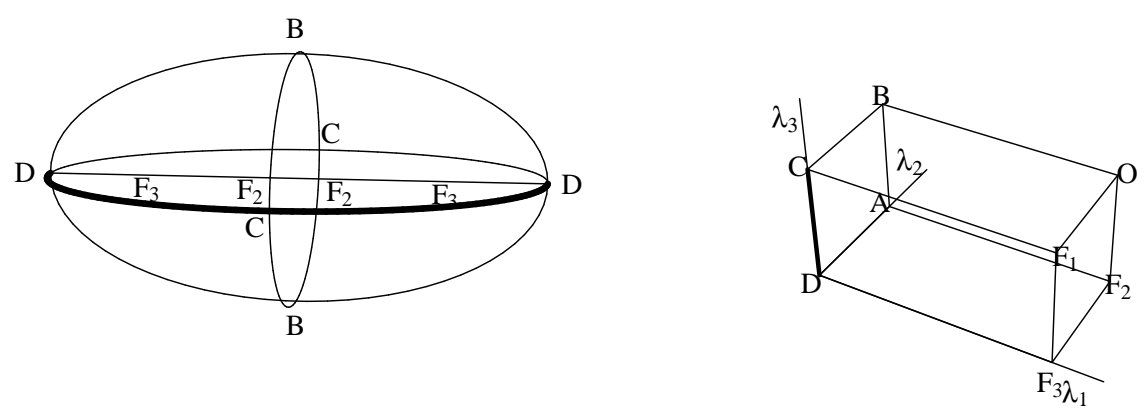

Figura 3.12: Kinks del tipo TK2 $\sigma_{2}$ embebidos en el Modelo $O(3)$ en coordenadas cartesianas y elípticas.

respecto al cambio de coordenadas que estamos utilizando. Hemos denominado kinks envolventes a los que pertenecen a ella y, para el caso $N=2$, se trataba de los kinks del tipo TK2. Como veremos a continuación, en el modelo tridimensional aparecen cuatro familias (desde el punto de vista "cartesiano", simplemente una en coordenadas elípticas) uniparamétricas de kinks sobre el elipsoide $\lambda_{1}=0$. Se tratará de kinks no topológicos y, de hecho, los kinks del tipo TK2 ya estudiados dentro de los Kinks Embebidos del modelo constituirán los límites singulares de dichas familias cuando los parámetros que las caracterizan tienden a sus valores asintóticos. Encontraremos, de esta manera, que las soluciones TK2 constituyen la intersección entre los Kinks Envolventes y los Embebidos.

Por otra parte, es de resaltar el hecho de que las soluciones envolventes, para $N=2$, son de tipo topológico mientras que para $N=3$ se trata de kinks no topológicos. Un análisis detallado de los modelos con $N \geq 4$ (que no hemos incluido en este trabajo) permitirá concluir que se trata de un hecho asociado al caracter par o impar de la dimensión en cada caso.

Consideraremos, por tanto, como superficie de prueba el elipsoide (3.30), cuya ecuación en coordenadas elípticas es simplemente $\lambda_{1}=0$ :

$$
M_{3} \equiv\left\{\left(\lambda_{1}, \lambda_{2}, \lambda_{3}\right) / \lambda_{1}=0\right\}
$$

El Hamiltoniano restringido a $M_{3}$ es

$$
\begin{aligned}
H= & \frac{1}{2} \frac{-4 A\left(\lambda_{2}\right)}{f_{2}\left(0, \lambda_{2}, \lambda_{3}\right)} \pi_{2}^{2}-\frac{1}{2} \frac{\lambda_{2}^{2}\left(\lambda_{2}-\bar{\sigma}_{2}^{2}\right)\left(\lambda_{2}-\bar{\sigma}_{3}^{2}\right)}{f_{2}\left(0, \lambda_{2}, \lambda_{3}\right)} \\
& +\frac{1}{2} \frac{-4 A\left(\lambda_{3}\right)}{f_{3}\left(0, \lambda_{2}, \lambda_{3}\right)} \pi_{3}^{2}-\frac{1}{2} \frac{\lambda_{3}^{2}\left(\lambda_{3}-\bar{\sigma}_{2}^{2}\right)\left(\lambda_{3}-\bar{\sigma}_{3}^{2}\right)}{f_{3}\left(0, \lambda_{2}, \lambda_{3}\right)}
\end{aligned}
$$


y tenemos, en definitiva, un tercer modelo bidimensional (desde el punto de vista elíptico, en coordenadas cartesianas las tres coordenadas son no constantes) dentro del modelo general, que a su vez es completamente integrable y Hamilton-Jacobi separable:

$$
H=\frac{H_{2}}{f_{2}\left(0, \lambda_{2}, \lambda_{3}\right)}+\frac{H_{3}}{f_{3}\left(0, \lambda_{2}, \lambda_{3}\right)}
$$

La resolución de la ecuación de Hamilton-Jacobi nos lleva a unas ecuaciones para las trayectorias:

$$
\begin{aligned}
e^{\sigma_{2} \sigma_{3}\left(\sigma_{3}^{2}-\sigma_{2}^{2}\right) \gamma_{2}=} & \left|\frac{\sqrt{1-\lambda_{2}}-\sigma_{2}}{\sqrt{1-\lambda_{2}}+\sigma_{2}}\right|^{\sigma_{3} \operatorname{sign}\left(\pi_{2}\right)} \cdot\left|\frac{\sqrt{1-\lambda_{2}}+\sigma_{3}}{\sqrt{1-\lambda_{2}}-\sigma_{3}}\right|^{\sigma_{2} \operatorname{sign}\left(\pi_{2}\right)} \\
& \left|\frac{\sqrt{1-\lambda_{3}}-\sigma_{2}}{\sqrt{1-\lambda_{3}}+\sigma_{2}}\right|^{\sigma_{3} \operatorname{sign}\left(\pi_{3}\right)} \cdot\left|\frac{\sqrt{1-\lambda_{3}}+\sigma_{3}}{\sqrt{1-\lambda_{3}}-\sigma_{3}}\right|^{\sigma_{2} \operatorname{sign}\left(\pi_{3}\right)}
\end{aligned}
$$

que podemos ver representadas en la Figura 3.13.
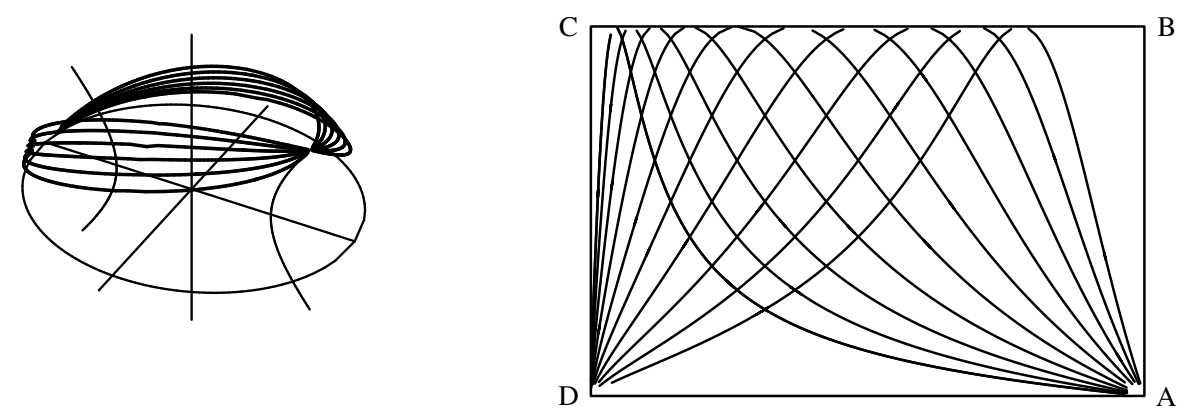

Figura 3.13: Kinks NTK3 en coordenadas cartesianas, (a), y en elípticas, (b).

La dependencia temporal para estas soluciones resulta ser:

$$
\begin{aligned}
e^{2\left(\gamma_{1}+x\right)\left(\sigma_{3}^{2}-\sigma_{2}^{2}\right) \sigma_{2} \sigma_{3}=} & \left|\frac{\sqrt{1-\lambda_{2}}-\sigma_{2}}{\sqrt{1-\lambda_{2}}+\sigma_{2}}\right|^{\sigma_{3} \bar{\sigma}_{2}^{2} \operatorname{sign}\left(\pi_{2}\right)} \cdot\left|\frac{\sqrt{1-\lambda_{2}}+\sigma_{3}}{\sqrt{1-\lambda_{2}}-\sigma_{3}}\right|^{\sigma_{2} \bar{\sigma}_{3}^{2} \operatorname{sign}\left(\pi_{2}\right)} . \\
& \left|\frac{\sqrt{1-\lambda_{3}}-\sigma_{2}}{\sqrt{1-\lambda_{3}}+\sigma_{2}}\right|^{\sigma_{3} \bar{\sigma}_{2}^{2} \operatorname{sign}\left(\pi_{3}\right)} \cdot\left|\frac{\sqrt{1-\lambda_{3}}+\sigma_{3}}{\sqrt{1-\lambda_{3}}-\sigma_{3}}\right|^{\sigma_{2} \bar{\sigma}_{3}^{2} \operatorname{sign}\left(\pi_{3}\right)}
\end{aligned}
$$

Las trayectorias son de tipo homoclínico, desde el punto de vista dinámico, y, por tanto, se trata de soluciones no topológicas que denotaremos como NTK3.

Como puede observarse, se trata de una familia uniparamétrica de trayectorias en elípticas que se corresponde con cuatro pares de familias en cartesianas. Dada una trayectoria en elípticas existen cuatro en cartesianas según se escoja el vacío, 
$\vec{v}^{+}$, o $\vec{v}^{-}$, y según se considere la trayectoria en el semielipsoide de inecuación $q_{3} \leq 0$ ó $q_{3} \geq 0$. Además, a cada trayectoria le corresponden, como es habitual, dos soluciones según el sentido en el que se recorre con respecto a $t$.

Todas las trayectorias convergen en el punto A (correspondiente en elípticas a los puntos umbílicos del elipsoide), que constituye así un punto conjugado al vacío D. Se observa una clara similitud entre estas soluciones y las estudiadas NTK2 $\sigma_{2}$ y NTK $2 \sigma_{3}$, si bien para las familias NTK3 son los puntos umbílicos los que juegan el papel de los focos de las elipses correspondientes.

\section{Kinks Genéricos}

Las trayectorias-solución generales del modelo son las correspondientes a valores finitos y no nulos de las constantes $C_{2}$ y $C_{3}$, o, alternativamente, a valores finitos de $\gamma_{2}$ y $\gamma_{3}$ en las ecuaciones (3.25) y (3.26).
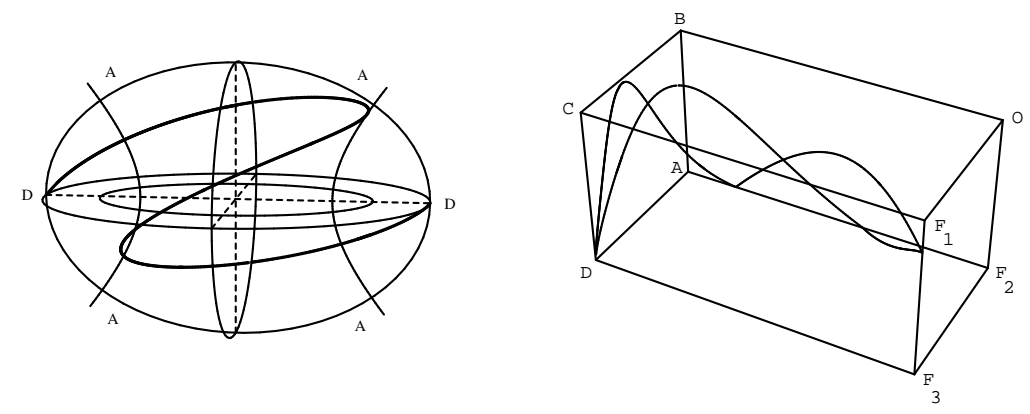

Figura 3.14: Ejemplo de Kink TK3 en el interior del elipsoide, (a), y en $P_{3}(0)$, (b).

Fijadas $C_{2}$ y $C_{3}\left(\right.$ ó $\gamma_{2}$ y $\gamma_{3}$ ) (ver como ejemplo la Figura 3.14), la trayectoria kink correspondiente es una curva alabeada, en el interior de $P_{3}(0)$, que comienza en el punto de vacío $\mathrm{D}$, alcanza la cara superior $\mathrm{BCF}_{1} \mathrm{O}$, corta a la arista $\mathrm{AF}_{2}$ para continuar hasta cortar a la arista $\mathrm{F}_{1} \mathrm{~F}_{3}$, volver a la cara superior, cortar una segunda vez a $\mathrm{AF}_{2}$, alcanzar por tercera vez la cara superior y terminar definitivamente en el punto D.

La traducción de este recorrido genérico a coordenadas cartesianas es delicada debido a la presencia de varias ambigüedades. La no unicidad del cambio de coordenadas (ocho a uno) complica, lógicamente, esta lectura. Un cuidado especial se hace necesario en los cortes con las cónicas (3.31) y (3.32), pues varias posibilidades son aparentemente válidas a priori. El requerimiento, para las soluciones, de continuidad y derivabilidad en los puntos de corte con dichas cónicas (aristas $\mathrm{AF}_{2}$ y 
$\mathrm{F}_{1} \mathrm{~F}_{3}$ de $\partial P_{3}(0)$, respectivamente), dentro del elipsoide (3.30), aclara notablemente el análisis del comportamiento de las trayectorias cartesianas. Si fijamos además el vacío de partida y el cuadrante del plano $q_{1}=0$ en el que se corta por primera vez la trayectoria con dicho plano ${ }^{9}$, entonces una única curva queda determinada por el cambio inverso de coordenadas.

La trayectoria representada en la Figura 3.14 se puede describir, por tanto, de la forma siguiente:

La curva parte del punto de vacío $\mathrm{D}=\vec{v}^{-} \mathrm{y}$, tras cruzar el plano $q_{1}=0$ en el cuadrante escogido, corta a la rama $q_{1}>0$ de la hipérbola característica (3.32), para retornar hacia la elipse (3.31); en este corte la curva pasa al semiespacio $q_{3}<0$ y continúa hasta cortar a la otra rama $\left(q_{1}<0\right)$ de la hipérbola, después de haber cortado de nuevo el plano $q_{1}=0$. Tras este corte con la otra rama de la hipérbola (donde corta de paso al plano $q_{2}=0$ ), la curva vuelve a cortar al plano $q_{1}=0$ para terminar definitivamente en el otro punto de vacío $\mathrm{D}=\vec{v}^{+}$.

Evidentemente se trata de kinks topológicos (en los sectores $\mathcal{C}^{(-,+)}$y $\mathcal{C}^{(+,-)}$), que denominaremos TK3 de manera general ${ }^{10}$.

Las elecciones realizadas nos recuerdan que una sóla trayectoria en coordenadas elípticas tiene asociadas ocho en cartesianas. Como veremos en el apartado 3.2.4, la descripción en coordenadas elípticas de las soluciones nos facilitará el análisis del Espacio Modular de Kinks del modelo, al estar considerado de manera natural en las mismas el cociente bajo la acción del grupo de simetría, $G=\mathbb{Z}_{2} \times \mathbb{Z}_{2} \times \mathbb{Z}_{2}$, del sistema.

Variando las constantes $\gamma_{2}$ y $\gamma_{3}$ encontraremos la totalidad de trayectorias de la familia TK3.

\section{Otras Parametrizaciones de los Kinks Genéricos}

De manera similar a lo ocurrido en el análisis del Modelo MSTB, en la sección 3.1, los parámetros $\gamma_{2}$ y $\gamma_{3}$ tienen una interpretación geométrica muy interesante, tanto en el espacio $\mathbb{R}^{3}$ como en $P_{3}(0)$. Es posible plantear unas ecuaciones similares a (3.18) que relacionen los parámetros con ángulos de incidencia sobre diferentes puntos (o curvas) distinguidos de la región $D^{3}$ (o $P_{3}(0)$ ). La complejidad de las mismas para este caso hace que nos centremos preferentemente en otros aspectos de la geometría de las soluciones.

\footnotetext{
${ }^{9} \mathrm{O}$ alternativamente el octante del elipsoide por el que la trayectoria comienza a trazarse.

${ }^{10} \mathrm{Si}$ se especifica el sector, la notación habitual impone que TK3 corresponda a $\mathcal{C}^{(-,+)}$y ATK3 al $\mathcal{C}^{(+,-)}$.
} 

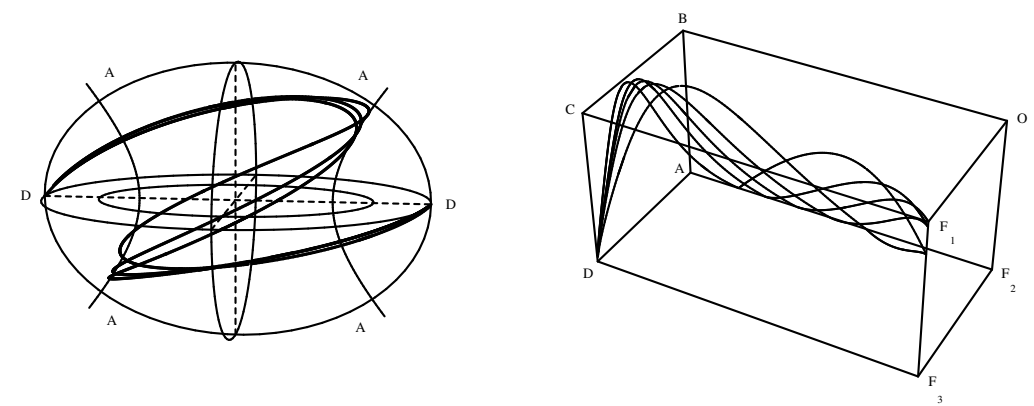

Figura 3.15: Varios kinks TK3 en el interior del elipsoide (a) y en $P_{3}(0)$ (b).

Como veremos, el estudio de otras parametrizaciones de los kinks genéricos aclarará en buena medida los límites asintóticos de la familia TK3 que analizaremos en la sección siguiente. El grado de complicación de los mismos será mucho mayor que en el caso bidimensional ya detallado en 3.1.

Partiendo de la parametrización inicial en las constantes $\gamma_{2}$ y $\gamma_{3}$, que establecen unívocamente una trayectoria TK3 (desde el punto de vista "elíptico", obviamente en cartesianas se corresponde con ocho trayectorias diferentes, como ya se ha comentado), tendremos que el espacio de soluciones TK3 es isomorfo a $\mathbb{R}^{2}$ o, equivalentemente, al plano complejo $\mathbb{C}$.

Introduciremos a continuación una nueva parametrización de la familia de kinks TK3, equivalente a la anterior, que se revelará muy útil a la hora de estudiar determinados límites asintóticos de los kinks, tanto analítica como geométricamente.

Definiremos las nuevas constantes, $\gamma$ y $\bar{\gamma}$, de la forma:

$$
\begin{array}{cc}
\gamma=\gamma_{3} \bar{\sigma}_{3}^{2}-\gamma_{2} ; & \bar{\gamma}=\gamma_{3} \bar{\sigma}_{2}^{2}-\gamma_{2} \\
\gamma_{2}=\frac{\bar{\sigma}_{2}^{2} \gamma-\bar{\sigma}_{3}^{2} \bar{\gamma}}{\sigma_{2}^{2}-\sigma_{3}^{2}} ; & \gamma_{3}=\frac{\gamma-\bar{\gamma}}{\sigma_{2}^{2}-\sigma_{3}^{2}}
\end{array}
$$

Podemos entonces manipular las ecuaciones $(3.25,3.26)$ para expresarlas en términos de $\gamma$ y $\bar{\gamma}$ de la forma:

$$
\begin{aligned}
e^{2 \sigma_{2} \bar{\sigma}_{2}^{2} \gamma=} & \left(\left|\frac{\sqrt{1-\lambda_{1}}-1}{\sqrt{1-\lambda_{1}}+1}\right|^{\sigma_{2}} \cdot\left|\frac{\sqrt{1-\lambda_{1}}+\sigma_{2}}{\sqrt{1-\lambda_{1}}-\sigma_{2}}\right|\right)^{\operatorname{sign}\left(\pi_{1}\right)} . \\
& \left(\left|\frac{\sqrt{1-\lambda_{2}}-1}{\sqrt{1-\lambda_{2}}+1}\right|^{\sigma_{2}} \cdot\left|\frac{\sqrt{1-\lambda_{2}}+\sigma_{2}}{\sqrt{1-\lambda_{2}}-\sigma_{2}}\right|\right)^{\operatorname{sign}\left(\pi_{2}\right)} . \\
& \left(\left|\frac{\sqrt{1-\lambda_{3}}-1}{\sqrt{1-\lambda_{3}}+1}\right|^{\sigma_{2}} \cdot\left|\frac{\sqrt{1-\lambda_{3}}+\sigma_{2}}{\sqrt{1-\lambda_{3}}-\sigma_{2}}\right|\right)^{\operatorname{sign}\left(\pi_{3}\right)} .
\end{aligned}
$$


y

$$
\begin{aligned}
e^{2 \sigma_{3} \bar{\sigma}_{3}^{2} \bar{\gamma}=} & \left(\left|\frac{\sqrt{1-\lambda_{1}}-1}{\sqrt{1-\lambda_{1}}+1}\right|^{\sigma_{3}} \cdot\left|\frac{\sqrt{1-\lambda_{1}}+\sigma_{3}}{\sqrt{1-\lambda_{1}}-\sigma_{3}}\right|\right)^{\operatorname{sign}\left(\pi_{1}\right)} . \\
& \left(\left|\frac{\sqrt{1-\lambda_{2}}-1}{\sqrt{1-\lambda_{2}}+1}\right|^{\sigma_{3}} \cdot\left|\frac{\sqrt{1-\lambda_{2}}+\sigma_{3}}{\sqrt{1-\lambda_{2}}-\sigma_{3}}\right|\right)^{\operatorname{sign}\left(\pi_{2}\right)} . \\
& \left(\left|\frac{\sqrt{1-\lambda_{3}}-1}{\sqrt{1-\lambda_{3}}+1}\right|^{\sigma_{3}} \cdot\left|\frac{\sqrt{1-\lambda_{3}}+\sigma_{3}}{\sqrt{1-\lambda_{3}}-\sigma_{3}}\right|\right)^{\operatorname{sign}\left(\pi_{3}\right)} .
\end{aligned}
$$

Veamos a continuación algunas de las interpretaciones geométricas que estas parametrizaciones admiten:

\section{Puntos de corte con la cara $\lambda_{3}=1$}

Cada uno de los kinks de la familia TK3 corta la cara superior de $\bar{P}_{3}(0)$ (plano $q_{1}=0$ en coordenadas cartesianas) tres veces a lo largo de su recorrido completo. Fijado uno de dichos puntos de corte (es decir los valores correspondientes de $\left.\begin{array}{lll}\lambda_{1} & \text { y } & \lambda_{2}\end{array}\right)$ los dos restantes quedan a su vez determinados. De esta manera, la arbitrariedad en los valores que puedan tomar las dos constantes que parametrizan a la familia de kinks (bien $\gamma_{2} \mathrm{y} \gamma_{3}$, bien $\gamma \mathrm{y} \bar{\gamma}$ ) es equivalente a fijar las coordenadas de uno de los puntos de corte con la cara $\mathrm{OF}_{1} \mathrm{BC}$ de $\partial P_{3}(0)$ (por ejemplo el segundo de ellos). Esta equivalencia se plasma analíticamente en las ecuaciones siguientes:

Dada una elección tomada de las constantes $\left(\gamma_{2}, \gamma_{3}\right)$, los puntos de corte con la cara $\lambda_{3}=1$ vienen determinados por las soluciones $\left(\lambda_{1}, \lambda_{2}\right)$ de las ecuaciones:

$$
\begin{aligned}
e^{2 \gamma_{2} \sigma_{2} \sigma_{3}\left(\sigma_{2}^{2}-\sigma_{3}^{2}\right)=} & \left(\left|\frac{\sqrt{1-\lambda_{1}}+\sigma_{2}}{\sqrt{1-\lambda_{1}}-\sigma_{2}}\right|^{\sigma_{3}} \cdot\left|\frac{\sqrt{1-\lambda_{1}}-\sigma_{3}}{\sqrt{1-\lambda_{1}}+\sigma_{3}}\right|^{\sigma_{2}}\right)^{\operatorname{sign}\left(\pi_{1}\right)} . \\
& \cdot\left(\left|\frac{\sqrt{1-\lambda_{2}}+\sigma_{2}}{\sqrt{1-\lambda_{2}}-\sigma_{2}}\right|^{\sigma_{3}} \cdot\left|\frac{\sqrt{1-\lambda_{2}}-\sigma_{3}}{\sqrt{1-\lambda_{2}}+\sigma_{3}}\right|^{\sigma_{2}}\right)^{\operatorname{sign}\left(\pi_{2}\right)}
\end{aligned}
$$

y

$$
\begin{aligned}
& e^{2 \gamma_{3} \sigma_{2} \sigma_{3} \bar{\sigma}_{2}^{2} \bar{\sigma}_{3}^{2}\left(\sigma_{2}^{2}-\sigma_{3}^{2}\right)}= \\
& =\left(\left|\frac{\sqrt{1-\lambda_{1}}-1}{\sqrt{1-\lambda_{1}}+1}\right|^{\sigma_{2} \sigma_{3}\left(\sigma_{2}^{2}-\sigma_{3}^{2}\right)} \cdot\left|\frac{\sqrt{1-\lambda_{1}}+\sigma_{2}}{\sqrt{1-\lambda_{1}}-\sigma_{2}}\right|^{\sigma_{3} \bar{\sigma}_{3}^{2}} \cdot\left|\frac{\sqrt{1-\lambda_{1}}-\sigma_{3}}{\sqrt{1-\lambda_{1}}+\sigma_{3}}\right|^{\sigma_{2} \bar{\sigma}_{2}^{2}}\right)^{\operatorname{sign}\left(\pi_{1}\right)} . \\
& \cdot\left(\left|\frac{\sqrt{1-\lambda_{2}}-1}{\sqrt{1-\lambda_{2}}+1}\right|^{\sigma_{2} \sigma_{3}\left(\sigma_{2}^{2}-\sigma_{3}^{2}\right)} \cdot\left|\frac{\sqrt{1-\lambda_{2}}+\sigma_{2}}{\sqrt{1-\lambda_{2}}-\sigma_{2}}\right|^{\sigma_{3} \bar{\sigma}_{3}^{2}} \cdot\left|\frac{\sqrt{1-\lambda_{2}}-\sigma_{3}}{\sqrt{1-\lambda_{2}}+\sigma_{3}}\right|^{\sigma_{2} \bar{\sigma}_{2}^{2}}\right)^{\mathrm{si} g\left(\lambda_{2}\right.}
\end{aligned}
$$


Equivalentemente, cada par $(\gamma, \bar{\gamma})$ conduce a unas ecuaciones con idénticas soluciones que $(3.50,3.51)$ :

$$
\begin{aligned}
e^{2 \sigma_{2} \bar{\sigma}_{2}^{2} \gamma=} & \left(\left|\frac{\sqrt{1-\lambda_{1}}-1}{\sqrt{1-\lambda_{1}}+1}\right|^{\sigma_{2}} \cdot\left|\frac{\sqrt{1-\lambda_{1}}+\sigma_{2}}{\sqrt{1-\lambda_{1}}-\sigma_{2}}\right|\right)^{\operatorname{sign}\left(\pi_{1}\right)} . \\
& \cdot\left(\left|\frac{\sqrt{1-\lambda_{2}}-1}{\sqrt{1-\lambda_{2}}+1}\right|^{\sigma_{2}} \cdot\left|\frac{\sqrt{1-\lambda_{2}}+\sigma_{2}}{\sqrt{1-\lambda_{2}}-\sigma_{2}}\right|\right)^{\operatorname{sign}\left(\pi_{2}\right)}
\end{aligned}
$$

$\mathrm{y}$

$$
\begin{aligned}
e^{2 \sigma_{3} \bar{\sigma}_{3}^{2} \bar{\gamma}=} & \left(\left|\frac{\sqrt{1-\lambda_{1}}-1}{\sqrt{1-\lambda_{1}}+1}\right|^{\sigma_{3}} \cdot\left|\frac{\sqrt{1-\lambda_{1}}+\sigma_{3}}{\sqrt{1-\lambda_{1}}-\sigma_{3}}\right|\right)^{\operatorname{sign}\left(\pi_{1}\right)} . \\
& \cdot\left(\left|\frac{\sqrt{1-\lambda_{2}}-1}{\sqrt{1-\lambda_{2}}+1}\right|^{\sigma_{3}} \cdot\left|\frac{\sqrt{1-\lambda_{2}}+\sigma_{3}}{\sqrt{1-\lambda_{2}}-\sigma_{3}}\right|\right)^{\operatorname{sign}\left(\pi_{2}\right)}
\end{aligned}
$$

Detallaremos en la próxima sección cómo se comportan las ecuaciones (3.50, $3.51)$ por un lado, y $(3.52,3.53)$, por otro, cuando alguno de los puntos de corte con $\lambda_{3}=1$ se considera muy cercano a alguna de las aristas de la cara $\mathrm{OF}_{1} \mathrm{BC}$ (se tratará evidentemente de un límite singular en el que una o varias de las constantes tiendan a $\pm \infty)$.

\section{Puntos de corte con las aristas $A F_{2}$ y $\mathbf{F}_{1} \mathbf{F}_{3}$}

El Modelo $O(3)$ presenta dos regiones especialmente distinguidas en el espacio de configuración del sistema mecánico: se trata de las cónicas que hemos denominado características, la elipse (3.31) y la hipérbola (3.32). Desde el punto de vista dinámico, se trata de los únicos conjuntos de puntos de $\bar{P}_{3}(0)$ (exceptuando los vacíos $D_{1}$ y $D_{2}$ ) que son atravesados por infinidad de soluciones kink. Desde esta perspectiva, su papel en el sistema es equivalente al representado por los focos de la elipse (3.10) en el modelo MSTB. Se tratará, por tanto, de curvas de puntos conjugados a los de vacío del sistema. En el Capítulo 6 de esta memoria volveremos sobre este tema a la hora de estudiar la Teoría de Morse del modelo.

Cada punto de la elipse (3.31), arista $\mathrm{F}_{1} \mathrm{~F}_{3}$ de $\bar{P}_{3}(0)$, es cortado una vez por cada trayectoria TK3 mientras que cada punto de (3.32), arista $\mathrm{AF}_{2}$ de $\bar{P}_{3}(0)$, lo es dos veces (en cartesianas se cortará una vez cada rama diferente de la hipérbola). Una vez fijado uno de los cortes con la arista $\mathrm{AF}_{2}$, el otro queda automáticamente determinado, se tienen por tanto dos libertades desde este punto de vista: el corte con $\mathrm{F}_{1} \mathrm{~F}_{3}$ y uno de los cortes con $\mathrm{AF}_{2}$.

De forma analítica, tendremos que la parametrización en las constantes $\gamma$ y $\bar{\gamma}$ es mucho más adecuada que la determinada por $\gamma_{2}$ y $\gamma_{3}$ para explicitar el cálculo 
de dichos puntos de corte y, en definitiva, la posibilidad de caracterizar cada una de las soluciones kink por los mismos.

Si tomamos el límite $\lambda_{2} \rightarrow \bar{\sigma}_{2}^{2}, \lambda_{3} \rightarrow \bar{\sigma}_{2}^{2}$ (límite que nos conduce a la arista $\mathrm{AF}_{2}$ en $\left.\bar{P}_{3}(0)\right)$ en las ecuaciones $(3.48,3.49)$ obtendremos, por un lado, la expresión:

$$
e^{2 \sigma_{3} \bar{\sigma}_{3}^{2} \bar{\gamma}}=\left(\left|\frac{\sqrt{1-\lambda_{1}}-1}{\sqrt{1-\lambda_{1}}+1}\right|^{\sigma_{3}} \cdot\left|\frac{\sqrt{1-\lambda_{1}}+\sigma_{3}}{\sqrt{1-\lambda_{1}}-\sigma_{3}}\right|\right)^{\operatorname{sign}\left(\pi_{1}\right)}
$$

cuyas soluciones denominaremos $\tilde{\lambda}_{1}$ de manera que $\left(\lambda_{1}, \lambda_{2}, \lambda_{3}\right)=\left(\tilde{\lambda}_{1}, \bar{\sigma}_{2}^{2}, \bar{\sigma}_{2}^{2},\right)$ serán los dos puntos de corte con la arista $\mathrm{AF}_{2}$, determinados en definitiva por el valor de la constante $\bar{\gamma}$. La otra ecuación nos conduce a una expresión de la forma (donde se ha de sustituir ya $\lambda_{1}$ por una de las soluciones de (3.54)):

$$
e^{2 \sigma_{2} \bar{\sigma}_{2}^{2} \gamma} \propto \lim _{\lambda_{2} \rightarrow \bar{\sigma}_{2}^{2} \lambda_{3} \rightarrow \bar{\sigma}_{2}^{2}} \frac{\sqrt{1-\lambda_{3}}-\sigma_{2}}{\sqrt{1-\lambda_{2}}-\sigma_{2}}
$$

de manera que la indeterminación del segundo miembro puede conducir a cualquier valor posible de la constante $\gamma$ (dado que se trata de dos límites en variables diferentes).

En lo que respecta a la elipse (3.31), de manera análoga a la anterior, deberemos tomar los límites $\lambda_{1} \rightarrow \bar{\sigma}_{3}^{2}$ y $\lambda_{2} \rightarrow \bar{\sigma}_{3}^{2}$ simultáneamente. Las ecuaciones $(3.48,3.49)$ nos conducen ahora a las expresiones:

$$
\begin{aligned}
e^{2 \sigma_{2} \bar{\sigma}_{2}^{2} \gamma}= & \left(\left|\frac{\sqrt{1-\lambda_{3}}-1}{\sqrt{1-\lambda_{3}}+1}\right|^{\sigma_{2}} \cdot\left|\frac{\sqrt{1-\lambda_{3}}+\sigma_{2}}{\sqrt{1-\lambda_{3}}-\sigma_{2}}\right|\right)^{\operatorname{sign}\left(\pi_{3}\right)} \\
& e^{2 \sigma_{3} \bar{\sigma}_{3}^{2} \gamma} \propto \lim _{\lambda_{1} \rightarrow \bar{\sigma}_{3}^{2} \lambda_{2} \rightarrow \bar{\sigma}_{3}^{2}} \frac{\sqrt{1-\lambda_{2}}-\sigma_{3}}{\sqrt{1-\lambda_{1}}-\sigma_{3}}
\end{aligned}
$$

de manera que, fijada $\gamma$, la ecuación (3.56) determina el punto de corte con $\mathrm{F}_{1} \mathrm{~F}_{3}$ : $\left(\lambda_{1}, \lambda_{2}, \lambda_{3}\right)=\left(\bar{\sigma}_{3}^{2}, \bar{\sigma}_{3}^{2}, \tilde{\lambda}_{3}\right)$, donde $\tilde{\lambda}_{3}$ es una raíz (única en este caso) de (3.56). Por su parte, la ecuación (3.57) permite que todos los valores de $\bar{\gamma}$ sean compatibles con dicho punto de corte.

Tenemos, en consecuencia, que una vez que se ha fijado el punto de corte con una rama de la hipérbola, mediante una elección de $\bar{\gamma}$, la segunda constante, $\gamma$, parametriza a toda una subfamilia de trayectorias TK3 que, manteniendo los cortes con la hipérbola fijos, intersecan con todos los puntos de la elipse $\mathrm{F}_{1} \mathrm{~F}_{3}$, exceptuando los propios $F_{1}$ y $F_{3}$. Una situación similar es la que se obtiene si se fija un valor constante de $\gamma$ de manera que queda determinado el punto de intersección con la elipse (3.31). Existe otra subfamilia, dependiente del parámetro 
$\bar{\gamma}$, o, equivalentemente, de los puntos de corte con las dos ramas de la hipérbola (3.32).

Evidentemente, el análisis anterior puede repetirse en función de las constantes $\gamma_{2}$ y $\gamma_{3}$. La dificultad que se plantea es que, al tomar los límites adecuados para alcanzar una de las dos cónicas características, las dos ecuaciones, (3.25) y (3.26), dependen de límites indeterminados. Es posible entonces manipular las ecuaciones resultantes de manera que en una de ellas se elimine la indeterminación, manteniéndose en la restante, el resultado que se obtiene es exactamente el sistema $(3.54,3.55)$ para los cortes con la hipérbola, y $(3.56,3.57)$ para la elipse. No incluiremos, por tanto, dichos cálculos en esta memoria puesto que conceptualmente no aportan ninguna novedad.

En lo que respecta a los extremos de las aristas estudiadas, es decir los vértices $\mathrm{A}, \mathrm{F}_{1}, \mathrm{~F}_{2}$ y $\mathrm{F}_{3}$, se trata de puntos no accesibles para las soluciones correspondientes a valores finitos de las constantes. Se alcanzan, como veremos en la próxima sección, cuando las constantes $\gamma_{2}$ y $\gamma_{3}$, o alternativamente $\gamma$ y $\bar{\gamma}$, tienden a sus límites asintóticos.

\subsubsection{Límites Especiales y Reglas de Suma}

La energía de cada solución kink, tal y como se estableció en la Sección 1.2, no es más que la acción de la trayectoria solución correspondiente en el sistema mecánico análogo (salvo constantes multiplicativas). De esta forma, los kinks genéricos (TK3) poseen una energía que se determina mediante el siguiente cálculo:

$$
\begin{gathered}
\frac{\sqrt{2} \lambda^{2}}{m^{3}} E_{\mathrm{TK} 3}=2 \mathcal{S}_{\lambda_{1}}\left(\lambda_{1}\right)+4 \mathcal{S}_{\lambda_{2}}\left(\lambda_{2}\right)+6 \mathcal{S}_{\lambda_{3}}\left(\lambda_{3}\right)= \\
=2 \int_{0}^{\bar{\sigma}_{3}^{2}} \frac{\lambda_{1}}{2 \sqrt{1-\lambda_{1}}} d \lambda_{1}+4 \int_{\bar{\sigma}_{3}^{2}}^{\bar{\sigma}_{2}^{2}} \frac{\lambda_{2}}{2 \sqrt{1-\lambda_{2}}} d \lambda_{2}+6 \int_{\bar{\sigma}_{2}^{2}}^{1} \frac{\lambda_{3}}{2 \sqrt{1-\lambda_{3}}} d \lambda_{3}= \\
=\frac{4}{3}+\frac{2}{3} \sigma_{3}\left(3-\sigma_{3}^{2}\right)+\frac{2}{3} \sigma_{2}\left(3-\sigma_{2}^{2}\right)
\end{gathered}
$$

donde, evidentemente, se ha considerado el número de veces que cada tramo es recorrido por una única trayectoria TK3 (dos veces para $\lambda_{1}$, cuatro para $\lambda_{2}$ y seis para $\left.\lambda_{3}\right)$.

Analizaremos en esta sección las energías de los diferentes tipos de kinks que hemos estudiado en la precedente. De manera análoga a lo ocurrido para el caso del Modelo MSTB, pero de una forma mucho más compleja, encontraremos una serie de reglas de suma para los diferentes tipos de kinks y demostraremos que 
dichas reglas no son en absoluto casuales; por el contrario, se corresponden con el hecho de que diferentes combinaciones de kinks envolventes y embebidos pueden ser considerados kinks genéricos si se pasa al límite asintótico en el espacio de los parámetros que caracterizan a estos últimos. Estos razonamientos nos llevarán, en la próxima sección, a presentar diferentes compactificaciones posibles del Espacio Modular de soluciones kink de la teoría.

Regla $E_{\mathrm{TK} 3}=E_{\mathrm{NTK} 2 \sigma_{3}}+E_{\mathrm{TK} 2 \sigma_{2}}$

Con un razonamiento similar al utilizado en (3.58), calcularemos la energía de los kinks de la familia embebida $\mathrm{NTK} 2 \sigma_{3}{ }^{11}$ :

$$
\begin{aligned}
E_{\mathrm{NTK} 2 \sigma_{3}}= & \frac{m^{3}}{\sqrt{2} \lambda^{2}}\left(2 \int_{0}^{\bar{\sigma}_{3}^{2}} \frac{\lambda_{1}}{2 \sqrt{1-\lambda_{1}}} d \lambda_{1}+4 \int_{\bar{\sigma}_{3}^{2}}^{\bar{\sigma}_{2}^{2}} \frac{\lambda_{2}}{2 \sqrt{1-\lambda_{2}}} d \lambda_{2}+\right. \\
& \left.+4 \int_{\bar{\sigma}_{2}^{2}}^{1} \frac{\lambda_{3}}{2 \sqrt{1-\lambda_{3}}} d \lambda_{3}\right)=\frac{m^{3}}{\sqrt{2} \lambda^{2}}\left(\frac{4}{3}+2 \sigma_{3}\left(1-\frac{\sigma_{3}^{2}}{3}\right)\right)
\end{aligned}
$$

Por su parte, para el kink TK2 $\sigma_{2}$ :

$$
E_{\mathrm{TK} 2 \sigma_{2}}=\frac{m^{3}}{\sqrt{2} \lambda^{2}}\left(4 \int_{\bar{\sigma}_{2}^{2}}^{1} \frac{\lambda_{3}}{2 \sqrt{1-\lambda_{3}}} d \lambda_{3}\right)=\frac{m^{3}}{\sqrt{2} \lambda^{2}} 2 \sigma_{2}\left(1-\frac{\sigma_{2}^{2}}{3}\right)
$$

de manera que se observa la regla de suma:

$$
E_{\mathrm{TK} 3}=E_{\mathrm{NTK} 2 \sigma_{3}}+E_{\mathrm{TK} 2 \sigma_{2}}
$$

Por otro lado, demostramos en la Sección 3.1 que la energía $E_{\mathrm{NTK} 2 \sigma_{3}}$ puede escribirse como:

$$
E_{\mathrm{NTK} 2 \sigma_{3}}=E_{\mathrm{TK} 2 \sigma_{3}}+E_{\mathrm{TK} 1}
$$

puesto que $E_{\mathrm{TK} 1}=\frac{m^{3}}{\sqrt{2} \lambda^{2}} \frac{4}{3}$ y $E_{\mathrm{TK} 2 \sigma_{3}}=\frac{m^{3}}{\sqrt{2} \lambda^{2}} 2 \sigma_{3}\left(1-\frac{\sigma_{3}^{2}}{3}\right)$.

La interpretación de la regla de suma (3.59) es clara: la suma de trayectorias NTK $2 \sigma_{3}+$ TK $2 \sigma_{2}$ tiene que ser, de alguna manera, un elemento límite de la familia TK3, con un "tiempo" reescalado, evidentemente. Calcularemos, por tanto, los límites asintóticos de la familia TK3, utilizando para ello las ecuaciones (3.48, 3.49).

En el límite $\gamma \rightarrow-\infty$ encontraremos kinks en la ecuación (3.48) en tres diferentes circunstancias:

\footnotetext{
${ }^{11} \mathrm{El}$ resultado, aunque calculado en otras coordenadas, es evidentemente igual al obtenido en la Sección 3.1 .
} 
- 1. Cuando el signo de $\pi_{1}$ se considera positivo $\left(\operatorname{sign}\left(\pi_{1}\right)=+1\right)$, el límite $\gamma \rightarrow-\infty$ se consigue en la ecuación (3.48) si $\lambda_{1} \rightarrow 0$. En esta situación, la ecuación (3.49) nos indica que $\bar{\gamma}$ podrá mantener un valor finito sólo cuando $\lambda_{2} \rightarrow \bar{\sigma}_{3}^{2}$ dentro de la elección $\operatorname{sign}\left(\pi_{2}\right)=+1$. Se trata de un límite no trivial en el que obtendremos dos veces el único tramo (arista DC) correspondiente al kink TK2 $\sigma_{2}{ }^{12}$ (según se considere $\operatorname{sign}\left(\pi_{3}\right)= \pm 1$ ), en el plano $q_{3}=0$, siempre y cuando $\lambda_{1}$ tienda a 0 y $\lambda_{2}$ tienda a $\bar{\sigma}_{3}^{2}, \forall \lambda_{3} \in\left(\bar{\sigma}_{2}^{2}, 1\right)$ de la siguiente forma concreta:

$$
\begin{aligned}
& \lim _{\left(\lambda_{1}, \lambda_{2}\right) \rightarrow\left(0, \bar{\sigma}_{3}^{2}\right)} \frac{\left(\sqrt{1-\lambda_{1}}-1\right)^{\sigma_{3}}}{\sqrt{1-\lambda_{2}}-\sigma_{3}}= \\
& =\frac{2^{\sigma_{3}}}{2 \sigma_{3}} \cdot e^{2 \sigma_{3} \bar{\sigma}_{3}^{2} \bar{\gamma}} \cdot\left(\left|\frac{\sqrt{1-\lambda_{3}}-1}{\sqrt{1-\lambda_{3}}+1}\right|^{\sigma_{3}} \cdot\left|\frac{\sqrt{1-\lambda_{3}}+\sigma_{3}}{\sqrt{1-\lambda_{3}}-\sigma_{3}}\right|\right)^{-\operatorname{sign}\left(\pi_{3}\right)}
\end{aligned}
$$

Existen otras posibilidades, para los casos:

$$
\left(\operatorname{sign}\left(\pi_{1}\right), \operatorname{sign}\left(\pi_{2}\right), \operatorname{sign}\left(\pi_{3}\right)\right)=(+1,-1, \pm 1)
$$

que no corresponden con límites kink en este caso $\gamma \rightarrow-\infty$. Serán relevantes, por contra, en el límite asociado $\gamma \rightarrow \infty$.

- 2. Si $\lambda_{2} \rightarrow \bar{\sigma}_{2}^{2}$ para los casos $\left(\operatorname{sign}\left(\pi_{1}\right), \operatorname{sign}\left(\pi_{2}\right), \operatorname{sign}\left(\pi_{3}\right)\right)=( \pm 1,-1, \pm 1)$, se obtiene directamente, sin más restricciones en la ecuación (3.49), que $\gamma \rightarrow-\infty$. De hecho, cuando $\lambda_{2} \rightarrow \bar{\sigma}_{2}^{2}$, la ecuación (3.49) se reduce a

$$
\begin{gathered}
\left|\frac{\sigma_{2}-1}{\sigma_{2}+1}\right|^{\sigma_{3}} \cdot\left|\frac{\sigma_{2}+\sigma_{3}}{\sigma_{2}-\sigma_{3}}\right| e^{2 \sigma_{3} \bar{\sigma}_{3}^{2} \bar{\gamma}}=\left(\left|\frac{\sqrt{1-\lambda_{1}}-1}{\sqrt{1-\lambda_{1}}+1}\right|^{\sigma_{3}} \cdot\left|\frac{\sqrt{1-\lambda_{1}}+\sigma_{3}}{\sqrt{1-\lambda_{1}}-\sigma_{3}}\right|\right)^{\operatorname{sign}\left(\pi_{1}\right)} \\
\cdot\left(\left|\frac{\sqrt{1-\lambda_{3}}-1}{\sqrt{1-\lambda_{3}}+1}\right|^{\sigma_{3}} \cdot\left|\frac{\sqrt{1-\lambda_{3}}+\sigma_{3}}{\sqrt{1-\lambda_{3}}-\sigma_{3}}\right|\right)^{\operatorname{sign}\left(\pi_{3}\right)}
\end{gathered}
$$

que no es otra que la ecuación de las trayectorias solución de la Ecuación de Hamilton-Jacobi para la parte de los kinks NTK2 $\sigma_{3}$ que pertenecen a la región $M_{2_{\sigma_{2}}}^{2}$.

- 3. Finalmente, el tercer caso relevante surge cuando $\lambda_{3}$ tiende a $\bar{\sigma}_{2}^{2}$ dentro de la elección de signos:

$$
\left(\operatorname{sign}\left(\pi_{1}\right), \operatorname{sign}\left(\pi_{2}\right), \operatorname{sign}\left(\pi_{3}\right)\right)=( \pm 1, \pm 1,-1)
$$

\footnotetext{
${ }^{12}$ Nótese que el kink completo precisa recorrer dos veces dicha arista.
} 
En esta situación, encontramos que $\gamma \rightarrow-\infty$ de nuevo directamente, sin más restricciones. La ecuación (3.49) se convierte entonces en

$$
\begin{gathered}
\left|\frac{\sigma_{2}-1}{\sigma_{2}+1}\right|^{\sigma_{3}} \cdot\left|\frac{\sigma_{2}+\sigma_{3}}{\sigma_{2}-\sigma_{3}}\right| e^{2 \sigma_{3} \bar{\sigma}_{3}^{2} \bar{\gamma}}=\left(\left|\frac{\sqrt{1-\lambda_{1}}-1}{\sqrt{1-\lambda_{1}}+1}\right|^{\sigma_{3}} \cdot\left|\frac{\sqrt{1-\lambda_{1}}+\sigma_{3}}{\sqrt{1-\lambda_{1}}-\sigma_{3}}\right|\right)^{\operatorname{sign}\left(\pi_{1}\right)} \\
\cdot\left(\left|\frac{\sqrt{1-\lambda_{2}}-1}{\sqrt{1-\lambda_{2}}+1}\right|^{\sigma_{2}} \cdot\left|\frac{\sqrt{1-\lambda_{2}}+\sigma_{3}}{\sqrt{1-\lambda_{2}}-\sigma_{3}}\right|\right)^{\operatorname{sign}\left(\pi_{2}\right)}
\end{gathered}
$$

que corresponde a la ecuación de la parte de los kinks NTK2 $\sigma_{3}$ que pertenece a $M_{2_{\sigma_{3}}}^{1}$.

Queda, por tanto, demostrado que, en el límite $\gamma \rightarrow-\infty$, puede verse la combinación NTK $2 \sigma_{3}+\mathrm{TK} 2 \sigma_{2}$ como un miembro de la familia TK3, tal y como apuntaba la regla de suma (3.59).

El análisis no estará completo, sin embargo, si no estudiamos qué ocurre en el límite $\gamma \rightarrow \infty$. El resultado es idéntico al ya tratado $\gamma \rightarrow-\infty$. Tomar el límite $\gamma \rightarrow \infty$ equivale, en el análisis anterior, a cambiar todas las elecciones de signos de $\pi_{1}, \pi_{2}$ y $\pi_{3}$ por las opuestas. Las tres opciones serían ahora:

- 1'. $\lambda_{1} \rightarrow 0$ produce $\gamma \rightarrow \infty$ si $\operatorname{sign}\left(\pi_{1}\right)=-1$. De nuevo $\bar{\gamma}$ finito nos proporciona la arista (doblemente recorrida) del kink TK2 $\sigma_{2}$, siempre y cuando $\lambda_{2} \rightarrow \bar{\sigma}_{3}^{2}$ en los casos con $\operatorname{sign}\left(\pi_{2}\right)=-1$, y con un comportamiento apropiado de los límites, dependiendo del valor de $\lambda_{3}$ en su recorrido por la arista DC.

- 2'. $\quad \lambda_{2} \rightarrow \bar{\sigma}_{2}^{2}$, para los casos $\left(\operatorname{sign}\left(\pi_{1}\right), \operatorname{sign}\left(\pi_{2}\right), \operatorname{sign}\left(\pi_{3}\right)\right)=( \pm 1,+1, \pm 1)$, reduce la ecuación (3.49) a la de la familia NTK2 $\sigma_{3}$ en $M_{2_{\sigma_{3}}}^{2}$.

- 3'. Y finalmente, $\lambda_{3} \rightarrow \bar{\sigma}_{2}^{2} \operatorname{con}\left(\operatorname{sign}\left(\pi_{1}\right), \operatorname{sign}\left(\pi_{2}\right), \operatorname{sign}\left(\pi_{3}\right)\right)=( \pm 1, \pm 1,+1)$, proporciona el límite $\gamma \rightarrow \infty$. La reducción de (3.49) es ahora la ecuación de NTK $2 \sigma_{3}$ en $M_{2_{\sigma_{3}}}^{1}$.

En definitiva, los límites $\gamma \rightarrow \pm \infty$ en la familia TK3 proporcionan como resultado la familia $\mathrm{NTK}_{2} \sigma_{3}$ más el kink TK2 $\sigma_{2}$. La regla de suma (3.59) queda completamente explicada.

Es posible estudiar ahora la combinación entre las reglas de suma (3.59) y (3.60):

$$
E_{\mathrm{TK} 3}=E_{\mathrm{TK} 2 \sigma_{2}}+E_{\mathrm{TK} 2 \sigma_{3}}+E_{\mathrm{TK} 1}
$$

Esta regla se puede demostrar considerando, dentro de los límites $\gamma \rightarrow \pm \infty$ ya tomados, la posibilidad $\bar{\gamma} \rightarrow \pm \infty$ en las ecuaciones reducidas $(3.61,3.62)$. 
Comenzando por el caso $\bar{\gamma} \rightarrow \infty$ encontraremos dos posibilidades en $M_{2_{\sigma_{3}}}^{2}$ y tres en $M_{2_{\sigma_{3}}}^{1}$ que conducen a diferentes tramos de soluciones kink:

- A. $\operatorname{En} M_{2_{\sigma_{3}}}^{2}\left(\lambda_{2}=\bar{\sigma}_{2}^{2}\right)$ :

- i. Si $\operatorname{sign}\left(\pi_{1}\right)=+1, \bar{\gamma} \rightarrow \infty$ cuando $\lambda_{1} \rightarrow \bar{\sigma}_{3}^{2}$ en la ecuación (3.61). En esta situación $\lambda_{1} \rightarrow \bar{\sigma}_{3}^{2}$ y $\lambda_{2}=\bar{\sigma}_{2}^{2}$ proporciona el tercer tramo del kink TK1, que hemos denotado anteriormente como TK1(III). Se obtiene, además, dos veces, como debe de ocurrir de cara a completar toda la trayectoria solución, de acuerdo con las dos posibilidades:

$$
\left(\operatorname{sign}\left(\pi_{1}\right), \operatorname{sign}\left(\pi_{2}\right), \operatorname{sign}\left(\pi_{3}\right)\right)=(+1,-1, \pm 1)
$$

- ii. Si $\operatorname{sign}\left(\pi_{1}\right)=-1, \bar{\gamma} \rightarrow \infty$ cuando $\lambda_{1}=0$. Encontramos en este caso el segundo tramo (arista AB) del kink topológico bidimensional en el plano $q_{2}=0, \mathrm{TK} 2 \sigma_{3}(\mathrm{II})$.

- B. $\operatorname{En} M_{2_{\sigma_{3}}}^{1}\left(\lambda_{3}=\bar{\sigma}_{2}^{2}\right)$ :

- iii. Para la posibilidad: $\operatorname{sign}\left(\pi_{1}\right)=+1$, se alcanza $\bar{\gamma} \rightarrow \infty$ si $\lambda_{1}=\bar{\sigma}_{3}^{2}$. Se obtiene, de manera análoga, el tramo TK1(II) (dos veces de nuevo).

- iv. Para $\operatorname{sign}\left(\pi_{1}\right)=-1, \bar{\gamma} \rightarrow \infty$ si $\lambda_{1}=0$. La contribución es ahora $\mathrm{TK} 2 \sigma_{3}(\mathrm{II})$.

- v. Por último, en la situación $\operatorname{sign}\left(\pi_{2}\right)=+1, \bar{\gamma}$ tiende a $\infty$ si $\lambda_{2}=\bar{\sigma}_{3}^{2}$, obteniéndose el tramo restante, TK1(I).

El límite $\bar{\gamma} \rightarrow-\infty$, después del $\gamma \rightarrow \pm \infty$, nos proporciona exactamente el mismo resultado. De esta manera hemos completado el análisis de la regla de suma (3.60): la combinación TK2 $\sigma_{3}$ más TK1 es un miembro límite de la familia $\mathrm{NTK} 2 \sigma_{3}$.

Ambas demostraciones nos prueban (desde esta perspectiva) la regla de suma conjunta, (3.63).

Desde el punto de vista geométrico, los límites anteriormente calculados tienen una interpretación muy clara e intuitiva: cada valor fijo y finito de la constante $\bar{\gamma}$ determina los puntos de corte de las correspondientes trayectorias TK3 con la arista $\mathrm{AF}_{2}$, por medio de la ecuación (3.54). El límite $\gamma \rightarrow \pm \infty$ conduce, por su parte, a que el punto de corte con la arista $\mathrm{F}_{1} \mathrm{~F}_{3}$ tienda al foco $\mathrm{F}_{3}$ (ver ecuación (3.56)). En lo que respecta a los puntos de corte con la cara $\lambda_{3}=1$ de $\partial P_{3}(0)$, 
el límite $\gamma \rightarrow \pm \infty$ hace que uno de los cortes tienda al punto $\mathrm{C}$ mientras que los otros dos restantes se sitúan en la arista BO (dependiendo los puntos concretos, evidentemente, del valor que tome $\bar{\gamma}$ ). La situación, por tanto, coincide con lo demostrado analíticamente, puesto que la combinación NTK2 $\sigma_{3}+$ TK2 $\sigma_{2}$ es la única posible que conduce a que todos sus miembros pasen por el punto $\mathrm{F}_{3}$, corten cuatro veces a la arista $\mathrm{AF}_{2}$, dos veces a la arista $\mathrm{BO}$ y pasen por el punto $\mathrm{C}$.

En la Figura 3.16 se muestra una trayectoria TK3 muy cercana al límite explicado.

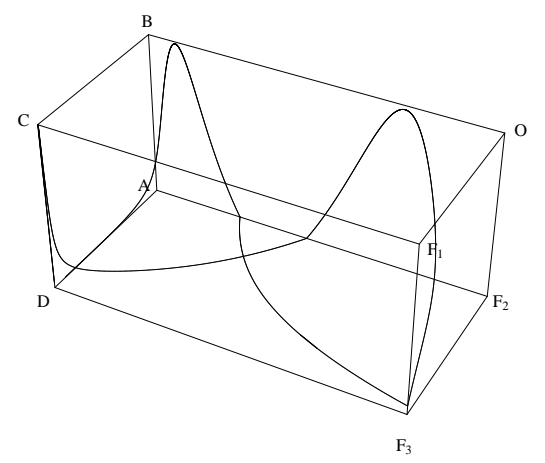

Figura 3.16: Trayectoria TK3 correspondiente a una valor grande de la constante $\gamma$.

Si posteriormente se toma el límite $\bar{\gamma} \rightarrow \pm \infty$ entonces, en (3.54), necesariamente los cortes con la arista $\mathrm{AF}_{2}$ se realizan en los extremos, $\mathrm{A} \mathrm{y}_{2}$, mientras que los cortes con BO se consiguen a su vez en B y en O. Dicha situación corresponde, evidentemente, a la sucesión de aristas que proporciona la configuración: TK2 $\sigma_{2}+$ TK2 $\sigma_{3}+$ TK1. En la Figura 3.19 está representado un kink cercano a este límite.

Regla $E_{\mathrm{TK} 3}=E_{\mathrm{NTK} 2 \sigma_{2}}+E_{\mathrm{TK} 2 \sigma_{3}}$

De manera similar al caso anterior, existen otras reglas de suma que involucran a las demás familias de kinks embebidos que hemos analizado en la sección precedente. La familia de kinks no topológicos del plano $q_{3}=0$ tiene la energía siguiente:

$$
\begin{aligned}
E_{\mathrm{NTK} 2 \sigma_{2}}= & \frac{m^{3}}{\sqrt{2} \lambda^{2}}\left(2 \int_{0}^{\bar{\sigma}_{3}^{2}} \frac{\lambda_{1}}{2 \sqrt{1-\lambda_{1}}} d \lambda_{1}+2 \int_{\bar{\sigma}_{3}^{2}}^{\bar{\sigma}_{2}^{2}} \frac{\lambda_{2}}{2 \sqrt{1-\lambda_{2}}} d \lambda_{2}+\right. \\
& \left.4 \int_{\bar{\sigma}_{2}^{2}}^{1} \frac{\lambda_{3}}{2 \sqrt{1-\lambda_{3}}} d \lambda_{3}\right)=\frac{m^{3}}{\sqrt{2} \lambda^{2}}\left(\frac{4}{3}+2 \sigma_{2}\left(1-\frac{\sigma_{2}^{2}}{3}\right)\right)
\end{aligned}
$$


Y conocemos, además, que la energía de los kinks TK2 $\sigma_{3}$ es:

$$
\begin{aligned}
E_{\mathrm{TK} 2 \sigma_{3}} & =\frac{m^{3}}{\sqrt{2} \lambda^{2}}\left(2 \int_{\bar{\sigma}_{3}^{2}}^{\bar{\sigma}_{2}^{2}} \frac{\lambda_{2}}{2 \sqrt{1-\lambda_{2}}} d \lambda_{2}+2 \int_{\bar{\sigma}_{2}^{2}}^{1} \frac{\lambda_{3}}{2 \sqrt{1-\lambda_{3}}} d \lambda_{3}\right)= \\
& =\frac{m^{3}}{\sqrt{2} \lambda^{2}} 2 \sigma_{3}\left(1-\frac{\sigma_{3}^{2}}{3}\right)
\end{aligned}
$$

Se verifican, por tanto, las reglas:

$$
\begin{aligned}
& E_{\mathrm{TK} 3}=E_{\mathrm{NTK} 2 \sigma_{2}}+E_{\mathrm{TK} 2 \sigma_{3}} \\
& E_{\mathrm{NTK} 2 \sigma_{2}}=E_{\mathrm{TK} 2 \sigma_{2}}+E_{\mathrm{TK} 1}
\end{aligned}
$$

análogas a las $(3.59,3.60)$.

Para demostrarlas (desde el punto de vista de los límites asintóticos) consideraremos primeramente, en las ecuaciones (3.48,3.49), el límite $\bar{\gamma} \rightarrow \infty$. Esto nos conducirá a tres posibilidades, diferentes de las encontradas en el anteriormente considerado caso $\gamma \rightarrow \pm \infty$ :

- 1. En todos los casos con $\operatorname{sign}\left(\pi_{1}\right)=-1$, el límite $\bar{\gamma} \rightarrow \infty$ se alcanza si $\lambda_{1}$ tiende a 0 . Sin embargo $\gamma$ permanece finita, de acuerdo con la ecuación (3.48), sólo en algunos de estos casos:

- a) en los casos $\left(\operatorname{sign}\left(\pi_{1}\right), \operatorname{sign}\left(\pi_{2}\right), \operatorname{sign}\left(\pi_{3}\right)\right)=(-1, \pm 1,-1)$, si $\lambda_{3}$ tiende a $\bar{\sigma}_{2}^{2}$ de tal manera que se verifique

$$
\begin{aligned}
& \lim _{\left(\lambda_{1}, \lambda_{3}\right) \rightarrow\left(0, \bar{\sigma}_{2}^{2}\right)} \frac{\left|\sqrt{1-\lambda_{3}}-\sigma_{2}\right|}{\left|\sqrt{1-\lambda_{1}}-1\right|^{\sigma_{2}}}= \\
& =\frac{2 \sigma_{2}}{2^{\sigma_{2}}} e^{2 \sigma_{2} \bar{\sigma}_{2}^{2} \gamma}\left(\left|\frac{\sqrt{1-\lambda_{2}}-1}{\sqrt{1-\lambda_{2}}+1}\right|^{\sigma_{2}} \cdot\left|\frac{\sqrt{1-\lambda_{2}}+\sigma_{2}}{\sqrt{1-\lambda_{2}}-\sigma_{2}}\right|\right)^{-\operatorname{sign}\left(\pi_{2}\right)}
\end{aligned}
$$

para cada valor concreto de $\lambda_{2}$ y para cada una de las dos posibles elecciones del signo de $\pi_{2}$, entonces se encuentra exactamente el primer tramo del kink bidimensional topológico en el plano $q_{2}=0$, es decir $\mathrm{TK} 2 \sigma_{3}(\mathrm{I})$ (obviamente dos veces, para $\operatorname{sign}\left(\pi_{2}\right)=+1$ y $\operatorname{para} \operatorname{sign}\left(\pi_{2}\right)=$ $-1)$.

- b) si, en los casos $\left(\operatorname{sign}\left(\pi_{1}\right), \operatorname{sign}\left(\pi_{2}\right), \operatorname{sign}\left(\pi_{3}\right)\right)=(-1,-1, \pm 1), \lambda_{2}$ tiende a $\bar{\sigma}_{2}^{2}$ de tal manera que

$$
\begin{aligned}
& \lim _{\left(\lambda_{1}, \lambda_{2}\right) \rightarrow\left(0, \bar{\sigma}_{2}^{2}\right)} \frac{\left|\sqrt{1-\lambda_{2}}-\sigma_{2}\right|}{\left|\sqrt{1-\lambda_{1}}-1\right|^{\sigma_{2}}}= \\
& =\frac{2 \sigma_{2}}{2^{\sigma_{2}}} e^{2 \sigma_{2} \bar{\sigma}_{2}^{2} \gamma}\left(\left|\frac{\sqrt{1-\lambda_{3}}-1}{\sqrt{1-\lambda_{3}}+1}\right|^{\sigma_{2}} \cdot\left|\frac{\sqrt{1-\lambda_{3}}+\sigma_{2}}{\sqrt{1-\lambda_{3}}-\sigma_{2}}\right|\right)^{-\operatorname{sign}\left(\pi_{3}\right)}
\end{aligned}
$$


para cada valor de $\lambda_{3}$ y cada signo de $\pi_{3}$, tenemos TK2 $\sigma_{3}$ (II).

- 2. En los casos con $\operatorname{sign}\left(\pi_{2}\right)=+1$, el límite $\lambda_{2} \rightarrow \bar{\sigma}_{3}^{2}$ implica que $\bar{\gamma}$ tienda a $\infty$ sin más restricciones en la ecuación (3.48). En este límite, $\lambda_{2}=\bar{\sigma}_{3}^{2}$, la ecuación (3.48) se reduce a:

$$
\begin{aligned}
\left|\frac{\sigma_{3}+1}{\sigma_{3}-1}\right|^{\sigma_{2}} \cdot\left|\frac{\sigma_{3}-\sigma_{2}}{\sigma_{3}+\sigma_{2}}\right| e^{2 \sigma_{2} \bar{\sigma}_{2}^{2} \gamma}= & \left(\left|\frac{\sqrt{1-\lambda_{1}}-1}{\sqrt{1-\lambda_{1}}+1}\right|^{\sigma_{2}} \cdot\left|\frac{\sqrt{1-\lambda_{1}}+\sigma_{2}}{\sqrt{1-\lambda_{1}}-\sigma_{2}}\right|\right)^{\operatorname{sign}\left(\pi_{1}\right)} \\
& \cdot\left(\left|\frac{\sqrt{1-\lambda_{3}}-1}{\sqrt{1-\lambda_{3}}+1}\right|^{\sigma_{2}} \cdot\left|\frac{\sqrt{1-\lambda_{3}}+\sigma_{2}}{\sqrt{1-\lambda_{3}}-\sigma_{2}}\right|\right)^{\operatorname{sign}\left(\pi_{3}\right)}(3.66)
\end{aligned}
$$

es decir, la ecuación de las trayectorias de la familia NTK2 $\sigma_{2}$ restringidas a la región $M_{2_{\sigma_{2}}}^{2}$.

- 3. La tercera posibilidad consiste en que $\operatorname{sign}\left(\pi_{1}\right)=+1$. Entonces $\lambda_{1}$ tendiendo a $\bar{\sigma}_{3}^{2}$ equivale a $\bar{\gamma} \rightarrow \infty$, y la ecuación (3.48) se reduce a:

$$
\begin{aligned}
\left|\frac{\sigma_{3}+1}{\sigma_{3}-1}\right|^{\sigma_{2}} \cdot\left|\frac{\sigma_{3}-\sigma_{2}}{\sigma_{3}+\sigma_{2}}\right| e^{2 \sigma_{2} \bar{\sigma}_{2}^{2} \gamma}= & \left(\left|\frac{\sqrt{1-\lambda_{2}}-1}{\sqrt{1-\lambda_{2}}+1}\right|^{\sigma_{2}} \cdot\left|\frac{\sqrt{1-\lambda_{2}}+\sigma_{2}}{\sqrt{1-\lambda_{2}}-\sigma_{2}}\right|\right)^{\operatorname{sign}\left(\pi_{2}\right)} \\
& \cdot\left(\left|\frac{\sqrt{1-\lambda_{3}}-1}{\sqrt{1-\lambda_{3}}+1}\right|^{\sigma_{2}} \cdot\left|\frac{\sqrt{1-\lambda_{3}}+\sigma_{2}}{\sqrt{1-\lambda_{3}}-\sigma_{2}}\right|\right)^{\operatorname{sign}\left(\pi_{3}\right)}(3.67)
\end{aligned}
$$

que da cuenta de la familia NTK2 $\sigma_{2}$ en el sector $M_{2_{\sigma_{2}}}^{1}$.

De forma similar al caso anterior, el límite $\bar{\gamma} \rightarrow-\infty$ proporciona los mismos resultados con el intercambio correspondiente de signos.

Las tres posibilidades son ahora:

- 1'. Para el caso $\operatorname{sign}\left(\pi_{1}\right)=-1, \lambda_{1}$ tendiendo a $\bar{\sigma}_{3}^{2}$ equivale a $\bar{\gamma} \rightarrow-\infty$. La ecuación (3.48) se reduce a ecuación de la familia NTK2 $\sigma_{2}$ en la región $M_{2_{\sigma_{2}}}^{1}$ de manera similar al caso 3 anterior.

- 2'. Si $\operatorname{sign}\left(\pi_{2}\right)=-1$, encontraremos $\bar{\gamma} \rightarrow-\infty$ para $\lambda_{2} \rightarrow \bar{\sigma}_{3}^{2}$. La ecuación (3.48) reducida no es otra que la ecuación de los kinks NTK2 $\sigma_{2}$ en $M_{2_{\sigma_{2}}}^{2}$, como en el caso 2 anterior.

- 3'. Por último, cuando $\operatorname{sign}\left(\pi_{1}\right)=+1, \bar{\gamma} \rightarrow-\infty$ se obtiene si $\lambda_{1} \rightarrow 0$. $\gamma$ permanece finito en dos casos diferentes: a) Si $\lambda_{3} \rightarrow \bar{\sigma}_{2}^{2}$ de la manera adecuada (y dependiente de $\lambda_{2}$ ), obteniéndose TK2 $\sigma_{3}(\mathrm{I})$. b) Si $\lambda_{2} \rightarrow \bar{\sigma}_{2}^{2}$ en un modo similar al caso 1.b) (salvo los signos intercambiados, obviamente), entonces el límite $\bar{\gamma} \rightarrow-\infty$ es el que proporciona TK2 $\sigma_{3}(\mathrm{II})$. 
Se ha demostrado, por tanto, que partiendo de las ecuaciones de la familia TK3 en la parametrización dada por las constantes $\gamma$ y $\bar{\gamma}$, se alcanza, en los límites $\bar{\gamma} \rightarrow \pm \infty$, la combinación: familia NTK2 $\sigma_{2}$ más TK2 $\sigma_{3}$. Queda probada así la regla de suma (3.64).

De manera similar al caso anterior, la regla de suma (3.64) puede conducirnos a su vez a la regla (3.63) si se calcula el límite $\gamma \rightarrow \pm \infty$ en las ecuaciones reducidas $(3.66,3.67)$, tras calcular el correspondiente límite $\bar{\gamma} \rightarrow \pm \infty$. Si combinamos, por ejemplo, los límites $\bar{\gamma} \rightarrow+\infty$ y $\gamma \rightarrow+\infty$, encontraremos dos posibilidades, tanto en $M_{2_{\sigma_{2}}}^{2}$ como en $M_{2_{\sigma_{2}}}^{1}$, que conducen a kinks:

- A. En la región $M_{2_{\sigma_{2}}}^{2}\left(\lambda_{2}=\bar{\sigma}_{3}^{2}\right)$ :

- i. Si $\operatorname{sign}\left(\pi_{1}\right)=-1$, se alcanza el límite $\gamma \rightarrow \infty$ cuando $\lambda_{1}$ tiende a 0 . $\lambda_{2}=\bar{\sigma}_{3}^{2}$ y $\lambda_{1}=0$ determinan el tramo TK2 $\sigma_{2}(\mathrm{I})$.

- ii. Si $\operatorname{sign}\left(\pi_{3}\right)=1, \lambda_{3} \rightarrow \bar{\sigma}_{2}^{2}$ equivale a $\gamma$ tendiendo a $\infty$ y ello nos proporciona TK1(I).

- B. Para $M_{2_{\sigma_{2}}}^{1}\left(\lambda_{1}=\bar{\sigma}_{3}^{2}\right)$ encontramos:

- iii. Si $\operatorname{sign}\left(\pi_{2}\right)=+1, \gamma \rightarrow \infty$ se corresponde con que $\lambda_{2}$ tienda a $\bar{\sigma}_{2}^{2}$. Obtenemos así el tramo TK1(III), para el que $\lambda_{1}=\bar{\sigma}_{3}^{2}$ y $\lambda_{2}=\bar{\sigma}_{2}^{2}$.

- iv. Para la posibilidad $\operatorname{sign}\left(\pi_{3}\right)=+1$ tendremos $\gamma \rightarrow \infty$ si $\lambda_{2} \rightarrow \bar{\sigma}_{2}^{2}$ y así obtenemos TK1(II) en este límite.

Si hubieramos considerado el límite $\gamma \rightarrow-\infty$ después de haber tomado $\bar{\gamma} \rightarrow$ $\pm \infty$ habríamos obtenido exactamente el mismo resultado final. Queda así probada la regla de suma (3.65), puesto que la combinación TK2 $\sigma_{2}+$ TK1 ha quedado establecida como un miembro de la familia NTK2 $\sigma_{2}$ en el límite $\gamma \rightarrow \pm \infty$. Combinando (3.64) y (3.65) se demuestra (3.63) y, en definitiva, se tiene a la combinación TK $2 \sigma_{2}+$ TK $2 \sigma_{3}+$ TK1 como un miembro de la familia TK3 en un límite de los parámetros muy particular.

Nuevamente es posible una interpretación geométrica clara de los cálculos anteriores. La consideración de $\bar{\gamma} \rightarrow \pm \infty$ (para un valor finito de $\gamma$ ) hace que mientras los cortes con la arista $\mathrm{AF}_{2}$ se sitúan en los extremos de la misma (ver ecuación (3.54)), los correspondientes (en esta situación se presentan dos puntos de corte) a la arista $\mathrm{F}_{1} \mathrm{~F}_{3}$ dependen directamente del valor de $\gamma$ (ver ecuación (3.56)).

Por su parte, en lo que respecta a los cortes con la cara $\lambda_{3}=1$, el límite considerado hace que uno de los cortes esté en el punto B mientras que los otros 
dos, de acuerdo con las ecuaciones $(3.52,3.53)$, pueden estar, bien en la arista $\mathrm{CF}_{1}$, bien en $\mathrm{F}_{1} \mathrm{O}$ (realmente pueden estar los dos en la primera o uno de ellos en la primera y el otro en la segunda, un análisis detallado de esas ecuaciones muestra que no pueden estar las dos soluciones en la arista $\mathrm{F}_{1} \mathrm{O}$ ), en función de los valores correspondientes de $\gamma$ y de las constantes $\sigma_{2}$ y $\sigma_{3}$ propias del modelo.

La unión de los dos razonamientos nos conduce inequívocamente a la configuración NTK $2 \sigma_{2}+$ TK $2 \sigma_{3}$, como se ha demostrado analíticamente. En la Figura 3.17 está representado un kink TK3 cercano a dicho límite.

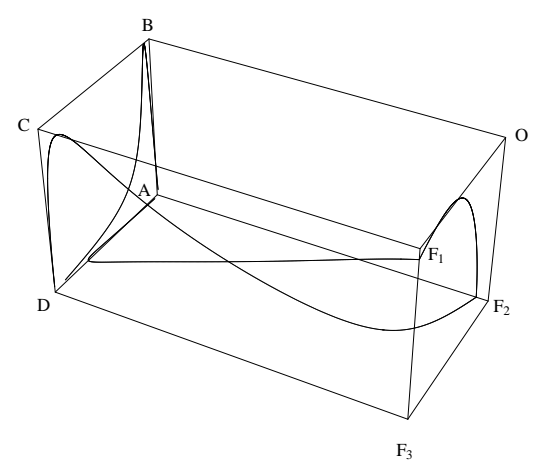

Figura 3.17: Trayectoria TK3 correspondiente a una valor grande de la constante $\bar{\gamma}$.

El posterior límite con $\gamma \rightarrow \pm \infty$ nos lleva a la misma situación geométrica del caso anterior y, en definitiva, a la combinación TK2 $\sigma_{2}+$ TK $2 \sigma_{3}+$ TK1.

\section{Regla $E_{\mathrm{TK} 3}=E_{\mathrm{NTK} 3}+E_{\mathrm{TK} 1}$}

Existe un tercer conjunto de reglas de suma de las energías de los kinks TK3, y de alguna manera más especial que los anteriormente expuestos.

La energía de los kinks envolventes (tridimensionales y no topológicos) que estudiábamos sobre el elipsoide $\lambda_{1}=0$, es decir las familias denotadas como NTK3, es:

$$
E_{\mathrm{NTK} 3}=\frac{m^{3}}{\sqrt{2} \lambda^{2}}\left(4 \mathcal{S}_{\lambda_{3}}\left(\lambda_{3}\right)+2 \mathcal{S}_{\lambda_{2}}\left(\lambda_{2}\right)\right)=\frac{2 m^{3}}{\sqrt{2} \lambda^{2}}\left(\sigma_{2}+\sigma_{3}-\frac{\sigma_{2}^{3}+\sigma_{3}^{3}}{3}\right)
$$

De manera que pueden comprobarse fácilmente las reglas de suma:

$$
E_{\mathrm{TK} 3}=E_{\mathrm{NTK} 3}+E_{\mathrm{TK} 1}
$$




$$
E_{\mathrm{NTK} 3}=E_{\mathrm{TK} 2 \sigma_{3}}+E_{\mathrm{TK} 2 \sigma_{2}}
$$

Esperaremos así que un proceso de paso al límite como los anteriores nos demuestre que, efectivamente, la combinación NTK3+TK1 puede ser vista como un miembro de la familia TK3 en las condiciones que dichos límites determinen. En esta ocasión, como veremos, las ecuaciones más adecuadas para efectuar dicho procedimiento serán las que se obtienen directamente de la ecuación de HamiltonJacobi del sistema, es decir las ecuaciones (3.25) y (3.26), parametrizadas por las constantes $\gamma_{2}$ y $\gamma_{3}$ (o alternativamente $C_{2}$ y $C_{3}$ ). Cuando se estudia en ellas el límite $\gamma_{3} \rightarrow-\infty$ o $C_{3}$ tendiendo a cero, resultan cinco casos relevantes:

- 1. $\operatorname{Si~} \operatorname{sign}\left(\pi_{1}\right)=+1$ y $\lambda_{1}$ tiende a 0 .

Encontramos entonces que $C_{3} \rightarrow 0$ en cualquiera de las cuatro opciones:

$$
\left(\operatorname{sign}\left(\pi_{1}\right), \operatorname{sign}\left(\pi_{2}\right), \operatorname{sign}\left(\pi_{3}\right)\right)=(+1, \pm 1, \pm 1)
$$

La ecuación (3.25) se reduce entonces a

$$
\begin{aligned}
& \left|\frac{1-\sigma_{2}}{1+\sigma_{2}}\right|^{\sigma_{3}}\left|\frac{1+\sigma_{3}}{1-\sigma_{3}}\right|^{\sigma_{2}} \cdot C_{2}=\left|\frac{\sqrt{1-\lambda_{2}}+\sigma_{2}}{\sqrt{1-\lambda_{2}}-\sigma_{2}}\right|^{\sigma_{3} \operatorname{sign}\left(\pi_{2}\right)} \cdot \\
& \cdot\left|\frac{\sqrt{1-\lambda_{2}}-\sigma_{3}}{\sqrt{1-\lambda_{2}}+\sigma_{3}}\right|^{\sigma_{2} \operatorname{sign}\left(\pi_{2}\right)} \cdot\left|\frac{\sqrt{1-\lambda_{3}}+\sigma_{2}}{\sqrt{1-\lambda_{3}}-\sigma_{2}}\right|^{\sigma_{3} \operatorname{sign}\left(\pi_{3}\right)} \cdot\left|\frac{\sqrt{1-\lambda_{3}}-\sigma_{3}}{\sqrt{1-\lambda_{3}}+\sigma_{3}}\right|^{\sigma_{2} \operatorname{sign}\left(\pi_{3}\right)}
\end{aligned}
$$

es decir, la ecuación de la familia NTK3 parametrizada por la constante $\gamma_{2}$ (o $\left.C_{2}\right)$.

- 2. Cuando $\operatorname{sign}\left(\pi_{1}\right)=+1$ y $\lambda_{1}$ tiende a $\bar{\sigma}_{3}^{2}$. El comportamiento de la ecuación (3.25), con $\gamma_{2}$ finita, nos conduce a tres subcasos diferentes:

- 2.a) Si $\lambda_{1} \rightarrow \bar{\sigma}_{3}^{2}$ y $\lambda_{2} \rightarrow \bar{\sigma}_{2}^{2}$ simultáneamente, para las opciones de signos:

$$
\left(\operatorname{sign}\left(\pi_{1}\right), \operatorname{sign}\left(\pi_{2}\right), \operatorname{sign}\left(\pi_{3}\right)\right)=(+1,+1, \pm 1)
$$

entonces la situación $\gamma_{3}$ tendiendo a $-\infty$, y al mismo tiempo $\gamma_{2}$ permaneciendo finita, se consigue de una forma no trivial en absoluto. Es necesario que el límite

$$
\lim _{\left(\lambda_{1}, \lambda_{2}\right) \rightarrow\left(\bar{\sigma}_{3}^{2}, \bar{\sigma}_{2}^{2}\right)} \frac{\left|\sqrt{1-\lambda_{1}}-\sigma_{3}\right|^{\sigma_{2} \bar{\sigma}_{2}^{2}}}{\left|\sqrt{1-\lambda_{2}}-\sigma_{2}\right|^{\sigma_{3} \bar{\sigma}_{3}^{2}}}
$$


sea nulo, para obtener $\gamma_{3} \rightarrow-\infty$, mientras que el límite

$$
\lim _{\left(\lambda_{1}, \lambda_{2}\right) \rightarrow\left(\bar{\sigma}_{3}^{2}, \bar{\sigma}_{2}^{2}\right)} \frac{\left|\sqrt{1-\lambda_{1}}-\sigma_{3}\right|^{\sigma_{2}}}{\left|\sqrt{1-\lambda_{2}}-\sigma_{2}\right|^{\sigma_{3}}}
$$

es necesariamente no nulo y dependiente del valor de $\lambda_{3}$ y del signo $\operatorname{sign}\left(\pi_{3}\right)$, para que pueda obtenerse el valor correcto de la constante $\gamma_{2}$ fijada. El límite tiene que ser exactamente igual a

$$
\begin{aligned}
& e^{2 \gamma_{2} \sigma_{2} \sigma_{3}\left(\sigma_{2}^{2}-\sigma_{3}^{2}\right)}\left|\frac{\sigma_{2}+\sigma_{3}}{\sigma_{2}-\sigma_{3}}\right|^{\sigma_{2}-\sigma_{3}} \frac{\left(2 \sigma_{3}\right)^{\sigma_{2}}}{\left(2 \sigma_{2}\right)^{\sigma_{3}}} \\
& \left(\left|\frac{\sqrt{1-\lambda_{3}}+\sigma_{2}}{\sqrt{1-\lambda_{3}}-\sigma_{2}}\right|^{\sigma_{3}} \cdot\left|\frac{\sqrt{1-\lambda_{3}}-\sigma_{3}}{\sqrt{1-\lambda_{3}}+\sigma_{3}}\right|^{\sigma_{2}}\right)^{-\operatorname{sign}\left(\pi_{3}\right)}
\end{aligned}
$$

Esta situación tan especial, en la que los dos límites tienen que valer simultáneamente los valores indicados, es posible gracias a que las constantes que determinan el modelo verifican que $\bar{\sigma}_{2}^{2}>\bar{\sigma}_{3}^{2}$.

La situación descrita se corresponde exactamente con el tramo TK1(III), y obviamente dos veces, como esperamos, de acuerdo con los dos posibles signos de $\pi_{3}$.

- 2.b) Si consideramos ahora $\lambda_{1} \rightarrow \bar{\sigma}_{3}^{2}$ y $\lambda_{2} \rightarrow \bar{\sigma}_{3}^{2}$ al mismo tiempo, para las posibilidades

$$
\left(\operatorname{sign}\left(\pi_{1}\right), \operatorname{sign}\left(\pi_{2}\right), \operatorname{sign}\left(\pi_{3}\right)\right)=(+1,-1, \pm 1)
$$

encontramos una situación diferente, el límite

$$
\lim _{\left(\lambda_{1}, \lambda_{2}\right) \rightarrow\left(\bar{\sigma}_{3}^{2}, \bar{\sigma}_{3}^{2}\right)} \frac{\left|\sqrt{1-\lambda_{1}}-\sigma_{3}\right|^{\sigma_{2} \bar{\sigma}_{2}^{2}}}{\left|\sqrt{1-\lambda_{2}}-\sigma_{3}\right|^{\sigma_{2} \bar{\sigma}_{2}^{2}}}
$$

es cero y, en consecuencia, el límite correspondiente en la ecuación (3.25):

$$
\lim _{\left(\lambda_{1}, \lambda_{2}\right) \rightarrow\left(\bar{\sigma}_{3}^{2}, \bar{\sigma}_{3}^{2}\right)} \frac{\left|\sqrt{1-\lambda_{1}}-\sigma_{3}\right|^{\sigma_{2}}}{\left|\sqrt{1-\lambda_{2}}-\sigma_{3}\right|^{\sigma_{2}}}
$$

es nulo también. Para que, en estas circunstancias, se mantenga la hipótesis de $\gamma_{3} \rightarrow-\infty$ y $\gamma_{2}$ fija y finita, la única posibilidad es que $\lambda_{3}$ tienda a $\bar{\sigma}_{2}^{2}$ junto con que $\operatorname{sign}\left(\pi_{3}\right)=-1$. Esto significa que el tramo de signos $(+1,-1,+1)$ del kink genérico TK3 original se convierte, en esta situación límite, en únicamente un punto, concretamente el $\mathrm{F}_{3}$. 
- 2.c) Cuando $\lambda_{1} \rightarrow \bar{\sigma}_{3}^{2}$ y $\lambda_{3} \rightarrow \bar{\sigma}_{2}^{2}$ simultáneamente, para las opciones de signos:

$$
\left(\operatorname{sign}\left(\pi_{1}\right), \operatorname{sign}\left(\pi_{2}\right), \operatorname{sign}\left(\pi_{3}\right)\right)=(+1, \pm 1,+1)
$$

Un análisis similar de este subcaso nos conduce al tramo TK1(II), siempre y cuando se verifique

$$
\lim _{\left(\lambda_{1}, \lambda_{3}\right) \rightarrow\left(\bar{\sigma}_{3}^{2}, \bar{\sigma}_{2}^{2}\right)} \frac{\left|\sqrt{1-\lambda_{1}}-\sigma_{3}\right|^{\sigma_{2} \bar{\sigma}_{2}^{2}}}{\left|\sqrt{1-\lambda_{3}}-\sigma_{2}\right|^{\sigma_{3} \bar{\sigma}_{3}^{2}}}=0
$$

mientras que el límite

$$
\lim _{\left(\lambda_{1}, \lambda_{3}\right) \rightarrow\left(\bar{\sigma}_{3}^{2}, \bar{\sigma}_{2}^{2}\right)} \frac{\left|\sqrt{1-\lambda_{1}}-\sigma_{3}\right|^{\sigma_{2}}}{\left|\sqrt{1-\lambda_{3}}-\sigma_{2}\right|^{\sigma_{3}}}
$$

permanece no nulo y dependiente del valor de $\lambda_{2}$ y del $\operatorname{signo} \operatorname{sign}\left(\pi_{2}\right)$ de una manera similar al límite analizado en el subcaso 2.a).

- 3. Para $\operatorname{sign}\left(\pi_{2}\right)=+1$ y $\lambda_{2}$ tendiendo a $\bar{\sigma}_{3}^{2}$, dos son los subcasos relevantes:

- 3.a) $\lambda_{1} \rightarrow \bar{\sigma}_{3}^{2}$ y $\lambda_{2} \rightarrow \bar{\sigma}_{3}^{2}$ simultáneamente, para las siguientes opciones en los signos:

$$
\left(\operatorname{sign}\left(\pi_{1}\right), \operatorname{sign}\left(\pi_{2}\right), \operatorname{sign}\left(\pi_{3}\right)\right)=(-1,+1, \pm 1)
$$

Esta situación presenta características análogas a las del subcaso 2.b). Los dos límites relevantes, en las ecuaciones (3.25) y (3.26), son nulos y así el valor de $\lambda_{3}$ estará fijado en $\bar{\sigma}_{2}^{2}$; se obtiene de esta manera que todo el tramo original se reduce, al pasar al límite, al punto $\mathrm{F}_{3}$.

- 3.b) $\lambda_{2} \rightarrow \bar{\sigma}_{3}^{2}$ y $\lambda_{3} \rightarrow \bar{\sigma}_{2}^{2}$ para la elección de signos:

$$
\left(\operatorname{sign}\left(\pi_{1}\right), \operatorname{sign}\left(\pi_{2}\right), \operatorname{sign}\left(\pi_{3}\right)\right)=( \pm 1,+1,+1)
$$

Se obtiene en este subcaso el tramo (I) del kink TK1 siempre y cuando se verifique, en la ecuación (3.25), que

$$
\begin{gathered}
\lim _{\left(\lambda_{2}, \lambda_{3}\right) \rightarrow\left(\bar{\sigma}_{3}^{2}, \bar{\sigma}_{2}^{2}\right)} \frac{\left|\sqrt{1-\lambda_{2}}-\sigma_{3}\right|^{\sigma_{2}}}{\left|\sqrt{1-\lambda_{3}}-\sigma_{2}\right|^{\sigma_{3}}}= \\
e^{2 \gamma_{2} \sigma_{2} \sigma_{3}\left(\sigma_{2}^{2}-\sigma_{3}^{2}\right)}\left|\frac{\sigma_{2}+\sigma_{3}}{\sigma_{2}-\sigma_{3}}\right|^{\sigma_{2}-\sigma_{3}} \frac{\left(2 \sigma_{3}\right)^{\sigma_{2}}}{\left(2 \sigma_{2}\right)^{\sigma_{3}}} \cdot \\
\left(\left|\frac{\sqrt{1-\lambda_{1}}+\sigma_{2}}{\sqrt{1-\lambda_{1}}-\sigma_{2}}\right|^{\sigma_{3}} \cdot\left|\frac{\sqrt{1-\lambda_{1}}-\sigma_{3}}{\sqrt{1-\lambda_{1}}+\sigma_{3}}\right|^{\sigma_{2}}\right)^{-\operatorname{sign}\left(\pi_{1}\right)}
\end{gathered}
$$


al mismo tiempo que

$$
\lim _{\left(\lambda_{2}, \lambda_{3}\right) \rightarrow\left(\bar{\sigma}_{3}^{2}, \bar{\sigma}_{2}^{2}\right)} \frac{\left|\sqrt{1-\lambda_{2}}-\sigma_{3}\right|^{\sigma_{2} \bar{\sigma}_{2}^{2}}}{\left|\sqrt{1-\lambda_{3}}-\sigma_{2}\right|^{\sigma_{3} \bar{\sigma}_{3}^{2}}}=0
$$

para asegurar que $\gamma_{3}$ tienda a $-\infty$.

- 4. En la situación $\operatorname{sign}\left(\pi_{2}\right)=-1$ y $\lambda_{2} \rightarrow \bar{\sigma}_{2}^{2}$, dos son los subcasos posibles:

- 4.a) Si $\lambda_{1} \rightarrow \bar{\sigma}_{3}^{2}$ y $\lambda_{2} \rightarrow \bar{\sigma}_{2}^{2}$, con signos:

$$
\left(\operatorname{sign}\left(\pi_{1}\right), \operatorname{sign}\left(\pi_{2}\right), \operatorname{sign}\left(\pi_{3}\right)\right)=(-1,-1, \pm 1)
$$

entonces no existe ninguna posibilidad que conduzca a los valores de las constantes $\gamma_{2}$ y $\gamma_{3}$ que hemos tomado.

- 4.b) Para el límite $\lambda_{2} \rightarrow \bar{\sigma}_{2}^{2}$ y $\lambda_{3} \rightarrow \bar{\sigma}_{2}^{2}$ con

$$
\left(\operatorname{sign}\left(\pi_{1}\right), \operatorname{sign}\left(\pi_{2}\right), \operatorname{sign}\left(\pi_{3}\right)\right)=( \pm 1,-1,+1)
$$

se obtiene, de una manera similar a los análisis precedentes, el punto $\mathrm{F}_{2}$.

- 5. Caso $\operatorname{sign}\left(\pi_{3}\right)=-1$ y $\lambda_{3} \rightarrow \bar{\sigma}_{2}^{2}$. Tres casos son ahora los relevantes, si bien ninguno de ellos produce soluciones kink.

- 5.a) $\lambda_{1} \rightarrow \bar{\sigma}_{3}^{2}$ y $\lambda_{3} \rightarrow \bar{\sigma}_{2}^{2}$, con signos:

$$
\left(\operatorname{sign}\left(\pi_{1}\right), \operatorname{sign}\left(\pi_{2}\right), \operatorname{sign}\left(\pi_{3}\right)\right)=(-1, \pm 1,-1)
$$

Se trata de unas hipótesis incompatibles con la elección de las constantes $\gamma_{2}$ y $\gamma_{3}$ realizada.

- 5.b) $\lambda_{2} \rightarrow \bar{\sigma}_{2}^{2}$ y $\lambda_{3} \rightarrow \bar{\sigma}_{2}^{2}$, con signos

$$
\left(\operatorname{sign}\left(\pi_{1}\right), \operatorname{sign}\left(\pi_{2}\right), \operatorname{sign}\left(\pi_{3}\right)\right)=( \pm 1,+1,-1)
$$

Esta situación conduce, de manera similar al caso previo 4.b), al punto $\mathrm{F}_{2}$.

- 5.c) $\lambda_{2} \rightarrow \bar{\sigma}_{3}^{2}$ y $\lambda_{3} \rightarrow \bar{\sigma}_{2}^{2}$, con una elección de signos:

$$
\left(\operatorname{sign}\left(\pi_{1}\right), \operatorname{sign}\left(\pi_{2}\right), \operatorname{sign}\left(\pi_{3}\right)\right)=( \pm 1,-1,-1)
$$

De nuevo nos encontramos con un subcaso imposible. 
Una vez completado el análisis de todas las posibilidades, es conveniente resaltar que el límite $\gamma_{3} \rightarrow+\infty$ conduce exactamente al mismo resultado, intercambiando en dicho cálculo todos los signos y, por tanto, unos subcasos con otros, de esta manera las situaciones antes mencionadas como imposibles se convierten ahora en los casos importantes, que nos conducen a los límites esperados.

Queda demostrado por tanto que, en los límites $\gamma_{3} \rightarrow \pm \infty$, la familia TK3 se convierte en la familia NTK3 más el kink TK1 y, en definitiva, la regla de suma (3.64) queda aclarada completamente.

La regla de suma (3.65) puede también obtenerse ahora si, tras haber tomado el límite $\gamma_{3} \rightarrow-\infty$ (o alternativamente $\gamma_{3} \rightarrow \infty$ ), consideramos el límite $\gamma_{2} \rightarrow$ $-\infty$. La condición $\lambda_{1}=0$ se alcanza en tres posibles subcasos que exponemos sucintamente:

- 1. $\operatorname{sign}\left(\pi_{2}\right)=+1, \lambda_{2}=\bar{\sigma}_{3}^{2}$. Se obtiene el único tramo de que consta el kink $\mathrm{TK} 2 \sigma_{2}$.

- 2. $\operatorname{sign}\left(\pi_{2}\right)=-1, \lambda_{2}=\bar{\sigma}_{2}^{2}$. Análogamente para el tramo TK2 $\sigma_{3}(\mathrm{II})$.

- 3. $\operatorname{sign}\left(\pi_{3}\right)=-1, \lambda_{3}=\bar{\sigma}_{2}^{2}$. Tramo TK2 $\sigma_{3}(\mathrm{I})$.

Y además dos veces cada uno de ellos, tal y como se espera. Se obtendría el mismo resultado si se toma el límite $\gamma_{2} \rightarrow \infty$ (tanto en el caso $\gamma_{3} \rightarrow \infty$ como en el $\left.\gamma_{3} \rightarrow-\infty\right)$. Se prueba, por tanto, que la combinación de los dos kink topológicos bidimensionales es una trayectoria límite de los kinks de la familia NTK3 y, en definitiva, la regla de suma (3.65). La combinación de las dos reglas de suma obtenidas, (3.64) y (3.65), nos proporciona una vez más la regla de suma (3.63).

Desde el punto de vista geométrico, $\gamma_{3} \rightarrow \pm \infty$ nos conduce, en las ecuaciones $(3.50,3.51)$, a que dos de los cortes con la cara $\lambda_{3}=1$ tengan lugar en la arista BC (en puntos concretos dependientes del valor de $\gamma_{2}$ ) mientras que el tercer punto de corte se sitúa en el punto O. Los puntos de corte con las aristas características se realizan en los extremos, $\mathrm{A}, \mathrm{F}_{2}$ y $\mathrm{F}_{3}$, debido a que $\gamma_{3} \rightarrow \pm \infty$ conduce a que $\gamma$ y $\bar{\gamma} \rightarrow \pm \infty$, y así las ecuaciones (3.54) y (3.56) determinan dichos puntos. La situación, por tanto, coincide con la calculada analíticamente.

El límite posterior $\gamma_{2} \rightarrow \pm \infty$ lleva a que los puntos de corte con BC se sitúen en los extremos del segmento y se reproduce la situación conocida.

En la Figura 3.18 está representada una trayectoria TK3 correspondiente a un valor grande de la constante $\gamma_{3}$. 


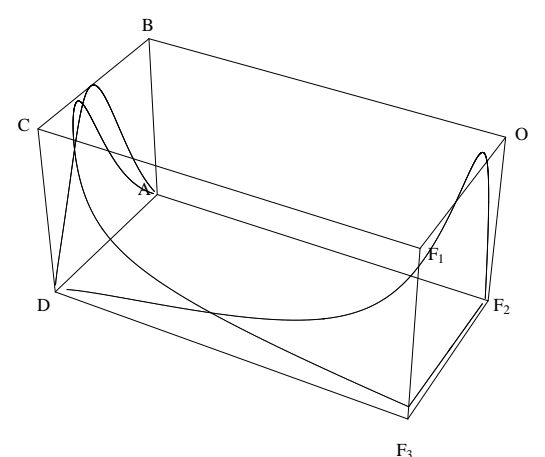

Figura 3.18: Trayectoria TK3 cercana al límite $\gamma_{3} \rightarrow \infty$ (con $\gamma_{2}$ finita).

\section{Regla $E_{\mathrm{TK} 3}=E_{\mathrm{TK} 2 \sigma_{2}}+E_{\mathrm{TK} 2 \sigma_{3}}+E_{\mathrm{TK} 1}$}

Como hemos visto en todos los apartados anteriores, las diferentes reglas de suma que hemos obtenido desembocan finalmente en la regla (3.63):

$$
E_{\mathrm{TK} 3}=E_{\mathrm{TK} 2 \sigma_{2}}+E_{\mathrm{TK} 2 \sigma_{3}}+E_{\mathrm{TK} 1}
$$

Sin embargo, no son éstos los únicos caminos posibles para obtener dicho resultado. Más aún, se trata de límites muy especiales y delicados en su comportamiento, como hemos detallado. La regla de suma (3.63) es, desde este punto de vista, la más general de todas, y puede obtenerse directamente del análisis de las ecuaciones de la familia TK3 por dos procedimientos generales.

En primer lugar, si se considera el límite $\gamma_{2} \rightarrow \pm \infty$ permaneciendo $\gamma_{3}$ finita, se obtiene, sorprendentemente, que, independientemente del valor de $\gamma_{3}$, el resultado es $\mathrm{NTK} 3=\mathrm{TK} 2 \sigma_{3}+\mathrm{TK} 2 \sigma_{2}+\mathrm{TK} 1$, de forma directa. Mostraremos de manera resumida esta situación para el caso concreto en el que $\gamma_{2} \rightarrow-\infty$. Se tienen en este caso cuatro contribuciones al límite:

- 1. Si $\lambda_{1} \rightarrow \bar{\sigma}_{3}^{2}$ en los tramos dados por:

$$
\left(\operatorname{sign}\left(\pi_{1}\right), \operatorname{sign}\left(\pi_{2}\right), \operatorname{sign}\left(\pi_{3}\right)\right)=(+1, \pm 1, \pm 1)
$$

tendremos los siguientes subcasos:

- 1.a) $\left(\lambda_{1}, \lambda_{2}\right) \rightarrow\left(\bar{\sigma}_{3}^{2}, \bar{\sigma}_{2}^{2}\right)$ en $\left(\operatorname{sign}\left(\pi_{1}\right), \operatorname{sign}\left(\pi_{2}\right), \operatorname{sign}\left(\pi_{3}\right)\right)=(+1,+1, \pm 1)$. Situación imposible.

- 1.b) $\left(\lambda_{1}, \lambda_{2}\right) \rightarrow\left(\bar{\sigma}_{3}^{2}, \bar{\sigma}_{2}^{2}\right)$ en $\left(\operatorname{sign}\left(\pi_{1}\right), \operatorname{sign}\left(\pi_{2}\right), \operatorname{sign}\left(\pi_{3}\right)\right)=(+1,-1, \pm 1)$. El punto $\mathrm{F}_{3}$. 
- 1.c) $\left(\lambda_{1}, \lambda_{3}\right) \rightarrow\left(\bar{\sigma}_{3}^{2}, \bar{\sigma}_{2}^{2}\right)$ en $\left(\operatorname{sign}\left(\pi_{1}\right), \operatorname{sign}\left(\pi_{2}\right), \operatorname{sign}\left(\pi_{3}\right)\right)=(+1, \pm 1,+1)$. Imposible.

- 2. Si $\lambda_{2} \rightarrow \bar{\sigma}_{3}^{2}$ en los tramos:

$$
\left(\operatorname{sign}\left(\pi_{1}\right), \operatorname{sign}\left(\pi_{2}\right), \operatorname{sign}\left(\pi_{3}\right)\right)=( \pm 1,+1, \pm 1)
$$

Subcasos:

- 2.a) $\left(\lambda_{1}, \lambda_{2}\right) \rightarrow\left(\bar{\sigma}_{3}^{2}, \bar{\sigma}_{3}^{2}\right)$ en $\left(\operatorname{sign}\left(\pi_{1}\right), \operatorname{sign}\left(\pi_{2}\right), \operatorname{sign}\left(\pi_{3}\right)\right)=(-1,+1, \pm 1)$. Imposible.

- 2.b) $\left(\lambda_{2}, \lambda_{3}\right) \rightarrow\left(\bar{\sigma}_{3}^{2}, \bar{\sigma}_{2}^{2}\right)$ en $\left(\operatorname{sign}\left(\pi_{1}\right), \operatorname{sign}\left(\pi_{2}\right), \operatorname{sign}\left(\pi_{3}\right)\right)=( \pm 1,+1,+1)$. Imposible.

- 2.c) $\left(\lambda_{1}, \lambda_{2}\right) \rightarrow\left(0, \bar{\sigma}_{3}^{2}\right)$ en $\left(\operatorname{sign}\left(\pi_{1}\right), \operatorname{sign}\left(\pi_{2}\right), \operatorname{sign}\left(\pi_{3}\right)\right)=(-1,+1, \pm 1)$. Se obtiene el kink TK2 $\sigma_{2}$ siempre y cuando se verifiquen los límites relativos adecuados.

- 3. Si $\lambda_{2} \rightarrow \bar{\sigma}_{2}^{2}$ en los tramos determinados por:

$$
\left(\operatorname{sign}\left(\pi_{1}\right), \operatorname{sign}\left(\pi_{2}\right), \operatorname{sign}\left(\pi_{3}\right)\right)=( \pm 1,-1, \pm 1)
$$

obtendremos los subcasos:

- 3.a) $\left(\lambda_{1}, \lambda_{2}\right) \rightarrow\left(\bar{\sigma}_{3}^{2}, \bar{\sigma}_{2}^{2}\right)$ en $\left(\operatorname{sign}\left(\pi_{1}\right), \operatorname{sign}\left(\pi_{2}\right), \operatorname{sign}\left(\pi_{3}\right)\right)=(-1,-1, \pm 1)$.

Esta situación proporciona, con los límites relativos adecuados, el tramo (III) del kink TK1.

- 3.b) $\left(\lambda_{2}, \lambda_{3}\right) \rightarrow\left(\bar{\sigma}_{2}^{2}, \bar{\sigma}_{2}^{2}\right)$ en $\left(\operatorname{sign}\left(\pi_{1}\right), \operatorname{sign}\left(\pi_{2}\right), \operatorname{sign}\left(\pi_{3}\right)\right)=( \pm 1,-1,+1)$. Se obtiene el punto $\mathrm{F}_{2}$.

- 3.c) $\left(\lambda_{1}, \lambda_{2}\right) \rightarrow\left(0, \bar{\sigma}_{2}^{2}\right)$ en $\left(\operatorname{sign}\left(\pi_{1}\right), \operatorname{sign}\left(\pi_{2}\right), \operatorname{sign}\left(\pi_{3}\right)\right)=(-1,-1, \pm 1)$. De manera análoga, el tramo (II) del kink TK2 $\sigma_{3}$.

- 4. Para $\lambda_{3} \rightarrow \bar{\sigma}_{2}^{2}$ con la elección:

$$
\left(\operatorname{sign}\left(\pi_{1}\right), \operatorname{sign}\left(\pi_{2}\right), \operatorname{sign}\left(\pi_{3}\right)\right)=( \pm 1, \pm 1,-1)
$$

tendremos:

- 4.a) $\left(\lambda_{1}, \lambda_{3}\right) \rightarrow\left(\bar{\sigma}_{3}^{2}, \bar{\sigma}_{2}^{2}\right)$ en $\left(\operatorname{sign}\left(\pi_{1}\right), \operatorname{sign}\left(\pi_{2}\right), \operatorname{sign}\left(\pi_{3}\right)\right)=(-1, \pm 1,-1)$. Con los límites adecuados se obtiene en tramo TK1(II). 
- 4.b) $\left(\lambda_{1}, \lambda_{3}\right) \rightarrow\left(0, \bar{\sigma}_{2}^{2}\right)$ en $\left(\operatorname{sign}\left(\pi_{1}\right), \operatorname{sign}\left(\pi_{2}\right), \operatorname{sign}\left(\pi_{3}\right)\right)=(-1, \pm 1,-1)$. El tramo $\mathrm{TK} 2 \sigma_{3}(\mathrm{I})$.

- 4.c) $\left(\lambda_{2}, \lambda_{3}\right) \rightarrow\left(\bar{\sigma}_{2}^{2}, \bar{\sigma}_{2}^{2}\right)$ en $\left(\operatorname{sign}\left(\pi_{1}\right), \operatorname{sign}\left(\pi_{2}\right), \operatorname{sign}\left(\pi_{3}\right)\right)=( \pm 1,+1,-1)$. De nuevo el punto $\mathrm{F}_{2}$.

-4 .d) $\left(\lambda_{2}, \lambda_{3}\right) \rightarrow\left(\bar{\sigma}_{3}^{2}, \bar{\sigma}_{2}^{2}\right)$ en $\left(\operatorname{sign}\left(\pi_{1}\right), \operatorname{sign}\left(\pi_{2}\right), \operatorname{sign}\left(\pi_{3}\right)\right)=( \pm 1,-1,-1)$. Finalmente el tramo restante: TK1(I).

La regla (3.63) queda, por tanto, explicada en la situación citada $\left(\gamma_{2} \rightarrow \pm \infty\right.$ con $\gamma_{3}$ finita). En la Figura 3.19 se representa un kink cercano a este comportamiento límite.

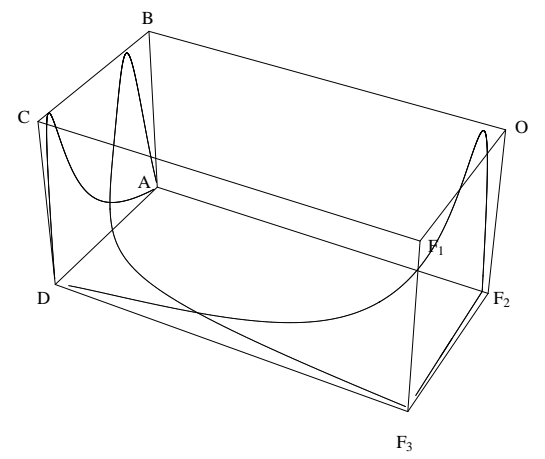

Figura 3.19: Trayectoria TK3 cercana al límite TK2 $\sigma_{3}+$ TK $2 \sigma_{2}+\mathrm{TK} 1$.

Por último, la situación más general de paso al límite, es decir, que ambas constantes, $\gamma_{2}$ y $\gamma_{3}$, tiendan a $\pm \infty$ sin que se verifique ninguna de las condiciones especiales que han sido expuestas ( $\sin$ que $\gamma$ ni $\bar{\gamma}$ sean finitas, y sin considerar los valores especiales de los límites relativos que han sido considerados anteriormente), conduce de manera trivial a la solución esperada: TK3 $=$ TK $2 \sigma_{3}+$ TK $2 \sigma_{2}+$ TK1.

Para finalizar esta sección, es interesante recordar todos los resultados que se han obtenido en la misma:

- $\gamma \rightarrow \pm \infty$, con $\bar{\gamma}$ finita: TK3 $=\mathrm{NTK} 2 \sigma_{3}+\mathrm{TK} 2 \sigma_{2}$. Tomando posteriormente $\bar{\gamma} \rightarrow \pm \infty$ se concluye en TK3 $=$ TK $2 \sigma_{3}+$ TK $2 \sigma_{2}+$ TK 1 .

- $\bar{\gamma} \rightarrow \pm \infty$, con $\gamma$ finita: TK3 $=$ NTK2 $\sigma_{2}+$ TK2 $\sigma_{3}$. El límite posterior $\bar{\gamma} \rightarrow \pm \infty$ produce el resultado esperado TK3 $=$ TK $2 \sigma_{3}+$ TK2 $\sigma_{2}+$ TK 1 . 
- $\gamma_{3} \rightarrow \pm \infty$, con $\gamma_{2}$ finita: TK3 $=$ NTK3 + TK1. Considerando después $\gamma_{2} \rightarrow \pm \infty$ se obtiene de nuevo TK3 $=$ TK $2 \sigma_{3}+$ TK2 $\sigma_{2}+\mathrm{TK} 1$.

- $\gamma_{2} \rightarrow \pm \infty$, con $\gamma_{3}$ finita: TK2 $\sigma_{3}+$ TK2 $\sigma_{2}+$ TK1 sin necesidad de considerar más límites.

- Todas las demás posibilidades de alcanzar el infinito en el espacio de los parámetros: TK3 $=$ TK $2 \sigma_{3}+\mathrm{TK} 2 \sigma_{2}+\mathrm{TK} 1$.

\subsubsection{Espacio "modular" de Kinks}

Como ya se ha comentado en la Sección 3.1, denominaremos Espacio Modular de kinks al espacio de ondas solitarias soluciones del modelo en estudio cociente por la acción del grupo de simetría del sistema. En el caso del modelo que nos ocupa, el grupo no será otro que $G=\mathbb{Z}_{2} \times \mathbb{Z}_{2} \times \mathbb{Z}_{2}$.

Tras la descripción de los diferentes tipos de kinks involucrados en el Modelo Sigma $O(3)$ Lineal Deformado que se ha realizado en los apartados anteriores, es fácil comprobar que los kinks que hemos denominado genéricos, descritos por la solución general de la ecuación de Hamilton-Jacobi para valores finitos de las constantes de integración, no son invariantes bajo la acción de ningún subgrupo no trivial de $G$. Por otro lado, un kink del tipo TK3, cuya órbita está descrita en coordenadas elípticas por las ecuaciones $(3.25,3.26)$ (o alternativamente por $(3.48,3.49))$, se corresponde, como ya se ha detallado, con ocho kinks de dicho tipo en coordenadas cartesianas debido a la invarianza bajo la acción de $G$ del cambio de coordenadas. Es entonces adecuado estudiar el espacio de soluciones TK3 en coordenadas elípticas, pues de esta forma el cociente bajo la acción de $G$ queda considerado automáticamente.

En el apartado 3.2.2 se demostró que el espacio de soluciones del tipo TK3 era isomorfo al plano $\mathbb{R}^{2} \simeq \mathbb{C}$, tenemos por tanto que:

$$
\mathcal{M}_{\mathrm{TK} 3}=\mathbb{R}^{2} \simeq \mathbb{C}
$$

En el apartado 3.2.3 se ha calculado el comportamiento de dicho espacio modular cuando se considera el límite para los valores no finitos de las constantes. El objetivo que perseguimos ahora es describir cómo los demás tipos de kinks presentes en el sistema (kinks embebidos y kinks envolventes) pueden ser incluidos en el espacio modular anterior, compactificando para ello el plano abierto $\mathbb{R}^{2}$ al incorporar al espacio el borde del mismo. Existirán, como veremos, diferentes maneras de realizar dicha compactificación no trivial, unas más adecuadas que otras de 
cara a incorporar unos u otros tipos de kinks. La razón de dicha variedad de posibilidades reside en las diferentes formas que tenemos en el plano para alcanzar el infinito, o, si se quiere, en la complejidad inherente al cálculo de límites con dos variables independientes.

La incorporación al espacio de kinks TK3 de otros tipos de soluciones requiere, evidentemente, que éstas posean la misma energía. Las reglas de suma deducidas anteriormente nos permitirán ahora establecer las combinaciones de kinks adecuados para dichos razonamientos.

Plantearemos, de esta manera, tres formas diferentes de describir $\mathcal{M}_{\text {TK3 }}$ y su compactificación:

- 1. Si se considera la pareja de constantes de integración $\left(\gamma_{2}, \gamma_{3}\right)$ como sistema de coordenadas ortogonales en $\mathbb{R}^{2} \simeq \mathbb{C}$, entonces $\mathcal{M}_{\mathrm{TK} 3}{ }^{13}$ puede verse como un cuadrado abierto a través de la proyección:

$$
\left(\gamma_{2}=\frac{\Gamma_{2}}{1-\left|\Gamma_{2}\right|}, \gamma_{3}=\frac{\Gamma_{3}}{1-\left|\Gamma_{3}\right|}\right) \longrightarrow\left(\Gamma_{2}, \Gamma_{3}\right)
$$

que se visualiza en la Figura 3.20.
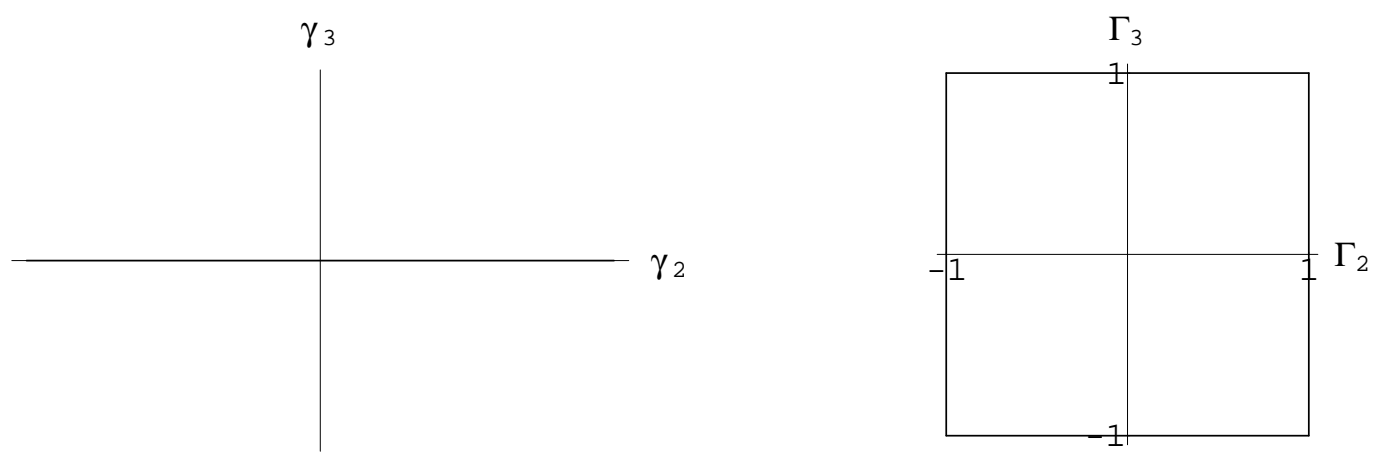

Figura 3.20: Proyección (3.70) en el plano.

Los lados superior e inferior, $\Gamma_{3}= \pm 1$, se corresponden con las líneas del infinito $\gamma_{3}= \pm \infty$; cada punto en estos lados, de acuerdo con los límites calculados en el apartado anterior, no es más que una combinación entre

\footnotetext{
${ }^{13}$ Consideraremos, siempre que no se especifique lo contrario, que $\mathcal{M}$ se refiere a espacio modular de soluciones tomadas en $P_{3}(0)$. El cociente bajo la acción del grupo $G$ está por tanto implícitamente tomado.
} 
un kink concreto de la familia de kinks no topológicos tridimensionales, NTK3, más el kink topológico unidimensional TK1. El espacio modular de kinks envolventes NTK3 está a su vez parametrizada por $\Gamma_{2} \in(-1,1)$, y así $\mathcal{M}_{\mathrm{NTK} 3+\mathrm{TK} 1} \simeq \mathbb{R P}^{1} \simeq S^{1} / \mathbb{Z}_{2}$, como se deduce del hecho de que ambos extremos, $\Gamma_{2}=1$ y $\Gamma_{2}=-1$, se corresponden exactamente con la misma situación: TK2 $\sigma_{2}+\mathrm{TK} 2 \sigma_{3}+\mathrm{TK} 1$.

Los lados laterales del cuadrado, $\Gamma_{2}= \pm 1$, se alcanzan cuando la constante $\gamma_{2}$ tiende a $\pm \infty$ y, como vimos, el límite en ese caso, indepentemente del valor tomado por la constante $\gamma_{3}$, es siempre la combinación: TK2 $\sigma_{2}+\mathrm{TK} 2 \sigma_{3}+$ TK1. Así, todo punto de $\Gamma_{2}= \pm 1$ se corresponde con dicha combinación.

La compactificación de $\mathcal{M}_{\mathrm{TK} 3}$ por medio de la incorporación de su frontera, $\mathcal{M}_{\mathrm{NTK} 3+\mathrm{TK} 1}$ y $\mathcal{M}_{\mathrm{TK} 2 \sigma_{2}+\mathrm{TK} 2 \sigma_{3}+\mathrm{TK} 1}=$ un punto, está representada en la Figura 3.21 (a). Las identificaciones naturales hacen que la primera de las compactificaciones de $\mathcal{M}_{\text {TK3 }}$ pueda verse como una curva elíptica "singular", de ecuación $y^{2}=x^{3}+x^{2}$ (ver por ejemplo [58]), que topológicamente es una esfera (ver Figura 3.21 (b)).

$$
\overline{\mathcal{M}}_{\mathrm{TK} 3}^{(1)}=\mathcal{M}_{\mathrm{TK} 3} \sqcup 2 \mathcal{M}_{\mathrm{NTK} 3+\mathrm{TK} 1} \sqcup 2 \mathcal{M}_{\mathrm{TK} 2 \sigma_{2}+\mathrm{TK} 2 \sigma_{3}+\mathrm{TK} 1} \cong S^{2}
$$
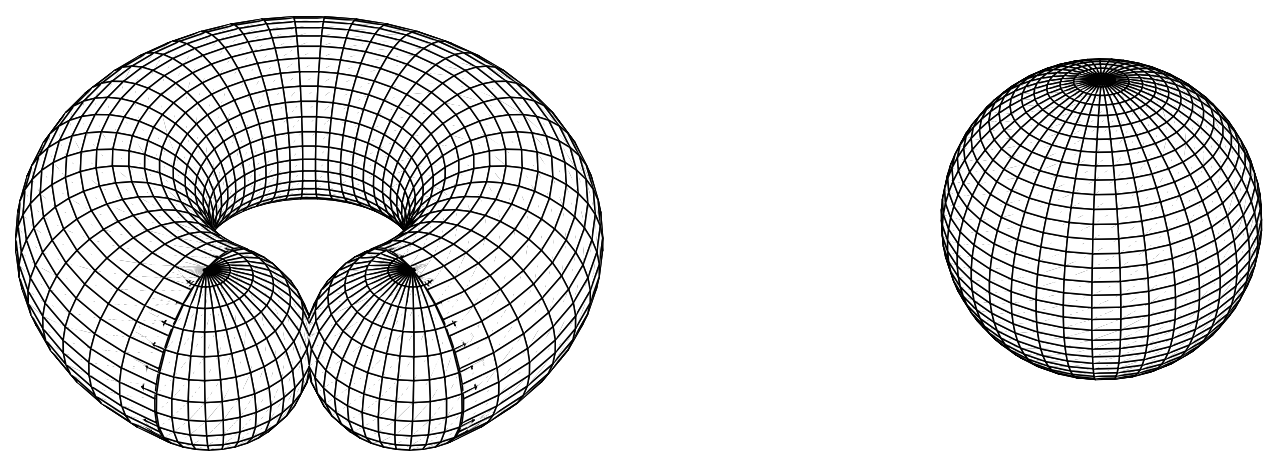

Figura 3.21: Compactificación tipo 1.

- 2. Utilizando como coordenadas ortogonales, en $\mathbb{R}^{2}$, la pareja de constantes $\gamma$ y $\bar{\gamma}$, tendremos que $\mathcal{M}_{\mathrm{TK} 3}$ puede ser visto también como un cuadrado abierto si proyectamos de la forma:

$$
\left(\gamma=\frac{\Gamma}{1-|\Gamma|}, \bar{\gamma}=\frac{\bar{\Gamma}}{1-|\bar{\Gamma}|}\right) \longrightarrow(\Gamma, \bar{\Gamma})
$$



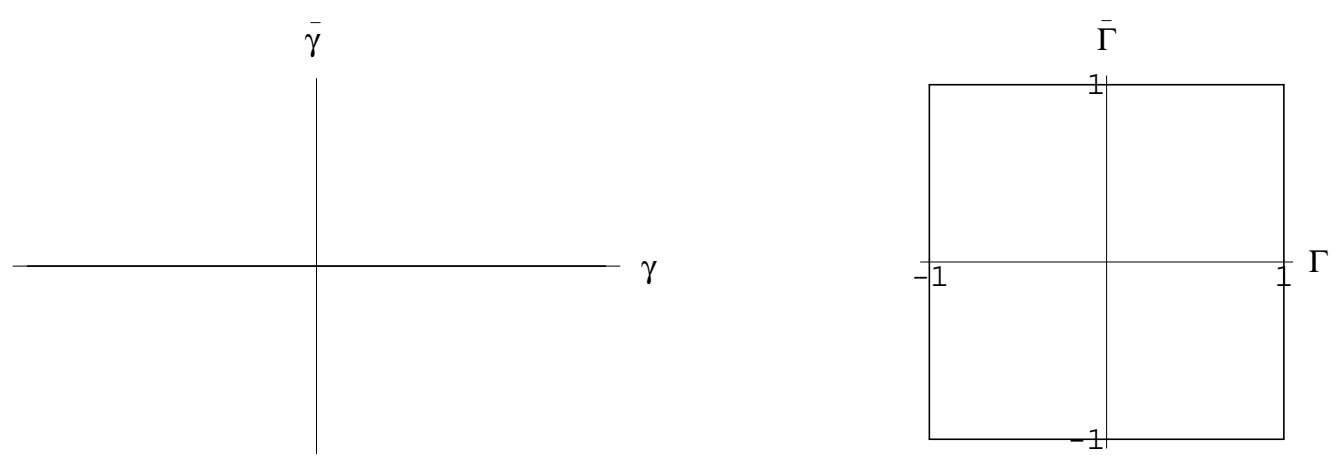

Figura 3.22: Proyección (3.71).

(ver Figura 3.22).

Los lados izquierdo y derecho, $\Gamma= \pm 1$, parametrizan, para los diferentes valores de $\bar{\Gamma}$, el espacio modular de kinks no topológicos bidimensionales del plano $q_{2}=0$ más el kink topológico de dos componentes en el plano $q_{3}=0$, es decir: $\mathcal{M}_{\mathrm{NTK} 2 \sigma_{3}+\mathrm{TK} 2 \sigma_{2}}=\mathbb{R P}^{1}$. De igual manera, los lados superior e inferior del cuadrado, $\bar{\Gamma}= \pm 1$, conducen a $\mathcal{M}_{\mathrm{NTK} 2 \sigma_{2}+\mathrm{TK} 2 \sigma_{3}}=\mathbb{R P}^{1}$, es decir, al espacio modular de los kinks embebidos restantes. Los cuatro vértices nos proporcionan el límite $\mathcal{M}_{\mathrm{TK} 2 \sigma_{3}+\mathrm{TK} 2 \sigma_{2}+\mathrm{TK} 1}=$ punto. Después de las identificaciones obvias, la segunda compactificación de $\mathcal{M}_{\mathrm{TK} 3}$ es un toro (ver Figura $3.23)$ :

$$
\begin{aligned}
\overline{\mathcal{M}}_{\mathrm{TK} 3}^{(2)}= & \mathcal{M}_{\mathrm{TK} 3} \sqcup 2 \mathcal{M}_{\mathrm{NTK} 2 \sigma_{3}+\mathrm{TK} 2 \sigma_{2}} \sqcup 2 \mathcal{M}_{\mathrm{NTK} 2 \sigma_{2}+\mathrm{TK} 2 \sigma_{3}} \sqcup \\
& \sqcup 4 \mathcal{M}_{\mathrm{TK} 2 \sigma_{3}+\mathrm{TK} 2 \sigma_{2}+\mathrm{TK} 1} \cong S^{1} \times S^{1}
\end{aligned}
$$

- 3. Finalmente, propondremos una tercera versión que se revelará la más eficaz a la hora de analizar cómo son alcanzados los límites singulares. Como acabamos de ver, la primera compactificación deja fuera los kinks embebidos y la segunda hace lo propio con los envolventes, conseguiremos ahora describir todos los tipos de kinks en una única parametrización.

La idea es introducir coordenadas polares en el plano $\mathcal{M}_{\mathrm{TK} 3}=\mathbb{R}^{2}$ de la forma:

$$
\rho=\sqrt{\gamma^{2}+\bar{\gamma}^{2}} ; \quad \theta=\arctan \frac{\bar{\gamma}}{\gamma}
$$

Podemos entonces proyectar, de manera análoga a las anteriores, si bien 


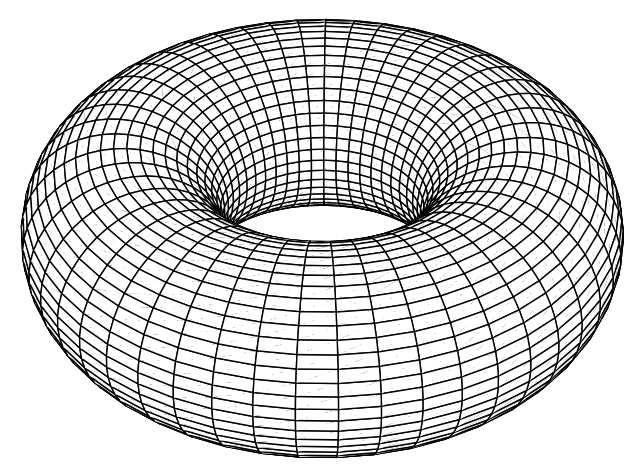

Figura 3.23: Compactificación tipo 2.

ahora el resultado de la proyección

$$
\left(\rho=\frac{R}{1-R}, \theta\right) \longrightarrow(R, \theta)
$$

será el disco abierto de radio $R<1$ centrado en (0,0) (ver Figura 3.24).
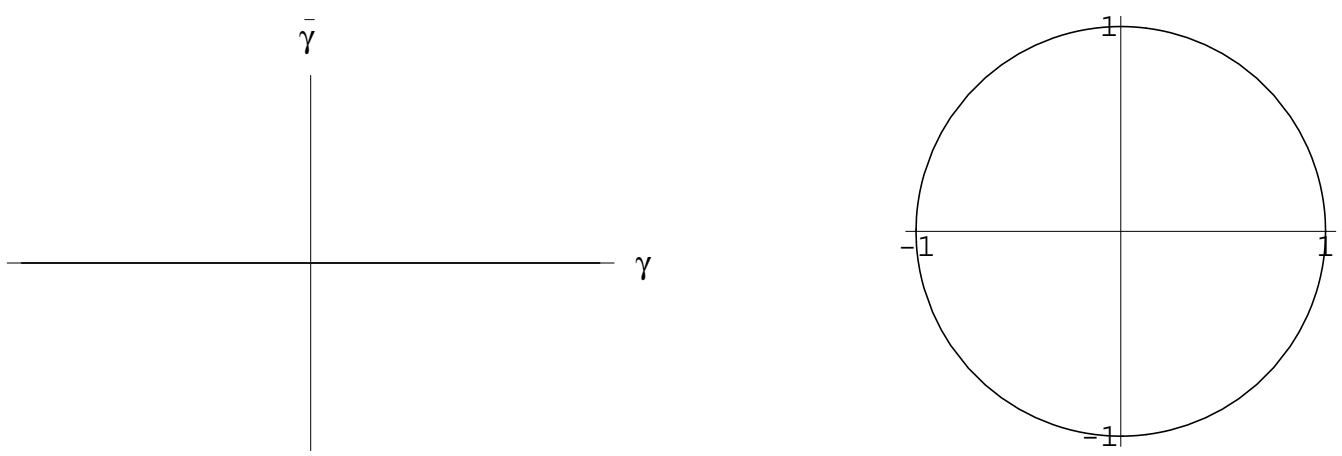

Figura 3.24: Proyección (3.72).

En el borde, $R=1$, del disco, encontraremos diferentes configuraciones dependiendo del camino por el cual accedamos a él. Dicho camino estará definido, de manera general, en el espacio de curvas sobre el espacio de soluciones. Si nos acercamos al borde a través de una dirección radial fijada, $\theta=\theta_{0}$, encontraremos la combinación TK2 $\sigma_{3}+$ TK $2 \sigma_{2}+$ TK1 para todo valor de $\theta_{0}$ exceptuando tres direcciones especiales:

- a) $\theta_{0}=0$ ó $\theta_{0}=\pi$. El proceso de alcanzar el borde a lo largo de 
esta dirección (en ambos sentidos) equivale a tomar el límite cuando $\Lambda$ tiende a $\pm \infty$ en la familia de rectas: $\gamma=\Lambda$, para $\Lambda \in \mathbb{R}$. Si pasamos a coordenadas polares proyectadas, las rectas $\gamma=\Lambda$ se convierten en las curvas:

$$
R=\frac{\Lambda}{\Lambda+\cos \theta}
$$

que pueden verse representadas en la Figura 3.25.

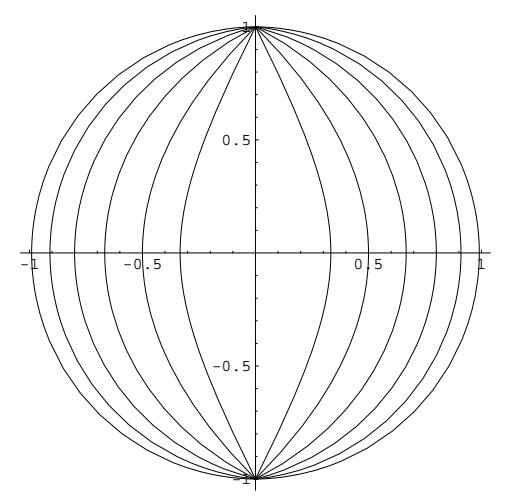

Figura 3.25: Curvas (3.73) para los valores $\Lambda= \pm \frac{1}{2}, \pm 1, \pm 2, \pm 4, \pm 10, \pm 100$.

Todos los puntos de cada una de esas curvas se corresponden con kinks del tipo TK3 que pasan por un mismo punto de la arista $\mathrm{F}_{1} \mathrm{~F}_{3}$ de $\partial P_{3}(0)$. Los distintos puntos de una misma curva parametrizan por tanto el punto de corte con la arista $\mathrm{AF}_{2}$. Encontramos entonces que valores muy grandes de la constante $\Lambda$ implican necesariamente que el punto de corte de los correspondientes kinks TK3 con la arista $\mathrm{F}_{1} \mathrm{~F}_{3}$ está muy cercano al foco $\mathrm{F}_{3}$ (ver Figura 3.16). En el límite $\Lambda \rightarrow \pm \infty$ se tiene $\gamma \rightarrow \infty \mathrm{y}$, en consecuencia, se alcanza el borde $R=1$ de tal manera que dicho borde describe exactamente el espacio modular de las configuraciones del tipo: NTK2 $\sigma_{3}+\mathrm{TK} 2 \sigma_{2}$. Todos los miembros de esta familia embebida en el Modelo $O(3)$ pasan por el foco $\mathrm{F}_{3}$ y están parametrizados por el punto de corte con la arista $\mathrm{AF}_{2}$.

La compactificación evidente que este proceso de paso al límite sugiere es la incorporación al espacio modular de las curvas $\Lambda \rightarrow \pm \infty$ en el borde del disco abierto. Teniendo en cuenta, no obstante, que los puntos antipodales de la circunferencia $R=1$ han de ser identificados, encontramos que la compactificación correcta del espacio modular es, 
en este caso, de la forma:

$$
\overline{\mathcal{M}}_{\mathrm{TK} 3}^{(3, \mathrm{I})}=\mathcal{M}_{\mathrm{TK} 3} \sqcup 2 \mathcal{M}_{\mathrm{NTK} 2 \sigma_{3}+\mathrm{TK} 2 \sigma_{2}} \cong \mathbb{R P}^{2}=\frac{S^{2}}{\mathbb{Z}_{2}}
$$

- b) $\theta_{0}=\frac{\pi}{2}$ ó $\theta_{0}=\frac{3 \pi}{2}$. Encontramos ahora una situación absolutamente similar a la anterior intercambiando $\gamma$ por $\bar{\gamma}$. Las rectas $\bar{\gamma}=\Lambda$ se convierten ahora, en coordenadas polares proyectadas, en las curvas:

$$
R=\frac{\Lambda}{\Lambda+\operatorname{sen} \theta}
$$

ver Figura 3.26.

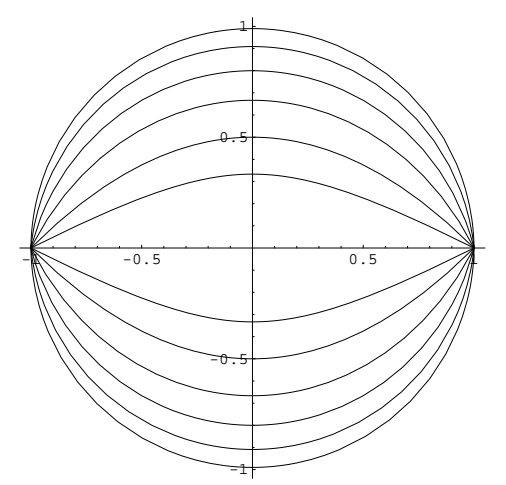

Figura 3.26: Curvas (3.74) para los valores $\Lambda= \pm \frac{1}{2}, \pm 1, \pm 2, \pm 4, \pm 10, \pm 100$.

En este caso encontramos que todos los kinks TK3 de una de las rectas $\bar{\gamma}=\Lambda$, es decir todos los puntos de la curva (3.74) para ese valor de $\Lambda$, pasan por un único punto de la arista $\mathrm{AF}_{2}{ }^{14}$. Se diferencian por tanto en el punto de corte con la otra arista singular, $\mathrm{F}_{1} \mathrm{~F}_{3}$. En este caso valores arbitrariamente grandes de $\Lambda$ nos indican que uno de los puntos de corte con $\mathrm{AF}_{2}$ está arbitrariamente cerca de $\mathrm{A}$ (y el otro evidentemente de $\mathrm{F}_{2}$ ) y, finalmente, el límite $\Lambda \rightarrow \infty$ nos lleva al borde $R=1$ identificado con el espacio modular de configuraciones NTK2 $\sigma_{2}$ $+\mathrm{TK} 2 \sigma_{3}$ (ver Figura 3.17) . La compactificación en este caso es, por tanto, de la forma:

$$
\overline{\mathcal{M}}_{\mathrm{TK} 3}^{(3, \mathrm{II})}=\mathcal{M}_{\mathrm{TK} 3} \sqcup 2 \mathcal{M}_{\mathrm{NTK} 2 \sigma_{2}+\mathrm{TK} 2 \sigma_{3}} \cong \mathbb{R P}^{2}=\frac{S^{2}}{\mathbb{Z}_{2}}
$$

\footnotetext{
${ }^{14}$ Recordemos que realmente la arista $\mathrm{AF}_{2}$ se alcanza dos veces por parte de cada solución, un punto de corte, no obstante, determina el otro inequívocamente. Por ello nos referimos únicamente a uno de los puntos de corte.
} 
- c) $\theta_{0}=\frac{\pi}{4}$ ó $\theta_{0}=\frac{5 \pi}{4}$. En este caso, el proceso de paso al límite hacia el borde del disco $R<1$ lo realizaremos a través de las rectas $\bar{\gamma}=\gamma-\Lambda$ $\mathrm{y}$, por tanto, tomaremos también $\Lambda \rightarrow \pm \infty$. Tenemos entonces que la constante relevante es $\gamma_{3}$, puesto que $\gamma_{3}\left(\bar{\sigma}_{2}^{2}-\bar{\sigma}_{3}^{2}\right)=\gamma-\bar{\gamma}=\Lambda$. A diferencia de lo que ocurre con la parametrización en $(\gamma, \bar{\gamma})$, que hemos tomado como coordenadas ortogonales en $\mathbb{R}^{2}$, las rectas $\gamma_{2}=$ cte no son ahora perpendiculares a las $\gamma_{3}=$ cte. puesto que las primeras corresponden a los valores: $\theta=\arctan \left(\frac{\bar{\sigma}_{2}}{\bar{\sigma}_{3}}\right)^{2}$ o $\arctan \left(\frac{\bar{\sigma}_{2}}{\bar{\sigma}_{3}}\right)^{2}+\pi$, que no difieren en general en $\frac{\pi}{2} \operatorname{del} \theta_{0}=\frac{\pi}{4}$ o $\theta_{0}=\frac{5 \pi}{4}$.

En coordenadas polares proyectadas las rectas $\bar{\gamma}=\gamma-\Lambda$ se traducen en las curvas:

$$
R=\frac{\Lambda}{\Lambda+\cos \theta-\operatorname{sen} \theta}
$$

que están representadas en la Figura 3.27.

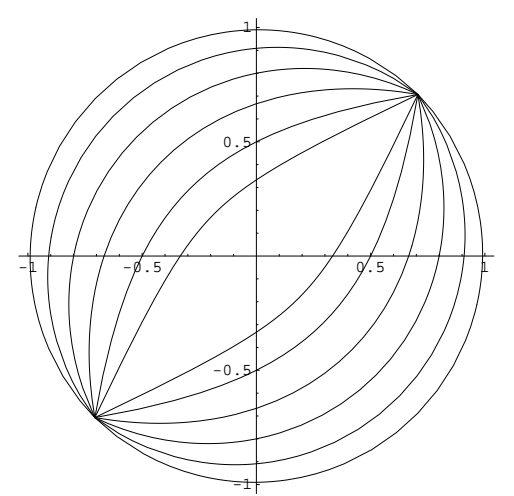

Figura 3.27: Curvas (3.75) para los valores $\Lambda= \pm \frac{1}{2}, \pm 1, \pm 2, \pm 4, \pm 10, \pm 100$.

Todos los kinks del tipo TK3 que pertenecen a una de esas curvas (para un valor fijo de $\Lambda$ ) tienen en común el valor de la constante $\gamma_{3}$, diferenciándose en el correspondiente de $\gamma_{2}$. La intuición geométrica de lo que esto significa es menos clara que en los casos anteriores. El límite para valores arbitrariamente grandes de $|\Lambda|$, sin embargo, tiene una implicación muy clara en la geometría de dichas soluciones: los puntos de corte con la cara $\mathrm{OF}_{1} \mathrm{BC}$ de $\partial P_{3}(0)$ tienden a situarse de la siguiente forma: dos de ellos se aproximan a la arista BC (los puntos límites de la misma concretos dependen del valor que tome la constante $\gamma_{2}$ ) mientras el punto de corte restante se aproximan al punto O (ver Figura 3.18). Tenemos, en consecuencia, que en el límite $R=1$ se alcanzan 
dichos cortes en $\mathrm{BC}$ y en $\mathrm{O}$, respectivamente, $\mathrm{y}$, necesariamente, se tiene el espacio modular de la combinación NTK3 + TK1 (parametrizado por el correspodiente valor de $\gamma_{2}$ ). De nuevo se deben identificar los puntos antipodales del borde, $R=1$, y así la compactificación de $\mathcal{M}_{3}$ correspondiente será:

$$
\overline{\mathcal{M}}_{\mathrm{TK} 3}^{(3, \mathrm{III})}=\mathcal{M}_{\mathrm{TK} 3} \sqcup 2 \mathcal{M}_{\mathrm{NTK} 3+\mathrm{TK} 1} \cong \mathbb{R} \mathbb{P}^{2}=\frac{S^{2}}{\mathbb{Z}_{2}}
$$

Como se ha dicho anteriormente, cualquier otro camino que se considere en el disco $R<1$ para alcanzar su borde (no necesariamente a través de curvas ortogonales a las rectas radiales $\theta=\theta_{0}$ ), nos conduce a la configuración TK2 $\sigma_{3}$ + TK $2 \sigma_{2}+$ TK1. De esta manera, tendremos una cuarta compactificación posible:

$$
\overline{\mathcal{M}}_{\mathrm{TK} 3}^{(3, \mathrm{IV})}=\mathcal{M}_{\mathrm{TK} 3} \sqcup \mathcal{M}_{\mathrm{TK} 2 \sigma_{3}+\mathrm{TK} 2 \sigma_{2}+\mathrm{TK} 1}
$$

Podemos resumir todos los aspectos relativos al espacio modular de soluciones kink del Modelo Sigma $O(3)$ Lineal Deformado que hemos detallado en los siguientes puntos significativos:

- Las soluciones naturales del sistema, tridimensionales y correspondientes a los valores regulares de las constantes que parametrizan a la totalidad de las soluciones, son kinks con las tres componentes no constantes, topológicos y tales que sus trayectorias, descritas en coordenadas elípticas, pertenecen al interior de del paralelepípedo $P_{3}(0)$. El espacio modular de las mismas es el plano abierto, si se considera la parametrización original, o bien el disco abierto o el cuadrado abierto si se toman las proyecciones descritas por las ecuaciones (3.70), (3.71) ó (3.72).

- Existen más tipos de kinks específicos del Modelo Sigma $O(3)$ Lineal Deformado, los kinks tridimensionales no topológicos. El calificativo tridimensional, como ya hemos comentado, se refiere a que sus tres componentes cartesianas son no constantes. Realmente se trata de soluciones sobre una superficie y, de hecho, en coordenadas elípticas vienen descritos únicamente por dos coordenadas no nulas: pertenecen a la cara $\lambda_{1}=0$ de $\partial P^{3}(0)$. Su espacio modular es la recta real (o el intervalo abierto $(-1,1)$, si se proyecta a través de (3.70)) y, si se añade a cada uno de sus miembros el kink TK1, tendremos que $\mathcal{M}_{\mathrm{TK} 3+\mathrm{TK} 1}$ pertenece al borde de $\overline{\mathcal{M}}_{\mathrm{TK} 3}^{(2)}$ y $\overline{\mathcal{M}}_{\mathrm{TK} 3}^{(3)}$. 
- Sobre dos de las caras singulares de $\partial P_{3}(0), \lambda_{3}=\bar{\sigma}_{2}^{2}$ y $\lambda_{2}=\bar{\sigma}_{2}^{2}$, el sistema posee como soluciones a los kink no topológicos de dos componentes que hemos denominado NTK2 $\sigma_{3}$. De manera análoga, en otras dos caras singulares, $\lambda_{1}=\bar{\sigma}_{3}^{2}$ y $\lambda_{2}=\bar{\sigma}_{3}^{2}$, aparece el otro tipo de kinks bidimensionales no topológicos existente en el modelo, la familia NTK $2 \sigma_{2}$. Ambas familias, $\mathrm{NTK} 2 \sigma_{3}$ y NTK2 $\sigma_{2}$, no son más que las soluciones regulares del Modelo Sigma $O(2)$ Lineal Deformado, de manera que se observa ahora cómo el Modelo bidimensional está inmerso dentro del tridimensional de dos formas no equivalentes. Los espacios modulares son, en ambos casos, la recta real o los intervalos abiertos si se toma la versión proyectada de los mismos. Si se añade el kink TK2 $\sigma_{2}$ a la familia NTK2 $\sigma_{3}$, o bien el kink TK2 $\sigma_{3}$ a la NTK2 $\sigma_{2}$, los espacios modulares resultantes son parte de la frontera de los espacios modulares totales compactificados, $\overline{\mathcal{M}}_{\text {TK3 }}^{(2)}$ y $\overline{\mathcal{M}}_{\mathrm{TK} 3}^{(3)}$.

- El kink topológico de dos componentes cartesianas no constantes TK2 $\sigma_{3}$ determina su trayectoria sobre las aristas (singulares) $\left(\lambda_{1}=0, \lambda_{3}=\bar{\sigma}_{2}^{2}\right) \mathrm{y}$ $\left(\lambda_{1}=0, \lambda_{2}=\bar{\sigma}_{2}^{2}\right)$ de $\partial P_{3}(0)$. El otro kink de similares características, que hemos denotado por TK2 $\sigma_{2}$, se traza por el contrario sobre una única arista de $\bar{P}_{3}(0)$, la de ecuaciones $\left(\lambda_{1}=0, \lambda_{2}=\bar{\sigma}_{3}^{2}\right)$. Cada uno de ellos forma parte del correspondiente Modelo Sigma $O(2)$ Lineal Deformado embebido dentro del Modelo tridimensional y sus espacios modulares son discretos, se reducen respectivamente a un sólo punto.

- Finalmente, el kink unidimensional topológico, TK1, se corresponde en coordenadas elípticas con una trayectoria sobre tres de las aristas de $\partial P_{3}(0)$ singulares restantes $\left(\lambda_{2}=\bar{\sigma}_{3}^{2}, \lambda_{3}=\bar{\sigma}_{2}^{2}\right),\left(\lambda_{1}=\bar{\sigma}_{3}^{2}, \lambda_{3}=\bar{\sigma}_{2}^{2}\right)$ y $\left(\lambda_{1}=\bar{\sigma}_{3}^{2}, \lambda_{2}=\bar{\sigma}_{2}^{2}\right)$. Puede verse como la solución única del Modelo Sigma $O(1)$ Lineal, que está inmerso simultáneamente en los dos sub-modelos $O(2)$ y en el total $O(3)$.

Como hemos visto, los tres kinks unidimensionales presentes en el modelo forman el "punto" TK $2 \sigma_{3}+$ TK $2 \sigma_{2}+$ TK1, que aparece varias veces dentro de la frontera de los espacios modulares compactificados $\overline{\mathcal{M}}_{\mathrm{TK} 3}^{(a)}, a=1,2,3$. Existe una analogía remarcable con la estructura del espacio modular de las superficies de Riemann; en la compactificación de Mumford-Deligne, [75], el borde de $\mathcal{M}_{g}$, una variedad compleja de dimensión $3 g-3$, incluye a todos los espacios modulares $\mathcal{M}_{g_{1}}, \forall g_{1}<g$, donde $g$ es el género de la curva algebraica. 


\section{Generalización a $N$ campos}

Como ya se ha comentado, no incluiremos en esta Memoria un análisis equivalente al realizado para los modelos $N=2$ y $N=3$ para el caso de $N \geq 4$ campos escalares. Es posible, no obstante, plantear las características principales de la estructura del espacio de soluciones, desde el punto de vista general, si se tiene en cuenta, por un lado, la sistemática que apuntan los casos estudiados y, por otro, los cálculos que hemos realizado a tal respecto.

Es evidente que la caracterización de los diferentes tipos de kinks en las categorías embebidos, envolventes y genéricos, continuará siendo natural para un número superior de componentes. De esta manera, en el Modelo Sigma $O(N)$ Lineal Deformado, encontraremos:

- Kinks Genéricos: Se tratará de ondas solitarias de tipo topológico si $N$ es un número impar y no-topológico para $N$ par. Estas soluciones, salvo en su punto inicial y final, permanecerán en el interior del hiperelipsoide de ecuación:

$$
q_{1}^{2}+\frac{q_{2}^{2}}{\bar{\sigma}_{2}^{2}}+\ldots+\frac{q_{N}^{2}}{\bar{\sigma}_{N}^{2}}=1
$$

en coordenadas cartesianas, o $\lambda_{1}=0$ en elípticas $N$-dimensionales. Sus ecuaciones se obtienen a partir de la integración de la ecuación de HamiltonJacobi descrita en el Capítulo 2 y las trayectorias dependerán de $N-1$ constantes arbitrarias.

Los diferentes kinks intersecarán en los puntos de las siguientes cuádricas singulares:

$$
\begin{array}{llcl}
\frac{q_{1}^{2}}{\bar{\sigma}_{N}^{2}}+\frac{q_{2}^{2}}{\bar{\sigma}_{N}^{2}-\bar{\sigma}_{2}^{2}}+ & \cdots+\frac{q_{N-1}^{2}}{\bar{\sigma}_{N}^{2}-\bar{\sigma}_{N-1}^{2}}=1, & q_{N}=0 ; \lambda_{1}=\lambda_{2}=\bar{\sigma}_{N}^{2} \\
\frac{q_{1}^{2}}{\bar{\sigma}_{N-1}^{2}}+\frac{q_{2}^{2}}{\bar{\sigma}_{N-1}^{2}-\bar{\sigma}_{2}^{2}}+\cdots & \cdots-\frac{q_{N}^{2}}{\bar{\sigma}_{N}^{2}-\bar{\sigma}_{N-1}^{2}}=1, & q_{N-1}=0 ; \lambda_{2}=\lambda_{3}=\bar{\sigma}_{N-1}^{2} \\
& \cdots & \cdots & \cdots \\
\frac{q_{1}^{2}}{\bar{\sigma}_{2}^{2}}-\frac{q_{3}^{2}}{\bar{\sigma}_{3}^{2}-\bar{\sigma}_{2}^{2}}- & \cdots & -\frac{q_{N}^{2}}{\bar{\sigma}_{N}^{2}-\bar{\sigma}_{2}^{2}}=1, & q_{2}=0 ; \lambda_{N-1}=\lambda_{N}=\bar{\sigma}_{2}^{2}
\end{array}
$$

- Kinks Envolventes: Sobre el hiperelipsoide anterior encontraremos $2^{N-1}$ familias (en coordenadas cartesianas, tan sólo una en elípticas) de kinks notopológicos si $N$ impar, y topológicos, si $N$ par. Las trayectorias de dichos kinks dependen de $N-2$ constantes arbitrarias y su comportamiento será tal que cada congruencia de kinks intersecará en las subvariedades "umbílicas":

$$
\lambda_{1}=0 \quad, \quad \lambda_{a}=\bar{\sigma}_{N-a+1}^{2}=\lambda_{a+1}, \forall a=2,3, \ldots, N-1
$$


- Kinks Embebidos. La estructura de los kinks embebidos es, posiblemente, la más compleja de todas. El modelo con $N$ campos admitirá, como submodelos, $N-1$ copias del Modelo Sigma $O(N-1)$ Lineal Deformado. Cada una de ellas, evidentemente, posee una estructura similar. La intersección de cada dos de estos sub-modelos consiste, precisamente, en una copia del Modelo Sigma $O(N-2)$ Lineal Deformado, embebido en ambos Modelos $O(N-1)$. Las diferentes intersecciones a su vez intersecan en copias del Modelo $O(N-3)$ y así sucesivamente hasta finalizar en una única copia del Modelo $O(1)$. Se tiene, por tanto, un proceso iterativo de tal manera que la variedad de kinks del Modelo Sigma $O(N-r)$ está incluida $\left(\begin{array}{c}N-1 \\ r\end{array}\right)$ en la variedad de kinks del sistema $O(N)$. 


\section{Capítulo 4}

\section{Mecánica Analítica en Variedades Riemannianas}

En la Sección 1.3 del primer capítulo de esta memoria se resumían los aspectos fundamentales de los Sistemas Dinámicos Integrables y del Método de HamiltonJacobi. Este último fue la técnica utilizada en el Capítulo 2 para obtener las ondas solitarias del Modelo Sigma $O(N)$ Lineal Deformado.

De cara a estudiar la estabilidad de las soluciones kink del modelo, analizaremos ahora uno de los aspectos ya citados de la Teoría de Sistemas Dinámicos, el planteamiento variacional de la Mecánica sobre una variedad riemanniana dada. En una segunda sección, detallaremos el Teorema de Jacobi acerca de la interpretación de las trayectorias solución de un sistema dinámico como las geodésicas en una variedad riemanniana, dependiente del sistema, y dotada de la que denominaremos "métrica de Jacobi" asociada. Esta técnica nos permitirá abordar el estudio de la estabilidad desde un punto de vista diferente al presente habitualmente en la literatura. Para finalizar este capítulo, se particularizan los resultados generales de las dos primeras secciones al caso en el que la teoría es pre-supersimétrica (entendiendo por tal carácter el que posee toda teoría que pueda ser extendida a una teoría supersimétrica), como ocurre en los modelos que estudiamos fundamentalmente en este trabajo.

Algunos de los teoremas que se incluyen en este capítulo son bien conocidos, hemos considerado oportuno detallar sus demostraciones sólo en aquellos casos en los que las mismas han sido reelaboradas en la notación que estamos utilizando o cuando las técnicas empleadas requieran una especial atención o interés. En los Apéndices C y D de esta memoria se han resumido algunos resultados fundamentales de la Geometría Riemanniana y del Cálculo Variacional aplicado a Geodésicas 
que se utilizan con frecuencia en este capítulo.

Se ha procurado adoptar una notación clara y estándar, con la evidente subjetividad que tales adjetivos implican. En cualquier caso, resaltaremos que se ha utilizado el Convenio de Einstein de suma en índices repetidos, así como la notación habitual de subíndices y superíndices que se intercambian por medio del tensor métrico.

\subsection{Cálculo Variacional en Sistemas Dinámicos}

El Principio Variacional de Acción de Hamilton establece que las trayectorias solución de un sistema dinámico son aquéllas que hacen extremal el valor del funcional de acción de dicho sistema:

$$
S[c]=\int_{t_{1}}^{t_{2}} \mathcal{L} d t
$$

donde $c(t)$ es una curva parametrizada en una variedad diferenciable $M$, y $\mathcal{L}$ es la función lagrangiana que caracteriza al sistema dinámico en estudio. Como ya hemos comentado, nos restringiremos a lo que se suele llamar en la literatura "lagrangianas naturales", de la forma:

$$
\mathcal{L}=\mathcal{L}(c, \dot{c})=\frac{1}{2}\langle\dot{c}, \dot{c}\rangle-U(c(t))
$$

donde se considera que $M$ es una variedad riemanniana $(M, g)$ y $\langle$,$\rangle denota el$ producto escalar en $T M: g(X, Y)=\langle X, Y\rangle$.

En un sistema de coordenadas locales $\mathbf{x} \equiv\left(x^{1}, \ldots, x^{n}\right)$ en $M$, tendremos:

$$
\begin{gathered}
c:\left[t_{1}, t_{2}\right] \rightarrow M \\
c(t)=\left(x^{1}(t), \ldots, x^{n}(t)\right)
\end{gathered}
$$

Tal y como se detalla en el Apéndice C, $M$ estará dotada de la correspondiente conexión de Levi-Civita. Por otro lado, en el Apéndice D se especifica la notación variacional escogida, de manera que $c(t, \xi)$ denota una variación de la curva $c(t)$ $(\xi \in(-\varepsilon, \varepsilon)), \dot{c}(t, \xi)$ y $V(t, \xi)$ son los campos sobre $c(t, \xi)$ siguientes:

$$
\dot{c}(t, \xi)=\frac{\partial c}{\partial t}(t, \xi) ; \quad V(t, \xi)=\frac{\partial c}{\partial \xi}(t, \xi)
$$

y, evidentemente:

$$
\dot{c}(t) \equiv \dot{c}(t, 0) ; \quad V(t) \equiv V(t, 0)
$$


$\dot{c}(t)$ es así el campo que asocia a cada punto de la curva el vector velocidad en ese punto, mientras que $V(t)$ es el campo asociado a la variación considerada.

Las Ecuaciones de Euler-Lagrange asociadas al Principio Variacional de Hamilton no son más que las ecuaciones de Newton del sistema mecánico. De cara a presentar de manera uniforme los resultados de esta sección, así como mostrar las técnicas que utilizaremos posteriormente para el cálculo del Hessiano del funcional de acción, incluiremos en la siguiente Proposición la demostración de este hecho.

Proposición 4.1. Para cada curva $c$ en $M$, la función $S[c](\xi)$ definida en $(-\varepsilon, \varepsilon)$ de la forma:

$$
S[c](\xi)=\int_{t_{1}}^{t_{2}} d t\left(\frac{1}{2}\langle\dot{c}(t, \xi), \dot{c}(t, \xi)\rangle-U(c(t, \xi))\right)
$$

es diferenciable con respecto a $\xi$, y además:

$$
\frac{d}{d \xi} S[c](0)=-\int_{t_{1}}^{t_{2}} d t\left\langle\frac{\mathrm{D}}{\partial t} \dot{c}(t, 0)+\operatorname{grad} U(c(t, 0)), V(t, 0)\right\rangle
$$

para toda variación propia definida sobre $c .^{1}$

\section{Demostración:}

$$
\begin{aligned}
\frac{d S[c](\xi)}{d \xi} & \stackrel{(a)}{=} \int_{t_{1}}^{t_{2}} d t\left(\frac{1}{2} \frac{\partial}{\partial \xi}\langle\dot{c}, \dot{c}\rangle-\frac{\partial U}{\partial \xi}\right) \stackrel{(b)}{=} \\
& =\int_{t_{1}}^{t_{2}} d t\left(\left\langle\frac{\mathrm{D}}{\partial \xi} \dot{c}, \dot{c}\right\rangle-\langle\operatorname{grad}(U), V\rangle\right) \stackrel{(c)}{=} \\
& =\int_{t_{1}}^{t_{2}} d t\left(\left\langle\frac{\mathrm{D}}{\partial t} V, \dot{c}\right\rangle-\langle\operatorname{grad}(U), V\rangle\right) \stackrel{(d)}{=} \\
& =\int_{t_{1}}^{t_{2}} d t\left(\frac{\partial}{\partial t}\langle V, \dot{c}\rangle-\left\langle V, \frac{\mathrm{D}}{\partial t} \dot{c}\right\rangle-\langle\operatorname{grad}(U), V\rangle\right)= \\
& =\left.\langle V, \dot{c}\rangle\right|_{t_{1}} ^{t_{2}}-\int_{t_{1}}^{t_{2}} d t\left\langle V, \frac{\mathrm{D}}{\partial t} \dot{c}+\operatorname{grad}(U)\right\rangle
\end{aligned}
$$

donde se ha utilizado:

- (a) La derivación bajo el signo de integral.

- (b) La conexión de Levi-Civita es métrica, y por tanto se verifica (C.8):

$$
\frac{\partial}{\partial \xi}\langle\dot{c}, \dot{c}\rangle=\left\langle\frac{\mathrm{D} \dot{c}}{\partial \xi}, \dot{c}\right\rangle+\left\langle\dot{c}, \frac{\mathrm{D} \dot{c}}{\partial \xi}\right\rangle
$$

\footnotetext{
${ }^{1}$ Nos remitimos al Apéndice D para las definiciones precisas de la variación uniparamétrica $c(t, \xi)$ de la curva $c(t)$, así como la definición de variación propia.
} 
y la derivada (C.17):

$$
\frac{\partial U}{\partial \xi}=\frac{\partial U}{\partial x^{i}} \frac{\partial x^{i}}{\partial \xi}=\left\langle\operatorname{grad}(U), \frac{\partial c}{\partial \xi}\right\rangle=\langle\operatorname{grad}(U), V\rangle
$$

siendo grad $(U)$ el gradiente de la función $U: M \rightarrow \mathbb{R}$, definido en (C.16):

$$
\operatorname{grad}(U)=g^{i j} \frac{\partial U}{\partial x^{i}} \frac{\partial}{\partial x^{j}}
$$

- (c) La conexión es simétrica (libre de torsión) y por tanto se verifica (C.12)(C.13):

$$
\frac{\mathrm{D}}{\partial \xi} \frac{\partial c}{\partial t}=\frac{\mathrm{D}}{\partial t} \frac{\partial c}{\partial \xi} \Rightarrow \frac{\mathrm{D}}{\partial \xi} \dot{c}=\frac{\mathrm{D}}{\partial t} V
$$

- (d) De nuevo (C.8):

$$
\left\langle\frac{\mathrm{D}}{\partial t} V, \dot{c}\right\rangle=\frac{\partial}{\partial t}\langle V, \dot{c}\rangle-\left\langle V, \frac{\mathrm{D}}{\partial t} \dot{c}\right\rangle
$$

Para $\xi=0$ tendremos:

$$
\frac{d S[c]}{d \xi}(0)=\left.\langle V(t), \dot{c}(t)\rangle\right|_{t_{1}} ^{t_{2}}-\int_{t_{1}}^{t_{2}} d t\left\langle V(t), \frac{\mathrm{D}}{d t} \dot{c}(t)+\operatorname{grad}(U)\right\rangle
$$

y, para el caso de variaciones propias: $V\left(t_{2}\right)=V\left(t_{1}\right)=0$, (D.1), y se obtiene el resultado buscado.

Q.E.D.

Aplicando el Teorema Fundamental del Cálculo Variacional (ver Apéndice D), las trayectorias extremales de la acción $S[c]$ verifican las ecuaciones:

$$
\frac{\mathrm{D}}{d t} \dot{c}+\operatorname{grad}(U)=\nabla_{\dot{c}} \dot{c}+\operatorname{grad}(U)=0
$$

que no son más que las ecuaciones de Newton del sistema dinámico considerado.

Para la variación segunda de la curva tendremos el teorema:

Teorema 4.2. Si $c(t)$ es una trayectoria extremal del funcional $S[c]$, entonces, para cada variación de $c$, se tiene:

$$
\begin{aligned}
\frac{d^{2} S[c]}{d \xi^{2}}(0)=- & \int_{t_{1}}^{t_{2}} d t\left(\left\langle\frac{\mathrm{D}^{2} V}{d t^{2}}+R(\dot{c}, V) \dot{c}, V\right\rangle+H(U)(V, V)\right)+ \\
& +\left.\left\langle\frac{\mathrm{D} V}{\partial \xi}, \dot{c}\right\rangle\right|_{t_{1}, \xi=0} ^{t_{2}, \xi=0}+\left.\left\langle\frac{\mathrm{D} \dot{c}}{\partial \xi}, V\right\rangle\right|_{t_{1}, \xi=0} ^{t_{2}, \xi=0}
\end{aligned}
$$


donde $H(U)$ es el hessiano de la función potencial $U$.

De la definición C.5 del Apéndice C se tiene: $H(U)=\nabla d U$, donde $\nabla d U$ denota la diferencial covariante de $d U$, que en coordenadas locales se escribe (C.18):

$$
H(U)=\nabla d U=\left(\nabla_{\frac{\partial}{\partial x^{j}}} d U\right) d x^{j}=\left(\frac{\partial^{2} U}{\partial x^{j} \partial x^{k}}-\frac{\partial U}{\partial x^{k}} \Gamma_{i j}^{k}\right) d x^{i} \otimes d x^{j}
$$

mientras que de forma intrínseca, utilizando la proposición (C.19):

$$
H(U)(X, Y)=\nabla d U(X, Y)=\left\langle\nabla_{X} \operatorname{grad}(U), Y\right\rangle
$$

para cualesquiera dos campos tangentes $X$ e $Y$.

\section{Demostración:}

$$
\begin{aligned}
& \frac{d^{2} S[c](\xi)}{d \xi^{2}}=\frac{d}{d \xi} \int_{t_{1}}^{t_{2}} d t\left(\left\langle\frac{\mathrm{D} V}{\partial t}, \dot{c}\right\rangle-\langle\operatorname{grad}(U), V\rangle\right)= \\
= & \int_{t_{1}}^{t_{2}} d t\left(\frac{\partial}{\partial \xi}\left\langle\frac{\mathrm{D} V}{\partial t}, \dot{c}\right\rangle-\frac{\partial}{\partial \xi}\langle\operatorname{grad}(U), V\rangle\right) \stackrel{(a)}{=} \\
= & \int_{t_{1}}^{t_{2}} d t\left(\left\langle\frac{\mathrm{D}}{\partial \xi} \frac{\mathrm{D}}{\partial t} V, \dot{c}\right\rangle+\left\langle\frac{\mathrm{D} V}{\partial t}, \frac{\mathrm{D} \dot{c}}{\partial \xi}\right\rangle-\left\langle\frac{\mathrm{D}}{\partial \xi} \operatorname{grad}(U), V\right\rangle-\left\langle\operatorname{grad}(U), \frac{\mathrm{D} V}{\partial \xi}\right\rangle\right) \stackrel{(b)}{=} \\
= & \int_{t_{1}}^{t_{2}} d t\left(\langle R(\dot{c}, V) V, \dot{c}\rangle+\left\langle\frac{\mathrm{D}}{\partial t} \frac{\mathrm{D} V}{\partial \xi}, \dot{c}\right\rangle+\left\langle\frac{\mathrm{D} V}{\partial t}, \frac{\mathrm{D} V}{\partial t}\right\rangle\right. \\
& \left.-\left\langle\nabla_{V} \operatorname{grad}(U), V\right\rangle-\left\langle\operatorname{grad}(U), \frac{\mathrm{D} V}{\partial \xi}\right\rangle\right) \stackrel{(c)}{=} \\
= & \int_{t_{1}}^{t_{2}} d t\left(\langle R(\dot{c}, V) V, \dot{c}\rangle+\frac{\partial}{\partial t}\left\langle\frac{\mathrm{D} V}{\partial \xi}, \dot{c}\right\rangle-\left\langle\frac{\mathrm{D} V}{\partial \xi}, \frac{\mathrm{D} \dot{c}}{\partial t}\right\rangle\right. \\
& \left.+\frac{\partial}{\partial t}\left\langle V, \frac{\mathrm{D} V}{\partial t}\right\rangle-\left\langle V, \frac{\mathrm{D}^{2} V}{\partial t^{2}}\right\rangle-\left\langle\nabla_{V} \operatorname{grad}(U), V\right\rangle-\left\langle\operatorname{grad}(U), \frac{\mathrm{D} V}{\partial \xi}\right\rangle\right)
\end{aligned}
$$

donde se ha utilizado:

- (a) La propiedad (C.8) del Apéndice C.

- (b) La propiedad (C.28), que para este caso se escribe:

$$
\frac{\mathrm{D}}{\partial \xi} \frac{\mathrm{D} V}{\partial t}-\frac{\mathrm{D}}{\partial t} \frac{\mathrm{D} V}{\partial \xi}=R(\dot{c}, V) V
$$

y la propiedad (C.12).

- (c) Las propiedades (C.8) y (C.12) de nuevo. 
Podemos aplicar a su vez la propiedad (C.26) del tensor de curvatura:

$$
\langle R(\dot{c}, V) V, \dot{c}\rangle=-\langle R(\dot{c}, V) \dot{c}, V\rangle
$$

Restringiendo para el caso $\xi=0$, y teniendo en cuenta que por hipótesis la curva $c(t, 0)=c(t)$ es una extremal de la acción $S[c]$, es decir:

$$
\frac{\mathrm{D} \dot{c}}{d t}+\operatorname{grad}(U)=0
$$

se obtiene:

$$
\begin{aligned}
\frac{d^{2} S[c]}{d \xi^{2}}(0)= & \int_{t_{1}}^{t_{2}} d t\left(-\left\langle\frac{\mathrm{D}^{2} V}{\partial t^{2}}+R(\dot{c}, V) \dot{c}, V\right\rangle-\nabla d U(V, V)\right) \\
& +\left.\left\langle\frac{\mathrm{D} \dot{c}}{\partial \xi}, V\right\rangle\right|_{t_{1}, \xi=0} ^{t_{2}, \xi=0}+\left.\left\langle\frac{\mathrm{D} V}{\partial \xi}, \dot{c}\right\rangle\right|_{t_{1}, \xi=0} ^{t_{2}, \xi=0}
\end{aligned}
$$

donde se ha usado finalmente la Proposición C.2 del apéndice C que nos dice, para este caso, que:

$$
H(U)(V, V)=\nabla d U(V, V)=\left\langle\nabla_{V} \operatorname{grad}(U), V\right\rangle
$$

Q.E.D.

Si recordamos la expresión de la curvatura seccional (ver Apéndice C), para cualesquiera dos campos tangentes $X, Y \in \Gamma(T M)$ se tenía:

$$
\begin{gathered}
K_{X}(Y)=R(X, Y) X \\
K(X, Y)=\left\langle K_{X}(Y), Y\right\rangle=\langle R(X, Y) X, Y\rangle
\end{gathered}
$$

y nos restringimos a variaciones propias; podemos escribir finalmente la expresión del funcional hessiano de un sistema dinámico como:

$$
\frac{d^{2} S[c]}{d \xi^{2}}(0)=\int_{t_{1}}^{t_{2}} d t\left\langle-\frac{\mathrm{D}^{2} V}{d t^{2}}-K_{\dot{c}}(V)-\nabla_{V} \operatorname{grad}(U), V\right\rangle
$$

\subsection{Métrica de Jacobi}

Presentaremos en esta sección el Teorema de Jacobi que permite calcular las curvas solución de un sistema dinámico, definido en una variedad riemanniana $(M, g)$, por medio de las ecuaciones de las curvas geodésicas en la variedad $(M, h)$, siendo $h$ la métrica de Jacobi asociada al sistema. La idea básica de dicho resultado radica en la utilización de la integral primera de la energía. 


\subsubsection{Teorema de Jacobi}

Antes de introducir el Teorema, demostraremos dos Lemas, relativos a trasformaciones conformes en una variedad riemanniana, el primero, y al comportamiento de las derivadas covariantes a lo largo de curvas bajo reparametrizaciones de las mismas, el segundo.

Lema 4.3. Dada una transformación conforme en una variedad riemanniana:

$$
(M, g) \rightarrow(M, \tilde{g}) ; \quad \tilde{g}=f(\mathbf{x}) g
$$

sean $\nabla$ y $\tilde{\nabla}$ las respectivas conexiones de Levi-Civita asociadas. Entonces se verifica, para cualesquiera campos tangentes $X, Y, Z \in \Gamma(T M)$

$$
\begin{aligned}
\tilde{\nabla}_{X} Y & =\nabla_{X} Y+\frac{1}{2}\langle F, Y\rangle X+\frac{1}{2}\langle F, X\rangle Y-\frac{1}{2}\langle X, Y\rangle F \\
\tilde{\nabla}_{X} \tilde{\nabla}_{Y} Z= & \nabla_{X} \nabla_{Y} Z+\frac{1}{2}\langle F, Z\rangle \nabla_{X} Y+\frac{1}{2}\langle F, Y\rangle \nabla_{X} Z \\
& -\frac{1}{2}\langle Y, Z\rangle \nabla_{X} F+\frac{1}{2}\langle F, X\rangle \nabla_{Y} Z+ \\
+ & \left(\frac{1}{2}\left\langle F, \nabla_{Y} Z\right\rangle+\frac{1}{2}\langle F, Z\rangle\langle F, Y\rangle-\frac{1}{4}\langle Y, Z\rangle\langle F, F\rangle\right) X+ \\
+ & \left(\frac{1}{2}\left\langle\nabla_{X} F, Z\right\rangle+\frac{1}{2}\left\langle F, \nabla_{X} Z\right\rangle+\frac{1}{4}\langle F, Z\rangle\langle F, X\rangle\right) Y+ \\
+ & \left(\frac{1}{2}\left\langle\nabla_{X} F, Y\right\rangle+\frac{1}{2}\left\langle F, \nabla_{X} Y\right\rangle+\frac{1}{4}\langle F, X\rangle\langle F, Y\rangle\right) Z+ \\
+ & \left(\frac{-1}{2}\left\langle\nabla_{X} Y, Z\right\rangle-\frac{1}{2}\left\langle Y, \nabla_{X} Z\right\rangle-\frac{1}{2}\left\langle X, \nabla_{Y} Z\right\rangle\right. \\
& \left.-\frac{1}{4}\langle F, Z\rangle\langle X, Y\rangle-\frac{1}{4}\langle F, Y\rangle\langle X, Z\rangle\right) F
\end{aligned}
$$

donde los productos escalares están tomados en la métrica $g$, y $F=\operatorname{grad}(\operatorname{Ln} f)$ (gradiente con respecto a $g$ ).

La expresión (4.3) puede reescribirse de la forma:

$$
\tilde{\nabla}_{X} Y=\nabla_{X} Y+\frac{1}{2}(Y(\ln f) X+X(\ln f) Y-\langle X, Y\rangle \operatorname{grad}(\ln f))
$$

\section{Demostración:}

Los símbolos de Christoffel de la métrica $\tilde{g}$, referidos a los de $g$, pueden obtenerse fácilmente por cálculo directo teniendo en cuenta su definición:

$$
\tilde{\Gamma}_{j k}^{i}=\frac{1}{2} \tilde{g}^{i r}\left(\partial_{k} \tilde{g}_{j r}-\partial_{r} \tilde{g}_{j k}+\partial_{j} \tilde{g}_{r k}\right)
$$


y la relación entre ambas métricas:

$$
\tilde{g}_{i j}=f g_{i j} ; \quad \tilde{g}^{i j}=\frac{1}{f} g^{i j}
$$

El resultado es:

$$
\tilde{\Gamma}_{j k}^{i}=\Gamma_{j k}^{i}+\frac{1}{2}\left[\operatorname{grad}(\ln f)^{m}\left(\delta_{j}^{i} g_{m k}+\delta_{k}^{i} g_{m j}\right)-g_{j k} \operatorname{grad}(\ln f)^{i}\right]
$$

De esta manera:

$$
\tilde{\nabla}_{X} Y=X^{j} \tilde{\nabla}_{j} Y=X^{j}\left(\frac{\partial Y^{i}}{\partial x^{j}}+\tilde{\Gamma}_{j k}^{i} Y^{k}\right) \frac{\partial}{\partial x^{i}}=\nabla_{X} Y+A_{j k}^{i} X^{j} Y^{k} \frac{\partial}{\partial x^{i}}
$$

donde

$$
\begin{gathered}
A_{j k}^{i} X^{j} Y^{k}=\frac{1}{2}\left[\operatorname{grad}(\ln f)^{m}\left(\delta_{j}^{i} g_{m k}+\delta_{k}^{i} g_{m j}\right)-g_{j k} \operatorname{grad}(\ln f)^{i}\right] X^{j} Y^{k}= \\
=\frac{1}{2}\left(\langle\operatorname{grad}(\ln f), Y\rangle X^{i}+\langle\operatorname{grad}(\ln f), X\rangle Y^{i}\right)-\frac{1}{2}\langle X, Y\rangle \operatorname{grad}(\ln f)^{i}
\end{gathered}
$$

y, en definitiva:

$$
\tilde{\nabla}_{X} Y=\nabla_{X} Y+\frac{1}{2}\langle\operatorname{grad}(\ln f), Y\rangle X+\frac{1}{2}\langle\operatorname{grad}(\ln f), X\rangle Y-\frac{1}{2}\langle X, Y\rangle \operatorname{grad}(\ln f)
$$

que no es otra que la expresión (4.3).

Para demostrar (4.4) tendremos simplemente que derivar covariantemente ambos miembros de (4.3) y aplicar de nuevo dicho resultado a la expresión resultante. Tras simplificar se obtiene directamente (4.4).

Q.E.D.

Lema 4.4. Dada una curva (diferenciable) $c:\left[t_{1}, t_{2}\right] \rightarrow M$ en una variedad riemanniana $M$, sea $c(s)=c(t(s))$ una reparametrización (admisible) de la misma, con $d s=f(\mathbf{x}(t)) d t\left(f(\mathbf{x}(t)) \neq 0, \forall t \in\left[t_{1}, t_{2}\right]\right)$. Entonces se verifican las igualdades siguientes, $\forall X \in \Gamma(T M)$ :

$$
\begin{gathered}
\nabla_{c^{\prime}} X=\frac{\mathrm{D} X}{d s}=\frac{1}{f(\mathbf{x})} \frac{\mathrm{D} X}{d t}=\nabla_{\dot{c}} X \\
\nabla_{c^{\prime}} c^{\prime}=\frac{1}{f(\mathbf{x})^{2}}\left(\nabla_{\dot{c}} \dot{c}-\langle\operatorname{grad}(\ln f), \dot{c}\rangle \dot{c}\right) \\
\nabla_{c^{\prime}} \nabla_{c^{\prime}} X=\frac{1}{f(\mathbf{x})^{2}}\left(\nabla_{\dot{c}} \nabla_{\dot{c}} X-\langle\operatorname{grad}(\ln f), \dot{c}\rangle \nabla_{\dot{c}} X\right)
\end{gathered}
$$


siendo $\dot{c}(t)=\frac{d c(t)}{d t}$ y $c^{\prime}(s)=\frac{d c(s)}{d s}$.

\section{Demostración:}

Las tres identidades se demuestran fácilmente por cálculo directo:

$\nabla_{c^{\prime}} X=\frac{\mathrm{D} X}{d s}=\left(\frac{d X^{i}}{d s}+\Gamma_{j k}^{i} x^{\prime j} X^{k}\right) \frac{\partial}{\partial x^{i}}=\left(\frac{d X^{i}}{d t} \frac{d t}{d s}+\Gamma_{j k}^{i} \dot{x}^{j} \frac{d t}{d s} X^{k}\right) \frac{\partial}{\partial x^{i}}=\frac{1}{f} \nabla_{\dot{c}} X$

Para (4.7):

$$
\begin{aligned}
\nabla_{c^{\prime}} c^{\prime} & =\frac{1}{f} \nabla_{\dot{c}} c^{\prime}=\frac{1}{f}\left(\frac{d x^{i}}{d t}+\Gamma_{j k}^{i} \dot{x}^{j} x^{\prime k}\right) \frac{\partial}{\partial x^{i}}=\left(\frac{1}{f} \frac{d}{d t}\left(\frac{\dot{x}^{i}}{f}\right)+\Gamma_{j k}^{i} \dot{x}^{j} \dot{x}^{k} \frac{1}{f^{2}}\right) \frac{\partial}{\partial x^{i}}= \\
& =\frac{1}{f^{2}}\left(\nabla_{\dot{c}} \dot{c}-\partial_{k} \ln f \dot{x}^{k} \dot{x}^{i} \frac{\partial}{\partial x^{i}}\right)=\frac{1}{f^{2}}\left(\nabla_{\dot{c}} \dot{c}-\langle\operatorname{grad}(\ln f), \dot{c}\rangle \dot{c}\right)
\end{aligned}
$$

Mientras que (4.8):

$$
\begin{aligned}
\nabla_{c^{\prime}} \nabla_{c^{\prime}} X & =\nabla_{c^{\prime}}\left(\frac{1}{f} \nabla_{\dot{c}} X\right)=\nabla_{c^{\prime}}\left(\frac{1}{f}\right) \nabla_{\dot{c}} X+\frac{1}{f^{2}} \nabla_{\dot{c}} \nabla_{\dot{c}} X= \\
& =\frac{d t}{d s} \frac{d}{d t}\left(\frac{1}{f}\right) \nabla_{\dot{c}} X+\frac{1}{f^{2}} \nabla_{\dot{c}} \nabla_{\dot{c}} X=\frac{1}{f^{2}}\left(\nabla_{\dot{c}} \nabla_{\dot{c}} X-\langle\operatorname{grad}(\ln f), \dot{c}\rangle \nabla_{\dot{c}} X\right)
\end{aligned}
$$

Q.E.D.

Una vez establecidos los Lemas anteriores, tenemos las herramientas que permitirán una demostración sencilla del Teorema de Jacobi que expondremos a continuación. La idea fundamental, como ya hemos comentado, se basa en la conservación de la energía mecánica sobre las trayectorias solución de un sistema mecánico.

El Teorema de Jacobi es conocido también como "Formulación de Jacobi del principio de mínima acción" (ver [65]), y, para Arnold, [9], es el "Principio de mínima acción en versión Maupertuis-Euler-Lagrange-Jacobi". Para el caso de movimiento libre de una partícula en una variedad riemanniana, se escribe [9]: "Una masa puntual reducida a una variedad riemanniana uniforme se desplaza por las líneas geodésicas". Se entiende entonces la métrica $g$ de la variedad $(M, g)$ como la "métrica cinemática" que describe Laugwitz, [65], escribiendo:

$$
d s^{2}=g_{i j} d x^{i} d x^{j}=g_{i j} \frac{d x^{i}}{d t} \frac{d x^{j}}{d t} d t^{2}
$$

Para el caso de movimiento en una variedad bajo una energía potencial, la métrica cinemática se ve sustituida por la métrica de Jacobi, como veremos a continuación. 
Definición. Dado un sistema dinámico determinado por el funcional de acción

$$
S[c]=\int_{t_{1}}^{t_{2}} d t\left(\frac{1}{2}\langle\dot{c}, \dot{c}\rangle-U(c(t))\right)
$$

sobre una variedad riemanniana $(M, g)$, llamaremos métrica de Jacobi asociada a dicho sistema a la métrica:

$$
d s^{2}=h_{i j} d x^{i} \otimes d x^{j} \equiv h_{i j} d x^{i} d x^{j}
$$

donde $h_{i j}=2\left(i_{1}-U\right) g_{i j}$ e $i_{1}$ es una constante que verifica $i_{1}>U$ en todo punto de $M^{2}{ }^{2}$

Por simplicidad, si $h$ es la métrica de Jacobi asociada a un sistema dinámico, denotaremos $\forall X, Y \in \Gamma(T M)$ :

$$
h(X, Y)=\langle X, Y\rangle^{J} ; \quad\|X\|^{J}=\sqrt{\langle X, X\rangle^{J}}
$$

y llamaremos grad ${ }^{J}$ al gradiente con respecto a la métrica $h$.

Teorema de Jacobi. Las curvas extremales del problema variacional asociado a la acción de un sistema dinámico:

$$
S[c]=\int_{t_{1}}^{t_{2}} d t\left(\frac{1}{2}\langle\dot{c}, \dot{c}\rangle-U(c(t))\right)
$$

donde $c:\left[t_{1}, t_{2}\right] \rightarrow M y(M, g)$ es una variedad riemanniana, correspondientes al valor constante $i_{1}$ de la integral primera de la energía, son las curvas geodésicas de la variedad $(M, h)$, donde $h$ es la métrica de Jacobi asociada al sistema.

\section{Demostración:}

Dado que la métrica de Jacobi se obtiene por medio de una transformación conforme y que las geodésicas de la misma vendrán expresadas en términos del parámetro-arco $s$ y no del parámetro $t$ del sistema dinámico, los dos Lemas anteriores son fundamentales para la demostración que nos ocupa.

Si llamamos $c(s)$ a una curva geodésica de la métrica $h$, la ecuación que la determina será, evidentemente:

$$
\frac{\mathrm{D}^{J} c}{d s}=\nabla_{c^{\prime}}^{J} c^{\prime}=0
$$

\footnotetext{
${ }^{2}$ De la definición se deduce que $d s_{h}^{2}=2\left(i_{1}-V\right) d s_{g}^{2}$, denotaremos en general $d s$ al elemento de $\operatorname{arco} d s_{h}$.
} 
donde se ha denotado $c^{\prime}=\frac{d c}{d s}$ y $\nabla^{J}$ es la conexión de Levi-Civita asociada a la métrica de Jacobi $h$.

Aplicando el Lema 4.3 (4.3) para la métrica de Jacobi: $f(\mathbf{x})=2\left(i_{1}-U\right),(4.9)$ se convierte en:

$$
\nabla_{c^{\prime}} c^{\prime}+\left\langle\operatorname{grad}\left(\operatorname{Ln}\left(2\left(i_{1}-U\right)\right)\right), c^{\prime}\right\rangle c^{\prime}-\frac{1}{2}\left\langle c^{\prime}, c^{\prime}\right\rangle \operatorname{grad}\left(\operatorname{Ln}\left(2\left(i_{1}-U\right)\right)\right)=0
$$

Por otro lado $d s^{2}=2\left(i_{1}-U\right) g_{i j} d x^{i} d x^{j}$, de manera que:

$$
\frac{d s}{d t}=\sqrt{2\left(i_{1}-U\right) g_{i j} \dot{x}^{i} \dot{x}^{j}}=\sqrt{2\left(i_{1}-U\right)\langle\dot{c}, \dot{c}\rangle}
$$

Dado que las trayectorias del sistema dinámico a las que se refiere el Teorema verifican $i_{1}=\frac{1}{2}\langle\dot{c}, \dot{c}\rangle+U$, tendremos, para este caso, $\langle\dot{c}, \dot{c}\rangle=2\left(i_{1}-U\right)$ de manera que la relación entre el parámetro arco de la métrica de Jacobi y el parámetro temporal del sistema dinámico, sobre las trayectorias de energía $i_{1}$, se escribe:

$$
\frac{d s}{d t}=2\left(i_{1}-U\right)
$$

La aplicación del Lema 4.4 (4.7) a la expresión (4.10) conduce a:

$$
\frac{1}{\left(2\left(i_{1}-V\right)\right)^{2}} \nabla_{\dot{c}} \dot{c}-\frac{1}{2\left(2\left(i_{1}-U\right)\right)^{2}}\langle\dot{c}, \dot{c}\rangle \operatorname{grad}\left(\operatorname{Ln}\left(2\left(i_{1}-U\right)\right)\right)=0
$$

que, aplicando de nuevo la restricción a energía constante: $\frac{1}{2}\langle\dot{c}, \dot{c}\rangle=i_{1}-U, \mathrm{y}$ simplificando (gracias a que $i_{1}$ es necesariamente mayor que $U$ ) finalmente produce:

$$
\nabla_{\dot{c}} \dot{c}+\operatorname{grad}(U)=0
$$

es decir, las ecuaciones de Newton el sistema dinámico.

Q.E.D.

Un primer hecho reseñable acerca del Teorema es que permitirá calcular no sólo las ecuaciones de las geodésicas y, por tanto, las ecuaciones de las trayectorias solución del sistema (como establece el Principio de Maupertuis propiamente dicho), sino que también se determina completamente la dependencia temporal sobre dichas trayectorias, usando el valor conocido de la integral primera de la energía. Una vez resueltas las ecuaciones de las geodésicas, la dependencia temporal se obtiene implícitamente integrando la ecuación:

$$
\frac{d s}{d t}=2 \sqrt{\left(i_{1}-U(\mathbf{x}(s))\right) T}=\left(i_{1}-U(\mathbf{x}(s))\right)
$$


(siendo $T$ evidentemente la energía cinética).

Otra precisión interesante es que la métrica de Jacobi de manera general dará lugar a curvatura no nula aún cuando la métrica inicial sea plana; volveremos a este hecho, y a su relación con el carácter pre-supersimétrico, en la tercera sección de este capítulo.

El Teorema de Jacobi abre multitud de posibilidades de aplicación de resultados de la Geometría Riemanniana a la Mecánica Analítica. En [65] se recogen algunas de ellas, como diversos teoremas sobre curvas braquistócronas o el estudio de la estabilidad de movimientos planetarios. En este último caso, se trasladan criterios sobre la estabilidad de las líneas geodésicas, gobernada por la Ecuación de desviación geodésica y, en definitiva, por el signo de la curvatura de la variedad (al menos para el caso bidimensional), al caso mecánico de trayectorias solución de un sistema mecánico. Demostraremos en el próximo apartado la relación que existe entre la que podemos llamar "estabilidad mecánica" y la "estabilidad geodésica". De manera general, no obstante, es preciso considerar que la métrica de Jacobi está definida para un valor concreto de la energía mecánica, y, por tanto, sólo sobre una hipersuperficie del espacio de fases del sistema. El criterio de estabilidad que pueda proporcionar, en definitiva, estará limitado a estabilidad frente otras trayectorias de igual energía y no frente a la totalidad de las soluciones del sistema considerado. En el caso de las soluciones de tipo kink, éste no será un inconveniente, como veremos, por estar restringida la existencia de ondas solitarias, como se especificó en el Capítulo 1 , al valor fijo de la energía $i_{1}=0$.

Desde el punto de vista del Cálculo Variacional, es interesante analizar la relación que existe entre el funcional de acción del sistema mecánico considerado y los funcionales de energía y longitud de la variedad $(M, h)$. Tal y como se detalla en el Apéndice D, las curvas extremales de la energía y de la longitud son las geodésicas de la variedad que unen dos puntos determinados. Como hemos demostrado en el Teorema de Jacobi, dichas curvas son exactamente las trayectorias solución del sistema dinámico parametrizadas en función de la longitud de arco de la métrica de Jacobi. En general, el valor de los funcionales de energía y longitud sobre dichas curvas no coinciden con el valor de la acción de las mismas. Sin embargo, para el caso de sistemas dinámicos del tipo que estudiamos en este trabajo, es decir sistemas procedentes de la búsqueda de soluciones de tipo kink en una teoría de campos, de manera que las condiciones de contorno de la teoría de campos nos imponen la restricción de que la energía mecánica de las trayectorias sea nula, tenemos el siguiente hecho reseñable: 
Proposición 4.5. Sea $c(t)$ una curva extremal del funcional de acción:

$$
S[c]=\int_{t_{1}}^{t_{2}} d t\left(\frac{1}{2}\langle\dot{c}, \dot{c}\rangle-U(c(t))\right)
$$

correspondiente a una energía mecánica $i_{1}=0$. Entonces, si $L^{J}[c]$ denota al funcional de longitud de la métrica de Jacobi asociada a la acción $S[c]$ :

$$
L^{J}[c]=\int_{s_{1}}^{s_{2}} \sqrt{\left\langle c^{\prime}, c^{\prime}\right\rangle^{J}} d s=\int_{s_{1}}^{s_{2}} d s ; \quad s_{1}=s_{1}\left(t_{1}\right), s_{2}=s_{2}\left(t_{2}\right)
$$

se verifica:

$$
S[c]=L^{J}[c]
$$

\section{Demostración:}

Si $i_{1}=0$, entonces se verifica: $\frac{1}{2}\langle\dot{c}, \dot{c}\rangle=-U(c)$, y, por tanto: $h_{i j}=(-2 U(c)) g_{i j}$. De esta manera: $d s=-2 U(c) d t$ sobre las soluciones, y así:

$$
L^{J}[c]=\int_{s_{1}}^{s_{2}} d s=\int_{t_{1}}^{t_{2}}(-2 U(c)) d t=\int_{t_{1}}^{t_{2}}\left(\frac{1}{2}\langle\dot{c}, \dot{c}\rangle-U(c)\right) d t=S[c]
$$

Q.E.D.

Teniendo en cuenta que las geodésicas de $(M, h)$ están parametrizadas por el arco de longitud, es evidente, a partir de la Proposición anterior, que el funcional energía de Jacobi, $E^{J}[c]$ :

$$
E^{J}[c]=\int_{s_{1}}^{s_{2}} \frac{1}{2}\left\langle c^{\prime}, c^{\prime}\right\rangle^{J} d s
$$

verificará, sobre las soluciones:

$$
E^{J}[c]=\frac{1}{2} L^{J}[c]=\frac{1}{2} S[c]
$$

Es adecuado comentar, a este respecto, que la definición de funcional energía que hemos tomado no siempre es la habitual en la literatura. En varios textos, por ejemplo [70], se define $E[c]$ como el doble de la expresión que aquí se ha planteado. Por otro lado, resaltamos que la fórmula (4.13) es sólo cierta si $s$ denota al parámetro longitud de arco y $c$ es una curva geodésica.

Varias precisiones son necesarias acerca de estos últimos resultados expuestos:

La consideración de energía mecánica nula sólo tiene sentido, desde el punto de vista de la métrica de Jacobi, si la energía potencial $U$ es definida negativa, 
pues en caso contrario la métrica de Jacobi no sería definida positiva, como se ha supuesto en todo el capítulo. Evidentemente, para los sistemas que estudiamos en esta memoria, asociados, vía la analogía mecánica, a la búsqueda de kinks en una teoría de campos, esto se verifica al menos parcialmente. La energía potencial $U$ es por definición $U=-V$, donde $V$ es el potencial de la teoría de campos subyacente, y está tomado de manera general semidefinido positivo (ver Capítulo 1).

Los resultados anteriores son por tanto válidos en toda la variedad $M$ considerada exceptuando los puntos en los que se anula la energía potencial, es decir, los vacíos de la teoría. Teniendo en cuenta el tipo de potenciales que se está estudiando, con un número finito de ceros aislados, tendremos que el paso del punto de vista dinámico al geométrico conlleva aplicar la variedad riemanniana $(M, g)$ (en general $M=\mathbb{R}^{N}$ y $g_{i j}=\delta_{i j}$ ) en la variedad riemanniana $\left(M^{\prime}, h\right)$, donde $M^{\prime}$ es la variedad $M$ con los ceros del potencial excluidos.

Por otro lado, las geodésicas correspondientes a los kinks del sistema serán las que unen precisamente estos puntos singulares de la variedad $M^{\prime}$, debido a las condiciones asintóticas ya expuestas ${ }^{3}$. Si se estudiara la totalidad de las soluciones del modelo (sin restringirnos a las de tipo onda solitaria, es decir incluyendo las soluciones periódicas), encontraríamos que, para las soluciones periódicas, la energía $i_{1}$ tomaría valores negativos, de tal manera que, al ser $-U \geq 0$, sólo estarán definidas las soluciones en la región determinada por $i_{1}-U>0$, excluyéndose en consecuencia los "puntos de retroceso" de dichas soluciones, determinados por $i_{1}-U=0$. El cálculo de las soluciones kink puede verse, desde este punto de vista, como un proceso de paso al límite singular en el espacio de curvas geodésicas sobre el espacio de variedades de Jacobi asociado al sistema dinámico.

En el Capítulo 5, dedicado, en su primera sección, al cálculo explícito de soluciones, analizaremos con detalle la delicada situación que presentan los puntos de vacío de la teoría para el Modelo Sigma Lineal Deformado.

\subsubsection{Hessiano Dinámico y Hessianos Geodésicos}

De cara a estudiar la estabilidad de las trayectorias solución de un sistema dinámico, surgen ahora varias posibilidades. Tal y como hemos comentado en la sección anterior, por un lado tenemos la expresión del funcional hessiano (4.2), ya deducida, que da cuenta de la variación segunda del funcional de acción del sistema consi-

\footnotetext{
${ }^{3}$ Esta situación está estudiada en la literatura, incluso en casos más complicados en los que las singularidades de la métrica de Jacobi no son puntos aislados, ver por ejemplo [95] y [96].
} 
derado. Por otro lado, por medio de la métrica de Jacobi, el sistema puede verse como un problema de cálculo de geodésicas y, en consecuencia, como un problema variacional asociado bien al funcional de energía (acción), $E^{J}[c]$, de las curvas en $(M, h)$, bien al funcional de longitud, $L^{J}[c]$. En el Apéndice D se detalla la relación y equivalencias entre ambos funcionales.

Demostraremos en los dos teoremas siguientes cómo ni siquiera en el caso que nos ocupa principalmente, con energía mecánica $i_{1}=0$, coinciden dichos funcionales sobre las curvas solución. Computaremos, no obstante, la diferencia existente entre ellos, y plantearemos un método para abordar el problema, basado en la diagonalización del tensor de curvatura sobre una base que se traslade paralelamente sobre las curvas solución. Esto proporcionará, en algunos casos, una separación de las ecuaciones espectrales en ecuaciones no acopladas que permitirá resolver completamente la estabilidad de las soluciones estudiadas, como se verá en el Capítulo 5.

Los funcionales hessianos de la energía y de la longitud (ver Apéndice D), en el caso de la métrica de Jacobi, y sobre variaciones propias, se escribirán:

$$
\begin{aligned}
\frac{d^{2} E^{J}[c]}{d \xi^{2}}(0) & =\int_{s_{1}}^{s_{2}} d s\left\langle-\nabla_{c^{\prime}}^{J} \nabla_{c^{\prime}}^{J} V-R^{J}\left(c^{\prime}, V\right) c^{\prime}, V\right\rangle^{J}= \\
& =\int_{s_{1}}^{s_{2}} d s\left\langle-\frac{\mathrm{D}^{J}}{d s} \frac{\mathrm{D}^{J} V}{d s}-K_{c^{\prime}}^{J}(V), V\right\rangle^{J} \\
\frac{d^{2} L^{J}[c]}{d \xi^{2}}(0) & =\int_{s_{1}}^{s_{2}} d s\left\langle-\nabla_{c^{\prime}}^{J} \nabla_{c^{\prime}}^{J} V^{\perp}-R^{J}\left(c^{\prime}, V^{\perp}\right) c^{\prime}, V^{\perp}\right\rangle^{J}= \\
& =\int_{s_{1}}^{s_{2}} d s\left\langle-\frac{\mathrm{D}^{J}}{d s} \frac{\mathrm{D}^{J} V^{\perp}}{d s}-K_{c^{\prime}}^{J}\left(V^{\perp}\right), V^{\perp}\right\rangle^{J}
\end{aligned}
$$

respectivamente. $V^{\perp}$ representa la componente ortogonal a la curva $c(s)$ del campo asociado a la variación:

$$
V^{\perp}=V-\left\langle\frac{c^{\prime}}{\left\|c^{\prime}\right\|^{J}}, V\right\rangle^{J} \frac{c^{\prime}}{\left\|c^{\prime}\right\|^{J}}=V-\left\langle c^{\prime}, V\right\rangle^{J} c^{\prime}
$$

teniendo en cuenta que $s$ es el parámetro arco y, por tanto, $\left\|c^{\prime}\right\|^{J}=1 . R^{J} \mathrm{y}$ $K^{J}$ denotan respectivamente a los tensores de curvatura y de curvatura seccional asociados a la métrica de Jacobi $h$.

Además de los Lemas 4.3 y 4.4 necesitaremos, en los teoremas posteriores, conocer cómo se comporta el tensor de curvatura bajo trasformaciones conformes, demostremos por tanto el siguiente Lema: 
Lema 4.6. Dada una trasformación conforme en una variedad riemanniana, $(M, g) \rightarrow(M, \tilde{g}), \tilde{g}=f(\mathbf{x}) g$, sean $R$ y $\tilde{R}$ los correspondientes tensores de curvatura asociados, entonces se verifica, para cualesquiera campos $X, Y, Z \in \Gamma(T M)$ :

$$
\begin{aligned}
\tilde{R}(X, Y) Z & =R(X, Y) Z-\frac{1}{2}\langle X, Z\rangle \nabla_{Y} F+\frac{1}{2}\langle Y, Z\rangle \nabla_{X} F+ \\
& +\left(\frac{1}{2}\left\langle\nabla_{Y} F, Z\right\rangle-\frac{1}{4}\langle F, Z\rangle\langle F, Y\rangle+\frac{1}{4}\langle Y, Z\rangle\langle F, F\rangle\right) X+ \\
& +\left(-\frac{1}{2}\left\langle\nabla_{X} F, Z\right\rangle+\frac{1}{4}\langle F, Z\rangle\langle F, X\rangle-\frac{1}{4}\langle X, Z\rangle\langle F, F\rangle\right) Y+ \\
& +\left(\frac{1}{2}\left\langle\nabla_{Y} F, X\right\rangle-\frac{1}{2}\left\langle\nabla_{X} F, Y\right\rangle\right) Z+ \\
& +\left(\frac{1}{4}\langle F, Y\rangle\langle X, Z\rangle-\frac{1}{4}\langle F, X\rangle\langle Y, Z\rangle\right) F
\end{aligned}
$$

siendo $\nabla$ la conexión de Levi-Civita asociada a la métrica $g, F=\operatorname{grad}(\operatorname{Ln} f)$ y donde los productos escalares y el gradiente están tomados con respecto a $g$.

\section{Demostración:}

Si recordamos la definición del tensor de curvatura:

$$
\tilde{R}(X, Y) Z=-\tilde{\nabla}_{X}\left(\tilde{\nabla}_{Y} Z\right)+\tilde{\nabla}_{Y}\left(\tilde{\nabla}_{X} Z\right)+\tilde{\nabla}_{[X, Y]} Z
$$

tendremos que aplicar la expresión (4.4) del Lema 4.3 a los términos $-\tilde{\nabla}_{X}\left(\tilde{\nabla}_{Y} Z\right)+$ $\tilde{\nabla}_{Y}\left(\tilde{\nabla}_{X} Z\right)$ para pasar de las derivadas covariantes segundas en función de $\tilde{g}$ a las correspondientes con respecto a $g$.

Para el término $\tilde{\nabla}_{[X, Y]} Z$ (con $[X, Y]=\nabla_{X} Y-\nabla_{Y} X$ por tratarse de la conexión de Levi-Civita y ser ésta libre de torsión ${ }^{4}$ ) aplicaremos (4.3).

Simplificando directamente todas las expresiones resultantes se obtiene el resultado buscado (4.16).

Q.E.D.

Teorema 4.7. Sea c una curva extremal del funcional de acción $S[c]$, y sea $E^{J}[c]$ el correspondiente funcional de energía en la métrica de Jacobi asociada a $S[c]$, entonces los funcionales hessianos correspondientes verifican:

$$
\frac{d^{2} E^{J}[c]}{d \xi^{2}}(0)=\frac{d^{2} S[c]}{d \xi^{2}}(0)+\int_{t_{1}}^{t_{2}} d t 2\left\langle\dot{c}, \frac{\mathrm{D} V}{d t}\right\rangle\langle F, V\rangle
$$

\footnotetext{
${ }^{4}$ Nótese que por aplicación directa de (4.3) se tiene que si $\nabla$ y $\tilde{\nabla}$ son conexiones libres de torsión, entonces:

$$
[X, Y]=\nabla_{X} Y-\nabla_{Y} X=\tilde{\nabla}_{X} Y-\tilde{\nabla}_{Y} X
$$
}


donde $F$ es el campo vectorial: $F=\operatorname{grad} \operatorname{Ln}\left(2\left(i_{1}-U\right)\right)$.

\section{Demostración:}

Partiendo de la expresión del hessiano (4.14) se trata, en primer lugar, de cambiar la métrica de Jacobi $h$ por la propia del sistema, $g$. En segundo lugar, se reparametrizarán las expresiones resultantes (dependientes de la longitud de arco en la métrica de Jacobi) en función del parámetro temporal $t$ del sistema dinámico.

La transformación $(M, g)$ a $(M, h)$ es conforme, como ya hemos visto. Podemos aplicar entonces el Lema 4.3 relativo al comportamiento de las derivadas covariantes bajo transformaciones conformes.

Considerando, por tanto, (4.4) para este caso tendremos:

$$
\begin{aligned}
\nabla_{X}^{J} \nabla_{X}^{J} Y= & \nabla_{X} \nabla_{X} Y+\frac{1}{2}\langle F, Y\rangle \nabla_{X} X+\langle F, X\rangle \nabla_{X} Y-\frac{1}{2}\langle X, Y\rangle \nabla_{X} F+ \\
& +\left(\left\langle F, \nabla_{X} Y\right\rangle+\frac{3}{4}\langle F, Y\rangle\langle F, X\rangle-\frac{1}{4}\langle X, Y\rangle\langle F, F\rangle+\frac{1}{2}\left\langle\nabla_{X} F, Y\right\rangle\right) X+ \\
& +\left(\frac{1}{2}\left\langle\nabla_{X} F, X\right\rangle+\frac{1}{2}\left\langle F, \nabla_{X} X\right\rangle+\frac{1}{4}\langle F, X\rangle^{2}\right) Y+ \\
& +\left(-\frac{1}{2}\left\langle\nabla_{X} X, Y\right\rangle-\left\langle X, \nabla_{X} Y\right\rangle-\frac{1}{4}\langle F, Y\rangle\langle X, X\rangle-\frac{1}{4}\langle F, X\rangle\langle X, Y\rangle\right) F
\end{aligned}
$$

De manera que:

$$
\begin{aligned}
\nabla_{c^{\prime}}^{J} \nabla_{c^{\prime}}^{J} V= & \nabla_{c^{\prime}} \nabla_{c^{\prime}} V+\frac{1}{2}\langle F, V\rangle \nabla_{c^{\prime}} c^{\prime}+\left\langle F, c^{\prime}\right\rangle \nabla_{c^{\prime}} V-\frac{1}{2}\left\langle c^{\prime}, V\right\rangle \nabla_{c^{\prime}} F+ \\
& +\left(\left\langle F, \nabla_{c^{\prime}} V\right\rangle+\frac{3}{4}\langle F, V\rangle\left\langle F, c^{\prime}\right\rangle-\frac{1}{4}\left\langle c^{\prime}, V\right\rangle\langle F, F\rangle+\frac{1}{2}\left\langle\nabla_{c^{\prime}} F, V\right\rangle\right) c^{\prime}+ \\
& +\left(\frac{1}{2}\left\langle\nabla_{c^{\prime}} F, c^{\prime}\right\rangle+\frac{1}{2}\left\langle F, \nabla_{c^{\prime}} c^{\prime}\right\rangle+\frac{1}{4}\left\langle F, c^{\prime}\right\rangle^{2}\right) V+ \\
& +\left(-\frac{1}{2}\left\langle\nabla_{c^{\prime}} c^{\prime}, V\right\rangle-\left\langle c^{\prime}, \nabla_{c^{\prime}} V\right\rangle-\frac{1}{4}\langle F, V\rangle\left\langle c^{\prime}, c^{\prime}\right\rangle-\frac{1}{4}\left\langle F, c^{\prime}\right\rangle\left\langle c^{\prime}, V\right\rangle\right) F
\end{aligned}
$$

En lo que respecta a la curvatura seccional, particularizando el Lema 4.6 al tensor de curvatura seccional se obtiene:

$$
\begin{aligned}
K_{c^{\prime}}^{J}(V)= & K_{c^{\prime}}(V)-\frac{1}{2}\left\langle c^{\prime}, c^{\prime}\right\rangle \nabla_{V} F+\frac{1}{2}\left\langle V, c^{\prime}\right\rangle \nabla_{c^{\prime}} F+ \\
& +\left(\frac{1}{2}\left\langle\nabla_{V} F, c^{\prime}\right\rangle-\frac{1}{4}\left\langle F, c^{\prime}\right\rangle\langle F, V\rangle+\frac{1}{4}\left\langle V, c^{\prime}\right\rangle\langle F, F\rangle\right. \\
& \left.+\frac{1}{2}\left\langle\nabla_{V} F, c^{\prime}\right\rangle-\frac{1}{2}\left\langle\nabla_{c^{\prime}} F, V\right\rangle\right) c^{\prime}+
\end{aligned}
$$




$$
\begin{aligned}
& +\left(\frac{-1}{2}\left\langle\nabla_{c^{\prime}} F, c^{\prime}\right\rangle+\frac{1}{4}\left\langle F, c^{\prime}\right\rangle^{2}-\frac{1}{4}\left\langle c^{\prime}, c^{\prime}\right\rangle\langle F, F\rangle\right) V+ \\
& +\left(\frac{1}{4}\langle F, V\rangle\left\langle c^{\prime}, c^{\prime}\right\rangle-\frac{1}{4}\left\langle F, c^{\prime}\right\rangle\left\langle V, c^{\prime}\right\rangle\right) F
\end{aligned}
$$

Sumando las expresiones anteriores y simplificando:

$$
\begin{aligned}
\nabla_{c^{\prime}}^{J} \nabla_{c^{\prime}}^{J} V+K_{c^{\prime}}^{J}(V)= & \nabla_{c^{\prime}} \nabla_{c^{\prime}} V+K_{c^{\prime}}(V)+\frac{1}{2}\langle F, V\rangle \nabla_{c^{\prime}} c^{\prime}+ \\
& +\left\langle F, c^{\prime}\right\rangle \nabla_{c^{\prime}} V-\frac{1}{2}\left\langle c^{\prime}, c^{\prime}\right\rangle \nabla_{V} F+ \\
& +\left(\left\langle F, \nabla_{c^{\prime}} V\right\rangle+\frac{1}{2}\langle F, V\rangle\left\langle F, c^{\prime}\right\rangle+\left\langle\nabla_{V} F, c^{\prime}\right\rangle\right) c^{\prime}+ \\
& +\left(\frac{1}{2}\left\langle F, \nabla_{c^{\prime}} c^{\prime}\right\rangle+\frac{1}{2}\left\langle F, c^{\prime}\right\rangle^{2}-\frac{1}{4}\left\langle c^{\prime}, c^{\prime}\right\rangle\langle F, F\rangle\right) V+ \\
& \left(\frac{-1}{2}\left\langle\nabla_{c^{\prime}} c^{\prime}, V\right\rangle-\left\langle c^{\prime}, \nabla_{c^{\prime}} V\right\rangle-\frac{1}{2}\left\langle F, c^{\prime}\right\rangle\left\langle V, c^{\prime}\right\rangle\right) F
\end{aligned}
$$

Una vez obtenida (4.18), que depende únicamente de la métrica $g$, es necesario reparametrizar en función del tiempo $t$ del sistema dinámico. Tenemos:

$$
d s=2\left(i_{1}-U\right) d t
$$

de manera que

$$
c^{\prime}=\frac{d c}{d s}=\frac{d c}{d t} \frac{d t}{d s}=\frac{1}{2\left(i_{1}-U\right)} \dot{c}=\frac{1}{f} \dot{c}
$$

donde se ha denotado, por simplicidad: $f=2\left(i_{1}-U\right)$.

Para los demás campos, que no dependen de derivadas con respecto a $s$, la reparametrización se obvia con el habitual abuso de notación:

$$
V=V(s, 0)=\left.\frac{\partial c(s, \xi)}{\partial \xi}\right|_{\xi=0}=V(s(t), 0) \equiv V(t, 0)=V
$$

y análogamente para el campo $F$.

La derivada covariante segunda, tal y como se calcula en el Lema 4.4, se trasforma:

$$
\nabla_{c^{\prime}} \nabla_{c^{\prime}} V=\frac{1}{f^{2}} \nabla_{\dot{c}} \nabla_{\dot{c}} V-\frac{1}{f^{2}}\langle F, \dot{c}\rangle \nabla_{\dot{c}} V
$$

mientras que la curvatura, debido al carácter tensorial, se comporta de la siguiente manera:

$$
K_{c^{\prime}}(V)=R\left(c^{\prime}, V\right) c^{\prime}=R\left(\frac{1}{f} \dot{c}, V\right) \frac{\dot{c}}{f}=\frac{1}{f^{2}} R(\dot{c}, V) \dot{c}=\frac{1}{f^{2}} K_{\dot{c}}(V)
$$


Sustituyendo en (4.18), se obtiene:

$$
\begin{aligned}
\nabla_{c^{\prime}}^{J} \nabla_{c^{\prime}}^{J} V+K_{c^{\prime}}^{J}(V)= & \frac{1}{f^{2}}\left[\nabla_{\dot{c}} \nabla_{\dot{c}} V+K_{\dot{c}}(V)+\frac{1}{2}\langle F, V\rangle \nabla_{\dot{c}} \dot{c}\right. \\
& -\frac{1}{2}\langle\dot{c}, \dot{c}\rangle \nabla_{V} F+\left\langle F, \nabla_{\dot{c}} V\right\rangle \dot{c}+\left\langle\nabla_{V} F, \dot{c}\right\rangle \dot{c} \\
& +\frac{1}{2}\left\langle F, \nabla_{\dot{c}} \dot{c}\right\rangle V-\frac{1}{4}\langle\dot{c}, \dot{c}\rangle\langle F, F\rangle V \\
& \left.-\frac{1}{2}\left\langle\nabla_{\dot{c}} \dot{c}, V\right\rangle F-\left\langle\dot{c}, \nabla_{\dot{c}} V\right\rangle F\right]
\end{aligned}
$$

Ahora bien, $F=\operatorname{grad} \operatorname{Ln}\left(2\left(i_{1}-U\right)\right)$, de manera que podemos calcular, $\forall X \in$ $\Gamma(T M)$ :

$$
\nabla_{X} F=\nabla_{X}\left(\frac{-\operatorname{grad} U}{i_{1}-U}\right)=\frac{-1}{i_{1}-U} \nabla_{X} \operatorname{grad} U-\frac{1}{\left(i_{1}-U\right)^{2}}\langle\operatorname{grad} U, X\rangle \operatorname{grad} U
$$

La conservación de la energía mecánica sobre las trayectorias a las que nos estamos restringiendo se traduce en que:

$$
\frac{1}{2}\langle\dot{c}, \dot{c}\rangle+U=i_{1}
$$

y finalmente, las curvas $c$ son soluciones del sistema, luego:

$$
\nabla_{\dot{c}} \dot{c}=-\operatorname{grad} U
$$

Teniendo en consideración todos estos resultados:

$$
\begin{aligned}
\nabla_{c^{\prime}}^{J} \nabla_{c^{\prime}}^{J} V+K_{c^{\prime}}^{J}(V)= & \frac{1}{f^{2}}\left[\nabla_{\dot{c}} \nabla_{\dot{c}} V+K_{\dot{c}}(V)+\nabla_{V} \operatorname{grad} U\right]+ \\
& +\frac{2}{f^{3}}\left[\langle\operatorname{grad} U, V\rangle \operatorname{grad} U-\left\langle\operatorname{grad} U, \nabla_{\dot{c}} V\right\rangle \dot{c}-\left\langle\nabla_{V} \operatorname{grad} U, \dot{c}\right\rangle \dot{c}\right. \\
& \left.-\frac{1}{\left(i_{1}-U\right)}\langle\operatorname{grad} U, V\rangle\langle\operatorname{grad} U, \dot{c}\rangle \dot{c}+\left\langle\dot{c}, \nabla_{\dot{c}} V\right\rangle \operatorname{grad} U\right]
\end{aligned}
$$

De esta manera, la forma cuadrática hessiano se escribirá:

$$
\begin{aligned}
& \left\langle\nabla_{c^{\prime}}^{J} \nabla_{c^{\prime}}^{J} V+K_{c^{\prime}}^{J}(V), V\right\rangle^{J}= \\
& =\frac{1}{2\left(i_{1}-U\right)}\left\langle\nabla_{\dot{c}} \nabla_{\dot{c}} V+K_{\dot{c}}(V)+\nabla_{V} \operatorname{grad} U, V\right\rangle+ \\
& +\frac{1}{2\left(i_{1}-U\right)}\left[\frac{1}{i_{1}-U}\langle\operatorname{grad} U, V\rangle^{2}-\frac{1}{i_{1}-U}\left\langle\operatorname{grad} U, \nabla_{\dot{c}} V\right\rangle\langle\dot{c}, V\rangle\right. \\
& -\frac{1}{i_{1}-U}\left\langle\nabla_{V} \operatorname{grad} U, \dot{c}\right\rangle\langle\dot{c}, V\rangle-\frac{1}{\left(i_{1}-U\right)^{2}}\langle\operatorname{grad} U, V\rangle\langle\operatorname{grad} U, \dot{c}\rangle\langle\dot{c}, V\rangle+ \\
& \left.+\frac{1}{i_{1}-U}\left\langle\dot{c}, \nabla_{\dot{c}} V\right\rangle\langle\operatorname{grad} U, V\rangle\right]
\end{aligned}
$$


La expresión (4.19) se puede simplificar notablemente si se utiliza la Proposición C.2 del Apéndice $\mathrm{C}:\left\langle\nabla_{V} \operatorname{grad} U, \dot{c}\right\rangle=\left\langle\nabla_{\dot{c} \operatorname{grad} U, V\rangle}\right.$, y se calcula la derivada:

$$
\frac{\partial}{\partial t}(\langle F, V\rangle\langle\dot{c}, V\rangle)
$$

Utilizando a su vez las ecuaciones de Newton, $\nabla_{\dot{c}} \dot{c}=-\operatorname{grad} U$, se concluye que:

$$
\begin{aligned}
& \left\langle\nabla_{c^{\prime}}^{J} \nabla_{c^{\prime}}^{J} V+K_{c^{\prime}}^{J}(V), V\right\rangle^{J}= \\
& =\frac{1}{2\left(i_{1}-U\right)}\left\langle\nabla_{\dot{c}} \nabla_{\dot{c}} V+K_{\dot{c}}(V)+\nabla_{V} \operatorname{grad} U, V\right\rangle+ \\
& +\frac{1}{2\left(i_{1}-U\right)} \frac{\partial}{\partial t}(\langle F, V\rangle\langle\dot{c}, V\rangle)+\frac{1}{\left(i_{1}-U\right)^{2}}\left\langle\dot{c}, \nabla_{\dot{c}} V\right\rangle\langle\operatorname{grad} U, U\rangle
\end{aligned}
$$

Finalmente, el funcional hessiano, utilizando que $d s=2\left(i_{1}-U\right) d t$ sobre las trayectorias solución, se escribe:

$$
\begin{aligned}
\frac{d^{2} E^{J}[c]}{d \xi^{2}}(0)= & -\int_{s_{1}}^{s_{2}} d s\left\langle\nabla_{c^{\prime}}^{J} \nabla_{c^{\prime}}^{J} V+K_{c^{\prime}}^{J}(V), V\right\rangle^{J}= \\
& -\int_{t_{1}}^{t_{2}} d t\left\langle\nabla_{\dot{c}} \nabla_{\dot{c}} V+K_{\dot{c}}(V)+\nabla_{V} \operatorname{grad} U, V\right\rangle+ \\
& +\int_{t_{1}}^{t_{2}} d t 2\left\langle\dot{c}, \nabla_{\dot{c}} V\right\rangle\langle F, V\rangle-\left.\langle F, V\rangle\langle\dot{c}, V\rangle\right|_{t_{1}} ^{t_{2}}
\end{aligned}
$$

que, para variaciones propias $\left(V\left(t_{1}\right)=V\left(t_{2}\right)=0\right)$, se reduce al resultado buscado:

$$
\frac{d^{2} E^{J}[c]}{d \xi^{2}}(0)=\frac{d^{2} S[c]}{d \xi^{2}}(0)+\int_{t_{1}}^{t_{2}} d t 2\left\langle\dot{c}, \nabla_{\dot{c}} V\right\rangle\langle F, V\rangle
$$

con $F=\operatorname{grad} \operatorname{Ln}\left(2\left(i_{1}-U\right)\right)$.

Q.E.D.

Si utilizamos un sistema de coordenadas locales en $(M, g)$ de manera que $c(t)=$ $\left(x^{1}(t), \ldots, x^{N}(t)\right)$, el teorema se expresa de la siguiente forma:

$\frac{d^{2} E^{J}[c]}{d \xi^{2}}(0)=\frac{d^{2} S[c]}{d \xi^{2}}(0)+\int_{t_{1}}^{t_{2}}\left[\frac{-2}{i_{1}-U} g_{i r} \dot{x}^{i} \frac{\partial U}{\partial x^{k}} V^{k} \frac{d V^{r}}{d t}-\frac{1}{i_{1}-U} \dot{x}^{i} \dot{x}^{j} \frac{\partial g_{i j}}{\partial x^{r}} \frac{\partial U}{\partial x^{k}} V^{k} V^{r}\right]$

Es interesante asimismo calcular la expresión recíproca de la (4.17) que acabamos de demostrar. Cambiando la parametrización y la métrica de nuevo y, simplificando los resultados adecuadamente, no es difícil demostrar que se verifica:

$$
\frac{d^{2} S[c]}{d \xi^{2}}(0)=\frac{d^{2} E^{J}[c]}{d \xi^{2}}(0)-\int_{s_{1}}^{s_{2}} d s\left[2\left\langle c^{\prime}, \nabla_{c^{\prime}}^{J} V\right\rangle^{J}\left\langle F^{J}, V\right\rangle^{J}-\left(\left\langle F^{J}, V\right\rangle^{J}\right)^{2}\right]
$$


donde $F^{J}=\operatorname{grad}^{J} \operatorname{Ln}\left(2\left(i_{1}-U\right)\right)$, es decir el gradiente con respecto a la métrica de Jacobi del logaritmo neperiano del factor métrico $2\left(i_{1}-U\right)$.

Para el funcional de longitud, el hessiano (4.15) conduce al siguiente teorema:

Teorema 4.8. Sea $c$ una curva extremal del funcional de acción $S[c]$ y sea $L^{J}[c]$ el correspondiente funcional de longitud en la métrica de Jacobi asociada a $S[c]$, entonces los funcionales hessianos correspondientes verifican:

$$
\frac{d^{2} L^{J}[c]}{d \xi^{2}}(0)=\frac{d^{2} S[c]}{d \xi^{2}}(0)-\int_{t_{1}}^{t_{2}} d t \frac{1}{2\left(i_{1}-U\right)}\left[\left\langle\nabla_{\dot{c}} \dot{c}, V\right\rangle-\left\langle\dot{c}, \nabla_{\dot{c}} V\right\rangle\right]^{2}
$$

\section{Demostración:}

En el Apéndice D está detallado el cálculo del funcional hessiano de la Longitud, tenemos así:

$$
\frac{d^{2} L^{J}[c]}{d \xi^{2}}(0)=-\int_{s_{1}}^{s_{2}} d s\left\langle\nabla_{c^{\prime}}^{J} \nabla_{c^{\prime}}^{J} V^{\perp}+K_{c^{\prime}}^{J}\left(V^{\perp}\right), V^{\perp}\right\rangle^{J}
$$

donde:

$$
V^{\perp}=V-\left\langle\frac{c^{\prime}}{\left\|c^{\prime}\right\|^{J}}, V\right\rangle^{J} \frac{c^{\prime}}{\left\|c^{\prime}\right\|^{J}}=V-\left\langle c^{\prime}, V\right\rangle c^{\prime}
$$

o, alternativamente:

$$
\begin{aligned}
\frac{d^{2} L^{J}[c]}{d \xi^{2}}(0) & =-\int_{s_{1}}^{s_{2}} d s\left\langle\nabla_{c^{\prime}}^{J} \nabla_{c^{\prime}}^{J} V+K_{c^{\prime}}^{J}(V), V\right\rangle^{J}-\int_{s_{1}}^{s_{2}} d s\left(\left\langle c^{\prime}, \nabla_{c^{\prime}}^{J} V\right\rangle^{J}\right)^{2} \\
& =\frac{d^{2} E^{J}[c]}{d \xi^{2}}(0)-\int_{s_{1}}^{s_{2}} d s\left(\left\langle c^{\prime}, \nabla_{c^{\prime}}^{J} V\right\rangle^{J}\right)^{2}
\end{aligned}
$$

Podemos por tanto utilizar el Teorema 4.7 anterior para el funcional hessiano de la energía y computar su diferencia con el de la Longitud en términos de la métrica $g$ y la parametrización en $t$.

$$
\begin{aligned}
\frac{d^{2} L^{J}[c]}{d \xi^{2}}(0) & =\frac{d^{2} E^{J}[c]}{d \xi^{2}}(0)-\int_{s_{1}}^{s_{2}} d s\left(\left\langle c^{\prime}, \nabla_{c^{\prime}}^{J} V\right\rangle^{J}\right)^{2}= \\
& =\frac{d^{2} S[c]}{d \xi^{2}}(0)+\int_{t_{1}}^{t_{2}} 2\left\langle\dot{c}, \nabla_{\dot{c}} V\right\rangle\langle F, V\rangle+\int_{t_{1}}^{t_{2}} A(t) d t
\end{aligned}
$$

donde, si denominamos $f=2\left(i_{1}-U\right), F=\operatorname{grad} \operatorname{Ln} f$, se tiene:

$$
\int_{t_{1}}^{t_{2}} A(t) d t=-\int_{s_{1}}^{s_{2}} d s\left(\left\langle c^{\prime}, \nabla_{c^{\prime}}^{J} V\right\rangle^{J}\right)^{2}=-\int_{t_{1}}^{t_{2}} d t f^{3}\left\langle c^{\prime}, \nabla_{c^{\prime}}^{J} V\right\rangle^{2}
$$


Aplicando el Lema 4.3, las ecuaciones de Newton, $\nabla_{\dot{c}} \dot{c}=-\operatorname{grad} U=\frac{f}{2} F, \mathrm{y}$ simplificando, la expresión anterior será:

$$
\int_{t_{1}}^{t_{2}} A(t) d t=-\int_{t_{1}}^{t_{2}} d t \frac{1}{f}\left(\left\langle\dot{c}, \nabla_{\dot{c}} V\right\rangle+\frac{f}{2}\langle F, V\rangle\right)^{2}
$$

y, finalmente:

$$
\frac{d^{2} L^{J}[c]}{d \xi^{2}}(0)=\frac{d^{2} S[c]}{d \xi^{2}}-\int_{t_{1}}^{t_{2}} d t \frac{1}{f}\left[\left\langle\dot{c}, \nabla_{\dot{c}} V\right\rangle-\left\langle\nabla_{\dot{c}} \dot{c}, V\right\rangle\right]^{2}
$$

Q.E.D.

De manera análoga al teorema anterior, podemos calcular la expresión recíproca de (4.22), obteniéndose:

$$
\frac{d^{2} S[c]}{d \xi^{2}}(0)=\frac{d^{2} L^{J}[c]}{d \xi^{2}}(0)+\int_{s_{1}}^{s_{2}} d s\left[\left\langle F^{J}, V\right\rangle^{J}-\left\langle c^{\prime}, \nabla_{c^{\prime}}^{J} V\right\rangle\right]^{2}
$$

con $F^{J}=\operatorname{grad}^{J} \operatorname{Ln}\left(2\left(i_{1}-U\right)\right)$.

Si se restringe el funcional hessiano de la acción del sistema dinámico al caso de variaciones propias ortogonales a las soluciones, es decir tales que: $\langle\dot{c}, V\rangle=0$ idénticamente, los teoremas anteriores se simplifican notablemente.

Dado que la métrica de Jacobi se define por medio de una transformación conforme con respecto a la métrica "dinámica" $g$, la ortogonalidad de las variaciones se mantiene en una con respecto a la otra; sin más que sustituir los resultados conocidos se tiene:

$$
V^{\perp}=V-\left\langle c^{\prime}, V\right\rangle^{J} c^{\prime}=V-\frac{1}{2\left(i_{1}-U\right)}\langle\dot{c}, V\rangle \dot{c}=V-\left\langle\frac{\dot{c}}{\|\dot{c}\|}, V\right\rangle \frac{\dot{c}}{\|\dot{c}\|}
$$

Si $V=V^{\perp}$, entonces evidentemente $\left\langle\dot{c}, V^{\perp}\right\rangle=0$ a lo largo de toda la trayectoria $c$ y, por tanto, $\frac{\partial}{\partial t}\left\langle\dot{c}, V^{\perp}\right\rangle=0$ que desarrollando nos conduce a:

$$
\left\langle\dot{c}, V^{\perp}\right\rangle=-\left\langle\nabla_{\dot{c}} \dot{c}, V^{\perp}\right\rangle
$$

Mientras que el mismo razonamiento sobre la geodésica $c(s)$ de la métrica de Jacobi nos proporciona:

$$
\left\langle\nabla_{c^{\prime}}^{J} V^{\perp}, c^{\prime}\right\rangle^{J}=-\left\langle\nabla_{c^{\prime}}^{J} c^{\prime}, V^{\perp}\right\rangle^{J}=0
$$

por ser $c(s)$ geodésica $\left(\nabla_{c^{\prime}}^{J} c^{\prime}=0\right)$. 
Con estas consideraciones es fácil obtener los resultados:

$$
\frac{d^{2} L^{J}[c]}{d \xi^{2}}(0)=\left.\frac{d^{2} S[c]}{d \xi^{2}}\right|_{V=V^{\perp}}-\int_{t_{1}}^{t_{2}} d t 2\left(i_{1}-U\right)\left\langle F, V^{\perp}\right\rangle^{2}
$$

o bien:

$$
\left.\frac{d^{2} S[c]}{d \xi^{2}}\right|_{V=V^{\perp}}=\frac{d^{2} L^{J}[c]}{d \xi^{2}}(0)+\int_{s_{1}}^{s_{2}} d s\left(\left\langle F^{J}, V^{\perp}\right\rangle^{J}\right)^{2}
$$

donde de nuevo $F^{J}=\operatorname{grad}^{J} \operatorname{Ln}\left(2\left(i_{1}-U\right)\right)$, es decir el gradiente calculado con respecto a la métrica de Jacobi $h$.

\subsubsection{Operador de Desviación Geodésica y Diagonalización de la Curvatura seccional}

Una vez establecidos los teoremas anteriores, el estudio de la estabilidad de las curvas solución pasa por resolver el problema espectral que nos proporciona cada uno de los funcionales hessianos que hemos calculado. De una manera general, podemos simplificar dicho problema si utilizamos una base que se traslade paralelamente a lo largo de dichas curvas.

Cualquiera de los hessianos calculados contiene el llamado operador de desviación geodésica:

$$
\nabla_{c^{\prime}} \nabla_{c^{\prime}}+R\left(c^{\prime}, \cdot\right) c^{\prime}=\nabla_{c^{\prime}} \nabla_{c^{\prime}}+K_{c^{\prime}}(\cdot)
$$

donde utilizamos una notación general que englobe todos los casos tratados, es decir $\nabla$ es la conexión de Levi-Civita y $K$ la curvatura seccional relativas a una métrica riemanniana $h$ genérica con respecto a la cual $c(s)$ es una curva geodésica de $(M, h)$ parametrizada en función del arco de longitud.

El núcleo del operador (4.24) está formado por los llamados Campos de Jacobi de la variedad, que corresponden a variaciones propias de la curva $c(s)$ tales que transforman geodésicas en geodésicas, como es bien conocido.

La idea crucial, que permitirá simplificar el análisis de los operadores hessianos, es considerar una base de los campos tangentes sobre la variedad que se traslade paralelamente a lo largo de la curva geodésica. Si $B_{0}=\left\{V_{1}\left(s_{0}\right), \ldots, V_{n}\left(s_{0}\right)\right\}$ es una base ortogonal del espacio tangente $T_{c\left(s_{0}\right)} M$, entonces, evidentemente, su extensión a los campos vectoriales paralelos sobre $c(s)$ (ver C.7, Apéndice C) $B=\left\{V_{1}(s), \ldots, V_{n}(s)\right\}$ constituye una base ortogonal de cada $T_{c(s)} M$ y, obviamente:

$$
\nabla_{c^{\prime}} V_{i}=\frac{\mathrm{D} V_{i}}{d s}=0, \quad \forall i=1, \ldots, n
$$


De esta manera todo campo $V$ sobre $c(s)$ puede escribirse en la base $B$ de la forma:

$$
V=\sum_{i=1}^{n} v^{i} V_{i}=v^{i} V_{i}
$$

utilizando el convenio de Einstein de suma de índices repetidos. Y así:

$$
\nabla_{c^{\prime}} V=\frac{d v^{i}}{d s} V_{i} ; \quad \nabla_{c^{\prime}} \nabla_{c^{\prime}} V=\frac{d^{2} v^{i}}{d s^{2}} V_{i}
$$

Por otro lado, escribiremos la curvatura seccional de un campo $V$ en términos de la base $B$ :

$$
K_{c^{\prime}}\left(V_{i}\right)=\rho_{i}^{j} V_{j}, \forall i \Rightarrow K_{c^{\prime}}(V)=v^{i} \rho_{i}^{j} V_{j}
$$

De esta manera, el operador (4.24) en la base paralela $B$ se reduce a:

$$
\nabla_{c^{\prime}} \nabla_{c^{\prime}} V+K_{c^{\prime}}(V)=\left(\frac{d^{2} v^{j}}{d s^{2}}+v^{i} \rho_{i}^{j}\right) V_{j}
$$

La Ecuación de Desviación Geodésica o Ecuación de Jacobi se descompone en $n$ ecuaciones ordinarias de segundo orden acopladas:

$$
\frac{d^{2} v^{j}}{d s^{2}}+v^{i} \rho_{i}^{j}=0, \quad \forall j=1, \ldots, n
$$

mientras que ocurre otro tanto con la ecuación espectral de (4.24):

$$
\nabla_{c^{\prime}} \nabla_{c^{\prime}} V+K_{c^{\prime}}(V)=\lambda V \Rightarrow \frac{d^{2} v^{j}}{d s^{2}}+v^{i} \rho_{i}^{j}=\lambda v^{j}, \quad \forall j=1, \ldots, n
$$

En el texto "Morse Theory" de J. Milnor [70], se considera la ecuación (4.25) para el caso en el que la variedad $M$ es un espacio riemanniano localmente simétrico (ver [50] para detalles). Utilizando el Teorema que establece que la condición necesaria y suficiente para que una variedad riemanniana sea un espacio localmente simétrico es que la curvatura seccional sea invariante bajo todas las traslaciones paralelas [50], Milnor propone la diagonalización de la curvatura seccional en un punto de la curva $c\left(s_{0}\right)$, es decir, construir $B_{0}$ tal que:

$$
K_{c^{\prime}}\left(V_{i}\left(s_{0}\right)\right)=\varepsilon_{i} V_{i}, \quad \forall i=1, \ldots, n
$$

donde, evidentemente, no hay sumación en índices repetidos. El teorema citado garantiza entonces que la base $B$, traslado paralelo sobre $c(s)$ de la base $B_{0}$ de $T_{c\left(s_{0}\right)} M$, sigue siendo una base de autovectores de $K_{c^{\prime}}$ para cada punto de la curva 
y con los mismos autovalores. Como consecuencia inmediata, las ecuaciones (4.25) se escriben:

$$
\frac{d^{2} v^{j}}{d s^{2}}+\varepsilon_{j} v^{j}=0, \forall j=1, \ldots, n
$$

es decir, un sistema de ecuaciones ordinarias no acopladas de segundo orden lineales y con coeficientes constantes (resaltemos una vez más que en (4.27) no se utiliza el convenio de suma). Es fácil concluir entonces que las ecuaciones del problema espectral, para el caso en el que $M$ es localmente simétrico, son del mismo tipo:

$$
\frac{d^{2} v^{j}}{d s^{2}}+\varepsilon_{j} v^{j}=\lambda v^{j}, \forall j=1, \ldots, n
$$

Desgraciadamente, como veremos en el próximo capítulo, la condición de espacio localmente simétrico es muy restrictiva y las variedades riemannianas $(M, h)$, siendo $h$ la métrica de Jacobi asociada a un problema dinámico, no lo verifican salvo en casos muy sencillos.

En cualquier caso, demostraremos que bajo la hipótesis más débil de que la curvatura seccional mantenga sus autovectores por traslado paralelo, aunque cambien los autovalores, las ecuaciones (4.26) podrán escribirse como un sistema de ecuaciones ordinarias no acopladas de segundo orden lineales con coeficientes variables.

Efectivamente, si partimos de:

$$
K_{c^{\prime}}\left(V_{i}\left(s_{0}\right)\right)=\varepsilon_{i} V_{i}, \quad \forall i=1, \ldots, n
$$

y trasladamos paralelamente la base de los autovectores $V_{i}\left(s_{0}\right)$ a lo largo de $c$ de tal manera que los campos $V_{i}(s)$ forman una base de autovectores de $K_{c^{\prime}}$ en el punto $c(s)$ tendremos:

$$
K_{c^{\prime}}\left(V_{i}(s)\right)=\varepsilon_{i}(s) V_{i}(s), \quad \forall i=1, \ldots, n
$$

y así las ecuaciones (4.26) serán, en esta base:

$$
\frac{d^{2} v^{j}(s)}{d s^{2}}+\varepsilon_{j}(s) v^{j}(s)=\lambda v^{j}(s), \forall j=1, \ldots, n
$$

Comprobaremos en el próximo capítulo que éste es precisamente el caso de la variedad $(M, h)$ asociada al sistema dinámico derivado del Modelo Sigma Lineal $O(N)$ Deformado.

\subsection{Sistemas Pre-Supersimétricos}

Finalizaremos este capítulo con una sección dedicada al estudio de los sistemas dinámicos asociados a la búsqueda de soluciones, de tipo onda solitaria, en una 
Teoría de Campos que admita una extensión supersimétrica (en principio, $\mathrm{N}=1$ supersimétrica). No entraremos en el análisis detallado de la teoría supersimétrica propiamente dicha, para la que nos remitiremos, entre las muchas posibles, a las referencias [3], [37] y [107]. Nos centraremos, por tanto, en las consecuencias que la supersimetría de la teoría tiene sobre el sistema mecánico asociado. Las teorías de campos que estamos estudiando constituyen, en este marco, la parte bosónica del modelo supersimétrico en consideración. Desde este punto de vista, llamaremos Sistema Dinámico Pre-Supersimétrico a áquel para el cual la energía potencial $U$ se puede escribir de la forma:

$$
U=-\frac{1}{2}\langle\operatorname{grad} W, \operatorname{grad} W\rangle
$$

para alguna función $W: M \rightarrow \mathbb{R}$.

Evidentemente, con respecto a la teoría de campos original, el potencial $V=$ $-U$ se escribirá:

$$
V=\frac{1}{2}\langle\operatorname{grad} W, \operatorname{grad} W\rangle
$$

y $W$ no será más que el superpotencial de la extensión supersimétrica.

En primer lugar analizaremos los sistemas dinámicos de este tipo, veremos cómo la expresión (4.30) permite calcular un sistema de ecuaciones de primer orden cuyas soluciones satisfacen el sistema de ecuaciones de segundo orden de Newton. En la segunda parte de la sección, detallaremos el caso de $\mathrm{N}=2$ supersimetría para sistemas dinámicos definidos en $\mathbb{R}^{2} \cong \mathbb{C}$, obtendremos un método de resolución directo de las ecuaciones de primer orden, y caracterizaremos, además, todos los sistemas que admiten extensiones supersimétricas de este tipo por medio de la curvatura de la métrica de Jacobi asociada a los mismos.

Como hemos comentado, si tenemos un sistema pre-supersimétrico, la energía potencial no es más que $-\frac{1}{2}\|\operatorname{grad} W\|^{2}$. Esto permite escribir la acción $S[c]$ del sistema (energía de las configuraciones estáticas de la teoría de campos original) en la llamada forma de Bogomol'nyi [13]:

$$
\begin{aligned}
S[c] & =\int_{t_{1}}^{t_{2}} d t \frac{1}{2}\langle\dot{c}+\epsilon \operatorname{grad}(W), \dot{c}+\epsilon \operatorname{grad}(W)\rangle-\epsilon \int_{t_{1}}^{t_{2}} d t\langle\dot{c}, \operatorname{grad}(W)\rangle= \\
& =\int_{t_{1}}^{t_{2}} d t \frac{1}{2}\|\dot{c}+\epsilon \operatorname{grad}(W)\|^{2}-\epsilon\left(W\left(c\left(t_{2}\right)\right)-W\left(c\left(t_{1}\right)\right)\right)
\end{aligned}
$$

donde $\epsilon= \pm 1$, y se ha supuesto, en principio, que la función $W: M \rightarrow \mathbb{R}$ es diferenciable en cualquier punto de la variedad riemanniana $M$. Veremos en el siguiente capítulo cómo esta suposición no siempre es adecuada y de qué manera la 
no diferenciabilidad de $W$ en determinados puntos puede alterar la descomposición de Bogomol'nyi (4.31) para un sistema concreto.

El sistema de ecuaciones de primer orden al que hemos aludido anteriormente no es otro que:

$$
\|\dot{c}+\epsilon \operatorname{grad}(W)\|=0 \Rightarrow \dot{c}+\epsilon \operatorname{grad}(W)=0
$$

cuyas soluciones verifican, evidentemente:

$$
S[c]=-\epsilon\left(W\left(c\left(t_{2}\right)\right)-W\left(c\left(t_{1}\right)\right)\right)
$$

es decir, la acción está determinada únicamente por los valores del superpotencial en los puntos inicial y final de la curva solución. Es obvio comprobar también que dichas soluciones satisfacen la condición de energía mecánica nula, tal y como imponían las condiciones asintóticas vistas en el Capítulo 2 de esta memoria, condiciones necesarias para que las soluciones del sistema dinámico se correspondieran con soluciones de tipo onda solitaria de la teoría de campos.

$$
\begin{aligned}
i_{1} & =\frac{1}{2}\langle\dot{c}, \dot{c}\rangle+U=\frac{1}{2}\langle\dot{c}, \dot{c}\rangle-\frac{1}{2}\langle\operatorname{grad}(W), \operatorname{grad}(W)\rangle \\
& =\frac{1}{2}\left((-\epsilon)^{2}-1\right)\langle\operatorname{grad}(W), \operatorname{grad}(W)\rangle \equiv 0
\end{aligned}
$$

Demostraremos a continuación que, además, las ecuaciones (4.32) satisfacen las ecuaciones de Newton del sistema. El enunciado recíproco no es cierto de manera general. Tal y como veremos en la sección siguiente, y en el Capítulo 6, un mismo sistema pre-supersimétrico puede admitir más de un superpotencial; de esta manera, diferentes sistemas de ecuaciones de primer orden conducen a soluciones distintas del modelo, todas ellas correspondientes a las mismas ecuaciones de segundo orden.

Calculemos en primer lugar las ecuaciones de Newton para un sistema presupersimétrico.

Proposición 4.9. Las ecuaciones de Newton de un sistema mecánico pre-supersimétrico se escriben de la forma:

$$
\frac{\mathrm{D} \dot{c}}{\partial t}-\nabla_{\operatorname{grad}(W)} \operatorname{grad}(W)=0
$$

\section{Demostración:}

Es posible demostrar el resultado (4.33) utilizando la demostración de la Proposición 4.1 acerca de las propias ecuaciones de Newton generales asociadas a un 
sistema dinámico en una variedad riemanniana dada. Por sustitución directa de la expresión (4.30) en la derivada variacional tendremos:

$$
\frac{\partial}{\partial \xi}\left(-\frac{1}{2}\langle\operatorname{grad} W, \operatorname{grad} W\rangle\right)=-\left\langle\frac{\mathrm{D}}{\partial \xi} \operatorname{grad} W, \operatorname{grad} W\right\rangle=-\left\langle\nabla_{\operatorname{grad} W} \operatorname{grad} W, c^{\prime}\right\rangle
$$

donde se ha usado la Proposición C.2 del Apéndice C. De esta manera, para variaciones propias, la primera variación de la acción se escribirá en la forma:

$$
\frac{d S}{d \xi}(0)=-\int_{t_{1}}^{t_{2}} d t\left\langle\frac{\mathrm{D}}{\partial t} \dot{c}-\nabla_{\operatorname{grad} W} \operatorname{grad} W, V\right\rangle
$$

que, aplicando el Teorema Fundamental del Cálculo Variacional al principio de acción extremal, conduce a las ecuaciones (4.33).

Q.E.D.

Utilizando un sistema de coordenadas locales en $M$, la ecuación (4.33) se convierte en el sistema de ecuaciones:

$$
\ddot{x}^{k}+\Gamma_{i l}^{k} \dot{x}^{i} \dot{x}^{l}-g^{k l}(\operatorname{grad}(W))^{i}[H(W)]_{i l}=0, \quad k=1, \ldots, N
$$

donde:

$$
[H(W)]_{i l}=\frac{\partial^{2} W}{\partial x^{i} \partial x^{l}}-\Gamma_{i l}^{r} \frac{\partial W}{\partial x^{r}}
$$

es el hessiano de $W$.

Proposición 4.10. En un sistema dinámico pre-supersimétrico las soluciones de las ecuaciones de primer orden (4.32) son soluciones de las ecuaciones de Newton (4.33).

\section{Demostración:}

La demostración es evidente por cálculo directo, si $c(t)$ es una solución de las ecuaciones (4.32):

$$
\dot{c}=-\epsilon \operatorname{grad}(W)
$$

entonces las ecuaciones de Newton (4.33) se escribirán de la forma:

$$
\begin{aligned}
& \nabla_{-\epsilon \operatorname{grad}(W)}(-\epsilon \operatorname{grad}(W))-\nabla_{\operatorname{grad}(W)} \operatorname{grad}(W)= \\
& =\nabla_{\operatorname{grad}(W)} \operatorname{grad}(W)-\nabla_{\operatorname{grad}(W)} \operatorname{grad}(W) \equiv 0
\end{aligned}
$$

Q.E.D. 


\subsection{1 $\mathrm{N}=2$ Pre-Supersimetría}

Una teoría de campos es $\mathrm{N}=2$ pre-supersimétrica si admite dos supercargas. No entraremos, una vez más, en los detalles de la teoría supersimétrica propiamente dicha (ver por ejemplo: [20], [94],[6], [3]), sino que nos centraremos en las repercusiones que la $\mathrm{N}=2$ pre-supersimetría tiene sobre el sistema mecánico asociado a la búsqueda de kinks en dichas teorías.

En particular, analizaremos en detalle los llamados modelos de Wess-Zumino (en 3+1 dimensiones), [108], que, por reducción dimensional, producen en $1+1$ dimensiones teorías de campos $\mathrm{N}=2$ supersimétricas, siempre y cuando el superpotencial de la teoría sea una función armónica de los campos (ver [94] y [3]).

Recientemente estos modelos han cobrado un renovado interés en el estudio de las intersecciones entre defectos de tipo pared o domain walls (ver Capítulo 1), dando lugar a un creciente número de publicaciones como [44], [92], [6] y las referencias allí recogidas.

Restringiéndonos inicialmente, como hemos comentado, al caso de dos campos escalares, podemos establecer como objeto de estudio un sistema dinámico bidimensional:

$$
S[c]=\int d t\left(\frac{1}{2}\langle\dot{c}, \dot{c}\rangle-U(c(t))\right)
$$

donde $U=-V$, y $V$ es el potencial de la teoría de campos $\mathrm{N}=2$ supersimétrica. Por tanto:

$$
V=\frac{1}{2}\|\operatorname{grad}(W)\|^{2}
$$

con $W$ una función armónica. Si, por simplicidad, partimos de una métrica euclídea en el espacio de configuración $\mathbb{R}^{2}$ y consideramos $\mathbb{R}^{2} \cong \mathbb{C}^{1}$ podremos escribir:

$$
z=q_{1}+i q_{2} ; \quad W\left(z, z^{*}\right)=\frac{1}{2}\left(W_{1}+i W_{2}\right)
$$

de manera que (4.35) se reduce a:

$$
-U=V=\frac{1}{2}\left[\left(\frac{\partial W}{\partial q_{1}}\right)^{2}+\left(\frac{\partial W}{\partial q_{2}}\right)^{2}\right]
$$

mientras que en términos de la variable compleja $z$ :

$$
-U=V=2 \frac{\partial W}{\partial z} \frac{\partial W}{\partial z^{*}}
$$

La ecuación de Laplace se escribe ahora de la forma:

$$
\frac{\partial}{\partial z} \frac{\partial W}{\partial z^{*}}=0
$$


Por otro lado, el potencial $U=-V$ es necesariamente real, de manera que el superpotencial $W$ ha de verificar, además de (4.37), la condición:

$$
\frac{\partial W_{1}}{\partial q_{1}} \frac{\partial W_{2}}{\partial q_{1}}+\frac{\partial W_{1}}{\partial q_{2}} \frac{\partial W_{2}}{\partial q_{2}}=0
$$

como se deduce de manera inmediata de (4.36).

Las soluciones de (4.37) son de la forma: $W\left(z, z^{*}\right)=f(z)+g\left(z^{*}\right)$. Si se impone a una solución de este tipo la condición de que $U$ sea real, tendremos que necesariamente $f^{\prime}(z)^{*}=g^{\prime}\left(z^{*}\right)$, condición que satisface automáticamente $f \equiv g, \mathrm{y}$, en consecuencia, consideraremos: $W\left(z, z^{*}\right)=f(z)+f\left(z^{*}\right)$. Esta solución verifica obviamente (4.38).

Podemos escribir entonces (4.36), para este tipo de soluciones, como:

$$
-U=V=2\left(\frac{\partial W}{\partial z}\right)\left(\frac{\partial W}{\partial z}\right)^{*}
$$

y, en definitiva, hechas todas estas consideraciones, podemos definir el superpotencial simplemente como una función holomorfa $W(z)$, que, utilizando (4.39), produce un potencial real y que satisface obviamente la ecuación de Laplace (4.37). Éste es precisamente el punto de partida habitual en la literatura relativa a estos modelos.

Con este criterio, es evidente que las funciones $W_{1}$ y $W_{2}$ satisfarán las ecuaciones de Cauchy-Riemann:

$$
\frac{\partial W_{1}}{\partial q_{1}}=\frac{\partial W_{2}}{\partial q_{2}} ; \quad \frac{\partial W_{1}}{\partial q_{2}}=-\frac{\partial W_{2}}{\partial q_{1}}
$$

de manera que es fácil comprobar que:

$-U=2\left(\frac{\partial W}{\partial z}\right)\left(\frac{\partial W}{\partial z}\right)^{*}=\frac{1}{2}\left[\left(\frac{\partial W_{1}}{\partial q_{1}}\right)^{2}+\left(\frac{\partial W_{1}}{\partial q_{2}}\right)^{2}\right]=\frac{1}{2}\left[\left(\frac{\partial W_{2}}{\partial q_{1}}\right)^{2}+\left(\frac{\partial W_{2}}{\partial q_{2}}\right)^{2}\right]$

y, en definitiva, tanto $W$ como el doble de su parte real y el doble de su parte imaginaria son superpotenciales válidos de la misma teoría $\mathrm{N}=2$ pre-supersimétrica. Es importante resaltar, en este punto, que si se multiplica el superpotencial $W$ por un número complejo de módulo uno, el resultado sigue siendo superpotencial de la teoría y, en consecuencia, el doble de su parte real y de su parte imaginaria otro tanto. Se dispone, por tanto, de toda una familia uniparamétrica de parejas de superpotenciales reales para toda teoría $\mathrm{N}=2$ pre-supersimétrica. 
Si se plantean las ecuaciones de primer orden (4.32), para un sistema de este tipo, tendremos dos posibilidades:

$$
\begin{aligned}
& \left\{\begin{array}{l}
\frac{d q_{1}}{d t}=-\epsilon \frac{\partial W_{1}}{\partial q_{1}} \\
\frac{d q_{2}}{d t}=-\epsilon \frac{\partial W_{1}}{\partial q_{2}}
\end{array}\right. \\
& \left\{\begin{array}{l}
\frac{d q_{1}}{d t}=-\epsilon \frac{\partial W_{2}}{\partial q_{1}} \\
\frac{d q_{2}}{d t}=-\epsilon \frac{\partial W_{2}}{\partial q_{2}}
\end{array}\right.
\end{aligned}
$$

que conducen, respectivamente, a las ecuaciones ordinarias de las órbitas de las soluciones:

$$
\frac{\partial W_{1}}{\partial q_{2}} d q_{1}-\frac{\partial W_{1}}{\partial q_{1}} d q_{2}=0
$$

para el caso de (4.41), y:

$$
\frac{\partial W_{2}}{\partial q_{2}} d q_{1}-\frac{\partial W_{2}}{\partial q_{1}} d q_{2}=0
$$

para (4.42). Las ecuaciones diferenciales (4.43) y (4.44) no sólo son sencillas sino que su integración resulta automática si se aplican a ellas las ecuaciones de CauchyRiemann (4.40).

Efectivamente, sustituyendo (4.40) en (4.43), se tiene:

$$
\frac{\partial W_{2}}{\partial q_{1}} d q_{1}+\frac{\partial W_{2}}{\partial q_{2}} d q_{2}=d W_{2}=0
$$

mientras que en (4.44):

$$
\frac{\partial W_{1}}{\partial q_{1}} d q_{1}+\frac{\partial W_{1}}{\partial q_{2}} d q_{2}=d W_{1}=0
$$

de forma que las trayectorias solución de las ecuaciones de primer orden asociadas al superpotencial $W_{1}$ resultan ser las curvas dadas por

$$
W_{2}=\gamma
$$

con $\gamma=$ cte. Análogamente, para el superpotencial $W_{2}$ las curvas solución son:

$$
W_{1}=\gamma_{\perp}
$$


con $\gamma_{\perp}$ constante. Es fácil observar, además, que las trayectorias (4.45) son exactamente las curvas ortogonales a las (4.46), como se deduce de manera evidente de (4.43), (4.44) y (4.40).

Con este razonamiento se han calculado recientemente (ver [6]) todas las soluciones de tipo kink de un modelo Wess-Zumino con un superpotencial de tipo polinómico propuesto por Gibbons y Townsend [44] para demostrar la existencia de intersecciones de defectos tipo pared.

\section{N=2 Pre-Supersimetría y Métrica de Jacobi}

Como ya hemos comentado anteriormente, existe una profunda y elegante relación entre la $\mathrm{N}=2$ pre-supersimetría de una teoría de dos campos escalares y la curvatura de la métrica de Jacobi del sistema dinámico asociado. Finalizaremos este capítulo explicitando dichas ideas.

La métrica de Jacobi del sistema dinámico bidimensional asociado al potencial (4.39) se escribe:

$$
h_{i j}=-2 U \delta_{i j}=2 V \delta_{i j}
$$

La pre-supersimetría de la teoría conduce a escribir (4.47) de la forma:

$$
h_{i j}=\left[\sum_{k=1}^{2}\left(\frac{\partial W}{\partial q^{k}}\right)^{2}\right] \delta_{i j}
$$

y, para el caso $\mathrm{N}=2$ pre-supersimétrico, dicha expresión será válida tanto para el doble de la parte real como para el doble de la parte imaginaria de uno de los superpotenciales holomorfos de la teoría.

Planteamos entonces el siguiente Teorema para este tipo de sistemas $\mathrm{N}=2$ presupersimétricos:

Teorema 4.11. La condición necesaria y suficiente para que una teoría de dos campos escalares sea $\mathrm{N}=2$ pre-supersimétrica es que la métrica de Jacobi del sistema dinámico asociado sea plana.

\section{Demostración:}

Las coordenadas cartesianas $\left(q^{1}, q^{2}\right)$ resultan ser, si la métrica de partida es la euclídea, como en este caso, un sistema de coordenadas isotermo para la métrica de Jacobi del sistema ${ }^{5}$.

No es difícil obtener la expresión del tensor de curvatura en este sistema de coordenadas. Hemos de tener en cuenta, por otro lado, que, para el caso de

\footnotetext{
${ }^{5}$ Un sistema de coordenadas locales se dice isotermo en una variedad riemanniana si la ex-
} 
dos dimensiones, la componente $R_{122}^{1}$, y las que resultan de los intercambios no triviales de sus índices, es la única no necesariamente nula del mismo. De esta forma tendremos que calcular únicamente la expresión:

$$
R_{122}^{1}=\frac{\partial^{2} \lambda}{\partial\left(q^{1}\right)^{2}}+\frac{\partial^{2} \lambda}{\partial\left(q^{2}\right)^{2}}=\Delta \lambda
$$

Tenemos que demostrar, por tanto, que la condición de $\mathrm{N}=2$ pre-supersimetría, es decir, la presencia de un superpotencial armónico $W$, es necesaria y suficiente para que $\lambda=\operatorname{Ln} \sqrt{2 V}$ sea una función armónica.

La condición necesaria se demuestra fácilmente por cálculo directo:

Si $\lambda=\operatorname{Ln} \sqrt{2 V}$ y $V=\frac{1}{2}\left[\left(\frac{\partial W}{\partial q^{1}}\right)^{2}+\left(\frac{\partial W}{\partial q^{2}}\right)^{2}\right]$ tendremos:

$$
\begin{aligned}
\Delta \lambda= & \frac{1}{2 V^{2}}\left[\frac{1}{2}\left(\left(\partial_{2} W\right)^{2}-\left(\partial_{1} W\right)^{2}\right)\left(\left(\partial_{1} \partial_{1} W\right)^{2}-\left(\partial_{2} \partial_{2} W\right)^{2}\right)\right]+ \\
& \frac{1}{2 V}\left[\partial_{1} W \partial_{1}(\Delta W)+\partial_{2} W \partial_{2}(\Delta W)\right]-\frac{1}{V^{2}} \partial_{1} \partial_{2} W \partial_{1} W \partial_{2} W \Delta W= \\
= & \frac{\Delta W}{V^{2}}\left[\frac{1}{4}\left(\left(\partial_{2} W\right)^{2}-\left(\partial_{1} W\right)^{2}\right)\left(\partial_{1} \partial_{1} W-\partial_{2} \partial_{2} W\right)-\partial_{1} \partial_{2} W \partial_{1} W \partial_{2} W\right]+ \\
& \frac{1}{2 V}\left[\partial_{1} W \partial_{1}(\Delta W)+\partial_{2} W \partial_{2}(\Delta W)\right]
\end{aligned}
$$

donde evidentemente $\partial_{i}=\frac{\partial}{\partial q^{i}}$.

Y entonces, trivialmente, la armonicidad de $W$ implica la de $\lambda$ y, en definitiva, la curvatura es nula si el superpotencial es armónico.

Es posible demostrar la suficiencia de varias formas equivalentes. Utilizaremos ahora un razonamiento de carácter más geométrico:

Tal y como demostraron el propio Riemann y Christoffel, la condición necesaria y suficiente para que una métrica dada sea isométrica a la euclídea es que su curvatura se anule ${ }^{6}$.

presión del tensor métrico en las mismas se escribe de la forma (ver por ejemplo [110]):

$$
d s^{2}=e^{2 \lambda}\left(d q^{1} \otimes d q^{1}+d q^{2} \otimes d q^{2}\right)
$$

Es evidente entonces, con $\lambda=\operatorname{Ln} \sqrt{2 V}$, que las coordenadas cartesianas del sistema mecánico son isotermas de $h_{i j}$.

${ }^{6}$ Realmente la condición de isometría que demostraron era general entre dos métricas cualesquiera, aquí la particularizamos al caso en el que una de ellas es la euclídea. Riemann demostró la condición necesaria en su famoso trabajo acerca de la conducción del calor, conocido habitualmente como Pariserarbeit (1861) por haber sido presentado a un premio de la Academia 
En consecuencia, la condición de curvatura plana implica la existencia de un cambio de coordenadas:

$$
\begin{aligned}
& u_{1}=u_{1}\left(q_{1}, q_{2}\right) \\
& u_{2}=u_{2}\left(q_{1}, q_{2}\right)
\end{aligned}
$$

de manera que:

$$
d s^{2}=2 V\left(d q_{1}^{2}+d q_{2}^{2}\right)=d u_{1}^{2}+d u_{2}^{2}
$$

y entonces, desarrollando esta ecuación, necesariamente se verifican las condiciones:

$$
\left(\frac{\partial u_{1}}{\partial q_{1}}\right)^{2}+\left(\frac{\partial u_{1}}{\partial q_{2}}\right)^{2}=\left(\frac{\partial u_{2}}{\partial q_{1}}\right)^{2}+\left(\frac{\partial u_{2}}{\partial q_{2}}\right)^{2}=2 \mathrm{~V}
$$

es decir, tanto $u_{1}$ como $u_{2}$ son superpotenciales de la teoría, y además:

$$
\frac{\partial u_{1}}{\partial q_{1}}=\frac{\partial u_{2}}{\partial q_{2}} ; \quad \frac{\partial u_{1}}{\partial q_{2}}=-\frac{\partial u_{2}}{\partial q_{1}}
$$

es decir, las funciones $u_{1}$ y $u_{2}$ son holomorfas conjugadas, y, por tanto, $W=$ $u_{1}+i u_{2}$ es un superpotencial armónico del sistema. La teoría es $\mathrm{N}=2$ presupersimétrica.

Q.E.D.

de Ciencias de París (que no ganó), fue publicado póstumamente en sus Obras Completas [89]. Christoffel demostró la condición necesaria en un trabajo en el que generalizó la invarianza bajo isometrías a tensores de cualquier rango, [21]. 


\section{Capítulo 5}

\section{De la Estabilidad de los Kinks del Modelo Sigma Lineal Deformado}

Dedicaremos este Capítulo al estudio de la estabilidad de las soluciones kink del Modelo Sigma $O(3)$ Lineal Deformado, obtenidas en el Capítulo 3. La técnica que se utilizará para ello será el análisis de los espectros correspondientes a los funcionales hessianos sobre las soluciones. Aplicaremos, de esta forma, los resultados generales que hemos obtenido en el Capítulo 4 acerca de la métrica de Jacobi asociada al sistema dinámico y de la relación existente entre los funcionales hessianos dinámicos y geométricos.

En el Capítulo 6 abordaremos este problema de la estabilidad desde dos puntos de vista diferentes pero relacionados con el que ahora nos ocupa.

La estructura de este capítulo es la siguiente: En una primera sección estudiaremos las geodésicas de la métrica de Jacobi para este modelo, comenzando con el caso $O(2)$ (modelo MSTB), contenido dos veces en el $O(3)$, como ya hemos visto. Dicha resolución se limitará a algunos kinks concretos puesto que el tratamiento general de las ecuaciones de las geodésicas conduce a un problema aún más complicado que el de enfrentarse directamente a las ecuaciones de segundo orden del sistema dinámico, al que por otra parte es absolutamente equivalente. Los cálculos realizados para el Modelo $O(2)$ se generalizarán fácilmente al Modelo $O(3)$ y al general con $N$ campos escalares.

En la segunda sección estudiaremos los funcionales hessianos y sus espectros, utilizando para ello tanto la "versión dinámica" como la "versión geométrica". Se establecerán de forma explícita las diferencias existentes entre una versión y otra, demostradas de manera general en el capítulo anterior (Teoremas 4.7 y 4.8). Una vez más, la complejidad de los cálculos restringirá los análisis a algunos tipos de 
kinks, tanto en el modelo de dos campos como en el tridimensional.

En lo que respecta a la notación utilizada, salvo que se especifique lo contrario, no se utilizará en este capítulo el convenio de Einstein de suma en los índices repetidos. Se adoptará, además, la notación vectorial (no usada en el Capítulo 4) en todos los casos en los que pueda presentarse confusión.

\subsection{Geodésicas de tipo Kink en la Métrica de Jacobi}

Tal y como hemos comentado, plantearemos en esta sección de manera general las ecuaciones de las curvas geodésicas en la Métrica de Jacobi asociada al Modelo Sigma $O(2)$ Lineal Deformado (Modelo MSTB), para calcular posteriormente, de manera explícita, la expresión (como segmentos geodésicos) de algunas de las ondas solitarias de dicho modelo. Finalmente, generalizaremos dichos resultados al caso del Modelo $O(3)$ y al modelo $N$-dimensional.

\subsubsection{Modelo $O(2)$ deformado}

\section{Coordenadas Cartesianas}

En el Capítulo 3 de esta memoria estudiamos el Modelo $O(2)$ Deformado (MSTB) con detalle. Utilizaremos ahora la notación propia del mismo introducida entonces, a pesar de que nuestro interés actual está en dicho modelo como parte embebida en el correspondiente modelo $O(3)$. Escribiremos así $q_{1}$ y $q_{2}$ como coordenadas y $\sigma$ como parámetro de la deformación; en el apartado 5.1.2 extenderemos dichos resultados a la situación $\left(q_{1}, q_{2}\right) \equiv\left(q_{1}, 0, q_{3}\right)$ y $\sigma \equiv \sigma_{3}$ por un lado, y $\left(q_{1}, q_{2}\right) \equiv$ $\left(q_{1}, q_{2}, 0\right)$ con $\sigma \equiv \sigma_{2}$ por otro.

Desde el punto de vista de la métrica de Jacobi, y en coordenadas cartesianas ${ }^{1}$, tendremos la siguiente situación:

La métrica de partida del sistema dinámico no es otra que la euclídea habitual:

$$
g_{i j}=\delta_{i j} \quad \forall i, j=1,2
$$

\footnotetext{
${ }^{1}$ Dado que la resolución completa de las ecuaciones para obtener todas las soluciones requiere el uso de coordenadas elípticas, la restricción al uso de coordenadas cartesianas nos permitirá analizar únicamente los kinks TK1, TK2 y un caso particular de la familia NTK2 para el cual los cálculos se simplifican notablemente.
} 
y, por tanto, todos los símbolos de Christoffel correspondientes son nulos:

$$
\Gamma_{j k}^{i}=0, \quad \forall i, j, k=1,2
$$

La métrica de Jacobi asociada al modelo es:

$$
h_{i j}=2\left(i_{1}-U\right) g_{i j}
$$

donde $i_{1}=0$ para las trayectorias del sistema asociadas a soluciones kink de la teoría de campos subyacente, y donde ${ }^{2}$ :

$$
U\left(q_{1}, q_{2}\right)=-\frac{1}{2}\left(q_{1}^{2}+q_{2}^{2}-1\right)^{2}-\frac{\sigma^{2}}{2} q_{2}^{2}
$$

por lo que resulta:

$$
h_{i j}=\left[\left(q_{1}^{2}+q_{2}^{2}-1\right)^{2}+\sigma^{2} q_{2}^{2}\right] \delta_{i j}
$$

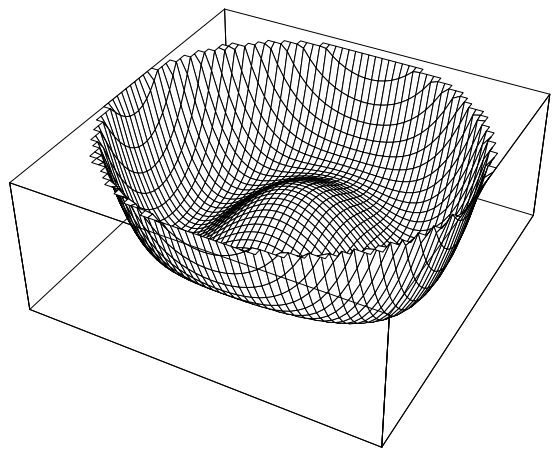

Figura 5.1: Representación gráfica del factor métrico $\left(q_{1}^{2}+q_{2}^{2}-1\right)^{2}+\sigma^{2} q_{2}^{2}$ frente a las coordenadas $q_{1}$ y $q_{2}$.

El cálculo de los símbolos de Christoffel de $h$ conduce a las expresiones:

$$
\begin{aligned}
\Gamma_{11}^{1 J} & =\frac{2 q_{1}\left(q_{1}^{2}+q_{2}^{2}-1\right)}{\left(q_{1}^{2}+q_{2}^{2}-1\right)^{2}+\sigma^{2} q_{2}^{2}} \\
\Gamma_{12}^{1 J} & =\frac{2 q_{2}\left(q_{1}^{2}+q_{2}^{2}-1\right)+\sigma^{2} q_{2}}{\left(q_{1}^{2}+q_{2}^{2}-1\right)^{2}+\sigma^{2} q_{2}^{2}}
\end{aligned}
$$

\footnotetext{
${ }^{2}$ Por simplicidad en la notación denominaremos, en el resto del Capítulo, $q_{1}$ y $q_{2}$ a las coordenadas $q^{1}$ y $q^{2}$. Como ya se ha comentado, no se utilizará, en ningún caso, el convenio de Einstein para suma de índices repetidos.
} 
mientras que el resto de los símbolos puede ser escrito en función de éstos:

$$
\begin{aligned}
& \Gamma_{22}^{1 J}=-\Gamma_{12}^{2 J}=-\Gamma_{11}^{1 J} \\
& \Gamma_{11}^{2 J}=-\Gamma_{22}^{2 J}=-\Gamma_{12}^{1 J}
\end{aligned}
$$

además de los deducibles trivialmente de la simetría $\Gamma_{j k}^{i J}=\Gamma_{k j}^{i J}$.

En definitiva, las ecuaciones de las geodésicas de este modelo, $\nabla_{c^{\prime}}^{J} c^{\prime}=0$, se escribirán de la forma (la prima denota, evidentemente, derivación con respecto al parámetro longitud de arco, $s)$ :

$$
\begin{gathered}
q_{1}^{\prime \prime}+\frac{2 q_{1}\left(q_{1}^{2}+q_{2}^{2}-1\right)}{\left(q_{1}^{2}+q_{2}^{2}-1\right)^{2}+\sigma^{2} q_{2}^{2}}\left(q_{1}^{\prime 2}-q_{2}^{\prime 2}\right)+2 \frac{2 q_{2}\left(q_{1}^{2}+q_{2}^{2}-1\right)+\sigma^{2} q_{2}}{\left(q_{1}^{2}+q_{2}^{2}-1\right)^{2}+\sigma^{2} q_{2}^{2}} q_{1}^{\prime} q_{2}^{\prime}=0 \\
q_{2}^{\prime \prime}+\frac{2 q_{2}\left(q_{1}^{2}+q_{2}^{2}-1\right)+\sigma^{2} q_{2}}{\left(q_{1}^{2}+q_{2}^{2}-1\right)^{2}+\sigma^{2} q_{2}^{2}}\left(q_{2}^{\prime 2}-q_{1}^{\prime 2}\right)+\frac{4 q_{1}\left(q_{1}^{2}+q_{2}^{2}-1\right)}{\left(q_{1}^{2}+q_{2}^{2}-1\right)^{2}+\sigma^{2} q_{2}^{2}} q_{1}^{\prime} q_{2}^{\prime}=0
\end{gathered}
$$

La resolución de este sistema de ecuaciones no es fácil, como ya se ha comentado en esta memoria. Podemos, sin embargo, resolverlas para algunos casos concretos, de una manera similar a la técnica de la órbita de prueba utilizada por Rajaraman. Analizaremos, con esta estrategia, los kinks TK1 y TK2 por un lado, y uno de los kinks de la familia NTK2, para el caso concreto en el que la constante $\sigma$ del modelo toma el valor $\frac{1}{2}$, por otro. Tal y como veremos, la ecuación de la órbita, muy complicada en general, se simplifica enormemente en las condiciones citadas.

\section{TK1}

Para obtener la solución kink TK1 consideraremos, lógicamente, las ecuaciones de las geodésicas $(5.3,5.4)$ en la métrica de Jacobi restringidas a la órbita:

$$
q_{2} \equiv 0
$$

La ecuación (5.3) se reduce a:

$$
q_{1}^{\prime \prime}+\frac{2 q_{1}}{q_{1}^{2}-1} q_{1}^{\prime 2}=0
$$

mientras que la ecuación (5.4) se trivializa a $0=0$.

(5.5) es una ecuación lineal de segundo orden que no contiene explícitamente a la variable independiente, es posible entonces reducirla a una ecuación de primer orden utilizando el cambio:

$$
p_{1}=\frac{d q_{1}}{d s}=q_{1}^{\prime}
$$




$$
\frac{d^{2} q_{1}}{d s^{2}}=\frac{d p_{1}}{d s}=\frac{d p_{1}}{d q_{1}} \frac{d q_{1}}{d s}=p_{1} \frac{d p_{1}}{d q_{1}}
$$

y así la ecuación se escribe de la forma:

$$
p_{1} \frac{d p_{1}}{d q_{1}}=\frac{2 q_{1}}{1-q_{1}^{2}} p_{1}^{2}
$$

Además de una solución evidente, $p_{1}=0$, tendremos la cuadratura:

$$
\int \frac{d p_{1}}{p_{1}}=\int \frac{2 q_{1}}{1-q_{1}^{2}} d q_{1}
$$

que produce la solución

$$
p_{1}=\frac{k}{1-q_{1}^{2}}
$$

siendo $k$ una constante de integración.

Si sustituimos ahora la expresión de $p_{1}$ obtenemos:

$$
\frac{d q_{1}}{d s}=\frac{k}{1-q_{1}^{2}}
$$

y, finalmente:

$$
\bar{q}_{1}-\frac{\bar{q}_{1}^{3}}{3}=k\left(s+s_{0}\right)
$$

donde se ha denotado $\bar{q}_{1}$ a la solución, criterio que mantendremos en lo que sigue.

La solución singular, $p_{1}=0$, de la ecuación (5.6), conduce al resultado $\bar{q}_{1}(s)=$ constante. La interpretación de esta solución es evidente: todo punto de la órbita considerada es en sí mismo una geodésica de la variedad $(M, h)$, son las geodésicas triviales. Sin embargo, desde el punto de vista dinámico, sólo los ceros de la función potencial son soluciones triviales de las ecuaciones de movimiento, dicho con otras palabras: en los demás puntos la partícula descrita por el sistema "no permanece" para todo instante de tiempo.

Este razonamiento se plasma analíticamente si tenemos en cuenta que el arco de longitud $s$ y el tiempo del sistema dinámico están relacionados, sobre esta órbita, por la ecuación: $d s=\left(1-q_{1}^{2}\right)^{2} d t$. Para todo punto $\bar{q}_{1}=$ cte $\neq \pm 1$ se verificará (por tratarse de una geodésica de longitud nula): $d s=0 \Rightarrow d t=0, \mathrm{y}$, por tanto, $\bar{q}_{1}$ es constante pero también el tiempo se ve reducido a un instante concreto. Por el contrario, para $\bar{q}_{1}= \pm 1$, tendremos $d s=0 d t$, y así la partícula "permanece" en dichos puntos para todo $t \in \mathbb{R}$.

Esta situación nos permite clasificar los puntos de la variedad $(M, h)$ en "puntos geodésicos dinámicos", es decir los ceros del potencial, y simplemente "puntos geodésicos" para el resto. 
El razonamiento anterior está intimamente relacionado con el hecho de que la métrica de Jacobi esté degenerada sobre los puntos geodésicos dinámicos, en este caso, como vemos, dos puntos aislados de $(M, h)$. En las referencias [95] y [96] se analizan con detalle las situaciones en las cuales las geodésicas de una métrica de Jacobi atraviesan regiones singulares de la propia métrica, para el caso Riemanniano como el que nos ocupa aquí y para métricas más generales, de tipo Lorentziano.

Para el resto de las soluciones de (5.6), es decir, para las soluciones incluidas en la expresión general (5.8), obtenemos, lógicamente, una dependencia en dos constantes arbitrarias, $k$ y $s_{0}$. El significado geométrico de las mismas es claro: las ecuaciones de las geodésicas $(5.3,5.4)$ son invariantes bajo reparametrizaciones afines de la forma: $s \rightarrow a s+b$, con $a$ y $b$ constantes. La presencia de la constante arbitraria $s_{0}$ corresponde a la invarianza bajo traslaciones en la longitud de arco y podemos fijarla nula determinando el origen de longitudes. El valor de la constante $k$, por el contrario, es significativo y determina el tipo de parametrización escogida de las curvas solución. Dado que buscamos soluciones parametrizadas en función de la longitud de arco, dicho valor quedará fijado por la condición de "velocidad uno" (utilizando (5.7)):

$$
\left\|c^{\prime}\right\|^{J}=1 \Rightarrow \sqrt{\frac{d \bar{q}_{1}}{d s} h_{11} \frac{d \bar{q}_{1}}{d s}}=\sqrt{k^{2}}=1 \Rightarrow k= \pm 1
$$

De esta manera, las soluciones (5.8), geodésicas de la métrica de Jacobi $h$ sobre la órbita $q_{2} \equiv 0$, se escriben:

$$
\bar{q}_{1}-\frac{\bar{q}_{1}^{3}}{3}= \pm s
$$

Las condiciones asintóticas del sistema dinámico, necesarias para que la energía de la teoría de campos original fuera finita, imponían que las trayectorias solución tendieran, en $t \rightarrow \pm \infty$, a los puntos de vacío del sistema, es decir, a los ceros del potencial, los antes denominados "puntos geodésicos dinámicos" del sistema: $\left(q_{1}, q_{2}\right)=( \pm 1,0)$. Estas condiciones, sobre la ecuación (5.9), se traducen entonces en que la longitud de arco $s$ variará en el intervalo $\left[s_{1}, s_{2}\right]=\left[-\frac{2}{3}, \frac{2}{3}\right]$ de tal manera que si escogemos el signo positivo en el segundo miembro de la ecuación (5.9) tendremos:

$$
\begin{gathered}
s=s_{1}=-\frac{2}{3} \Rightarrow \bar{q}_{1}\left(s_{1}\right)=-1 \\
s=s_{2}=\frac{2}{3} \Rightarrow \bar{q}_{1}\left(s_{2}\right)=1
\end{gathered}
$$


es decir, la solución TK1, mientras que la elección del signo negativo en (5.9) produce el antikink ATK1.

En ambos casos, y de acuerdo con lo demostrado en el capítulo anterior, se verifica que la longitud del segmento geodésico considerado coincide exactamente con la acción mecánica sobre la solución (y, salvo las constantes multiplicativas, con la energía de dichas ondas solitarias):

$$
L^{J}\left[c_{\mathrm{TK} 1}\right]=\int_{s_{1}}^{s_{2}} d s=s_{2}-s_{1}=\frac{4}{3}=S\left[c_{\mathrm{TK} 1}\right]=\frac{\lambda^{2} \sqrt{2}}{m^{3}} E_{\mathrm{TK} 1}
$$

Para recuperar la expresión de las soluciones TK1 y ATK1 en función del parámetro "temporal" del sistema dinámico tenemos que considerar, en la ecuación (5.7), la expresión de $d s$ en función de $d t$ ya escrita: $d s=\left(1-q_{1}^{2}\right)^{2} d t$. De esta forma tendremos:

$$
\frac{d q_{1}}{d s}=\frac{d q_{1}}{d t}\left(1-q_{1}^{2}\right)^{-2}=\frac{ \pm 1}{1-q_{1}^{2}} \Rightarrow \frac{d q_{1}}{1-q_{1}^{2}}= \pm d t
$$

y en definitiva:

$$
\bar{q}_{1}(t)= \pm \tanh \left(t+t_{0}\right)
$$

La ecuación (5.9) nos proporciona la expresión de $\bar{q}_{1}(s)$ de forma implícita. $\mathrm{Su}$ resolución, por medio de las fórmulas de Cardano-Tartaglia (ver por ejemplo [2]), nos permitirá obtener la forma explícita del kink "visto" como segmento geodésico de $h$. Si tenemos en cuenta que la solución está restringida al intervalo $\bar{q}_{1} \in[-1,1]$, sólo una de las tres raíces de la cúbica (5.9) toma valores reales entre $s_{1}=-\frac{2}{3} \mathrm{y}$ $s_{2}=\frac{2}{3}$ :

$$
q_{1}^{\mathrm{TK} 1}(s)=\bar{q}_{1}(s)=-\cos \frac{\theta}{3}+\sqrt{3} \operatorname{sen} \frac{\theta}{3}
$$

siendo $\theta=\arctan \frac{\sqrt{4-9 s^{2}}}{-3 s}$ (y donde la función arcotangente se considera valorada en el intervalo $[0, \pi])$. Ambas expresiones, $\bar{q}_{1}(t)$ y $\bar{q}_{1}(s)$, para el kink TK1, están representadas en la Figura 5.2.

Por último, un comentario interesante acerca del cálculo realizado es el siguiente: la ecuación (5.9), que proporciona la longitud de arco sobre la geodésica en términos de la variable $\bar{q}_{1}$, coincide exactamente con la expresión del superpotencial $W$ que esta teoría admite, restringido a la órbita del kink TK1. Estudiaremos con detalle el carácter pre-supersimétrico del modelo en el Capítulo 6, precisaremos aquí simplemente que este resultado es evidente si se tiene en cuenta que la longitud en la métrica de Jacobi se escribe $L^{J}=\int d s$ y que la acción del sistema dinámico, que coincide con la longitud $L^{J}$ sobre las soluciones, se escribe, para los sistemas 

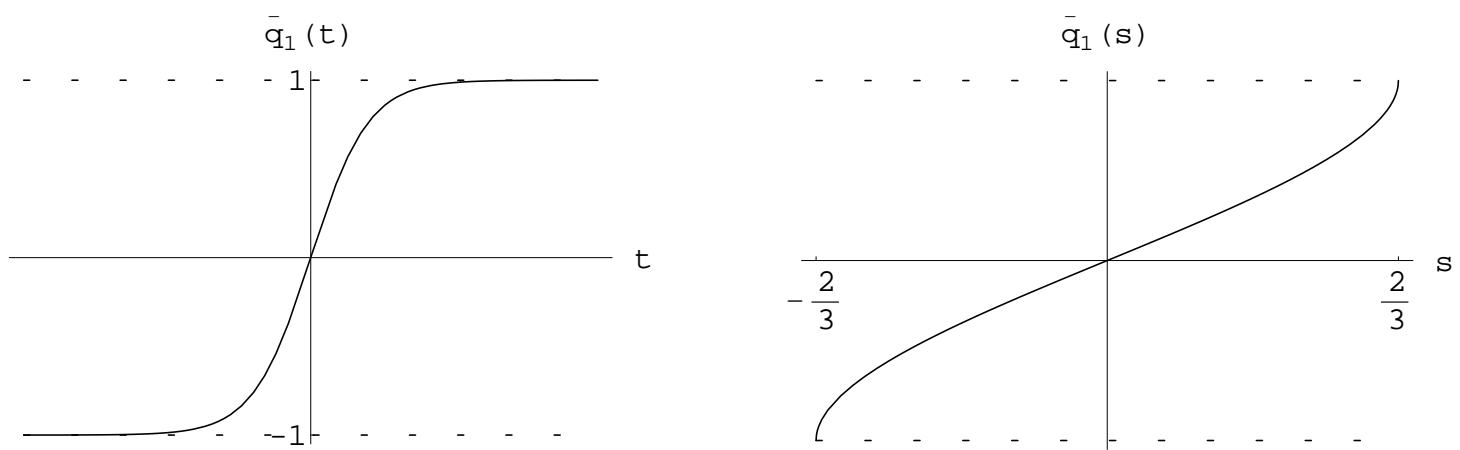

Figura 5.2: Representación gráfica de las funciones $\bar{q}_{1}(t)$ y $\bar{q}_{1}(s)$ para el kink TK1.

pre-supersimétricos, y de acuerdo con la descomposición de Bogomol'nyi expuesta en el Capítulo 4, como $S=\int d W$, sobre las soluciones de las ecuaciones de primer orden del sistema. Como ya hemos dicho, demostraremos en el Capítulo 6 que el Modelo Sigma $O(N)$ Lineal Deformado es un modelo pre-supersimétrico. En particular, para el caso del Modelo $O(2)$, puede comprobarse que el potencial $V$ admite una pareja de superpotenciales dados por las expresiones (realmente son cuatro los superpotenciales si se tiene en cuenta la posibilidad de cambiar el signo globalmente):

$$
W^{ \pm}\left(q_{1}, q_{2}\right)=\sqrt{q_{1}^{2}+q_{2}^{2} \pm 2 \sigma q_{1}+\sigma^{2}}\left[\frac{1}{3}\left(q_{1}^{2}+q_{2}^{2} \mp \sigma q_{1}+\sigma^{2}\right)-1\right]
$$

que, para la órbita $\left(q_{1}, q_{2}\right)=\left(\bar{q}_{1}, 0\right)$, se reducen a:

$$
W^{ \pm}\left(\bar{q}_{1}, 0\right)=-\left(\bar{q}_{1}-\frac{\bar{q}_{1}^{3}}{3}\right) \pm\left(-\sigma+\frac{\sigma^{3}}{3}\right)
$$

es decir, coincide con la expresión del arco $s$ (5.9) sobre la curva TK1 salvo en una constante aditiva.

\section{TK2}

Estudiaremos ahora las ecuaciones $(5.3,5.4)$ para el caso de las soluciones del tipo TK2. La órbita de prueba que consideramos en este caso no es otra que la elipse de ecuación:

$$
q_{1}^{2}+\frac{q_{2}^{2}}{1-\sigma^{2}}=1
$$


Como ya se demostró en el Capítulo 3, sobre esta elipse existen cuatro soluciones de tipo kink: TK2, TK2*, ATK2 y ATK2*. Restringiremos el análisis a aquellas soluciones que transcurren sobre la semielipse superior (TK2 y ATK2):

$$
q_{2}=\bar{\sigma} \sqrt{1-q_{1}^{2}}
$$

donde $\bar{\sigma}=\sqrt{1-\sigma^{2}}$.

La métrica de Jacobi

$$
h_{i j}=\left[\left(q_{1}^{2}+q_{2}^{2}-1\right)^{2}+\sigma^{2} q_{2}^{2}\right] \delta_{i j}
$$

sobre dicha curva se escribirá de la forma:

$$
h_{i j}=\left[\sigma^{2}\left(1-q_{1}^{2}\right)\left(1-\sigma^{2} q_{1}^{2}\right)\right] \delta_{i j}
$$

Los símbolos de Christoffel de $h$ restringidos a esta órbita por medio de la sustitución (5.12) se reducen a:

$$
\begin{gathered}
\Gamma_{11}^{1 J}=\frac{-2 q_{1}}{1-\sigma^{2} q_{1}^{2}}=-\Gamma_{22}^{1 J}=\Gamma_{12}^{2 J} \\
\Gamma_{12}^{1 J}=\frac{\bar{\sigma}\left(2 q_{1}^{2}-1\right)}{\sqrt{1-q_{1}^{2}}\left(1-\sigma^{2} q_{1}^{2}\right)}=-\Gamma_{11}^{2 J}=\Gamma_{22}^{2 J}
\end{gathered}
$$

De esta manera, las ecuaciones de las geodésicas (5.3, 5.4), utilizando (5.12) y sus derivadas primera y segunda, se reducen a una única ecuación para la variable $q_{1}$ :

$$
q_{1}^{\prime \prime}-\frac{2 \sigma^{2} q_{1}}{1-\sigma^{2} q_{1}^{2}} q_{1}^{\prime 2}=0
$$

La resolución de (5.13) se realiza de manera análoga a la de (5.5). La sustitución adecuada será:

$$
q_{1}^{\prime}=p_{1} ; \quad q_{1}^{\prime \prime}=p_{1} \frac{d p_{1}}{d q_{1}}
$$

de manera que se obtiene trivialmente (además de $p_{1}=0$, similar a la obtenida en el caso TK1):

$$
p_{1}=\frac{k}{1-\sigma^{2} q_{1}^{2}} \Rightarrow q_{1}^{\prime}=\frac{d q_{1}}{d s}=\frac{k}{1-\sigma^{2} q_{1}^{2}}
$$

para alguna constante de integración $k$. La integración de esta última ecuación es evidente y conduce a la solución:

$$
\bar{q}_{1}-\frac{\sigma^{2} \bar{q}_{1}^{3}}{3}=k\left(s-s_{0}\right)
$$


Una vez más la exigencia de que la geodésica esté parametrizada en función de la longitud de arco fijará el valor de la constante $k$ :

$$
\left\|c^{\prime}\right\|^{J}=1 \Rightarrow \sqrt{\bar{q}_{1}^{\prime} h_{11} \bar{q}_{1}^{\prime}+\bar{q}_{2}^{\prime} h_{22} \bar{q}_{2}^{\prime}}=1
$$

Conocemos $\bar{q}_{1}^{\prime}=\frac{k}{1-\sigma^{2} \bar{q}_{1}^{2}}$ y es trivial calcular:

$$
\bar{q}_{2}=\bar{\sigma} \sqrt{1-\bar{q}_{1}^{2}} \Rightarrow \bar{q}_{2}^{\prime}=\frac{-\bar{\sigma} \bar{q}_{1}}{\sqrt{1-\bar{q}_{1}^{2}}} \bar{q}_{1}^{\prime}
$$

de manera que:

$$
\left\|c^{\prime}\right\|^{J}=1 \Rightarrow k^{2} \sigma^{2}=1
$$

y, en definitiva: $k= \pm \sigma^{-1}$

Sustituyendo el valor $k=\frac{1}{\sigma}$ y tomando $s_{0}=0$ en (5.14) obtendremos:

$$
\sigma\left(\bar{q}_{1}-\frac{\sigma^{2} \bar{q}_{1}^{3}}{3}\right)=s
$$

es decir, una cúbica, similar a (5.8), que nos proporciona implícitamente la componente $\bar{q}_{1}$ de la solución TK2 en función del parámetro arco de la métrica de Jacobi, que evoluciona, de acuerdo con las condiciones asintóticas, en el intervalo $s \in\left[s_{1}, s_{2}\right]$, con:

$$
s_{1}=-\sigma\left(1-\frac{\sigma^{2}}{3}\right) ; \quad s_{2}=\sigma\left(1-\frac{\sigma^{2}}{3}\right)
$$

Evidentemente, $k=-\frac{1}{\sigma}$ conduce a la solución ATK2. Para la componente $q_{2}$, a partir de la expresión (5.12), no es difícil encontrar la ecuación cuasi-cúbica:

$$
\frac{\sigma}{\bar{\sigma}^{3}} \sqrt{\bar{\sigma}^{2}-\bar{q}_{2}^{2}}\left(\bar{\sigma}^{2}-\frac{\sigma^{2}}{3}\left(\bar{\sigma}^{2}-\bar{q}_{2}^{2}\right)\right)=s
$$

La longitud del segmento geodésico TK2 no es más, como en el kink TK1, que la acción de la correspondiente onda solitaria:

$$
L^{J}\left[c_{\mathrm{TK} 2}\right]=\int_{s_{1}}^{s_{2}} d s=2 \sigma\left(1-\frac{\sigma^{2}}{3}\right)=S\left[c_{\mathrm{TK} 2}\right]
$$

Para obtener la expresión del kink en función del tiempo $t$, tenemos que recordar que en este caso: $d s=\sigma^{2}\left(1-q_{1}^{2}\right)\left(1-\sigma^{2} q_{1}^{2}\right) d t$, de tal manera que resulta la cuadratura:

$$
\int \frac{d q_{1}}{1-q_{1}^{2}}=\int \pm \sigma d t
$$


y en definitiva:

$$
\bar{q}_{1}= \pm \tanh \left(\sigma\left(t-t_{0}\right)\right)
$$

que, tomando $t_{0}=0$ y el signo positivo, se reduce a la citada solución TK2:

$$
q_{1}^{\mathrm{TK} 2}(t)=\bar{q}_{1}(t)=\tanh (\sigma t)
$$

Recordando (5.12) tendremos:

$$
q_{2}^{\mathrm{TK} 2}(t)=\bar{q}_{2}(t)=\sqrt{1-\sigma^{2}} \operatorname{sech}(\sigma t)
$$

La solución ATK2 se obtiene, como ya hemos dicho, tomando el otro signo posible para la constante $k$. Los kinks TK2* y ATK2* son el resultado de considerar la semielipse inferior, y, por tanto, el signo opuesto al tomado en (5.12).

En la Figuras 5.3 y 5.4 puede visualizarse el kink TK2 en función del parámetro longitud de arco y del parámetro "tiempo" del sistema dinámico.
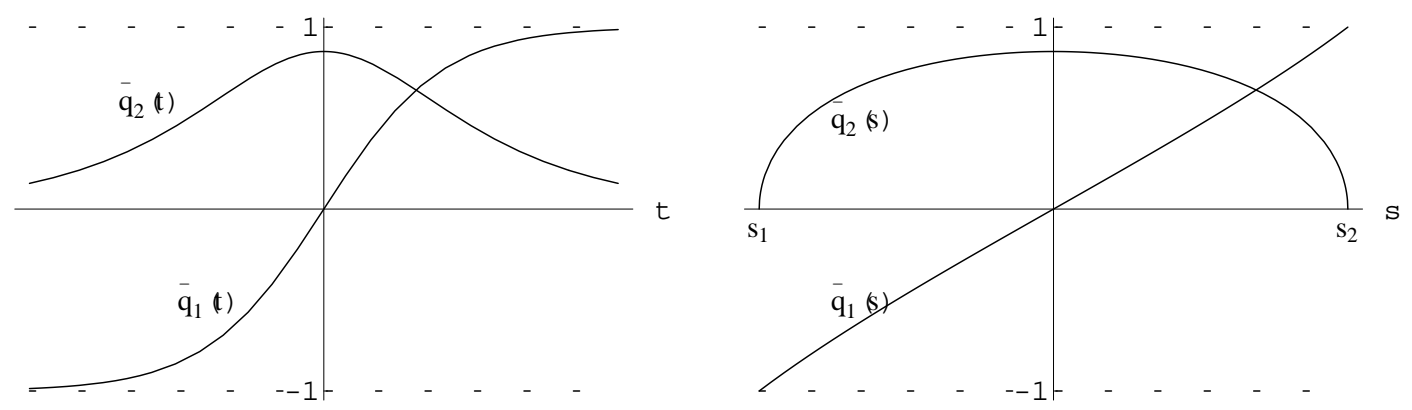

Figura 5.3: Gráficas de las componentes del kink TK2 en función de: (a) parámetro temporal, y (b) parámetro arco.

Por último, las ecuaciones (5.15) y (5.16) pueden ser resueltas por las fórmulas de Cardano-Tartaglia, de manera similar a la utilizada en el caso TK1, obteniéndose, para el rango $s \in\left[s_{1}, s_{2}\right]$, las expresiones:

$$
\begin{aligned}
& q_{1}^{\mathrm{TK} 2}(s)=\bar{q}_{1}(s)=-\frac{1}{\sigma} \cos \frac{\theta}{3}+\frac{\sqrt{3}}{\sigma} \operatorname{sen} \frac{\theta}{3} \\
& q_{2}^{\mathrm{TK} 2}(s)=\bar{q}_{2}(s)=\frac{\bar{\sigma}}{\sigma} \sqrt{-2+\sigma^{2}+\cos \frac{2 \theta}{3}+\sqrt{3} \operatorname{sen} \frac{2 \theta}{3}}
\end{aligned}
$$

siendo $\theta=\arctan \frac{\sqrt{4-9 s^{2}}}{-3 s}$ (y donde de nuevo se considera que la función arcotangente toma valores en el intervalo $[0, \pi])$. 

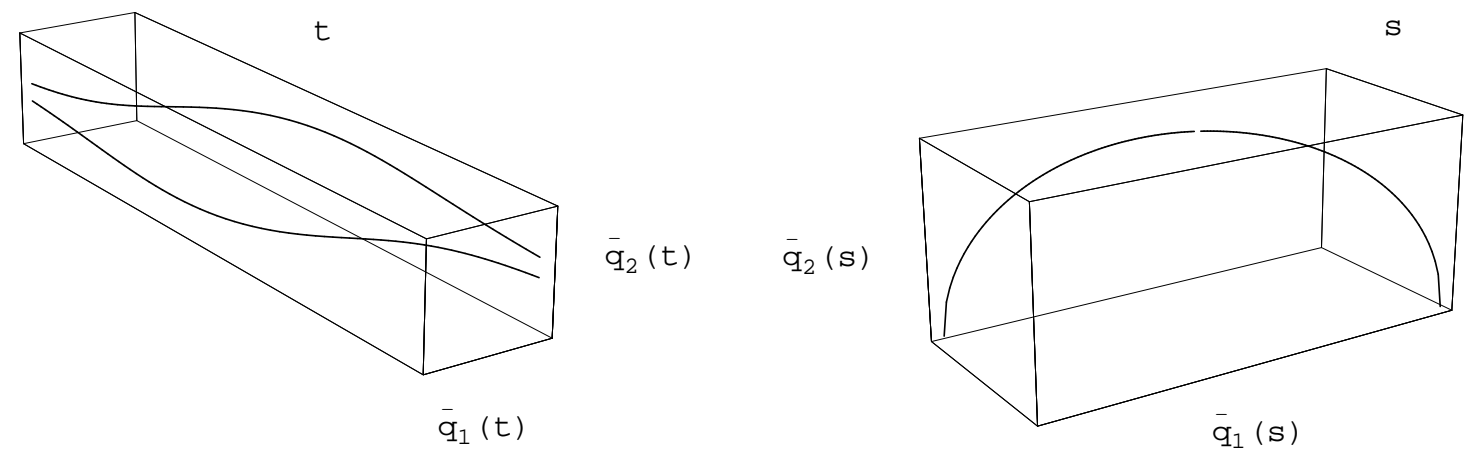

Figura 5.4: (a) Gráficas del kink TK2 y TK2* frente al tiempo $t$. (b) Gráfica del kink TK2 frente a la longitud de arco $s$.

Si calculamos la expresión de los superpotenciales $W^{ \pm}$restringidos a la elipse, por medio de la sustitución (5.12), tendremos ahora:

$$
W^{ \pm}\left(\bar{q}_{1}, \bar{\sigma} \sqrt{1-\bar{q}_{1}^{2}}\right)=-\sigma\left(\bar{q}_{1}-\frac{\sigma^{2} \bar{q}_{1}^{2}}{3}\right) \mp \frac{2}{3}
$$

de forma que, salvo constantes aditivas, encontramos de nuevo el resultado antes comentado: la longitud de arco sobre el segmento geodésico coincide con el superpotencial de la teoría sobre la órbita solución.

\section{$\operatorname{NTK2(0)} \operatorname{con} \sigma=\frac{1}{2}$}

Las soluciones no topológicas del modelo MSTB, analizadas en el Capítulo 3, constituyen una familia uniparamétrica de kinks (NTK2) determinada por las ecuaciones (3.16) para las órbitas y (3.17) para la dependencia temporal de las mismas. Si recordamos la ecuación (3.16):

$$
\begin{aligned}
& \left(\left|\frac{\sqrt{1-\mu_{1}}-\sigma}{\sqrt{1-\mu_{1}}+\sigma}\right| \cdot\left|\frac{\sqrt{1-\mu_{1}}+1}{\sqrt{1-\mu_{1}}-1}\right|^{\sigma}\right)^{\operatorname{sign}\left(\pi_{1}\right)} \cdot \\
& \left(\left|\frac{\sqrt{1-\mu_{2}}-\sigma}{\sqrt{1-\mu_{2}}+\sigma}\right| \cdot\left|\frac{\sqrt{1-\mu_{2}}+1}{\sqrt{1-\mu_{2}}-1}\right|^{\sigma}\right)^{\operatorname{sign}\left(\pi_{2}\right)}=e^{2 \sigma \bar{\sigma}^{2} \gamma_{2}}
\end{aligned}
$$

observamos que los diferentes valores de la constante $\gamma_{2}$ determinan los diferentes kinks dentro de la familia. Para cada valor de $\gamma_{2}$ tendremos, según la elección de los $\operatorname{signos} \operatorname{sign}\left(\pi_{1}\right)$ y $\operatorname{sign}\left(\pi_{2}\right)$ cuatro tramos cuya sucesión continua y diferenciable proporciona la solución completa (recordemos que las coordenadas elípticas 
bidimensionales $\mu_{1}$ y $\mu_{2}$ representan el plano "cartesiano" mediante una correspondencia $2^{2}=4$ a 1 , de manera que la exigencia de continuidad y derivabilidad entre los diferentes tramos nos conduce a que cada uno de ellos está situado en un cuadrante diferente). Para el caso particular en el que la constante $\gamma_{2}$ es nula, las cuatro elecciones de signos se reducen a dos, se trata de la solución "simétrica" con respecto al cambio $q_{2} \rightarrow-q_{2}$. Si además consideramos el caso $\sigma=\frac{1}{2}$ tendremos que la ecuación (3.16) se reduce a:

$$
\left|\frac{\sqrt{1-\mu_{1}}-\frac{1}{2}}{\sqrt{1-\mu_{1}}+\frac{1}{2}}\right| \cdot\left|\frac{\sqrt{1-\mu_{1}}+1}{\sqrt{1-\mu_{1}}-1}\right|^{\frac{1}{2}} \cdot\left|\frac{\sqrt{1-\mu_{2}}-\frac{1}{2}}{\sqrt{1-\mu_{2}}+\frac{1}{2}}\right| \cdot\left|\frac{\sqrt{1-\mu_{2}}+1}{\sqrt{1-\mu_{2}}-1}\right|^{\frac{1}{2}}=1
$$

para la elección de signos iguales, $\operatorname{sign}\left(\pi_{1}\right)=\operatorname{sign}\left(\pi_{2}\right), \mathrm{y}$ :

$$
\left|\frac{\sqrt{1-\mu_{1}}-\frac{1}{2}}{\sqrt{1-\mu_{1}}+\frac{1}{2}}\right| \cdot\left|\frac{\sqrt{1-\mu_{1}}+1}{\sqrt{1-\mu_{1}}-1}\right|^{\frac{1}{2}} \cdot\left|\frac{\sqrt{1-\mu_{2}}+\frac{1}{2}}{\sqrt{1-\mu_{2}}-\frac{1}{2}}\right| \cdot\left|\frac{\sqrt{1-\mu_{2}}-1}{\sqrt{1-\mu_{2}}+1}\right|^{\frac{1}{2}}=1
$$

para el caso de signos diferentes: $\operatorname{sign}\left(\pi_{1}\right)=-\operatorname{sign}\left(\pi_{2}\right)$.

Si simplificamos (5.18) y (5.19) adecuadamente se convierten en:

$$
\sqrt{1-\mu_{1}}\left(\frac{1}{4}-\mu_{1}\right)+\sqrt{1-\mu_{2}}\left(\frac{1}{4}-\mu_{2}\right)=0
$$

para (5.18) y

$$
\sqrt{1-\mu_{1}}\left(\frac{1}{4}-\mu_{1}\right)-\sqrt{1-\mu_{2}}\left(\frac{1}{4}-\mu_{2}\right)=0
$$

para (5.19). Ambas ecuaciones pueden escribirse conjuntamente de la forma:

$$
\mu_{1}\left(4 \mu_{1}-3\right)^{2}-\mu_{2}\left(4 \mu_{2}-3\right)^{2}=0
$$

Finalmente, no es difícil comprobar que (5.20) puede simplificarse a la expresión:

$$
\left(\mu_{1}-\mu_{2}\right)\left(\left(4 \mu_{1}-3\right)^{2}+\left(4 \mu_{2}-3\right)^{2}-9+16 \mu_{1} \mu_{2}\right)=0
$$

de manera que la órbita se reduce por un lado a un punto: $\mu_{1}=\mu_{2}=1-\sigma^{2}=\frac{3}{4}$ (el foco de la elipse propia del sistema) y, por otro, a la expresión:

$$
\left(4 \mu_{1}-3\right)^{2}+\left(4 \mu_{2}-3\right)^{2}-9+16 \mu_{1} \mu_{2}=0
$$

que, en coordenadas cartesianas (recordemos que $\sigma=\frac{1}{2}$ ):

$$
\begin{aligned}
& q_{1}^{2}=4\left(1-\mu_{1}\right)\left(1-\mu_{2}\right) \\
& q_{2}^{2}=-4\left(\frac{3}{4}-\mu_{1}\right)\left(\frac{3}{4}-\mu_{2}\right)
\end{aligned}
$$


se escribe:

$$
\left(q_{1} \pm \frac{1}{4}\right)^{2}+q_{2}^{2}=\frac{9}{16}
$$

y, por tanto, en coordenadas cartesianas, la órbita en cuestión se traduce en dos circunferencias. Evidentemente, el signo positivo en (5.21) nos conduce a que la solución pertenezca al sector topológico $\mathcal{C}^{--}$mientras que el negativo está asociado a $\mathcal{C}^{++}$.

Continuaremos nuestros razonamientos refiriéndonos a la primera de las posibilidades. Consideraremos por tanto la circunferencia:

$$
\left(q_{1}+\frac{1}{4}\right)^{2}+q_{2}^{2}=\frac{9}{16}
$$

(ver Figura 5.5).

En la referencia [87] se obtuvo esta órbita tanteando sobre las ecuaciones de segundo orden del sistema.

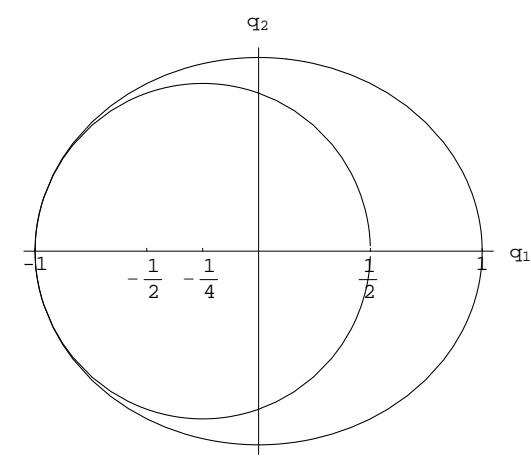

Figura 5.5: Gráfica de la circunferencia (5.22) interior a la elipse (5.12).

Podemos ahora calcular la ecuación del correspondiente kink como geodésica de la métrica de Jacobi, de igual manera que lo hemos realizado para los kinks TK1 y TK2. La métrica de Jacobi se restringe a (5.22) de la forma:

$$
h_{11}=h_{22}=\frac{3}{8}\left(1+q_{1}\right) ; \quad h_{12}=h_{21}=0
$$

mientras que los símbolos de Christoffel se reducen a:

$$
\Gamma_{11}^{1 J}=-\frac{8}{3} q_{1} ; \quad \Gamma_{12}^{1 J}= \pm \frac{-\sqrt{2}}{3} \sqrt{1-q_{1}-2 q_{1}^{2}} \frac{4 q_{1}+3}{q_{1}+1}
$$


donde la ambigüedad de signos se corresponde con la elección de una de las semicircunferencias: $q_{2}= \pm \frac{1}{\sqrt{2}} \sqrt{1-q_{1}-2 q_{1}^{2}}$, y donde el resto de los símbolos no nulos dependen de los anteriores de la forma ya explicitada en las secciones precedentes.

Las ecuaciones de las geodésicas $(5.3,5.4)$, restringidas a la órbita $(5.22)$, se reducen a una única ecuación en la variable $q_{1}$ :

$$
q_{1}^{\prime \prime}+\frac{q_{1}^{\prime 2}}{1-2 q_{1}}=0
$$

cuya resolución se realiza de manera análoga a las correspondientes a los kinks TK1 y TK2 realizadas anteriormente. Se obtiene:

$$
\frac{d q_{1}}{d s}=k \sqrt{1-2 q_{1}}
$$

$\mathrm{y}$, tras integrar la cuadratura correspondiente:

$$
k s+s_{0}=-\sqrt{1-2 \bar{q}_{1}}
$$

La determinación de la constante $k$ se realiza de forma análoga a los casos anteriores:

$$
\left\|c^{\prime}\right\|^{J}=1 \Rightarrow \sqrt{\frac{d \bar{q}_{1}}{d s} h_{11} \frac{d \bar{q}_{1}}{d s}+\frac{d \bar{q}_{2}}{d s} h_{22} \frac{d \bar{q}_{2}}{d s}}=1
$$

donde evidentemente:

$$
\frac{d \bar{q}_{2}}{d s}= \pm \frac{1}{2 \sqrt{2}} \frac{-\left(1+4 \bar{q}_{1}\right)}{\sqrt{1-\bar{q}_{1}-2 \bar{q}_{1}^{2}}} \frac{d \bar{q}_{1}}{d s}
$$

Simplificando (5.25) obtenemos:

$$
k^{2}=\frac{64}{27} \Rightarrow k= \pm \frac{8}{3 \sqrt{3}}
$$

por tanto la solución buscada se escribe de la forma ${ }^{3}$ :

$$
\pm \frac{8}{3 \sqrt{3}} s=-\sqrt{1-2 \bar{q}_{1}}
$$

y, en definitiva:

$$
\begin{aligned}
& \bar{q}_{1}(s)=\frac{1}{2}-\frac{32}{27} s^{2} \\
& \bar{q}_{2}(s)= \pm \frac{4}{27} s \sqrt{81-64 s^{2}}
\end{aligned}
$$

\footnotetext{
${ }^{3}$ Evidentemente la elección del signo del primer miembro depende del correspondiente signo del parámetro $s$.
} 
Las condiciones asintóticas tomadas imponen que la solución parte y llega al punto $\left(q_{1}, q_{2}\right)=(-1,0)$ de manera que (5.27) obliga a que el rango de variación tomado para $s$ sea $\left[s_{1}, s_{2}\right]$, con:

$$
s_{1}=-\frac{9}{8}, \quad s_{2}=+\frac{9}{8}
$$

La longitud del segmento geodésico proporciona, una vez más, la acción del sistema mecánico sobre esta trayectoria y, en consecuencia, la energía de la onda solitaria:

$$
L^{J}\left[c_{\mathrm{NTK} 2}\right]=\int_{s_{1}}^{s_{2}} d s=\frac{9}{4}=\frac{\lambda^{2} \sqrt{2}}{m^{3}} E_{\mathrm{NTK} 2}
$$

coincidente con el valor calculado en el Capítulo 3 de esta memoria (para el caso $\left.\sigma=\frac{1}{2}\right)$.

Si queremos obtener la expresión de la solución en función del parámetro temporal $t$, recordemos: $d s=\frac{3}{8}\left(1+q_{1}\right) d t$, utilizando (5.24) y el valor calculado de $k$, conduce a la cuadratura:

$$
\int \frac{ \pm \sqrt{3} d q_{1}}{\left(1+q_{1}\right) \sqrt{1-2 q_{1}}}=\int d t
$$

y, finalmente, a la solución:

$$
\begin{aligned}
\bar{q}_{1}(t) & =\frac{3}{1+\cosh t}-1 \\
\bar{q}_{2}(t) & = \pm \sqrt{\frac{1}{1+\cosh t}\left(\frac{1}{2}-\frac{1}{1+\cosh t}\right)}
\end{aligned}
$$

Podemos ver representadas estas expresiones en las figuras 5.6 y 5.7.

Finalmente es interesante estudiar en este caso el comportamiento de los dos superpotenciales del modelo. La elección realizada de la órbita (5.22), en el sector topológico $\mathcal{C}^{--}$, y, por tanto, soluciones tales que parten y llegan al punto de vacío $\left(q_{1}, q_{2}\right)=(-1,0)$, determina que sólo uno de los superpotenciales es válido para la descripción de dichas soluciones. Este hecho quedará completamente de manifiesto en el estudio de las ecuaciones de primer orden que realizaremos en el Capítulo 6. De esta manera, la solución calculada verificará la identidad entre la longitud de arco de la métrica de Jacobi y el superpotencial del modelo sobre la solución únicamente para la función $W^{-}$, mientras que las correspondientes soluciones de $\mathcal{C}^{++}$tendrán como superpotencial asociado a $W^{+}$. 

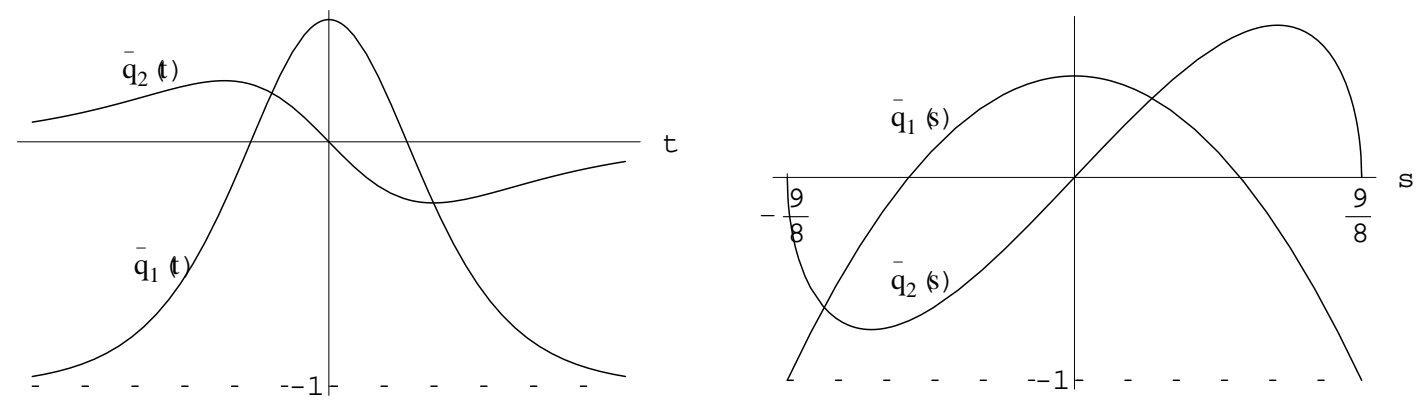

Figura 5.6: Gráficas de las funciones $\left(\bar{q}_{1}(t), \bar{q}_{2}(t)\right)$ y de las correspondientes $\left(\bar{q}_{1}(s), \bar{q}_{2}(s)\right)$ para el kink NTK2(0) $\left(\sigma=\frac{1}{2}\right)$.

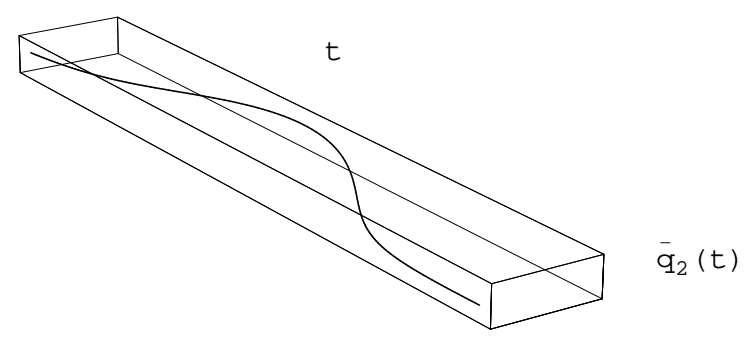

$\bar{q}_{1}(t)$

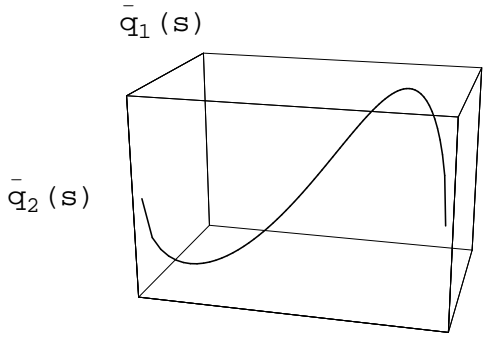

$\mathrm{S}$

Figura 5.7: Representación tridimensional de la curva (5.22) frente al parámetro $t$ (en (a)) y $s$ (en (b)) con las parametrizaciones $\left(\bar{q}_{1}(t), \bar{q}_{2}(t)\right)$ y $\left(\bar{q}_{1}(s), \bar{q}_{2}(s)\right)$ respectivamente. 
Si calculamos $W^{-}$restringido a la órbita (5.22) tendremos ${ }^{4}$ :

$$
W^{-}\left(\bar{q}_{1}, \pm \sqrt{\frac{9}{16}-\left(\left(\bar{q}_{1}+\frac{1}{4}\right)^{2}\right.}\right)=-\frac{3 \sqrt{3}}{8} \sqrt{1-2 \bar{q}_{1}}
$$

y, en definitiva, $W^{-}$coincide con $s$ sobre las soluciones.

\section{Coordenadas Elípticas}

Por último, plantearemos en este apartado la situación que se presenta si se utilizan, en el Modelo MSTB, las coordenadas elípticas bidimensionales.

De acuerdo con lo establecido en el Capítulo 3, denominaremos $\mu_{1}$ y $\mu_{2}$ a dichas coordenadas.

La métrica en elípticas se escribía (ver Capítulo 2) de la forma:

$$
g=\left(\begin{array}{cc}
g_{11} & 0 \\
0 & g_{22}
\end{array}\right)
$$

donde:

$$
g_{11}=\frac{-1}{4} \frac{\mu_{1}-\mu_{2}}{\left(\mu_{1}-1\right)\left(\mu_{1}-\bar{\sigma}^{2}\right)} ; \quad g_{22}=\frac{-1}{4} \frac{\mu_{2}-\mu_{1}}{\left(\mu_{2}-1\right)\left(\mu_{2}-\bar{\sigma}^{2}\right)}
$$

y donde la definición de las coordenadas establecía los rangos de variación siguientes: $0 \leq \mu_{1} \leq \bar{\sigma}^{2}, \bar{\sigma}^{2} \leq \mu_{2} \leq 1$.

El Lagrangiano del sistema dinámico se escribirá entonces de la forma:

$$
L=\frac{1}{2} g_{11} \dot{\mu}_{1}^{2}+\frac{1}{2} g_{22} \dot{\mu}_{2}^{2}+\frac{1}{2\left(\mu_{2}-\mu_{1}\right)}\left(-\mu_{1}^{3}+\bar{\sigma}^{2} \mu_{1}^{2}+\mu_{2}^{3}-\bar{\sigma}^{2} \mu_{2}^{2}\right)
$$

Los símbolos de Christoffel de la métrica (5.30) son:

$$
\begin{aligned}
\Gamma_{11}^{1} & =\frac{1}{2\left(\mu_{1}-\mu_{2}\right)}-\frac{2 \mu_{1}-\left(1+\bar{\sigma}^{2}\right)}{2\left(\mu_{1}-1\right)\left(\mu_{1}-\bar{\sigma}^{2}\right)} \\
\Gamma_{12}^{1} & =\frac{-1}{2\left(\mu_{1}-\mu_{2}\right)} \\
\Gamma_{22}^{1} & =\frac{\left(\mu_{1}-1\right)\left(\mu_{1}-\bar{\sigma}^{2}\right)}{2\left(\mu_{2}-1\right)\left(\mu_{2}-\bar{\sigma}^{2}\right)\left(\mu_{1}-\mu_{2}\right)}
\end{aligned}
$$

\footnotetext{
${ }^{4}$ Es apropiado recordar, dada la definición de superpotencial que se hizo en el Capítulo 4, que si $W$ es un superpotencial válido para una teoría, entonces $-W$ también lo es. De esta manera (5.29) coincide completamente con la expresión (5.26).
} 


$$
\begin{aligned}
\Gamma_{11}^{2} & =\frac{\left(\mu_{2}-1\right)\left(\mu_{2}-\bar{\sigma}^{2}\right)}{2\left(\mu_{1}-1\right)\left(\mu_{1}-\bar{\sigma}^{2}\right)\left(\mu_{2}-\mu_{1}\right)} \\
\Gamma_{12}^{2} & =\frac{-1}{2\left(\mu_{2}-\mu_{1}\right)} \\
\Gamma_{22}^{2} & =\frac{1}{2\left(\mu_{2}-\mu_{1}\right)}-\frac{2 \mu_{2}-\left(1+\bar{\sigma}^{2}\right)}{2\left(\mu_{2}-1\right)\left(\mu_{2}-\bar{\sigma}^{2}\right)}
\end{aligned}
$$

La métrica de Jacobi para este caso se escribirá:

$$
h=\frac{1}{\mu_{2}-\mu_{1}}\left[-\left(\mu_{1}^{3}-\bar{\sigma}^{2} \mu_{1}^{2}\right)+\left(\mu_{2}^{3}-\bar{\sigma}^{2} \mu_{2}^{2}\right)\right] g
$$

mientras que los símbolos de Christoffel son:

$$
\begin{aligned}
\Gamma_{11}^{1 J}= & \frac{\mu_{1}^{4}-2 \mu_{1}^{3}\left(1+\bar{\sigma}^{2}\right)+\mu_{1}^{2} \bar{\sigma}^{2}\left(\bar{\sigma}^{2}+4\right)-2 \mu_{1} \bar{\sigma}^{4}}{\left.2\left(\mu_{1}-1\right)\left(\mu_{1}-\bar{\sigma}^{2}\right)\left(\mu_{1}-\mu_{2}\right)\left(\mu_{1}\left(\mu_{1}-\bar{\sigma}^{2}\right)\right)+\mu_{1} \mu_{2}+\mu_{2}\left(\mu_{2}-\bar{\sigma}^{2}\right)\right)} \\
& +\frac{-\mu_{2}^{3}\left(1+\bar{\sigma}^{2}\right)+\mu_{2}^{2}\left(1+\bar{\sigma}^{2}\right) \bar{\sigma}^{2}+2 \mu_{1}\left(\mu_{2}^{3}-\mu_{2}^{2} \bar{\sigma}^{2}\right)}{\left.2\left(\mu_{1}-1\right)\left(\mu_{1}-\bar{\sigma}^{2}\right)\left(\mu_{1}-\mu_{2}\right)\left(\mu_{1}\left(\mu_{1}-\bar{\sigma}^{2}\right)\right)+\mu_{1} \mu_{2}+\mu_{2}\left(\mu_{2}-\bar{\sigma}^{2}\right)\right)} \\
\Gamma_{12}^{1 J}= & \frac{-\mu_{2}\left(3 \mu_{2}-2 \bar{\sigma}^{2}\right)}{2\left(\mu_{1}-\mu_{2}\right)\left(\mu_{1}\left(\mu_{1}-\bar{\sigma}^{2}\right)+\mu_{2}\left(\mu_{2}-\bar{\sigma}^{2}\right)+\mu_{1} \mu_{2}\right)} \\
\Gamma_{22}^{1 J}= & \frac{\mu_{1}\left(\mu_{1}-1\right)\left(\mu_{1}-\bar{\sigma}^{2}\right)\left(3 \mu_{1}-2 \bar{\sigma}^{2}\right)}{2\left(\mu_{1}-\mu_{2}\right)\left(\mu_{2}-1\right)\left(\mu_{2}-\bar{\sigma}^{2}\right)\left(\mu_{1}\left(\mu_{1}-\bar{\sigma}^{2}\right)+\mu_{2}\left(\mu_{2}-\bar{\sigma}^{2}\right)+\mu_{1} \mu_{2}\right)} \\
\Gamma_{11}^{2 J}= & \frac{-\mu_{2}\left(\mu_{2}-1\right)\left(\mu_{2}-\bar{\sigma}^{2}\right)\left(3 \mu_{2}-2 \bar{\sigma}^{2}\right)}{2\left(\mu_{1}-\mu_{2}\right)\left(\mu_{1}-1\right)\left(\mu_{1}-\bar{\sigma}^{2}\right)\left(\mu_{1}\left(\mu_{1}-\bar{\sigma}^{2}\right)+\mu_{2}\left(\mu_{2}-\bar{\sigma}^{2}\right)+\mu_{1} \mu_{2}\right)} \\
\Gamma_{12}^{2 J}= & \frac{\mu_{1}\left(3 \mu_{1}-2 \bar{\sigma}^{2}\right)}{2\left(\mu_{1}-\mu_{2}\right)\left(\mu_{1}\left(\mu_{1}-\bar{\sigma}^{2}\right)+\mu_{2}\left(\mu_{2}-\bar{\sigma}^{2}\right)+\mu_{1} \mu_{2}\right)} \\
\Gamma_{22}^{2 J}= & \frac{\mu_{2}\left(\mu_{2}-2\right)\left(\mu_{2}-\bar{\sigma}^{2}\right)^{2}+\left(2 \mu_{2}-1-\bar{\sigma}^{2}\right) \mu_{1}^{2}\left(\mu_{1}-\bar{\sigma}^{2}\right)}{2\left(\mu_{2}-1\right)\left(\mu_{2}-\bar{\sigma}^{2}\right)\left(-\mu_{1}^{2}\left(\mu_{1}-\bar{\sigma}^{2}\right)+\mu_{2}^{2}\left(\mu_{2}-\bar{\sigma}^{2}\right)\right)}
\end{aligned}
$$

además de los resultantes de las simetrías obvias de los mismos.

Las ecuaciones de las geodésicas serán, en estas coordenadas:

$$
\begin{aligned}
& \mu_{1}^{\prime \prime}+\Gamma_{11}^{1 J} \mu_{1}^{\prime 2}+2 \Gamma_{12}^{1 J} \mu_{1}^{\prime} \mu_{2}^{\prime}+\Gamma_{22}^{1 J} \mu_{2}^{\prime 2}=0 \\
& \mu_{2}^{\prime \prime}+\Gamma_{11}^{2 J} \mu_{1}^{\prime 2}+2 \Gamma_{12}^{2 J} \mu_{1}^{\prime} \mu_{2}^{\prime}+\Gamma_{22}^{2 J} \mu_{2}^{\prime 2}=0
\end{aligned}
$$

De la expresión de los símbolos de Christoffel se intuye que la resolución de (5.31, 5.32) es complicada y, por tanto, no nos detendremos en realizarla, habida cuenta, por otra parte, que conocemos sus soluciones, obtenidas en el Capítulo 3 por el método de Hamilton-Jacobi.

En el caso particular de los kinks tipo TK2, las ecuaciones $(5.31,5.32)$ se simplifican notablemente. Mostraremos a continuación dicho cálculo. 


\section{TK2}

La elipse $q_{1}^{2}+\frac{q_{2}^{2}}{\bar{\sigma}^{2}}=1$, sobre la que yacen los kinks de este tipo, tiene por ecuación en coordenadas elípticas:

$$
\mu_{1}=0
$$

La métrica de Jacobi restringida a la elipse se escribe de la forma sencilla:

$$
h_{i j}=\left(\mu_{2}^{2}-\bar{\sigma}^{2} \mu_{2}\right) g_{i j}
$$

y los símbolos de Christoffel sobre la misma se reducen a:

$$
\begin{aligned}
\Gamma_{11}^{1 J} & =\frac{1+\bar{\sigma}^{2}}{2 \bar{\sigma}^{2}} \\
\Gamma_{12}^{1 J} & =\frac{3 \mu_{2}-2 \bar{\sigma}^{2}}{2 \mu_{2}\left(\mu_{2}-\bar{\sigma}^{2}\right)} \\
\Gamma_{22}^{1 J} & =0 \\
\Gamma_{11}^{2 J} & =\frac{\left(\mu_{2}-1\right)\left(3 \mu_{2}-2 \bar{\sigma}^{2}\right)}{2 \bar{\sigma}^{2} \mu_{2}} \\
\Gamma_{12}^{2 J} & =0 \\
\Gamma_{22}^{2 J} & =\frac{\mu_{2}-2}{2 \mu_{2}\left(\mu_{2}-1\right)}
\end{aligned}
$$

De esta manera, las ecuaciones de las geodésicas (5.31, 5.32), sobre la elipse, se simplifican a una única ecuación:

$$
\mu_{2}^{\prime \prime}+\frac{\mu_{2}-2}{2 \mu_{2}\left(\mu_{2}-1\right)} \mu_{2}^{\prime 2}=0
$$

con $\mu_{2}^{\prime}=\frac{d \mu_{2}}{d s}$.

La resolución de (5.33) se realiza con el mismo cambio de variable utilizado en las secciones anteriores:

$$
p_{2}=\mu_{2}^{\prime} ; \quad \mu_{2}^{\prime \prime}=p_{2} \frac{d p_{2}}{d \mu_{2}}
$$

que nos conduce a la ecuación de primer orden:

$$
\frac{d p_{2}}{d \mu_{2}}=-\frac{\mu_{2}-2}{2 \mu_{2}\left(\mu_{2}-1\right)}
$$

cuya integral general es:

$$
p_{2}=\frac{k \sqrt{1-\mu_{2}}}{\mu_{2}}
$$


Sustituyendo e integrando obtenemos:

$$
\frac{-2}{3 k} \sqrt{1-\bar{\mu}_{2}}\left(2+\bar{\mu}_{2}\right)=s-s_{0}
$$

ecuación que nos proporciona de manera implícita la dependencia de la solución en el parámetro arco de la métrica de Jacobi.

La constante $s_{0}$ puede ser fijada de nuevo, sin pérdica de generalidad. Tomaremos de esta forma $s_{0}=0$. En lo que respecta a la constante $k$ encontramos, una vez más, que la condición de parámetro longitud de arco para la variable $s$ determina su valor inequívocamente:

$$
\sqrt{\frac{d \bar{\mu}_{2}}{d s} h_{22} \frac{d \bar{\mu}_{2}}{d s}}=1 \Rightarrow k= \pm 2
$$

y, en consecuencia, escribiremos (5.34) de la forma:

$$
\pm \frac{1}{3} \sqrt{1-\bar{\mu}_{2}}\left(2+\bar{\mu}_{2}\right)=s
$$

Una vez más encontramos que la longitud de arco, dada por la ecuación (5.35), coincide con el valor del superpotencial sobre la solución, en este caso en coordenadas elípticas (que será calculado en el Capítulo 6). Teniendo en cuenta que $W^{ \pm}$ se expresa en términos de las coordenadas elípticas como ${ }^{5}$ :

$$
W^{ \pm}\left(\mu_{1}, \mu_{2}\right)=\frac{1}{3} \sqrt{1-\mu_{1}}\left(\mu_{1}+2\right) \pm \frac{1}{3} \sqrt{1-\mu_{2}}\left(\mu_{2}+2\right)
$$

obtendremos:

$$
W^{ \pm}\left(0, \bar{\mu}_{2}\right)= \pm \frac{1}{3} \sqrt{1-\bar{\mu}_{2}}\left(2+\bar{\mu}_{2}\right)
$$

como habíamos comentado.

Si se considera que la relación entre la longitud de arco de la métrica de Jacobi y el parámetro temporal del sistema se escribe, para este caso, en la forma:

$$
d s=\mu_{2}\left(\mu_{2}-\bar{\sigma}^{2}\right) d t
$$

es fácil deducir la expresión de $\mu_{2}$ en términos del parámetro $t$. Integrando:

$$
d \mu_{2}= \pm 2 \sqrt{1-\mu_{2}}\left(\mu_{2}-\bar{\sigma}^{2}\right) d t
$$

el resultado coincide, evidentemente, con el obtenido en la sección 3.1:

$$
\bar{\mu}_{2}(t)=1-\sigma^{2} \tanh ^{2}(\sigma t)
$$

\footnotetext{
${ }^{5}$ De nuevo salvo signos globales.
} 


\subsubsection{Modelos Sigma $O(3)$ y $O(N)$ Deformados}

En el apartado anterior hemos restringido la resolución de las ecuaciones de las geodésicas al Modelo Sigma Lineal $O(2)$ Deformado. Generalizaremos ahora dichos resultados al caso tridimensional, el Modelo $O(3)$, y al caso general con $N$ campos escalares. Como ya se ha comentado, la resolución general, aunque interesante, es difícil y no aporta resultados cualitativamente novedosos. Por esta razón limitaremos nuestro análisis al planteamiento del problema y a las particularizaciones triviales, como son el cálculo de las soluciones TK1, TK2 $\sigma_{2}$ y TK2 $\sigma_{3}$.

\section{Modelo $O(3)$}

La métrica de Jacobi para el modelo tridimensional se escribe en la forma:

$$
h_{i j}=\left[\left(q_{1}^{2}+q_{2}^{2}+q_{3}^{2}-1\right)^{2}+\sigma_{2}^{2} q_{2}^{2}+\sigma_{3}^{2} q_{3}^{2}\right] \delta_{i j}
$$

Los símbolos de Cristofell en este caso son:

$$
\begin{aligned}
\Gamma_{11}^{1 J} & =\frac{2 q_{1}\left(q_{1}^{2}+q_{2}^{2}+q_{3}^{2}-1\right)}{\left(q_{1}^{2}+q_{2}^{2}+q_{3}^{2}-1\right)^{2}+\sigma_{2}^{2} q_{2}^{2}+\sigma_{3}^{2} q_{3}^{2}} \\
\Gamma_{12}^{1 J} & =\frac{q_{2}\left(2\left(q_{1}^{2}+q_{2}^{2}+q_{3}^{2}-1\right)+\sigma_{2}^{2}\right)}{\left(q_{1}^{2}+q_{2}^{2}+q_{3}^{2}-1\right)^{2}+\sigma_{2}^{2} q_{2}^{2}+\sigma_{3}^{2} q_{3}^{2}} \\
\Gamma_{13}^{1 J} & =\frac{q_{3}\left(2\left(q_{1}^{2}+q_{2}^{2}+q_{3}^{2}-1\right)+\sigma_{3}^{2}\right)}{\left(q_{1}^{2}+q_{2}^{2}+q_{3}^{2}-1\right)^{2}+\sigma_{2}^{2} q_{2}^{2}+\sigma_{3}^{2} q_{3}^{2}} \\
\Gamma_{22}^{1 J} & =\frac{-2 q_{1}\left(q_{1}^{2}+q_{2}^{2}+q_{3}^{2}-1\right)}{\left(q_{1}^{2}+q_{2}^{2}+q_{3}^{2}-1\right)^{2}+\sigma_{2}^{2} q_{2}^{2}+\sigma_{3}^{2} q_{3}^{2}} \\
\Gamma_{23}^{1 J} & =0 \\
\Gamma_{33}^{1 J} & =\frac{-2 q_{1}\left(q_{1}^{2}+q_{2}^{2}+q_{3}^{2}-1\right)}{\left(q_{1}^{2}+q_{2}^{2}+q_{3}^{2}-1\right)^{2}+\sigma_{2}^{2} q_{2}^{2}+\sigma_{3}^{2} q_{3}^{2}} \\
\Gamma_{11}^{2 J} & =\frac{-q_{2}\left(2\left(q_{1}^{2}+q_{2}^{2}+q_{3}^{2}-1\right)+\sigma_{2}^{2}\right)}{\left(q_{1}^{2}+q_{2}^{2}+q_{3}^{2}-1\right)^{2}+\sigma_{2}^{2} q_{2}^{2}+\sigma_{3}^{2} q_{3}^{2}} \\
\Gamma_{12}^{2 J} & =\frac{2 q_{1}\left(q_{1}^{2}+q_{2}^{2}+q_{3}^{2}-1\right)}{\left(q_{1}^{2}+q_{2}^{2}+q_{3}^{2}-1\right)^{2}+\sigma_{2}^{2} q_{2}^{2}+\sigma_{3}^{2} q_{3}^{2}} \\
\Gamma_{13}^{2 J} & =0 \\
\Gamma_{22}^{2 J} & =\frac{q_{2}\left(2\left(q_{1}^{2}+q_{2}^{2}+q_{3}^{2}-1\right)+\sigma_{2}^{2}\right)}{\left(q_{1}^{2}+q_{2}^{2}+q_{3}^{2}-1\right)^{2}+\sigma_{2}^{2} q_{2}^{2}+\sigma_{3}^{2} q_{3}^{2}} \\
\Gamma_{23}^{2 J} & =\frac{q_{3}\left(2\left(q_{1}^{2}+q_{2}^{2}+q_{3}^{2}-1\right)+\sigma_{3}^{2}\right)}{\left(q_{1}^{2}+q_{2}^{2}+q_{3}^{2}-1\right)^{2}+\sigma_{2}^{2} q_{2}^{2}+\sigma_{3}^{2} q_{3}^{2}}
\end{aligned}
$$




$$
\begin{aligned}
\Gamma_{33}^{2 J} & =\frac{-q_{2}\left(2\left(q_{1}^{2}+q_{2}^{2}+q_{3}^{2}-1\right)+\sigma_{2}^{2}\right)}{\left(q_{1}^{2}+q_{2}^{2}+q_{3}^{2}-1\right)^{2}+\sigma_{2}^{2} q_{2}^{2}+\sigma_{3}^{2} q_{3}^{2}} \\
\Gamma_{11}^{3 J} & =\frac{-q_{3}\left(2\left(q_{1}^{2}+q_{2}^{2}+q_{3}^{2}-1\right)+\sigma_{3}^{2}\right)}{\left(q_{1}^{2}+q_{2}^{2}+q_{3}^{2}-1\right)^{2}+\sigma_{2}^{2} q_{2}^{2}+\sigma_{3}^{2} q_{3}^{2}} \\
\Gamma_{12}^{3 J} & =0 \\
\Gamma_{13}^{3 J} & =\frac{2 q_{1}\left(q_{1}^{2}+q_{2}^{2}+q_{3}^{2}-1\right)}{\left(q_{1}^{2}+q_{2}^{2}+q_{3}^{2}-1\right)^{2}+\sigma_{2}^{2} q_{2}^{2}+\sigma_{3}^{2} q_{3}^{2}} \\
\Gamma_{22}^{3 J} & =\frac{-q_{3}\left(2\left(q_{1}^{2}+q_{2}^{2}+q_{3}^{2}-1\right)+\sigma_{3}^{2}\right)}{\left(q_{1}^{2}+q_{2}^{2}+q_{3}^{2}-1\right)^{2}+\sigma_{2}^{2} q_{2}^{2}+\sigma_{3}^{2} q_{3}^{2}} \\
\Gamma_{23}^{3 J} & =\frac{q_{2}\left(2\left(q_{1}^{2}+q_{2}^{2}+q_{3}^{2}-1\right)+\sigma_{2}^{2}\right)}{\left(q_{1}^{2}+q_{2}^{2}+q_{3}^{2}-1\right)^{2}+\sigma_{2}^{2} q_{2}^{2}+\sigma_{3}^{2} q_{3}^{2}} \\
\Gamma_{33}^{3 J} & =\frac{q_{2}\left(2\left(q_{1}^{2}+q_{2}^{2}+q_{3}^{2}-1\right)+\sigma_{2}^{2}\right)}{\left(q_{1}^{2}+q_{2}^{2}+q_{3}^{2}-1\right)^{2}+\sigma_{2}^{2} q_{2}^{2}+\sigma_{3}^{2} q_{3}^{2}}
\end{aligned}
$$

Escribiremos en consecuencia las ecuaciones de las geodésicas de manera compacta:

$$
\begin{aligned}
& q_{1}^{\prime \prime}+\Gamma_{11}^{1 J} q_{1}^{\prime 2}+2 \Gamma_{12}^{1 J} q_{1}^{\prime} q_{2}^{\prime}+2 \Gamma_{13}^{1 J} q_{1}^{\prime} q_{3}^{\prime}+\Gamma_{22}^{1 J} q_{2}^{\prime 2}+\Gamma_{33}^{1 J} q_{3}^{\prime 2}=0 \\
& q_{2}^{\prime \prime}+\Gamma_{11}^{2 J} q_{1}^{\prime 2}+2 \Gamma_{12}^{2 J} q_{1}^{\prime} q_{2}^{\prime}+2 \Gamma_{23}^{2 J} q_{2}^{\prime} q_{3}^{\prime}+\Gamma_{22}^{2 J} q_{2}^{\prime 2}+\Gamma_{33}^{2 J} q_{3}^{\prime 2}=0 \\
& q_{3}^{\prime \prime}+\Gamma_{11}^{3 J} q_{1}^{\prime 2}+2 \Gamma_{13}^{3 J} q_{1}^{\prime} q_{3}^{\prime}+2 \Gamma_{23}^{3 J} q_{2}^{\prime} q_{3}^{\prime}+\Gamma_{22}^{3 J} q_{2}^{\prime 2}+\Gamma_{33}^{3 J} q_{3}^{\prime 2}=0
\end{aligned}
$$

Particularizaremos a continuación las ecuaciones (5.36) a los tipos de kinks que se han descrito en la sección anterior.

\section{TK1}

Las ecuaciones (5.36), restringidas a la órbita $q_{2}=q_{3}=0$, se reducen exactamente a la misma ecuación que obteníamos en el apartado anterior.

La primera de la ecuaciones (5.36) se escribe sobre la órbita TK1 de la forma:

$$
q_{1}^{\prime \prime}-\frac{2 q_{1}}{1-q_{1}^{2}} q_{1}^{\prime 2}=0
$$

mientras que las dos restantes se trivializan a la identidad $0=0$.

La resolución de este caso es, por tanto, la realizada para el modelo MSTB.

\section{TK2 $\sigma_{2}$}

La restricción a la elipse

$$
q_{1}^{2}+\frac{q_{2}^{2}}{1-\sigma_{2}^{2}}=1 ; \quad q_{3}=0
$$


reduce los símbolos de Christoffel a los correspondientes del Modelo $O(2)$ y las ecuaciones (5.36) reproducen las ya escritas para los kinks del tipo TK2. En este caso se tiene el plano coordenado por $\left(q_{1}, q_{2}, 0\right)$ y la constante $\sigma$ es ahora $\sigma_{2}$.

\section{$\mathrm{TK} 2 \sigma_{3}$}

Finalmente, la órbita:

$$
q_{1}^{2}+\frac{q_{3}^{2}}{1-\sigma_{3}^{2}}=1 ; \quad q_{2}=0
$$

conduce a idénticos resultados en el plano coordenado por $\left(q_{1}, 0, q_{3}\right)$ y con constante asociada: $\sigma=\sigma_{3}$.

\section{Modelo $O(N)$}

La generalización al caso tridimensional de los cálculos realizados para el modelo MSTB conduce fácilmente a la extensión de dichos resultados al caso de $N$ campos escalares.

La métrica de Jacobi será ahora, de manera genérica:

$$
h=\left[\left(\sum_{a=1}^{N} q_{a}^{2}-1\right)^{2}+\sum_{b=2}^{N} \sigma_{b}^{2} q_{b}^{2}\right] g
$$

siendo $g$ la métrica euclídea.

Los símbolos de Christoffel asociados son

$$
\begin{aligned}
& \Gamma_{a b}^{c J}=0 ; \quad \forall a \neq b, b \neq c, c \neq a \\
& \Gamma_{a a}^{a J}=\frac{q_{a}\left(2\left(\sum_{b=1}^{N} q_{b}^{2}-1\right)+\sigma_{a}^{2}\right)}{\left(\sum_{c=1}^{N} q_{c}^{2}-1\right)^{2}+\sum_{b=2}^{N} \sigma_{b}^{2} q_{b}^{2}} ; \forall a=1, \ldots, N \\
& \Gamma_{a b}^{a J}=\frac{q_{b}\left(2\left(\sum_{c=1}^{N} q_{c}^{2}-1\right)+\sigma_{b}^{2}\right)}{\left(\sum_{c=1}^{N} q_{c}^{2}-1\right)^{2}+\sum_{d=2}^{N} \sigma_{d}^{2} q_{d}^{2}} ; \quad \forall a, b \\
& \Gamma_{b b}^{a J}=-\Gamma_{a a}^{a J} ; \quad \forall a, b
\end{aligned}
$$

\subsection{Funcional Segunda Variación. Modelo $O(2)$}

Calcularemos en esta sección los funcionales hessianos de la acción y de la longitud y energía, en la métrica de Jacobi, asociada correspondientes a los kinks TK1 y 
TK2. Las expresiones generales de dichos funcionales así como los teoremas que relacionan unos con otros han sido detallados en el Capítulo 4.

La determinación del espectro de estos funcionales será llevada a cabo utilizando las técnicas desarrolladas en el capítulo precedente acerca de la diagonalización de la curvatura seccional en una base que se traslade paralelamente a lo largo de la curva solución. Este razonamiento geométrico, aplicado en el caso del funcional variación segunda de la acción del sistema dinámico, nos permitirá establecer analíticamente la estabilidad de la solución TK2 y la inestabilidad del TK1, para el Modelo MSTB.

Con respecto a la notación utilizada, de cara a evitar posibles confusiones hemos estimado oportuno cambiar algunos criterios con respecto a los adoptados en el Capítulo 4, donde se demostraban los resultados generales. De esta manera hemos incluido el símbolo de vector sobre los campos asociados a las variaciones de las curvas y hemos abandonado el criterio de Einstein de suma en índices repetidos, como en la sección anterior. La notación tensorial de índices arriba y abajo, de acuerdo con el carácter covariante o contravariante, ha sido también abandonada de manera general.

\subsubsection{Funcional Hessiano Dinámico: TK1}

Según se estableció en el Teorema 4.2, el funcional hessiano $\frac{d^{2} S[c]}{d \xi^{2}}(0)$, para variaciones propias, se escribe:

$$
\begin{aligned}
\frac{d^{2} S[c]}{d \xi^{2}}(0) & =\quad-\int_{t_{1}}^{t_{2}} d t\left(\left\langle\frac{\mathrm{D}^{2} \vec{V}}{\partial t^{2}}+K_{\dot{c}}(\vec{V}), \vec{V}\right\rangle+H(U)(\vec{V}, \vec{V})\right)= \\
& =-\int_{t_{1}}^{t_{2}} d t\left\langle\frac{\mathrm{D}^{2} \vec{V}}{\partial t^{2}}+K_{\dot{c}}(\vec{V})+\nabla_{\vec{V}} \operatorname{grad}(U), \vec{V}\right\rangle= \\
& =\int_{t_{1}}^{t_{2}} d t\langle\Delta \vec{V}, \vec{V}\rangle
\end{aligned}
$$

donde $\Delta$ es el operador diferenciable (operador Hessiano):

$$
\Delta \vec{V}=-\frac{\mathrm{D}^{2} \vec{V}}{\partial t^{2}}-K_{\dot{c}}(\vec{V})-\nabla_{\vec{V}} \operatorname{grad}(U)
$$

y donde además, de acuerdo con lo antedicho, se ha adoptado la notación vectorial $(\vec{V})$ para los campos de cara a una mayor claridad. 
Si tenemos en cuenta que para el sistema en estudio, en coordenadas cartesianas, la métrica es euclídea, se verificará:

$$
g_{i j}=\delta_{i j} \quad \Rightarrow \quad \Gamma_{j k}^{i}=0 ; \quad R_{i j k}^{l}=0 ; \quad \frac{\mathrm{D}}{d t}=\frac{d}{d t}
$$

$\forall i, j, k, l$, y de esta manera podemos calcular:

$$
\nabla_{\vec{V}} \operatorname{grad}(U)=\sum_{i=1}^{2} \sum_{j=1}^{2} V^{i} \partial_{i}(\operatorname{grad} U)^{j} \partial_{j}=\sum_{i=1}^{2} \sum_{j=1}^{2} \frac{\partial^{2} U}{\partial q^{i} \partial q^{j}} V^{i} \delta^{j k} \vec{e}_{k}
$$

donde se ha denotado $\vec{e}_{j}=\partial_{j}=\frac{\partial}{\partial q^{j}}$ a la base de campos natural, en $\Gamma\left(T \mathbb{R}^{3}\right)$, del sistema de coordenadas tomado. De acuerdo con los criterios de notación que hemos mencionado, escribiremos en adelante para toda variación $\vec{V}: \vec{V}=$ $v_{1} \vec{e}_{1}+v_{2} \vec{e}_{2}$, y así (5.38) se reescribe de la forma:

$$
\nabla_{\vec{V}} \operatorname{grad}(U)=\sum_{i=1}^{2} \sum_{j=1}^{2} \frac{\partial^{2} U}{\partial q_{i} \partial q_{j}} v_{i} \vec{e}_{j}
$$

La solución TK1 nos venía dada por las expresiones:

$$
\bar{q}_{1}(t)=\tanh t ; \quad \bar{q}_{2}(t)=0
$$

con $t \in(-\infty, \infty)$.

Tal y como comprobaremos en el próximo apartado, es interesante considerar, además de la parametrización "natural" 6 en el "tiempo mecánico" $t$, otra parametrización de las soluciones, en términos de su propia primera componente, $\bar{q}_{1}$. La razón es clara, cuando pasemos a estudiar los funcionales Hessianos en la métrica de Jacobi asociada, el parámetro "natural" es la longitud de arco $s$ en dicha métrica. De acuerdo con los cálculos efectuados en la sección anterior, la obtención de la ecuación explícita que relacione $t$ y $s$ requiere la sustitución de las fórmulas de Cardano-Tartaglia en las expresiones (5.39), con la consiguiente dificultad de cálculo. Sin embargo, las expresiones de $\bar{q}_{1}$, tanto en términos de $t$ como de $s$, son sencillas. De esta manera escribiremos, junto con (5.39), las ecuaciones:

$$
\bar{q}_{1} \equiv \bar{q}_{1} ; \quad \bar{q}_{2}=0
$$

de tal manera que

$$
\dot{\bar{q}}_{1}=1-\bar{q}_{1}^{2} ; \quad \dot{\bar{q}}_{2}=0
$$

\footnotetext{
6 "Natural", evidentemente, en tanto en cuanto se trata de un sistema dinámico con un "tiempo" definido.
} 
$\operatorname{con} \bar{q}_{1} \in(-1,1)$.

Con todas estas consideraciones, podemos finalmente escribir las componentes del operador hessiano sobre la solución TK1 en la forma:

$$
\begin{gathered}
-\frac{d^{2} v_{1}}{d t^{2}}-\frac{\partial^{2} U}{\partial q_{1}^{2}} v_{1}-\frac{\partial^{2} U}{\partial q_{1} \partial q_{2}} v_{2}=-\frac{d^{2} v_{1}}{d t^{2}}+\left(6 \bar{q}_{1}^{2}-2\right) v_{1} \\
-\frac{d^{2} v_{2}}{d t^{2}}-\frac{\partial^{2} U}{\partial q_{2}^{2}} v_{2}-\frac{\partial^{2} U}{\partial q_{1} \partial q_{2}} v_{1}=-\frac{d^{2} v_{2}}{d t^{2}}+\left(2 \bar{q}_{1}^{2}-2+\sigma^{2}\right) v_{2}
\end{gathered}
$$

donde $\bar{q}_{1}=\bar{q}_{1}(t)=\tanh t$. Y, en definitiva:

$$
\begin{array}{r}
\Delta \vec{V}=\left(-\frac{d^{2}}{d t^{2}}+4-\frac{6}{\cosh ^{2} t}\right) v_{1} \vec{e}_{1}+\left(-\frac{d^{2}}{d t^{2}}+\sigma^{2}-\frac{2}{\cosh ^{2} t}\right) v_{2} \vec{e}_{2} \\
\Delta \vec{V} \equiv\left(\begin{array}{cc}
-\frac{d^{2}}{d t^{2}}+4-\frac{6}{\cosh ^{2} t} & 0 \\
0 & -\frac{d^{2}}{d t^{2}}+\sigma^{2}-\frac{2}{\cosh ^{2} t}
\end{array}\right)\left(\begin{array}{l}
v_{1} \\
v_{2}
\end{array}\right)
\end{array}
$$

O bien, en términos de $\bar{q}_{1}$ :

$$
\begin{aligned}
\Delta \vec{V} & =\left(-\left(1-\bar{q}_{1}^{2}\right)^{2} \frac{d^{2}}{d \bar{q}_{1}^{2}}+2 \bar{q}_{1}\left(1-\bar{q}_{1}^{2}\right) \frac{d}{d \bar{q}_{1}}+6 \bar{q}_{1}^{2}-2\right) v_{1} \vec{e}_{1}+ \\
& +\left(-\left(1-\bar{q}_{1}^{2}\right)^{2} \frac{d^{2}}{d \bar{q}_{1}^{2}}+2 \bar{q}_{1}\left(1-\bar{q}_{1}^{2}\right) \frac{d}{d \bar{q}_{1}}+2 \bar{q}_{1}^{2}-2+\sigma^{2}\right) v_{2} \vec{e}_{2}
\end{aligned}
$$

Se observa, por tanto, que el operador hessiano $\Delta$ es diagonal para este caso. De esta forma, la determinación de su espectro de autovalores y autofunciones separa en dos problemas independientes, cada uno de ellos equivalente a un sistema unidimensional determinado por una ecuación de Schrödinger con un potencial de tipo Pösch-Teller, de espectro bien conocido.

Las características especiales del caso TK1 (la solución permanece sobre la recta $q_{2}=0$ ) nos conducen, además, a considerar independientemente variaciones $\vec{V}^{\text {tg }}$ tangentes a la solución y variaciones $\vec{V}^{\perp}$ ortogonales a las mismas de manera natural, sin más que escribir:

$$
\begin{aligned}
& \vec{V}=v_{1} \vec{e}_{1}+v_{2} \vec{e}_{2}=\vec{V}^{\mathrm{tg}}+\vec{V}^{\perp} \\
& \vec{V}^{\mathrm{tg}}=v_{1} \vec{e}_{1} ; \quad \vec{V}^{\perp}=v_{2} \vec{e}_{2}
\end{aligned}
$$

En este caso, por tanto, la base ordinaria de los campos tangentes a la variedad está formada por un vector tangente a la solución y otro ortogonal a la misma. En general, para otro tipo de soluciones, esto, evidentemente, no ocurre. Por analogía con el método empleado para estudiar la ecuación de desviación geodésica, o si se quiere, el problema espectral de los funcionales de energía y de longitud geométricos, expresaremos siempre las variaciones en una base de estas características, a las que denominaremos, por razones evidentes, bases de Frenet. 


\section{Variaciones Tangentes}

Dada una variación tangente a la solución: $\vec{V}^{\mathrm{tg}}=v_{1} \vec{e}_{1}$, tendremos:

$$
\Delta \vec{V}^{\mathrm{tg}}=\left(-\frac{d^{2}}{d t^{2}}+4-\frac{6}{\cosh ^{2} t}\right) v_{1} \vec{e}_{1}
$$

o bien

$$
\Delta \vec{V}^{\operatorname{tg}}=\left(-\left(1-\bar{q}_{1}^{2}\right)^{2} \frac{d^{2}}{d \bar{q}_{1}^{2}}+2 \bar{q}_{1}\left(1-\bar{q}_{1}^{2}\right) \frac{d}{d \bar{q}_{1}}+6 \bar{q}_{1}^{2}-2\right) v_{1} \vec{e}_{1}
$$

El espectro de (5.44) es bien conocido, como ya hemos dicho (ver por ejemplo [68], y [73] para el caso general). De hecho, la ecuación espectral del operador $\Delta$, escrito de la forma (5.45), se reduce a la ecuación que determina los polinomios de Legendre o, alternativamente, a la correspondiente a los polinomios de Jacobi (los polinomios de Jacobi dependientes de la variable tanh $t$ son equivalentes a los polinomios de Legendre en la variable $t$, ver [2] y [28]).

El espectro consta de una parte discreta y otra continua ${ }^{7}$ :

- Espectro discreto. Formado por dos autofunciones:

$$
\begin{aligned}
& -v_{1}^{(0)}(t)=\operatorname{sech}^{2} t, \text { con autovalor } \lambda=0 . \\
& -v_{1}^{(3)}(t)=\frac{\tanh t}{\cosh t}, \text { con } \lambda=3 .
\end{aligned}
$$

- Espectro continuo:

$$
\tilde{v}_{1}^{\left(\lambda_{k}\right)}(t)=e^{i k t}\left(3 \tanh ^{2} t-1-3 i k \tanh t-k^{2}\right)
$$

con autovalores correspondientes $\lambda_{k}=k^{2}+4$, con $k \in \mathbb{R}$. La autofunción correspondiente al caso $k=0(\lambda=4)$ es:

$$
v_{1}^{(4)}(t)=1-\frac{3}{2 \cosh ^{2} t}
$$

y frecuentemente es denominada "estado semi-ligado", por tratarse de la primera autofunción del continuo.

En la expresión anterior, se han escrito las autofunciones del continuo de manera explícita. Como ya se ha comentado, dichas funciones admiten una expresión

\footnotetext{
${ }^{7}$ Denominaremos, en este caso y en los siguientes, $v_{i}^{(\lambda)}$ a la autofunción de autovalor $\lambda$, es decir: $\Delta\left(v_{i}^{(\lambda)} \vec{e}_{i}\right)=\lambda\left(v_{i}^{(\lambda)} \vec{e}_{i}\right)$
} 
general en términos de los polinomios de Jacobi de segundo orden y también en términos de las funciones hipergeométricas:

$$
\tilde{v}_{1}^{\left(\lambda_{k}\right)}(t)=\frac{1}{\cosh ^{k} t}{ }_{2} F_{1}\left(k-2, k+3, k+1, \frac{e^{t}}{e^{t}-e^{-t}}\right)=e^{i k t} P_{2}(\tanh t)
$$

donde es necesario precisar que se ha denominado (como es habitual, por otra parte) $P_{2}$ al polinomio de Jacobi $P_{2}^{(-i k, i k)}$, correspondiente a la notación general $P_{n}^{(a, b)}(x)$, soluciones de la ecuación:

$$
\left(1-x^{2}\right) y^{\prime \prime}+(b-a-(a+b+2) x) y^{\prime}+(n(n+a+b+1)) y=0
$$

Las expresiones anteriormente escritas para $\tilde{v}_{1}^{\left(\lambda_{k}\right)}$ constituyen la solución más general del espectro continuo de este problema. Si imponemos que las soluciones del problema se correspondan con funciones reales, tendremos que considerar, de forma estándar, combinaciones lineales de estas autofunciones y sus complejas conjugadas, de tal manera que se cancele la parte imaginaria de las mismas. Consideraremos, por tanto, en lugar de las autofunciones $\tilde{v}_{1}^{\left(\lambda_{k}\right)}$, las expresiones:

$$
v_{1}^{\left(\lambda_{k}\right)}(t)=\left(3 \tanh ^{2} t-1-k^{2}\right) \cos (k t)+3 k \tanh t \operatorname{sen}(k t)
$$

o, alternativamente:

$$
v_{1}^{\left(\lambda_{k}\right)}(t)=\left(3 \tanh ^{2} t-1-k^{2}\right) \operatorname{sen}(k t)-3 k \tanh t \cos (k t)
$$

Puede comprobarse fácilmente que la primera de las autofunciones anteriores se corresponde con una función par mientras que la segunda es una función impar.

La interpretación del espectro obtenido es clara si tenemos en cuenta que las variaciones tangentes a la solución representan las reparametrizaciones de la misma. El modo cero da cuenta de la invarianza bajo traslaciones temporales y, evidentemente, la autofunción no es más que la derivada de la solución, es decir, el vector velocidad:

$$
\vec{V}(t)=v_{1}^{(0)}(t) \vec{e}_{1}+0 \vec{e}_{2}=\frac{1}{\cosh ^{2} t} \vec{e}_{1}=\dot{c}_{\mathrm{TK} 1}
$$

Los restantes modos, todos de autovalor positivo, nos indican que las demás reparametrizaciones posibles aumentan la acción del sistema.

Es fácil escribir el espectro expuesto en términos del parámetro $\bar{q}_{1}$. Tendremos:

- $v_{1}^{(0)}\left(\bar{q}_{1}\right)=1-\bar{q}_{1}^{2}$. 
- $v_{1}^{(3)}\left(\bar{q}_{1}\right)=\bar{q}_{1} \sqrt{1-\bar{q}_{1}^{2}}$.

- $v_{1}^{\left(\lambda_{k}\right)}\left(\bar{q}_{1}\right)=\left(3 \bar{q}_{1}^{2}-1-k^{2}\right) \cos \left(k \operatorname{arctanh} \bar{q}_{1}\right)+3 k \bar{q}_{1} \operatorname{sen}\left(k \operatorname{arctanh} \bar{q}_{1}\right)$, o bien: $v_{1}^{\left(\lambda_{k}\right)}\left(\bar{q}_{1}\right)=\left(3 \bar{q}_{1}^{2}-1-k^{2}\right) \operatorname{sen}\left(k \operatorname{arctanh} \bar{q}_{1}\right)-3 k \bar{q}_{1} \cos \left(k \operatorname{arctanh} \bar{q}_{1}\right)$

Es necesario precisar que las autofunciones anteriores no se corresponden en general con variaciones propias del sistema. Es fácil comprobar que únicamente $\vec{V}^{\mathrm{tg}}=v_{1}^{(0)} \vec{e}_{1}$ y $\vec{V}^{\mathrm{tg}}=v_{1}^{(3)} \vec{e}_{1}$ se anulan en los extremos de la solución TK1, es decir, en $t \rightarrow \pm \infty$, o alternativamente en $\bar{q}_{1}= \pm 1$. Por otro lado, es posible construir variaciones propias, a partir de las autofunciones del espectro continuo, sin más que restringir el rango de variación de $t$ a un intervalo finito $\left[-\frac{L}{2}, \frac{L}{2}\right]$, imponer que la variación se anule en sus extremos, y considerar, posteriormente, el límite cuando la constante $L$ tiende a infinito.

Siguiendo el procedimiento habitual (ver por ejemplo [87]) impondremos que, para valores grandes de $|t|$, las variaciones (5.46) admitidas verifiquen:

$$
v_{1}^{\left(\lambda_{k_{n}}\right)}(t) \cong \frac{1}{\sqrt{k_{n}^{2}+4}} \cos \left[k_{n} t+\frac{1}{2} \delta\left(k_{n}\right)\right]
$$

donde $\delta\left(k_{n}\right)$ recibe el nombre de defasaje. Teniendo en cuenta que para $|t|$ grande se tiene $\tanh t \rightarrow \pm 1$, obtendremos:

$$
v_{1}^{\left(\lambda_{k_{n}}\right)}(t) \rightarrow\left(2-k_{n}^{2}\right) \cos \left(k_{n} t\right) \pm 3 k_{n} \operatorname{sen}\left(k_{n} t\right)
$$

y de esta manera, igualando las dos expresiones anteriores, resulta:

$$
\delta\left(k_{n}\right)= \pm 2 \arctan \frac{3 k_{n}}{2-k_{n}^{2}}
$$

La condición de variación propia se obtiene imponiendo:

$$
0=v_{1}^{\left(\lambda_{k_{n}}\right)}\left( \pm \frac{L}{2}\right)=\frac{1}{\sqrt{k_{n}^{2}+4}} \cos \left[ \pm k_{n} \frac{L}{2}+\frac{1}{2} \delta\left(k_{n}\right)\right]
$$

de manera que, finalmente, serán admisibles como variaciones propias las correspondientes a los valores de $k_{n}$ solución de la ecuación trascendente:

$$
\pm k_{n} L \pm 2 \arctan \frac{3 k_{n}}{2-k_{n}^{2}}=(2 n+1) \pi
$$

$\forall n \in \mathbb{Z}$ 
Por su parte, para las variaciones (5.47), correspondientes a funciones impares, consideraremos, de manera análoga:

$$
v_{1}^{\left(\lambda_{k_{n}}\right)}(t) \cong \frac{1}{\sqrt{k_{n}^{2}+4}} \operatorname{sen}\left[k_{n} t+\frac{1}{2} \delta\left(k_{n}\right)\right]
$$

que conduce a la misma expresión, (5.48), para el defasaje $\delta\left(k_{n}\right)$. En este caso los valores de $k_{n}$ válidos serán las soluciones de la ecuación:

$$
\pm k_{n} L \pm 2 \arctan \frac{3 k_{n}}{2-k_{n}^{2}}=2 n \pi
$$

$\forall n \in \mathbb{Z}$

Para las dos variaciones discretas y propias que obtuvimos, el funcional hessiano verifica:

$$
\begin{aligned}
\frac{d^{2} S\left[c_{\mathrm{TK} 1}\right]}{d \xi^{2}}(0) & =\int_{\infty}^{\infty} d t\left\langle\Delta\left(v_{1}^{(0)} \vec{e}_{1}\right), v_{1}^{(0)} \vec{e}_{1}\right\rangle= \\
& =\int_{-\infty}^{\infty} d t \quad 0 \frac{1}{\cosh ^{4} t}=0 \\
\frac{d^{2} S\left[c_{\mathrm{TK} 1}\right]}{d \xi^{2}}(0) & =\int_{\infty}^{\infty} d t\left\langle\Delta\left(v_{1}^{(3)} \vec{e}_{1}\right), v_{1}^{(3)} \vec{e}_{1}\right\rangle= \\
& =\int_{-\infty}^{\infty} d t 3 \frac{\tanh ^{2} t}{\cosh ^{2} t}=2>0
\end{aligned}
$$

lo cual confirma la estabilidad mencionada en la dirección tangente.

\section{Variaciones Ortogonales}

Para las variaciones ortogonales a la recta $q_{2}=0$ :

$$
\vec{V}^{\perp}=0 \vec{e}_{1}+v_{2} \vec{e}_{2}
$$

tendremos

$$
\Delta \vec{V}^{\perp}=\left(-\frac{d^{2}}{d t^{2}}+\sigma^{2}-\frac{2}{\cosh ^{2} t}\right) v_{2} \vec{e}_{2}
$$

En este caso el espectro está formado por un modo discreto, de autovalor negativo, y un espectro continuo. Veamos:

- Modo discreto:

con autovalor $\lambda=-1+\sigma^{2}$.

$$
v_{2}^{\left(-1+\sigma^{2}\right)}(t)=\frac{1}{\cosh t}
$$


- Espectro continuo ${ }^{8}$ :

$$
\tilde{v}_{2}^{\left(\lambda_{k}\right)}(t)=e^{i k t}(i k-\tanh t)
$$

con autovalores respectivos: $\lambda_{k}=k^{2}+\sigma^{2}, k \in \mathbb{R}$. Si consideramos funciones reales, de manera análoga al caso anteriormente descrito, tendremos entonces que

$$
v_{2}^{\left(\lambda_{k}\right)}(t)=\operatorname{sen}(k t) \tanh t-k \cos (k t)
$$

son autofunciones pares y

$$
v_{2}^{\left(\lambda_{k}\right)}(t)=\cos (k t) \tanh t+k \sin (k t)
$$

son las impares.

La "traducción" de este espectro al parámetro $\bar{q}_{1}$ es trivial:

- Modo negativo:

$$
v_{2}^{\left(-1+\sigma^{2}\right)}\left(\bar{q}_{1}\right)=\sqrt{1-\bar{q}_{1}^{2}}
$$

- Espectro continuo:

$$
v_{2}^{\left(\lambda_{k}\right)}\left(\bar{q}_{1}\right)=\bar{q}_{1} \operatorname{sen}\left(k \operatorname{arctanh} \bar{q}_{1}\right)-k \cos \left(k \operatorname{arctanh} \bar{q}_{1}\right)
$$

$\mathrm{y}$

$$
v_{2}^{\left(\lambda_{k}\right)}\left(\bar{q}_{1}\right)=\bar{q}_{1} \cos \left(k \operatorname{arctanh} \bar{q}_{1}\right)+k \operatorname{sen}\left(k \operatorname{arctanh} \bar{q}_{1}\right)
$$

Es necesario precisar, en este punto, que el operador hessiano tiene, además, un "modo" cero ortogonal, dado por la expresión:

$$
v_{2}^{(0)}(t)=e^{\sigma t}(\sigma-\tanh t)
$$

y con autovalor evidentemente nulo. No se trata de una variación propia y, por tanto, no está incluida en el espectro discreto propiamente dicho. Sin embargo, se trata de una autofunción que se revelará muy importante en el siguiente apartado, pues se corresponderá, desde el punto de vista geométrico, con un campo de Jacobi sobre la geodésica. Esta variación se anula en el punto inicial de la trayectoria,

\footnotetext{
${ }^{8} \mathrm{Al}$ igual que en el caso de las variaciones tangentes, la expresión general del espectro continuo puede escribirse en términos de funciones hipergeométricas o utilizando polinomios de Jacobi. De esta manera:

$$
\tilde{v}_{2}^{\left(\lambda_{k}\right)}(t)=e^{i k t}(i k-\tanh t)=e^{i k t} P_{1}(\tanh t)
$$
}


$\left(q_{1}, q_{2}\right)=(-1,0)$, y nuevamente en el foco de la elipse $q_{1}^{2}+\frac{q_{2}^{2}}{\bar{\sigma}^{2}}=1$, de coordenadas ${ }^{9}$ : $\left(q_{1}, q_{2}\right)=(\sigma, 0)$.

Con respecto al parámetro $\bar{q}_{1}$, se escribe:

$$
v_{2}^{(0)}\left(\bar{q}_{1}\right)=e^{\sigma \operatorname{arctanh} \bar{q}_{1}}\left(\sigma-\bar{q}_{1}\right)
$$

La presencia de un modo negativo (única variación propia genuina de todo el espectro, por otra parte) nos indica la inestabilidad de la solución TK1 con respecto a las variaciones ortogonales a la misma. Si calculamos el Funcional Hessiano para esta variación tendremos:

$$
\frac{d^{2} S\left[c_{\mathrm{TK} 1}\right]}{d \xi^{2}}(0)=\int_{-\infty}^{\infty} \frac{-1+\sigma^{2}}{\cosh ^{2} t} d t=-2 \bar{\sigma}^{2}<0
$$

Esto demuestra analíticamente lo que observamos en el Capítulo 3 al estudiar las energías de las diferentes soluciones, TK1 tiene más energía (desde el punto de vista de la teoría de campos, acción para el sistema dinámico) que la solución TK2, y ambas pertenecen al mismo sector topológico de la teoría, la solución estable será, en consecuencia, la de energía mínima. Demostraremos analíticamente en la siguiente sección que el kink TK2 es estable, particularmente con respecto a las variaciones dadas en la misma dirección que las $\vec{V}^{\perp}$ que aquí producen la inestabilidad; se tratará, por tanto, de la dirección "más peligrosa", en el espacio de variaciones, con respecto a la estabilidad.

$\mathrm{Al}$ igual que en el caso de las variaciones tangentes, el espectro continuo calculado puede ser "discretizado" de tal manera que podamos admitir como propias variaciones que realmente lo son en un proceso límite. Si consideramos ahora que las variaciones $(5.50), v_{2}^{\left(\lambda_{k}\right)}(t)$, para valores muy grandes de $|t|$, se escriben de la forma:

$$
v_{2}^{\left(\lambda_{k_{n}}\right)} \cong \frac{1}{\sqrt{k_{n}^{2}+4}} \cos \left[k_{n} t+\frac{1}{2} \delta\left(k_{n}\right)\right]
$$

y teniendo en cuenta que en el límite se verifica:

$$
v_{2}^{\left(\lambda_{k_{n}}\right)} \rightarrow \pm \operatorname{sen}\left(k_{n} t\right)-k_{n} \cos \left(k_{n} t\right)
$$

es fácil deducir que el defasaje, en este caso, se expresa de la forma:

$$
\delta\left(k_{n}\right)= \pm 2 \arctan \frac{1}{k_{n}}
$$

\footnotetext{
${ }^{9}$ Es conveniente precisar que se está describiendo únicamente el caso en el cual se considera el sector topológico $\mathcal{C}^{(-,+)}$, es decir la solución TK1. Si se tomara en cuenta la solución ATK1, nos aparecería un modo cero equivalente de la forma $e^{-\sigma t}(\sigma+\tanh t)$, que se anula en $\left(q_{1}, q_{2}\right)=(1,0)$ y en el foco $\left(q_{1}, q_{2}\right)=(-\sigma, 0)$.
} 
Por su parte, la imposición de variación propia conduce a que los valores de $k_{n}$ válidos son precisamente las soluciones de la ecuación:

$$
\pm k_{n} L \pm 2 \arctan \frac{1}{k_{n}}=(2 n+1) \pi
$$

con $n \in \mathbb{Z}$.

Para las variaciones impares, (5.51), la ecuación a satisfacer por los valores de $k_{n}$ admisibles será:

$$
\pm k_{n} L \pm 2 \arctan \frac{1}{k_{n}}=2 n \pi
$$

con $n \in \mathbb{Z}$.

\subsubsection{Funcionales Hessianos Geométricos: TK1}

Según se demostró en el Capítulo 4, las expresiones de los funcionales variación segunda de la Energía y de la Longitud en un sistema dinámico (vía la métrica de Jacobi asociada) se escriben respectivamente de la forma:

$$
\begin{aligned}
\frac{d^{2} E^{J}[c]}{d \xi^{2}}(0) & =\int_{s_{1}}^{s_{2}} d s\left\langle-\frac{\mathrm{D}^{J}}{d s} \frac{\mathrm{D}^{J} \vec{V}}{d s}-K_{c^{\prime}}^{J}(\vec{V}), \vec{V}\right\rangle^{J}= \\
& =\int_{s_{1}}^{s_{2}} d s\left\langle\Delta^{J} \vec{V}, \vec{V}\right\rangle^{J} \\
\frac{d^{2} L^{J}[c]}{d \xi^{2}}(0) & =\int_{s_{1}}^{s_{2}} d s\left\langle-\frac{\mathrm{D}^{J}}{d s} \frac{\mathrm{D}^{J} \vec{V}^{\perp}}{d s}-K_{c^{\prime}}^{J}\left(\vec{V}^{\perp}\right), \vec{V}^{\perp}\right\rangle^{J}= \\
& =\int_{s_{1}}^{s_{2}} d s\left\langle\Delta^{J} \vec{V}^{\perp}, \vec{V}^{\perp}\right\rangle^{J}
\end{aligned}
$$

para toda variación propia $\vec{V}$, y donde $\Delta^{J}$ es el operador hessiano (operador de desviación geodésica, en el lenguaje propio de la Geometría Riemanniana):

$$
\Delta^{J} \vec{V}=-\frac{\mathrm{D}^{J}}{d s} \frac{\mathrm{D}^{J} \vec{V}}{d s}-K_{c^{\prime}}^{J}(\vec{V})
$$

Como puede observarse, la expresión de los dos funcionales Hessianos geométricos es formalmente idéntica exceptuando el hecho de que el correspondiente a la longitud sólo actúa sobre variaciones ortogonales a la geodésica solución (o, si se quiere, sobre la componente ortogonal de una variación genérica). Desde un punto de vista geométrico es fácilmente entendible la razón por la cual el hessiano de la 
longitud no se ve afectado por las variaciones tangentes. Las variaciones de las curvas solución en la dirección tangente representan reparametrizaciones de las mismas y la longitud de las curvas geodésicas es independiente de la parametrización tomada.

Tal y como se demostró en los Teoremas 4.7 y 4.8, los funcionales Hessianos geométricos verifican, con respecto al funcional hessiano dinámico, las ecuaciones (4.16) y (4.21):

$$
\begin{aligned}
\frac{d^{2} E^{J}[c]}{d \xi^{2}}(0) & =\frac{d^{2} S[c]}{d \xi^{2}}(0)+\int_{t_{1}}^{t_{2}} d t 2\left\langle\dot{c}, \frac{\mathrm{D} \vec{V}}{d t}\right\rangle\langle\vec{F}, \vec{V}\rangle \\
\frac{d^{2} L^{J}[c]}{d \xi^{2}}(0) & =\frac{d^{2} S[c]}{d \xi^{2}}(0)+\int_{t_{1}}^{t_{2}} d t \frac{1}{2\left(i_{1}-U\right)}\left[\left\langle\nabla_{\dot{c}} \dot{c}, \vec{V}\right\rangle-\left\langle\dot{c}, \nabla_{\dot{c}} \vec{V}\right\rangle\right]^{2}
\end{aligned}
$$

donde $\vec{F}=\operatorname{grad} \operatorname{Ln}\left(2\left(i_{1}-U\right)\right)$.

Para variaciones ortogonales a la trayectoria solución estas expresiones se simplificaban notablemente, resultando:

$$
\frac{d^{2} L^{J}[c]}{d \xi^{2}}(0)=\left.\frac{d^{2} S[c]}{d \xi^{2}}(0)\right|_{\vec{V}=\vec{V} \perp}-\int_{t_{1}}^{t_{2}} d t 2\left(i_{1}-U\right)\left\langle\vec{F}, \vec{V}^{\perp}\right\rangle^{2}
$$

y recíprocamente:

$$
\left.\frac{d^{2} S[c]}{d \xi^{2}}(0)\right|_{\vec{V}=\vec{V} \perp}=\frac{d^{2} L^{J}[c]}{d \xi^{2}}(0)+\int_{s_{1}}^{s_{2}} d s\left(\left\langle\vec{F}^{J}, \vec{V}^{\perp}\right\rangle^{J}\right)^{2}
$$

donde: $\vec{F}^{J}=\operatorname{grad}^{J} \operatorname{Ln}\left(2\left(i_{1}-U\right)\right)$.

Una vez recordadas las expresiones demostradas en el capítulo anterior podemos analizar ahora los espectros de estos funcionales. Todos los elementos necesarios relativos a la métrica de Jacobi han sido ya calculados en las secciones precedentes, salvo el tensor de curvatura, cuyas componentes son

$$
\begin{gathered}
R_{122}^{1 J}=\left(4-\sigma^{2}\right) \frac{-\left(q_{1}^{2}+q_{2}^{2}-1\right)^{2}+\sigma^{2} q_{2}^{2}}{\left(\left(q_{1}^{2}+q_{2}^{2}-1\right)^{2}+\sigma^{2} q_{2}^{2}\right)^{2}}=-R_{212}^{1 J} \\
R_{121}^{2 J}=-R_{211}^{2 J}=-R_{122}^{1 J}
\end{gathered}
$$

mientras que todas las demás componentes son nulas.

Ya hemos calculado la solución TK1 como segmento geodésico de la variedad $(M, h)$, donde $h$ es la métrica de Jacobi asociada al modelo en estudio. Como se ha explicado en el apartado anterior, usaremos como parámetro a la componente $q_{1}$ 
sobre la solución TK1, que hemos denotado $\bar{q}_{1}$, obviando por tanto la dependencia en la longitud de arco $\bar{q}_{1}=\bar{q}_{1}(s)$, dada por la fórmula de Cardano-Tartaglia (5.10).

El tensor de curvatura seccional sobre la solución TK1 será:

$$
\begin{gathered}
K_{c_{\mathrm{TK} 1}^{\prime}}^{J}(\vec{V})=R^{J}\left(c^{\prime}, \vec{V}\right) c^{\prime}=\bar{q}_{1}^{\prime} \bar{q}_{1}^{\prime} R_{121}^{2 J} v_{2} \vec{e}_{2}=\frac{4-\sigma^{2}}{\left(1-\bar{q}_{1}^{2}\right)^{4}} v_{2} \vec{e}_{2} \\
K_{c_{\mathrm{TK} 1}^{\prime}}^{J}(\vec{V}) \equiv\left(\begin{array}{cc}
0 & 0 \\
0 & \frac{4-\sigma^{2}}{\left(1-\bar{q}_{1}^{2}\right)^{4}}
\end{array}\right)\left(\begin{array}{c}
v_{1} \\
v_{2}
\end{array}\right)
\end{gathered}
$$

donde ahora: $\bar{q}_{1}^{\prime}=\frac{1}{1-\bar{q}_{1}^{2}}$.

Como ya se ha comentado, para el caso del kink TK1, las variaciones tangentes y normales a la solución son de la forma $\vec{V}^{\text {tg }}=v_{1} \vec{e}_{1}$ y $\vec{V}^{\perp}=v_{2} \vec{e}_{2}$, respectivamente. Como puede observarse, la curvatura seccional sólo actúa de manera no trivial sobre la componente ortogonal a la trayectoria. Evidentemente este hecho no es una propiedad particular ni de este modelo y ni de esta solución, la antisimetría del tensor de curvatura conduce de manera inmediata a que la curvatura seccional $K_{c^{\prime}}\left(\alpha c^{\prime}\right)=0$ para cualquier campo $\alpha c^{\prime}$ tangente a la curva $c(s)$.

El cálculo directo del funcional hessiano nos proporciona la siguiente expresión para el operador hessiano $\Delta^{J}$ :

$$
\begin{aligned}
\Delta^{J}\left(v_{1} \vec{e}_{1}+v_{2} \vec{e}_{2}\right)= & -\frac{\mathrm{D}^{2} v_{1}}{d s^{2}} \vec{e}_{1}+\left(-\frac{\mathrm{D} v_{2}}{d s^{2}}-\frac{4-\sigma^{2}}{\left(1-\bar{q}_{1}^{2}\right)^{4}} v_{2}\right) \vec{e}_{2}= \\
= & \left(-\frac{d^{2} v_{1}}{d s^{2}}+\frac{4 \bar{q}_{1}}{\left(1-\bar{q}_{1}^{2}\right)^{2}} \frac{d v_{1}}{d s}+\frac{2 \bar{q}_{1}^{2}+2}{\left(1-\bar{q}_{1}^{2}\right)^{4}} v_{1}\right) \vec{e}_{1}+ \\
& +\left(-\frac{d^{2} v_{2}}{d s^{2}}+\frac{4 \bar{q}_{1}}{\left(1-\bar{q}_{1}^{2}\right)^{2}} \frac{d v_{2}}{d s}+\frac{2 \bar{q}_{1}^{2}-2+\sigma^{2}}{\left(1-\bar{q}_{1}^{2}\right)^{4}} v_{2}\right) \vec{e}_{2}
\end{aligned}
$$

con $\bar{q}_{1}=\bar{q}_{1}(s)$, o bien en función únicamente de $\bar{q}_{1}$ :

$$
\begin{aligned}
\Delta^{J}\left(v_{1} \vec{e}_{1}+v_{2} \vec{e}_{2}\right)= & \left(\frac{-1}{\left(1-\bar{q}_{1}^{2}\right)^{2}} \frac{d^{2} v_{1}}{d \bar{q}_{1}^{2}}+\frac{2 \bar{q}_{1}}{\left(1-\bar{q}_{1}^{2}\right)^{3}} \frac{d v_{1}}{d \bar{q}_{1}}+\frac{2 \bar{q}_{1}^{2}+2}{\left(1-\bar{q}_{1}^{2}\right)^{4}} v_{1}\right) \vec{e}_{1}+ \\
& +\left(\frac{-1}{\left(1-\bar{q}_{1}^{2}\right)^{2}} \frac{d^{2} v_{2}}{d \bar{q}_{1}^{2}}+\frac{2 \bar{q}_{1}}{\left(1-\bar{q}_{1}^{2}\right)^{3}} \frac{d v_{2}}{d \bar{q}_{1}}+\frac{2 \bar{q}_{1}^{2}-2+\sigma^{2}}{\left(1-\bar{q}_{1}^{2}\right)^{4}} v_{2}\right) \vec{e}_{2}
\end{aligned}
$$

Tal y como se comentó en el Capítulo 4, para el caso de variedades localmente simétricas la diagonalización paralela de la curvatura seccional permite resolver de forma casi trivial la ecuación de desviación geodésica y, en definitiva, el espectro del operador hessiano. En este caso, el espacio no es localmente simétrico, sin embargo, el procedimiento descrito en la sección 4.2.3 nos permitirá simplificar dicho análisis. 
Si queremos expresar las variaciones en una base que se traslade paralelamente a lo largo de la solución TK1, tendremos que resolver las ecuaciones del traslado paralelo, $\nabla_{c^{\prime}}^{J} \vec{U}=\overrightarrow{0}$, sobre el TK1:

$$
\begin{aligned}
& \frac{d U_{1}}{d s}+\Gamma_{11}^{1 J} \bar{q}_{1}^{\prime} U_{1}+\Gamma_{12}^{1 J} \bar{q}_{1}^{\prime} U_{2}=0 \\
& \frac{d U_{2}}{d s}+\Gamma_{11}^{2 J} \bar{q}_{1}^{\prime} U_{1}+\Gamma_{12}^{2 J} \bar{q}_{1}^{\prime} U_{2}=0
\end{aligned}
$$

que se reducen a:

$$
\begin{aligned}
& U_{1}^{\prime}-\frac{2 \bar{q}_{1}}{\left(1-\bar{q}_{1}^{2}\right)^{2}} U_{1}=0 \\
& U_{2}^{\prime}-\frac{2 \bar{q}_{1}}{\left(1-\bar{q}_{1}^{2}\right)^{2}} U_{2}=0
\end{aligned}
$$

Manteniendo la parametrización en la componente $\bar{q}_{1}$ y recordando que:

$$
\frac{d \bar{q}_{1}}{d s}=\bar{q}_{1}^{\prime}=\frac{1}{1-\bar{q}_{1}^{2}}
$$

las ecuaciones son fáciles de resolver y obtenemos:

$$
U_{1}=\frac{a}{1-\bar{q}_{1}^{2}} \quad, \quad U_{2}=\frac{b}{1-\bar{q}_{1}^{2}}
$$

para constantes arbitrarias $a$ y $b$.

El tensor de curvatura seccional es diagonal en la base tomada $\left\{\overrightarrow{e_{1}}, \vec{e}_{2}\right\}$ de manera que $\vec{e}_{1}$ es un vector propio con autovalor nulo $\left(\lambda_{1}=0\right)$ y $\vec{e}_{2}$ es un autovector con autovalor $\lambda_{2}=\frac{4-\sigma^{2}}{\left(1-\bar{q}_{1}^{2}\right)^{4}}, \forall \bar{q}_{1} \in(-1,1)$. Tenemos por tanto que el autovector $\vec{e}_{2}$ lo es en cada punto de la curva con un autovalor diferente.

Podemos entonces construir el traslado paralelo de dicha base de diagonalización a lo largo de la curva solución. Para ello tomaremos como condiciones iniciales:

$$
\vec{U}_{1}(0)=\vec{e}_{1} \Rightarrow\left(\begin{array}{c}
U_{1}(0) \\
U_{2}(0)
\end{array}\right)=\left(\begin{array}{l}
1 \\
0
\end{array}\right)
$$

$\mathrm{y}$

$$
\vec{U}_{2}(0)=\vec{e}_{2} \Rightarrow\left(\begin{array}{c}
U_{1}(0) \\
U_{2}(0)
\end{array}\right)=\left(\begin{array}{l}
0 \\
1
\end{array}\right)
$$

que conducen a la base paralela:

$$
\vec{U}_{1}=\frac{1}{1-\bar{q}_{1}^{2}} \vec{e}_{1} \equiv\left(\begin{array}{c}
\frac{1}{1-\bar{q}_{1}^{2}} \\
0
\end{array}\right), \quad \vec{U}_{2}=\frac{1}{1-\bar{q}_{1}^{2}} \vec{e}_{2} \equiv\left(\begin{array}{c}
0 \\
\frac{1}{1-\bar{q}_{1}^{2}}
\end{array}\right)
$$


de tal manera que $\vec{U}_{1}$ es un vector propio de la curvatura seccional con autovalor $\lambda_{1}=0$ y $\vec{U}_{2}$ lo es con autovalor $\lambda_{2}=\frac{4-\sigma^{2}}{\left(1-\bar{q}_{1}^{2}\right)^{4}}$. Evidentemente nos encontramos en la situación descrita en el Capítulo 4, el traslado paralelo de los autovectores de la curvatura seccional a lo largo de la trayectoria solución nos produce un campo de autovectores con diferente autovalor en cada punto de la curva.

Se verifica, por otro lado, que $\vec{U}_{1}$ es exactamente $c_{\text {TK1 }}^{\prime}$, es decir, el vector tangente a la geodésica, mientras que $\vec{U}_{2}$ es $c_{\text {TK1 }}^{\prime \perp}$. Ambos son, por tanto, unitarios en la métrica de Jacobi.

Podemos considerar, de esta manera, las variaciones propias $\vec{V}$ expresadas en función de la base paralela de autovectores de la curvatura seccional en la forma:

$$
\vec{V}(s)=w_{1}(s) \vec{U}_{1}(s)+w_{2}(s) \vec{U}_{2}(s)
$$

y, por tanto, al ser $\vec{V}$ paralelo, tendremos:

$$
-\nabla_{c^{\prime}}^{J} \nabla_{c^{\prime}}^{J} \vec{V}=-\frac{d^{2} w_{1}}{d s^{2}} \vec{U}_{1}-\frac{d^{2} w_{2}}{d s^{2}} \vec{U}_{2}
$$

de manera que podemos escribir el operador hessiano del funcional de energía en la métrica de Jacobi como:

$$
\Delta^{J} \vec{V}=\left(-\frac{d^{2} w_{1}}{d s^{2}}\right) \vec{U}_{1}+\left(-\frac{d^{2} w_{2}}{d s^{2}}-\frac{4-\sigma^{2}}{\left(1-\bar{q}_{1}^{2}\right)^{4}} w_{2}\right) \vec{U}_{2}
$$

donde $\bar{q}_{1}=\bar{q}_{1}(s)$, o bien, finalmente en el parámetro $\bar{q}_{1}$ :

$$
\begin{aligned}
\Delta^{J} \vec{V}= & \left(\frac{-1}{\left(1-\bar{q}_{1}^{2}\right)^{2}} \frac{d^{2} w_{1}}{d \bar{q}_{1}^{2}}-\frac{2 \bar{q}_{1}}{\left(1-\bar{q}_{1}^{2}\right)^{3}} \frac{d w_{1}}{d \bar{q}_{1}}\right) \vec{U}_{1}+ \\
& +\left(\frac{-1}{\left(1-\bar{q}_{1}^{2}\right)^{2}} \frac{d^{2} w_{2}}{d \bar{q}_{1}^{2}}-\frac{2 \bar{q}_{1}}{\left(1-\bar{q}_{1}^{2}\right)^{3}} \frac{d w_{2}}{d \bar{q}_{1}}-\frac{4-\sigma^{2}}{\left(1-\bar{q}_{1}^{2}\right)^{4}} w_{2}\right) \vec{U}_{2}
\end{aligned}
$$

Para el funcional de Longitud $L^{J}$, el operador hessiano es formalmente idéntico pero restringido a actuar sobre variaciones ortogonales a la solución únicamente, de manera que se tiene:

$$
\Delta^{J} \vec{V}^{\perp}=\Delta^{J}\left(w_{2} \vec{U}_{2}\right)=\left(-\frac{d^{2} w_{2}}{d s^{2}}-\frac{4-\sigma^{2}}{\left(1-\bar{q}_{1}^{2}\right)^{4}} w_{2}\right) \vec{U}_{2}
$$

con $\bar{q}_{1}=\bar{q}_{1}(s)$, o bien, en términos de $\bar{q}_{1}$ :

$$
\Delta^{J} \vec{V}^{\perp}=\left(\frac{-1}{\left(1-\bar{q}_{1}^{2}\right)^{2}} \frac{d^{2} w_{2}}{d \bar{q}_{1}^{2}}-\frac{2 \bar{q}_{1}}{\left(1-\bar{q}_{1}^{2}\right)^{3}} \frac{d w_{2}}{d \bar{q}_{1}}-\frac{4-\sigma^{2}}{\left(1-\bar{q}_{1}^{2}\right)^{4}} w_{2}\right) \vec{U}_{2}
$$




\section{Variaciones Tangentes}

Como ya hemos comentado, las variaciones tangentes sólo aparecen en el estudio del Hessiano de la Energía. Según (5.52), en términos de la base paralela $\left\{\vec{U}_{1}(s), \vec{U}_{2}(s)\right\}$, tenemos que, sobre una variación $\vec{V}^{\text {tg }}=w_{1}(s) \vec{U}_{1}(s)$, el operador Hessiano se escribe en la forma:

$$
\Delta^{J} \vec{V}^{\mathrm{tg}}=-\frac{d^{2} w_{1}}{d s^{2}} \vec{U}_{1}
$$

o bien en términos de $\bar{q}_{1}$ :

$$
\Delta^{J} \vec{V}^{\mathrm{tg}}=\left(\frac{-1}{\left(1-\bar{q}_{1}^{2}\right)^{2}} \frac{d^{2} w_{1}}{d \bar{q}_{1}^{2}}-\frac{2 \bar{q}_{1}}{\left(1-\bar{q}_{1}^{2}\right)^{3}} \frac{d w_{1}}{d \bar{q}_{1}}\right) \vec{U}_{1}
$$

Antes de analizar el operador (5.56), o equivalentemente el (5.57), es fácil calcular los términos que diferencian el funcional Hessiano geométrico de la energía del correspondiente funcional dinámico. De acuerdo con la fórmula (4.16) antes escrita tendremos que computar

$$
\int_{t_{1}}^{t_{2}} 2\left\langle\dot{c}, \nabla_{\dot{c}} \vec{V}\right\rangle\langle F, \vec{V}\rangle
$$

para obtener la relación siguiente entre ambos hessianos:

$$
\frac{d^{2} E^{J}}{d \xi^{2}}(0)=\frac{d^{2} S}{d \xi^{2}}(0)+\int_{t_{1}}^{t_{2}} d t 4\left(1-\tanh ^{2} t\right) v_{1}^{2}
$$

y por tanto la diferencia entre ambos afecta únicamente a las componentes tangentes de las variaciones, mientras que la parte ortogonal de ambos coincide.

De esta manera podemos escribir fácilmente el funcional hessiano de la energía para variaciones tangentes a la solución y en términos del parámetro temporal dinámico $t, \vec{V}^{\operatorname{tg}}(t)=v_{1}(t) \vec{e}_{1}$, de la forma:

$$
\frac{d^{2} E^{J}\left[c_{\mathrm{TK} 1}\right]}{d \xi^{2}}(0)=\int_{-\infty}^{\infty} d t\left\langle\Delta^{J} \vec{V}^{\mathrm{tg}}(t), \vec{V}^{\mathrm{tg}}(t)\right\rangle
$$

con

$$
\Delta^{J} \vec{V}^{\mathrm{tg}}=\left(-\frac{d^{2}}{d t^{2}}+2 \bar{q}_{1}^{2}+2\right) v_{1} \vec{e}_{1}=\left(-\frac{d^{2}}{d t^{2}}+4-\frac{2}{\cosh ^{2} t}\right) v_{1} \vec{e}_{1}
$$

El espectro de (5.58) es del mismo tipo que los ya analizados. De hecho si denotamos por $Q$ y $Q^{+}$a los operadores diferenciales:

$$
Q=-\frac{d}{d t}+2 \tanh t ; \quad Q^{+}=\frac{d}{d t}+2 \tanh t
$$


tendremos que el operador hessiano dinámico asociado al kink TK1 no es otro que $Q Q^{+}$mientras que el operador geométrico correspondiente, $\Delta^{J}$, es exactamente $Q^{+} Q$. Es así muy fácil computar el espectro de $\Delta^{J}$, obteniéndose:

- Un modo discreto positivo:

$$
v_{1}^{(3)}(t)=\frac{1}{\cosh t}
$$

con autovalor $\lambda=3$.

- Una parte continua:

$$
v_{1}^{\left(\lambda_{k}\right)}(t)=\cos (k t) \tanh t+k \operatorname{sen}(k t)
$$

o bien:

$$
v_{1}^{\left(\lambda_{k}\right)}(t)=\operatorname{sen}(k t) \tanh t-k \cos (k t)
$$

con autovalores respectivos $\lambda_{k}=k^{2}+4, k \in \mathbb{R}$.

Los autovalores de este operador coinciden con los del correspondiente dinámico salvo en el primero, el modo traslacional. Tenemos, en definitiva, que la energía geométrica "pierde" el modo cero tangente frente a la acción mecánica. La explicación de este hecho reside en que el funcional de energía en la métrica de Jacobi no es invariante más que bajo las reparametrizaciones afines del parámetro arco. De esta manera, en función de $s$ siempre existirá un modo cero tangente (correspondiente a la variación dada por el vector tangente $c^{\prime}$ ). Dado que el parámetro $t$ no es afín frente a $s$, dicho modo cero se pierde, el primer modo propio tiene autovalor 3 (energía que ha "costado" la reparametrización).

Plasmar analíticamente este razonamiento es una tarea delicada que abordaremos a continuación. Si se analiza el funcional Hessiano de la energía para variaciones tangentes a la solución en la base ordinaria:

$$
\frac{d^{2} E^{J}\left[c_{\mathrm{TK} 1}\right]}{d \xi^{2}}(0)=\int_{s_{1}}^{s_{2}} d s\left\langle-\nabla_{c_{\mathrm{TK} 1}^{\prime}} \nabla_{c_{\mathrm{TK} 1}^{\prime}} \vec{V}^{\mathrm{tg}}-K_{c_{\mathrm{TK} 1}}\left(\vec{V}^{\mathrm{tg}}\right), \vec{V}^{\mathrm{tg}}\right\rangle
$$

resulta evidente que la variación $\vec{V}^{\operatorname{tg}}(s)=c_{\mathrm{TK} 1}^{\prime}(s)=\vec{U}_{1}(s)$ es propia y de autovalor nulo (por ser la solución geodésica de la métrica y por las propiedades ya expuestas de la curvatura seccional), es el modo cero citado.

El espectro de (5.56), por otro lado, es trivial, se trata de las ondas planas $e^{i k s}$. ¿Cómo se "traduce" entonces el espectro ahora calculado sobre el operador (5.56) o 
(5.57)? La respuesta es que la "traducción" se realiza sobre las formas cuadráticas hessianas (los funcionales hessianos), no sobre los operadores correspondientes.

La variación $\vec{V}^{\operatorname{tg}}(s)=1 \vec{U}_{1}(s)$, es decir $w_{1}(s)=1$, es un modo nulo también de (5.56):

$$
\frac{d^{2} E^{J}\left[c_{\mathrm{TK} 1}\right]}{d \xi^{2}}(0)=\int_{-\frac{2}{3}}^{\frac{2}{3}} d s\left\langle-\frac{d^{2} 1}{d s^{2}} \vec{U}_{1}, \vec{U}_{1}\right\rangle^{J}=0
$$

La variación propia $\vec{V}^{\mathrm{tg}}=v_{1}^{(3)} \vec{e}_{1}=\frac{1}{\cosh t} \vec{e}_{1}$ de (5.58) se escribe de la forma:

$$
\vec{V}^{\operatorname{tg}}\left(\bar{q}_{1}\right)=\left(1-\bar{q}_{1}^{2}\right)^{\frac{3}{2}} \vec{U}_{1}\left(\bar{q}_{1}\right)
$$

en términos de $\bar{q}_{1}$ y en la base paralela de autovectores de la curvatura. Si calculamos $\Delta^{J} \vec{V}^{\operatorname{tg}}\left(\bar{q}_{1}\right)$ con la expresión (5.57) tendremos:

$\Delta^{J} \vec{V}^{\operatorname{tg}}\left(\bar{q}_{1}\right)=\Delta^{J}\left(\left(1-\bar{q}_{1}^{2}\right)^{\frac{3}{2}} \vec{U}_{1}\left(\bar{q}_{1}\right)\right)=\frac{3}{\left(1-\bar{q}_{1}^{2}\right)^{4}}\left(1-\bar{q}_{1}^{2}\right)^{\frac{3}{2}} \vec{U}_{1}\left(\bar{q}_{1}\right)=\frac{3}{\left(1-\bar{q}_{1}^{2}\right)^{4}} \vec{V}^{\operatorname{tg}}\left(q_{1}\right)$

con lo que estrictamente hablando no se trata de una autofunción. Sin embargo se verifica:

$$
\begin{aligned}
\frac{d^{2} E^{J}\left[c_{\mathrm{TK} 1}\right]}{d \xi^{2}}(0) & =\int_{-\frac{2}{3}}^{\frac{2}{3}} d s\left\langle\Delta^{J} \vec{V}^{\operatorname{tg}}\left(\bar{q}_{1}\right), \vec{V}^{\operatorname{tg}}\left(\bar{q}_{1}\right)\right\rangle^{J}= \\
& =\int_{-1}^{1}\left(1-\bar{q}_{1}^{2}\right) d \bar{q}_{1}\left\langle\frac{3}{\left(1-\bar{q}_{1}^{2}\right)^{4}} \vec{V}^{\operatorname{tg}}\left(\bar{q}_{1}\right), \vec{V}^{\operatorname{tg}}\left(\bar{q}_{1}\right)\right\rangle^{J}= \\
& =\int_{-1}^{1}\left(1-\bar{q}_{1}^{2}\right) d \bar{q}_{1} \frac{3}{\left(1-\bar{q}_{1}^{4}\right)}\left(1-\bar{q}_{1}^{2}\right)^{3}=\int_{-1}^{1} d \bar{q}_{1} 3=6>0
\end{aligned}
$$

utilizando que $\left\|\vec{U}_{1}\right\|_{J}=1$.

Si calculamos tomando el parámetro $t: \vec{V}^{\operatorname{tg}}(t)=\frac{1}{\cosh t} \vec{e}_{1}$, tendremos:

$$
\begin{aligned}
\frac{d^{2} E^{J}\left[c_{\mathrm{TK} 1}\right]}{d \xi^{2}}(0) & =\int_{-\infty}^{\infty} d t\left\langle\Delta^{J} \vec{V}^{\operatorname{tg}}(t), \vec{V}^{\mathrm{tg}}(t)\right\rangle= \\
& =\int_{-\infty}^{\infty} d t\left\langle 3 \vec{V}^{\mathrm{tg}}(t), \vec{V}^{\mathrm{tg}}(t)\right\rangle= \\
& =\int_{\infty}^{\infty} d t \frac{3}{\cosh ^{2} t}=6>0
\end{aligned}
$$




\section{Variaciones Ortogonales}

Ya hemos calculado que el funcional hessiano de la energía se diferenciaba del dinámico únicamente en la parte del mismo que afectaba a las variaciones tangentes, sobre las variaciones ortogonales, por tanto, coinciden. Para el funcional hessiano de la longitud, dado que coincide con el de la energía sobre éstas últimas, se verificará otro tanto. No es difícil, no obstante, aplicar la fórmula (4.21) y computar el término:

$$
-\int_{t_{1}}^{t_{2}} d t f(t)\left\langle\vec{F}, \vec{V}^{\perp}\right\rangle^{2}
$$

donde $f=\left.2\left(i_{1}-U\right)\right|_{\mathrm{TK} 1}=\left(1-\bar{q}_{1}^{2}\right)^{2}$ y $\vec{F}=\operatorname{grad} \operatorname{Ln} f$. De esta forma $\vec{F} \propto \vec{e}_{1} \mathrm{y}$ así:

$$
\left\langle\operatorname{grad} \operatorname{Ln}\left(1-\bar{q}_{1}^{2}\right)^{2}, \vec{V}^{\perp}\right\rangle=0
$$

es decir, no existe diferencia entre el funcional hessiano de la longitud y el dinámico restringido a variaciones ortogonales.

Si utilizamos el parámetro $\bar{q}_{1} \mathrm{y}$, en principio, escribimos una variación ortogonal en términos de la base ordinaria, $\vec{V}^{\mathrm{tg}}\left(\bar{q}_{1}\right)=v_{2}\left(\bar{q}_{1}\right) \vec{e}_{2}$, tendremos:

$$
\left.\frac{d^{2} E^{J}\left[c_{\mathrm{TK} 1}\right]}{d \xi^{2}}\right|_{\vec{V}=\vec{V} \perp}(0)=\frac{d^{2} L^{J}\left[c_{\mathrm{TK} 1}\right]}{d \xi^{2}}(0)=\int_{-1}^{1}\left(1-\bar{q}_{1}^{2}\right) d \bar{q}_{1}\left\langle\Delta^{J}\left(v_{2}\left(\bar{q}_{1}\right) \vec{e}_{2}\right), v_{2}\left(\bar{q}_{1}\right) \vec{e}_{2}\right\rangle^{J}
$$

donde:

$$
\begin{aligned}
\Delta^{J}\left(v_{2}\left(\bar{q}_{1}\right) \vec{e}_{2}\right) & =\left[-\frac{1}{\left(1-\bar{q}_{1}^{2}\right)^{2}} \frac{d^{2} v_{2}}{d \bar{q}_{1}^{2}}+\frac{2 \bar{q}_{1}}{\left(1-\bar{q}_{1}^{2}\right)^{3}} \frac{d v_{2}}{d \bar{q}_{1}}+\frac{2 \bar{q}_{1}^{2}-2+\sigma^{2}}{\left(1-\bar{q}_{1}^{2}\right)^{4}} v_{2}\right] \vec{e}_{2}= \\
& =\frac{1}{\left(1-\bar{q}_{1}^{2}\right)^{4}} \Delta\left(v_{2} \vec{e}_{2}\right)
\end{aligned}
$$

siendo $\Delta$ el correspondiente operador hessiano dinámico para variaciones ortogonales, descrito en la sección anterior (y expresado en la parametrización $\bar{q}_{1}$ ).

Los autovalores y las autofunciones ortogonales de $\Delta$ son, por tanto, trasladables a $\Delta^{J}$, resultando:

$$
\begin{aligned}
\Delta^{J}\left(v_{2}^{\left(-1+\sigma^{2}\right)} \vec{e}_{2}\right) & =\Delta^{J}\left(\sqrt{1-\bar{q}_{1}^{2}} \vec{e}_{2}\right)=\frac{-1+\sigma^{2}}{\left(1-\bar{q}_{1}^{2}\right)^{4}} \sqrt{1-\bar{q}_{1}^{2}} \vec{e}_{2} \\
\Delta^{J}\left(v_{2}^{\left(\lambda_{k}\right)} \vec{e}_{2}\right) & =\Delta^{J}\left(\left(\bar{q}_{1} \operatorname{sen}\left(k \operatorname{arctanh} \bar{q}_{1}\right)-k \cos \left(k \operatorname{arctanh} \bar{q}_{1}\right)\right) \vec{e}_{2}\right)= \\
& =\frac{k^{2}+\sigma^{2}}{\left(1-\bar{q}_{1}^{2}\right)^{4}}\left(\bar{q}_{1} \operatorname{sen}\left(k \operatorname{arctanh} \bar{q}_{1}\right)-k \cos \left(k \operatorname{arctanh} \bar{q}_{1}\right)\right) \vec{e}_{2} \\
\Delta^{J}\left(v_{2}^{\left(\lambda_{k}\right)} \vec{e}_{2}\right) & =\Delta^{J}\left(\left(\bar{q}_{1} \cos \left(k \operatorname{arctanh} \bar{q}_{1}\right)+k \operatorname{sen}\left(k \operatorname{arctanh} \bar{q}_{1}\right)\right) \vec{e}_{2}\right)= \\
& =\frac{k^{2}+\sigma^{2}}{\left(1-\bar{q}_{1}^{2}\right)^{4}}\left(\bar{q}_{1} \cos \left(k \operatorname{arctanh} \bar{q}_{1}\right)+k \operatorname{sen}\left(k \operatorname{arctanh} \bar{q}_{1}\right)\right) \vec{e}_{2}
\end{aligned}
$$


siendo el modo negativo la única variación propia (estrictamente hablando, recordemos que las autofunciones del continuo pueden ser "discretizadas" de tal manera que puedan admitirse como componentes de variaciones propias). Una vez más, por tanto, las autofunciones del operador dinámico dejan de serlo al pasar al operador geométrico, puesto que los autovalores dejan de ser constantes al aparecer el factor $\left(1-\bar{q}_{1}^{2}\right)^{-4}$. El problema se resuelve, de nuevo, sobre la forma cuadrática hessiano.

Para el modo negativo, tendremos, sobre el funcional:

$$
\begin{aligned}
\frac{d^{2} L^{J}\left[c_{\mathrm{TK} 1}\right]}{d \xi^{2}}(0) & =\left.\frac{d^{2} E^{J}\left[c_{\mathrm{TK} 1}\right]}{d \xi^{2}}\right|_{\vec{V}=\vec{V}^{\perp}}(0)= \\
& =\int_{-1}^{1}\left(1-\bar{q}_{1}^{2}\right) d \bar{q}_{1}\left\langle\Delta^{J}\left(v_{2}^{\left(-1+\sigma^{2}\right)}\left(\bar{q}_{1}\right) \vec{e}_{2}\right), v_{2}^{\left(-1+\sigma^{2}\right)}\left(\bar{q}_{1}\right) \vec{e}_{2}\right\rangle^{J}= \\
& =\int_{-1}^{1}\left(1-\bar{q}_{1}^{2}\right) d \bar{q}_{1} \frac{-1+\sigma^{2}}{\left(1-\bar{q}_{1}^{2}\right)^{4}}\left(1-\bar{q}_{1}^{2}\right)\left\langle\vec{e}_{2}, \vec{e}_{2}\right\rangle^{J}=2\left(-1+\sigma^{2}\right)<0
\end{aligned}
$$

al verificarse: $\left\langle\vec{e}_{2}, \vec{e}_{2}\right\rangle^{J}=\left(1-\bar{q}_{1}^{2}\right)^{2}$.

Si utilizamos la base paralela $\left\{\vec{U}_{1}, \vec{U}_{2}\right\}$, el operador $\Delta^{J}$ se escribe ahora:

$$
\begin{aligned}
&\left.\frac{d^{2} E^{J}\left[c_{\mathrm{TK} 1}\right]}{d \xi^{2}}\right|_{\vec{V}=\vec{V}^{\perp}}(0)=\frac{d^{2} L^{J}\left[c_{\mathrm{TK} 1}\right]}{d \xi^{2}}(0)= \\
&= \int_{-1}^{1}\left(1-\bar{q}_{1}^{2}\right) d \bar{q}_{1}\left\langle\Delta^{J}\left(w_{2}\left(\bar{q}_{1}\right) \vec{U}_{2}\right), w_{2}\left(\bar{q}_{1}\right) \vec{U}_{2}\right\rangle^{J} \\
& \Delta^{J}\left(w_{2}\left(\bar{q}_{1}\right) \vec{U}_{2}\right)=\left[-\frac{1}{\left(1-\bar{q}_{1}^{2}\right)^{2}} \frac{d^{2} w_{2}}{d \bar{q}_{1}^{2}}-\frac{2 \bar{q}_{1}}{\left(1-\bar{q}_{1}^{2}\right)^{3}} \frac{d w_{2}}{d \bar{q}_{1}}-\frac{4-\sigma^{2}}{\left(1-\bar{q}_{1}^{2}\right)^{4}} w_{2}\right] \vec{U}_{2}
\end{aligned}
$$

o bien, en términos de la longitud de arco $s$ :

$$
\Delta^{J}\left(w_{2}(s) \vec{U}_{2}(s)\right)=\left[-\frac{d^{2} w_{2}(s)}{d s^{2}}-\frac{4-\sigma^{2}}{\left(1-\bar{q}_{1}^{2}(s)\right)^{4}} w_{2}(s)\right] \vec{U}_{2}(s)
$$

El espectro de $\Delta$ se "traduce" ahora, teniendo en cuenta que

$$
\vec{V}^{\perp}=w_{2} \vec{U}_{2} \Rightarrow w_{2}=v_{2}\left(1-\bar{q}_{1}^{2}\right)
$$

en la forma:

$$
\begin{aligned}
& \Delta^{J}\left(\left(1-\bar{q}_{1}^{2}\right)^{\frac{3}{2}} \vec{U}_{2}\right)=\frac{-1+\sigma^{2}}{\left(1-\bar{q}_{1}^{2}\right)^{4}}\left(1-\bar{q}_{1}^{2}\right)^{\frac{3}{2}} \vec{U}_{2} \\
& \Delta^{J}\left(\left(1-\bar{q}_{1}^{2}\right)\left(\bar{q}_{1} \operatorname{sen}\left(k \operatorname{arctanh} \bar{q}_{1}\right)-k \cos \left(k \operatorname{arctanh} \bar{q}_{1}\right)\right) \vec{U}_{2}\right)
\end{aligned}
$$




$$
\begin{aligned}
& =\frac{k^{2}+\sigma^{2}}{\left(1-\bar{q}_{1}^{2}\right)^{4}}\left(1-\bar{q}_{1}^{2}\right)\left(\bar{q}_{1} \operatorname{sen}\left(k \operatorname{arctanh} \bar{q}_{1}\right)-k \cos \left(k \operatorname{arctanh} \bar{q}_{1}\right)\right) \vec{U}_{2} \\
& \Delta^{J}\left(\left(1-\bar{q}_{1}^{2}\right)\left(\bar{q}_{1} \cos \left(k \operatorname{arctanh} \bar{q}_{1}\right)+k \operatorname{sen}\left(k \operatorname{arctanh} \bar{q}_{1}\right)\right) \vec{U}_{2}\right)= \\
& =\frac{k^{2}+\sigma^{2}}{\left(1-\bar{q}_{1}^{2}\right)^{4}}\left(1-\bar{q}_{1}^{2}\right)\left(\bar{q}_{1} \cos \left(k \operatorname{arctanh} \bar{q}_{1}\right)+k \operatorname{sen}\left(k \operatorname{arctanh} \bar{q}_{1}\right)\right) \vec{U}_{2}
\end{aligned}
$$

Y los funcionales geométricos verifican, para el modo negativo, el resultado antes calculado

$$
\begin{aligned}
& \left.\frac{d^{2} E^{J}}{d \xi^{2}}(0)\right|_{\vec{V}=\vec{V} \perp}=\frac{d^{2} L^{J}}{d \xi^{2}}(0)= \\
& =\int_{-1}^{+1} d \bar{q}_{1}\left(-1+\sigma^{2}\right)\left\langle\vec{U}_{2}, \vec{U}_{2}\right\rangle^{J}=2\left(-1+\sigma^{2}\right)<0
\end{aligned}
$$

Por último, y desde un punto de vista puramente geométrico, es interesante comentar que el operador de desviación geodésica, $\Delta^{J}$, posee un campo de Jacobi ${ }^{10}$ no trivial:

$$
\begin{gathered}
\vec{V}^{\perp}=\left(1-\bar{q}_{1}^{2}\right) e^{\sigma \operatorname{arctanh} \bar{q}_{1}}\left(\sigma-\bar{q}_{1}\right) \vec{U}_{2} \\
\Delta^{J}\left(\left(1-\bar{q}_{1}^{2}\right) e^{\sigma \operatorname{arctanh} \bar{q}_{1}}\left(\sigma-\bar{q}_{1}\right) \vec{U}_{2}\right)=0 \vec{U}_{2}
\end{gathered}
$$

Este campo se anula cuando $\bar{q}_{1} \rightarrow-1$ y en $\bar{q}_{1}=\sigma$. Tenemos por tanto que el punto de vacío $(-1,0)$ y el foco $(\sigma, 0)$ de la elipse $q_{1}^{2}+\bar{\sigma}^{-2} q_{2}^{2}=1$ son puntos conjugados $^{11}$. Demostramos por tanto un hecho que utilizaremos en el próximo Capítulo 6 al estudiar la Teoría de Morse en el espacio de soluciones del sistema.

\subsubsection{Funcional Hessiano Dinámico: TK2}

Analizaremos en este apartado el espectro del Funcional:

$$
\frac{d^{2} S\left[c_{\mathrm{TK} 2}\right]}{d \xi^{2}}(0)=\int_{-\infty}^{\infty} d t\langle\Delta \vec{V}, \vec{V}\rangle
$$

donde $\Delta$ es ahora el operador hessiano dinámico sobre la solución TK2.

El kink TK2 nos venía dado por las expresiones:

$$
\bar{q}_{1}(t)=\tanh \sigma t ; \quad \bar{q}_{2}(t)=\bar{\sigma} \operatorname{sech} \sigma t
$$

\footnotetext{
${ }^{10}$ Recordemos que los campos de Jacobi se definen como las soluciones no triviales de la llamada "ecuación de Jacobi" o ecuación de desviación geodésica: $\nabla_{c^{\prime}} \nabla_{c^{\prime}} \vec{V}-K_{c^{\prime}}(\vec{V})=\overrightarrow{0}$ en una variedad riemanniana dada. Constituyen, por tanto, el núcleo del operador de desviación geodésica.

${ }^{11}$ De acuerdo con la definición, dos puntos pertenecientes a una geodésica son conjugados precisamente si existe un campo de Jacobi no trivial que se anula simultáneamente en ambos.
} 
que parametrizan a la elipse ${ }^{12}$ :

$$
q_{1}^{2}+\frac{q_{2}^{2}}{\bar{\sigma}^{2}}=1
$$

$\operatorname{con} \bar{\sigma}=\sqrt{1-\sigma^{2}}$.

En función del parámetro $\bar{q}_{1}$ las expresiones anteriores se reescriben como:

$$
\bar{q}_{1} \equiv \bar{q}_{1} ; \quad \bar{q}_{2}\left(\bar{q}_{1}\right)=\bar{\sigma} \sqrt{1-\bar{q}_{1}^{2}}
$$

donde $\bar{q}_{1} \in[-1,1]$.

Base ordinaria. Si calculamos el operador $\Delta$ sobre esta solución en la base $B=\left\{\vec{e}_{1}, \vec{e}_{2}\right\}$ de los campos, tendremos la siguiente expresión resultante:

$$
\begin{aligned}
\Delta\left(v_{1} \vec{e}_{1}+v_{2} \vec{e}_{2}\right) & =\left[\left(-\frac{d^{2}}{d t^{2}}+4-\frac{2\left(2+\sigma^{2}\right)}{\cosh ^{2} \sigma t}\right) v_{1}+4 \bar{\sigma} \frac{\tanh \sigma t}{\cosh \sigma t} v_{2}\right] \vec{e}_{1}+ \\
& +\left[4 \bar{\sigma} \frac{\tanh \sigma t}{\cosh \sigma t} v_{1}+\left(-\frac{d^{2}}{d t^{2}}+\sigma^{2}-\frac{2\left(3 \sigma^{2}-2\right)}{\cosh ^{2} \sigma t}\right) v_{2}\right] \vec{e}_{2}(5.5
\end{aligned}
$$

mientras que el mismo operador, en la misma base, pero con parámetro $\bar{q}_{1}$, se escribe:

$$
\begin{aligned}
\Delta \vec{V}= & {\left[\left(-\sigma^{2}\left(1-\bar{q}_{1}^{2}\right)^{2} \frac{d^{2}}{d \bar{q}_{1}^{2}}+2 \sigma^{2} \bar{q}_{1}\left(1-\bar{q}_{1}^{2}\right) \frac{d}{d \bar{q}_{1}}+2\left(2+\sigma^{2}\right) \bar{q}_{1}^{2}-2 \sigma^{2}\right) v_{1}+\right.} \\
& \left.+4 \bar{\sigma} \bar{q}_{1} \sqrt{1-\bar{q}_{1}^{2}} v_{2}\right] \vec{e}_{1}+\left[4 \bar{\sigma} \bar{q}_{1} \sqrt{1-\bar{q}_{1}^{2}} v_{1}+\right. \\
& \left.\left(-\sigma^{2}\left(1-\bar{q}_{1}^{2}\right)^{2} \frac{d^{2}}{d \bar{q}_{1}^{2}}+2 \sigma^{2} \bar{q}_{1}\left(1-\bar{q}_{1}^{2}\right) \frac{d}{d \bar{q}_{1}}+4-5 \sigma^{2}-2\left(2-3 \sigma^{2}\right) \bar{q}_{1}^{2}\right) v_{2}\right] \vec{e}_{2}
\end{aligned}
$$

Como podemos observar el operador en este caso no es diagonal, el análisis directo del espectro del mismo conduce a un sistema de ecuaciones acopladas y, por tanto, ya no equivalente a dos ecuaciones de Schrödinger unidimensionales independientes. Construiremos a continuación bases de Frenet dadas por vectores tangentes y ortogonales a la solución, y por tanto a la elipse, de manera que se pueda abordar el estudio del espectro de $\Delta$ al menos para un determinado tipo de variaciones, que serán precisamente las importantes desde el punto de vista de la estabilidad dado que el resto de las variaciones mueven los puntos de vacío y, por tanto, llevarían las soluciones a curvas no pertenecientes al espacio de configuración.

\footnotetext{
${ }^{12}$ Realmente estas ecuaciones sólo parametrizan a la semielipse superior, recuérdese que nos estamos limitando a estudiar sólo el kink TK2 y no sus kinks asociados TK2*, ATK2 y ATK2*.
} 
Bases de Frenet $^{13}$. Para obtener un vector tangente unitario a la semielipse sobre la que transcurre la solución TK2 calculamos directamente:

$$
\vec{u}^{\operatorname{tg}}(t)=\frac{\dot{c}_{\mathrm{TK} 2}}{\left\|\dot{c}_{\mathrm{TK} 2}\right\|}=\frac{1}{\cosh \sigma t \sqrt{1-\sigma^{2} \tanh ^{2} \sigma t}}\left(\vec{e}_{1}-\bar{\sigma} \operatorname{senh} \sigma t \vec{e}_{2}\right)
$$

mientras que un vector ortogonal unitario será:

$$
\vec{u}^{\perp}(t)=\frac{1}{\cosh \sigma t \sqrt{1-\sigma^{2} \tanh ^{2} \sigma t}}\left(-\bar{\sigma} \operatorname{senh} \sigma t \vec{e}_{1}-\vec{e}_{2}\right)
$$

La expresión del operador $\Delta$ en la base unitaria $B_{F}=\left\{\vec{u}^{\operatorname{tg}}(t), \vec{u}^{\perp}(t)\right\}$ resulta extraordinariamente complicada e inmanejable a efectos de cálculo. Por otro lado, de cara a obtener las variaciones propias del espectro de $\Delta$, no es necesario en absoluto que la base considerada sea unitaria. De esta manera, podemos tomar como base la determinada por los vectores $\vec{a}^{\text {tg }}(t)$ y $\vec{a}^{\perp}(t)$ definidos de la forma:

$$
\vec{a}^{\operatorname{tg}}(t)=\vec{e}_{1}-\bar{\sigma} \operatorname{senh} \sigma t \vec{e}_{2} ; \quad \vec{a}^{\perp}(t)=\bar{\sigma} \operatorname{senh} \sigma t \vec{e}_{1}+\vec{e}_{2}
$$

y tendremos que $B_{F}^{\prime}=\left\{\vec{a}^{\operatorname{tg}}(t), \vec{a}^{\perp}(t)\right\}$ es una base ortogonal pero no ortonormal.

Varias son las razones que nos conducen a tomar esta base de Frenet, $B_{F}^{\prime}$. Como veremos, si expresamos una variación genérica tangente a la curva, $\vec{V}^{\mathrm{tg}}$, en la forma:

$$
\vec{V}^{\operatorname{tg}}(t)=v_{1}(t) \vec{a}^{\operatorname{tg}}(t)
$$

y una ortogonal como:

$$
\vec{V}^{\perp}(t)=v_{2}(t) \vec{a}^{\perp}(t)
$$

encontraremos que de manera general no existen autofunciones del operador con estas características. Sin embargo, dada la forma concreta de los vectores de $B_{F}^{\prime}$, si proyectamos las variaciones tangentes y ortogonales sobre las direcciones más peligrosas, desde el punto de vista de la estabilidad, esto es, las direcciones de menor coste en energía con respecto a las soluciones, obtendremos variaciones bien definidas en las que, además, el comportamiento de las autofunciones compensa el hecho de que los vectores de $B_{F}^{\prime}$ tengan norma infinita en los extremos de la trayectoria. De esta manera, las variaciones "proyectadas" serán propias y su contribución será finita.

\footnotetext{
${ }^{13}$ Como ya se ha comentado, denominaremos Base de Frenet sobre una curva a toda base de los campos tangentes a la variedad tal que, para cada punto de la curva, uno de los vectores sea tangente y el otro ortogonal a la misma.
} 


\section{Variaciones Tangentes}

Consideremos una variación tangente genérica y escrita de la forma (5.61), el operador hessiano $\Delta$ sobre ella será:

$$
\begin{aligned}
\Delta\left(v_{1} \vec{a}^{\mathrm{tg}}\right)= & {\left[-\frac{d^{2}}{d t^{2}}+4 \sigma^{2}-\frac{6 \sigma^{2}}{\cosh ^{2} \sigma t}\right] v_{1} \vec{e}_{1}+} \\
& +(-\bar{\sigma} \operatorname{senh} \sigma t)\left[-\frac{d^{2}}{d t^{2}}-2 \sigma \frac{\cosh \sigma t}{\operatorname{senh} \sigma t} \frac{d}{d t}-\frac{6 \sigma^{2}}{\cosh ^{2} \sigma t}\right] v_{1} \vec{e}_{2}
\end{aligned}
$$

cuya segunda componente puede ser simplificada si se integra por partes el funcional para el caso de variaciones propias, obteniéndose:

$$
\begin{aligned}
\Delta\left(v_{1} \vec{a}^{\mathrm{tg}}\right)= & {\left[-\frac{d^{2}}{d t^{2}}+4 \sigma^{2}-\frac{6 \sigma^{2}}{\cosh ^{2} \sigma t}\right] v_{1} \vec{e}_{1}+} \\
& +(-\bar{\sigma} \operatorname{senh} \sigma t)\left[-\frac{d^{2}}{d t^{2}}+2 \sigma^{2}-\frac{6 \sigma^{2}}{\cosh ^{2} \sigma t}+\frac{\sigma^{2}}{\operatorname{senh}^{2} \sigma t}\right] v_{1} \vec{e}_{2}(5.63)
\end{aligned}
$$

Como puede observarse en esta expresión, (5.63), en cada componente nos aparece un operador de tipo Schrödinger. En la primera se trata, una vez más, de un potencial de tipo Pösch-Teller, sin embargo, en la segunda, aparece un término singular, la cosecante cuadrado, que produce una barrera infinita en $t=0$; evidentemente esto es un reflejo de que las variaciones generales en esta dirección suponen un incremento infinito de energía. Evitaremos esta singularidad proyectando el operador sobre la primera componente.

Si definimos los operadores de proyección $\pi_{1} \mathrm{y} \pi_{2}$ en la forma:

$$
\pi_{1}\left(x_{1} \vec{e}_{1}+x_{2} \vec{e}_{2}\right)=x_{1} \vec{e}_{1} ; \quad \pi_{2}\left(x_{1} \vec{e}_{1}+x_{2} \vec{e}_{2}\right)=x_{2} \vec{e}_{2}
$$

tendremos entonces que el hessiano que presenta un buen comportamiento es:

$$
\pi_{1}\left(\Delta\left(v_{1} \vec{a}^{\mathrm{tg}}\right)\right)=\left[-\frac{d^{2}}{d t^{2}}+4 \sigma^{2}-\frac{6 \sigma^{2}}{\cosh ^{2} \sigma t}\right] v_{1} \vec{e}_{1}
$$

El espectro de (5.64) es muy similar al de las variaciones tangentes del caso TK1, antes descrito. Tendremos:

- Un modo cero:

$$
v_{1}^{(0)}(t)=\frac{1}{\cosh ^{2} \sigma t}
$$

- Un modo positivo:

con autovalor $\lambda=3 \sigma^{2}$.

$$
v_{1}^{\left(3 \sigma^{2}\right)}(t)=\frac{\tanh \sigma t}{\cosh \sigma t}
$$


- Un espectro continuo:

$$
\tilde{v}_{1}^{\left(\lambda_{k}\right)}(t)=e^{i k \sigma t} P_{2}(\tanh \sigma t)=e^{i k \sigma t}\left(3 \tanh ^{2} \sigma t-1-3 i k \tanh \sigma t-k^{2}\right)
$$

con autovalores respectivos $\lambda_{k}=\sigma^{2}\left(k^{2}+4\right)$, que comienza $(\operatorname{con} k=0)$ en la autofunción:

$$
v_{1}^{\left(4 \sigma^{2}\right)}(t)=1-\frac{3}{2 \cosh ^{2} \sigma t}
$$

de autovalor $\lambda_{0}=4 \sigma^{2}$. Restringiéndonos a variaciones reales, reescribiremos el espectro continuo en la forma:

$$
v_{1}^{\left(\lambda_{k}\right)}(t)=\left(3 \tanh ^{2}(\sigma t)-1-k^{2}\right) \cos (k \sigma t)+3 k \tanh (\sigma t) \operatorname{sen}(k \sigma t)
$$

para autofunciones pares y, para las impares:

$$
v_{1}^{\left(\lambda_{k}\right)}(t)=\left(3 \tanh ^{2}(\sigma t)-1-k^{2}\right) \operatorname{sen}(k \sigma t)-3 k \tanh (\sigma t) \operatorname{sen}(k \sigma t)
$$

Como puede observarse, sólo las dos primeras autofunciones, $v_{1}^{(0)}$ y $v_{1}^{\left(3 \sigma^{2}\right)}$, conducen a variaciones propias, el resto de las presentes no se anulan en $t \rightarrow \pm \infty$.

Una vez más, es posible "discretizar" las autofunciones del continuo y restringir las mismas a las que son propias por medio de un proceso de paso al límite, similar al aplicado en el caso del kink TK1, en la sección anterior. Como puede deducirse fácilmente de la expresión de $v_{1}^{\left(\lambda_{k}\right)}(t)$, los resultados que se obtienen son idénticos a los del caso citado sin más que sustituir $t$ por $\sigma t$.

La presencia del modo cero se interpreta de manera análoga al caso del kink TK1, es decir, representa la invarianza bajo traslaciones temporales, que no altera la acción del sistema. Como ya hemos comentado en el caso del kink TK1, se trata de la derivada de la solución (salvo una constante multiplicativa):

$$
\vec{V}^{\mathrm{tg}}(t)=v_{1}^{(0)} \vec{a}^{\mathrm{tg}}=\frac{1}{\cosh ^{2} \sigma t} \vec{e}_{1}-\bar{\sigma} \frac{\operatorname{senh} \sigma t}{\cosh ^{2} \sigma t} \vec{e}_{2}=\frac{1}{\sigma} \dot{c}_{\mathrm{TK} 2}
$$

La ausencia de modos negativos indica, evidentemente, estabilidad con respecto a las variaciones en esa dirección.

Podemos definir ahora el funcional hessiano "proyectado", o forma cuadrática "proyectada", $\pi_{1}\left(\frac{d^{2} S[c]}{d \xi^{2}}(0)\right)$, como el funcional hessiano correspondiente al operador hessiano proyectado, es decir:

$$
\pi_{1}\left(\frac{d^{2} S[c]}{d \xi^{2}}(0)\right) \stackrel{\text { def }}{=} \int_{t_{1}}^{t_{2}} d t\left\langle\pi_{1}(\Delta \vec{V}), \vec{V}\right\rangle
$$


y, de esta manera, para la variación $\vec{V}^{\mathrm{tg}}=\frac{1}{\cosh ^{2} \sigma t} \vec{a}^{\mathrm{tg}}$, tendremos:

$$
\pi_{1}\left(\frac{d^{2} S[c]}{d \xi^{2}}(0)\right)=\int_{-\infty}^{\infty} d t\left\langle\pi_{1}\left(\Delta\left(\frac{1}{\cosh ^{2} \sigma t} \vec{a}^{\operatorname{tg}}\right)\right), \frac{1}{\cosh ^{2} \sigma t} \vec{a}^{\operatorname{tg}}\right\rangle=0
$$

mientras que para la variación $\vec{V}^{\operatorname{tg}}=\frac{\operatorname{senh} \sigma t}{\cosh ^{2} \sigma t} \vec{a}^{\text {tg }}$ resultará:

$$
\begin{aligned}
\pi_{1}\left(\frac{d^{2} S[c]}{d \xi^{2}}(0)\right) & =\int_{-\infty}^{\infty} d t\left\langle\pi_{1}\left(\Delta\left(\frac{\operatorname{senh} \sigma t}{\cosh ^{2} \sigma t} \vec{a}^{\operatorname{tg}}\right)\right), \frac{\operatorname{senh} \sigma t}{\cosh ^{2} \sigma t} \vec{a}^{\operatorname{tg}}\right\rangle= \\
& =\int_{-\infty}^{\infty} d t 3 \sigma^{2} \frac{\operatorname{senh}^{2} \sigma t}{\cosh ^{4} \sigma t}=2 \sigma>0
\end{aligned}
$$

Si consideramos el parámetro $\bar{q}_{1}$, el espectro anterior se escribe:

- Modo cero:

$$
v_{1}^{(0)}\left(\bar{q}_{1}\right)=1-\bar{q}_{1}^{2}
$$

- Modo positivo:

$$
v_{1}^{\left(3 \sigma^{2}\right)}\left(\bar{q}_{1}\right)=\bar{q}_{1} \sqrt{1-\bar{q}_{1}^{2}}
$$

- Espectro continuo, autofunciones pares:

$$
v_{1}^{\left(\lambda_{k}\right)}(t)=\left(3 \bar{q}_{1}^{2}-1-k^{2}\right) \cos \left(\frac{k}{\sigma} \operatorname{arctanh} \bar{q}_{1}\right)+3 k \bar{q}_{1} \operatorname{sen}\left(\frac{k}{\sigma} \operatorname{arctanh} \bar{q}_{1}\right)
$$

- Espectro continuo, autofunciones impares:

$$
v_{1}^{\left(\lambda_{k}\right)}(t)=\left(3 \bar{q}_{1}^{2}-1-k^{2}\right) \operatorname{sen}\left(\frac{k}{\sigma} \operatorname{arctanh} \bar{q}_{1}\right)+3 k \bar{q}_{1} \cos \left(\frac{k}{\sigma} \operatorname{arctanh} \bar{q}_{1}\right)
$$

\section{Variaciones Ortogonales}

Para las variaciones ortogonales que hemos considerado, $\vec{V}^{\perp}(t)=v_{2}(t) \vec{a}^{\perp}(t)$, el operador hessiano dinámico resulta:

$$
\begin{aligned}
& \Delta\left(v_{2}(t) \vec{a}^{\perp}(t)\right)=\Delta\left(v_{2}(t) \bar{\sigma} \operatorname{senh} \sigma t \vec{e}_{1}+v_{2}(t) \vec{e}_{2}\right)= \\
& =(\bar{\sigma} \operatorname{senh} \sigma t)\left[-\frac{d^{2}}{d t^{2}}-2 \sigma \frac{\cosh \sigma t}{\operatorname{senh} \sigma t} \frac{d}{d t}+4-\sigma^{2}-\frac{2 \sigma^{2}}{\cosh ^{2} \sigma t}\right] v_{2}(t) \vec{e}_{1}+ \\
& +\left[-\frac{d^{2}}{d t^{2}}+4-3 \sigma^{2}-\frac{2 \sigma^{2}}{\cosh ^{2} \sigma t}\right] v_{2}(t) \vec{e}_{2}
\end{aligned}
$$


o bien, si se integra por partes en la expresión del funcional para eliminar la derivada primera (y sólo para variaciones propias, claro está), se obtiene:

$$
\begin{aligned}
\Delta\left(v_{2}(t) \vec{a}^{\perp}(t)\right)= & (\bar{\sigma} \operatorname{senh} \sigma t)\left[-\frac{d^{2}}{d t^{2}}+4+\sigma^{2}-\frac{2 \sigma^{2}}{\cosh ^{2} \sigma t}+\frac{\sigma^{2}}{\operatorname{senh}^{2} \sigma t}\right] v_{2}(t) \vec{e}_{1}+ \\
& +\left[-\frac{d^{2}}{d t^{2}}+4-3 \sigma^{2}-\frac{2 \sigma^{2}}{\cosh ^{2} \sigma t}\right] v_{2}(t) \vec{e}_{2}
\end{aligned}
$$

Observamos nuevamente que para una de las componentes (la primera, en este caso) el operador de Schrödinger que resulta presenta una barrera infinita, una singularidad, en $t=0$, mientras que para la segunda componente se obtiene un potencial de tipo Pösch-Teller.

Proyectaremos entonces en la segunda componente, de igual manera que se hizo con las variaciones tangentes sobre la primera de ellas. Por otro lado, como ya se ha citado, si recordamos la situación que encontrábamos en el caso TK1, la dirección del vector $\vec{e}_{2}$ era precisamente en la que aparecía el modo negativo que nos indicaba la inestabilidad del kink.

Escribiremos, por tanto, el operador hessiano proyectado en la forma:

$$
\pi_{2}\left(\Delta\left(v_{2}(t) \vec{a}^{\perp}(t)\right)\right)=\left(-\frac{d^{2}}{d t^{2}}+4-3 \sigma^{2}-\frac{2 \sigma^{2}}{\cosh ^{2} \sigma t}\right) v_{2}(t) \vec{e}_{2}
$$

cuyo espectro presenta un único modo discreto y una parte continua:

- Modo discreto:

$$
v_{2}^{\left(4 \bar{\sigma}^{2}\right)}(t)=\frac{1}{\cosh \sigma t}
$$

con autovalor $\lambda=4\left(1-\sigma^{2}\right)=4 \bar{\sigma}^{2}$.

- Espectro continuo:

$$
\tilde{v}_{2}^{\left(\lambda_{k}\right)}(t)=e^{i k \sigma t} P_{1}(\tanh \sigma t)=e^{i k \sigma t}(i k-\tanh \sigma t)
$$

con autovalores respectivos $\lambda_{k}=\left(k^{2}-3\right) \sigma^{2}+4$. Las autofunciones reales serán ahora:

$$
v_{2}^{\left(\lambda_{k}\right)}(t)=\tanh (\sigma t) \cos (k t)+k \operatorname{sen}(k t)
$$

$\mathrm{y}$

$$
v_{2}^{\left(\lambda_{k}\right)}(t)=\tanh (\sigma t) \operatorname{sen}(k t)-k \cos (k t)
$$


Como podemos observar, la única autofunción que conduce a una variación propia es el modo discreto, el espectro continuo no lo hace así por tratarse de funciones que no se anulan en $t \rightarrow \pm \infty$. De nuevo es posible aumentar el espacio de funciones por el proceso límite ya descrito. En este caso los resultados son idénticos a los obtenidos para las variaciones ortogonales al kink TK1 si se intercambia $t$ por $\sigma t$ en los mismos.

Si calculamos el funcional "proyectado" sobre la variación: $\vec{V}^{\perp}=v_{2}^{\left(4 \bar{\sigma}^{2}\right)} \vec{a}^{\perp}(t)$, tendremos:

$$
\begin{aligned}
\pi_{2}\left(\frac{d^{2} S[c]}{d \xi^{2}}(0)\right) & =\int_{-\infty}^{\infty} d t\left\langle\pi_{2}\left(\Delta \vec{V}^{\perp}\right), \vec{V}^{\perp}\right\rangle= \\
& =\int_{-\infty}^{\infty} d t \frac{4\left(1-\sigma^{2}\right)}{\cosh ^{2} \sigma t}=\frac{8 \bar{\sigma}^{2}}{\sigma}>0
\end{aligned}
$$

que nos confirma la estabilidad de la solución TK2.

Por último, este espectro es trivialmente calculable en función del parámetro $\bar{q}_{1}$, resultando:

- Modo discreto:

$$
v_{2}^{\left(4 \bar{\sigma}^{2}\right)}\left(\bar{q}_{1}\right)=\sqrt{1-\bar{q}_{1}^{2}}
$$

- Espectro continuo, autofunciones pares:

$$
v_{2}^{\left(\lambda_{k}\right)}\left(\bar{q}_{1}\right)=\bar{q}_{1} \operatorname{sen}\left(\frac{k}{\sigma} \operatorname{arctanh} \bar{q}_{1}\right)-k \cos \left(\frac{k}{\sigma} \operatorname{arctanh} \bar{q}_{1}\right)
$$

- Espectro continuo, autofunciones impares:

$$
v_{2}^{\left(\lambda_{k}\right)}\left(\bar{q}_{1}\right)=\bar{q}_{1} \cos \left(\frac{k}{\sigma} \operatorname{arctanh} \bar{q}_{1}\right)+k \operatorname{sen}\left(\frac{k}{\sigma} \operatorname{arctanh} \bar{q}_{1}\right)
$$

\subsubsection{Funcionales Hessianos Geométricos: TK2}

Analizaremos en este apartado los funcionales Hessianos geométricos sobre la solución TK2.

Las expresiones del kink TK2 visto como segmento geodésico en la métrica de Jacobi se han deducido ya en la sección anterior. La métrica $h$, sobre estas soluciones, se escribe:

$$
h_{i j}=\left(\sigma^{2}\left(1-\bar{q}_{1}^{2}(s)\right)\left(1-\sigma \bar{q}_{1}^{2}(s)\right)\right) \delta_{i j}
$$


donde $\bar{q}_{1}(s)$ viene dado por una de las ecuaciones de Cardano-Tartaglia. Evidentemente, usaremos de nuevo $\bar{q}_{1}$ como parámetro para simplificar los cálculos, teniendo en cuenta que

$$
\bar{q}_{1} \equiv \bar{q}_{1} ; \quad \bar{q}_{2}=\bar{\sigma} \sqrt{1-\bar{q}_{1}^{2}} ; \quad d s=\sigma\left(1-\sigma^{2} \bar{q}_{1}^{2}\right) d \bar{q}_{1}
$$

El tensor de curvatura de la métrica de Jacobi restringido al kink TK2, y en función ya del parámetro $\bar{q}_{1}$, viene determinado, como se especificó en el apartado 5.2 .2 , por una única componente (las demás no triviales son iguales, en valor absoluto, a ésta):

$$
R_{122}^{1 J}=\left(4-\sigma^{2}\right) \frac{1-2 \sigma^{2}+\sigma^{2} \bar{q}_{1}^{2}}{\sigma^{2}\left(1-\bar{q}_{1}^{2}\right)\left(1-\sigma^{2} \bar{q}_{1}^{2}\right)^{2}}
$$

y, de esta manera, el tensor de curvatura seccional sobre el TK2 se escribe:

$$
K_{c^{\prime}}(\vec{V}) \equiv \frac{\left(4-\sigma^{2}\right)\left(\sigma^{2} \bar{q}_{1}^{2}-2 \sigma^{2}+1\right)}{\sigma^{4}\left(1-\bar{q}_{1}^{2}\right)\left(1-\sigma^{2} \bar{q}_{1}^{2}\right)^{4}}\left(\begin{array}{cc}
-\frac{\bar{\sigma}^{2} \bar{q}_{1}^{2}}{1-\bar{q}_{1}^{2}} & -\frac{\bar{\sigma} \bar{q}_{1}}{\sqrt{1-\bar{q}_{1}^{2}}} \\
-\frac{\bar{\sigma} \bar{q}_{1}}{\sqrt{1-\bar{q}_{1}^{2}}} & -1
\end{array}\right)\left(\begin{array}{l}
v_{1} \\
v_{2}
\end{array}\right)
$$

siendo $\vec{V}=v_{1} \vec{e}_{1}+v_{2} \vec{e}_{2}$ un campo tangente a la curva TK2.

Base ordinaria. Podemos calcular directamente la expresión del operador de desviación geodésica $\Delta^{J}$ para esta solución, de manera análoga a lo expuesto para el TK1:

$$
\begin{aligned}
\frac{d^{2} E^{J}\left[c_{\mathrm{TK} 2}\right]}{d \xi^{2}}(0) & =\int_{s_{1}}^{s_{2}} d s\left\langle\Delta^{J} \vec{V}(s), \vec{V}(s)\right\rangle^{J}= \\
& =\int_{-1}^{1} \sigma\left(1-\sigma^{2} \bar{q}_{1}^{2}\right) d \bar{q}_{1}\left\langle\Delta^{J} \vec{V}\left(\bar{q}_{1}\right), \vec{V}\left(\bar{q}_{1}\right)\right\rangle^{J} \\
\frac{d^{2} L^{J}\left[c_{\mathrm{TK} 2}\right]}{d \xi^{2}}(0) & =\int_{s_{1}}^{s_{2}} d s\left\langle\Delta^{J} \vec{V}^{\perp}(s), \vec{V}^{\perp}(s)\right\rangle^{J}= \\
& =\int_{-1}^{1} \sigma\left(1-\sigma^{2} \bar{q}_{1}^{2}\right) d \bar{q}_{1}\left\langle\Delta^{J} \vec{V}^{\perp}\left(\bar{q}_{1}\right), \vec{V}^{\perp}\left(\bar{q}_{1}\right)\right\rangle^{J}
\end{aligned}
$$

de forma que se obtiene, para una variación $\vec{V}\left(\bar{q}_{1}\right)=v_{1}\left(\bar{q}_{1}\right) \vec{e}_{1}+v_{2}\left(\bar{q}_{1}\right) \vec{e}_{2}$ cualquiera:

$$
\begin{aligned}
\Delta^{J} \vec{V}\left(\bar{q}_{1}\right)= & {\left[\frac{-1}{\sigma^{2}\left(1-\sigma^{2} \bar{q}_{1}^{2}\right)^{2}} \frac{d^{2} v_{1}}{d \bar{q}_{1}^{2}}+\frac{2 \bar{q}_{1}}{\sigma^{2}\left(1-\bar{q}_{1}^{2}\right)\left(1-\sigma^{2} \bar{q}_{1}^{2}\right)^{2}} \frac{d v_{1}}{d \bar{q}_{1}}+\right.} \\
& +\frac{2\left(2 \bar{q}_{1}^{2}-2 \sigma^{4} \bar{q}_{1}^{2}\left(\bar{q}_{1}^{2}-2\right)+\sigma^{6} \bar{q}_{1}^{4}\left(\bar{q}_{1}^{2}-1\right)+\sigma^{2}\left(1-7 \bar{q}_{1}^{2}+2 \bar{q}_{1}^{4}\right)\right)}{\sigma^{4}\left(1-\bar{q}_{1}^{2}\right)^{2}\left(1-\sigma^{2} \bar{q}_{1}^{2}\right)^{4}} v_{1}+ \\
& \left.+\frac{2 \bar{\sigma}}{\sigma^{2} \sqrt{1-\bar{q}_{1}^{2}}\left(1-\sigma^{2} \bar{q}_{1}^{2}\right)^{3}} \frac{d v_{2}}{d \bar{q}_{1}}-\frac{2 \bar{\sigma}\left(\sigma^{2}-2\right) \bar{q}_{1}\left(1+\sigma^{2}\left(\bar{q}_{1}^{2}-2\right)\right)}{\sigma^{4}\left(1-\bar{q}_{1}^{2}\right)^{\frac{3}{2}}\left(1-\sigma^{2} \bar{q}_{1}^{2}\right)^{4}} v_{2}\right] \vec{e}_{1}+
\end{aligned}
$$




$$
\begin{aligned}
& {\left[\frac{-1}{\sigma^{2}\left(1-\sigma^{2} \bar{q}_{1}^{2}\right)^{2}} \frac{d^{2} v_{2}}{d \bar{q}_{1}^{2}}+\frac{2 \bar{q}_{1}}{\sigma^{2}\left(1-\bar{q}_{1}^{2}\right)\left(1-\sigma^{2} \bar{q}_{1}^{2}\right)^{2}} \frac{d v_{2}}{d \bar{q}_{1}}+\right.} \\
& +\frac{4-4 \bar{q}_{1}^{2}+\sigma^{2}\left(-7+12 \bar{q}_{1}^{2}-4 \bar{q}_{1}^{4}\right)+2 \sigma^{4}\left(1-3 \bar{q}_{1}^{2}+\bar{q}_{1}^{4}\right)}{\sigma^{4}\left(1-\bar{q}_{1}^{2}\right)^{2}\left(1-\sigma^{2} \bar{q}_{1}^{2}\right)^{4}}+ \\
& +\frac{\sigma^{6} \bar{q}_{1}^{2}\left(2-3 \bar{q}_{1}^{2}+2 \bar{q}_{1}^{4}\right)}{\sigma^{4}\left(1-\bar{q}_{1}^{2}\right)^{2}\left(1-\sigma^{2} \bar{q}_{1}^{2}\right)^{4}} v_{2} \\
& \left.-\frac{2 \bar{\sigma}}{\sigma^{2} \sqrt{1-\bar{q}_{1}^{2}}\left(1-\sigma^{2} \bar{q}_{1}^{2}\right)^{3}} \frac{d v_{1}}{d \bar{q}_{1}}+\frac{4 \bar{\sigma} \bar{q}_{1}\left(1+\sigma^{2}\left(\bar{q}_{1}^{2}-2\right)\right)}{\sigma^{4}\left(1-\bar{q}_{1}^{2}\right)^{\frac{3}{2}}\left(1-\sigma^{2} \bar{q}_{1}^{2}\right)^{4}} v_{1}\right] \vec{e}_{2}
\end{aligned}
$$

El operador no es diagonal, evidentemente, y su análisis claramente requiere utilizar una base más adecuada en la que resulte una expresión más sencilla.

Base paralela de autovectores de la curvatura. Procederemos de manera similar al caso del TK1. Calculemos en primer lugar las soluciones de la ecuación de traslado paralelo sobre el TK2.

Las ecuaciones del traslado paralelo son

$$
\nabla_{c_{\mathrm{TK} 2}^{\prime}} \vec{U}=\overrightarrow{0}
$$

Una solución evidente es el propio campo vectorial $c_{\mathrm{TK} 2}^{\prime}$, puesto que, como sabemos, las lineas geodésicas son las autoparalelas. La otra solución que buscamos es la dada por el vector normal a la curva solución. Escritas las ecuaciones en la variable $\bar{q}_{1}$ tendremos:

$$
\begin{aligned}
& \frac{d U_{1}}{d \bar{q}_{1}}+\frac{\bar{q}_{1}\left(2 \sigma^{2} \bar{q}_{1}^{2}-1-\sigma^{2}\right)}{\left(1-\sigma^{2} \bar{q}_{1}^{2}\right)\left(1-\bar{q}_{1}^{2}\right)} U_{1}-\frac{\bar{\sigma}}{\sqrt{1-\bar{q}_{1}^{2}}\left(1-\sigma^{2} \bar{q}_{1}^{2}\right)} U_{2}=0 \\
& \frac{d U_{2}}{d \bar{q}_{1}}+\frac{\bar{\sigma}}{\sqrt{1-\bar{q}_{1}^{2}}\left(1-\sigma^{2} \bar{q}_{1}^{2}\right)} U_{1}+\frac{\bar{q}_{1}\left(2 \sigma^{2} \bar{q}_{1}^{2}-1-\sigma^{2}\right)}{\left(1-\sigma^{2} \bar{q}_{1}^{2}\right)\left(1-\bar{q}_{1}^{2}\right)} U_{2}=0
\end{aligned}
$$

despejando en una ecuación y sustituyendo en la otra $U_{2}$, obtenemos a partir del sistema anterior una ecuación de segundo orden:

$$
\sqrt{1-\bar{q}_{1}^{2}}\left(1-\sigma^{2} \bar{q}_{1}^{2}\right) \frac{d^{2} U_{1}}{d \bar{q}_{1}^{2}}+\frac{\bar{q}_{1}}{\sqrt{1-\bar{q}_{1}^{2}}}\left(7 \sigma^{2} \bar{q}_{1}^{2}-3-4 \sigma^{2}\right) \frac{d U_{1}}{d \bar{q}_{1}^{2}}-\frac{2 \sigma^{2}\left(1-4 \bar{q}_{1}^{2}\right)}{\sqrt{1-\bar{q}_{1}^{2}}} U_{1}=0
$$

cuya integración no es difícil, a pesar de su aspecto, y conduce finalmente a las soluciones:

$$
\begin{aligned}
U_{1} & =-\frac{C_{1} \bar{q}_{1}+C_{2} \sqrt{1-\bar{q}_{1}^{2}}}{\sqrt{1-\bar{q}_{1}^{2}}\left(1-\sigma^{2} \bar{q}_{1}^{2}\right)} \\
U_{2} & =\frac{C_{1}\left(1-\bar{q}_{1}^{2}\right)+C_{2} \bar{\sigma}^{2} \bar{q}_{1} \sqrt{1-\bar{q}_{1}^{2}}}{\bar{\sigma} \sqrt{1-\bar{q}_{1}^{2}}\left(1-\sigma^{2} \bar{q}_{1}^{2}\right)}
\end{aligned}
$$


para constantes arbitrarias $C_{1}$ y $C_{2}$.

Si tomamos ahora $C_{1}=0$ y $C_{2}=\frac{-1}{\sigma}$ obtenemos exactamente el campo tangente a la curva:

$$
\vec{U}_{1}=c_{\mathrm{TK} 2}^{\prime}=\frac{1}{\sigma\left(1-\sigma^{2} \bar{q}_{1}^{2}\right)} \vec{e}_{1}-\frac{\bar{\sigma} \bar{q}_{1}}{\sigma \sqrt{1-\bar{q}_{1}^{2}}\left(1-\sigma^{2} \bar{q}_{1}^{2}\right)} \vec{e}_{2}
$$

paralelo y unitario $\left(\left\|\vec{U}_{1}\right\|_{J}=1\right)$. Para obtener una base ortonormal imponemos que el campo $\vec{U}_{2}$ verifique: $\left\langle\vec{U}_{1}, \vec{U}_{2}\right\rangle^{J}=0$ y $\left\|\vec{U}_{2}\right\|_{J}=1$, de manera que obtenemos $C_{1}=\frac{\bar{\sigma}}{\sigma}, C_{2}=0$ y así:

$$
\vec{U}_{2}=\frac{-\bar{\sigma} \bar{q}_{1}}{\sigma \sqrt{1-\bar{q}_{1}^{2}}\left(1-\sigma^{2} \bar{q}_{1}^{2}\right)} \vec{e}_{1}-\frac{1}{\sigma\left(1-\sigma^{2} \bar{q}_{1}^{2}\right)} \vec{e}_{2}
$$

Si se diagonaliza ahora la curvatura seccional, que hemos escrito anteriormente, se obtiene un campo de autovectores con autovalor nulo determinado por la dirección del vector $\vec{U}_{1}$ y un segundo campo, con autovalor dependiente del punto de la curva en el que nos encontremos, precisamente en la dirección de $\vec{U}_{2}\left(\bar{q}_{1}\right)$. Tenemos por tanto, $\forall \bar{q}_{1}$, el resultado:

$$
\begin{aligned}
& K_{c^{\prime}}^{J}\left(\vec{U}_{1}\right)=0 \vec{U}_{1} \\
& K_{c^{\prime}}^{J}\left(\vec{U}_{2}\right)=-\frac{\left(4-\sigma^{2}\right)\left(1+\sigma^{2}\left(\bar{q}_{1}^{2}-2\right)\right)}{\sigma^{4}\left(1-\bar{q}_{1}^{2}\right)^{2}\left(1-\sigma^{2} \bar{q}_{1}^{2}\right)^{3}} \vec{U}_{2}
\end{aligned}
$$

Con todos estos resultados podemos escribir finamente la expresión del operador hessiano correspondiente al funcional de energía en la métrica de Jacobi en función de la base paralela ortonormal de autovectores de la curvatura seccional $\left\{\vec{U}_{1}, \vec{U}_{2}\right\}$ :

$$
\begin{aligned}
\Delta^{J}\left(w_{1} \vec{U}_{1}+w_{2} \vec{U}_{2}\right)= & -\frac{d^{2} w_{1}}{d s^{2}} \vec{U}_{1}+\left[-\frac{d^{2} w_{2}}{d s^{2}}+\frac{\left(4-\sigma^{2}\right)\left(1+\sigma^{2}\left(\bar{q}_{1}^{2}-2\right)\right)}{\sigma^{4}\left(1-\bar{q}_{1}^{2}\right)^{2}\left(1-\sigma^{2} \bar{q}_{1}^{2}\right)^{3}} w_{2}\right] \vec{U}_{2}= \\
= & {\left[\frac{-1}{\sigma^{2}\left(1-\sigma^{2} \bar{q}_{1}^{2}\right)^{2}} \frac{d^{2} w_{1}}{d \bar{q}_{1}^{2}}-\frac{2 \bar{q}_{1}}{\left(1-\sigma^{2} \bar{q}_{1}^{2}\right)^{3}} \frac{d w_{1}}{d \bar{q}_{1}}\right] \vec{U}_{1}+} \\
& +\left[\frac{-1}{\sigma^{2}\left(1-\sigma^{2} \bar{q}_{1}^{2}\right)^{2}} \frac{d^{2} w_{2}}{d \bar{q}_{1}^{2}}-\frac{2 \bar{q}_{1}}{\left(1-\sigma^{2} \bar{q}_{1}^{2}\right)^{3}} \frac{d w_{2}}{d \bar{q}_{1}}+\right. \\
& \left.+\frac{\left(4-\sigma^{2}\right)\left(1+\sigma^{2}\left(\bar{q}_{1}^{2}-2\right)\right)}{\sigma^{4}\left(1-\bar{q}_{1}^{2}\right)^{2}\left(1-\sigma^{2} \bar{q}_{1}^{2}\right)^{3}} w_{2}\right] \vec{U}_{2}
\end{aligned}
$$

Como puede observarse en la expresión anterior, la parte del operador hessiano que actúa sobre las variaciones tangentes a la curva solución es muy simple y, de hecho, similar a la obtenida en el caso del kink TK1. Centraremos por tanto nuestra atención en la parte más compleja del operador, la que actúa sobre las componentes ortogonales a la solución. 


\section{Variaciones Ortogonales}

Para una variación ortogonal: $\vec{V}^{\perp}(s)=w_{2}(s) \vec{U}_{2}(s)$, o bien $\vec{V}^{\perp}\left(\bar{q}_{1}\right)=w_{2}\left(\bar{q}_{1}\right) \vec{U}_{2}\left(\bar{q}_{1}\right)$ tendremos:

$$
\begin{aligned}
\Delta^{J}\left(w_{2} \vec{U}_{2}\right)= & {\left[-\frac{d^{2} w_{2}}{d s^{2}}+\frac{\left(4-\sigma^{2}\right)\left(1+\sigma^{2}\left(\bar{q}_{1}^{2}(s)-2\right)\right)}{\sigma^{4}\left(1-\bar{q}_{1}^{2}(s)\right)^{2}\left(1-\sigma^{2} \bar{q}_{1}^{2}(s)\right)^{3}} w_{2}\right] \vec{U}_{2}=} \\
= & {\left[\frac{-1}{\sigma^{2}\left(1-\sigma^{2} \bar{q}_{1}^{2}\right)^{2}} \frac{d^{2} w_{2}}{d \bar{q}_{1}^{2}}-\frac{2 \bar{q}_{1}}{\left(1-\sigma^{2} \bar{q}_{1}^{2}\right)^{3}} \frac{d w_{2}}{d \bar{q}_{1}}+\right.} \\
& \left.+\frac{\left(4-\sigma^{2}\right)\left(1+\sigma^{2}\left(\bar{q}_{1}^{2}-2\right)\right)}{\sigma^{4}\left(1-\bar{q}_{1}^{2}\right)^{2}\left(1-\sigma^{2} \bar{q}_{1}^{2}\right)^{3}} w_{2}\right] \vec{U}_{2}
\end{aligned}
$$

La variación propia deducida en el hessiano proyectado dinámico:

$$
\vec{V}^{\perp}=v_{2}^{\left(4 \bar{\sigma}^{2}\right)} \vec{a}^{\perp}=\sqrt{1-\bar{q}_{1}^{2}} \vec{a}^{\perp}
$$

se escribe, en la base $\left\{\vec{U}_{1}, \vec{U}_{2}\right\}$, de la forma:

$$
\vec{V}^{\perp}=w_{2}^{\left(4 \bar{\sigma}^{2}\right)} \vec{U}_{2}=-\sigma \sqrt{1-\bar{q}_{1}^{2}}\left(1-\sigma^{2} \bar{q}_{1}^{2}\right) \vec{U}_{2}
$$

de manera que:

$$
\Delta^{J}\left(w_{2}^{\left(4 \bar{\sigma}^{2}\right)} \vec{U}_{2}\right)=\frac{4 \bar{\sigma}^{4}}{\sigma^{4}\left(1-\bar{q}_{1}^{2}\right)^{2}\left(1-\sigma^{2} \bar{q}_{1}^{2}\right)^{4}} w_{2}^{\left(4 \bar{\sigma}^{2}\right)} \vec{U}_{2}
$$

Tal y como ocurría con la solución TK1, la traducción de una autofunción del hessiano dinámico no lo es estrictamente del geométrico.

El valor de las formas cuadráticas hessianas geométricas sobre dicha variación es infinito, como puede comprobarse fácilmente:

$$
\begin{aligned}
& \frac{d^{2} L^{J}\left[c_{\mathrm{TK} 2}\right]}{d \xi^{2}}(0)=\left.\frac{d^{2} L^{J}\left[c_{\mathrm{TK} 2}\right]}{d \xi^{2}}(0)\right|_{\vec{V}=\vec{V}^{\perp}}= \\
& =\int_{-1}^{1} \sigma\left(1-\sigma^{2} \bar{q}_{1}^{2}\right) d \bar{q}_{1}\left\langle\Delta^{J}\left(w_{2}^{\left(4 \bar{\sigma}^{2}\right)} \vec{U}_{2}\right), w_{2}^{\left(4 \bar{\sigma}^{2}\right)} \vec{U}_{2}\right\rangle^{J}= \\
& =\int_{-1}^{1} d \bar{q}_{1} \frac{4 \bar{\sigma}^{4}}{\sigma\left(1-\bar{q}_{1}^{2}\right)\left(1-\sigma^{2} \bar{q}_{1}^{2}\right)}=\infty
\end{aligned}
$$

Sin embargo, si proyectamos, de manera análoga a lo realizado en el caso del hessiano dinámico, obtendremos:

$$
\begin{aligned}
\pi_{2}\left(\Delta^{J}\left(w_{2}^{\left(4 \bar{\sigma}^{2}\right)} \vec{U}_{2}\right)\right)= & \frac{4 \bar{\sigma}^{4}}{\sigma^{4}\left(1-\bar{q}_{1}^{2}\right)^{2}\left(1-\sigma^{2} \bar{q}_{1}^{2}\right)^{4}} w_{2}^{\left(4 \bar{\sigma}^{2}\right)} \pi_{2}\left(\vec{U}_{2}\right)= \\
& =\frac{4 \bar{\sigma}^{4}}{\sigma^{4}\left(1-\bar{q}_{1}^{2}\right)^{2}\left(1-\sigma^{2} \bar{q}_{1}^{2}\right)^{4}} w_{2}^{\left(4 \bar{\sigma}^{2}\right)} \frac{-\vec{e}_{2}}{\sigma\left(1-\sigma^{2} \bar{q}_{1}^{2}\right)}
\end{aligned}
$$


y de esta manera:

$$
\begin{aligned}
& \pi_{2}\left(\frac{d^{2} L^{J}\left[c_{\mathrm{TK} 2}\right]}{d \xi^{2}}(0)\right)=\pi_{2}\left(\left.\frac{d^{2} L^{J}\left[c_{\mathrm{TK} 2}\right]}{d \xi^{2}}(0)\right|_{\vec{V}=\vec{V}^{\perp}}\right)= \\
& =\int_{-1}^{1} \sigma\left(1-\sigma^{2} \bar{q}_{1}^{2}\right) d \bar{q}_{1}\left\langle\pi_{2}\left(\Delta^{J}\left(w_{2}^{\left(4 \bar{\sigma}^{2}\right)} \vec{U}_{2}\right)\right), w_{2}^{\left(4 \bar{\sigma}^{2}\right)} \vec{U}_{2}\right\rangle^{J}= \\
& =\int_{-1}^{1} d \bar{q}_{1} \frac{4 \bar{\sigma}^{4}}{\sigma\left(1-\sigma^{2} \bar{q}_{1}^{2}\right)^{2}}= \\
& =\frac{4 \bar{\sigma}^{2}}{\sigma}+\frac{4 \bar{\sigma}^{4}}{\sigma^{2}} \operatorname{arctanh} \sigma>0
\end{aligned}
$$

Queda probada, por tanto, la estabilidad, tanto dinámica como geométricamente, de la solución TK2. Una vez más insistiremos en que este hecho estaba demostrado ya utilizando el argumento que nos proporciona la energía de los diferentes tipos de kinks.

\subsection{Funcional Segunda Variación. Modelo $O(3)$}

Finalizaremos este capítulo con una sección dedicada a generalizar los resultados obtenidos en el caso del Modelo MSTB al caso tridimensional, el Modelo Sigma $O(3)$ Lineal Deformado.

Como veremos, para los kinks TK1, TK2 $\sigma_{2}$ y TK2 $\sigma_{3}$, la generalización citada no será difícil en absoluto dado que introducirá, en los operadores hessianos correspondientes, únicamente una componente desacoplada con las demás, es decir, un término diagonal. Además, dicho término conduce a un problema espectral conocido y similar a los ya expuestos en la sección anterior.

\subsubsection{Funcionales Hessianos para el kink TK1}

En el caso del kink TK1, los resultados que se obtuvieron en la sección anterior serán válidos ahora sin más que abordar el problema desde el punto de vista tridimensional. Manteniendo los criterios de notación ya utilizados, escribiremos:

$$
\vec{V}=v_{1} \vec{e}_{1}+v_{2} \vec{e}_{2}+v_{3} \vec{e}_{3}
$$

para toda variación propia considerada.

Es entonces trivial demostrar que la forma cuadrática hessiana dinámica sobre la solución TK1, $\left(\bar{q}_{1}, \bar{q}_{2}, \bar{q}_{3}\right)=(\tanh t, 0,0)$, determinada por el funcional:

$$
\frac{d^{2} S\left[c_{\mathrm{TK} 1}\right]}{d \xi^{2}}(0)=\int_{-\infty}^{\infty} d t\langle\Delta \vec{V}, \vec{V}\rangle
$$


tiene asociado el operador hessiano $\Delta$ que actúa en la forma:

$$
\begin{aligned}
\Delta \vec{V}= & \left(-\frac{d^{2}}{d t^{2}}+4-\frac{6}{\cosh ^{2} t}\right) v_{1} \vec{e}_{1}+ \\
& +\left(-\frac{d^{2}}{d t^{2}}+\sigma_{2}^{2}-\frac{2}{\cosh ^{2} t}\right) v_{2} \vec{e}_{2}+ \\
& +\left(-\frac{d^{2}}{d t^{2}}+\sigma_{3}^{2}-\frac{2}{\cosh ^{2} t}\right) v_{3} \vec{e}_{3}
\end{aligned}
$$

Se trata por tanto de un operador diagonal y, en consecuencia, tendremos que el problema espectral separa en tres ecuaciones de Schödinger con potencial de Pösch-Teller, en lugar de las dos ecuaciones que obteníamos para el Modelo $O(2)$.

El espectro de variaciones tangentes que ya analizamos se repite ahora de manera idéntica, mientras que el espectro de variaciones ortogonales aparece tanto en la dirección de $\vec{e}_{2}$ como en la de $\vec{e}_{3}$ (evidentemente sustituyendo la constante $\sigma$ por $\sigma_{2}$, para la dirección $\vec{e}_{2}$, y por $\sigma_{3}$ para $\vec{e}_{3}$ ) .

Ninguno de los resultados, como hemos dicho, difiere de los obtenidos y, por tanto, no incluiremos los mismos en este apartado. Nos remitimos a la sección 5.2.1 con las sustituciones antedichas.

De esta forma, tendremos ahora dos direcciones de inestabilidad para el kink TK1. Las variaciones $\vec{V}=\frac{1}{\cosh t} \vec{e}_{2}$ y $\vec{V}=\frac{1}{\cosh t} \vec{e}_{3}$, ambas ortogonales a la trayectoria solución, verificarán:

$$
\frac{d^{2} S\left[c_{\mathrm{TK} 1}\right]}{d \xi^{2}}(0)=\int_{-\infty}^{\infty} \frac{-1+\sigma_{2}^{2}}{\cosh ^{2} t} d t=-2 \bar{\sigma}_{2}^{2}<0
$$

para la primera $y$ :

$$
\frac{d^{2} S\left[c_{\mathrm{TK} 1}\right]}{d \xi^{2}}(0)=\int_{-\infty}^{\infty} \frac{-1+\sigma_{3}^{2}}{\cosh ^{2} t} d t=-2 \bar{\sigma}_{3}^{2}<0
$$

para la segunda. En el primer caso el resultado nos informa de la pérdida de energía presente en la dirección que conduce al kink TK2 $\sigma_{2}$ y, en el segundo, de la correspondiente al kink TK2 $\sigma_{3}$.

En lo que respecta a las formas cuadráticas hessianas geométricas, encontramos la siguiente situación: la curvatura seccional se escribe de manera sencilla sobre la solución, en la forma:

$$
K_{c_{\mathrm{TK} 1}^{\prime}}^{J}(\vec{V})=\left(\begin{array}{ccc}
0 & 0 & 0 \\
0 & \frac{4-\sigma_{2}^{2}}{\left(1-\bar{q}_{1}^{2}\right)^{4}} & 0 \\
0 & 0 & \frac{4-\sigma_{3}^{2}}{\left(1-\bar{q}_{1}^{2}\right)^{4}}
\end{array}\right)\left(\begin{array}{l}
v_{1} \\
v_{2} \\
v_{3}
\end{array}\right)
$$


y, por tanto, es fácil comprobar que se reproducen los resultados obtenidos para las variaciones tangentes, mientras que los correspondientes a las variaciones ortogonales son ahora válidos tanto para la componente segunda como para la tercera.

Desde el punto de vista geométrico tendremos en este caso dos campos de Jacobi independientes para la correspondiente ecuación de desviación geodésica, por un lado encontramos:

$$
\vec{V}=e^{\sigma_{2} \arctan \bar{q}_{1}}\left(\sigma_{2}-\bar{q}_{1}\right) \vec{e}_{2}
$$

que nos muestra que el foco $\mathrm{F}_{2}$ es un punto conjugado del de vacío, $\vec{v}^{-}$. Y, por otro:

$$
\vec{V}=e^{\sigma_{3} \arctan \bar{q}_{1}}\left(\sigma_{3}-\bar{q}_{1}\right) \vec{e}_{3}
$$

que hace otro tanto con el foco $\mathrm{F}_{3}$. Este hecho demuestra que el índice de Morse del kink TK1, considerado como integrante del espacio de soluciones del Modelo $O(3)$, es dos. Utilizaremos este resultado en el Capítulo 6.

\subsubsection{Funcional Hessiano Dinámico: TK2 $\sigma_{2}$ y TK2 $\sigma_{3}$}

Analizaremos a continuación los funcionales hessianos sobre las soluciones que hemos denominado TK2 $\sigma_{2}$ y TK $2 \sigma_{3}$ que, como vimos en el Capítulo 3, se corresponden con los kinks envolventes de cada uno de los Modelos $O(2)$ que constituyen los kinks embebidos del Modelo Sigma $O(3)$ Lineal Deformado. Con respecto a los resultados obtenidos en la sección anterior para el kink TK2, encontraremos ahora que una parte del hessiano coincide con la calculada entonces (intercambiando la constante $\sigma$ por la que corresponda en cada caso), mientras que la perspectiva tridimensional se refleja en la aparición de una nueva componente.

\section{TK2 $\sigma_{2}$}

En el plano $q_{3}=0$ encontrábamos, entre otras, la solución TK2 $\sigma_{2}$ :

$$
\bar{q}_{1}=\tanh \left(\sigma_{2} t\right) ; \quad \bar{q}_{2}=\bar{\sigma}_{2} \operatorname{sech}\left(\sigma_{2} t\right) ; \quad \bar{q}_{3}=0
$$

Si $\vec{V}=v_{1} \vec{e}_{1}+v_{2} \vec{e}_{2}+v_{3} \vec{e}_{3}$ denota una variación propia cualquiera, tendremos que el funcional

$$
\frac{d^{2} S\left[c_{\mathrm{TK} 2 \sigma_{2}}\right]}{d \xi^{2}}=\int_{t_{1}}^{t_{2}} d t\langle\Delta \vec{V}, \vec{V}\rangle
$$


presenta la siguiente expresión para el operador $\Delta$ :

$$
\begin{aligned}
\Delta \vec{V}= & {\left[\left(-\frac{d^{2}}{d t^{2}}+4-\frac{2\left(2+\sigma_{2}^{2}\right)}{\cosh ^{2}\left(\sigma_{2} t\right)}\right) v_{1}+\left(4 \sqrt{1-\sigma_{2}^{2}} \frac{\tanh \left(\sigma_{2} t\right)}{\cosh \left(\sigma_{2} t\right)}\right) v_{2}\right] \vec{e}_{1}+} \\
& +\left[\left(4 \sqrt{1-\sigma_{2}^{2}} \frac{\tanh \left(\sigma_{2} t\right)}{\cosh \left(\sigma_{2} t\right)}\right) v_{1}+\left(-\frac{d^{2}}{d t^{2}}+\sigma_{2}^{2}-\frac{2\left(3 \sigma_{2}^{2}-2\right)}{\cosh ^{2}\left(\sigma_{2} t\right)}\right) v_{2}\right] \vec{e}_{2}+ \\
& +\left[\left(-\frac{d^{2}}{d t^{2}}+\sigma_{3}^{2}-\frac{2 \sigma_{2}^{2}}{\cosh ^{2}\left(\sigma_{2} t\right)}\right) v_{3}\right] \vec{e}_{3}
\end{aligned}
$$

$$
\begin{aligned}
& \Delta \vec{V} \equiv \\
& \left(\begin{array}{ccc}
-\frac{d^{2}}{d t^{2}}+4-\frac{2\left(2+\sigma_{2}^{2}\right)}{\cosh ^{2}\left(\sigma_{2} t\right)} & 4 \sqrt{1-\sigma_{2}^{2} \frac{\tanh \left(\sigma_{2} t\right)}{\cosh \left(\sigma_{2} t\right)}} & 0 \\
4 \sqrt{1-\sigma_{2}^{2} \frac{\tanh \left(\sigma_{2} t\right)}{\cosh \left(\sigma_{2} t\right)}} & -\frac{d^{2}}{d t^{2}}+\sigma_{2}^{2}-\frac{2\left(3 \sigma_{2}^{2}-2\right)}{\cosh ^{2}\left(\sigma_{2} t\right)} & 0 \\
0 & 0 & -\frac{d^{2}}{d t^{2}}+\sigma_{3}^{2}-\frac{2 \sigma_{2}^{2}}{\cosh ^{2}\left(\sigma_{2} t\right)}
\end{array}\right)\left(\begin{array}{l}
v_{1} \\
v_{2} \\
v_{3}
\end{array}\right)
\end{aligned}
$$

El problema espectral asociado a este operador es obviamente separable en dos partes desacopladas. Las variaciones de la forma: $\vec{V}=v_{1} \vec{e}_{1}+v_{2} \vec{e}_{2}$ conducen a un problema espectral similar al tratado para el modelo MSTB y, en consecuencia, no repetiremos aquí los resultados obtenidos.

Por su parte, las variaciones de la forma $\vec{V}=v_{3} \vec{e}_{3}$ proporcionan un problema espectral nuevo consistente en una ecuación de Schrödinger idéntica a algunas de las ya analizadas.

\section{Variaciones en la dirección $\vec{e}_{3}$}

Restringiéndonos a variaciones en la dirección del vector $\vec{e}_{3}$ :

$$
\vec{V}=v_{3} \vec{e}_{3}
$$

el operador $\Delta$ actuará de la manera siguiente:

$$
\Delta\left(v_{3} \vec{e}_{3}\right)=\left[\left(-\frac{d^{2}}{d t^{2}}+\sigma_{3}^{2}-\frac{2 \sigma_{2}^{2}}{\cosh ^{2}\left(\sigma_{2} t\right)}\right) v_{3}\right] \vec{e}_{3}
$$

El espectro es fácilmente calculable y se obtiene:

- Modo discreto:

$$
v_{3}=\frac{1}{\cosh \left(\sigma_{2} t\right)}
$$

con autovalor asociado: $\lambda=\sigma_{3}^{2}-\sigma_{2}^{2}$. 
- Espectro continuo. Las autofunciones del continuo son:

$$
\tilde{v}_{3}^{\left(\lambda_{k}\right)}=e^{i k \sigma_{2} t}\left(i k-\tanh \left(\sigma_{2} t\right)\right)
$$

con

$$
\lambda_{k}=k^{2} \sigma_{2}^{2}+\sigma_{3}^{2}
$$

$\forall k \in \mathbb{R}$. Una vez más, las autofunciones del continuo complejas antedichas pueden combinarse para determinar variaciones reales de tipo par e impar. Tendremos así, para las variaciones pares:

$$
v_{3}^{\left(\lambda_{k}\right)}=\operatorname{sen}\left(k \sigma_{2} t\right) \tanh \left(\sigma_{2} t\right)-k \cos \left(\sigma_{2} t\right)
$$

y, para las impares:

$$
v_{3}^{\left(\lambda_{k}\right)}=\cos \left(k \sigma_{2} t\right) \tanh \left(\sigma_{2} t\right)+k \operatorname{sen}\left(\sigma_{2} t\right)
$$

De manera análoga a lo que encontramos en el caso del kink TK1, el operador hessiano, en esta dirección, posee un "modo" cero (variación no propia) dado por la autofunción ${ }^{14}$ :

$$
v_{3}=e^{\sigma_{3} t}\left(\frac{\sigma_{3}}{\sigma_{2}}-\tanh \left(\sigma_{2} t\right)\right)
$$

Si bien únicamente el modo discreto conduce a una variación propia genuina, es posible admitir como propias a las variaciones que resultan del proceso límite de discretización que puede realizarse sobre las autofunciones del continuo, de manera absolutamente idéntica a lo efectuado en la sección anterior.

Para la variación propia: $v_{3}=\frac{1}{\cosh \left(\sigma_{2} t\right)}$, tendremos que la forma cuadrática hessiana toma el valor:

$$
\frac{d^{2} S\left[c_{\mathrm{TK} 2 \sigma_{2}}\right]}{d \xi^{2}}=\int_{-\infty}^{\infty} d t \frac{\sigma_{3}^{2}-\sigma_{2}^{2}}{\cosh ^{2}\left(\sigma_{2} t\right)}=2 \frac{\sigma_{3}^{2}-\sigma_{2}^{2}}{\sigma_{2}}>0
$$

que nos confirma el resultado que obteníamos por medio del análisis de las energías, en el Capítulo 3, se trata de una solución estable.

\footnotetext{
${ }^{14}$ Como en el caso citado, realmente son dos las autofunciones de autovalor nulo puesto que

$$
v_{3}=e^{-\sigma_{3} t}\left(-\frac{\sigma_{3}}{\sigma_{2}}-\tanh \left(\sigma_{2} t\right)\right)
$$

relacionada con la otra por medio de inversión "temporal", también es un "modo" nulo. Evidentemente, una de ellas está asociada al kink TK2 $\sigma_{2}$ y la otra al correspondiente antikink.
} 


\section{$\mathrm{TK} 2 \sigma_{3}$}

En el caso de la solución TK2 $\sigma_{3}$, de ecuaciones:

$$
\bar{q}_{1}=\tanh \left(\sigma_{3} t\right) ; \quad \bar{q}_{2}=0 ; \quad \bar{q}_{3}=\bar{\sigma}_{3} \operatorname{sech}\left(\sigma_{3} t\right)
$$

el cálculo anterior se repite sin más que intercambiar los papeles de la segunda y la tercera componentes en todas las expresiones, además de sustituir $\sigma_{2}$ por $\sigma_{3}, \mathrm{y}$ recíprocamente.

El operador hessiano actuará, en este caso, en la forma:

$$
\begin{aligned}
& \Delta \vec{V} \equiv \\
& \left(\begin{array}{ccc}
-\frac{d^{2}}{d t^{2}}+4-\frac{2\left(2+\sigma_{3}^{2}\right)}{\cosh ^{2}\left(\sigma_{3} t\right)} & 0 & 4 \sqrt{1-\sigma_{3}^{2}} \frac{\tanh \left(\sigma_{3} t\right)}{\cosh \left(\sigma_{3} t\right)} \\
0 & -\frac{d^{2}}{d t^{2}}+\sigma_{2}^{2}-\frac{2 \sigma_{3}^{2}}{\cosh ^{2}\left(\sigma_{3} t\right)} & 0 \\
4 \sqrt{1-\sigma_{3}^{2} \frac{\tanh \left(\sigma_{3} t\right)}{\cosh \left(\sigma_{3} t\right)}} & 0 & -\frac{d^{2}}{d t^{2}}+\sigma_{3}^{2}-\frac{2\left(3 \sigma_{3}^{2}-2\right)}{\cosh ^{2}\left(\sigma_{3} t\right)}
\end{array}\right)\left(\begin{array}{l}
v_{1} \\
v_{2} \\
v_{3}
\end{array}\right)
\end{aligned}
$$

y, en definitiva, el problema espectral separa nuevamente en dos problemas desacoplados. Las variaciones del tipo $\vec{V}=v_{1} \vec{e}_{1}+v_{3} \vec{e}_{3}$ producirán el espectro encontrado para el caso del Modelo MSTB (cambiando $\sigma$ por $\sigma_{3}$ y recordando que se plantea ahora el problema en las componentes primera y tercera).

Por su parte, las variaciones del tipo $\vec{V}=v_{2} \vec{e}_{2}$ son ahora las novedosas.

\section{Variaciones en la dirección $\vec{e}_{2}$}

Una vez más no será necesario realizar nuevos cálculos sino que el apartado anterior nos proporciona directamente los resultados:

$$
\Delta\left(v_{2} \vec{e}_{2}\right)=\left[\left(-\frac{d^{2}}{d t^{2}}+\sigma_{2}^{2}-\frac{2 \sigma_{3}^{2}}{\cosh ^{2}\left(\sigma_{3} t\right)}\right) v_{2}\right] \vec{e}_{2}
$$

para el operador y, el espectro:

- Modo discreto:

$$
v_{2}=\frac{1}{\cosh \left(\sigma_{3} t\right)}
$$

con autovalor asociado: $\lambda=\sigma_{2}^{2}-\sigma_{3}^{2}$.

- Espectro continuo:

$$
v_{2}^{\left(\lambda_{k}\right)}=\operatorname{sen}\left(k \sigma_{3} t\right) \tanh \left(\sigma_{3} t\right)-k \cos \left(\sigma_{3} t\right)
$$

$\mathrm{y}$

$$
v_{2}^{\left(\lambda_{k}\right)}=\cos \left(k \sigma_{3} t\right) \tanh \left(\sigma_{3} t\right)+k \operatorname{sen}\left(\sigma_{3} t\right)
$$


con

$$
\lambda_{k}=k^{2} \sigma_{3}^{2}+\sigma_{2}^{2}
$$

$\forall k \in \mathbb{R}$

Para la variación propia: $v_{2}=\frac{1}{\cosh \left(\sigma_{3} t\right)}$, obtendremos:

$$
\frac{d^{2} S\left[c_{\mathrm{TK} 2 \sigma_{3}}\right]}{d \xi^{2}}=\int_{-\infty}^{\infty} d t \frac{\sigma_{2}^{2}-\sigma_{3}^{2}}{\cosh ^{2}\left(\sigma_{3} t\right)}=2 \frac{\sigma_{2}^{2}-\sigma_{3}^{2}}{\sigma_{3}}<0
$$

En este caso, el hecho de que $\sigma_{2}^{2}$ sea menor que $\sigma_{3}^{2}$ nos determina inestabilidad de esta solución y, precisamente, en la dirección que conduce hacia el kink estable, TK $2 \sigma_{2}$.

Nuevamente tenemos autofunciones nulas del operador hessiano en esta dirección, en este caso:

$$
v_{2}=e^{\sigma_{2} t}\left(\frac{\sigma_{2}}{\sigma_{3}}-\tanh \left(\sigma_{3} t\right)\right)
$$

y

$$
v_{2}=e^{-\sigma_{2} t}\left(-\frac{\sigma_{2}}{\sigma_{3}}-\tanh \left(\sigma_{3} t\right)\right)
$$

Observando el comportamiento asintótico de ambas, es trivial deducir que la primera de ellas nos va a proporcionar, en la versión geométrica, un campo de Jacobi asociado a la solución kink, mientras que la segunda estará asociada a la solución antikink.

\subsubsection{Funcional Hessiano Geométrico: TK2 $\sigma_{2}$ y TK2 $\sigma_{3}$}

Computaremos a continuación las formas cuadráticas hessianas geométricas para las variaciones en la dirección $\vec{e}_{3}$, en el caso del kink TK2 $\sigma_{2}$, y para la dirección $\vec{e}_{2}$, en el del TK2 $\sigma_{3}$. Dado que en ambos casos se trata de variaciones ortogonales, plantearemos únicamente la forma cuadrática asociada al funcional de longitud, dado que, para ese tipo de variaciones, coincide con el de la energía.

El funcional será por tanto:

$$
\frac{d^{2} L^{J}\left[c_{\mathrm{TK} 2 \sigma_{2}}\right]}{d \xi^{2}}=\int_{s_{1}}^{s_{2}} d s\left\langle\Delta^{J} \vec{V}, \vec{V}\right\rangle
$$

con

$$
\Delta^{J} \vec{V}=-\nabla_{c_{\mathrm{TK} 2 \sigma_{2}}^{\prime}}^{J} \nabla_{c_{\mathrm{TK} 2 \sigma_{2}}^{\prime}}^{J} \vec{V}-K_{c_{\mathrm{TK} 2 \sigma_{2}}^{\prime}}^{J}(\vec{V})
$$


Si computamos la curvatura seccional de la métrica de Jacobi sobre el kink TK $2 \sigma_{2}$, tendremos (para $\vec{V}=v_{1} \vec{e}_{1}+v_{2} \vec{e}_{2}+v_{3} \vec{e}_{3}$ ):

$$
\begin{aligned}
& K_{c_{\mathrm{T} K 2 \sigma_{2}}^{J}}^{J}(\vec{V})= \\
& =\left[-\frac{\left(4-\sigma_{2}^{2}\right) \bar{\sigma}_{2}^{2} \bar{q}_{1}^{2}\left(1+\sigma_{2}^{2} \bar{q}_{1}^{2}-2 \sigma_{2}^{2}\right)}{\sigma_{2}^{4}\left(1-\bar{q}_{1}^{2}\right)^{2}\left(1-\sigma_{2}^{2} \bar{q}_{1}^{2}\right)^{4}} v_{1}-\frac{\left(4-\sigma_{2}^{2}\right) \bar{\sigma}_{2} \bar{q}_{1}\left(1+\sigma_{2}^{2} \bar{q}_{1}^{2}-2 \sigma_{2}^{2}\right)}{\sigma_{2}^{4}\left(1-\bar{q}_{1}^{2}\right)^{\frac{3}{2}}\left(1-\sigma_{2}^{2} \bar{q}_{1}^{2}\right)^{4}} v_{2}\right] \vec{e}_{1}+ \\
& +\left[-\frac{\left(4-\sigma_{2}^{2}\right) \bar{\sigma}_{2} \bar{q}_{1}\left(1+\sigma_{2}^{2} \bar{q}_{1}^{2}-2 \sigma_{2}^{2}\right)}{\sigma_{2}^{4}\left(1-\bar{q}_{1}^{2}\right)^{\frac{3}{2}}\left(1-\sigma_{2}^{2} \bar{q}_{1}^{2}\right)^{4}} v_{1}-\frac{\left(4-\sigma_{2}^{2}\right)\left(1+\sigma_{2}^{2} \bar{q}_{1}^{2}-2 \sigma_{2}^{2}\right)}{\sigma_{2}^{4}\left(1-\bar{q}_{1}^{2}\right)\left(1-\sigma_{2}^{2} \bar{q}_{1}^{2}\right)^{4}} v_{2}\right] \vec{e}_{2}+ \\
& +\left[\frac{-\sigma_{3}^{2}+\sigma_{2}^{4}\left(4-\sigma_{2}^{2}\right)\left(1-\bar{q}_{1}^{2}\right)^{2}+\sigma_{2}^{2}\left(3 \bar{\sigma}_{2}^{2}+\sigma_{2}^{4}\right)+\sigma_{2}^{2} \bar{\sigma}_{3}^{2} \bar{q}_{1}^{2}\left(\sigma_{2}^{2} \bar{q}_{1}^{2}-2\right)}{\sigma_{2}^{4}\left(1-\bar{q}_{1}^{2}\right)^{2}\left(1-\sigma_{2}^{2} \bar{q}_{1}^{4}\right)^{4}} v_{3}\right] \vec{e}_{3}
\end{aligned}
$$

Repitiendo los argumentos que se utilizaron en la sección anterior, calcularemos a continuación una base que se traslade paralelamente sobre la curva $c_{\mathrm{TK} 2 \sigma_{2}}$. De esta manera, si escribimos $\vec{V}=w_{1} \vec{U}_{1}+w_{2} \vec{U}_{2}+w_{3} \vec{U}_{3}$, siendo $\left\{\vec{U}_{1}, \vec{U}_{2}, \vec{U}_{3}\right\}$ tal base, tendremos:

$$
\nabla_{c_{\mathrm{TK} 2 \sigma_{2}}^{\prime}}^{J} \nabla_{c_{\mathrm{TK} 2 \sigma_{2}}^{\prime}}^{J} \quad \vec{V}=\frac{d^{2} w_{1}}{d s^{2}} \vec{U}_{1}+\frac{d^{2} w_{3}}{d s^{2}} \vec{U}_{3}+\frac{d^{2} w_{3}}{d s^{2}} \vec{U}_{3}
$$

La resolución de la ecuación del traslado paralelo:

$$
\nabla_{c_{\mathrm{TK} 2 \sigma_{2}}^{\prime}} \vec{U}=\overrightarrow{0}
$$

fijando como condición que las soluciones sean autovectores de la curvatura seccional sobre la curva, nos proporciona la base paralela de autovectores ortonormales:

$$
\begin{aligned}
\vec{U}_{1} & =\frac{1}{\sigma_{2}\left(1-\sigma_{2}^{2} \bar{q}_{1}^{2}\right)} \vec{e}_{1}-\frac{\sqrt{1-\sigma^{2}} \bar{q}_{1}}{\sigma_{2} \sqrt{1-\bar{q}_{1}^{2}}\left(1-\sigma_{2}^{2} \bar{q}_{1}^{2}\right)} \vec{e}_{2} \\
\vec{U}_{2} & =-\frac{\sqrt{1-\sigma^{2}} \bar{q}_{1}}{\sigma_{2} \sqrt{1-\bar{q}_{1}^{2}}\left(1-\sigma_{2}^{2} \bar{q}_{1}^{2}\right)} \vec{e}_{1}-\frac{1}{\sigma_{2}\left(1-\sigma_{2}^{2} \bar{q}_{1}^{2}\right)} \vec{e}_{2} \\
\vec{U}_{3} & =\frac{1}{\sigma_{2} \sqrt{1-\bar{q}_{1}^{2}} \sqrt{1-\sigma_{2}^{2} \bar{q}_{1}^{2}}} \vec{e}_{3}
\end{aligned}
$$

tales que $\vec{U}_{1}$ es autovector de la curvatura con autovalor nulo, $\vec{U}_{2}$ tiene autovalor:

$$
\lambda_{2}=-\frac{\left(4-\sigma_{2}^{2}\right)\left(1+\sigma_{2}^{2}\left(\bar{q}_{1}^{2}-2\right)\right)}{\sigma_{2}^{4}\left(1-\bar{q}_{1}^{2}\right)^{2}\left(1-\sigma_{2}^{2} \bar{q}_{1}^{2}\right)^{3}}
$$

y $\vec{U}_{3}$ tiene el valor propio asociado:

$$
\lambda_{3}=\frac{-\sigma_{3}^{2}+\sigma_{2}^{4}\left(4-\sigma_{2}^{2}\right)\left(1-\bar{q}_{1}^{2}\right)^{2}+\sigma_{2}^{2}\left(3 \bar{\sigma}_{2}^{2}+\sigma_{2}^{4}\right)+\sigma_{2}^{2} \bar{\sigma}_{3}^{2} \bar{q}_{1}^{2}\left(\sigma_{2}^{2} \bar{q}_{1}^{2}-2\right)}{\sigma_{2}^{4}\left(1-\bar{q}_{1}^{2}\right)^{2}\left(1-\sigma_{2}^{2} \bar{q}_{1}^{2}\right)^{4}}
$$


El operador $\Delta^{J}$, sobre esta base, se escribe:

$$
\begin{aligned}
& \Delta^{J}\left(w_{1} \vec{U}_{1}+w_{2} \vec{U}_{2}+w_{3} \vec{U}_{3}\right)=-\frac{d^{2} w_{1}}{d s^{2}} \vec{U}_{1}+ \\
& +\left[-\frac{d^{2} w_{2}}{d s^{2}}+\frac{\left(4-\sigma_{2}^{2}\right)\left(1+\sigma_{2}^{2}\left(\bar{q}_{1}^{2}-2\right)\right)}{\sigma_{2}^{4}\left(1-\bar{q}_{1}^{2}\right)^{2}\left(1-\sigma_{2}^{2} \bar{q}_{1}^{2}\right)^{3}} w_{2}\right] \vec{U}_{2}+ \\
& {\left[-\frac{d^{2} w_{3}}{d s^{2}}-\frac{-\sigma_{3}^{2}+\sigma_{2}^{4}\left(4-\sigma_{2}^{2}\right)\left(1-\bar{q}_{1}^{2}\right)^{2}+\sigma_{2}^{2}\left(3 \bar{\sigma}_{2}^{2}+\sigma_{2}^{4}\right)+\sigma_{2}^{2} \bar{\sigma}_{3}^{2} \bar{q}_{1}^{2}\left(\sigma_{2}^{2} \bar{q}_{1}^{2}-2\right)}{\sigma_{2}^{4}\left(1-\bar{q}_{1}^{2}\right)^{2}\left(1-\sigma_{2}^{2} \bar{q}_{1}^{2}\right)^{4}} w_{3}\right] \vec{U}_{3}}
\end{aligned}
$$

mientras que si utilizamos el parámetro $\bar{q}_{1}$ :

$$
\frac{d^{2}}{d s^{2}}=\frac{1}{\sigma_{2}^{2}\left(1-\sigma_{2}^{2} \bar{q}_{1}^{2}\right)^{2}} \frac{d^{2}}{d \bar{q}_{1}^{2}}+\frac{2 \bar{q}_{1}}{\left(1-\sigma_{2}^{2} \bar{q}_{1}^{2}\right)^{3}} \frac{d}{d \bar{q}_{1}}
$$

El problema vuelve a ser separable. El análisis de las variaciones en las direcciones $\vec{U}_{1}$ y $\vec{U}_{2}$ es el ya realizado para el modelo $O(2)$. Para la componente en $\vec{U}_{3}$ (variaciones binormales a la geodésica) se tiene:

$$
\begin{gathered}
\vec{V}=\frac{1}{\cosh \sigma_{2} t} \vec{e}_{3} \equiv w_{3} \vec{U}_{3} ; \quad w_{3}=\sigma_{2}\left(1-\bar{q}_{1}^{2}\right) \sqrt{1-\sigma_{2}^{2} \bar{q}_{1}^{2}} \\
\Delta^{J}\left(w_{3} \vec{U}_{3}\right)=\frac{\sigma_{3}^{2}-\sigma_{2}^{2}}{\sigma_{2}^{4}\left(1-\bar{q}_{1}^{2}\right)^{2}\left(1-\sigma_{2}^{2} \bar{q}_{1}^{2}\right)^{2}} w_{3} \vec{U}_{3}
\end{gathered}
$$

de manera que, para la variación discreta, la de mínimo autovalor, tendremos:

$$
\begin{aligned}
\frac{d^{2} L^{J}\left[c_{\mathrm{TK} 2 \sigma_{2}}\right]}{d \xi^{2}} & =\int_{-1}^{1} \sigma_{2}\left(1-\sigma_{2}^{2} \bar{q}_{1}^{2}\right) d \bar{q}_{1} \frac{\sigma_{3}^{2}-\sigma_{2}^{2}}{\sigma_{2}^{4}\left(1-\bar{q}_{1}^{2}\right)^{2}\left(1-\sigma_{2}^{2} \bar{q}_{1}^{2}\right)^{2}} w_{3}^{2}\left\|\vec{U}_{3}\right\|_{J}^{2}= \\
& =\frac{\sigma_{3}^{2}-\sigma_{2}^{2}}{\sigma_{2}} \int_{-1}^{1} d \bar{q}_{1}=2 \frac{\sigma_{3}^{2}-\sigma_{2}^{2}}{\sigma_{2}}>0
\end{aligned}
$$

Como vemos, el funcional hessiano dinámico y el geométrico coinciden para las variaciones en la dirección de $\vec{e}_{3}$ (o de $\vec{U}_{3}$ ). Este hecho no es, obviamente, casual. Si calculamos el término que diferenciaba ambos funcionales, para esta geodésica, tendremos que el campo $\vec{F}^{J}$ verifica:

$$
\begin{aligned}
\left.\vec{F}^{J}\right|_{\mathrm{TK} 2 \sigma_{2}}= & \left.\operatorname{grad}^{J} \ln f\right|_{\mathrm{TK} 2 \sigma_{2}}=\frac{\sigma_{2}^{2}}{\left(\sigma_{2}^{2}\left(1-\bar{q}_{1}^{2}\right)\left(1-\sigma_{2}^{2} \bar{q}_{1}^{2}\right)\right)^{2}}\left[-4 \bar{q}_{1}\left(1-\bar{q}_{1}^{2}\right) \vec{e}_{1}+\right. \\
& \left.+\left(-4 \bar{\sigma}_{2}\left(1-\bar{q}_{1}^{2}\right)^{\frac{3}{2}}+2 \bar{\sigma}_{2} \sqrt{1-\bar{q}_{1}^{2}}\right) \vec{e}_{2}+0 \vec{e}_{3}\right]
\end{aligned}
$$

con $f=\left(\bar{q}_{1}^{2}+\bar{q}_{2}^{2}+\bar{q}_{3}^{2}-1\right)^{2}+\sigma_{2}^{2} \bar{q}_{2}^{2}+\sigma_{3}^{2} \bar{q}_{3}^{2}$. De esta forma:

$$
\left\langle\vec{F}^{J}, w_{3} \vec{U}_{3}\right\rangle_{J}=0
$$


y, en consecuencia, el término de diferencia se anula.

Si repetimos ahora los cálculos para el kink TK2 $\sigma_{3}$ tendremos idénticos resultados salvo el intercambio entre las constantes $\sigma_{2}$ y $\sigma_{3}$. Dicho intercambio produce, obviamente, que la forma cuadrática sea definida negativa y, por tanto, se confirma la inestabilidad también desde el punto de vista de la métrica de Jacobi.

Finalmente, hemos obtenido en el apartado anterior que existen modos nulos en la dirección del vector binormal, tanto para el kink TK2 $\sigma_{2}$ como para el TK2 $\sigma_{3}$. Dado que los funcionales hessianos dinámico y geométrico coinciden, en este caso, dicho hallazgo se traduce en la existencia de campos de Jacobi no triviales.

Para el caso TK2 $\sigma_{2}$ tendremos el campo de Jacobi:

$$
\vec{V}=e^{\sigma_{3} t}\left(\frac{\sigma_{3}}{\sigma_{2}}-\tanh \left(\sigma_{2} t\right)\right) \vec{e}_{3}
$$

que se anula en el punto de partida, $\vec{v}^{-}=(-1,0)$, en $t \rightarrow-\infty$, y no se anula en más puntos, dado que $\sigma_{3}>\sigma_{2}$. No existe, en consecuencia, ningún punto conjugado del de vacío a lo largo de dicha solución. Análogamente, la variación $\vec{V}=e^{-\sigma_{3} t}\left(-\frac{\sigma_{3}}{\sigma_{2}}-\tanh \left(\sigma_{2} t\right)\right) \vec{e}_{3}$ nos condce a la misma situación con respecto al antikink ATK $2 \sigma_{2}$. Hemos demostrado, en consecuencia, que, como veremos en el Capítulo 6, el índice de Morse de la trayectoria TK2 $\sigma_{2}$ es nulo.

En lo que se refiere al kink TK2 $\sigma_{3}$, los campos de Jacobi serán ahora:

$$
\vec{V}=e^{\sigma_{2} t}\left(\frac{\sigma_{2}}{\sigma_{3}}-\tanh \left(\sigma_{3} t\right)\right) \vec{e}_{2}
$$

y

$$
\vec{V}=e^{-\sigma_{2} t}\left(-\frac{\sigma_{2}}{\sigma_{3}}-\tanh \left(\sigma_{3} t\right)\right) \vec{e}_{2}
$$

El primero de ellos se anula en el punto de vacío $\vec{v}^{-}$, cuando $t \rightarrow-\infty$, y en uno de los puntos umbílicos del elipsoide, cuando $t=\frac{1}{\sigma_{3}} \operatorname{arctanh} \frac{\sigma_{2}}{\sigma_{3}}$. Un cálculo elemental nos muestra que se trata del segundo de los punto umbílicos que la solución TK $2 \sigma_{3}$ atraviesa (correspondiente a $\bar{q}_{1}>0$ ). Demostramos, por tanto, que dicho punto es conjugado de $\vec{v}^{-}$. Análogamente, el segundo campo de Jacobi establece una situación idéntica referida ahora al anti-kink ATK2 $\sigma_{3}$, el otro punto umbílico atravesado (con $\bar{q}_{1}<0$ ) es conjugado de $\vec{v}^{+}=(1,0)$. 


\section{Capítulo 6}

\section{Teoría de Morse de Kinks}

En el Capítulo 5 hemos establecido la estabilidad o inestabilidad de las soluciones kink del Modelo Sigma $O(3)$ Lineal Deformado por medio del estudio de los espectros de los funcionales Hessianos correspondientes. Abordaremos ahora el análisis de la Teoría de Morse aplicada a dicho problema. La Teoría de Morse presenta, de una manera elegante y precisa, todos los aspectos relativos a la estabilidad y a las características de las soluciones kink que se han obtenido. Establece, además, una relación entre dichos aspectos y la topología del espacio de configuración. Determinaremos, por tanto, cómo la caracterización de las soluciones en kinks BPS y no-BPS (que realizaremos en este capítulo) y los espectros de los operadores Hessianos (obtenidos en el anterior), se sintetizan ahora por medio de la aplicación del Teorema del Índice de Morse, estableciéndose, a través de las desigualdades de Morse, las propiedades topológicas del espacio de configuración de la teoría de campos.

Como hemos visto, el análisis de los operadores Hessianos podía ser replanteado en términos del operador de desviación geodésica en la métrica de Jacobi asociada. Se demostró, de manera general, que el funcional de acción mecánica y los funcionales de energía y longitud en la métrica de Jacobi asociada, compartían los puntos críticos (curvas solución), en lo que respecta a la primera variación de los mismos. En lo que respecta a la segunda variación, si bien los funcionales no coincidían (puesto que la curvatura en las regiones próximas a las trayectorias críticas así lo determinaba, aunque se mantuviera el signo de la curvatura seccional), sí que lo hacían las dimensiones de los subespacios de estabilidad e inestabilidad. Utilizaremos de nuevo estas consideraciones en el estudio de la Teoría de Morse.

El método que aquí plantearemos apareció por primera vez en la literatura en [53] y fue desarrollado completamente para para el Modelo Sigma $O(2)$ Lineal 
Deformado (modelo MSTB) en [67] y [68]. Consiste esencialmente en calcular el grado de inestabilidad (índice de Morse de la geodésica, desde esta perspectiva), por medio del Teorema del Índice de Morse, como el número de veces que la curva pasa por puntos conjugados al punto inicial de la misma, contados con su multiplicidad.

Estructuraremos el Capítulo 6 de la siguiente forma: En la sección 6.1 demostraremos que el Modelo Sigma $O(3)$ Lineal Deformado es un modelo presupersimétrico como los que se han estudiado de manera general en la Sección 4.3 del Capítulo 4. Para ello, calcularemos explícitamente la función superpotencial correspondiente y obtendremos ocho soluciones posibles. El carácter presupersimétrico del modelo permite una descomposición à la Bogomol'nyi de la acción del mismo, que conduce a las correspondientes ecuaciones de primer orden. Encontraremos, además, que la existencia de puntos singulares de los superpotenciales (puntos de no diferenciabilidad) permite clasificar las soluciones kink en las de tipo BPS y tipo no BPS. En la sección 6.2 se resolverán las ecuaciones de primer orden. Las soluciones son idénticas a las obtenidas, aplicando el método de Hamilton-Jacobi, en el Capítulo 3. El interés de este planteamiento reside en que permite una mejor interpretación del espacio de soluciones del sistema, así como una correcta determinación de los puntos conjugados respecto de los puntos de vacío del modelo. Podremos además computar las energías de las diferentes familias o tipos de kinks como cotas de Bogomol'nyi. Estos resultados nos permitirán abordar en la sección final el estudio de la Teoría de Morse y analizar así la estabilidad o inestabilidad de las soluciones desde el punto de vista antedicho.

\subsection{El Superpotencial y las Ecuaciones de primer orden}

Como ya hemos comentado, calcularemos en esta sección un superpotencial (realmente todo un conjunto de superpotenciales equivalentes) para el Modelo Sigma $O(3)$ Lineal Deformado.

Tal y como se definió en el Capítulo 4, decimos que un sistema mecánico es pre-supersimétrico si existe una función $W: M \rightarrow \mathbb{R}$ tal que su energía potencial verifique (4.30):

$$
U=-\frac{1}{2}\langle\operatorname{grad} W, \operatorname{grad} W\rangle
$$

donde $M$ es la variedad riemanniana sobre la que está definido el sistema, es decir 
el espacio de configuración del mismo. Si el sistema mecánico en estudio es el resultado de aplicar el método de la analogía mecánica en una teoría de campos en la que se buscan soluciones del tipo onda solitaria, como en la situación que aquí planteamos, el potencial $V=-U$ de la teoría de campos verificará:

$$
V=\frac{1}{2}\langle\operatorname{grad} W, \operatorname{grad} W\rangle
$$

En nuestro caso, $M=\mathbb{R}^{3}$ y la métrica es la euclídea, con lo que la búsqueda de un superpotencial se reduce a la resolución de la ecuación en derivadas parciales siguiente:

$$
\left(\frac{\partial W}{\partial q_{1}}\right)^{2}+\left(\frac{\partial W}{\partial q_{2}}\right)^{2}+\left(\frac{\partial W}{\partial q_{3}}\right)^{2}=\left(q_{1}^{2}+q_{2}^{2}+q_{3}^{2}-1\right)^{2}+\sigma_{2}^{2} q_{2}^{2}+\sigma_{3}^{2} q_{3}^{2}
$$

En la sección 4.3 del Capítulo 4 se detallaron las propiedades de los sistemas pre-supersimétricos, la descomposición de Bogomol'nyi que en ellos se plantea conduce a la existencia de ecuaciones de primer orden para el sistema en estudio, ecuaciones que evidentemente satisfacen (sus soluciones) las ecuaciones de segundo orden (de Euler-Lagrange, o si se quiere, de Newton).

La descomposición de Bogomol'nyi se realizaba de la forma (4.31) para el funcional de acción del sistema:

$$
S[c]=\int_{-\infty}^{\infty} d t \frac{1}{2}\langle\dot{c}+\epsilon \operatorname{grad}(W), \dot{c}+\epsilon \operatorname{grad}(W)\rangle-\epsilon \int_{-\infty}^{\infty} d t\langle\dot{c}, \operatorname{grad}(W)\rangle
$$

donde $c(t) \equiv\left(q_{1}(t), \ldots, q_{N}(t)\right)$ y $\epsilon= \pm 1$.

Podemos escribir (6.2) para el caso de la métrica euclídea estándar en $\mathbb{R}^{3}$. Si fijamos, por simplicidad, $\epsilon=1$, tendremos:

$$
S[c]=\frac{1}{2} \int_{-\infty}^{\infty} d t \sum_{i=1}^{3}\left(\frac{d q_{i}}{d t}+\frac{\partial W}{\partial q_{i}}\right)\left(\frac{d q_{i}}{d t}+\frac{\partial W}{\partial q_{i}}\right)-\int_{c(-\infty)}^{c(\infty)} \sum_{i=1}^{3} d q_{i} \frac{\partial W}{\partial q_{i}}
$$

y las ecuaciones de primer orden: $\dot{c}=-\epsilon \operatorname{grad} W$, se escribirán entonces de la forma:

$$
\frac{d q_{i}}{d t}=-\frac{\partial W}{\partial q_{i}}
$$

Resulta evidente que si $c(t)$ es una solución de las ecuaciones de primer orden y $W$ es diferenciable en todos los puntos de dicha solución $c(t)$, entonces el integrando del segundo término de (6.2) es una diferencial exacta: $\langle\dot{c}, \operatorname{grad}(W)\rangle=\frac{d W}{d t}$, y, por aplicación del Teorema de Stokes, la acción de dicha solución (la energía de la teoría de campos) depende únicamente de los valores que toma el superpotencial 
en los puntos inicial y final de la trayectoria, determinados, por otra parte, por las condiciones asintóticas del sistema y, en definitiva, por las propiedades topológicas de las soluciones. Es habitual en la literatura denominar a este tipo de soluciones kinks BPS (de Bogomol'nyi-Prasad-Sommerfeld).

La acción de la solución dinámica (energía del kink desde el punto de vista de la teoría de campos original) es entonces una cota topológica (llamada Cota de Bogomol'nyi) y los kinks de este tipo serán mínimos absolutos del funcional de energía y estables frente a pequeñas fluctuaciones.

$$
S[c]=|W(c(\infty))-W(c(-\infty))|
$$

Esta situación cambia si la función $W$ no es diferenciable a lo largo de todas las curvas solución del sistema. Tal y como veremos, existen soluciones (para el modelo en estudio) tales que el superpotencial $W$ presenta un conjunto discreto de puntos de no diferenciabilidad a lo largo de las mismas. En este caso, $W$ sólo es, por tanto, diferenciable a trozos, y así la aplicación del Teorema de Stokes conduce a que $S[c]$ depende de los valores que tome $W$ en dichos puntos de no diferenciabilidad. La energía deja de ser entonces una cota topológica y los correspondientes kinks no son estables. Estrictamente hablando, por otra parte, y tal y como detallaremos en las secciones siguientes, para dichas soluciones no existen unas ecuaciones de primer orden propiamente dichas, sino un conjunto de diferentes ecuaciones de primer orden que se satisfacen en los diferentes intervalos de derivabilidad del superpotencial. En este caso denominaremos a dichas soluciones kinks no-BPS. Dado que las singularidades de los superpotenciales pueden ser "genuinas", dinámicas, o debidas a los cambios de coordenadas que se realizarán, pueden surgir equívocos acerca del carácter BPS o no-BPS de las diferentes soluciones. Por ello definiremos kink BPS de una manera no ambigua: Llamaremos kink BPS a toda solución de tipo onda solitaria de una teoría pre-supersimétrica tal que su energía dependa únicamente de la diferencia entre los valores que alcanza el superpotencial de la teoría en los extremos de dicha solución.

Analizaremos en este capítulo estos aspectos que, como veremos, atañen no sólo a los kinks no topológicos sino también a algunos de los topológicos. Demostraremos cómo, de hecho, los únicos kinks que satisfacen unas ecuaciones de primer orden propiamente dichas y, por tanto, son estables (y BPS), son los del tipo TK $2 \sigma_{2}$, lo cual concuerda con los resultados de los análisis de los espectros de los funcionales hessianos realizado en el capítulo precedente. 


\subsubsection{Cálculo del Superpotencial}

Para resolver la ecuación (6.1) utilizaremos, una vez más, coordenadas elípticas de Jacobi. Si utilizamos el cambio de coordenadas deducido en el Capítulo 3 para el caso tridimensional tendremos:

$$
\begin{aligned}
& q_{1}^{2}=\frac{\left(1-\lambda_{1}\right)\left(1-\lambda_{2}\right)\left(1-\lambda_{3}\right)}{\sigma_{2}^{2} \sigma_{3}^{2}} \\
& q_{2}^{2}=\frac{\left(\bar{\sigma}_{2}^{2}-\lambda_{1}\right)\left(\bar{\sigma}_{2}^{2}-\lambda_{2}\right)\left(\bar{\sigma}_{2}^{2}-\lambda_{3}\right)}{-\sigma_{2}^{2}\left(\sigma_{3}^{2}-\sigma_{2}^{2}\right)} \\
& q_{3}^{2}=\frac{\left(\bar{\sigma}_{3}^{2}-\lambda_{1}\right)\left(\bar{\sigma}_{3}^{2}-\lambda_{2}\right)\left(\bar{\sigma}_{3}^{2}-\lambda_{3}\right)}{\sigma_{3}^{2}\left(\sigma_{3}^{2}-\sigma_{2}^{2}\right)}
\end{aligned}
$$

donde las constantes de separación tomadas eran, respectivamente, $\bar{\sigma}_{2}^{2}=1-\sigma_{2}^{2}$, $\bar{\sigma}_{3}^{2}=1-\sigma_{3}^{2}$ y 1 . De esta forma, el espacio $\mathbb{R}^{3}$ se representaba (ocho a uno) en el paralelepípedo infinito $\bar{P}_{3}(\infty)=\partial \bar{P}_{3}(\infty) \sqcup P_{3}(\infty)$ formado por los puntos $\left(\lambda_{1}, \lambda_{2}, \lambda_{3}\right)$, donde:

$$
-\infty<\lambda_{1} \leq \bar{\sigma}_{3}^{2} \leq \lambda_{2} \leq \bar{\sigma}_{2}^{2} \leq \lambda_{3} \leq 1
$$

El cambio (6.4) conducía a la siguiente expresión para el potencial del modelo:

$$
V(\vec{\lambda})=\frac{1}{2}\left(\frac{\lambda_{1}^{2}\left(\lambda_{1}-\bar{\sigma}_{2}^{2}\right)\left(\lambda_{1}-\bar{\sigma}_{3}^{2}\right)}{\left(\lambda_{1}-\lambda_{2}\right)\left(\lambda_{1}-\lambda_{3}\right)}+\frac{\lambda_{2}^{2}\left(\lambda_{2}-\bar{\sigma}_{2}^{2}\right)\left(\lambda_{2}-\bar{\sigma}_{3}^{2}\right)}{\left(\lambda_{2}-\lambda_{1}\right)\left(\lambda_{2}-\lambda_{3}\right)}+\frac{\lambda_{3}^{2}\left(\lambda_{3}-\bar{\sigma}_{2}^{2}\right)\left(\lambda_{3}-\bar{\sigma}_{3}^{2}\right)}{\left(\lambda_{3}-\lambda_{1}\right)\left(\lambda_{3}-\lambda_{2}\right)}\right)
$$

con $\vec{\lambda} \equiv\left(\lambda_{1}, \lambda_{2}, \lambda_{3}\right)$.

Y la métrica euclídea asociada a las coordenadas cartesianas se convertía, por medio de (6.4), en la métrica "elíptica":

$$
\begin{aligned}
& g_{11}=-\frac{1}{4} \frac{\left(\lambda_{1}-\lambda_{2}\right)\left(\lambda_{1}-\lambda_{3}\right)}{\left(\lambda_{1}-1\right)\left(\lambda_{1}-\bar{\sigma}_{2}^{2}\right)\left(\lambda_{1}-\bar{\sigma}_{3}^{2}\right)}=\frac{-\Lambda\left(\lambda_{1}\right)}{4 A\left(\lambda_{1}\right)} \\
& g_{22}=-\frac{1}{4} \frac{\left(\lambda_{2}-\lambda_{1}\right)\left(\lambda_{2}-\lambda_{3}\right)}{\left(\lambda_{2}-1\right)\left(\lambda_{2}-\bar{\sigma}_{2}^{2}\right)\left(\lambda_{2}-\bar{\sigma}_{3}^{2}\right)}=\frac{-\Lambda\left(\lambda_{2}\right)}{4 A\left(\lambda_{2}\right)} \\
& g_{33}=-\frac{1}{4} \frac{\left(\lambda_{3}-\lambda_{1}\right)\left(\lambda_{3}-\lambda_{2}\right)}{\left(\lambda_{3}-1\right)\left(\lambda_{3}-\bar{\sigma}_{2}^{2}\right)\left(\lambda_{3}-\bar{\sigma}_{3}^{2}\right)}=\frac{-\Lambda\left(\lambda_{3}\right)}{4 A\left(\lambda_{3}\right)}
\end{aligned}
$$

y $g_{i j}=0, \forall i \neq j$.

Si se escribe el primer miembro de (6.1) en forma de producto escalar

$$
\left(\frac{\partial W}{\partial q_{1}}\right)^{2}+\left(\frac{\partial W}{\partial q_{2}}\right)^{2}+\left(\frac{\partial W}{\partial q_{3}}\right)^{2}=\langle\operatorname{grad} W, \operatorname{grad} W\rangle
$$


es fácil calcular de forma directa la expresión de la ecuación (6.1) en coordenadas elípticas:

$$
2 V=\langle\operatorname{grad} W, \operatorname{grad} W\rangle=g^{11}\left(\frac{\partial W}{\partial \lambda_{1}}\right)^{2}+g^{22}\left(\frac{\partial W}{\partial \lambda_{2}}\right)^{2}+g^{33}\left(\frac{\partial W}{\partial \lambda_{3}}\right)^{2}
$$

o explícitamente:

$$
\sum_{a=1}^{3} \frac{\lambda_{a}^{2}\left(\lambda_{a}-\bar{\sigma}_{2}^{2}\right)\left(\lambda_{a}-\bar{\sigma}_{3}^{2}\right)}{f_{a}(\vec{\lambda})}=\sum_{a=1}^{3} \frac{-4\left(\lambda_{a}-1\right)\left(\lambda_{a}-\bar{\sigma}_{2}^{2}\right)\left(\lambda_{a}-\bar{\sigma}_{3}^{2}\right)}{f_{a}(\vec{\lambda})}\left(\frac{\partial W}{\partial \lambda_{a}}\right)^{2}
$$

Si buscamos soluciones separadas de la forma: $W\left(\lambda_{1}, \lambda_{2}, \lambda_{3}\right)=W_{1}\left(\lambda_{1}\right)+$ $W_{2}\left(\lambda_{2}\right)+W_{3}\left(\lambda_{3}\right)$, entonces las soluciones del sistema de tres ecuaciones ordinarias:

$$
\left(\frac{d W_{a}}{d \lambda_{a}}\right)^{2}=\frac{\lambda_{a}^{2}}{4\left(1-\lambda_{a}\right)} ; \quad \forall a=1,2,3
$$

satisfacen de forma evidente la ecuación (6.6).

La integración de (6.7) es trivial:

$$
W_{a}^{\left(\alpha_{a}\right)}=-(-1)^{\alpha_{a}} \int \frac{\lambda_{a} d \lambda_{a}}{2 \sqrt{1-\lambda_{a}}}=(-1)^{\alpha_{a}} \frac{1}{3}\left(\lambda_{a}+2\right) \sqrt{1-\lambda_{a}}, \quad \alpha_{a}=0,1
$$

salvo constantes (aditivas) arbitrarias que hemos hecho nulas en esa expresión.

Tenemos en definitiva 8 soluciones de (6.6):

$$
\begin{aligned}
& W^{\left(\alpha_{1}, \alpha_{2}, \alpha_{3}\right)}\left(\lambda_{1}, \lambda_{2}, \lambda_{3}\right)=\frac{1}{3}\left((-1)^{\alpha_{1}}\left(\lambda_{1}+2\right) \sqrt{1-\lambda_{1}}\right. \\
& \left.+(-1)^{\alpha_{2}}\left(\lambda_{2}+2\right) \sqrt{1-\lambda_{2}}+(-1)^{\alpha_{3}}\left(\lambda_{3}+2\right) \sqrt{1-\lambda_{3}}\right)
\end{aligned}
$$

según se consideren las diferentes combinaciones posibles de los parámetros arbitrarios $\alpha_{1}, \alpha_{2}$ y $\alpha_{3}$ (y, en consecuencia, los signos relativos $(-1)^{\alpha_{1}},(-1)^{\alpha_{2}}$ y $\left.(-1)^{\alpha_{3}}\right)$.

Un comentario importante en este punto es el siguiente: La ecuación (6.6) no es otra que la ecuación de Hamilton-Jacobi (ecuación (2.46) del Capítulo 2) restringida a considerar todas las constantes de separación nulas (como imponen las condiciones asintóticas del modelo) y donde se debe identificar el superpotencial $W$ con la parte de la acción $\mathcal{S}$ independiente del tiempo ( $W$ es así la frecuentemente llamada función característica de Hamilton). De esta forma, las soluciones $W_{a}$ coinciden con las soluciones $\mathcal{S}_{a}$ deducidas entonces y, en definitiva, la completa integrabilidad (y separabilidad Hamilton-Jacobi) del sistema equivale a su carácter pre-supersimétrico. 
En el Apéndice B de esta memoria hemos calculado la expresión de $W^{\left(\alpha_{1}, \alpha_{2}, \alpha_{3}\right)}$ en coordenadas cartesianas. Hemos decidido separar dicho cálculo del discurso general por razones de claridad. La dificultad de cálculo que plantea el cambio de coordenadas inverso a (6.4) hace que la obtención del superpotencial en coordenadas cartesianas sea compleja.

Dada la complicación de la expresión del superpotencial en coordenadas cartesianas que se obtiene en el Apéndice B, no es trivial observar en qué puntos del espacio dicha función no es diferenciable. Para realizar este cálculo seguiremos utilizando las coordenadas elípticas: Si se tienen en cuenta los rangos de variación de las coordenadas elípticas, se tiene que los superpotenciales $W^{\left(\alpha_{1}, \alpha_{2}, \alpha_{3}\right)}\left(\lambda_{1}, \lambda_{2}, \lambda_{3}\right)$ son funciones diferenciables en todo punto del "espacio elíptico" excepto en el "plano" $\lambda_{3}=1$. La presencia de la métrica no euclídea en la expresión (6.6) obliga a analizar, además, las regiones $f_{a}(\vec{\lambda})=\Lambda^{\prime}\left(\lambda_{i}\right)=0$ como singulares.

En coordenadas cartesianas la métrica no introduce, evidentemente, ningún punto de singularidad.

$\lambda_{3}=1$ se corresponde en cartesianas con el plano $q_{1}=0$ sobre el cual $W^{\left(\alpha_{1}, \alpha_{2}, \alpha_{3}\right)}\left(q_{1}, q_{2}, q_{3}\right)$ es diferenciable, y, por tanto, se trata de una singularidad inducida por el cambio de coordenadas, no de una singularidad genuina. Por su parte, $f_{i}(\vec{\lambda})=\Lambda^{\prime}\left(\lambda_{i}\right)=0$ comprende las siguientes zonas:

- a)

$$
f_{1}(\vec{\lambda})=\Lambda^{\prime}\left(\lambda_{1}\right)=\left(\lambda_{1}-\lambda_{2}\right)\left(\lambda_{1}-\lambda_{3}\right)=0 \Rightarrow \lambda_{1}=\lambda_{2}=\bar{\sigma}_{3}^{2}
$$

- b)

$$
f_{3}(\vec{\lambda})=\Lambda^{\prime}\left(\lambda_{3}\right)=\left(\lambda_{3}-\lambda_{1}\right)\left(\lambda_{3}-\lambda_{2}\right)=0 \Rightarrow \lambda_{3}=\lambda_{2}=\bar{\sigma}_{2}^{2}
$$

- c) Finalmente $f_{2}(\vec{\lambda})=\Lambda^{\prime}\left(\lambda_{2}\right)=\left(\lambda_{2}-\lambda_{1}\right)\left(\lambda_{2}-\lambda_{3}\right)=0$ reproduce las dos situaciones anteriores.

Observamos entonces cómo las singularidades de la ecuación (6.6) se corresponden exactamente con las regiones de $\partial P_{3}(\infty)$ en las que dos de las coordenadas elípticas se hacen iguales. Tenemos entonces dos aristas "singulares" de $\partial P_{3}(\infty)$ :

- La arista $\mathrm{F}_{1} \mathrm{~F}_{3}$, correspondiente a la situación a). En coordenadas cartesianas dicha arista se traduce en la que hemos llamado (ver Capítulo 3) "elipse característica" del cambio de coordenadas, de ecuación (sobre el plano $q_{3}=$ $0)$ :

$$
\frac{q_{1}^{2}}{\sigma_{3}^{2}}+\frac{q_{2}^{2}}{\sigma_{3}^{2}-\sigma_{2}^{2}}=1
$$


- La arista (infinita) $\lambda_{2}=\lambda_{3}=\bar{\sigma}_{2}^{2}$ se corresponde, en coordenadas cartesianas, con la "hipérbola característica". Dicha hipérbola representa el lugar geométrico de los puntos umbílicos de cada uno de los elipsoides que resultan de tomar $\lambda_{1}=$ cte. En particular, para el elipsoide $\lambda_{1}=0$, frontera "dinámica" del paralelepípedo $\bar{P}_{3}(0)$ determinado en el Capítulo 2 , se restringe a la arista (finita) $\mathrm{AF}_{2}$. La ecuación de toda la hipérbola, en el plano $q_{2}=0$, es:

$$
\frac{q_{1}^{2}}{\sigma_{2}^{2}}-\frac{q_{3}^{2}}{\sigma_{3}^{2}-\sigma_{2}^{2}}=1
$$

No es difícil comprobar que las funciones $W^{\left(\alpha_{1}, \alpha_{2}, \alpha_{3}\right)}\left(q_{1}, q_{2}, q_{3}\right)$ no son diferenciables precisamente sobre estas dos curvas, (6.9) y (6.10).

Por otro lado, este hecho pasa a ser casi una trivialidad, sin necesidad de realizar cálculos tediosos (aunque sencillos), si se tiene en cuenta el punto de vista de la teoría general de resolución de las ecuaciones cúbicas. La función $W^{\left(\alpha_{1}, \alpha_{2}, \alpha_{3}\right)}\left(\lambda_{1}, \lambda_{2}, \lambda_{3}\right)$ es diferenciable, como ya hemos dicho, $\forall\left(\lambda_{1}, \lambda_{2}, \lambda_{3}\right) \in \bar{P}_{3}(\infty)$ (con la excepción comentada del caso $\lambda_{3}=1$ ). Por tanto, $W^{\left(\alpha_{1}, \alpha_{2}, \alpha_{3}\right)}\left(q_{1}(\vec{\lambda})\right.$, $\left.q_{2}(\vec{\lambda}), q_{3}(\vec{\lambda})\right)$ será diferenciable cuando lo sean las ecuaciones del cambio de coordenadas (B.3). Evidentemente, si tenemos en cuenta la expresión de $\theta$, las singularidades de dichas ecuaciones aparecen exactamente en la región $q^{3}+r^{2}=0$ (ver Apéndice B). Ahora bien, que $q^{3}+r^{2}$ se anule significa que dos de las raíces de (B.2) coinciden. De hecho, si calculamos el límite

$$
\lim _{q^{3}+r^{2} \rightarrow 0, r<0} \theta=0
$$

entonces, sustituyendo en (B.3), obtenemos $\lambda_{1}=\lambda_{2}=\bar{\sigma}_{3}^{2}$, la elipse (6.9).

Y de manera análoga:

$$
\lim _{q^{3}+r^{2} \rightarrow 0, r>0} \theta=\pi
$$

conduce a $\lambda_{2}=\lambda_{3}=\bar{\sigma}_{2}^{2}$, la hipérbola (6.10).

Por cualquiera de los dos caminos queda demostrado por tanto que $W^{\left(\alpha_{1}, \alpha_{2}, \alpha_{3}\right)}(\vec{q})$ es no diferenciable sobre ambas curvas de $\mathbb{R}^{3}$.

\subsubsection{Ecuaciones de primer orden}

Si escribimos la expresión de la energía de la teoría de campos original del Modelo Sigma $O(3)$ Lineal Deformado para las configuraciones estáticas, utilizando los 
superpotenciales que hemos deducido en la sección anterior, tendremos ${ }^{1}$ :

$$
E=\frac{m^{3}}{2 \lambda^{2} \sqrt{2}} \int_{-\infty}^{\infty} d x \sum_{a, b=1}^{3}\left\{g_{a b}(\vec{\lambda}) \frac{d \lambda_{a}}{d x} \cdot \frac{d \lambda_{b}}{d x}+g_{a b}^{-1}(\vec{\lambda}) \frac{\partial W^{\left(\alpha_{1}, \alpha_{2}, \alpha_{3}\right)}}{\partial \lambda_{a}} \frac{\partial W^{\left(\alpha_{1}, \alpha_{2}, \alpha_{3}\right)}}{\partial \lambda_{b}}\right\}
$$

y donde:

$$
\frac{\partial W^{\left(\alpha_{1}, \alpha_{2}, \alpha_{3}\right)}}{\partial \lambda_{a}}=(-1)^{\alpha_{a}} \frac{\lambda_{a}}{2 \sqrt{1-\lambda_{a}}}, \quad a=1,2,3
$$

Podemos escribir entonces, como ya hemos comentado, la energía en forma de Bogomol'nyi:

$$
\begin{aligned}
E= & \frac{m^{3}}{2 \lambda^{2} \sqrt{2}}\left[\int d x \sum_{a, b=1}^{3} g_{a b}(\vec{\lambda})\left[\frac{d \lambda_{a}}{d x}+\sum_{c=1}^{3} g_{a c}^{-1}(\vec{\lambda}) \frac{\partial W^{\left(\alpha_{1}, \alpha_{2}, \alpha_{3}\right)}}{\partial \lambda_{c}}\right] .\right. \\
& \left.\cdot\left[\frac{d \lambda_{b}}{d x}+\sum_{d=1}^{3} g_{b d}^{-1}(\vec{\lambda}) \frac{\partial W^{\left(\alpha_{1}, \alpha_{2}, \alpha_{3}\right)}}{\partial \lambda_{d}}\right]\right]+ \\
+ & \frac{m^{3}}{\lambda^{2} \sqrt{2}}\left[\left|\int d x \sum_{a=1}^{3} \frac{\partial W^{\left(\alpha_{1}, \alpha_{2}, \alpha_{3}\right)}}{\partial \lambda_{a}}[\vec{\lambda}] \frac{d \lambda_{a}}{d x}\right|\right]
\end{aligned}
$$

donde la primera de las dos integrales proporciona una contribución semidefinida positiva y, por tanto, existe una cota a la energía de un kink, que satisface la desigualdad:

$$
E \geq \frac{m^{3}}{\lambda^{2} \sqrt{2}}\left|\int_{P_{K}} \sum_{a=1}^{3} \frac{\partial W^{\left(\alpha_{1}, \alpha_{2}, \alpha_{3}\right)}}{\partial \lambda_{a}}[\vec{\lambda}] d \lambda_{a}\right|
$$

con la integral calculada sobre la trayectoria $P_{K}$ del kink correspondiente.

De aquí se deduce, de manera evidente, que si se satisfacen las ecuaciones de primer orden ${ }^{2}$ :

$$
\begin{aligned}
& \frac{d \lambda_{1}}{d x}=(-1)^{\alpha_{1}} 2 \frac{\lambda_{1}\left(\lambda_{1}-\bar{\sigma}_{2}^{2}\right)\left(\lambda_{1}-\bar{\sigma}_{3}^{2}\right)}{\left(\lambda_{1}-\lambda_{2}\right)\left(\lambda_{1}-\lambda_{3}\right)} \cdot \sqrt{1-\lambda_{1}} \\
& \frac{d \lambda_{2}}{d x}=(-1)^{\alpha_{2}} 2 \frac{\lambda_{2}\left(\lambda_{2}-\bar{\sigma}_{2}^{2}\right)\left(\lambda_{2}-\bar{\sigma}_{3}^{2}\right)}{\left(\lambda_{2}-\lambda_{1}\right)\left(\lambda_{2}-\lambda_{3}\right)} \cdot \sqrt{1-\lambda_{2}} \\
& \frac{d \lambda_{3}}{d x}=(-1)^{\alpha_{3}} 2 \frac{\lambda_{3}\left(\lambda_{3}-\bar{\sigma}_{2}^{2}\right)\left(\lambda_{3}-\bar{\sigma}_{3}^{2}\right)}{\left(\lambda_{3}-\lambda_{1}\right)\left(\lambda_{3}-\lambda_{2}\right)} \cdot \sqrt{1-\lambda_{3}}
\end{aligned}
$$

\footnotetext{
${ }^{1}$ Dado que adoptaremos en este apartado, y en las secciones siguientes, el punto de vista de la Teoría de Campos original, escribiremos la energía $E$ de la misma en lugar de la acción $S$ del sistema dinámico asociado. Consecuentemente, volveremos a utilizar el "tiempo" $x^{1} \equiv x$ en lugar del equivalente "tiempo dinámico" $t$.

${ }^{2}$ Mantendremos, como hicimos en la ecuación (6.3), el criterio de tomar el signo arbitrario positivo, $\epsilon=1$.
} 
entonces la desigualdad (6.13) se convierte en igualdad, decimos entonces que la cota de Bogomol'nyi está saturada.

Dado que, en el sistema (6.14), los parámetros $\alpha_{1}, \alpha_{2}$ y $\alpha_{3}$ pueden tomar arbitrariamente los valores 0 ó 1 , realmente (6.14) constituye un conjunto de ocho sistemas de ecuaciones ordinarias de primer orden diferentes ${ }^{3}$. Como veremos en la Sección 6.2, la integración de dichos sistemas conduce a la obtención de todas las soluciones kink de la teoría. Sin embargo, la presencia de singularidades del superpotencial, demostrada en el apartado anterior, hace que dicha integración no sea en absoluto trivial, como detallaremos.

Es interesante resaltar, por otro lado, que (6.14) se podría haber obtenido resolviendo (algebraicamente) el sistema de ecuaciones no lineales que se determina cuando se impone que las integrales primeras del sistema (en coordenadas elípticas) tomen los valores que para ellas determinan las condiciones asintóticas que resultan de considerar soluciones de tipo kink. El mismo razonamiento es válido en coordenadas cartesianas. Para el caso del modelo bidimensional (el ya referido modelo MSTB), este punto de vista, muy directo a partir de las expresiones de las integrales primeras $I_{1}$ e $I_{2}$, permite demostrar de manera casi trivial el carácter pre-supersimétrico del mismo. Para el modelo que nos ocupa, tridimensional, dicho tratamiento es casi inabordable en coordenadas cartesianas, habida cuenta la gran complejidad de la expresión del superpotencial deducido en el Apéndice B.

Antes de proceder a la resolución del sistema (6.14) analizaremos brevemente el mismo.

Si escribimos el sistema (6.14) de la forma:

$$
\frac{d \lambda_{a}}{(-1)^{\alpha_{a}} 2 \lambda_{a}\left(\lambda_{a}-\bar{\sigma}_{2}^{2}\right)\left(\lambda_{a}-\bar{\sigma}_{3}^{2}\right) \sqrt{1-\lambda_{a}}}=\frac{d x}{f_{a}(\vec{\lambda})}
$$

y sumamos las tres ecuaciones, obtenemos

$$
\sum_{a=1}^{3} \frac{d \lambda_{a}}{(-1)^{\alpha_{a}} 2 \lambda_{a}\left(\lambda_{a}-\bar{\sigma}_{2}^{2}\right)\left(\lambda_{a}-\bar{\sigma}_{3}^{2}\right) \sqrt{1-\lambda_{a}}}=0
$$

\footnotetext{
${ }^{3}$ La existencia de ocho superpotenciales diferentes según las elecciones de signos que se realicen equivale en la práctica a las diferentes descomposiciones de Bogomolny posibles. En (6.12) se ha tomado un signo concreto para la descomposición y se ha utilizado genéricamente $W^{\left(\alpha_{1}, \alpha_{2}, \alpha_{3}\right)}$. Alternativamente podemos fijar uno de los superpotenciales concretos y considerar todas las descomposiciones posibles. Cualquiera de las dos técnicas conducen evidentemente a los mismos sistemas (6.14).
} 
Por otro lado, si multiplicamos cada una de las ecuaciones (6.15) por $\lambda_{a}-\lambda_{b}$, $a \neq b$ y sumamos las seis ecuaciones resultantes tendremos:

$$
\sum_{a=1}^{3} \frac{\lambda_{a} d \lambda_{a}}{(-1)^{\alpha_{a}} 2 \lambda_{a}\left(\lambda_{a}-\bar{\sigma}_{2}^{2}\right)\left(\lambda_{a}-\bar{\sigma}_{3}^{2}\right) \sqrt{1-\lambda_{a}}}=0
$$

donde se han usado para la simplificación las ecuaciones (6.15).

Por último, multiplicando cada ecuación de (6.15) por la correspondiente función $f_{a}(\vec{\lambda})$ y sumando los resultados, se obtiene:

$$
\sum_{a=1}^{3} \frac{\lambda_{a}^{2} d \lambda_{a}}{(-1)^{\alpha_{a}} 2 \lambda_{a}\left(\lambda_{a}-\bar{\sigma}_{2}^{2}\right)\left(\lambda_{a}-\bar{\sigma}_{3}^{2}\right) \sqrt{1-\lambda_{a}}}=d x
$$

Las expresiones (6.16) y (6.17) que hemos obtenido son exactamente las ecuaciones cuya integración proporciona las trayectorias solución que fueron obtenidas en el Capítulo 3 por medio de la separación de la ecuación de Hamilton-Jacobi del sistema, tras imponer que las integrales primeras fueran nulas, como consecuencia de las condiciones asintóticas del mismo. La dependencia "temporal" de las soluciones viene determinada, evidentemente, por la ecuación (6.18), coincidente también con la obtenida por el método de Hamilton-Jacobi.

Las posibilidad de realizar las elegantes simplificaciones anteriores, que nos han permitido pasar del sistema (6.14) al nuevo sistema ((6.16), (6.17), (6.18)) en el que las variables están separadas, no se debe, evidentemente, al azar. La "magia" de estos cálculos radica en el Lema de Jacobi demostrado en la sección 2.2 del Capítulo 2, y, de hecho, son cálculos fácilmente generalizables al caso $N$ dimensional. Se trata, en definitiva, de simplificaciones similares a las realizadas por el propio Jacobi en sus cálculos de las geodésicas del elipsoide triaxial (ver [81]).

Las ecuaciones (6.14) permiten también deducir fácilmente otro resultado importante que ya obtuvimos por el método de Hamilton-Jacobi. Se trata de la demostración de que el movimiento, para las soluciones kink de este sistema, está restringido a la región limitada por el elipsoide de ecuación:

$$
q_{1}^{2}+\frac{q_{2}^{2}}{\bar{\sigma}_{2}^{2}}+\frac{q_{3}^{2}}{\bar{\sigma}_{3}^{2}}=1
$$

en $\mathbb{R}^{3}$, o, equivalentemente, $\lambda_{1}=0$ en $\bar{P}_{3}(\infty)$, que queda reducido entonces al paralelepípedo finito $\bar{P}_{3}(0)$. 
Si se eleva al cuadrado la primera de las ecuaciones presentes en (6.15), y se recuerda que el momento canónico asociado a la variable $\lambda_{1}$ es: $\pi_{1}=g_{11}(\vec{\lambda}) \frac{d \lambda_{1}}{d x}$, tendremos entonces que dicha ecuación equivale a:

$$
\frac{1}{2} \pi_{1}^{2}+\frac{1}{8} \frac{\lambda_{1}^{2}}{\lambda_{1}-1}=0
$$

La ecuación (6.19), como ya se expuso para el caso general $N$-dimensional en el Capítulo 2, describe el movimiento de una partícula con energía nula bajo la influencia de un potencial:

$$
\begin{aligned}
\mathcal{V}\left(\lambda_{1}\right) & =\frac{1}{8} \frac{\lambda_{1}^{2}}{\lambda_{1}-1}, & & -\infty<\lambda_{1} \leq \bar{\sigma}_{3}^{2} \\
& =\infty, & & \bar{\sigma}_{3}^{2}<\lambda_{1}<\infty
\end{aligned}
$$

$\mathcal{V}\left(\lambda_{1}\right)$ tiene un máximo en $\lambda_{1}=0$ y tiende a $-\infty$ cuando $\lambda_{1}$ tiende a $-\infty$; por tanto, el movimiento acotado ocurrirá únicamente en el intervalo $\lambda_{1} \in\left[0, \bar{\sigma}_{3}^{2}\right]$.

Si consideramos además las condiciones asintóticas que conducen a energía finita de los kinks, y que en coordenadas elípticas se escriben de la forma:

$\lim _{x \rightarrow \pm \infty} \frac{d \lambda_{a}}{d x}=0, \quad \lim _{x \rightarrow \pm \infty} \lambda_{1}(x)=0, \quad \lim _{x \rightarrow \pm \infty} \lambda_{2}(x)=\bar{\sigma}_{3}^{2}, \quad \lim _{x \rightarrow \pm \infty} \lambda_{3}(x)=\bar{\sigma}_{2}^{2}$

tendremos demostrado entonces que las configuraciones kink permanecen en el paralelepípedo $\bar{P}_{3}(0): 0 \leq \lambda_{1} \leq \bar{\sigma}_{3}^{2} \leq \lambda_{2} \leq \bar{\sigma}_{2}^{2} \leq \lambda_{3} \leq 1$.

\subsection{Solución general de las ecuaciones de primer orden}

Plantearemos en esta sección la resolución de las ecuaciones de primer orden (6.14), o, más exactamente, como ya hemos comentado, de los diferentes sistemas de ecuaciones de primer orden que se incluyen en (6.14) debido a las ocho posibilidades de elección de signos relativos con las que nos encontramos.

Por otro lado, y dado que el cambio de coordenadas (6.4) es singular en la frontera de $P_{3}(0), \partial P_{3}(0)$, con la excepción de la que hemos llamado "frontera dinámica", la cara $\lambda_{1}=0$ (es decir, el elipsoide $q_{1}^{2}+\bar{\sigma}_{2}^{-2} q_{2}^{2}+\bar{\sigma}_{3}^{-2} q_{3}^{2}=1$ en coordenadas cartesianas), comenzaremos el análisis de las ecuaciones (6.14) estudiando su comportamiento límite sobre $\partial P_{3}(0)$. El sistema se reduce dimensionalmente sobre diferentes caras de $\partial P_{3}(0)$ y observaremos, de acuerdo con lo calculado en 
el Capítulo 3, cómo aparecen sobre esta frontera los kinks embebidos y los kinks envolventes del modelo. Existe una excepción: el plano $q_{1}=0$ de $\mathbb{R}^{3}$ o, equivalentemente, la cara $\lambda_{3}=1$ de $\partial P_{3}(0)$; quedará fuera de este análisis por una razón evidente, no contiene a los puntos de vacío. Como ya se comentó en el Capítulo 3 , es una cara que será cortada por todas las soluciones (excepto las soluciones constantes, es decir los propios puntos $\vec{v}^{-}$y $\vec{v}^{+}$, o el punto D de $\left.\bar{P}_{3}(0)\right)$ una o varias veces, pero no existen soluciones totalmente contenidas en ella. No es una región singular si se considera en coordenadas cartesianas en $\mathbb{R}^{3}$.

Posteriormente analizaremos los puntos regulares, del interior de $\bar{P}_{3}(0)$, para obtener las familias de kinks TK3, soluciones que hemos denominado genéricas de la teoría en estudio.

Mostraremos cómo las ecuaciones (6.14) conducen a idénticos resultados a los obtenidos en el Capítulo 3 de esta memoria y nos centraremos en la obtención de la energía de los diferentes tipos de kinks como cotas de Bogomol'nyi en distintas situaciones, hecho que nos conducirá a clasificar los kinks en BPS y no-BPS de acuerdo con el criterio establecido anteriormente. Esta clasificación permite, a su vez, establecer un nuevo criterio de estabilidad, ya comentado, que proporciona el mismo resultado obtenido, desde el punto de vista del análisis espectral de los Hessianos, en el capítulo anterior.

Como ya establecimos en la sección anterior, las soluciones de las ecuaciones de primer orden (6.14) saturan la cota de Bogomol'nyi:

$$
E\left[\vec{\lambda}_{K}\right]=\frac{m^{3}}{\lambda^{2} \sqrt{2}}\left|\int_{P_{K}} d W^{\left(\alpha_{1}, \alpha_{2}, \alpha_{3}\right)}\right| .
$$

De esta manera, si el superpotencial es una función diferenciable a lo largo de todo el camino $P_{K}$ (trayectoria del kink), entonces $\left|\int_{P_{K}} d W^{\left(\alpha_{1}, \alpha_{2}, \alpha_{3}\right)}\right|$ dependerá únicamente de los valores que alcance $W^{\left(\alpha_{1}, \alpha_{2}, \alpha_{3}\right)}$ en los extremos de la misma; es habitual, en la literatura, referirse a este tipo de situaciones diciendo que $\left|\int_{P_{K}} d W^{\left(\alpha_{1}, \alpha_{2}, \alpha_{3}\right)}\right|$ es entonces una cantidad topológica. En este caso, la energía del kink será un mínimo absoluto en el sector topológico correspondiente del espacio de configuración; la onda solitaria será absolutamente estable y tendremos estados topológicos BPS (ver [13], [10]). Como veremos, en nuestro sistema únicamente un tipo de kink pertenece a esta categoría a pesar de que la totalidad de los kinks satisfacen ecuaciones de primer orden. La razón, ya citada, está en el hecho de que, para los demás kinks, las trayectorias atraviesan puntos de no diferenciabilidad del superpotencial, de esta manera $\left|\int_{P_{K}} d W^{\left(\alpha_{1}, \alpha_{2}, \alpha_{3}\right)}\right|$ no es una cantidad topológica, puesto que depende de los valores que tome $W^{\left(\alpha_{1}, \alpha_{2}, \alpha_{3}\right)}$ en los puntos singulares. 
Por último, la resolución que realizaremos pondrá de manifiesto explícitamente la relación existente entre los puntos singulares que cada trayectoria kink atraviesa, singularidades del superpotencial, y los puntos conjugados a los puntos de vacío que el modelo presenta y que serán estudiados en la próxima sección a la hora de aplicar el Teorema del Índice de Morse al modelo que estamos estudiando.

\section{Cara $\lambda_{1}=0$. Kinks no topológicos envolventes}

Como se ha comentado, la cara $\lambda_{1}=0$ de $\partial P_{3}(0)$ se corresponde en coordenadas cartesianas con el elipsoide de ecuación

$$
q_{1}^{2}+\frac{q_{2}^{2}}{\bar{\sigma}_{2}^{2}}+\frac{q_{3}^{2}}{\bar{\sigma}_{3}^{2}}=1
$$

en $\mathbb{R}^{3}$. Sobre esta superficie el superpotencial se reduce a la expresión:

$$
W^{\left(\alpha_{1}, \alpha_{2}\right)}(\vec{\mu})=\frac{1}{3}\left((-1)^{\alpha_{1}}\left(\mu_{1}+2\right) \sqrt{1-\mu_{1}}+(-1)^{\alpha_{2}}\left(\mu_{2}+2\right) \sqrt{1-\mu_{2}}\right)
$$

donde se ha denotado $\lambda_{2}=\mu_{1}$ y $\lambda_{3}=\mu_{2}$ como las componentes del vector bidimensional $\vec{\mu}=\left(\mu_{1}, \mu_{2}\right)$ que parametriza el paralelogramo $\bar{P}_{2}\left(\bar{\sigma}_{3}^{2}, \bar{\sigma}_{2}^{2}\right): \bar{\sigma}_{3}^{2} \leq \mu_{1} \leq$ $\bar{\sigma}_{2}^{2} \leq \mu_{2} \leq 1$.

La métrica reducida a $\bar{P}_{2}\left(\bar{\sigma}_{3}^{2}, \bar{\sigma}_{2}^{2}\right)$ se escribe de la forma:

$$
\begin{aligned}
g_{11}(\vec{\mu}) & =-\frac{1}{4} \frac{\mu_{1}\left(\mu_{1}-\mu_{2}\right)}{\left(\mu_{1}-1\right)\left(\mu_{1}-\bar{\sigma}_{2}^{2}\right)\left(\mu_{1}-\bar{\sigma}_{3}^{2}\right)} \\
g_{22}(\vec{\mu}) & =-\frac{1}{4} \frac{\mu_{2}\left(\mu_{2}-\mu_{1}\right)}{\left(\mu_{2}-1\right)\left(\mu_{2}-\bar{\sigma}_{2}^{2}\right)\left(\mu_{2}-\bar{\sigma}_{3}^{2}\right)}
\end{aligned}
$$

y así las ecuaciones de primer orden, (6.14), para este sistema bidimensional se reducen a las ecuaciones:

$$
\begin{aligned}
& \frac{d \mu_{1}}{d x}=(-1)^{\alpha_{1}} 2 \frac{\left(\mu_{1}-\bar{\sigma}_{2}^{2}\right)\left(\mu_{1}-\bar{\sigma}_{3}^{2}\right)}{\left(\mu_{1}-\mu_{2}\right)} \cdot \sqrt{1-\mu_{1}} \\
& \frac{d \mu_{2}}{d x}=(-1)^{\alpha_{2}} 2 \frac{\left(\mu_{2}-\bar{\sigma}_{2}^{2}\right)\left(\mu_{2}-\bar{\sigma}_{3}^{2}\right)}{\left(\mu_{2}-\mu_{1}\right)} \cdot \sqrt{1-\mu_{2}}
\end{aligned}
$$

Con razonamientos similares a los empleados en la sección anterior, no es difícil obtener, a partir de las ecuaciones (6.22), las correspondientes expresiones en variables separadas cuya integración proporciona las ecuaciones de la órbitas de los kinks, por un lado, y la dependencia temporal de los mismos, por otro. Así encontramos:

$$
\sum_{a=1}^{2} \frac{d \mu_{a}}{(-1)^{\alpha_{a}} 2\left(\mu_{a}-\bar{\sigma}_{2}^{2}\right)\left(\mu_{a}-\bar{\sigma}_{3}^{2}\right) \sqrt{1-\mu_{a}}}=0
$$


para las órbitas, y:

$$
\sum_{a=1}^{2} \frac{\mu_{a} d \mu_{a}}{(-1)^{\alpha_{a}} 2\left(\mu_{a}-\bar{\sigma}_{2}^{2}\right)\left(\mu_{a}-\bar{\sigma}_{3}^{2}\right) \sqrt{1-\mu_{a}}}=d x
$$

en lo que respecta a la dependencia frente al "tiempo" $x$.

La integración de (6.23) proporciona la ecuación (3.46) del Capítulo 3 (con el cambio de notación comentado, por supuesto), mientras que la integración de (6.24) conduce a la ecuación (3.47).

Como ya se explicó en dicho capítulo, la familia de kinks resultante sobre esta superficie, $\lambda_{1}=0$, está compuesta por soluciones no topológicas que pasan, todas ellas, por el punto A de $\partial P_{3}(0)$. En el espacio "cartesiano" $\mathbb{R}^{3}$ esto se traduce en cuatro familias de kinks no-topológicos que salen y llegan a uno de los dos puntos de vacío y pasan respectivamente por cada uno de los cuatro puntos umbílicos del elipsoide, representados en coordenadas elípticas por el único punto A. Dado que se trata de curvas alabeadas, con las tres componentes no nulas, recibieron en el Capítulo 3 el nombre de NTK3, de acuerdo con la notación que estamos utilizando en esta memoria. Se les clasificó, también, como los kinks envolventes del sistema, dado que permanecen en el elipsoide citado (cara $\lambda_{1}=0$ ), fuera del cual no existen soluciones kink.

La energía de cada uno de los miembros de estas familias es idéntica y fue calculada también en el Capítulo 3, obteniéndose:

$$
\begin{aligned}
E(\mathrm{NTK} 3) & =2 E_{\lambda_{2}}\left(\lambda_{2}\right)+4 E_{\lambda_{3}}\left(\lambda_{3}\right) \\
& =\frac{m^{3}}{\lambda^{2} \sqrt{2}}\left(2\left(\sigma_{2}+\sigma_{3}-\frac{\sigma_{2}^{2}+\sigma_{3}^{2}}{3}\right)\right)
\end{aligned}
$$

debido a que los rangos correspondientes de las variables $\lambda_{2}$ y $\lambda_{3}$ se recorren tres y cuatro veces, respectivamente, en cada uno de los miembros de la familia NTK3.

Nos centraremos en este momento en la obtención de la energía citada desde el punto de vista de las ecuaciones de primer orden y las cotas de Bogomol'nyi correspondientes. Para ello, conviene estudiar con detalle la órbita de un kink NTK3 cualquiera, desde un punto de vista no incluido en el análisis que realizamos en el Capítulo 3.

Si consideramos el punto inicial de la trayectoria tomada, evidentemente el punto $\mathrm{D}$, que se alcanza en $x \rightarrow-\infty$, sus coordenadas en el paralelogramo $\bar{P}_{2}\left(\bar{\sigma}_{3}^{2}, \bar{\sigma}_{2}^{2}\right)$ son $\mathrm{D} \equiv\left(\bar{\sigma}_{3}^{2}, \bar{\sigma}_{2}^{2}\right)$ y así, evidentemente, las derivadas $\frac{d \mu_{1}}{d x}$ y $\frac{d \mu_{2}}{d x}$ son, en las cercanías de dicho punto, necesariamente positivas. Como puede observarse en la Figura 3.13 esta situación se mantiene hasta el punto $\mathrm{P}_{1}$ donde el kink alcanza la 
arista superior de $\bar{P}_{2}\left(\bar{\sigma}_{3}^{2}, \bar{\sigma}_{2}^{2}\right)$, que corresponde a $\mu_{2}=1$ (o, si se quiere $\lambda_{3}=1$ ). A partir de este punto la derivada $\frac{d \mu_{2}}{d x}$ pasa a ser necesariamente negativa, puesto que $\mu_{2}$ decrece desde el valor 1 que alcanza en $\mathrm{P}_{1}$, como hemos dicho, hasta el valor $\mu_{2}=\bar{\sigma}_{2}^{2}$ que recupera en el punto umbílico A. A partir de A, la situación se repite, pero ahora con $\frac{d \mu_{1}}{d x}$ negativo. Si denominamos $x_{1}$ al valor que la variable $x$ toma en $\mathrm{P}_{1}$ (y que obviamente es arbitrario al existir invarianza bajo traslaciones en la coordenada espacial), $x_{0}$ al correspondiente al punto A y $x_{2}$ al relativo al punto $\mathrm{P}_{2}$, en el que el kink "toca" la arista $\mathrm{BC}$ por segunda vez, en el "camino de vuelta" hacia el punto $\mathrm{D}$, tendremos que las ecuaciones que se verifican en cada tramo son las siguientes:

- a) $\mathrm{D} \rightarrow \mathrm{P}_{1} ; x \in\left(-\infty, x_{1}\right]$. Las ecuaciones (6.22) que se verifican en este tramo se corresponden con la elección de signos: $\alpha_{1}=0, \alpha_{2}=0$.

- b) $\mathrm{P}_{1} \rightarrow \mathrm{A} ; x \in\left[x_{1}, x_{0}\right]$. En este caso los signos adecuados en (6.22) son $\alpha_{1}=0, \alpha_{2}=1$.

- c) $\mathrm{A} \rightarrow \mathrm{P}_{2} ; x \in\left[x_{0}, x_{2}\right]$. Ahora encontramos $\alpha_{1}=1, \alpha_{2}=0$.

- d) $\mathrm{P}_{2} \rightarrow \mathrm{D} ; x \in\left[x_{2}, \infty\right)$. Finalmente, la posibilidad restante: $\alpha_{1}=1, \alpha_{2}=1$.

Dado que encontramos cuatro sistemas de ecuaciones de primer orden para este tipo de kinks, la descomposición de Bogomol'nyi puede escribirse de la forma ${ }^{4}$

$$
\begin{aligned}
\frac{\lambda^{2} \sqrt{2}}{m^{3}} E(\mathrm{NTK} 3)= & -\int_{\mathrm{NTK} 3} d\left(W_{1}^{\left(\alpha_{1}\right)}+W_{2}^{\left(\alpha_{2}\right)}\right)= \\
= & -\int_{\mathrm{D}}^{\mathrm{P}_{1}} d\left(W_{1}^{(0)}+W_{2}^{(0)}\right)-\int_{\mathrm{P}_{1}}^{\mathrm{A}} d\left(W_{1}^{(0)}+W_{2}^{(1)}\right) \\
& -\int_{\mathrm{A}}^{\mathrm{P}_{2}} d\left(W_{1}^{(1)}+W_{2}^{(0)}\right)-\int_{\mathrm{P}_{2}}^{\mathrm{D}} d\left(W_{1}^{(1)}+W_{3}^{(1)}\right)=
\end{aligned}
$$

\footnotetext{
${ }^{4}$ Como ya se comentó en la sección precedente, existe una ambigüedad en la elección global del signo a considerar en la descomposición de Bogomol'nyi, ambigüedad, por otra parte, equivalente a la invarianza de los cálculos si se cambia el signo de la función superpotencial en su definición. Tal y como se han escogido las ecuaciones de primer orden en este caso, con $\epsilon=1$, resulta evidente que la energía será:

$$
E(\mathrm{NTK} 3)=-\frac{m^{3}}{\lambda^{2} \sqrt{2}} \int_{\mathrm{NTK} 3} d W^{\left(\alpha_{1}, \alpha_{2}\right)}
$$

En general, se puede considerar $E(\mathrm{NTK} 3)=\frac{m^{3}}{\lambda^{2} \sqrt{2}}\left|\int_{\mathrm{NTK} 3} d W^{\left(\alpha_{1}, \alpha_{2}\right)}\right|$ y despreocuparse de dicho signo global, dado que el resultado es necesariemente positivo.
} 


$$
\begin{aligned}
= & -\left[W_{1}^{(0)}\left(\vec{\mu}_{\mathrm{P}_{1}}\right)-W_{1}^{(0)}\left(\vec{\mu}_{\mathrm{D}}\right)+W_{1}^{(0)}\left(\vec{\mu}_{\mathrm{A}}\right)-W_{1}^{(0)}\left(\vec{\mu}_{\mathrm{P}_{1}}\right)+\right. \\
& +W_{1}^{(1)}\left(\vec{\mu}_{\mathrm{P}_{2}}\right)-W_{1}^{(1)}\left(\vec{\mu}_{\mathrm{A}}\right)+W_{1}^{(1)}\left(\vec{\mu}_{\mathrm{D}}\right)-W_{1}^{(1)}\left(\vec{\mu}_{\mathrm{P}_{2}}\right)+ \\
& +W_{2}^{(0)}\left(\vec{\mu}_{\mathrm{P}_{1}}\right)-W_{2}^{(0)}\left(\vec{\mu}_{\mathrm{D}}\right)+W_{2}^{(1)}\left(\vec{\mu}_{\mathrm{A}}\right)-W_{2}^{(1)}\left(\vec{\mu}_{\mathrm{P}_{1}}\right)+ \\
& \left.+W_{2}^{(0)}\left(\vec{\mu}_{\mathrm{P}_{2}}\right)-W_{2}^{(0)}\left(\vec{\mu}_{\mathrm{A}}\right)+W_{2}^{(1)}\left(\vec{\mu}_{\mathrm{D}}\right)-W_{2}^{(1)}\left(\vec{\mu}_{\mathrm{P}_{2}}\right)\right]= \\
= & -\left[W_{1}^{(1)}\left(\vec{\mu}_{\mathrm{D}}\right)-W_{1}^{(0)}\left(\vec{\mu}_{\mathrm{D}}\right)+W_{1}^{(0)}\left(\vec{\mu}_{\mathrm{A}}\right)-W_{1}^{(1)}\left(\vec{\mu}_{\mathrm{A}}\right)+\right. \\
& W_{2}^{(1)}\left(\vec{\mu}_{\mathrm{D}}\right)-W_{2}^{(0)}\left(\vec{\mu}_{\mathrm{D}}\right)+W_{2}^{(0)}\left(\vec{\mu}_{\mathrm{P}_{1}}\right)-W_{2}^{(1)}\left(\vec{\mu}_{\mathrm{P}_{1}}\right)+ \\
& \left.+W_{2}^{(1)}\left(\vec{\mu}_{\mathrm{A}}\right)-W_{2}^{(0)}\left(\vec{\mu}_{\mathrm{A}}\right)+W_{2}^{(0)}\left(\vec{\mu}_{\mathrm{P}_{2}}\right)-W_{2}^{(1)}\left(\vec{\mu}_{\mathrm{P}_{2}}\right)\right]= \\
= & 2\left(\sigma_{2}-\frac{\sigma_{2}^{3}}{3}\right)+2\left(\sigma_{3}-\frac{\sigma_{3}^{3}}{3}\right)
\end{aligned}
$$

Concluimos por tanto que $E$ (NTK3) no es una cantidad topológica, dado que en su cómputo final intervienen los valores que alcanza en un punto intermedio, A, de la trayectoria, y no sólo de los valores alcanzados en los puntos inicial y final de las soluciones ${ }^{5}$. Los kinks NTK3 serán en consecuencia inestables, del tipo no-BPS.

\section{Plano $q_{3}=0$. Modelo $O(2)$ embebido en el modelo $O(3): \mathbf{I}$}

La reducción del sistema al plano $q_{3}=0$ de $\mathbb{R}^{3}$ se convierte, sobre $\bar{P}_{3}(0)$, en reducción a dos caras del mismo, la cara $\lambda_{1}=\bar{\sigma}_{3}^{2}$ y la cara $\lambda_{2}=\bar{\sigma}_{3}^{2}$, como se detalló en el Capítulo 3. La arista de unión entre ambas no es otra que $\lambda_{1}=\lambda_{2}=\bar{\sigma}_{3}^{2}$, es decir, la línea $\mathrm{F}_{1} \mathrm{~F}_{3}$ o, equivalentemente en coordenadas cartesianas, la elipse característica del plano $q_{3}=0$ que pasa por $\mathrm{F}_{1}$ y $\mathrm{F}_{3}$ y tiene focos en $\mathrm{F}_{2}$.

A. Sobre la cara $\lambda_{1}=\bar{\sigma}_{3}^{2}$ tomaremos coordenadas

$$
\lambda_{2}=\nu_{1}^{I}, \lambda_{3}=\nu_{2}^{I} \quad \Rightarrow \quad \vec{\nu}^{I}=\left(\nu_{1}^{I}, \nu_{2}^{I}\right)
$$

de manera que el superpotencial reducido se escribe:

$$
W^{\left(\alpha_{1}, \alpha_{2}\right)}\left(\vec{\nu}^{I}\right)=\frac{1}{3}\left((-1)^{\alpha_{1}}\left(\nu_{1}^{I}+2\right) \sqrt{1-\nu_{1}^{I}}+(-1)^{\alpha_{2}}\left(\nu_{2}^{I}+2\right) \sqrt{1-\nu_{2}^{I}}\right)
$$

\footnotetext{
${ }^{5}$ Es interesante apuntar que los diferentes superpotenciales se anulan en la arista BC, es decir, en los puntos $\mathrm{P}_{1}$ y $\mathrm{P}_{2}$. Su contribución al cálculo de la energía se reduce, por tanto, al cambio en los signos relativos que produce el paso por estos puntos. Evidentemente, el diferente comportamiento de estos puntos frente a los umbílicos, A, radica en el hecho de que éstos últimos son puntos de no diferenciabilidad genuinos del superpotencial, mientras que la existencia de los primeros se debe a las especiales características del cambio de coordenadas. Intuitivamente, por otra parte, se observa que la energía no puede depender de dichos puntos puesto que en tal caso no coincidirían las energías de los diferentes kinks de la misma familia.
} 
La métrica:

$$
\begin{aligned}
g_{11}\left(\vec{\nu}^{I}\right) & =-\frac{1}{4} \frac{\left(\nu_{1}^{I}-\nu_{2}^{I}\right)}{\left(\nu_{1}^{I}-1\right)\left(\nu_{1}^{I}-\bar{\sigma}_{2}^{2}\right)} \\
g_{22}\left(\vec{\nu}^{I}\right) & =-\frac{1}{4} \frac{\left(\nu_{2}^{I}-\nu_{1}^{I}\right)}{\left(\nu_{2}^{I}-1\right)\left(\nu_{2}^{I}-\bar{\sigma}_{2}^{2}\right)}
\end{aligned}
$$

y, finalmente, las ecuaciones de primer orden reducidas:

$$
\begin{aligned}
\frac{d \nu_{1}^{I}}{d x} & =(-1)^{\alpha_{1}} 2 \frac{\nu_{1}^{I}\left(\nu_{1}^{I}-\bar{\sigma}_{2}^{2}\right)}{\left(\nu_{1}^{I}-\nu_{2}^{I}\right)} \cdot \sqrt{1-\nu_{1}^{I}} \\
\frac{d \nu_{2}^{I}}{d x} & =(-1)^{\alpha_{2}} 2 \frac{\nu_{2}^{I}\left(\nu_{2}^{I}-\bar{\sigma}_{2}^{2}\right)}{\left(\nu_{2}^{I}-\nu_{1}^{I}\right)} \cdot \sqrt{1-\nu_{2}^{I}}
\end{aligned}
$$

De esta forma es fácil deducir que las ecuaciones de las trayectorias kink están determinadas por la integración de:

$$
\sum_{a=1}^{2} \frac{d \nu_{a}^{I}}{(-1)^{\alpha_{a}} 2 \nu_{a}^{I}\left(\nu_{a}^{I}-\bar{\sigma}_{2}^{2}\right) \sqrt{1-\nu_{a}^{I}}}=0
$$

y la dependencia temporal por la ecuación

$$
\sum_{a=1}^{2} \frac{\nu_{a}^{I} d \nu_{a}^{I}}{(-1)^{\alpha_{a}} 2 \nu_{a}^{I}\left(\nu_{a}^{I}-\bar{\sigma}_{2}^{2}\right) \sqrt{1-\nu_{a}^{I}}}=d x
$$

Por analogía con la notación del caso anterior denotaremos a esta cara (paralelogramo): $\bar{P}_{2}\left(\bar{\sigma}_{3}^{2}, \bar{\sigma}_{2}^{2}\right): \bar{\sigma}_{3}^{2} \leq \nu_{1}^{I} \leq \bar{\sigma}_{2}^{2} \leq \nu_{2}^{I} \leq 1$.

B. Para la cara $\lambda_{2}=\bar{\sigma}_{3}^{2}$, tenemos resultados análogos:

$$
\begin{gathered}
\lambda_{1}=\nu_{1}^{I I}, \lambda_{3}=\nu_{2}^{I I}, \quad \Rightarrow \quad \vec{\nu}^{I I}=\left(\nu_{1}^{I I}, \nu_{2}^{I I}\right) \\
W^{\left(\alpha_{1}, \alpha_{2}\right)}\left(\vec{\nu}^{I I}\right)=\frac{1}{3}\left((-1)^{\alpha_{1}}\left(\nu_{1}^{I I}+2\right) \sqrt{1-\nu_{1}^{I I}}+(-1)^{\alpha_{2}}\left(\nu_{2}^{I I}+2\right) \sqrt{1-\nu_{2}^{I I}}\right) \\
g_{11}\left(\vec{\nu}^{I I}\right)=-\frac{1}{4} \frac{\left(\nu_{1}^{I I}-\nu_{2}^{I I}\right)}{\left(\nu_{1}^{I I}-1\right)\left(\nu_{1}^{I I}-\bar{\sigma}_{2}^{2}\right)} \\
g_{22}\left(\vec{\nu}^{I I}\right)=-\frac{1}{4} \frac{\left(\nu_{2}^{I I}-\nu_{1}^{I I}\right)}{\left(\nu_{2}^{I I}-1\right)\left(\nu_{2}^{I I}-\bar{\sigma}_{2}^{2}\right)} \\
\frac{d \nu_{1}^{I I}}{d x}=(-1)^{\alpha_{1}} 2 \frac{\nu_{1}^{I I}\left(\nu_{1}^{I I}-\bar{\sigma}_{2}^{2}\right)}{\left(\nu_{1}^{I I}-\nu_{2}^{I I}\right)} \cdot \sqrt{1-\nu_{1}^{I I}} \\
\frac{d \nu_{2}^{I I}}{d x}=(-1)^{\alpha_{2}} 2 \frac{\nu_{2}^{I I}\left(\nu_{2}^{I I}-\bar{\sigma}_{2}^{2}\right)}{\left(\nu_{2}^{I I}-\nu_{1}^{I I}\right)} \cdot \sqrt{1-\nu_{2}^{I I}}
\end{gathered}
$$




$$
\begin{aligned}
& \sum_{a=1}^{2} \frac{d \nu_{a}^{I I}}{(-1)^{\alpha_{a}} 2 \nu_{a}^{I I}\left(\nu_{a}^{I I}-\bar{\sigma}_{2}^{2}\right) \sqrt{1-\nu_{a}^{I I}}}=0 \\
& \sum_{a=1}^{2} \frac{\nu_{a}^{I I} d \nu_{a}^{I I}}{(-1)^{\alpha_{a}} 2 \nu_{a}^{I I}\left(\nu_{a}^{I I}-\bar{\sigma}_{2}^{2}\right) \sqrt{1-\nu_{a}^{I I}}}=d x
\end{aligned}
$$

ahora sobre el paralelogramo $\bar{P}_{2}\left(0, \bar{\sigma}_{2}^{2}\right): 0 \leq \nu_{1}^{I I} \leq \bar{\sigma}_{3}^{2}, \bar{\sigma}_{2}^{2} \leq \nu_{2}^{I I} \leq 1$.

La integración de las ecuaciones (6.28) y (6.32) nos proporcionará, evidentemente, las ecuaciones de los kinks (3.39)-(3.40), que hemos denominado familia NTK $2 \sigma_{2}$ en el Capítulo 3 por tratarse de kinks no topológicos de dos componentes (cartesianas) no nulas. De manera análoga, (6.29) y (6.33) nos conducen a la dependencia temporal de dichas soluciones, (3.41)-(3.42). Las soluciones que se obtienen son obviamente las mismas que las obtenidas en el Modelo $O(2)$, si bien ahora expresadas en términos de las coordenadas elípticas tridimensionales y no en las bidimensionales. La familia resultante depende de dos constantes arbitrarias, una de ellas es la responsable de la invarianza bajo traslaciones en la variable $x$, mientras que la otra, que aparece en las ecuaciones de las órbitas, es la que nos diferencia entre unos y otros kinks de la misma familia uniparamétrica. Por supuesto, en coordenadas cartesianas existen dos familias de este tipo, la perteneciente al sector topológico $\mathcal{C}^{(-,-)}$y la del sector $\mathcal{C}^{(+,+)}$.

La energía de cualquiera de los kinks de estas familias coincide, ya fue calculada en el Capítulo 3, y resultaba

$$
\begin{aligned}
E\left(\mathrm{NTK} 2 \sigma_{2}\right) & =2 E_{\lambda_{1}}\left(\lambda_{1}\right)+2 E_{\lambda_{2}}\left(\lambda_{2}\right)+4 E_{\lambda_{3}}\left(\lambda_{3}\right) \\
& =\frac{m^{3}}{\lambda^{2} \sqrt{2}}\left[\frac{4}{3}+2 \sigma_{2}\left(1-\frac{\sigma_{2}^{2}}{3}\right)\right] .
\end{aligned}
$$

debido a que los rangos de variación de cada una de las variables $\left(\lambda_{1}, \lambda_{2}, \lambda_{3}\right)$ se recorren 2,2 y 4 veces respectivamente sobre una solución cualquiera de las familias.

De la misma manera que hicimos en el caso de los kinks NTK3, describiremos ahora con detalle cómo esta energía puede verse como cotas de Bogomol'nyi a través de las ecuaciones de primer orden. Para ello detallemos la trayectoria de un kink cualquiera de estas familias.

$\mathrm{Si}$, partiendo del punto $\mathrm{D}$, denominamos $\mathrm{P}_{1}$ y $\mathrm{P}_{2}$ a los puntos de intersección entre la trayectoria y las aristas $\mathrm{CF}_{1}$ y $\mathrm{F}_{1} \mathrm{~F}_{3}$ respectivamente, que el kink alcanza antes de pasar por el foco $\mathrm{F}_{2} \mathrm{y}$, de manera análoga, llamamos $\mathrm{P}_{3}$ y $\mathrm{P}_{4}$ a los pasos por las mismas aristas en sentido inverso, en el "camino de retorno" al punto D, tendremos los siguientes tramos a considerar, en sentido cronológico: 
- En la cara $\lambda_{2}=\bar{\sigma}_{3}^{2}$ :

1. $\mathrm{D} \rightarrow \mathrm{P}_{1}$. En este tramo, evidentemente, $\frac{d \nu_{1}^{I I}}{d x} \mathrm{y} \frac{d \nu_{2}^{I I}}{d x}$ son necesariamente positivos, puesto que en el mismo $\nu_{1}^{I I} \equiv \lambda_{1}$ crece desde su valor mínimo $\nu_{1}^{I I}=0$ hasta el valor que alcanza en $\mathrm{P}_{1}$, mientras que $\nu_{2}^{I I} \equiv \lambda_{3}$ pasa desde $\bar{\sigma}_{2}^{2}$ hasta 1 . En consecuencia las ecuaciones de primer orden que se verifican en este tramo son las correspondientes a la elección de signos tales que $\alpha_{1}=0$ y $\alpha_{2}=0$. El intervalo de tiempo empleado será entonces $\left(-\infty, x_{1}\right]$ siendo $x_{1}$ el valor (arbitrario, como ya hemos comentado) en el cual la solución alcanza el punto $\mathrm{P}_{1}$.

2. $\mathrm{P}_{1} \rightarrow \mathrm{P}_{2}$. En este caso la coordenada $\nu_{1}^{I I}$ continúa siendo creciente pero $\nu_{2}^{I I}$ decrece desde su valor máximo 1 hasta el correspondiente al punto $\mathrm{P}_{2}$. Se tiene, por tanto, que las ecuaciones que se satisfacen ahora serán las correspondientes a los valores $\alpha_{1}=0$ y $\alpha_{2}=1$, en el intervalo de tiempo $\left[x_{1}, x_{2}\right]$.

- En la cara $\lambda_{1}=\bar{\sigma}_{3}^{2}$ :

3. $\mathrm{P}_{2} \rightarrow \mathrm{F}_{2}$. En este caso la derivada $\frac{d \nu_{1}^{I}}{d x}$ es positiva, puesto que $\nu_{1}^{I} \equiv \lambda_{2}$ crece hasta alcanzar su valor máximo $\bar{\sigma}_{2}^{2}$ mientras que $\frac{d \nu_{2}^{I}}{d x}$ es negativa, al decrecer $\nu_{2}^{I} \equiv \lambda_{3}$ hasta su mínimo $\bar{\sigma}_{2}^{2}$. Tendremos así, en las ecuaciones (6.27), la elección $\alpha_{1}=0$ y $\alpha_{2}=1$. El tiempo empleado en este tramo será $\left[x_{2}, x_{0}\right]$.

4. $\mathrm{F}_{2} \rightarrow \mathrm{P}_{3}$. Se trata evidentemente de la situación opuesta, $\frac{d \nu_{1}^{I}}{d x}<0 \mathrm{y}$ $\frac{d \nu_{1}^{I}}{d x}>0$. La elección es $\alpha_{1}=1$ y $\alpha_{2}=0$ en $\left[x_{0}, x_{3}\right]$.

- Nuevamente en la cara $\lambda_{2}=\bar{\sigma}_{3}^{2}$ :

5. $\mathrm{P}_{3} \rightarrow \mathrm{P}_{4} . \frac{d \nu_{1}^{I I}}{d x}<0 \mathrm{y} \frac{d \nu_{2}^{I I}}{d x}>0$. Consecuentemente $\alpha_{1}=1, \alpha_{2}=0$ en $\left[x_{3}, x_{4}\right]$.

6. $\mathrm{P}_{4} \rightarrow \mathrm{D}$. Finalmente, en $\left[x_{4}, \infty\right)$, tendremos $\frac{d \nu_{1}^{I I}}{d x}<0$ y $\frac{d \nu_{2}^{I I}}{d x}<0$ y así $\alpha_{1}=1=\alpha_{2}$.

Con todas estas consideraciones, podemos calcular la energía separando la integral general en los tramos cronológicos descritos:

$$
\frac{\lambda^{2} \sqrt{2}}{m^{3}} E\left(\mathrm{NTK} 2 \sigma_{2}\right)=-\int_{\mathrm{NTK} 2 \sigma_{2}} d\left(W^{\left(\alpha_{1}, \alpha_{2}, \alpha_{3}\right)}\right)=
$$




$$
\begin{aligned}
= & -\int_{\mathrm{D}}^{\mathrm{P}_{1}} d W^{(0,0)}\left(\vec{\nu}^{I I}\right)-\int_{\mathrm{P}_{1}}^{\mathrm{P}_{2}} d W^{(0,1)}\left(\vec{\nu}^{I I}\right)-\int_{\mathrm{P}_{2}}^{\mathrm{F}_{2}} d W^{(0,1)}\left(\vec{\nu}^{I}\right) \\
& -\int_{\mathrm{F}_{2}}^{\mathrm{P}_{3}} d W^{(1,0)}\left(\vec{\nu}^{I}\right)-\int_{\mathrm{P}_{3}}^{\mathrm{P}_{4}} d W^{(1,0)}\left(\vec{\nu}^{I I}\right)-\int_{\mathrm{P}_{4}}^{\mathrm{D}} d W^{(1,1)}\left(\vec{\nu}^{I I}\right)= \\
= & \frac{4}{3}+2 \sigma_{2}\left(1-\frac{\sigma_{2}^{2}}{3}\right)
\end{aligned}
$$

Y concluimos, finalmente, que $E\left(\mathrm{NTK} 2 \sigma_{2}\right)$, al igual que $E(\mathrm{NTK} 3)$, no es una cantidad topológica puesto que depende de los valores que alcanza el superpotencial en puntos intermedios de la trayectoria. Se trata, por tanto, de kinks inestables, no-BPS.

Además de los kinks NTK2 $\sigma_{2}$ en el plano $q_{3}=0$ existen dos tipos de kinks, que ya hemos estudiado, se trata del tipo TK2 $\sigma_{2}$ (que incluyen el TK2 $\sigma_{2}$ propiamente dicho y el ATK $2 \sigma_{2}$, TK $2 \sigma_{2}{ }^{*}$ y ATK $2 \sigma_{2}{ }^{*}$ ) y el kink TK1 (y su correspondiente antikink ATK1). Nos detendremos brevemente en analizarlos desde la perspectiva que estamos adoptando en esta sección:

- La órbita del kink TK2 $\sigma_{2}$ se reduce, en coordenadas elípticas, a la arista DC de $\partial P_{3}(0): \lambda_{1}=0$ y $\lambda_{2}=\bar{\sigma}_{3}^{2}$. Sobre ella, el sistema de ecuaciones de primer orden (6.14) se reducen a una única ecuación:

$$
\frac{d \lambda_{3}}{d x}=(-1)^{\beta}\left(\lambda_{3}-\bar{\sigma}_{2}^{2}\right) \sqrt{1-\lambda_{3}}
$$

cuya integración directa nos proporciona la dependencia temporal de la coordenada $\lambda_{3}$ y así escribimos el kink explícitamente de la forma:

$$
\lambda_{1}^{\mathrm{TK} 2 \sigma_{2}}(x)=0, \quad \lambda_{2}^{\mathrm{TK} 2 \sigma_{2}}(x)=\bar{\sigma}_{3}^{2}, \quad \lambda_{3}^{\mathrm{TK} 2 \sigma_{2}}(x)=1-\bar{\sigma}_{2}^{2} \tanh ^{2}\left(\sigma_{2} x\right)
$$

Su energía es trivialmente calculable:

$$
E\left(\operatorname{TK} 2 \sigma_{2}\right)=2 E_{\lambda_{3}}\left(\lambda_{3}\right)=\frac{m^{3}}{\lambda^{2} \sqrt{2}}\left[2 \sigma_{2}\left(1-\frac{\sigma_{2}^{2}}{3}\right)\right]
$$

Y esta expresión puede calcularse como una cota de Bogomol'nyi si tenemos en cuenta que el kink TK2 $\sigma_{2}$ recorre la arista DC dos veces, en primer lugar $\frac{d \lambda_{3}}{d x}$ es positivo en $\mathrm{D} \rightarrow \mathrm{C}$, mientras que en $\mathrm{C} \rightarrow \mathrm{D}$ será negativo al decrecer $\lambda_{3}$ desde su valor máximo, 1 , hasta su mínimo $\bar{\sigma}_{2}^{2}$. Las ecuaciones de primer orden se verifican en ambos tramos con signos opuestos. Existe, sin embargo, una diferencia sustancial entre esta "singularidad" (en $\lambda_{3}=1$ ) y las 
observadas en los puntos umbílicos para el caso NTK3, o el foco $\mathrm{F}_{2}$ para los kinks NTK2 $\sigma_{2}$. La única singularidad que presenta el kink TK2 $\sigma_{2}$ es debida al cambio de coordenadas, es decir, $\lambda_{3}=1$ no es una región de singularidad en las coordenadas cartesianas y, de hecho, los valores que alcanza el superpotencial en el punto $\mathrm{C}$ no influyen en la energía, al ser nulos. Tenemos de esta manera:

$$
\begin{aligned}
E\left(\mathrm{TK} 2 \sigma_{2}\right) & =-\frac{m^{3}}{\lambda^{2} \sqrt{2}} \int_{\mathrm{TK} 2 \sigma_{2}} d W_{3}^{\left(\alpha_{3}\right)}=-\frac{m^{3}}{\lambda^{2} \sqrt{2}}\left[\int_{\mathrm{D}}^{\mathrm{C}} d W_{3}^{(0)}+\int_{\mathrm{C}}^{\mathrm{D}} d W_{3}^{(1)}\right]= \\
& =\frac{m^{3}}{\lambda^{2} \sqrt{2}} 2\left[\left|W_{3}^{\left(\alpha_{3}\right)}\left(\lambda_{3}=\bar{\sigma}_{2}^{2}\right)\right|\right]=\frac{m^{3}}{\lambda^{2} \sqrt{2}}\left[2 \sigma_{2}\left(1-\frac{\sigma_{2}^{2}}{3}\right)\right]
\end{aligned}
$$

Podemos afirmar así que $E\left(\mathrm{TK} 2 \sigma_{2}\right)$ es una cantidad topológica y, en consecuencia, los kinks tipo TK2 $\sigma_{2}$ son absolutamente estables y, por tanto, defectos topológicos de tipo BPS.

- En lo que respecta al kink TK1, contenido tanto en el plano $q_{3}=0$ como en el $q_{2}=0$, su órbita en $\partial P_{3}(0)$ consta de tres tramos, cada uno de los cuales se recorren dos veces. Partiendo del punto $\mathrm{D}$, el primero de los tramos, la arista $\mathrm{DF}_{3}$ corresponde a $\lambda_{2}=\bar{\sigma}_{3}^{2}$ y $\lambda_{3}=\bar{\sigma}_{2}^{2}$, de manera que $0 \leq \lambda_{1} \leq \bar{\sigma}_{3}^{2}$. El segundo tramo consiste en la arista $\mathrm{F}_{3} \mathrm{~F}_{2}, \lambda_{1}=\bar{\sigma}_{3}^{2}, \lambda_{3}=\bar{\sigma}_{2}^{2}, \bar{\sigma}_{3}^{2} \leq \lambda_{2} \leq \bar{\sigma}_{2}^{2}$. Finalmente el tercero será $\mathrm{F}_{3} \mathrm{O}$, con $\lambda_{1}=\bar{\sigma}_{3}^{2}, \lambda_{2}=\bar{\sigma}_{2}^{2}, \bar{\sigma}_{2}^{2} \leq \lambda_{3} \leq 1$. Las ecuaciones de primer orden en cada tramo, así como su integración explícita, se detallan a continuación (y donde las dos opciones de signo, + o -, están asociadas, evidentemente, a la primera y segunda vez que se recorre el tramo, respectivamente):

1. $\lambda_{2}=\bar{\sigma}_{3}^{2}$ and $\lambda_{3}=\bar{\sigma}_{2}^{2}$.

$$
\begin{gathered}
\frac{d \lambda_{1}}{d x}= \pm 2 \lambda_{1} \sqrt{1-\lambda_{1}} \\
x \in\left(-\infty,-\operatorname{arctanh} \sigma_{3}\right] \sqcup\left[\operatorname{arctanh} \sigma_{3}, \infty\right) \\
\lambda_{1}^{\mathrm{TK} 1}(x)=1-\tanh ^{2} x, \quad \lambda_{2}^{\mathrm{TK} 1}(x)=\bar{\sigma}_{3}^{2}, \quad \lambda_{3}^{\mathrm{TK} 1}(x)=\bar{\sigma}_{2}^{2}
\end{gathered}
$$

2. $\lambda_{1}=\bar{\sigma}_{3}^{2}$ and $\lambda_{3}=\bar{\sigma}_{2}^{2}$.

$$
\begin{gathered}
\frac{d \lambda_{2}}{d x}= \pm 2 \lambda_{2} \sqrt{1-\lambda_{2}} \\
x \in\left[-\operatorname{arctanh} \sigma_{3},-\operatorname{arctanh} \sigma_{2}\right] \sqcup\left[\operatorname{arctanh} \sigma_{2}, \operatorname{arctanh} \sigma_{3}\right]
\end{gathered}
$$




$$
\lambda_{1}^{\mathrm{TK} 1}(x)=\bar{\sigma}_{3}^{2}, \quad \lambda_{2}^{\mathrm{TK} 1}(x)=1-\tanh ^{2} x, \quad \lambda_{3}^{\mathrm{TK} 1}(x)=\bar{\sigma}_{2}^{2}
$$

3. $\lambda_{1}=\bar{\sigma}_{3}^{2}$ and $\lambda_{2}=\bar{\sigma}_{2}^{2}$.

$$
\begin{gathered}
\frac{d \lambda_{3}}{d x}= \pm 2 \lambda_{3} \sqrt{1-\lambda_{3}} \\
x \in\left[-\operatorname{arctanh} \sigma_{2}, 0\right] \sqcup\left[0, \operatorname{arctanh} \sigma_{2}\right] \\
\lambda_{1}^{\mathrm{TK} 1}(x)=\bar{\sigma}_{3}^{2}, \quad \lambda_{2}^{\mathrm{TK} 1}(x)=\bar{\sigma}_{2}^{2}, \quad \lambda_{3}^{\mathrm{TK} 1}(x)=1-\tanh ^{2} x
\end{gathered}
$$

Como vemos, las tres componentes elípticas son no nulas (aunque cada una de ellas lo es en un tramo diferente). En coordenadas cartesianas, como ya se ha detallado anteriormente, se tiene de una única componente no nula, $q_{1}$. Se trata, por otro lado, de la solución kink del Modelo Sigma Lineal $O(1)$.

La energía que se computó en el Capítulo 3:

$$
E(\mathrm{TK} 1)=2 E_{\lambda_{1}}\left(\lambda_{1}\right)+2 E_{\lambda_{2}}\left(\lambda_{2}\right)+2 E_{\lambda_{3}}\left(\lambda_{3}\right)=\frac{m^{3}}{\lambda^{2} \sqrt{2}} \cdot \frac{4}{3}
$$

puede verse ahora desde el punto de vista de la cota de Bogomol'nyi. Siguiendo los razonamientos que se han detallado en los anteriores casos estudiados tendremos la siguiente expresión:

$$
\begin{aligned}
\frac{\lambda^{2} \sqrt{2}}{m^{3}} E(\mathrm{TK} 1)= & -\int_{\mathrm{TK} 1} d\left(W_{1}^{\left(\alpha_{1}\right)}+W_{2}^{\left(\alpha_{2}\right)}+W_{3}^{\left(\alpha_{3}\right)}\right)= \\
= & -\int_{\mathrm{D}}^{\mathrm{F}_{3}} d W_{1}^{(0)}-\int_{\mathrm{F}_{3}}^{\mathrm{F}_{2}} d W_{2}^{(0)}-\int_{\mathrm{F}_{2}}^{\mathrm{O}} d W_{3}^{(0)} \\
& -\int_{\mathrm{O}}^{\mathrm{F}_{2}} d W_{3}^{(1)}-\int_{\mathrm{F}_{2}}^{\mathrm{F}_{3}} d W_{2}^{(1)}-\int_{\mathrm{F}_{3}}^{\mathrm{D}} d W_{1}^{(1)}= \\
= & \frac{4}{3}
\end{aligned}
$$

de manera que el kink TK1 satisface seis sistemas de ecuaciones de primer orden diferentes en los seis tramos de que consta la solución:

$$
\begin{aligned}
& x \in\left(-\infty,-\operatorname{arctanh} \sigma_{3}\right], x \in\left[-\operatorname{arctanh} \sigma_{3},-\operatorname{arctanh} \sigma_{2}\right] \\
& x \in\left[-\operatorname{arctanh} \sigma_{2}, 0\right], x \in\left[0, \operatorname{arctanh} \sigma_{2}\right] \\
& x \in\left[\operatorname{arctanh} \sigma_{2}, \operatorname{arctanh} \sigma_{3}\right], x \in\left[\operatorname{arctanh} \sigma_{3}, \infty\right)
\end{aligned}
$$

Su energía depende de los valores que el superpotencial alcanza en los puntos singulares $\mathrm{F}_{2} \mathrm{y}_{3}$, además del punto $\mathrm{D}$ y, en definitiva, se trata de una solución inestable, no-BPS. 


\section{Plano $q_{2}=0$. Modelo $O(2)$ embebido en el modelo $O(3)$ : II}

De manera similar al caso anterior, nos encontramos ahora con que el plano $q_{2}=0$ de $\mathbb{R}^{3}$ se corresponde con dos caras de $\partial P_{3}(0)$, en este caso se trata de $\lambda_{2}=\bar{\sigma}_{2}^{2}$ y $\lambda_{3}=\bar{\sigma}_{2}^{2}$. La arista común es ahora la hipérbola característica que pasa por los puntos umbílicos y por el $\mathrm{F}_{2}$, teniendo como focos a los puntos $\mathrm{F}_{3}$. Escribiremos a continuación únicamente las expresiones resultantes en ambas caras:

- A. Cara $\lambda_{2}=\bar{\sigma}_{2}^{2}$

$$
\begin{gathered}
\lambda_{1}=\rho_{1}^{I}, \lambda_{3}=\rho_{2}^{I}, \quad \Rightarrow \quad \vec{\rho}^{I}=\left(\rho_{1}^{I}, \rho_{2}^{I}\right) \\
W^{\left(\alpha_{1}, \alpha_{2}\right)}\left(\vec{\rho}^{I}\right)=\frac{1}{3}\left((-1)^{\alpha_{1}}\left(\rho_{1}^{I}+2\right) \sqrt{1-\rho_{1}^{I}}+(-1)^{\alpha_{2}}\left(\rho_{2}^{I}+2\right) \sqrt{1-\rho_{2}^{I}}\right) \\
g_{11}\left(\vec{\rho}^{I}\right)=-\frac{1}{4} \frac{\left(\rho_{1}^{I}-\rho_{2}^{I}\right)}{\left(\rho_{1}^{I}-1\right)\left(\rho_{1}^{I}-\bar{\sigma}_{3}^{2}\right)} \\
g_{22}\left(\vec{\rho}^{I}\right)=-\frac{1}{4} \frac{\left(\rho_{2}^{I}-\rho_{1}^{I}\right)}{\left(\rho_{2}^{I}-1\right)\left(\rho_{2}^{I}-\bar{\sigma}_{3}^{2}\right)} \\
\frac{d \rho_{1}^{I}}{d x}=(-1)^{\alpha_{1}} 2 \frac{\rho_{1}^{I}\left(\rho_{1}^{I}-\bar{\sigma}_{3}^{2}\right)}{\left(\rho_{1}^{I}-\rho_{2}^{I}\right)} \cdot \sqrt{1-\rho_{1}^{I}} \\
\frac{d \rho_{2}^{I}}{d x}=(-1)^{\alpha_{2}} 2 \frac{\rho_{2}^{I}\left(\rho_{2}^{I}-\bar{\sigma}_{2}^{2}\right)}{\left(\rho_{2}^{I}-\rho_{1}^{I}\right)} \cdot \sqrt{1-\rho_{2}^{I}} \\
\sum_{a=1}^{2} \frac{d \rho_{a}^{I}}{(-1)^{\alpha_{a}} 2 \rho_{a}^{I}\left(\rho_{a}^{I}-\bar{\sigma}_{3}^{2}\right) \sqrt{1-\rho_{a}^{I}}}=0 \\
\sum_{a=1}^{2} \frac{\rho_{a}^{I} d \rho_{a}^{I}}{(-1)^{\alpha_{a}} 2 \rho_{a}^{I}\left(\rho_{a}^{I}-\bar{\sigma}_{3}^{2}\right) \sqrt{1-\rho_{a}^{I}}}=d x
\end{gathered}
$$

en el paralelogramo $\left.\bar{P}_{2}\left(0, \bar{\sigma}_{2}^{2}\right)\right): 0 \leq \rho_{1}^{I} \leq \bar{\sigma}_{3}^{2}, \bar{\sigma}_{2}^{2} \leq \rho_{2}^{I} \leq 1$.

- B. Cara $\lambda_{3}=\bar{\sigma}_{2}^{2}$

$$
\begin{gathered}
\lambda_{1}=\rho_{1}^{I I}, \lambda_{2}=\rho_{2}^{I I}, \quad \Rightarrow \quad \vec{\rho}^{I I}=\left(\rho_{1}^{I I}, \rho_{2}^{I I}\right) \\
W^{\left(\alpha_{1}, \alpha_{2}\right)}\left(\vec{\rho}^{I I}\right)=\frac{1}{3}\left((-1)^{\alpha_{1}}\left(\rho_{1}^{I I}+2\right) \sqrt{1-\rho_{1}^{I I}}+(-1)^{\alpha_{2}}\left(\rho_{2}^{I I}+2\right) \sqrt{1-\rho_{2}^{I I}}\right)
\end{gathered}
$$




$$
\begin{gathered}
g_{11}\left(\vec{\rho}^{I I}\right)=-\frac{1}{4} \frac{\left(\rho_{1}^{I I}-\rho_{2}^{I I}\right)}{\left(\rho_{1}^{I I}-1\right)\left(\rho_{1}^{I I}-\bar{\sigma}_{2}^{2}\right)} \\
g_{22}\left(\vec{\rho}^{I I}\right)=-\frac{1}{4} \frac{\left(\rho_{2}^{I I}-\rho_{1}^{I I}\right)}{\left(\rho_{2}^{I I}-1\right)\left(\rho_{2}^{I I}-\bar{\sigma}_{2}^{2}\right)} \\
\frac{d \rho_{1}^{I I}}{d x}=(-1)^{\alpha_{1}} 2 \frac{\rho_{1}^{I I}\left(\rho_{1}^{I I}-\bar{\sigma}_{2}^{2}\right)}{\left(\rho_{1}^{I I}-\rho_{2}^{I I}\right)} \cdot \sqrt{1-\rho_{1}^{I I}} \\
\frac{d \rho_{2}^{I I}}{d x}=(-1)^{\alpha_{2}} 2 \frac{\rho_{2}^{I I}\left(\rho_{2}^{I I}-\bar{\sigma}_{2}^{2}\right)}{\left(\rho_{2}^{I I}-\rho_{1}^{I I}\right)} \cdot \sqrt{1-\rho_{2}^{I I}} \\
\sum_{a=1}^{2} \frac{d \rho_{a}^{I I}}{(-1)^{\alpha_{a}} 2 \rho_{a}^{I I}\left(\rho_{a}^{I I}-\bar{\sigma}_{2}^{2}\right) \sqrt{1-\rho_{a}^{I I}}}=0 \\
\sum_{a=1}^{2} \frac{\rho_{a}^{I I} d \rho_{a}^{I I}}{(-1)^{\alpha_{a}} 2 \rho_{a}^{I I}\left(\rho_{a}^{I I}-\bar{\sigma}_{2}^{2}\right) \sqrt{1-\rho_{a}^{I I}}}=d x
\end{gathered}
$$

para el paralelogramo $\bar{P}_{2}\left(0, \bar{\sigma}_{3}^{2}\right): 0 \leq \rho_{1}^{I I} \leq \bar{\sigma}_{3}^{2} \leq \rho_{2}^{I I} \leq \bar{\sigma}_{2}^{2}$.

La integración de (6.39) y (6.43) nos proporcionará las ecuaciones (3.34)-(3.35) de las familias de kinks NTK2 $\sigma_{3}$ obtenidas en el Capítulo 3. Se trata de dos familias (en coordenadas cartesianas) uniparamétricas de kinks no topológicos, similares a los descritos en el plano $q_{3}=0$. La integración de (6.40) y (6.44) conduce a las ecuaciones de la dependencia temporal (3.36)-(3.37).

Aunque se trata de kinks similares a los NTK $2 \sigma_{2}$, como hemos dicho, existen diferencias en la energía debidas a los diferentes valores de las constantes $\sigma_{2}$ y $\sigma_{3}$. De hecho, la descripción de las soluciones $\mathrm{NTK} 2 \sigma_{3}$ en coordenadas elípticas tridimensionales es muy diferente a la de las NTK2 $\sigma_{2}$, a pesar de ser formalmente idéntica en elípticas bidimensionales. La razón es esencialmente que los puntos singulares que estas soluciones atraviesan, en el plano $q_{2}=0$, son los correspondientes a la hipérbola característica, y no a la elipse, esto hace que el número de tramos a considerar sea superior ( 8 en lugar de los 6 del caso $q_{3}=0$ ), aunque, finalmente, las energías de ambos tipos de kink coincidan formalmente si se cambia un parámetro por otro.

$$
\begin{aligned}
E\left(\mathrm{NTK} 2 \sigma_{3}\right) & =2 E_{\lambda_{1}}\left(\lambda_{1}\right)+4 E_{\lambda_{2}}\left(\lambda_{2}\right)+4 E_{\lambda_{3}}\left(\lambda_{3}\right) \\
& =\frac{m^{3}}{\lambda^{2} \sqrt{2}}\left[\frac{4}{3}+2 \sigma_{3}\left(1-\frac{\sigma_{3}^{2}}{3}\right)\right]
\end{aligned}
$$

Si denominamos $\mathrm{P}_{1}, \mathrm{P}_{3}, \mathrm{P}_{4}$ y $\mathrm{P}_{6}$ a los puntos de corte de una solución cualquiera de este tipo con la hipérbola citada (arista $\mathrm{AF}_{3}$ de $\partial \bar{P}_{3}(0)$ ), y $\mathrm{P}_{2}$ y $\mathrm{P}_{5}$ a los de 
corte con la arista $\mathrm{BO}$ (eje $q_{1}=q_{2}=0$ en cartesianas) respectivamente, podemos describir dicho kink, cronológicamente en el "tiempo" $x$, de la forma siguiente:

- En la cara $\lambda_{3}=\bar{\sigma}_{2}^{2}$ :

1. $\mathrm{D} \rightarrow \mathrm{P}_{1}$. En este primer tramo, las derivadas $\frac{d \rho_{1}^{I I}}{d x}$ y $\frac{d \rho_{2}^{I I}}{d x}$ son evidentemente positivas, las ecuaciones de primer orden (6.42) que se satisfacen son las correspondientes a los valores $\alpha_{1}=\begin{array}{llll}0 & \alpha_{2}=1 & \text { (nótese que }\end{array}$ las características de la segunda de las ecuaciones, (6.42), hace que se obtenga el signo positivo considerando $\alpha_{2}=1$ ).

- En la cara $\lambda_{2}=\bar{\sigma}_{2}^{2}$ :

2. $\mathrm{P}_{1} \rightarrow \mathrm{P}_{2}$. $\rho_{1}^{I} \equiv \lambda_{1}$ continúa siendo creciente y $\rho_{2}^{I} \equiv \lambda_{3}$ crece también, en este caso desde su valor mínimo $\bar{\sigma}_{2}^{2}$ hasta su máximo 1 . Las ecuaciones a considerar, (6.38), son las relativas a $\alpha_{1}=0, \alpha_{2}=0$.

3. $\mathrm{P}_{2} \rightarrow \mathrm{P}_{3}$. De manera análoga al anterior se deduce que los valores a considerar serán: $\alpha_{1}=0, \alpha_{2}=1$.

- En la cara $\lambda_{3}=\bar{\sigma}_{2}^{2}$ :

4. $\mathrm{P}_{3} \rightarrow \mathrm{F}_{2}$. En el tramo en el que se alcanza el foco $\mathrm{F}_{2}$ se tiene: $\alpha_{1}=0$, $\alpha_{2}=0$ en las ecuaciones (6.42).

5. $\mathrm{F}_{2} \rightarrow \mathrm{P}_{4} \cdot \alpha_{1}=1, \alpha_{2}=1$.

- De nuevo en la cara $\lambda_{2}=\bar{\sigma}_{2}^{2}$ :

6. $\mathrm{P}_{4} \rightarrow \mathrm{P}_{5} . \alpha_{1}=1, \alpha_{2}=0$.

7. $\mathrm{P}_{5} \rightarrow \mathrm{P}_{6} . \alpha_{1}=0, \alpha_{2}=0$.

- Finalmente en $\lambda_{3}=\bar{\sigma}_{2}^{2}$ :

8. $\mathrm{P}_{6} \rightarrow$ D. $\alpha_{1}=1, \alpha_{2}=0$. 
Con todas estas consideraciones podemos calcular la energía:

$$
\begin{aligned}
\frac{\lambda^{2} \sqrt{2}}{m^{3}} E\left(\mathrm{NTK} 2 \sigma_{3}\right)= & -\int_{\mathrm{NTK}_{2} \sigma_{2}} d\left(W^{\left(\alpha_{1}, \alpha_{2}, \alpha_{3}\right)}\right)= \\
= & -\int_{\mathrm{D}}^{\mathrm{P}_{1}} d W^{(0,1)}\left(\vec{\rho}^{I I}\right)-\int_{\mathrm{P}_{1}}^{\mathrm{P}_{2}} d W^{(0,0)}\left(\vec{\rho}^{I}\right)-\int_{\mathrm{P}_{2}}^{\mathrm{P}_{3}} d W^{(0,1)}\left(\vec{\rho}^{I}\right) \\
& -\int_{\mathrm{P}_{3}}^{\mathrm{F}_{2}} d W^{(0,0)}\left(\vec{\rho}^{I I}\right)-\int_{\mathrm{F}_{2}}^{\mathrm{P}_{4}} d W^{(1,1)}\left(\vec{\rho}^{I I}\right)-\int_{\mathrm{P}_{4}}^{\mathrm{P}_{5}} d W^{(1,0)}\left(\vec{\rho}^{I}\right) \\
& -\int_{\mathrm{P}_{5}}^{\mathrm{P}_{6}} d W^{(0,0)}\left(\vec{\rho}^{I}\right)-\int_{\mathrm{P}_{6}}^{\mathrm{D}} d W^{(1,0)}\left(\vec{\rho}^{I}\right)=\frac{4}{3}+2 \sigma_{3}\left(1-\frac{\sigma_{3}^{2}}{3}\right)
\end{aligned}
$$

Una vez más, la energía, en este caso $E\left(\mathrm{NTK} 2 \sigma_{3}\right)$ no es una cantidad topológica puesto que depende del valor que el superpotencial alcanza en el punto intermedio $\mathrm{F}_{2}$ (las contribuciones de los demás puntos "singulares" se cancelan mutuamente, cuando no son nulas). Estos kinks son nuevamente inestables, no-BPS.

De manera similar a lo ocurrido en el plano $q_{3}=0$, encontraremos ahora dos tipos más de kinks. Por un lado, el kink TK1, compartido por ambos planos, y que no repetiremos, y, por otro, el kink topológico TK2 $\sigma_{3}$ (cuatro kinks de nuevo si se observa desde $\mathbb{R}^{3}$ ).

En este caso, el kink TK2 $\sigma_{3}$ tiene cuatro tramos y, como veremos, va a ser inestable al atravesar su órbita uno de los puntos umbílicos del elipsoide $\lambda_{1}=0$.

El kink TK $2 \sigma_{3}$ recorre la arista DA, para pasar a la arista $\mathrm{AB} y$, posteriormente, repetir dicho camino en sentido inverso.

En primer lugar, tendremos la arista DA, $\lambda_{1}=0$ y $\lambda_{3}=\bar{\sigma}_{2}^{2}$. Sobre ella el sistema de ecuaciones de primer orden (6.14) se reduce a la ecuación:

$$
\frac{d \lambda_{2}}{d x}= \pm 2\left(\lambda_{2}-\bar{\sigma}_{3}^{2}\right) \sqrt{1-\lambda_{2}}
$$

donde los dos signos corresponden a las dos veces que se recorre dicha arista en sentidos opuestos. Integrando (6.47) tendremos la solución:

$$
\lambda_{1}^{\mathrm{TK} 2 \sigma_{3}}(x)=0, \quad \lambda_{2}^{\mathrm{TK} 2 \sigma_{3}}(x)=1-\sigma_{3}^{2} \tanh ^{2}\left(\sigma_{3} x\right), \quad \lambda_{3}^{\mathrm{TK} 2 \sigma_{3}}(x)=\bar{\sigma}_{2}^{2}
$$

Esta solución será válida en los intervalos de "tiempo": $x \in\left(-\infty, \frac{-1}{\sigma_{3}} \operatorname{arctanh} \frac{\sigma_{2}}{\sigma_{3}}\right]$ $\sqcup\left[\frac{1}{\sigma_{3}} \operatorname{arctanh} \frac{\sigma_{2}}{\sigma_{3}}, \infty\right)$.

Para la arista $\mathrm{AB}, \lambda_{1}=0, \lambda_{2}=\bar{\sigma}_{2}^{2}$, tendremos que las ecuaciones se reducen en este caso a

$$
\frac{d \lambda_{3}}{d x}= \pm 2\left(\lambda_{3}-\bar{\sigma}_{3}^{2}\right) \sqrt{1-\lambda_{3}}
$$


cuya integración nos proporciona:

$$
\lambda_{1}^{\mathrm{TK} 2 \sigma_{3}}(x)=0, \quad \lambda_{2}^{\mathrm{TK} 2 \sigma_{3}}(x)=\bar{\sigma}_{2}^{2}, \quad \lambda_{3}^{\mathrm{TK} 2 \sigma_{3}}(x)=1-\sigma_{3}^{2} \tanh ^{2}\left(\sigma_{3} x\right)
$$

para $x \in\left[\frac{-1}{\sigma_{3}} \operatorname{arctanh} \frac{\sigma_{2}}{\sigma_{3}}, \frac{1}{\sigma_{3}} \operatorname{arctanh} \frac{\sigma_{2}}{\sigma_{3}}\right]$.

En coordenadas cartesianas tendremos, obviamente, cuatro kinks: TK2 $\sigma_{3}$, $\mathrm{TK}^{*} \sigma_{3}$ y los correspondientes antikinks. La energía de todos ellos es:

$$
E\left(\mathrm{TK} 2 \sigma_{3}\right)=2 E_{\lambda_{2}}\left(\lambda_{2}\right)+2 E_{\lambda_{3}}\left(\lambda_{3}\right)=\frac{m^{3}}{\lambda^{2} \sqrt{2}}\left[2 \sigma_{3}\left(1-\frac{\sigma_{3}^{2}}{3}\right)\right] .
$$

Como se ha indicado, en el tramo $\mathrm{D} \rightarrow \mathrm{A}$ tendremos la ecuación (6.47) con signo positivo. Igualmente ocurrirá para $\mathrm{A} \rightarrow \mathrm{B}$, mientras que los signos de los dos tramos restantes son negativos. De esta forma, la energía puede calcularse por medio de la expresión:

$$
\begin{aligned}
E\left(\mathrm{TK} 2 \sigma_{3}\right)= & -\frac{m^{3}}{\lambda^{2} \sqrt{2}} \int_{\mathrm{TK} 2 \sigma_{3}} d\left(W_{2}^{\left(\alpha_{2}\right)}+W_{3}^{\left(\alpha_{3}\right)}\right)= \\
= & -\frac{m^{3}}{\lambda^{2} \sqrt{2}}\left[\int_{\mathrm{D}}^{\mathrm{A}} d W_{2}^{(0)}+\int_{\mathrm{A}}^{\mathrm{B}} d W_{3}^{(0)}+\right. \\
& \left.+\int_{\mathrm{B}}^{\mathrm{A}} d W_{3}^{(1)}+\int_{\mathrm{A}}^{\mathrm{D}} d W_{2}^{(1)}\right]= \\
= & \frac{m^{3}}{\lambda^{2} \sqrt{2}}\left[2 \sigma_{3}\left(1-\frac{\sigma_{3}^{2}}{3}\right)\right]
\end{aligned}
$$

En definitiva, las soluciones TK $2 \sigma_{3}$ verifican dos sistemas diferentes de ecuaciones de primer orden, se trata de un tipo de kinks inestables, no-BPS.

\section{Kinks Genéricos en el interior de $\overline{\mathbf{P}}_{3}(0)$}

La integración de (6.16) y (6.17):

$$
\begin{gathered}
\sum_{a=1}^{3} \frac{(-1)^{\alpha_{a}}}{2} \int \frac{d \lambda_{a}}{\left(\lambda_{a}-\bar{\sigma}_{2}^{2}\right)\left(\lambda_{a}-\bar{\sigma}_{3}^{2}\right) \sqrt{1-\lambda_{a}}}=\gamma_{2} \\
\sum_{a=1}^{3} \frac{(-1)^{\alpha_{a}}}{2} \int \frac{d \lambda_{a}}{\lambda_{a}\left(\lambda_{a}-\bar{\sigma}_{2}^{2}\right)\left(\lambda_{a}-\bar{\sigma}_{3}^{2}\right) \sqrt{1-\lambda_{a}}}=\gamma_{3},
\end{gathered}
$$

donde $\gamma_{2}$ y $\gamma_{3}$ son constantes de integración reales, conduce a las ecuaciones generales de las órbitas de los kinks TK3, (3.25) y (3.26), deducidas por medio del método de Hamilton-Jacobi en el Capítulo 3. Nótese que $(6.52,6.53)$ no son más 
que las cuadraturas $(3.23,3.24)$. Por su parte, la integración de (6.18) proporciona la dependencia temporal de las mismas:

$$
\sum_{a=1}^{3} \frac{(-1)^{\alpha_{a}}}{2} \int \frac{\lambda_{a} d \lambda_{a}}{\left(\lambda_{a}-\bar{\sigma}_{2}^{2}\right)\left(\lambda_{a}-\bar{\sigma}_{3}^{2}\right) \sqrt{1-\lambda_{a}}}=\gamma_{1}+x
$$

que depende de la constante de integración $\gamma_{1}$ y que coincide con la expresión (3.27) deducida en el Capítulo 3.

No repetiremos aquí las ecuaciones resultantes de las cuadraturas (6.52), (6.53) y (6.54). Dichas ecuaciones, así como la descripción detallada del comportamiento de los kink TK3 tanto en el espacio "cartesiano" $\mathbb{R}^{3}$ como en el paralelepípedo elíptico $\bar{P}_{3}(0)$ están desarrolladas en el Capítulo 3. Nos centraremos ahora en la deducción de la energía (o acción mecánica) de dichos kinks como una cota de Bogomol'nyi.

Tal y como se calculó en el Capítulo 3 , tenemos que cada rango de variación posible de las coordenadas elípticas $\left(\lambda_{1}, \lambda_{2}, \lambda_{3}\right)$ se recorre, por una solución TK3 cualquiera, 2, 4 y 6 veces respectivamente. En consecuencia:

$$
\begin{aligned}
E_{\lambda_{1}}\left(\lambda_{1}\right) & =\frac{m^{3}}{\lambda^{2} \sqrt{2}} \int_{0}^{\bar{\sigma}_{3}^{2}} \frac{\lambda_{1} d \lambda_{1}}{2 \sqrt{1-\lambda_{1}}} \\
E_{\lambda_{2}}\left(\lambda_{2}\right) & =\frac{m^{3}}{\lambda^{2} \sqrt{2}} \int_{\bar{\sigma}_{3}^{2}}^{\bar{\sigma}_{2}^{2}} \frac{\lambda_{2} d \lambda_{2}}{2 \sqrt{1-\lambda_{2}}} \\
E_{\lambda_{3}}\left(\lambda_{3}\right) & =\frac{m^{3}}{\lambda^{2} \sqrt{2}} \int_{\bar{\sigma}_{2}^{2}}^{1} \frac{\lambda_{3} d \lambda_{3}}{2 \sqrt{1-\lambda_{3}}}
\end{aligned}
$$

y así:

$$
\begin{aligned}
E(\mathrm{TK} 3) & =2 E_{\lambda_{1}}\left(\lambda_{1}\right)+4 E_{\lambda_{2}}\left(\lambda_{2}\right)+6 E_{\lambda_{3}}\left(\lambda_{3}\right) \\
& =\frac{m^{3}}{\lambda^{2} \sqrt{2}}\left(\frac{4}{3}+\frac{2}{3}\left[\sigma_{2}\left(3-\sigma_{2}^{2}\right)+\sigma_{3}\left(3-\sigma_{3}^{2}\right)\right]\right)
\end{aligned}
$$

y es, evidentemente, la misma cantidad para cualquiera de los kinks de la familia. Como ya se comentó, en coordenadas cartesianas se trata de dos familias biparamétricas de kinks topológicos, en los sectores $\mathcal{C}^{(-,+)}$y $\mathcal{C}^{(+,-)}$respectivamente.

Analicemos con detalle los diferentes superpotenciales y, en consecuencia, las diferentes ecuaciones de primer orden, (6.14), que están asociados a cada tramo de un kink cualquiera de la familia.

En orden "cronológico": 
1. El primer tramo une el punto de vacío D con un punto de la cara superior $\lambda_{3}=1$ de $\partial P_{3}(0)$ al que denominaremos $\mathrm{P}_{1}$. Evidentemente, las tres coordenadas son crecientes en este tramo, particularmente $\lambda_{3}$, que recorre todo su rango de variación. En definitiva: para $x \in\left(-\infty, x_{1}\right]$ tendremos que, necesariamente: $\alpha_{1}=\alpha_{2}=\alpha_{3}=0$, y así el superpotencial a considerar será: $W^{(0,0,0)}$.

2. A continuación, el kink pasa a través del interior de $\bar{P}_{3}(0)$ hasta llegar a la arista $\mathrm{AF}_{2}$, en un punto que llamaremos $\mathrm{P}_{2}$. En este caso tendremos el superpotencial $W^{(0,0,1)}$ puesto que $\lambda_{3}$ es decreciente en este intervalo. El tramo transcurre en el intervalo $\left[x_{1}, x_{2}\right]$.

3. $\mathrm{P}_{2} \rightarrow \mathrm{P}_{3}$. El kink pasa de la arista $\mathrm{AF}_{2}$ a la arista $\mathrm{F}_{1} \mathrm{~F}_{3} ; \lambda_{2}$ decrece pero $\lambda_{1}$ y $\lambda_{3}$ aumentan. En consecuencia, en el intervalo $\left[x_{2}, x_{3}\right]$, tendremos las ecuaciones de primer orden correspondientes a $\alpha_{1}=0, \alpha_{2}=1$ y $\alpha_{3}=0$ y así $W^{(0,1,0)}$.

4. $\mathrm{P}_{3} \rightarrow \mathrm{P}_{4}$. Nuevamente alcanzamos la cara superior del paralelepípedo y así $\alpha_{3}=0$. En este caso $\alpha_{1}=1$ y $\alpha_{2}=0$ en $\left[x_{3}, x_{4}\right]$.

5. $\mathrm{P}_{4} \rightarrow \mathrm{P}_{5}$. Se trata de un tramo similar al segundo explicado, pero con $\lambda_{1}$ decreciente. Tendremos, por tanto, $W^{(1,0,1)}$ en $\left[x_{4}, x_{5}\right]$.

6. $\mathrm{P}_{5} \rightarrow \mathrm{P}_{6}$. Pasamos ahora de la arista $\mathrm{AF}_{2}$ a la cara superior, se trata de un ramo idéntico al segundo considerado pero recorrido en sentido inverso, de esta manera se tiene $\alpha_{1}=1, \alpha_{2}=1$ y $\alpha_{3}=0$ en $\left[x_{5}, x_{6}\right]$.

7. Por último, desde $\mathrm{P}_{6}$ se retorna a $\mathrm{D}$ de manera similar al primer tramo pero con sentido opuesto. Se finaliza por tanto con $W^{(1,1,1)}$ en $\left[x_{6}, \infty\right)$.

Con todo ello, concluiremos que la energía se puede calcular de la forma.

$$
\begin{aligned}
\frac{\lambda^{2} \sqrt{2}}{m^{3}} E(\mathrm{TK} 3)= & -\int_{\mathrm{TK} 3} d W^{\left(\alpha_{1}, \alpha_{2}, \alpha_{3}\right)}=-\int_{\mathrm{TK} 3} d\left(W_{1}^{\left(\alpha_{1}\right)}+W_{2}^{\left(\alpha_{2}\right)}+W_{3}^{\left(\alpha_{3}\right)}\right) \\
= & -\int_{\mathrm{D}}^{\mathrm{P}_{1}} d W^{(0,0,0)}-\int_{\mathrm{P}_{1}}^{\mathrm{P}_{2}} d W^{(0,0,1)}-\int_{\mathrm{P}_{2}}^{\mathrm{P}_{3}} d W^{(0,1,0)} \\
& -\int_{\mathrm{P}_{3}}^{\mathrm{P}_{4}} d W^{(1,0,0)}-\int_{\mathrm{P}_{4}}^{\mathrm{P}_{5}} d W^{(1,0,1)}-\int_{\mathrm{P}_{5}}^{\mathrm{P}_{6}} d W^{(1,1,0)} \\
& -\int_{\mathrm{P}_{6}}^{\mathrm{D}} d W^{(1,1,1)}=\frac{4}{3}+\frac{2}{3}\left[\sigma_{2}\left(3-\sigma_{2}^{2}\right)+\sigma_{3}\left(3-\sigma_{3}^{2}\right)\right]
\end{aligned}
$$


Una vez más, concluimos que $E$ (TK3) no es una cantidad topológica y así los kinks TK3 son ondas solitarias no-BPS y, en definitiva, inestables.

\subsection{Teoría de Morse de Kinks}

Aplicaremos en esta sección la Teoría de Morse à la Bott en el espacio de soluciones kink del Modelo Sigma O(3) Lineal Deformado. Los aspectos fundamentales de la misma se han expuesto sucintamente en el Apéndice D.

Para conseguir dicho objetivo, y de acuerdo con la notación introducida en el Apéndice D, realizaremos las siguientes identificaciones:

1. $M$ denotará a la variedad riemanniana $P_{3}(0)$, considerada con la métrica de Jacobi asociada al sistema

$$
h_{a b}(\vec{\lambda})=2 U(\vec{\lambda}) g_{a b}(\vec{\lambda})
$$

2. $\Omega M$ (espacio de caminos cerrados con un punto fijo en la variedad $M$ ) es el espacio de configuración de los kinks, $\mathcal{C}$. Es necesario precisar que, como ya se explicó en el Capítulo 3, en coordenadas elípticas dicho espacio sólo tiene una componente conexa como consecuencia de que el cambio de coordenadas es ocho a uno. Los cuatro sectores desconectados aparecen únicamente cuando se considera el problema en coordenadas cartesianas. De esta manera, $\mathcal{C}$ será:

$$
\mathcal{C}=\mathcal{C}^{(-,-)} \sqcup \mathcal{C}^{(-,+)} \sqcup \mathcal{C}^{(+,-)} \sqcup \mathcal{C}^{(+,+)} .
$$

3. $m_{0}$ (punto fijo de los caminos considerados en $\Omega M$ ) será evidentemente el punto (único en elípticas) de vacío D en $P_{3}(0)$.

4. El papel del funcional de Longitud $L$ del espacio de lazos lo representará el funcional $E^{J}[\vec{\lambda}]$ y, por tanto, las geodésicas (kinks) estarán parametrizadas necesariamente por el arco de longitud en la métrica de Jacobi.

El primer objetivo a abordar es la determinación de los puntos conjugados a D, punto de vacío. En la sección 6.1.2 hemos demostrado que la elipse característica (6.9) (arista $\mathrm{F}_{1} \mathrm{~F}_{3}$ de $\partial P_{3}(0)$ ) y la hipérbola característica (6.10) (arista $\mathrm{AF}_{2}$ ) eran los puntos singulares de la ecuación (6.1) ó (6.6), de manera que, en ellos, las ecuaciones de primer orden no estaban definidas. Si escribimos ahora las ecuaciones de los flujos de las trayectorias solución, comprobaremos que dichas curvas constituyen la totalidad de los puntos conjugados al punto D. 
Los flujos de las trayectorias se deducen de las ecuaciones de primer orden de manera trivial y resultan ser:

$$
\begin{aligned}
& \frac{d \lambda_{2}}{d \lambda_{1}}=-\frac{\lambda_{2}\left(\lambda_{2}-\bar{\sigma}_{2}^{2}\right)\left(\lambda_{2}-\bar{\sigma}_{3}^{2}\right)}{\lambda_{1}\left(\lambda_{1}-\bar{\sigma}_{2}^{2}\right)\left(\lambda_{1}-\bar{\sigma}_{3}^{2}\right)} \cdot \frac{\left(\lambda_{1}-\lambda_{3}\right)}{\left(\lambda_{2}-\lambda_{3}\right)} \cdot \sqrt{\frac{1-\lambda_{2}}{1-\lambda_{1}}} \cdot \frac{(-1)^{\alpha_{2}}}{(-1)^{\alpha_{1}}} \\
& \frac{d \lambda_{3}}{d \lambda_{1}}=-\frac{\lambda_{3}\left(\lambda_{3}-\bar{\sigma}_{2}^{2}\right)\left(\lambda_{3}-\bar{\sigma}_{3}^{2}\right)}{\lambda_{1}\left(\lambda_{1}-\bar{\sigma}_{2}^{2}\right)\left(\lambda_{1}-\bar{\sigma}_{3}^{2}\right)} \cdot \frac{\left(\lambda_{1}-\lambda_{2}\right)}{\left(\lambda_{3}-\lambda_{2}\right)} \cdot \sqrt{\frac{1-\lambda_{3}}{1-\lambda_{1}}} \cdot \frac{(-1)^{\alpha_{3}}}{(-1)^{\alpha_{1}}}
\end{aligned}
$$

Dichos flujos no están definidos en las siguientes regiones:

- (1). Si $\lambda_{1}=\bar{\sigma}_{3}^{2}=\lambda_{2}, \frac{d \lambda_{2}}{d \lambda_{1}}=\frac{0}{0}$. Así, cada punto de la arista abierta $\mathrm{F}_{1} \mathrm{~F}_{3}$ es un punto conjugado de D. Por cada uno de los puntos de la línea focal $\mathrm{F}_{1} \mathrm{~F}_{3}$ pasa una congruencia de kinks parametrizados por un círculo $S^{1}$ alredor del punto en el plano en el que el flujo está indefinido. En consecuencia, la multiplicidad de los puntos conjugados en la arista $\mathrm{F}_{1} \mathrm{~F}_{3}$ es 1. Esto implica, según se ha comentado, la existencia de un único modo cero ortogonal no trivial (campo de Jacobi) del operador hessiano geométrico del funcional $E^{J}$ (operador de desviación geodésica).

- (2). Si $\lambda_{2}=\bar{\sigma}_{2}^{2}=\lambda_{3}, \frac{d \lambda_{3}}{d \lambda_{1}}=\frac{0}{0}$. Cada punto de la arista abierta $\mathrm{AF}_{2}$ es asimismo un punto conjugado a $\mathrm{D} . \mathrm{AF}_{2}$ es una línea focal formada por puntos conjugados de multiplicidad 1 por las mismas razones expuestas en el caso anterior.

- (3). Si $\lambda_{1}=0, \lambda_{2}=\bar{\sigma}_{3}^{2}, \lambda_{3}=\bar{\sigma}_{2}^{2}$, ambos flujos estarán indefinidos: $\frac{d \lambda_{2}}{d \lambda_{1}}=\frac{0}{0} \mathrm{y}$ $\frac{d \lambda_{3}}{d \lambda_{1}}=\frac{0}{0}$. Por tanto, el propio punto D es conjugado de sí mismo con multiplicidad 2; el flujo está indefinido en una variedad de dimensión dos rodeando a $\mathrm{D}$, es decir, existe una congruencia de trayectorias kink que, partiendo del punto D y parametrizadas por una sección bidimensional, vuelven al punto D. Desde el punto de vista del operador de desviación geodésica, existen dos campos de Jacobi independientes que se anulan en D.

- (4). Los dos flujos están también indefinidos en los puntos $\mathrm{A}, \mathrm{F}_{2}$ y $\mathrm{F}_{3}$. La razón, en este caso, estriba en el hecho de que por estos puntos pasan dos familias unidimensionales de kinks. Las trayectorias de los espacios modulares $\mathcal{M}_{\mathrm{NTK} 3+\mathrm{TK} 1}$ y $\mathcal{M}_{\mathrm{NTK} 2 \sigma_{3}+\mathrm{TK} 2 \sigma_{2}}$ atraviesan el foco $\mathrm{F}_{3}$. De manera análoga las trayectorias kink en $\mathcal{M}_{\mathrm{NTK} 3+\mathrm{TK} 1}$ y $\mathcal{M}_{\mathrm{NTK} 2 \sigma_{2}+\mathrm{TK} 2 \sigma_{3}}$ convergen en el punto umbílico A y en el foco $\mathrm{F}_{2}$. En la Sección 5.3 se calcularon explícitamente 
los campos de Jacobi que demuestran que estos tres puntos son conjugados de D.

\subsubsection{La serie de Morse de $\mathcal{C}$}

Una vez establecidos los puntos conjugados a tener en cuenta, aplicaremos ahora el Teorema del Índice de Morse para computar el índice de cada una de las trayectorias kink de la teoría. Dado que estamos estudiando la teoría de Morse para espacios de lazos, tendremos que calcular dicho índice considerando kinks no topológicos necesariamente o bien, como haremos, tomar la "unión" de dos kinks topológicos del mismo tipo. Desde el punto de vista de las coordenadas elípticas, como ya se ha comentado, la distinción entre kink topológico y no-topológico carece en buen medida de sentido, dado que los puntos de vacío son indistinguibles. Sin embargo, el cómputo del número de veces que una solución kink incide sobre la cara superior del paralelepípedo $\bar{P}_{3}(0)$, sí que permite que dicha distinción sea planteada. Por otro lado, es necesario precisar que cuando nos referimos al concepto de "trayectoria kink" estamos aludiendo a dos soluciones kink que transitan sobre la misma curva; evidentemente, las dos soluciones se diferencian en el "orden cronológico" en el que recorren dicha trayectoria, es decir, están relacionadas por medio de inversión temporal, o, desde el punto de vista de la teoría de campos, por inversión espacial. Queda plenamente justificado, por tanto, el considerar la "unión" de dos soluciones kink aún en el caso de la perspectiva "elíptica".

Otra cuestión a advertir es que "deformaremos" la arista DC al punto D en $\bar{P}_{3}(0)$ (de manera análoga a las referencias [67], [68] y [69] para el caso del Modelo MSTB). Ello no cambiará la topología del espacio de trayectorias en $\bar{P}_{3}(0)$ con la métrica de Jacobi, y únicamente dejará fuera de la cuenta en la serie de Morse de $\mathcal{C}$ a los kinks de tipo TK $2 \sigma_{2}$ que, por otro lado, son estables.

Veamos entonces:

I. Vacío: D. Para las trayectorias constantes, reducidas a un punto, y, por tanto, únicamente el punto D, el índice de Morse es evidentemente nulo: $\mu(\mathrm{D})=0$.

II. $\mathrm{TK} 2 \sigma_{3} \sqcup \mathrm{ATK} 2 \sigma_{3}$.

La órbita sobre las aristas DA y AB (y "vuelta") en $\bar{P}_{3}(0)$ incluye, en el sentido comentado anteriormente, dos soluciones de tipo kink:

- 1) El kink TK2 $\sigma_{3}$ : Esta solución parte del punto D en el "instante" $x=-\infty$ y alcanza el punto umbílico A en $x=\frac{-1}{\sigma_{3}} \operatorname{arctanh} \frac{\sigma_{2}}{\sigma_{3}}$, habiendo permanecido 
para ello en DA. En el punto A la solución "gira" un ángulo recto y, a través de la arista $\mathrm{AB}$, llega al punto $\mathrm{B}$ en $x=0$. El kink se completa, en el semieje $x \in \mathbb{R}^{+}$, realizando el "camino" inverso: pasará por A en $x=\frac{1}{\sigma_{3}} \operatorname{arctanh} \frac{\sigma_{2}}{\sigma_{3}}$ y finaliza en D a "tiempo" $x=+\infty$.

- 2) El kink ATK2 $\sigma_{3}$ : el proceso es idéntico al anterior con el cambio de $x$ por $-x$ (inversión temporal o espacial según se considere un punto de vista u otro de los citados)

Aunque sólo en el segundo paso de los kinks por el punto umbílico A éste es un punto conjugado a $\mathrm{D}$, pues es entonces cuando estas soluciones se encuentran con las congruencias correspondientes de kinks NTK3 (y ANTK3), la órbita, o trayectoria kink, TK2 $\sigma_{3} \sqcup \mathrm{ATK} 2 \sigma_{3}$, considerada como un todo, tiene índice de Morse dos:

$$
\mu\left(\mathrm{TK} 2 \sigma_{3} \sqcup \mathrm{ATK} 2 \sigma_{3}\right)=2
$$

Los razonamientos anteriores pueden simplificarse si, desde el punto de vista de las coordenadas elípticas, planteamos la órbita o trayectoria TK2 $\sigma_{3}$ y tenemos en cuenta que, en sus dos "pasos" por el punto A, éste debe ser computado como punto conjugado al punto D.

III. TK1 $\sqcup$ AK1.

La órbita sobre las aristas $\mathrm{DF}_{3}, \mathrm{~F}_{3} \mathrm{~F}_{2}$ y $\mathrm{F}_{2} \mathrm{O}$ de $\bar{P}_{3}(0)$, incluye también dos soluciones de tipo kink:

- 1) El kink TK1: parte del punto D en $x=-\infty$, recorre la arista $\mathrm{DF}_{3}$ hasta alcanzar $\mathrm{F}_{3}$ en $x=-\operatorname{arctanh} \sigma_{3}$, continúa por $\mathrm{F}_{3} \mathrm{~F}_{2}$ hasta $\mathrm{F}_{2}$, donde "llega" en $x=-\operatorname{arctanh} \sigma_{2}$. El tercer tramo es $\mathrm{F}_{2} \mathrm{O}$ y pasa por $\mathrm{O}$ a "tiempo" $x=0$. La trayectoria se completa, en $x \in \mathbb{R}^{+}$, con el camino inverso hasta alcanzar $\mathrm{D}$ en $x=+\infty$.

- 2) El kink ATK1: de nuevo se trata del mismo proceso intercambiando $x$ por $-x$.

$\mathrm{Al}$ igual que en el caso del kink TK2 $\sigma_{3}$, el índice de Morse se computa con el razonamiento anterior, teniendo en cuenta que los puntos conjugados $\left(\mathrm{F}_{2} \mathrm{y}\right.$ $\mathrm{F}_{3}$ ) lo son únicamente la segunda de las veces que se pasa por ellos, pues ahí es donde inciden las congruencias correspondientes de kinks NTK2 $\sigma_{2}$ y NTK2 $\sigma_{2}$ (y los antikinks asociados). La combinación TK1 $\sqcup$ ATK1 tendrá, en definitiva, índice cuatro.

$$
\mu(\mathrm{TK} 1 \sqcup \mathrm{ATK} 1)=4
$$




\section{TK3 ப ATK3.}

Una trayectoria TK3 pasa por la arista $\mathrm{F}_{1} \mathrm{~F}_{3}$ una vez y dos por la correspondiente $\mathrm{AF}_{2}$. Por su parte, el antikink ATK3 posee un comportamiento idéntico en el sentido opuesto. Tendremos, por tanto, que:

$$
\mu(\mathrm{TK} 3 \sqcup \mathrm{ATK} 3)=6
$$

La variedad crítica formada por este tipo de configuraciones, que denotaremos $\mathcal{N}_{c}=\overline{\mathcal{M}}_{\mathrm{TK} 3}^{(3)} \sqcup \overline{\mathcal{M}}_{\mathrm{ATK} 3}^{(3)}$, ha sido escogida en la tercera de las compactificaciones analizadas en el Capítulo 3, y debe ser entendido, por tanto, que las congruencias de configuraciones NTK3+TK1, NTK $2 \sigma_{3}+\mathrm{TK} 2 \sigma_{2}$ y NTK $2 \sigma_{2}+\mathrm{TK} 2 \sigma_{3}$ se tienen en cuenta al considerar las trayectorias que atraviesan los puntos $\mathrm{A}, \mathrm{F}_{2}$ y $\mathrm{F}_{3}$. La inclusión de las configuraciones en el borde de la variedad de módulos está en el espíritu del principio de complección de las variedades críticas, crucial para la demostración de que el funcional de longitud de un camino en un grupo de Lie y el funcional de Yang-Mills sobre una superficie de Riemann son funcionales de Morse perfectos (ver [15]).

Encontraremos, de esta manera, que el polinomio de Poincaré de $\mathcal{N}_{c}$ será: $P_{t}\left(\mathcal{N}_{c}\right)=1+t^{2}, \mathrm{y}$, por tanto, la contribución de la combinación TK3 $\sqcup$ ATK3 al polinomio de Morse será: $\left(1+t^{2}\right) t^{6}$.

Las combinaciones analizadas son realmente las únicas que deberían ser incluidas debido a que son las únicas que comienzan y finalizan en el punto D. Estrictamente hablando, D no puede ser atravesado por ninguna trayectoria puesto que la métrica de Jacobi está degenerada en dicho punto. Desde el punto de vista dinámico, el punto D sólo puede ser alcanzado, una segunda vez, en tiempo infinito.

Si admitimos la posibilidad de enlazar varias soluciones kink (lo que denotaremos por el símbolo \#), obviando el razonamiento anterior (siguiendo el criterio adoptado en las referencias [67], [68] y [69]), encontraremos que las siguientes variedades críticas a considerar serán:

V. $\left(\mathrm{TK} 2 \sigma_{3} \# \operatorname{TK} 3(1)\right) \sqcup\left(\operatorname{ATK} 2 \sigma_{3} \# \operatorname{ATK} 3(1)\right)$.

La trayectoria TK2 $\sigma_{3}$ seguida de un único kink TK3, precisamente TK3(1)= TK $2 \sigma_{2}+$ TK $2 \sigma_{3}+$ TK1. Las dos trayectorias están unidas en el punto D, que contribuye, por tanto, con dos unidades al índice de Morse, dada su multiplicidad. Lógicamente se considera, simultáneamente, la composición de anti-kinks correspondiente. Con estas consideraciones se tiene:

$$
\mu\left(\left(\operatorname{TK} 2 \sigma_{3} \# \operatorname{TK} 3(1)\right) \sqcup\left(\operatorname{ATK} 2 \sigma_{3} \# \operatorname{ATK} 3(1)\right)\right)=10
$$


VI. (TK1 \# TK3(1)) $\sqcup($ ATK1 \# ATK3(1)).

Aplicando idénticos razonamientos obtendremos ahora:

$$
\mu((\operatorname{TK} 1 \# \operatorname{TK} 3(1)) \sqcup(\operatorname{ATK} 1 \# \operatorname{ATK} 3(1)))=12
$$

VII. (TK3 \# TK3) $\sqcup$ (ATK3 \# ATK3).

El enlace de dos familias de kinks TK3 requiere, una vez más, la aplicación de la Teoría de Morse degenerada. El índice de Morse de un miembro concreto de la familia combinada es:

$$
\mu((\mathrm{TK} 3 \# \mathrm{TK} 3) \sqcup(\mathrm{ATK} 3 \# \mathrm{ATK} 3))=14
$$

pero el Polinomio de Poincaré de la variedad crítica es:

$$
P_{t}\left(\left(\overline{\mathcal{M}}_{\mathrm{TK} 3}^{(3)} \# \overline{\mathcal{M}}_{\mathrm{TK} 3}^{(3)}\right) \sqcup\left(\overline{\mathcal{M}}_{\mathrm{ATK} 3}^{(3)} \# \overline{\mathcal{M}}_{\mathrm{ATK} 3}^{(3)}\right)\right)=1+t^{2}
$$

Iterando los elementos explicados obtendremos la serie de Morse completa:

$$
\begin{aligned}
\mathcal{M}_{t}\left(\left.E\right|_{\mathcal{C}}\right) & =1+\left(1+t^{2}\right) t^{2}+\left(1+t^{2}\right) t^{6}+\left(1+t^{2}\right) t^{10}+\left(1+t^{2}\right) t^{14}+\ldots \\
& =1+\left(1+t^{2}\right) \sum_{k=0}^{\infty} t^{2(2 k+1)}=\frac{1}{1-t^{2}}
\end{aligned}
$$

Dado que los coeficientes de las potencias impares en $t$ se anulan, el Principio Lacunario establece que las Desigualdades de Morse se convierten en igualdades (ver [14]), y así tendremos:

$$
\mathcal{M}_{t}\left(\left.E\right|_{\mathcal{C}}\right)=P_{t}(\mathcal{C})=\frac{1}{1-t^{2}}=P_{t}\left(\Omega S^{3}\right)
$$

Es decir, el espacio de configuración de la teoría de campos es del mismo tipo homológico que $\Omega S^{3}$, el espacio de lazos en la esfera $S^{3}$. Existe, de esta manera, una universalidad topológica y los primeros términos de $\mathcal{M}_{t}\left(\left.E\right|_{\mathcal{C}}\right)$ nos informan de que la existencia de los kinks $\mathrm{TK} 2 \sigma_{3} \sqcup \mathrm{AK} 2 \sigma_{3}$ se debe al hecho de que el grupo de holonomía $H_{2}(\mathcal{C}, \mathbb{R})=\mathbb{R}$ es no trivial, los kinks TK1 $\sqcup$ AK1 están asociados a que $H_{4}(\mathcal{C}, \mathbb{R})=\mathbb{R}$, los correspondientes TK3 $\sqcup \mathrm{AK} 3$ lo estarán con que $H_{6}(\mathcal{C}, \mathbb{R})=\mathbb{R}$ y así sucesivamente. 


\subsubsection{Análisis de la estabilidad por el Teorema del Índice de Morse}

El índice de Morse de cualquier trayectoria kink puede ser computado por medio del Teorema del Índice de Morse, tal y como hemos hecho anteriormente. Aplicaremos ahora este hecho al estudio de la estabilidad de las ondas solitarias en los diferentes sectores topológicos del espacio de configuración $\mathcal{C}$. Utilizaremos, por tanto, en este análisis, el punto de vista de las coordenadas cartesianas. Como veremos, los resultados confirmarán lo obtenido desde las demás perspectivas abordadas en esta memoria.

Si recordamos la jerarquía en las energías, dentro de cada sector topológico, como referente de los resultados ya obtenidos acerca de la estabilidad, tendremos:

A. Sectores topológicos, $\mathcal{C}^{(-,+)}$y $\mathcal{C}^{(+,-)}$:

$$
\begin{aligned}
& E(\mathrm{TK} 3)>E(\mathrm{TK} 1)>E\left(\mathrm{TK} 2 \sigma_{3}\right)>E\left(\mathrm{TK} 2 \sigma_{2}\right) \\
& E(\mathrm{AK} 3)>E(\mathrm{AK} 1)>E\left(\mathrm{AK} 2 \sigma_{3}\right)>E\left(\mathrm{AK} 2 \sigma_{2}\right)
\end{aligned}
$$

B. Sectores no-topológicos, $\mathcal{C}^{(-,-)}$y $\mathcal{C}^{(+,+)}$:

$$
E\left(\mathrm{NTK} 2 \sigma_{3}( \pm)\right)>E\left(\operatorname{NTK} 2 \sigma_{2}( \pm)\right)>E(\operatorname{NTK} 3( \pm))>E\left(\vec{v}^{ \pm}\right)
$$

donde los signos, evidentemente, denotan el vacío asociado al sector correspondiente.

Los resultados obtenidos por el análisis de las energías nos han demostrado que en los sectores topológicos $\mathcal{C}^{(-,+)}$y $\mathcal{C}^{(+,-)}$únicamente los kinks del tipo TK2 $\sigma_{2}$ son estables (se trata, recordemos, del kink TK2 $\sigma_{2}$ propiamente dicho y de los kinks TK $2 \sigma_{2}{ }^{*}, \operatorname{ATK} 2 \sigma_{2}$ y ATK $\left.2 \sigma_{2}{ }^{*}\right)$. En los sectores no-topológicos, $\mathcal{C}^{(-,-)}$y $\mathcal{C}^{(+,+)}$, sólo eran estables los estados fundamentales, $\vec{v}^{-}$y $\vec{v}^{+}$, respectivamente.

El grado de inestabilidad de cada tipo de kinks viene marcado, por otra parte, por su energía frente a la de los demás, y está medida por su correspondiente índice de Morse. Comprobaremos ahora este hecho computando el índice de cada uno de los tipos de soluciones, para ello aplicaremos el Teorema del Índice de Morse en el espacio $\mathbb{R}^{3}$, es decir, en coordenadas cartesianas, como ya se ha comentado.

1. Kinks en el sector topológico $\mathcal{C}^{(-,+)}$:

A. $\mu($ TK3 $)=3$. El Teorema se aplica a una trayectoria de tipo TK3 de la siguiente manera: La curva parte del punto de vacío $\vec{v}^{-}$, atraviesa una rama de la 
hipérbola focal $\frac{q_{1}^{2}}{\sigma_{2}^{2}}-\frac{q_{3}^{2}}{\sigma_{3}^{2}-\sigma_{2}^{2}}=1$, continúa hasta la elipse focal $\frac{q_{1}^{2}}{\sigma_{3}^{2}}+\frac{q_{2}^{2}}{\sigma_{3}^{2}-\sigma_{2}^{2}}=$ 1, alcanza de nuevo la hipérbola característica (esta vez en la otra rama de la misma), y finalmente llega a $\vec{v}^{+}$. Se cruzan, por tanto, tres puntos conjugados, cada uno de ellos de multiplicidad uno, del punto $\vec{v}^{-}$(D) y tenemos, en consecuencia, tres direcciones ortogonales de inestabilidad en $\mathcal{C}$ para cada uno de los kinks TK3.

B. $\mu(\mathrm{TK} 1)=2$. Con los mismos argumentos que se presentaron en coordenadas elípticas es fácil concluir que la trayectoria TK1 atraviesa dos veces los focos $\mathrm{F}_{2}$ y $\mathrm{F}_{3}$ del elipsoide $q_{1}^{2}+\frac{q_{2}^{2}}{\bar{\sigma}_{2}^{2}}+\frac{q_{3}^{2}}{\bar{\sigma}_{3}^{2}}=1$, sin embargo, únicamente en el segundo de dichos pasos se deben computar como puntos conjugados a $\vec{v}^{-}$, puesto que en ese caso es cuando convergen en los mismos congruencias, en este caso de NTK2 $\sigma_{2}$ y NTK $2 \sigma_{3}$ respectivamente, asociadas al mismo punto de partida $\vec{v}^{-}$. El índice es por tanto 2, existen únicamente dos direcciones de inestabilidad en un entorno del kink TK1 en $\mathcal{C}$, como se ha demostrado explícitamente en el Capítulo 5.

C. $\mu\left(\mathrm{TK} 2 \sigma_{3}\right)=1$. Una trayectoria TK2 $\sigma_{3}$ pasa por dos puntos umbílicos del elipsoide. Por un razonamiento análogo al anterior, es únicamente en el segundo de dichos puntos donde debe computarse el índice de Morse, pues es allí donde converge una familia de kinks NTK3 asociada al mismo punto inicial. El índice es uno y existe por tanto una sóla dirección de inestabilidad, de acuerdo además con lo demostrado en el Capítulo 5 por medio del análisis del funcional hessiano correspondiente.

D. $\mu\left(\mathrm{TK} 2 \sigma_{2}\right)=0$. El kink TK2 $\sigma_{2}$ no cruza inguna de las líneas focales, como ya hemos comentado, se trata de la única solución topológica estable, su índice es nulo y no tiene ninguna dirección de inestabilidad asociada, su forma cuadrática hessiana, como vimos, es semidefinida positiva. En la Sección 5.3 obtuvimos, además, que ningún campo de Jacobi asociado al operador de desviación geodésica, para esta solución, se anulaba simultáneamente en dos puntos de la misma.

No repetiremos este análisis para el sector topológico $\mathcal{C}^{(+,-)}$, es evidentemente idéntico en sus resultados al expuesto.

2. Kinks en el sector topológico $\mathcal{C}^{(-,-)}$:

A. $\mu\left(\mathrm{NTK} 2 \sigma_{3}\right)=3$. Toda trayectoria del tipo NTK2 $\sigma_{3}$ alcanza la hipérbola focal $\frac{q_{1}^{2}}{\sigma_{2}^{2}}-\frac{q_{3}^{2}}{\sigma_{3}^{2}-\sigma_{2}^{2}}=1$ en un total de cuatro puntos. Únicamente el segundo y el 
cuarto de dichos cortes pueden ser considerados puntos conjugados a $\vec{v}^{-}$. El recorrido de una trayectoria de este tipo se realiza de la siguiente manera: 1) el segundo corte con la hipérbola característica coincide con el primer corte a la misma de una congruencia de kinks TK3; 2) El kink NTK2 $\sigma_{3}$ pasa por el foco $\mathrm{F}_{3}$ al mismo tiempo que todos los demás kinks de la misma familia, $\left.\mathrm{NTK} 2 \sigma_{3} ; 3\right) \mathrm{El}$ cuarto punto de incidencia con la hipérbola focal (en la otra rama de la misma, evidentemente) es simultáneo al realizado por la familia TK3 antedicha.

Estos razonamientos aclaran la afirmación inicial, el índice de Morse de los kinks NTK2 $\sigma_{3}$ es 3, es decir, existen tres direcciones de inestabilidad en el espacio $T_{\mathrm{NTK} 2 \sigma_{3}} \mathcal{C}$.

B. $\mu\left(\mathrm{NT} 2 \sigma_{2}\right)=2$. Un kink del tipo NTK2 $\sigma_{2}$ corta a la elipse focal de ecuación: $\frac{q_{1}^{2}}{\sigma_{3}^{2}}+\frac{q_{2}^{2}}{\sigma_{3}^{2}-\sigma_{2}^{2}}=1$ dos veces. Evidentemente, sólo el segundo de esos cortes es un punto conjugado de $\vec{v}^{-}$. El recorrido es, por tanto, el siguiente: 1) Todos los kinks de la familia NTK2 $\sigma_{2}$ inciden en el foco $\mathrm{F}_{2} . \mathrm{F}_{2}$ es, por tanto, un punto conjugado del punto de partida con multiplicidad uno; 2) El segundo corte de un miembro concreto de la familia con la elipse focal, como hemos dicho, es también un punto conjugado. La razón, en este caso, es la presencia en dicho punto de una congruencia uniparamétrica de kinks TK3, la multiplicidad, en consecuencia, será también uno.

El índice de Morse es, en definitiva, 2 y así existen dos direcciones de inestabilidad en $T_{\mathrm{NTK} 2 \sigma_{2}} \mathcal{C}$.

C. $\mu($ NTK3 $)=1$. Cada kink de una familia NTK3 interseca con todos los demás en un punto umbílico del elipsoide. Dicho punto es, por tanto, conjugado del de vacío $\vec{v}^{-}$y, además, con multiplicidad uno. El índice es, de esta forma, uno.

D. $\mu\left(\vec{v}^{-}\right)=0$. Se trata, obviamente, de la única solución estable dentro del sector $\mathcal{C}^{(-,-)}$.

De nuevo omitimos el análisis correspondiente al otro sector, en este caso, de kinks no topológicos, $\mathcal{C}^{(+,+)}$, por tratarse de una situación absolutamente análoga.

Resumimos, en definitiva, el análisis realizado, comentando que se dispone de una estratificación de cada componente conexa del espacio de configuración $\mathcal{C}$ por medio de los "puntos críticos" del funcional E. La Teoría de Morse relaciona, de 
esta manera, la estructura de tales puntos críticos con la homología del propio espacio de configuración. 


\section{Conclusiones}

Las conclusiones más importantes que hemos obtenido tras la realización de este trabajo, poniendo énfasis en lo que respecta a las aportaciones originales acerca de los temas tratados, son las siguientes:

1 Resolución de la Ecuación de Hamilton-Jacobi del Sistema Dinámico asociado, vía la analogía mecánica, al problema del cálculo de las soluciones kink del Modelo Sigma $O(N)$ Lineal Deformado.

2 Demostración de que las soluciones de dicho sistema están restringidas a una región acotada del espacio de configuración, de manera general un hiperelipsoide y su interior. Este hecho estaba observado en la literatura para el Modelo $O(2)$, si bien no explicado.

3 Descripción completa del espacio de soluciones kink del Modelo Sigma $O(3)$ Lineal Deformado, incluyendo la rica estructura de límites especiales que el mismo presenta y que conducen, finalmente, a diferentes posibilidades de compactificación del espacio modular de ondas solitarias.

4 Planteamiento del problema de la búsqueda de soluciones de tipo onda solitaria como el cálculo de curvas geodésicas en una variedad riemanniana dotada de la métrica de Jacobi asociada. Demostración de los Teoremas que relacionan la estabilidad de las soluciones desde el punto de vista dinámico y geométrico.

5 Descripción de las ecuaciones de un sistema dinámico asociado a la búsqueda de kinks en una teoría pre-supersimétrica. Enunciado y demostración, en el caso de $\mathrm{N}=2$ pre-supersimetría, del teorema que relaciona dicho carácter con la curvatura de la métrica de Jacobi asociada. Este teorema proporciona un criterio para clasificar las teorías $\mathrm{N}=2$ pre-supersimétricas.

6 Análisis de la estabilidad de las soluciones kink del Modelo Sigma $O(3)$ Lineal Deformado en términos de los espectros de las correspondientes formas cuadráticas hessianas, tanto desde el punto de vista dinámico como geométrico. 
7 Planteamiento del Modelo Sigma $O(3)$ Lineal Deformado como un modelo presupersimétrico, incluyendo el cálculo explícito del conjunto de superpotenciales válidos para este modelo. Resolución de las ecuaciones de primer orden asociadas (equivalentes a la integración de la ecuación de Hamilton-Jacobi anteriormente realizada) y análisis completo de las soluciones, incluyendo la clasificación en kinks BPS y no-BPS, con las consiguientes conclusiones acerca de la estabilidad que dicho carácter proporciona.

8 Estudio de la Teoría de Morse del espacio de soluciones del Modelo y aplicación del Teorema del Índice de Morse a las mismas, como mecanismo sintetizador de todos los resultados obtenidos acerca de la estabilidad de dichas soluciones.

9 Fuera de los objetivos marcados por este trabajo, pero relacionados con los mismos: resolución completa del Modelo Elíptico-Cilíndrico, caso "intermedio" entre los Modelos Sigma $O(2)$ Lineal Deformado y el correspondiente Modelo $O(3)$, y cálculo de las correcciones cuánticas a la masa de los Kinks del Modelo $O(3)$. 


\section{Apéndice A}

\section{Modelo Elíptico-Cilíndrico}

Dedicaremos el primero de los apéndices a exponer someramente el modelo que hemos denominado Elíptico-Cilíndrico, por razones que se revelarán evidentes al detallar la estructura del mismo. Como ya se comentó en la Introducción de la Memoria, se trata de un modelo de alguna manera "intermedio" entre el MSTB y el Modelo Sigma $O(3)$ Lineal Deformado. A pesar de ser un modelo no perteneciente estrictamente a la familia estudiada en este trabajo, constituye una buena muestra de otras posibilidades a analizar dentro del esquema general de teorías de tres campos escalares con sistema dinámico asociado completamente integrables. Por otra parte, se trata de un modelo no tratado en la literatura al respecto.

\section{A.1 Planteamiento del Modelo Elíptico-Cilíndrico}

Llamaremos Modelo Elíptico-Cilíndrico a la deformación del Modelo Sigma $O(2)$ Lineal Deformado siguiente:

$$
\begin{aligned}
S[\chi]= & \int d^{2} y\left\{\frac{1}{2}\left(\partial_{\mu} \chi_{1} \partial^{\mu} \chi_{1}+\partial_{\mu} \chi_{2} \partial^{\mu} \chi_{2}+\partial_{\mu} \chi_{3} \partial^{\mu} \chi_{3}\right)\right. \\
& \left.-\frac{\lambda^{2}}{4}\left(\chi_{1}^{2}+\chi_{2}^{2}-\frac{m^{2}}{\lambda^{2}}\right)^{2}-\frac{\beta_{1}^{2}}{4} \chi_{1}^{2}-\frac{\beta_{2}^{2}}{4} \chi_{2}^{2}-\frac{\lambda^{2}}{4}\left(\chi_{3}^{2}-\frac{m^{2} a^{2}}{\lambda^{2}}\right)^{2}\right\}(\mathrm{A} .1)
\end{aligned}
$$

Como vemos, se trata de añadir al potencial del Modelo Sigma $O(2)$ Lineal Deformado un término dependiente del tercer campo, $\frac{\lambda^{2}}{4}\left(\chi_{3}^{2}-\frac{m^{2} a^{2}}{\lambda^{2}}\right)^{2}$ (siendo $a$ una constante positiva), que no es más que el correspondiente al Modelo Sigma $O(1)$ Lineal, el citado modelo $\phi^{4}$. 
Pasando a variables sin dimensiones, obtendremos:

$$
\begin{array}{r}
S[\vec{\Phi}]=\frac{m^{2}}{\lambda^{2}} \int_{-\infty}^{+\infty} d x^{0} \int_{-\infty}^{+\infty} d x^{1}\left\{\frac{1}{2} \partial_{\mu} \vec{\Phi} \cdot \partial^{\mu} \vec{\Phi}-V\left(\phi_{1}, \phi_{2}, \phi_{3}\right)\right\} \\
V\left(\phi_{1}, \phi_{2}, \phi_{3}\right)=\frac{1}{2}\left(\phi_{1}^{2}+\phi_{2}^{2}-1\right)^{2}+\frac{\sigma_{1}^{2}}{2} \phi_{1}^{2}+\frac{\sigma_{2}^{2}}{2} \phi_{2}^{2}+\frac{1}{2}\left(\phi_{3}^{2}-a^{2}\right)^{2}
\end{array}
$$

Y, al igual que en el Modelo Sigma $O(2)$ Lineal Deformado, tomaremos:

$$
\sigma_{1}=0<\sigma_{2}=\sigma<1
$$

De esta manera, la energía de las configuraciones estáticas, $\vec{\Phi}\left(x^{0}, x^{1}\right)=\vec{q}\left(x^{1}\right) \equiv$ $\left(q_{1}\left(x^{1}\right), q_{2}\left(x^{1}\right), q_{3}\left(x^{1}\right)\right)$, será ahora:

$$
\begin{gathered}
E[\vec{q}]=\frac{m^{3}}{\sqrt{2} \lambda^{2}} \int_{-\infty}^{+\infty} d x^{1}\left\{\frac{1}{2}\left(\left(\frac{d q_{1}}{d x^{1}}\right)^{2}+\left(\frac{d q_{2}}{d x^{1}}\right)^{2}+\left(\frac{d q_{3}}{d x^{1}}\right)^{2}\right)+V\left(q_{1}, q_{2}, q_{3}\right)\right\} \\
V\left(q_{1}, q_{2}, q_{3}\right)=\frac{1}{2}\left(q_{1}^{2}+q_{2}^{2}-1\right)^{2}+\frac{\sigma^{2}}{2} q_{2}^{2}+\frac{1}{2}\left(q_{3}^{2}-a^{2}\right)^{2}
\end{gathered}
$$

La variedad de vacío para este modelo está compuesta por cuatro puntos:

$$
\mathcal{V}=\left\{\vec{v}^{1}=(-1,0, a), \vec{v}^{2}=(1,0, a), \vec{v}^{3}=(-1,0,-a), \vec{v}^{4}=(1,0,-a)\right\}
$$

El modelo es invariante bajo la acción del grupo discreto $G=\mathbb{Z}_{2} \times \mathbb{Z}_{2} \times \mathbb{Z}_{2}$ generado por las trasformaciones $q_{a} \rightarrow(-1)^{\delta_{a b}} q_{a}, \forall b=1,2,3$, mientras que el grupo de isotropía de uno cualquiera de los elementos de $\mathcal{V}$ es $H=\mathbb{Z}_{2}$, de manera que $\mathcal{V}$ puede verse, de manera similar a los modelos analizados en el Capítulo 2, como la órbita de un elemento bajo la acción del grupo $G$ cociente el grupo de isotropía de dicho elemento:

$$
\mathcal{V}=G / H=\mathbb{Z}_{2} \times \mathbb{Z}_{2}
$$

El espacio modular de vacíos $\mathcal{V}^{0}=\mathcal{V} / G$ consta de un único elemento, es así el mismo que en el Modelo Sigma $O(N)$ Deformado. Evidentemente:

$$
\mathcal{V}^{0}=\mathcal{V} / G=e
$$

El espacio de configuración que se considera para las soluciones de tipo kink es el conjunto de aplicaciones $\vec{q}: \mathbb{R} \rightarrow \mathbb{R}^{3}$ para las cuales el funcional de energía (A.3) es finito:

$$
\mathcal{C}=\left\{\vec{q}\left(x^{1}\right) / E[\vec{q}]<+\infty\right\}
$$


lo cual conduce a las condiciones asintóticas siguientes:

$$
\lim _{x^{1} \rightarrow \pm \infty} \frac{d \vec{q}}{d x^{1}}=\overrightarrow{0}, \quad \lim _{x^{1 \rightarrow \pm \infty}} \vec{q}\left(x^{1}\right)=\vec{v}
$$

para algún $\vec{v} \in \mathcal{V}$.

El espectro de partículas, matriz de masa en cada punto crítico $\vec{v}^{i}, \forall i=1, \ldots, 4$, será:

$$
M^{2}\left(\vec{v}^{i}\right)=\left(\begin{array}{ccc}
4 & 0 & 0 \\
0 & \sigma^{2} & 0 \\
0 & 0 & 4
\end{array}\right) \cdot \frac{m^{2}}{2}
$$

Dada la estructura de la variedad de vacío, $\mathcal{V}$, el espacio de configuración $\mathcal{C}$ será la unión de $16\left(2^{4}\right)$ sectores topológicamente disconexos:

$$
\mathcal{C}=\bigsqcup_{i, j=1}^{4} \mathcal{C}^{(i, j)}
$$

donde el superíndice $(i, j)$ significa obviamente que los elementos de dicho sector alcanzan el vacío $\vec{v}^{i}$ en $x^{1} \rightarrow-\infty$ y el $\vec{v}^{j}$ en $x^{1} \rightarrow+\infty$.

La carga topológica de cada sector será ahora un vector de tres componentes (siendo la segunda de ellas nula permanentemente, al ocurrir otro tanto con la segunda componente de los puntos de vacío). Tendremos, en definitiva, las siguientes posibilidades:

\begin{tabular}{|l|c|}
\hline Sector & $Q^{T}$ \\
\hline $\mathcal{C}^{(1,1)}$ & $(0,0,0)$ \\
$\mathcal{C}^{(1,2)}$ & $(1,0,0)$ \\
$\mathcal{C}^{(1,3)}$ & $(0,0,-1)$ \\
$\mathcal{C}^{(1,4)}$ & $(1,0,-1)$ \\
$\mathcal{C}^{(2,1)}$ & $(-1,0,0)$ \\
$\mathcal{C}^{(2,2)}$ & $(0,0,0)$ \\
$\mathcal{C}^{(2,3)}$ & $(-1,0,-1)$ \\
$\mathcal{C}^{(2,4)}$ & $(0,0,-1)$ \\
\hline
\end{tabular}

\begin{tabular}{|l|c|}
\hline Sector & $Q^{T}$ \\
\hline $\mathcal{C}^{(3,1)}$ & $(0,0,1)$ \\
$\mathcal{C}^{(3,2)}$ & $(1,0,1)$ \\
$\mathcal{C}^{(3,3)}$ & $(0,0,0)$ \\
$\mathcal{C}^{(3,4)}$ & $(1,0,0)$ \\
$\mathcal{C}^{(4,1)}$ & $(-1,0,1)$ \\
$\mathcal{C}^{(4,2)}$ & $(0,0,1)$ \\
$\mathcal{C}^{(4,3)}$ & $(-1,0,0)$ \\
$\mathcal{C}^{(4,4)}$ & $(0,0,0)$ \\
\hline
\end{tabular}

y denominaremos, como es habitual, kinks no-topológicos a los que pertenecen a sectores cuya carga sea el vector nulo y kinks topológicos a los de sectores con carga no nula. La distinción kink-antikink es, una vez más, arbitraria, especialmente en este modelo. Utilizaremos el nombre de kink para las configuraciones con cargas de componentes positivas y antikink para las de componentes negativas. En las 
configuraciones con ambos signos en la carga, el signo de la primera componente será el que nos marque el criterio de distinción.

Utilizando la analogía mecánica, el sistema dinámico asociado al Modelo ElípticoCilíndrico vendrá determinado por la funcional de acción:

$$
\begin{gathered}
J[\vec{q}]=\int_{-\infty}^{+\infty} \mathcal{L}\left(\vec{q}, \frac{d \vec{q}}{d t}\right) d t \\
\mathcal{L}=\frac{1}{2}\left(\left(\frac{d q_{1}}{d t}\right)^{2}+\left(\frac{d q_{2}}{d t}\right)^{2}+\left(\frac{d q_{3}}{d t}\right)^{2}\right)-U\left(q_{1}, q_{2}, q_{3}\right)
\end{gathered}
$$

siendo $t=x^{1}$, y el potencial:

$$
U\left(q_{1}, q_{2}, q_{3}\right)=-\frac{1}{2}\left(q_{1}^{2}+q_{2}^{2}-1\right)^{2}-\frac{\sigma^{2}}{2} q_{2}^{2}-\frac{1}{2}\left(q_{3}^{2}-a^{2}\right)^{2}
$$

$J[\vec{q}]$ es evidentemente igual a la energía de la configuración $\vec{q}$ en la teoría de campos, salvo las constantes multiplicativas, que se han obviado.

El sistema es de nuevo completamente integrable, se trata, por tanto, de otra deformación "integrable" del modelo sigma lineal. Las coordenadas adecuadas para estudiar la separabilidad de la ecuación de Hamilton-Jacobi serán las elípticocilíndricas, es decir, las coordenadas elípticas bidimensionales más la tercera coordenada, $q_{3}$, que se mantiene "cartesiana":

$$
\begin{aligned}
q_{1}^{2} & =\frac{1}{\sigma^{2}}\left(1-\mu_{1}\right)\left(1-\mu_{2}\right) \\
q_{2}^{2} & =\frac{1}{\sigma^{2}}\left(\bar{\sigma}^{2}-\mu_{1}\right)\left(\mu_{2}-\bar{\sigma}^{2}\right) \\
q_{3} & =q_{3}
\end{aligned}
$$

donde, al igual que en el Modelo MSTB, se ha adoptado el nombre $\mu$ en lugar del $\lambda$ habitual con el fin de establecer una inequívoca distinción entre las coordenadas elípticas bidimensionales y las coordenadas tridimensionales restringidas a bidimensionales, que no son equivalentes en sentido estricto. Las constantes tomadas en la definición (A.10) siguen la misma notación que el caso general ya estudiado, de manera que las coordenadas variarán en los rangos:

$$
\mu_{1}<\bar{\sigma}^{2}=1-\sigma^{2}=1-\sigma_{2}^{2}<\mu_{2}<1=\bar{\sigma}_{1}^{2}=1-\sigma_{1}^{2}
$$

Los vacíos se escriben, en las coordenadas (A.10), de la forma: $\left(\mu_{1}, \mu_{2}, q_{3}\right)=$ $\left(0, \sigma^{2}, a\right)$ para $\vec{v}^{1}$ y $\vec{v}^{2},\left(\mu_{1}, \mu_{2}, q_{3}\right)=\left(0, \sigma^{2},-a\right)$ para $\vec{v}^{3}$ y $\vec{v}^{4}$. El cambio de coordenadas, como vemos, no es biunívoco sino cuatro a uno, como le corresponde a dos coordenadas de tipo elíptico. 
La correspondencia se establece por tanto del espacio "cartesiano" $\mathbb{R}^{3}$ a la región infinita $P_{3}(-\infty, \pm \infty)$ :

$$
P_{3}(-\infty, \pm \infty)=\left\{\left(\mu_{1}, \mu_{2}, q_{3}\right) /-\infty<\mu_{1}<\bar{\sigma}^{2}, \bar{\sigma}^{2}<\mu_{2}<1,-\infty<q_{3}<+\infty\right\}
$$

Sin embargo, y con los mismos razonamientos que se utilizaron en la sección 2.4 para el caso general $N$-dimensional, las soluciones del modelo que consideramos no estarán en todo $P_{3}(-\infty, \pm \infty)$ (o equivalentemente en todo $\mathbb{R}^{3}$ ). El análisis en este caso se repite dos veces, la separación del problema en un sistema análogo al del Modelo Sigma $O(2)$ Deformado más un modelo Sigma $O(1)$ determina en este caso la restricción a una subregión $P_{3}(0, \pm a)$ de $P_{3}(-\infty, \pm \infty)$, el paralelepípedo:

$$
P_{3}(0, \pm a)=\left\{\left(\mu_{1}, \mu_{2}, q_{3}\right) / 0 \leq \mu_{1}<\bar{\sigma}^{2}, \bar{\sigma}^{2}<\mu_{2}<1,-a \leq q_{3} \leq a\right\}
$$

puesto que las soluciones del sistema dinámico exteriores a esa región se irán al infinito asintóticamente, no pudiendo verificar las condiciones (A.6).

El paralelepípedo "elíptico-cilíndrico" $P_{3}(0, \pm a)$ se corresponde en coordenadas cartesianas, mediante el cambio (A.10), con la frontera y el interior del cilindro elíptico de ecuaciones:

$$
\begin{aligned}
& q_{1}^{2}+\frac{q_{2}^{2}}{\bar{\sigma}^{2}}=1 \\
& -a \leq q_{3} \leq+a
\end{aligned}
$$

La notación escogida para reflejar las equivalencias es la siguiente:

- Los vacíos $\vec{v}^{1}$ y $\vec{v}^{2}$ se corresponden con el vértice $\vec{v}^{+}$de $P_{3}(0, \pm a)$ mientras que $\vec{v}^{3}$ y $\vec{v}^{4}$ con el denotado $\vec{v}^{-}$.

- Los focos de la elipse (A.11) situada a la "altura" $q_{3}=a$ se identifican con el punto $\mathrm{F}^{+a}$, mientras que los relativos a $q_{3}=-a$ se corresponden con $\mathrm{F}^{-a}$. Lógicamente todos los focos correspondientes a elipses intermedias (las líneas focales) se aplican a la arista $\mathrm{F}^{+a} \mathrm{~F}^{-a}$.

- El segmento del eje $q_{3}$ del cilindro delimitado por las elipses superior $\left(q_{3}=a\right)$ e inferior $\left(q_{3}=-a\right)$ está en correspondencia uno-uno con la arista $\mathrm{O}^{+a} \mathrm{O}^{-a}$.

- Los segmentos del cilindro determinados por los semiejes menores de la elipse (A.11) se aplican en la arista $\mathrm{B}^{+a} \mathrm{~B}^{-a}$. 
Es conveniente en este punto, de cara a obtener una notación clara, recordar las funciones $A$ y $\Lambda$ introducidas en el Capítulo 2, y particularizarlas para el caso de dos coordenadas elípticas y de las constantes que hemos considerado:

$$
A(\mu)=(\mu-1)\left(\mu-\bar{\sigma}^{2}\right) ; \quad \Lambda(\mu)=\left(\mu-\mu_{1}\right)\left(\mu-\mu_{2}\right)
$$

Se puede escribir así:

$$
\begin{aligned}
& q_{1}^{2}=\frac{\Lambda(1)}{A^{\prime}(1)} \\
& q_{2}^{2}=\frac{\Lambda\left(\bar{\sigma}^{2}\right)}{A^{\prime}\left(\bar{\sigma}^{2}\right)}
\end{aligned}
$$

Las coordenadas elíptico-cilíndricas son obviamente ortogonales y es trivial deducir que la métrica estándar en coordenadas cartesianas pasa a ser, en estas coordenadas:

$$
d s^{2}=d q_{1}^{2}+d q_{2}^{2}+d q_{3}^{2}=-\frac{1}{4} \frac{\Lambda^{\prime}\left(\mu_{1}\right)}{A\left(\mu_{1}\right)} d \mu_{1}^{2}-\frac{1}{4} \frac{\Lambda^{\prime}\left(\mu_{2}\right)}{A\left(\mu_{2}\right)} d \mu_{2}^{2}+d q_{3}^{2}
$$

definida positiva a pesar de los aparentes signos negativos.

El lagrangiano $\mathcal{L}$ se escribe:

$$
\begin{gathered}
\mathcal{L}=\frac{1}{2}\left[-\frac{1}{4} \frac{\Lambda^{\prime}\left(\mu_{1}\right)}{A\left(\mu_{1}\right)}\left(\frac{d \mu_{1}}{d t}\right)^{2}-\frac{1}{4} \frac{\Lambda^{\prime}\left(\mu_{2}\right)}{A\left(\mu_{2}\right)}\left(\frac{d \mu_{2}}{d t}\right)^{2}+\left(\frac{d q_{3}}{d t}\right)^{2}\right]-U\left(\mu_{1}, \mu_{2}, q_{3}\right) \\
U\left(\mu_{1}, \mu_{2}, q_{3}\right)=-\left[\frac{1}{2} \frac{\mu_{1}^{2}\left(\mu_{1}-\bar{\sigma}^{2}\right)}{\Lambda^{\prime}\left(\mu_{1}\right)}+\frac{1}{2} \frac{\mu_{2}^{2}\left(\mu_{2}-\bar{\sigma}^{2}\right)}{\Lambda^{\prime}\left(\mu_{2}\right)}+\frac{1}{2}\left(q_{3}^{2}-a^{2}\right)^{2}\right]
\end{gathered}
$$

El paso al formalismo hamiltoniano requiere el cálculo de los momentos generalizados:

$$
\pi_{1}=\frac{\partial \mathcal{L}}{\partial \frac{d \mu_{1}}{d t}}=-\frac{1}{4} \frac{\Lambda^{\prime}\left(\mu_{1}\right)}{A\left(\mu_{1}\right)} \frac{d \mu_{1}}{d t} ; \quad \pi_{2}=\frac{\partial \mathcal{L}}{\partial \frac{d \mu_{2}}{d t}}=-\frac{1}{4} \frac{\Lambda^{\prime}\left(\mu_{2}\right)}{A\left(\mu_{2}\right)} \frac{d \mu_{2}}{d t} ; \quad p_{3}=\frac{\partial \mathcal{L}}{\partial \frac{d q_{3}}{d t}}=\frac{d q_{3}}{d t}
$$

que conducen a una expresión para el Hamiltoniano:

$$
\begin{aligned}
H= & \frac{1}{2}\left[\frac{-4 A\left(\mu_{1}\right)}{\Lambda^{\prime}\left(\mu_{1}\right)} \pi_{1}^{2}+\frac{-4 A\left(\mu_{2}\right)}{\Lambda^{\prime}\left(\mu_{2}\right)} \pi_{2}^{2}+p_{3}^{2}\right] \\
& -\frac{1}{2} \frac{\mu_{1}^{2}\left(\mu_{1}-\bar{\sigma}^{2}\right)}{\Lambda^{\prime}\left(\mu_{1}\right)}-\frac{1}{2} \frac{\mu_{2}^{2}\left(\mu_{2}-\bar{\sigma}^{2}\right)}{\Lambda^{\prime}\left(\mu_{2}\right)}-\frac{1}{2}\left(q_{3}^{2}-a^{2}\right)^{2}
\end{aligned}
$$

que, teniendo en cuenta las expresiones de $\Lambda^{\prime}\left(\mu_{1}\right)$ y $\Lambda^{\prime}\left(\mu_{2}\right)$ para este caso $^{1}$ :

$$
\Lambda^{\prime}\left(\mu_{1}\right)=-\Lambda^{\prime}\left(\mu_{2}\right)=\mu_{1}-\mu_{2}
$$

\footnotetext{
${ }^{1}$ Nótese que de acuerdo con la notación introducida en el Capítulo 2 tendremos: $\Lambda^{\prime}\left(\mu_{a}\right)=$ $f_{a}(\vec{\mu})=\mu_{a}-\mu_{b}$. De esta forma: $f_{1}(\vec{\mu})=-f_{2}(\vec{\mu})=\mu_{1}-\mu_{2}$.
} 
se puede escribir de la forma:

$$
H\left(\pi_{1}, \pi_{2}, p_{3}, \mu_{1}, \mu_{2}, q_{3}\right)=\frac{1}{\mu_{1}-\mu_{2}}\left(H_{\mu_{1}}-H_{\mu_{2}}\right)+H_{3}
$$

donde:

$$
\begin{aligned}
H_{\mu_{1}} & =\frac{-1}{2} 4 A\left(\mu_{1}\right) \pi_{1}^{2}-\frac{1}{2} \mu_{1}^{2}\left(\mu_{1}-\bar{\sigma}^{2}\right) \\
H_{\mu_{2}} & =\frac{-1}{2} 4 A\left(\mu_{2}\right) \pi_{2}^{2}-\frac{1}{2} \mu_{2}^{2}\left(\mu_{2}-\bar{\sigma}^{2}\right) \\
H_{3} & =\frac{1}{2} p_{3}^{2}-\frac{1}{2}\left(q_{3}^{2}-a^{2}\right)^{2}
\end{aligned}
$$

Es evidente, por tanto, que la ecuación de Hamilton-Jacobi va a ser separable en estas coordenadas. La forma sencilla del hamiltoniano permite que la parte elíptica del mismo separe de manera inmediata, sin necesidad de recurrir a las fórmulas de Jacobi, obligadas para el caso $N$-dimensional, como vimos en el Capítulo 2. Buscaremos de esta manera una solución del tipo:

$$
\mathcal{S}\left(t ; \mu_{1}, \mu_{2}, q_{3}\right)=-I_{1} t+\mathcal{S}_{1}\left(\mu_{1}\right)+\mathcal{S}_{2}\left(\mu_{2}\right)+\mathcal{S}_{3}\left(q_{3}\right)
$$

para la ecuación de Hamilton-Jacobi:

$$
\frac{\partial \mathcal{S}}{\partial t}+H\left(\frac{\partial \mathcal{S}}{\partial \mu_{1}}, \frac{\partial \mathcal{S}}{\partial \mu_{2}}, \frac{\partial \mathcal{S}}{\partial q_{3}}, \mu_{1}, \mu_{2}, q_{3}\right)=0
$$

y pasaremos así de una ecuación en derivadas parciales a ecuaciones diferenciales ordinarias.

El primer paso del proceso de separación nos conduce evidentemente a la ecuación:

$$
I_{1}=H\left(\frac{d \mathcal{S}_{1}}{d \mu_{1}}, \frac{d \mathcal{S}_{2}}{d \mu_{2}}, \frac{d \mathcal{S}_{3}}{d q_{3}}, \mu_{1}, \mu_{2}, q_{3}\right)
$$

donde la constante $I_{1}$ no es más que el valor de la integral primera de la energía del sistema dinámico.

Varias posibilidades equivalentes pueden ser escogidas para continuar el proceso de separación de variables en la ecuación (A.18), tomaremos:

$$
I_{1}-H_{3}\left(\frac{d \mathcal{S}_{3}}{d q_{3}}, q_{3}\right)=\frac{1}{\mu_{1}-\mu_{2}}\left(H_{\mu_{1}}\left(\frac{d \mathcal{S}_{1}}{d \mu_{1}}, \mu_{1}\right)-H_{\mu_{2}}\left(\frac{d \mathcal{S}_{2}}{d \mu_{2}}, \mu_{2}\right)\right)=I_{2}
$$

donde se ha introducido una nueva constante de separación $I_{2}$. La ecuación (A.19) descompone en dos ecuaciones independientes. La primera de ellas puede escribirse como

$$
\left(\frac{d \mathcal{S}_{3}}{d q_{3}}\right)^{2}=-2 I_{2}+2 I_{1}+\left(q_{3}^{2}-a^{2}\right)^{2}
$$


y nos proporciona la solución para $\mathcal{S}_{3}$ mediante la cuadratura:

$$
\mathcal{S}_{3}\left(q_{3}\right)=\operatorname{sign}\left(\frac{d \mathcal{S}_{3}}{d q_{3}}\right) \int \sqrt{2 I_{1}-2 I_{2}+\left(q_{3}-a^{2}\right)^{2}} d q_{3}
$$

mientras que la segunda es una ecuación acoplada:

$$
-4 A\left(\mu_{1}\right)\left(\frac{d \mathcal{S}_{1}}{d \mu_{1}}\right)^{2}+4 A\left(\mu_{2}\right)\left(\frac{d \mathcal{S}_{2}}{d \mu_{2}}\right)^{2}-\mu_{1}^{2}\left(\mu_{1}-\bar{\sigma}^{2}\right)+\mu_{2}^{2}\left(\mu_{2}-\bar{\sigma}^{2}\right)=2 I_{2}\left(\mu_{1}-\mu_{2}\right)
$$

pero separable en las dos ecuaciones siguientes:

$$
\begin{aligned}
& 4 A\left(\mu_{1}\right)\left(\frac{d \mathcal{S}_{1}}{d \mu_{1}}\right)^{2}+\mu_{1}^{2}\left(\mu_{1}-\bar{\sigma}^{2}\right)+2 I_{2} \mu_{1}=I_{3} \\
& 4 A\left(\mu_{2}\right)\left(\frac{d \mathcal{S}_{2}}{d \mu_{2}}\right)^{2}+\mu_{2}^{2}\left(\mu_{2}-\bar{\sigma}^{2}\right)+2 I_{2} \mu_{2}=I_{3}
\end{aligned}
$$

ecuaciones formalmente idénticas y que conducen a la cuadratura:

$$
\mathcal{S}_{i}\left(\mu_{i}\right)=\frac{1}{2} \operatorname{sign}\left(\frac{d \mathcal{S}_{i}}{d \mu_{i}}\right) \int \sqrt{\left|\frac{I_{3}-\mu_{i}\left(\mu_{i}-\bar{\sigma}^{2}\right)-2 I_{2} \mu_{i}}{A\left(\mu_{i}\right.}\right|} d \mu_{i}
$$

para $i=1,2$.

Podemos, finalmente, escribir la solución general de la ecuación de HamiltonJacobi, dependiente de las tres constantes arbitrarias $I_{1}, I_{2}$ y $I_{3}$ :

$$
\begin{aligned}
\mathcal{S}=\quad & -I_{1} t+\sum_{i=1}^{2}\left(\frac{1}{2} \operatorname{sign}\left(\frac{d \mathcal{S}_{i}}{d \mu_{i}}\right) \int \sqrt{\mid \frac{I_{3}-\mu_{i}\left(\mu_{i}-\bar{\sigma}^{2}\right)-2 I_{2} \mu_{i} \mid}{A\left(\mu_{i}\right)}} d \mu_{i}\right) \\
& +\operatorname{sign}\left(\frac{d \mathcal{S}_{3}}{d q_{3}}\right) \int \sqrt{2 I_{1}-2 I_{2}+\left(q_{3}-a^{2}\right)^{2}} d q_{3}
\end{aligned}
$$

Las ecuaciones de las trayectorias solución, así como la dependencia temporal de las mismas, vienen dadas por las derivadas parciales de la función generatriz (A.26) con respecto a las constantes de movimiento $I_{1}, I_{2}$ y $I_{3}$ de la forma:

$$
\zeta_{1}=\frac{\partial \mathcal{S}}{\partial I_{1}} ; \quad \zeta_{2}=\frac{\partial \mathcal{S}}{\partial I_{2}} ; \quad \zeta_{3}=\frac{\partial \mathcal{S}}{\partial I_{3}}
$$

con nuevas constantes arbitrarias $\zeta_{1}, \zeta_{2}$ y $\zeta_{3}$. Las ecuaciones de las trayectorias se escribirán, por tanto:

$$
\zeta_{2}=\frac{\partial \mathcal{S}}{\partial I_{2}}=\frac{1}{2} \operatorname{sign}\left(\frac{d \mathcal{S}_{1}}{d \mu_{1}}\right) \int \frac{-\mu_{1} d \mu_{1}}{\sqrt{\left|A\left(\mu_{1}\right)\left(I_{3}-\mu_{1}^{2}\left(\mu_{1}-\bar{\sigma}^{2}\right)-2 I_{2} \mu_{1}\right)\right|}}
$$




$$
\begin{aligned}
& +\frac{1}{2} \operatorname{sign}\left(\frac{d \mathcal{S}_{2}}{d \mu_{2}}\right) \int \frac{-\mu_{2} d \mu_{2}}{\sqrt{\left|A\left(\mu_{2}\right)\left(I_{3}-\mu_{2}^{2}\left(\mu_{2}-\bar{\sigma}^{2}\right)-2 I_{2} \mu_{2}\right)\right|}} \\
& -\operatorname{sign}\left(\frac{d \mathcal{S}_{3}}{d q_{3}}\right) \int \frac{d q_{3}}{\sqrt{\left|2 I_{1}-2 I_{2}+\left(q_{3}^{2}-a^{2}\right)^{2}\right|}} \\
\zeta_{3}= & \frac{\partial \mathcal{S}}{\partial I_{3}}=\frac{1}{4} \operatorname{sign}\left(\frac{d \mathcal{S}_{1}}{d \mu_{1}}\right) \int \frac{d \mu_{1}}{\sqrt{\left|A\left(\mu_{1}\right)\left(I_{3}-\mu_{1}^{2}\left(\mu_{1}-\bar{\sigma}^{2}\right)-2 I_{2} \mu_{1}\right)\right|}} \\
& +\frac{1}{4} \operatorname{sign}\left(\frac{d \mathcal{S}_{2}}{d \mu_{2}}\right) \int \frac{d \mu_{2}}{\sqrt{\left|A\left(\mu_{2}\right)\left(I_{3}-\mu_{2}^{2}\left(\mu_{2}-\bar{\sigma}^{2}\right)-2 I_{2} \mu_{2}\right)\right|}}
\end{aligned}
$$

mientras que la dependencia temporal viene determinada por la ecuación:

$$
t+\zeta_{1}=\frac{\partial \mathcal{S}}{\partial I_{1}}=\operatorname{sign}\left(\frac{d \mathcal{S}_{3}}{d q_{3}}\right) \int \frac{d q_{3}}{\sqrt{\left|2 I_{1}-2 I_{2}+\left(q_{3}^{2}-a^{2}\right)^{2}\right|}}
$$

La integración general de estas cuadraturas requiere la utilización de funciones hiperelípticas de género 2 (ver [81]), dado que están involucradas raíces de polinomios de quinto grado. Sin embargo, no será necesario realizar tales integraciones si tenemos en cuenta que las soluciones que buscamos, es decir, las soluciones clásicas que corresponden a soluciones solitónicas de la teoría clásica de campos subyacente a nuestro modelo mecánico, han de verificar las condiciones asintóticas (A.6), lo cual impone unos valores concretos de las constantes de movimiento del sistema dinámico. Como ya se comentó en el Capítulo 2 este tipo de cuadraturas conducen a funciones theta que, para los valores particulares de las constantes de integración que deben considerarse de cara a la obtención de soluciones de tipo onda solitaria, se reducen a funciones más sencillas, del tipo trigonométricas hiperbólicas.

Efectivamente, las condiciones (A.6) nos restringen el análisis al caso $I_{1}=I_{2}=$ $I_{3}=0^{2}$, para el cual las ecuaciones (A.27) (A.28) y (A.29) serán, respectivamente:

$$
\begin{aligned}
-\zeta_{2}= & \frac{1}{2} \operatorname{sign}\left(\pi_{1}\right) \int \frac{d \mu_{1}}{\left(\bar{\sigma}^{2}-\mu_{1}\right) \sqrt{1-\mu_{1}}} \\
& +\frac{1}{2} \operatorname{sign}\left(\pi_{2}\right) \int \frac{d \mu_{2}}{\left(\mu_{2}-\bar{\sigma}^{2}\right) \sqrt{1-\mu_{2}}} \\
& +\operatorname{sign}\left(p_{3}\right) \int \frac{d q_{3}}{q_{3}^{2}-a^{2}}
\end{aligned}
$$

\footnotetext{
${ }^{2}$ Este hecho puede comprobarse muy fácilmente sin más que sustituir las condiciones asintóticas (A.6) en la definición de las coordenadas elíptico-cilíndricas y posteriormente éstas en la definición de $I_{1}, I_{2}$ y $I_{3}$.
} 


$$
\begin{aligned}
\zeta_{3}= & \frac{1}{4} \operatorname{sign}\left(\pi_{1}\right) \int \frac{d \mu_{1}}{\mu_{1}\left(\bar{\sigma}^{2}-\mu_{1}\right) \sqrt{1-\mu_{1}}} \\
& +\frac{1}{2} \operatorname{sign}\left(\pi_{2}\right) \int \frac{d \mu_{2}}{\mu_{2}\left(\mu_{2}-\bar{\sigma}^{2}\right) \sqrt{1-\mu_{2}}}
\end{aligned}
$$

y para la dependencia temporal:

$$
t+\zeta_{1}=\operatorname{sign}\left(p_{3}\right) \int \frac{d q_{3}}{q_{3}^{2}-a^{2}}
$$

\section{A.2 Tipos de Soluciones}

\section{Kinks Unidimensionales}

Llamaremos kinks unidimensionales a aquellas soluciones que poseen sólamente una componente no constante. Consideraremos así una "órbita de prueba" con esas características en el espacio determinado por las variables $\left(q_{1}, q_{2}, q_{3}\right)$. Encontraremos, de esta manera, que existen este tipo de soluciones en ocho de los sectores topológicos que hemos descrito anteriormente, si bien los agruparemos en cuatro tipos, dado que por cada kink perteneciente a un sector topológico $\mathcal{C}^{(i, j)}$ con $i \neq j$ tendremos su correspondiente antikink en el sector $\mathcal{C}^{(j, i)}$.

- Kinks unidimensionales en los sectores $\mathcal{C}^{(1,2)}$ y $\mathcal{C}^{(2,1)}$.

La órbita de prueba será:

$$
\mathcal{M}_{1}^{+a}=\left\{\left(q_{1}, q_{2}, q_{3}\right) \in \mathbb{R}^{3} /-1 \leq q_{1} \leq 1, q_{2}=0, q_{3}=a\right\}
$$

Las condiciones asintóticas nos restringen, evidentemente, al intervalo $[-1,1]$ dentro del eje $q_{1}$. El lagrangiano del sistema dinámico restringido será:

$$
\mathcal{L}=\frac{1}{2}\left(\frac{d q_{1}}{d t}\right)^{2}+\frac{1}{2}\left(q_{1}^{2}-1\right)^{2}
$$

mientras que la energía, única integral primera no trivial para este sistema restringido será:

$$
I=\frac{1}{2}\left(\frac{d q_{1}}{d t}\right)^{2}-\frac{1}{2}\left(q_{1}^{2}-1\right)^{2}
$$

que, debido a las condiciones asintóticas, tomará el valor constante cero sobre las trayectorias solución, como ya se ha comentado anteriormente.

La ecuación $I=0$ se despeja de manera evidente:

$$
\frac{d q_{1}}{d t}= \pm\left(q_{1}^{2}-1\right)
$$


y su integración nos proporciona las soluciones del tipo TK1 siguientes:

$$
\vec{q}_{\mathrm{TK} 1(+a)}(t)=\left(\begin{array}{c}
\tanh \left(t-t_{0}\right) \\
0 \\
a
\end{array}\right) ; \quad \vec{q}_{\mathrm{ATK} 1(+a)}(t)=\left(\begin{array}{c}
-\tanh \left(t-t_{0}\right) \\
0 \\
a
\end{array}\right)
$$

donde hemos tomado la ya mencionda convención arbitraria de denominar a la primera solución (perteneciente al sector $\mathcal{C}^{(1,2)}$ ) como Kink y a la segunda (perteneciente a $\mathcal{C}^{(2,1)}$ ) como Antikink, atendiendo al signo del vector carga topológica.

- Kinks unidimensionales en los sectores $\mathcal{C}^{(3,4)}$ y $\mathcal{C}^{(4,3)}$.

La órbita de prueba para este caso será:

$$
\mathcal{M}_{1}^{-a}=\left\{\left(q_{1}, q_{2}, q_{3}\right) \in \mathbb{R}^{3} /-1 \leq q_{1} \leq 1, q_{2}=0, q_{3}=-a\right\}
$$

Observamos, por tanto, que se repite el caso anterior sin más que intercambiar el signo de la tercera componente, $q_{3}$. No repetiremos las expresiones pues, exceptuando el signo citado, son absolutamente análogas a las escritas para $\mathcal{M}_{1}^{+a}$.

- Kinks unidimensionales en los sectores $\mathcal{C}^{(1,3)}$ y $\mathcal{C}^{(3,1)}$.

En este caso, la órbita de prueba es el segmento vertical

$$
\mathcal{M}_{1}^{-1}=\left\{\left(q_{1}, q_{2}, q_{3}\right) \in \mathbb{R}^{3} / q_{1}=-1, q_{2}=0,-a \leq q_{3} \leq a\right\}
$$

El Lagrangiano del sistema restringido a $\mathcal{M}_{1}^{-1}$ se escribe de la forma:

$$
\mathcal{L}=\frac{1}{2}\left(\frac{d q_{3}}{d t}\right)^{2}+\frac{1}{2}\left(q_{3}^{2}-a^{2}\right)^{2}
$$

Y, de nuevo, el valor constante y nulo de la única integral primera no trivial, la energía:

$$
I=\frac{1}{2}\left(\frac{d q_{3}}{d t}\right)^{2}-\frac{1}{2}\left(q_{3}^{2}-a^{2}\right)^{2}
$$

proporciona una ecuación de primer orden separable y, en definitiva, las soluciones:

$$
\vec{q}_{\mathrm{TK} 1(-1)}(t)=\left(\begin{array}{c}
-1 \\
0 \\
a \tanh \left(a t-t_{0}\right)
\end{array}\right) ; \quad \vec{q}_{\mathrm{ATK} 1(-1)}(t)=\left(\begin{array}{c}
-1 \\
0 \\
-a \tanh \left(a t-t_{0}\right)
\end{array}\right)
$$

- Kinks unidimensionales en los sectores $\mathcal{C}^{(2,4)}$ y $\mathcal{C}^{(4,2)}$.

De manera análoga al caso anterior, tendremos:

$$
\mathcal{M}_{1}^{+1}=\left\{\left(q_{1}, q_{2}, q_{3}\right) \in \mathbb{R}^{3} / q_{1}=+1, q_{2}=0,-a \leq q_{3} \leq a\right\}
$$

luego en coordenadas cartesianas el único cambio será, con respecto al caso anterior, el del signo de la coordenada $q_{1}$. 


\section{Kinks Bidimensionales}

Una vez establecidos los kinks (denotados genéricamente como K1) con una única componente cartesiana no nula, estudiaremos los bidimensionales (K2), que aparecerán tanto en sectores topológicos (TK2 y ATK2), como no-topológicos (NTK2 y ANTK2).

- Kinks Bidimensionales Topológicos.

Existen dos tipos de kinks bidimensionales topológicos en este modelo, por un lado encontraremos kinks en las aristas de $P_{3}(0, \pm a)$ correspondientes en coordenadas cartesianas a las elipses determinadas por las secciones $q_{3}= \pm a$ del cilindro elíptico. Por otro lado, la restricción al plano $q_{2}=0$ nos reduce el sistema a uno bidimensional en el que las soluciones tienen carga topológica no nula, apareciendo así toda una familia uniparamétrica de kinks topológicos bidimensionales.

I. La primera región de prueba que tomaremos es la sección elíptica del cilindro que estamos considerando, tanto la correspondiente a $q_{3}=a$, como la de $q_{3}=-a$. En el primer caso, tenemos una curva que nos conecta los vacíos $\vec{v}^{1}$ y $\vec{v}^{2}$, mientras que, en el segundo, se tratará de $\vec{v}^{3}$ y $\vec{v}^{4}$. Vamos a obtener, por tanto, kinks y antikinks en los sectores $\mathcal{C}^{(1,2)}, \mathcal{C}^{(2,1)}, \mathcal{C}^{(3,4)}$ y $\mathcal{C}^{(4,3)}$.

Describiendo ambas situaciones simultáneamente, podemos escribir:

$$
\mathcal{M}_{1}^{\mathrm{e}( \pm a)}=\left\{\left(q_{1}, q_{2}, q_{3}\right) \in \mathbb{R}^{3} / q_{1}^{2}+\frac{q_{2}^{2}}{\bar{\sigma}^{2}}=1, \quad q_{3}= \pm a\right\}
$$

donde $\mathrm{e}( \pm a)$ denota a la elipse correspondiente al valor $+a \mathrm{o}-a$ de la coordenada $q_{3}$. En coordenadas elíptico-cilíndricas nos encontramos con que toda la elipse se traduce en una única arista del paralelepípedo $P_{3}(0, \pm a), v^{+} \mathrm{B}^{+a}$ para la elipse $\mathrm{e}(+a)$ y $v^{-} \mathrm{B}^{-a}$ para $\mathrm{e}(-a)$.

De esta forma, resulta especialmente fácil en este caso buscar las soluciones directamente en coordenadas elíptico-cilíndricas, la restricción a $\mathcal{M}_{1}^{\mathrm{e}( \pm a)}$ se traduce, como hemos dicho, en que sólo la coordenada $\mu_{2}$ es no constante $\left(\mu_{1}=0, q_{3}= \pm a\right)$.

Los resultados que se obtienen son, evidentemente, dos copias de los kinks envolventes del Modelo Sigma $O(2)$ Lineal Deformado. Denotaremos así a dichos kinks como: TK2 $( \pm a)$ para los kinks topológicos pertenecientes a la semielipse dada por la inecuación $q_{1} \geq 0$, y $\operatorname{ATK} 2( \pm a)$ para sus correspondientes antikinks, mientras que llamaremos: $\operatorname{TK} 2^{*}( \pm a)$ y $\operatorname{ATK} 2^{*}( \pm a)$ a los kinks y antikinks cuyas 
trayectorias coinciden con la semielipse $q_{1} \leq 0$.

$$
\begin{aligned}
& \vec{q}_{\mathrm{TK} 2( \pm a)}=\left(\begin{array}{c}
\tanh \sigma\left(t-t_{0}\right) \\
\bar{\sigma} \operatorname{sech} \sigma\left(t-t_{0}\right) \\
\pm a
\end{array}\right), \quad \vec{q}_{\mathrm{ATK} 2( \pm a)}=\left(\begin{array}{c}
-\tanh \sigma\left(t-t_{0}\right) \\
-\bar{\sigma} \operatorname{sech} \sigma\left(t-t_{0}\right) \\
\pm a
\end{array}\right) \\
& \vec{q}_{\mathrm{TK} 2^{*}( \pm a)}=\left(\begin{array}{c}
\tanh \sigma\left(t-t_{0}\right) \\
-\bar{\sigma} \operatorname{sech} \sigma\left(t-t_{0}\right) \\
\pm a
\end{array}\right), \quad \vec{q}_{\mathrm{ATK} 2^{*}( \pm a)}=\left(\begin{array}{c}
-\tanh \sigma\left(t-t_{0}\right) \\
\bar{\sigma} \operatorname{sech} \sigma\left(t-t_{0}\right) \\
\pm a
\end{array}\right)
\end{aligned}
$$

Los kinks denotados TK2 $(a)$ pertenecen al sector topológico $\mathcal{C}^{(1,2)}$, al igual que los $\operatorname{TK} 2^{*}(a)$. Los antikinks $\operatorname{ATK} 2(a)$ y $\operatorname{ATK}^{*}(a)$ pertenecen a $\mathcal{C}^{(2,1)}$. De igual forma TK2 $(-a)$ y $\operatorname{TK} 2^{*}(a)$ están en $\mathcal{C}^{(3,4)}$, mientras que $\operatorname{ATK} 2(-a)$ y $\operatorname{ATK} 2^{*}(-a)$ lo hacen en $\mathcal{C}^{(4,3)}$.

II. Consideraremos en segundo lugar, como ya hemos comentado, la superficie de prueba determinada por la ecuación: $q_{2}=0$. En coordenadas elíptico-cilíndricas se trata de dos caras del paralelepípedo $P_{3}(0, \pm a)$, la determinada por $\mu_{1}=\bar{\sigma}^{2}$ y la de ecuación $\mu_{2}=\bar{\sigma}^{2}$.

Es fácil comprobar, en este caso, que las coordenadas más adecuadas para plantear la resolución del sistema son las cartesianas, para las cuales se encuentra un sistema de ecuaciones directamente separado. El Lagrangiano se escribirá:

$$
\mathcal{L}=\frac{1}{2}\left(\frac{d q_{1}}{d t}\right)^{2}+\frac{1}{2}\left(q_{1}^{2}-1\right)^{2}+\frac{1}{2}\left(\frac{d q_{3}}{d t}\right)^{2}+\frac{1}{2}\left(q_{3}^{2}-a\right)^{2}
$$

En este sistema el paso al formalismo hamiltoniano es trivial y obtenemos una función Hamiltoniana:

$$
H=\frac{1}{2} p_{1}^{2}-\frac{1}{2}\left(q_{1}^{2}-1\right)^{2}+\frac{1}{2} p_{3}^{2}-\frac{1}{2}\left(q_{3}^{2}-a\right)^{2}=H_{1}+H_{3}
$$

donde $p_{1}=\frac{d q_{1}}{d t}$ y $p_{3}=\frac{d q_{3}}{d t}$. Es fácil también comprobar que los hamiltonianos $H_{1}$ y $H_{3}$ son a su vez integrales primeras en involución del sistema. Las condiciones asintóticas (A.6) fijan de nuevo su valor en 0 y, en definitiva, las ecuaciones:

$$
\begin{aligned}
& \frac{1}{2}\left(\frac{d q_{1}}{d t}\right)^{2}-\frac{1}{2}\left(q_{1}^{2}-1\right)^{2}=0 \\
& \frac{1}{2}\left(\frac{d q_{3}}{d t}\right)^{2}-\frac{1}{2}\left(q_{3}^{2}-a\right)^{2}=0
\end{aligned}
$$

nos proporcionan directamente las cuadraturas ya resueltas en anteriores apartados y, por tanto, las soluciones. No es necesario, de esta forma, aplicar el Método de Hamilton-Jacobi, que conduciría evidentemente a idénticas soluciones. 
Tenemos en este caso cuatro familias de soluciones, pertenecientes a los sectores $\mathcal{C}^{(1,4)}$ y $\mathcal{C}^{(2,3)}$, para los kinks, y a los sectores $\mathcal{C}^{(4,1)}$ y $\mathcal{C}^{(3,2)}$, para los antikinks. Los denotaremos como TK2 $i$, TK2ii, ATK2i y ATK2ii respectivamente.

$$
\begin{aligned}
& \vec{q}_{\mathrm{TK} 2 i}=\left(\begin{array}{c}
\tanh \left(t-t_{0}\right) \\
0 \\
-a \tanh \left(a t-t_{1}\right)
\end{array}\right), \quad \vec{q}_{\mathrm{TK} 2 i i}=\left(\begin{array}{c}
-\tanh \left(t-t_{0}\right) \\
0 \\
-a \tanh \left(a t-t_{1}\right)
\end{array}\right) \\
& \vec{q}_{\mathrm{ATK} 2 i}=\left(\begin{array}{c}
-\tanh \left(t-t_{0}\right) \\
0 \\
a \tanh \left(a t-t_{1}\right)
\end{array}\right), \quad \vec{q}_{\mathrm{ATK} 2 i i}=\left(\begin{array}{c}
\tanh \left(t-t_{0}\right) \\
0 \\
a \tanh \left(a t-t_{1}\right)
\end{array}\right)
\end{aligned}
$$

Las constantes de integración $t_{0}$ y $t_{1}$ nos proporcionan la parametrización de cada una de las familias. No se trata, sin embargo, de familias biparamétricas. Si tenemos en cuenta que el criterio que se ha adoptado en este trabajo ha sido el de denominar solución kink al conjunto de infinitas soluciones equivalentes por reparametrizaciones de tipo traslación en el tiempo $t$, nos encontraremos en este caso con que la presencia de dos constantes significa realmente que cada familia está parametrizada realmente por una sóla constante, que no es otra que la diferencia entre $t_{0}$ y $t_{1}$. Si calculamos la ecuación implícita de las trayectorias, eliminando el tiempo de las expresiones anteriores ${ }^{3}$, obtendremos la ecuación:

$$
e^{2 \gamma}=\left|\frac{q_{3}-a}{q_{3}+a}\right|^{\frac{-\operatorname{sign}\left(p_{3}\right)}{a}} \cdot\left|\frac{q_{1}-1}{q_{1}+1}\right|^{\operatorname{sign} p_{1}}
$$

donde la constante $\gamma$ es exactamente $\gamma=t_{1}-t_{0}$, y donde las diferentes elecciones de los signos relativos nos proporcionan los kinks de los diferentes sectores.

En coordenadas elíptico-cilíndricas la región que estamos estudiando, es decir el plano $q_{2}=0$, se traduce en dos de las caras del paralelepípedo $P_{3}(0, \pm a)$, la determinada por $\mu_{1}=\bar{\sigma}^{2}$, y la correspondiente a $\mu_{2}=\bar{\sigma}^{2}$. Las expresiones de las familias TK2 $i$ y TK $2 i i^{4}$ en estas coordenadas serán:

$$
\mu_{\mathrm{TK} 2}=\left(\begin{array}{c}
1-\tanh ^{2}\left(t-t_{0}\right) \\
\bar{\sigma}^{2} \\
-a \tanh \left(a t-t_{1}\right)
\end{array}\right)
$$

\footnotetext{
${ }^{3} \mathrm{O}$ alternativamente resolviendo el sistema por el método de Hamilton-Jacobi.

${ }^{4}$ Nótese que en coordenadas elíptico-cilíndricas los vacíos $\vec{v}^{1}$ y $\vec{v}^{2}$ se corresponden con un mismo punto, y otro tanto con $\vec{v}^{3}$ y $\vec{v}^{4}$. Las expresiones para TK2i y TK2ii son por tanto indistinguibles en estas coordenadas.
} 
para el rango

$$
t \in\left(-\infty, t_{0}+\operatorname{arctanh}(-\sigma)\right] \sqcup\left[t_{0}+\operatorname{arctanh} \sigma, \infty\right)
$$

mientras que la expresión válida en el rango:

$$
t \in\left[t_{0}+\operatorname{arctanh}(-\sigma), t_{0}+\operatorname{arctanh} \sigma\right]
$$

será:

$$
\mu_{\mathrm{TK} 2}=\left(\begin{array}{c}
\bar{\sigma}^{2} \\
1-\tanh ^{2}\left(t-t_{0}\right) \\
-a \tanh \left(a t-t_{1}\right)
\end{array}\right)
$$

Los sectores de antikinks tienen, lógicamente, las mismas ecuaciones cambiando el signo de la tercera coordenada.

- Kinks Bidimensionales no-Topológicos.

La restricción del modelo a los planos $q_{3}= \pm a$ reduce el mismo al ya comentado, y bien conocido, Modelo MSTB, es decir al Modelo Sigma $O(2)$ Lineal Deformado, los kinks TK1 $( \pm a)$ y los TK2 $( \pm a)$ son también soluciones pertenecientes a estas superficies de prueba, si bien de carácter topológico. Los Kinks no topológicos en estas regiones se corresponden con las soluciones generales de la ecuación de Hamilton-Jacobi para este sistema reducido. Una vez más, no repetiremos los resultados y nos remitiremos a lo expuesto en el Capítulo 3.

\section{Kinks Tridimensionales}

Finalmente, existen dos tipos de kinks con las tres componentes cartesianas no nulas, por un lado tendremos dos pares de familias (kinks y antikinks) uniparamétricas de soluciones topológicas que permanecen en la superficie del cilindro elíptico de ecuación $\mu_{1}=0$ y que, en cierto modo, son una generalización de los kinks TK2 $( \pm a)$ antes estudiados. Por otro lado, encontraremos otros dos pares de familias, en este caso biparamétricas, de soluciones también topológicas que se corresponden con las trayectorias generales del sistema, es decir, las soluciones que se obtienen para valores no singulares de las constantes de integración en la resolución de la ecuación de Hamilton-Jacobi para el sistema completo. 
- Kinks tridimensionales sobre la superficie cilíndrica.

La restricción del sistema a la superficie del cilindro elíptico de ecuaciones (A.11) y (A.12) se realiza de una manera fácil si se utilizan coordenadas elípticocilíndricas. Como ya hemos comentado en estas coordenadas la superficie cilíndrica se reduce a la ecuación $\mu_{1}=0$ dentro del rango para $q_{3}$ determinado por (A.12).

El Lagrangiano restringido se escribe:

$$
\mathcal{L}=\frac{1}{8} \frac{\mu_{2}}{\left(1-\mu_{2}\right)\left(\mu_{2}-\bar{\sigma}^{2}\right)}\left(\frac{d \mu_{2}}{d t}\right)^{2}+\frac{1}{2} \mu_{2}\left(\mu_{2}-\bar{\sigma}^{2}\right)+\frac{1}{2}\left(\frac{d q_{3}}{d t}\right)^{2}+\frac{1}{2}\left(q_{3}^{2}-a^{2}\right)^{2}
$$

mientras que el Hamiltoniano puede separarse de la forma:

$$
\begin{gathered}
H=H_{\mu_{2}}+H_{3} \\
H_{\mu_{2}}=2 \frac{\left(1-\mu_{2}\right)\left(\mu_{2}-\bar{\sigma}^{2}\right)}{\mu_{2}} \pi_{2}^{2}-\frac{1}{2} \mu_{2}\left(\mu_{2}-\bar{\sigma}^{2}\right) \\
H_{3}=\frac{1}{2} p_{3}^{2}-\frac{1}{2}\left(q_{3}^{2}-a^{2}\right)^{2}
\end{gathered}
$$

La ecuación de Hamilton-Jacobi resulta ser, obviamente, separable y no es difícil obtener su solución:

$$
\begin{aligned}
\mathcal{S}= & -E t+\operatorname{sign}\left(\pi_{2}\right) \int \sqrt{\frac{F \mu_{2}}{2\left(1-\mu_{2}\right)\left(\mu_{2}-\bar{\sigma}^{2}\right)}+\frac{1}{4} \frac{\mu_{2}^{2}}{1-\mu_{2}}} d \mu_{2} \\
& +\operatorname{sign}\left(p_{3}\right) \int \sqrt{2 E-2 F+\left(q_{3}^{2}-a^{2}\right)^{2}} d q_{3}
\end{aligned}
$$

donde $E$ y $F$ son las constantes de separación, cuyo valor viene fijado por las condiciones asintóticas (A.6), de manera que las soluciones que nos interesan, asociadas a soluciones kinks de la teoría de campos subyacente, se obtendrán para el caso $E=F=0$.

Las ecuaciones $\epsilon_{1}=\frac{\partial \mathcal{S}}{\partial E}$ y $\epsilon_{2}=\frac{\partial \mathcal{S}}{\partial F}$ nos proporcionarán la dependencia temporal y las ecuaciones de las trayectorias, respectivamente, de estas soluciones, que denominaremos como TK3c. Particularizando los resultados para la hipersuperficie $E=F=0$ obtendremos:

$$
\left|\frac{q_{3}-a}{q_{3}+a}\right|^{\operatorname{sign}\left(p_{3}\right)}=e^{2 a\left(\epsilon_{1}+t\right)}
$$

para la evolución temporal, y:

$$
\left|\frac{q_{3}-a}{q_{3}+a}\right|^{-\frac{\operatorname{sign}\left(p_{3}\right)}{a}} \cdot\left|\frac{\sqrt{1-\mu_{2}}-\sigma}{\sqrt{1-\mu_{2}}+\sigma}\right|^{\frac{\operatorname{sign}\left(\pi_{2}\right)}{\sigma}}=e^{2 \epsilon_{2}}
$$


para las ecuaciones de las órbitas.

Es posible combinar ambas ecuaciones, (A.47) y (A.48), de manera que obtenemos explícitamente las ecuaciones paramétricas de esta familia de soluciones TK3 $c$ en función del tiempo $t$ :

$$
\mu_{\mathrm{TK} 3 c}=\left(\begin{array}{c}
0 \\
1-\sigma^{2} \tanh ^{2}\left(-\operatorname{sign}\left(p_{3}\right)(t+\epsilon)\right) \\
a \tanh \left(-\operatorname{sign}\left(p_{3}\right) a\left(t+\epsilon_{1}\right)\right)
\end{array}\right)
$$

y donde, evidentemente, la elección del signo de $p_{3}$ determina el carácter kink o antikink de la trayectoria.

Una vez más, tenemos una familia uniparamétrica de soluciones, o, para ser más precisos, de trayectorias, recordemos que hemos adoptado el criterio de considerar las familias por las órbitas, de forma que cada órbita o trayectoria, determinada en este caso por un valor concreto de la constante $\epsilon_{2}\left(\epsilon=\epsilon_{1}+\epsilon_{2}\right)$, representa realmente a toda una familia de soluciones de las ecuaciones, parametrizadas a su vez por la constante "temporal" $\epsilon_{1}$.

Los sectores topológicos involucrados en este caso, $\mathcal{C}^{(1,3)}, \mathcal{C}^{(3,1)}, \mathcal{C}^{(2,4)}$ y $\mathcal{C}^{(4,2)}$, se ponen de manifiesto al pasar las soluciones (A.49) a coordenadas cartesianas, cada elección de signos nos proporcionará uno de los sectores. La expresión general que engloba todas las posibilidades será:

$$
\vec{q}_{\mathrm{TK} 3 c}=\left(\begin{array}{c} 
\pm \tanh \left(-\operatorname{sign}\left(\pi_{2}\right)(t+\epsilon)\right) \\
\bar{\sigma} \operatorname{sech}\left(-\operatorname{sign}\left(\pi_{2}\right)(t+\epsilon)\right) \\
a \tanh \left(-\operatorname{sign}\left(p_{3}\right) a\left(t+\epsilon_{1}\right)\right)
\end{array}\right)
$$

- Kinks generales.

Por último, presentaremos el caso general, las cuatro familias biparamétricas de trayectorias solución de la ecuación de Hamilton-Jacobi asociada al sistema dinámico que estamos considerando.

La integración de las expresiones (A.30) y (A.31) nos proporciona las ecuaciones:

$$
\begin{aligned}
&\left|\frac{\sqrt{1-\mu_{1}}-\sigma}{\sqrt{1-\mu_{1}}+\sigma}\right|^{-\frac{\operatorname{sign}\left(\pi_{1}\right)}{\sigma}} \cdot\left|\frac{\sqrt{1-\mu_{2}}-\sigma}{\sqrt{1-\mu_{2}}+\sigma}\right|^{\frac{\operatorname{sign}\left(\pi_{2}\right)}{\sigma}} \cdot\left|\frac{q_{3}-a}{q_{3}+a}\right|^{\frac{\operatorname{sign}\left(p_{3}\right)}{a}}=e^{-2 \zeta_{2}} \\
&\left|\frac{\sqrt{1-\mu_{1}}-1}{\sqrt{1-\mu_{1}}+1}\right|^{\operatorname{sign}\left(\pi_{1}\right)} \cdot\left|\frac{\sqrt{1-\mu_{1}}+\sigma}{\sqrt{1-\mu_{1}}-\sigma}\right|^{\frac{\operatorname{sign}\left(\pi_{1}\right)}{\sigma}} \cdot \\
&\left|\frac{\sqrt{1-\mu_{2}}+1}{\sqrt{1-\mu_{2}}-1}\right|^{\operatorname{sign}\left(\pi_{2}\right)} \cdot\left|\frac{\sqrt{1-\mu_{2}}-\sigma}{\sqrt{1-\mu_{2}}+\sigma}\right|^{\frac{\operatorname{sign}\left(\pi_{2}\right)}{\sigma}}=e^{2 \bar{\sigma}^{2} \zeta_{3}}
\end{aligned}
$$


y tenemos, en definitiva, kinks pertenecientes al interior de la región cilíndrica, con las trayectorias parametrizadas por dos constantes arbitrarias, $\zeta_{2}$ y $\zeta_{3}$.

Se tratará de kinks topológicos pertenecientes a los sectores $\mathcal{C}^{(1,4)}$ y $\mathcal{C}^{(4,1)}$, por un lado, y a $\mathcal{C}^{(2,3)}$ y $\mathcal{C}^{(3,2)}$, por otro. Los denominaremos de manera genérica kinks TK3. 


\section{Apéndice B}

\section{Superpotencial del Modelo $O(3)$ en Coordenadas Cartesianas}

Hemos incluido en este apéndice los cálculos que conducen a determinar la expresión del superpotencial del Modelo Sigma $O(N)$ Lineal Deformado en coordenadas cartesianas. En el Capítulo 6 hemos obtenido dicho superpotencial en coordenadas elípticas, (6.8):

$$
\begin{aligned}
& W^{\left(\alpha_{1}, \alpha_{2}, \alpha_{3}\right)}\left(\lambda_{1}, \lambda_{2}, \lambda_{3}\right)=\frac{1}{3}\left((-1)^{\alpha_{1}}\left(\lambda_{1}+2\right) \sqrt{1-\lambda_{1}}\right. \\
& \left.+(-1)^{\alpha_{2}}\left(\lambda_{2}+2\right) \sqrt{1-\lambda_{2}}+(-1)^{\alpha_{3}}\left(\lambda_{3}+2\right) \sqrt{1-\lambda_{3}}\right)
\end{aligned}
$$

\section{B.1 Cambio de coordenadas inverso}

Para obtener la expresión de $W^{\left(\alpha_{1}, \alpha_{2}, \alpha_{3}\right)}$ en coordenadas cartesianas necesitaremos, en primer lugar, conocer las expresiones que determinan el cambio de coordenadas inverso al dado por:

$$
\begin{aligned}
q_{1}^{2} & =\frac{\left(1-\lambda_{1}\right)\left(1-\lambda_{2}\right)\left(1-\lambda_{3}\right)}{\sigma_{2}^{2} \sigma_{3}^{2}} \\
q_{2}^{2} & =\frac{\left(\bar{\sigma}_{2}^{2}-\lambda_{1}\right)\left(\bar{\sigma}_{2}^{2}-\lambda_{2}\right)\left(\bar{\sigma}_{2}^{2}-\lambda_{3}\right)}{-\sigma_{2}^{2}\left(\sigma_{3}^{2}-\sigma_{2}^{2}\right)} \\
q_{3}^{2} & =\frac{\left(\bar{\sigma}_{3}^{2}-\lambda_{1}\right)\left(\bar{\sigma}_{3}^{2}-\lambda_{2}\right)\left(\bar{\sigma}_{3}^{2}-\lambda_{3}\right)}{\sigma_{3}^{2}\left(\sigma_{3}^{2}-\sigma_{2}^{2}\right)}
\end{aligned}
$$

Para ello cambiaremos ligeramente la notación con el propósito de simplificar las ecuaciones que resultan en dicho cálculo. 
Denotaremos:

$$
\begin{aligned}
\alpha & =\bar{\sigma}_{1}^{2}+\bar{\sigma}_{2}^{2}+\bar{\sigma}_{3}^{2}=1+\bar{\sigma}_{2}^{2}+\bar{\sigma}_{3}^{2} \\
\beta & =\bar{\sigma}_{1}^{2} \bar{\sigma}_{2}^{2}+\bar{\sigma}_{1}^{2} \bar{\sigma}_{3}^{2}+\bar{\sigma}_{2}^{2} \bar{\sigma}_{3}^{2}=\bar{\sigma}_{2}^{2}+\bar{\sigma}_{3}^{2}+\bar{\sigma}_{2}^{2} \bar{\sigma}_{3}^{2} \\
\gamma & =\bar{\sigma}_{1}^{2} \bar{\sigma}_{2}^{2} \bar{\sigma}_{3}^{2}=\bar{\sigma}_{2}^{2} \bar{\sigma}_{3}^{2} \\
u & =q_{1}^{2}+q_{2}^{2}+q_{3}^{2} \\
v & =q_{1}^{2}+\bar{\sigma}_{2}^{2} q_{2}^{2}+\bar{\sigma}_{3}^{2} q_{3}^{2} \\
w & =q_{1}^{2}+\bar{\sigma}_{2}^{4} q_{2}^{2}+\bar{\sigma}_{2}^{4} q_{3}^{2}
\end{aligned}
$$

Tal y como se explicó en el Capítulo 2, las coordenadas elípticas se construyen a partir de la ecuación:

$$
\sum_{i=1}^{3} \frac{q_{i}^{2}}{a_{i}-\lambda}=1
$$

Se demostró en dicho capítulo que (B.1) tiene exactamente tres raíces reales distintas en la incógnita $\lambda$, raíces que no son otras que las coordenadas elípticas $\lambda_{1}, \lambda_{2}$ y $\lambda_{3}$ presentadas anteriormente.

Manipulando trivialmente (B.1), esta ecuación puede escribirse como una ecuación cúbica en $\lambda$ :

$$
\lambda^{3}+(\alpha-u) \lambda^{2}+(\beta-\alpha u+v) \lambda+(\beta u-\alpha v-w-\gamma)=\lambda^{3}+c_{2} \lambda^{2}+c_{1} \lambda+c_{0}=0
$$

Para resolver (B.2), por las fórmulas de Cardano-Tartaglia (ver [2]), definiremos las cantidades:

$$
\begin{aligned}
q & =\frac{1}{3} c_{1}-\frac{1}{9} c_{2}^{2}=\frac{1}{9}\left(3 \beta-\alpha^{2}-\alpha u+3 v-u^{2}\right) \\
r & =\frac{1}{6}\left(c_{1} c_{2}-3 c_{0}\right)-\frac{1}{27} c_{2}^{3}= \\
& =\frac{1}{9}\left(\frac{1}{3} \alpha^{3}-\frac{3}{2} \alpha \beta+\frac{9}{2} \gamma\right)+\frac{1}{18}\left(\alpha^{2}-6 \beta\right) u+\frac{1}{3} v-\frac{1}{2} w-\frac{\alpha}{18} u^{2}+\frac{1}{6} u v-\frac{1}{27} u^{3}
\end{aligned}
$$

Como hemos comentado, (B.2) tiene tres raíces reales diferentes. Según la teoría general de resolución de las ecuaciones cúbicas ([2]) ello implica necesariamente que $q^{3}+r^{2}<0$. Un cálculo simplificado de esta expresión proporciona el resultado:

$$
\begin{aligned}
& q^{3}+r^{2}=\frac{1}{108}\left(4 \beta^{3}-\alpha^{2} \beta^{2}+4 \alpha^{3} \gamma-18 \alpha \beta \gamma+27 \gamma^{2}\right)+ \\
& +\frac{1}{54}\left(-\alpha \beta\left(\alpha^{2}-4 \beta\right)+3 \gamma\left(\alpha^{2}-6 \beta\right)\right) u+\frac{1}{27}\left(\alpha^{4}-5 \alpha^{2} \beta+3 \beta^{2}+9 \alpha \gamma\right) v+
\end{aligned}
$$




$$
\begin{aligned}
& +\frac{1}{108}\left(-\alpha^{4}+2 \alpha^{2} \beta+8 \beta^{2}-6 \alpha \gamma\right)+\frac{1}{27}\left(2 \alpha^{2}+3 \beta\right) v^{2}+ \\
& +\frac{1}{54}\left(4 \alpha^{3}-19 \alpha \beta+9 \gamma\right) u v+\frac{1}{54}\left(-\alpha^{3}+4 \alpha \beta-2 \gamma\right) u^{3}+\frac{1}{54}\left(\alpha^{2}-10 \beta\right) u^{2} v+ \\
& +\frac{2 \alpha}{27} u v^{2}+\frac{1}{27} v^{3}+\frac{1}{108}\left(-\alpha^{2}+4 \beta\right) u^{4}-\frac{1}{108} u^{2} v^{2}-\frac{\alpha}{54} u^{3} v+\frac{1}{27} u^{3} w+\frac{1}{4} w^{2} \\
& -\frac{1}{9}\left(\frac{1}{3} \alpha^{3}-\frac{3}{2} \alpha \beta+\frac{9}{2} \gamma\right) w-\frac{1}{18}\left(\alpha^{2}-6 \beta\right) u w+\frac{1}{18} \alpha u^{2} w-\frac{1}{3} \alpha v w-\frac{1}{6} u v w
\end{aligned}
$$

y, necesariamente, como ya hemos dicho, toma valores negativos.

Definiendo entonces las cantidades $s_{1}$ y $s_{2}$ en la forma:

$$
s_{1}=\left(r+\sqrt{q^{3}+r^{2}}\right)^{\frac{1}{3}} ; \quad s_{2}=\left(r-\sqrt{q^{3}+r^{2}}\right)^{\frac{1}{3}}
$$

tendremos finalmente las raíces:

$$
\begin{aligned}
\lambda_{i} & =s_{1}+s_{2}-\frac{c_{2}}{3} \\
\lambda_{i i} & =-\frac{1}{2}\left(s_{1}+s_{2}\right)-\frac{c_{2}}{3}+i \frac{\sqrt{3}}{2}\left(s_{1}-s_{2}\right) \\
\lambda_{i i i} & =-\frac{1}{2}\left(s_{1}+s_{2}\right)-\frac{c_{2}}{3}-i \frac{\sqrt{3}}{2}\left(s_{1}-s_{2}\right)
\end{aligned}
$$

que, para nuestro caso, con $q^{3}+r^{2}<0$, se escribirán:

$$
s_{1}=\left(r+i \sqrt{\left|q^{3}+r^{2}\right|}\right)^{\frac{1}{3}} ; \quad s_{2}=\left(r-i \sqrt{\left|q^{3}+r^{2}\right|}\right)^{\frac{1}{3}}
$$

y, en definitiva:

$$
\begin{aligned}
& \lambda_{1}=-\sqrt{|q|}\left(\cos \frac{\theta}{3}+\sqrt{3} \sin \frac{\theta}{3}\right)-\frac{u-\alpha}{3} \\
& \lambda_{2}=-\sqrt{|q|}\left(\cos \frac{\theta}{3}-\sqrt{3} \sin \frac{\theta}{3}\right)-\frac{u-\alpha}{3} \\
& \lambda_{3}=2 \sqrt{|q|} \cos \frac{\theta}{3}-\frac{u-\alpha}{3}
\end{aligned}
$$

con:

$$
\theta=\arctan \frac{\sqrt{\left|q^{3}+r^{2}\right|}}{r}
$$

y donde se ha considerado únicamente el caso $\theta \in[0, \pi]$ (las demás posibilidades conducen a una redistribución de los índices: $\lambda_{1}$ pasa a ser $\lambda_{2}, \lambda_{2}$ pasa a $\lambda_{3}$, etc.) 


\section{B.2 Superpotencial en coordenadas cartesianas}

Una vez encontrado el cambio de coordenadas (B.3), su sustitución en la expresión (6.8) nos conduce a los superpotenciales en coordenadas cartesianas:

$$
\begin{aligned}
& W^{\left(\alpha_{1}, \alpha_{2}, \alpha_{3}\right)}\left(q_{1}, q_{2}, q_{3}\right)=\frac{1}{3 \sqrt{3}}\left[(-1)^{\alpha_{1}} \sqrt{3-a+u-6 \sqrt{|q|} \cos \frac{\theta}{3}} \cdot\right. \\
& \cdot\left(6+a-u+6 \sqrt{|q|} \cos \frac{\theta}{3}\right)+(-1)^{\alpha_{2}} \sqrt{3-a+u+3 \sqrt{|q|}\left(\cos \frac{\theta}{3}-\sqrt{3} \sin \frac{\theta}{3}\right)} \cdot \\
& \cdot\left(6+a-u+3 \sqrt{|q|}\left(-\cos \frac{\theta}{3}+\sqrt{3} \sin \frac{\theta}{3}\right)\right) \\
& +(-1)^{\alpha_{3}} \sqrt{3-a+u+3 \sqrt{|q|}\left(\cos \frac{\theta}{3}+\sqrt{3} \sin \frac{\theta}{3}\right)} \cdot \\
& \left.\cdot\left(6+a-u-3 \sqrt{|q|}\left(\cos \frac{\theta}{3}+\sqrt{3} \sin \frac{\theta}{3}\right)\right)\right]
\end{aligned}
$$

Volviendo a escribir en términos de las variables $q_{1}, q_{2}$ y $q_{3}$ se tiene:

$$
\begin{aligned}
& W^{\left(\alpha_{1}, \alpha_{2}, \alpha_{3}\right)}\left(q_{1}, q_{2}, q_{3}\right)=\frac{1}{3 \sqrt{3}}\left[(-1)^{\alpha_{1}} \sqrt{\sigma_{2}^{2}+\sigma_{3}^{2}+\left(q_{1}^{2}+q_{2}^{2}+q_{3}^{2}\right)-6 \sqrt{|q|} \cos \frac{\theta}{3}}\right. \\
& \cdot\left(9-\sigma_{2}^{2}-\sigma_{3}^{2}-\left(q_{1}^{2}+q_{2}^{2}+q_{3}^{2}\right)+6 \sqrt{|q|} \cos \frac{\theta}{3}\right)+ \\
& +(-1)^{\alpha_{2}} \sqrt{\sigma_{2}^{2}+\sigma_{3}^{2}+\left(q_{1}^{2}+q_{2}^{2}+q_{3}^{2}\right)+3 \sqrt{|q|}\left(\cos \frac{\theta}{3}-\sqrt{3} \sin \frac{\theta}{3}\right)} . \\
& +\left(9-\sigma_{2}^{2}-\sigma_{3}^{2}-\left(q_{1}^{2}+q_{2}^{2}+q_{3}^{2}\right)+3 \sqrt{|q|}\left(-\cos \frac{\theta}{3}+\sqrt{3} \sin \frac{\theta}{3}\right)\right) \\
& \cdot\left(9-\sigma^{\alpha_{3}} \sqrt{\sigma_{2}^{2}+\sigma_{3}^{2}+\left(q_{1}^{2}+q_{2}^{2}+q_{3}^{2}\right)+3 \sqrt{|q|}\left(\cos \frac{\theta}{3}+\sqrt{3} \sin \frac{\theta}{3}\right)} .\right. \\
& (9.5) \\
& \left.\left(\sigma_{3}^{2}-\left(q_{1}^{2}+q_{2}^{2}+q_{3}^{2}\right)-3 \sqrt{|q|}\left(\cos \frac{\theta}{3}+\sqrt{3} \sin \frac{\theta}{3}\right)\right)\right]
\end{aligned}
$$

y donde $\theta=\arctan \frac{\sqrt{\left|q^{3}+r^{2}\right|}}{r}$, con

$$
\begin{aligned}
|q|=-q= & \frac{1}{9}\left(\left(q_{1}^{2}+q_{2}^{2}+q_{3}^{2}\right)^{2}-\left(\sigma_{2}^{2}+\sigma_{3}^{2}\right) q 1^{2}+\left(2 \sigma_{2}^{2}-\sigma_{3}^{2}\right) q_{2}^{2}+\right. \\
& \left.+\left(2 \sigma_{3}^{2}-\sigma_{2}^{2}\right) q_{3}^{2}+\sigma_{2}^{4}+\sigma_{3}^{4}-\sigma_{2}^{2} \sigma_{3}^{2}\right)
\end{aligned}
$$


y

$$
\begin{aligned}
r= & \frac{-1}{54}\left(2\left(\sigma_{2}^{6}+\sigma_{3}^{6}\right)-3\left(\sigma_{2}^{4} \sigma_{3}^{2}+\sigma_{2}^{2} \sigma_{3}^{4}\right)\right)+\frac{1}{18}\left(\sigma_{2}^{4}+\sigma_{3}^{4}-4 \sigma_{2}^{2} \sigma_{3}^{2}\right) \cdot \\
& \cdot\left(q_{1}^{2}+q_{2}^{2}+q_{3}^{2}\right)+\frac{1}{18}\left(\sigma_{2}^{2}+\sigma_{3}^{2}\right)\left(q_{1}^{2}+q_{2}^{2}+q_{3}^{2}\right)^{2} \\
& -\frac{1}{6}\left(q_{1}^{2}+q_{2}^{2}+q_{3}^{2}\right)\left(\sigma_{2}^{2} q_{2}^{2}+\sigma_{3}^{2} q_{3}^{2}\right)+\frac{1}{6}\left(2 \sigma_{2}^{2} \sigma_{3}^{2}-\sigma_{2}^{4}\right) q_{2}^{2}+ \\
& +\frac{1}{6}\left(2 \sigma_{2}^{2} \sigma_{3}^{2}-\sigma_{3}^{4}\right) q_{3}^{2}-\frac{1}{27}\left(q_{1}^{2}+q_{2}^{2}+q_{3}^{2}\right)^{3}
\end{aligned}
$$




\section{Apéndice $\mathrm{C}$}

\section{Recopilación Breve de Geometría Riemanniana}

Incluimos en esta Memoria un apéndice en el que se resumen algunos de los conceptos básicos de la Geometría Riemanniana. El objetivo de esta inclusión es presentar, por un lado, la notación que se ha adoptado a lo largo de este trabajo y, por otro, las propiedades y teoremas que se han utilizado en los diferentes capítulos del mismo. De las muchas y excelentes referencias que existen en la literatura sobre este tópico, se han seguido esencialmente las siguientes: [50], [59], [57], [65] y [70].

Dado el carácter recopilatorio de este apéndice, hemos obviado las demostraciones de las Proposiciones y Teoremas que se han incluido (y que pueden encontrarse, evidentemente, en las referencias citadas). Se ha hecho una excepción con la Proposición C.2 debido a que su demostración no está recogida en ninguno de los textos citados y, además, se trata de un resultado que se utiliza repetidas veces en el Capítulo 4 de esta Memoria.

\section{C.1 Conexiones y derivadas covariantes}

Sea $M$ una variedad riemanniana (i.e. variedad diferenciable tal que en cada espacio tangente $T_{p} M, \forall p \in M$, está definida una métrica riemanniana $g=g_{i j} d x^{i} \otimes$ $\left.d x^{j}\right)$.

Prescindiendo, por innecesaria en este contexto, de la noción general de conexión en un fibrado principal ${ }^{1}$, nos centraremos en la definición de conexión en un fibrado

\footnotetext{
${ }^{1}$ Ver por ejemplo [59] para la teoría general.
} 
vectorial sobre una variedad (particularmente en el fibrado tangente a la variedad riemanniana dada $M$ ).

Definición C.1. Una conexión (o conexión afín) sobre el fibrado vectorial $V$ sobre la variedad diferenciable $M$ es toda aplicación $\nabla$ de $\Gamma(V) \otimes \Gamma(T M)$ en $\Gamma(V)$ que verifica las propiedades ${ }^{2}$ :

$$
\nabla(K, X)=\nabla_{X} K, \quad \forall X \in \Gamma(T M), \quad \forall K \in \Gamma(V)
$$

- $\nabla_{f X+g Y} K=f \nabla_{X} K+g \nabla_{Y} K, \forall X, Y \in \Gamma(T M), \forall f, g \in C^{\infty}(M), \forall K \in$ $\Gamma(V)$.

- $\nabla_{X}(f K)=f \nabla_{X} K+X(f) K, \forall X \in \Gamma(T M), \forall f \in C^{\infty}(M), \forall K \in \Gamma(V)$.

$$
\nabla_{X}\left(K+K^{\prime}\right)=\nabla_{X} K+\nabla_{X} K^{\prime}, \forall X \in \Gamma(T M), \forall K, K^{\prime} \in \Gamma(V) .
$$

$\nabla_{X} K$ recibe el nombre de derivada covariante de $K$ con respecto a $X$.

La Definición C.1 puede ser reinterpretada de la forma:

$$
\nabla: \Gamma(V) \rightarrow \Gamma(V) \otimes \Gamma\left(T^{*} M\right)
$$

de manera que $\nabla(K) \equiv \nabla K$ actúa sobre un campo vectorial tangente $X \in \Gamma(T M)$ de la forma:

$$
\nabla K(X) \equiv \nabla_{X} K \in \Gamma(V)
$$

Desde este punto de vista, se denomina a $\nabla K$ diferencial covariante de la sección $K$.

Dos son los casos en los que nos centraremos fundamentalmente: en primer lugar cuando el fibrado vectorial no es otro que el fibrado tangente a la variedad $M(V=T M)$, y en segundo, y generalizando al anterior, el caso del fibrado tensorial sobre $M\left(V=T_{s}^{r}(M)\right)$.

Conexiones en el Fibrado Tangente. Para el caso de los campos vectoriales, la conexión se convierte en una ley de composición interna: $\nabla: \Gamma(T M) \otimes \Gamma(T M) \rightarrow$ $\Gamma(T M)$ de manera que

$$
\nabla(X, Y) \equiv \nabla_{Y} X
$$

y la diferencial covariante de un campo vectorial $X \in \Gamma(T M)$ es un tensor $\left(\begin{array}{l}1 \\ 1\end{array}\right)$ que actúa de la forma:

$$
\nabla X(Y)=\nabla_{Y} X \in \Gamma(T M)
$$

\footnotetext{
${ }^{2}$ Evidentemente, $\Gamma(V)$ denota a las secciones de dicho fibrado.
} 
Si $\left(x^{1}, \ldots, x^{n}\right)$ son coordenadas locales en un abierto $U$ de $M$, entonces la base de los campos vectoriales en $M$ será: $\left\{\frac{\partial}{\partial x^{1}}, \ldots, \frac{\partial}{\partial x^{n}}\right\}$, de manera que la conexión $\nabla$ está determinada por los símbolos de Christoffel de la forma:

$$
\nabla_{\frac{\partial}{\partial x^{i}}} \frac{\partial}{\partial x^{j}}=\Gamma_{i j}^{k} \frac{\partial}{\partial x^{k}}
$$

De esta manera, si $X=X^{i} \frac{\partial}{\partial x^{i}}$ e $Y=Y^{j} \frac{\partial}{\partial x^{j}}$ son dos campos en $M$, tendremos que la derivada covariante se escribe en componentes de la siguiente manera:

$$
\nabla_{X} Y=X^{i}\left(\frac{\partial Y^{k}}{\partial x^{i}}+\Gamma_{i j}^{k} Y^{j}\right) \frac{\partial}{\partial x^{k}}=X^{i} \nabla_{i} Y
$$

donde se ha denotado $\nabla_{i}=\nabla_{\frac{\partial}{\partial x^{i}}}$.

Por su parte, para la diferencial covariante, tendremos: $\nabla Y=\nabla_{j} Y \otimes d x^{j}, \mathrm{y}$ así:

$$
\left(\nabla_{j} Y \otimes d x^{j}\right)\left(X^{i} \frac{\partial}{\partial x^{i}}\right)=\nabla_{j} Y X^{i} \delta_{i}^{j}=\nabla_{j} Y X^{j}=\nabla_{X} Y
$$

Conexiones en el Fibrado Tensorial. De cara a particularizar la Definición C.1 para el caso de campos tensoriales, es conveniente entender un campo tensorial $K$ de tipo $\left(\begin{array}{l}r \\ s\end{array}\right)$ como una aplicación multilineal tal que, $\forall x \in M$, actúa en $T_{x} M \times . s$. $\times T_{x} M$ y toma valores en $\mathbf{T}_{0}^{r}(x)$ (tensores tipo $\left(\begin{array}{l}r \\ 0\end{array}\right)$ ), es decir, $K \in \Gamma\left(T_{s}^{r} M\right)$

$$
K: \Gamma(T M) \times \stackrel{s}{.} \times \Gamma(T M) \rightarrow \mathbf{T}_{0}^{r}
$$

De esta manera, la derivada covariante $\nabla_{X} K$ con respecto a un campo $X$ del campo tensorial $K$ es un nuevo campo tensorial del mismo tipo que $K$.

La diferencial covariante de $K \in \Gamma\left(T_{s}^{r} M\right)$ se definirá, en consecuencia, como un campo tensorial $\nabla K$ de tipo $\left(\begin{array}{c}r \\ s+1\end{array}\right)$ de la forma:

$$
(\nabla K)\left(X_{1}, \ldots, X_{s} ; X\right)=\left(\nabla_{X} K\right)\left(X_{1}, \ldots, X_{s}\right)
$$

Es trivial entonces demostrar la proposición siguiente (ver [59]):

Proposición C.1. Con las definiciones anteriores se verifica:

$$
\begin{aligned}
(\nabla K)\left(X_{1}, \ldots, X_{s} ; X\right)= & \nabla_{X}\left(K\left(X_{1}, \ldots, X_{s}\right)\right) \\
& -\sum_{i=1}^{s} K\left(X_{1}, \ldots, \nabla_{X} X_{i}, \ldots, X_{s}\right)
\end{aligned}
$$


Para el caso de uno-formas (tensores $\left.\left(\begin{array}{l}0 \\ 1\end{array}\right)\right): K=\omega=\omega_{i} d x^{i} ; \omega: \Gamma(T M) \rightarrow \mathbf{T}_{0}^{0}$. $\nabla \omega$ será un tensor $\left(\begin{array}{l}0 \\ 2\end{array}\right)$ de manera que:

$$
(\nabla \omega)(Y ; X)=\left(\nabla_{X} \omega\right)(Y)
$$

que, de acuerdo con la proposición anterior verificará:

$$
\nabla \omega(Y ; X)=\left(\nabla_{X} \omega\right)(Y)=\nabla_{X}(\omega(Y))-\omega\left(\nabla_{X} Y\right)
$$

En componentes:

$$
\begin{gathered}
\nabla \omega(Y ; X)=\nabla_{X}\left(\omega_{i} Y^{i}\right)-\omega\left(\nabla_{X} Y\right)=X^{j} \partial_{j}\left(\omega_{i} Y^{i}\right)-\omega\left(X^{i} \nabla_{i} Y\right)= \\
X^{j} Y^{i} \partial_{j} \omega_{i}+X^{j} \omega_{i} \partial_{j} Y^{i}-\omega_{k} X^{i}\left(\partial_{i} Y^{k}+\Gamma_{i l}^{k} Y^{l}\right)=X^{j} Y^{i}\left(\partial_{j} \omega_{i}-\Gamma_{j i}^{k} \omega_{k}\right)=X^{j}\left(\nabla_{j} \omega\right)(Y)
\end{gathered}
$$

de manera que:

$$
\nabla_{X} \omega=X^{j} \nabla_{j} \omega=X^{j}\left(\partial_{j} \omega_{i}-\Gamma_{j i}^{k} \omega_{k}\right) d x^{i}
$$

En el caso en el que $\omega=d f=\partial_{l} f d x^{l}$, tendremos:

$$
\left(\nabla_{X} \omega\right)(Y)=X^{j} Y^{l}\left(\frac{\partial^{2} f}{\partial x^{j} \partial x^{l}}-\Gamma_{j l}^{k} \partial_{k} f\right)
$$

Curvas y traslado paralelo. Si $c:\left[t_{1}, t_{2}\right] \rightarrow M$ es una curva (diferenciable) en $M$, un campo $X$ a lo largo de $c(t)$ es una función que asigna a cada $t \in\left[t_{1}, t_{2}\right]$ un vector tangente $X_{t} \in T_{c(t)} M$.

Todo campo de vectores a lo largo de una curva tiene asociado otro, su derivada covariante a lo largo de la curva, de la forma:

$$
\nabla_{\frac{d}{d t}} X \equiv \frac{\mathrm{D} X}{d t}=\left(\frac{d X^{k}}{d t}+\frac{d c^{i}}{d t} \Gamma_{i j}^{k} X^{j}\right) \frac{\partial}{\partial x^{k}}=\frac{d c^{i}(t)}{d t} \nabla_{i} X=\dot{c}^{i} \nabla_{i} X=\nabla_{\dot{c}} X
$$

donde $c(t)=\left(c^{1}(t), \ldots, c^{n}(t)\right)$ en coordenadas locales en un abierto de $M$, y donde utilizamos, evidentemente, el abuso de notación habitual al identificar $\frac{d}{d t} \in \Gamma(T \mathbb{R})$ con $\frac{d}{d t} \equiv \dot{c} \equiv \frac{d c}{d t} \equiv c_{*}\left(\frac{d}{d t}\right) \in \Gamma(T M)$.

Un campo vectorial $X$ a lo largo de una curva $c(t)$ se dice paralelo si $\frac{\mathrm{D} X}{d t}=0$ idénticamente.

En general, dada una curva $c$ sobre una variedad diferenciable dotada de una conexión y un vector tangente $X_{0}$ en $c\left(t_{0}\right)$, existe un campo de vectores paralelo $X$ a lo largo de $c$ y sólo uno que extienda al vector $X_{0}$. Las componentes de dicho campo $X$ serán, lógicamente, las soluciones del sistema de ecuaciones:

$$
\frac{d X^{k}}{d t}+\Gamma_{i j}^{k} \frac{d c^{i}}{d t} X^{j}=0
$$


con las condiciones iniciales $X_{0}^{k}$.

El vector $X_{t}$ será el traslado paralelo de $X_{0}$ a lo largo de $c$.

Conexiones métricas y libres de torsión. Llamaremos en general conexión en una variedad riemanniana $M$ a toda conexión definida sobre su fibrado tensorial. Definición C.2. Una conexión $\nabla$ definida en una variedad riemanniana $M$ se dice "compatible con la métrica" o simplemente conexión-métrica si el traslado paralelo conserva el producto escalar.

Esto equivale a decir que para cualquier curva en $M$ y cualquier par de campos vectoriales $X$ e $Y$ paralelos a lo largo de $c$, el producto escalar $\langle X, Y\rangle$ es constante $\forall t \in\left[t_{1}, t_{2}\right]$.

La condición de conexión-métrica tiene las siguientes consecuencias fáciles de demostrar:

- Con la notación de la Definición C.2:

$$
\frac{d}{d t}\langle X, Y\rangle=\left\langle\frac{\mathrm{D} X}{d t}, Y\right\rangle+\left\langle X, \frac{\mathrm{D} Y}{d t}\right\rangle
$$

- De manera general:

$$
X(\langle Y, Z\rangle)=\left\langle\nabla_{X} Y, Z\right\rangle+\left\langle Y, \nabla_{X} Z\right\rangle, \quad \forall X, Y, Z \in \Gamma(T M)
$$

- Si $g$ es la métrica riemanniana definida en $T M$, es decir: $\langle X, Y\rangle=g_{i j} X^{i} Y^{j}$, entonces:

$$
\nabla g=0
$$

es decir, la diferencial covariante del tensor $g$ es nula idénticamente ( $g$ es paralela con respecto a $\nabla)$.

Definición C.3. Dada una variedad $M$ con conexión $\nabla$ sobre $T M$, se define el tensor de torsión $T_{\nabla}$ de la forma:

$$
T_{\nabla}(X, Y)=\nabla_{X} Y-\nabla_{Y} X-[X, Y]
$$

para $X, Y \in \Gamma(T M)$.

Se dice que la conexión $\nabla$ es libre de torsión o conexión simétrica si se verifica que $T_{\nabla} \equiv 0$ idénticamente.

Para las conexiones libres de torsión, si $X$ e $Y$ son dos campos vectoriales cuyo paréntesis de Lie se anule, es evidente que

$$
\nabla_{X} Y=\nabla_{Y} X
$$


En particular, si $s: \mathbb{R}^{2} \rightarrow M, s(u, v) \in M$, es una superficie (parametrizada) sobre una variedad riemanniana y denotamos, de manera similar al caso de las curvas, $\frac{\partial}{\partial u} \equiv s_{*}\left(\frac{\partial}{\partial u}\right) \equiv \frac{\partial s}{\partial u}$ y $\frac{\partial}{\partial v} \equiv s_{*}\left(\frac{\partial}{\partial v}\right) \equiv \frac{\partial s}{\partial v}$, tendremos que

$$
\frac{\mathrm{D}}{\partial u} \frac{\partial s}{\partial v}=\frac{\mathrm{D}}{\partial v} \frac{\partial s}{\partial u}
$$

como consecuencia directa de que $\left[\frac{\partial}{\partial u}, \frac{\partial}{\partial v}\right]=0$, y donde $\frac{\mathrm{D}}{\partial u}$ y $\frac{\mathrm{D}}{\partial v}$ denotan a las derivadas covariantes sobre la superficie $s$, generalización evidente de la derivada covariante a lo largo de una curva: Si $X$ es un campo sobre $s$, es decir un campo que asocia a cada $(u, v) \in \mathbb{R}^{2}$ un vector $X_{(u, v)} \in T_{s(u, v)} M$, entonces, para $v=v_{0}$ fijo, $\left.X\right|_{s\left(u, v_{0}\right)}$ es un campo a lo largo de la curva $s\left(u, v_{0}\right)$ y se puede definir

$$
\frac{\mathrm{D} X}{\partial u} \equiv \frac{\left.\mathrm{D} X\right|_{s\left(u, v_{0}\right)}}{d u}
$$

y análogamente para $\frac{\mathrm{D} X}{\partial v}$.

Si denotamos $\dot{s}=\frac{\partial s}{\partial u}$ y $s^{\prime}=\frac{\partial s}{\partial v}$, entonces (C.12) se escribe en la forma:

$$
\nabla_{\dot{s}} s^{\prime}=\nabla_{s^{\prime}} \dot{s}
$$

Teorema Fundamental. Sea $M$ una variedad riemanniana, entonces existe una única conexión definida en $M$ que sea simultáneamente compatible con la métrica de $M$ y libre de torsión. Dicha conexión recibe usualmente el nombre de conexión de Levi-Civita.

Una consecuencia directa del Teorema anterior es que los símbolos de Christoffel de la conexión de Levi-Civita están determinados de manera única por las componentes del tensor métrico y sus derivadas. En un sistema de coordenadas locales:

$$
\Gamma_{i j}^{l}=\frac{1}{2} g^{k l}\left(\frac{\partial g_{j k}}{\partial x^{i}}+\frac{\partial g_{i k}}{\partial x^{j}}-\frac{\partial g_{i j}}{\partial x^{k}}\right)
$$

donde se observa trivialmente la simetría de $\Gamma_{i j}^{l}$ con respecto a los índices $i j$ (simetría derivada de ser la conexión libre de torsión).

Es fácil demostrar, por cálculo directo, que las derivadas de las componentes del tensor métrico se escriben de la forma:

$$
\frac{\partial g_{l p}}{\partial x^{j}}=g_{p i} \Gamma_{j l}^{i}+g_{l i} \Gamma_{j p}^{i}
$$

En el resto del Apéndice nos referiremos, siempre que no se especifique lo contrario, a la conexión de Levi-Civita. 


\section{Gradientes y Hessianos.}

Definición C.4. Dada una variedad riemanniana $(M, g)$, sea $\left(x^{1}, \ldots, x^{n}\right)$ un sistema de coordenadas locales en $M$. Se define el gradiente de toda función diferenciable $f \in C^{1}(M)$ como el campo vectorial $\operatorname{grad} f \in \Gamma(T M)$ siguiente:

$$
\operatorname{grad} f=g^{i j} \frac{\partial f}{\partial x^{j}} \frac{\partial}{\partial x^{i}}
$$

Es evidente, a partir de la definición, que si $X=X^{i} \frac{\partial}{\partial x^{i}}$ es un campo en $M$, entonces la derivada covariante de una función con respecto a $X$ no es más que la correspondiente derivada direccional:

$$
\nabla_{X} f=X(f)=\langle X, \operatorname{grad} f\rangle
$$

Definición C.5. Dada una función dos veces diferenciable $f: M \rightarrow \mathbb{R}(f \in$ $C^{2}(M)$ ) en una variedad riemanianna $(M, g)$, se llama Hessiano de $f$ a la forma bilineal $\nabla d f$. En un sistema de coordenadas locales $\left(x^{1}, \ldots, x^{N}\right)$ se tiene (según (C.5)):

$$
\nabla d f=\left(\frac{\partial^{2} f}{\partial x^{i} \partial x^{j}}-\frac{\partial f}{\partial x^{k}} \Gamma_{i j}^{k}\right) d x^{i} \otimes d x^{j}
$$

Proposición C.2. Sea $f$ una función dos veces diferenciable en una variedad riemanniana $(M, g)$, y sean $X$ e $Y$ dos campos vectoriales cualesquiera en $M$, entonces se verifica:

$$
\nabla d f(X, Y)=\left\langle\nabla_{X} \operatorname{grad}(f), Y\right\rangle=\left\langle\nabla_{Y} \operatorname{grad}(f), X\right\rangle
$$

Demostración. Es evidente a partir de la expresión (C.4) y de tratarse de la conexión de Levi-Civita que

$$
(\nabla d f)(Y ; X)=(\nabla d f)(X ; Y)
$$

De esta manera basta con demostrar la primera de las igualdades. De acuerdo con la definición de gradiente: $\partial_{k} f=g_{k p}(\operatorname{grad}(f))^{p}$, y así:

$$
\nabla d f(X, Y)=X^{j} Y^{l}\left(\frac{\partial}{\partial x^{j}}\left(g_{l p}(\operatorname{grad}(f))^{p}\right)-\Gamma_{j l}^{k} g_{k p}(\operatorname{grad}(f))^{p}\right)=
$$




$$
=X^{j} Y^{l}\left(\frac{\partial g_{l p}}{\partial x^{j}}(\operatorname{grad}(f))^{p}+g_{l p} \frac{\partial(\operatorname{grad}(f))^{p}}{\partial x^{j}}-\Gamma_{j l}^{k} g_{k p}(\operatorname{grad}(f))^{p}\right)
$$

que, utilizando (C.15), conduce a:

$$
X^{j} Y^{l} g_{l i}\left(\partial_{j}(\operatorname{grad}(f))^{i}+\Gamma_{j p}^{i}(\operatorname{grad}(f))^{p}\right)=\left\langle\nabla_{X} \operatorname{grad}(f), Y\right\rangle
$$

Q.E.D.

\section{C.2 Tensor de Curvatura}

Tal y como ya hemos comentado, nos restringimos a la conexión de Levi-Civita en una variedad riemanniana $(M, g)$, definimos en consecuencia el tensor de curvatura de dicha conexión, obviando la definición general de curvatura de una conexión cualquiera.

Definición C.6. El Tensor de Curvatura $R$ de la conexión de Levi-Civita $\nabla$ de una variedad riemanniana $(M, g)$ se define de la forma ${ }^{3}$ :

$$
R(X, Y) Z=-\nabla_{X}\left(\nabla_{Y} Z\right)+\nabla_{Y}\left(\nabla_{X} Z\right)+\nabla_{[X, Y]} Z
$$

para cualesquiera tres campos $X, Y, Z \in \Gamma(T M)$.

En un sistema de coordenadas locales en $M$, tomemos $X=X^{i} \frac{\partial}{\partial x^{i}}, Y=Y^{j} \frac{\partial}{\partial x^{j}}$, $Z=Z^{k} \frac{\partial}{\partial x^{k}}$. Entonces:

$$
R(X, Y) Z=X^{i} Y^{j} Z^{k} R\left(\frac{\partial}{\partial x^{i}}, \frac{\partial}{\partial x^{j}}\right) \frac{\partial}{\partial x^{k}}
$$

como consecuencia de las propiedades ya mostradas de la conexión. Es habitual denotar:

$$
R\left(\frac{\partial}{\partial x^{i}}, \frac{\partial}{\partial x^{j}}\right) \frac{\partial}{\partial x^{k}}=R_{i j k}^{l} \frac{\partial}{\partial x^{l}}
$$

de manera que, por cálculo directo, se obtiene:

$$
R_{i j k}^{l}=\frac{\partial \Gamma_{i k}^{l}}{\partial x^{j}}-\frac{\partial \Gamma_{j k}^{l}}{\partial x^{i}}+\Gamma_{i k}^{r} \Gamma_{j r}^{l}-\Gamma_{j k}^{r} \Gamma_{i r}^{l}
$$

Es usual asimismo la siguiente definición:

$$
R_{i j k l}=g_{h l} R_{i j k}^{h}
$$

\footnotetext{
${ }^{3}$ Existen dos criterios diferentes a la hora de definir $R$ según el signo que se considere, hemos seguido en este caso la notación de [70].
} 
que conduce a que

$$
R_{i j k l}=\left\langle R\left(\frac{\partial}{\partial x^{i}}, \frac{\partial}{\partial x^{j}}\right) \frac{\partial}{\partial x^{k}}, \frac{\partial}{\partial x^{l}}\right\rangle
$$

El tensor de curvatura de la conexión de Levi-Civita verifica las siguientes identidades:

$$
\begin{gathered}
R(X, Y) Z+R(Y, X) Z=0 ; \quad R_{i j k}^{l}=-R_{j i k}^{l} \\
R(X, Y) Z+R(Y, Z) X+R(Z, X) Y=0 \\
\langle R(X, Y) Z, W\rangle+\langle R(X, Y) W, Z\rangle=0 ; \quad R_{i j k l}=-R_{i j l k} \\
\langle R(X, Y) Z, W\rangle=\langle R(Z, W) X, Y\rangle ; \quad R_{i j k l}=R_{k l i j}
\end{gathered}
$$

Por último, si $X$ es un campo sobre una superficie $s(u, v)$ definida en $M$ (ver sección anterior), es trivial obtener la expresión:

$$
\frac{\mathrm{D}}{\partial v} \frac{\mathrm{D} X}{\partial u}-\frac{\mathrm{D}}{\partial u} \frac{\mathrm{D} X}{\partial v}=R\left(\frac{\partial s}{\partial u}, \frac{\partial s}{\partial v}\right) X
$$

Definición C.7. La curvatura seccional de un plano generado por los vectores tangentes $X, Y \in T_{p} M$ se define como:

$$
K(X, Y)=\langle R(X, Y) X, Y\rangle
$$

De esta manera, si definimos $K_{X}: T_{p} M \rightarrow T_{p} M$ como la transformación lineal $K_{X}(Y)=R(X, Y) X$, podremos expresar la curvatura seccional de la forma:

$$
K(X, Y)=\left\langle K_{X}(Y), Y\right\rangle
$$




\section{Apéndice D}

\section{Cálculo Variacional Aplicado a Geodésicas}

Hemos incluido un Apéndice dedicado al Cálculo Variacional, y particularmente a Cálculo Variacional aplicado a geodésicas, con el ánimo de resumir los elementos fundamentales del mismo que han sido utilizados en las demostraciones y razonamientos de los capítulos de la memoria. Por razones evidentes se restringe el apéndice al caso de funcionales expresables como una integral simple de una sóla variable en la que el integrando depende de una función (en general vectorial) y de su derivada primera. Asimismo nos centraremos en los casos de variaciones propias, es decir, en problemas variacionales con fronteras fijas.

De manera análoga al Apéndice $\mathrm{C}$, omitiremos las demostraciones de la mayor parte de los resultados aquí recogidos, que pueden encontrarse en multitud de textos sobre el tema. Las referencias fundamentales a las que nos remitimos serán [31], [84] y [70].

\section{D.1 Conceptos Básicos del Cálculo Variacional}

Como es bien sabido, el cálculo variacional estudia los métodos que permiten obtener los valores extremales de los funcionales.

El primero que se planteó el cálculo de variaciones fue Newton al determinar el sólido de revolución de resistencia mínima en un fluido (publicado en 1687 en su libro "Philosophiae Naturalis principia mathematica"). La verdadera formulación del problema, sin embargo, se debe a Johann Bernouilli, que en 1696 propuso a la atención de los matemáticos determinar la curva que debe recorrer un cuerpo 
pesado en el menor tiempo posible, es decir la línea de deslizamiento más rápido o braquistócrona. El propio Johann Bernouilli, Jakob Bernouilli, Newton, Leibnitz y L'Hôpital resolvieron el problema. El mismo Johann Bernouilli propuso, en 1697, el problema de calcular las líneas geodésicas o de menor longitud en una superficie dada, resuelto por él mismo en 1698 y de una manera general por L. Euler en 1728. Un tercer problema discutido por los hermanos Bernouilli (Johann y Jakob), el llamado problema isoperimétrico (determinación de la curva cerrada que contiene área máxima), cuya solución se conocía desde la Grecia Clásica, y que asimismo fue resuelto de manera general por Euler, contribuyó al desarrollo definitivo del Cálculo Variacional tal y como lo entendemos hoy en día. Puede citarse, por tanto, a Leonard Euler como el fundador del Cálculo Variacional y a J. Lagrange como el primero en establecer una técnica sistemática de resolución de los problemas variacionales. Hacia 1832 Gauss estudió el cálculo variacional para funcionales determinados por integrales dobles, necesario para sus estudios sobre la capilaridad, y Poisson extendió más tarde dichos estudios al caso de integrales de cualquier orden.

\section{D.2 Variaciones y Teorema Fundamental}

En una notación similar a la del Apéndice C, sea $c(t)$ una curva en una variedad riemanniana $(M, g)^{1}$.

$$
c:\left[t_{1}, t_{2}\right] \rightarrow M
$$

Definición D.1. Una variación de la curva $c(t)$ es toda función:

$$
\varphi:\left[t_{1}, t_{2}\right] \times(-\epsilon, \epsilon) \rightarrow M
$$

diferenciable y tal que

$$
\varphi(t, 0)=c(t), \quad \forall t \in\left[t_{1}, t_{2}\right]
$$

Evidentemente $\varphi(t, \xi)$ define una superficie en $M$.

Denominaremos variación propia (o variación con fronteras fijas) a aquélla que verifica:

$$
\varphi\left(t_{1}, \xi\right)=c\left(t_{1}\right), \quad \varphi\left(t_{2}, \xi\right)=c\left(t_{2}\right) ; \quad \forall \xi \in(-\epsilon, \epsilon)
$$

\footnotetext{
${ }^{1}$ Entenderemos por curva, si no se especifica lo contrario, a toda función de clase $C^{\infty}$ dependiente de un parámetro real en la variedad $(M, g)$.
} 
En cada punto $\varphi(t, \xi)$ de $M$, la variación define dos vectores tangentes: $\frac{\partial \varphi}{\partial t}(t, \xi)$ y $\frac{\partial \varphi}{\partial \xi}(t, \xi)$. En particular, sobre cada punto de la curva $c(t)$ tendremos los vectores: $\frac{\partial \varphi}{\partial t}(t, 0)$ y $\frac{\partial \varphi}{\partial \xi}(t, 0)$.

$\frac{\partial \varphi}{\partial t}(t, 0)$ no es más que el vector tangente $\dot{c}(t)$ a la curva en $c(t)$, mientras que $\frac{\partial \varphi}{\partial \xi}(t, 0)$ será un campo vectorial sobre la curva al que se denomina campo asociado a la variación ${ }^{2} \varphi$. No es difícil demostrar la proposición siguiente (ver [84]):

Proposición D.1. Si $\varphi$ es una variación propia de la curva $c(t)$, entonces se verifica:

$$
\frac{\partial \varphi}{\partial \xi}\left(t_{1}, 0\right)=\frac{\partial \varphi}{\partial \xi}\left(t_{2}, 0\right)=0
$$

Es cierta además la proposición recíproca:

Si $X$ es un campo sobre la curva $c(t)$ tal que $X\left(t_{1}\right)=X\left(t_{2}\right)=0$, entonces existe una variación propia de $c(t)$ definida en $\left[t_{1}, t_{2}\right] \times\left(-\epsilon_{0}, \epsilon_{0}\right)$ (para algún $\left.\epsilon_{0}\right)$ tal que $X$ sea su campo vectorial asociado.

Con el fin de simplificar la notación, utilizaremos la siguiente equivalencia:

$$
c(t, \xi) \equiv \varphi(t, \xi)
$$

de manera que denotaremos:

$$
\begin{aligned}
\dot{c}(t, \xi) & \equiv \frac{\partial \varphi}{\partial t}(t, \xi) ; & \dot{c}(t) & \equiv \frac{\partial \varphi}{\partial t}(t, 0) \\
V(t, \xi) & \equiv \frac{\partial \varphi}{\partial \xi}(t, \xi) ; & V(t) & \equiv \frac{\partial \varphi}{\partial \xi}(t, 0)
\end{aligned}
$$

Completamos la presentación de los ingredientes básicos del Cálculo de Variaciones aplicado a geodésicas con el Teorema Fundamental del Cálculo Variacional (ver por ejemplo [31] para la demostración):

Teorema Fundamental. Si para cada función continua $\eta(x)$ se tiene:

$$
\int_{x_{1}}^{x_{2}} d x \Phi(x) \eta(x)=0
$$

siendo $\Phi(x)$ una función continua en el intervalo $\left[x_{1}, x_{2}\right]$, entonces se verifica:

$$
\Phi(x) \equiv 0
$$

$\forall x \in\left[x_{1}, x_{2}\right]$.

\footnotetext{
${ }^{2}$ Tal y como se especificó en el Apéndice $\mathrm{C}$, se utiliza en la práctica un abuso de notación frecuente al identificar $\dot{c}(t) \equiv \frac{d}{d t} \equiv c_{*}\left(\frac{d}{d t}\right)$.
} 


\section{D.3 Cálculo Variacional aplicado a Geodésicas}

Energía y Longitud. Consideremos el espacio de curvas de la variedad $(M, g)$ que pasan por los puntos $P$ y $Q$ de $M, \Omega_{P}^{Q}$. Para cada curva $c \in \Omega_{P}^{Q}$ :

$$
c:\left[t_{1}, t_{2}\right] \rightarrow M ; \quad c\left(t_{1}\right)=P, c\left(t_{2}\right)=Q
$$

se define el funcional de energía, $E[c]$, de la forma:

$$
E[c]=\int_{t_{1}}^{t_{2}} d t \frac{1}{2}\langle\dot{c}(t), \dot{c}(t)\rangle
$$

donde $\dot{c}(t) \in T_{c(t)} M$ es el vector tangente a la curva en $c(t)$. En un sistema de coordenadas locales en $M$, de forma que $c(t)=\left(x^{1}(t), \ldots, x^{n}(t)\right)$, tendremos:

$$
\dot{c}(t)=\frac{d x^{i}}{d t} \frac{\partial}{\partial x^{i}}=\dot{x}^{i} \frac{\partial}{\partial x^{i}}
$$

El funcional de longitud se define de manera análoga como:

$$
L[c]=\int_{t_{1}}^{t_{2}} d t\langle\dot{c}(t), \dot{c}(t)\rangle^{\frac{1}{2}}=\int_{t_{1}}^{t_{2}}\|\dot{c}\| d t
$$

Reparametrizaciones. El funcional de longitud es invariante bajo reparametrizaciones de la curva, algo que no ocurre con el de energía.

Proposición D.2. Sea $\phi=\phi(t)$ una reparametrización admisible ${ }^{3}$ de la curva $c(t)$, entonces $L[c(t)]=L[c(\phi)]$.

El funcional de longitud nos permite construir el parámetro-arco para cada curva $c(t)$ de $\Omega_{P}^{Q}$ :

$$
s(t)=\int_{t_{1}}^{t} d t\langle\dot{c}(t), \dot{c}(t)\rangle^{\frac{1}{2}}
$$

para cada $t \in\left[t_{1}, t_{2}\right]^{4}$. Evidentemente $s\left(t_{2}\right)=L[c]$. Aplicando el Teorema Fundamental del Cálculo tenemos:

$$
d s=\langle\dot{c}(t), \dot{c}(t)\rangle^{\frac{1}{2}} d t
$$

de manera que, para curvas regulares (es decir, tales que $\dot{c}(t) \neq 0, \forall t \in\left[t_{1}, t_{2}\right]$ ), el parámetro-arco será una reparametrización admisible. En estas condiciones:

$$
L[c]=\int_{0}^{s\left(t_{2}\right)} d s
$$

\footnotetext{
${ }^{3}$ Entenderemos por reparametrización admisible aquella tal que $\phi^{\prime}(t) \neq 0, \forall t \in\left[t_{1}, t_{2}\right]$.

${ }^{4}$ Una notación más rigurosa, pero engorrosa, sería $s_{c}(t)$.
} 
y es trivial demostrar la proposición:

Proposición D.3. Sea $c(s)$ una curva definida en una variedad riemanniana $M$ y tal que el parámetro $s \in\left[s_{1}, s_{2}\right]$ es la longitud de arco, entonces

$$
\left\|c^{\prime}(s)\right\|=\left\langle c^{\prime}(s), c^{\prime}(s)\right\rangle^{\frac{1}{2}}=1
$$

$\forall s \in\left[s_{1}, s_{2}\right]$.

\section{Variación primera de $E$ y $L$.}

El teorema que presentamos a continuación permite caracterizar las curvas geodésicas de la variedad $M$ como extremales de los funcionales anteriormente definidos con respecto a variaciones propias consideradas en el espacio de curvas $\Omega$.

Teorema D.5. Para cada curva $c$ en $M$, las funciones $E[c](\xi)$ y $L[c](\xi)$ definidas de la forma:

$$
\begin{aligned}
& E[c](\xi)=\int_{t_{1}}^{t_{2}} d t \frac{1}{2}\langle\dot{c}(t, \xi), \dot{c}(t, \xi)\rangle \\
& L[c](\xi)=\int_{t_{1}}^{t_{2}} d t\langle\dot{c}(t, \xi), \dot{c}(t, \xi)\rangle^{\frac{1}{2}}
\end{aligned}
$$

son diferenciables con respecto a $\xi$ y además:

$$
\begin{gathered}
\frac{d}{d \xi} E[c](0)=\left.\left\langle c^{\prime}, \dot{c}\right\rangle\right|_{t=t_{1}, \xi=0} ^{t=t_{2}, \xi=0}-\int_{t_{1}}^{t_{2}} d t\left\langle\frac{\mathrm{D}}{\partial t} \dot{c}(t, 0), c^{\prime}(t, 0)\right\rangle \\
\frac{d}{d \xi} L[c](0)=\int_{t_{1}}^{t_{2}} d t\left(\frac{\frac{\partial}{\partial t}\left\langle c^{\prime}, \dot{c}\right\rangle}{\langle\dot{c}, \dot{c}\rangle^{\frac{1}{2}}}-\frac{\left\langle c^{\prime}, \frac{\mathrm{D}}{\partial t} \dot{c}\right\rangle}{\langle\dot{c}, \dot{c}\rangle^{\frac{1}{2}}}\right)
\end{gathered}
$$

La demostración, en el caso del funcional de energía, es similar a la correspondiente a la Proposición 4.1, del Capítulo 4, relativa a la variación primera del funcional de acción de un sistema dinámico. Para el funcional de longitud se procede de manera análoga (ver por ejemplo [57] y [70]).

Para el caso de variaciones propias, el teorema anterior conduce a la siguiente expresión de la variación de la energía:

$$
\frac{d}{d \xi} E[c](0)=-\int_{t_{1}}^{t_{2}} d t\left\langle\frac{\mathrm{D}}{\partial t} \dot{c}(t), c^{\prime}(t)\right\rangle
$$

La condición de extremal, $\frac{d}{d \xi} E[c](0)=0$, según el Teorema Fundamental antes expuesto, se verifica si y sólo si

$$
\frac{\mathrm{D}}{\partial t} \dot{c}(t)=0
$$


es decir, si la curva es autoparalela o geodésica. Queda demostrado, por tanto, que toda curva extremal del funcional de energía es una geodésica de la variedad riemanniana, y recíprocamente.

Si consideramos curvas parametrizadas en función del arco de longitud, la variación de la longitud (para variaciones propias) conduce a idéntico resultado. Llegamos así a la siguiente conclusión: Las curvas extremales del funcional energía son las curvas autoparalelas de la conexión de Levi-Civita (a las que denominamos geodésicas $^{5}$ ) y son, a su vez, curvas extremales del funcional de longitud siendo su parámetro la longitud de arco. En este punto, el lenguaje de la Dinámica es clarificador: Los extremales de la energía son las curvas de longitud extremal recorridas a velocidad constante (parametrizadas en función del arco o funciones afines del arco, es decir $\left\|\frac{d c}{d t}\right\|=$ cte $\mathrm{y}$, por tanto, $t=a s+b$, con $a$ y $b$ constantes arbitrarias), mientras que los extremales del funcional longitud son las curvas de longitud extremal, independientemente de la velocidad con la que son recorridas.

No es difícil demostrar ${ }^{6}$ que los extremales del funcional longitud (en cualquier parametrización) satisfacen las ecuaciones:

$$
\frac{\mathrm{D} c(t)}{d t}=\lambda \dot{c}(t)
$$

donde $\lambda=-\frac{d^{2} t}{d s^{2}} \cdot\left(\frac{d s}{d t}\right)^{2}$, siendo $t$ el parámetro del que depende la curva y $s$ el arco de longitud sobre dicha curva. Es ahora evidente que para el caso de parametrizaciones afines, las ecuaciones escritas son exactamente las de las curvas geodésicas.

\section{D.4 Desviación Geodésica}

Si tomamos ahora una curva geodésica $c(t)$ en $M$ (curva que une los puntos $c\left(t_{0}\right)$ y $\left.c\left(t_{1}\right)\right)$, podemos calcular la variación segunda de los funcionales $E$ y $L:^{7}$

Teorema D.6. Sea $c:\left[t_{1}, t_{2}\right] \rightarrow M$ una curva geodésica, entonces la variación segunda del funcional energía sobre dicha curva es:

$$
\frac{d^{2}}{d \xi^{2}} E[c](0)=-\int_{t_{1}}^{t_{2}} d t\left\langle\frac{\mathrm{D}^{2} c^{\prime}}{\partial t^{2}}+R\left(\dot{c}, c^{\prime}\right) \dot{c}, c^{\prime}\right\rangle+
$$

\footnotetext{
${ }^{5}$ Conviene precisar que la definición de geodésica no es única, algunos autores llaman curvas geodésicas a los extremales del funcional de longitud, independientemente de la parametrización que se considere. Tal y como estamos demostrando, ambas definiciones no son totalmente equivalentes.

${ }^{6}$ Sustituyendo $s=s(t)$ en las ecuaciones $\frac{\mathrm{D} c}{d s}=0$ y aplicando la regla de la cadena.

${ }^{7}$ Milnor realiza este cálculo utilizando una variación dos-paramétrica de los funcionales, los resultados son equivalentes a los mostrados aquí.
} 


$$
+\left.\left\langle\frac{\mathrm{D} c^{\prime}}{\partial \xi}, \dot{c}\right\rangle\right|_{t_{1}} ^{t_{2}}+\left.\left\langle c^{\prime}, \frac{\mathrm{D} \dot{c}}{\partial \xi}\right\rangle\right|_{t_{1}} ^{t_{2}}
$$

La variación segunda del funcional de longitud requiere un cálculo más detallado:

Teorema D.7. Sea $c:\left[t_{1}, t_{2}\right] \rightarrow M$ una curva geodésica donde además $t$ es una parametrización afín $(t=a s+b$ siendo $s$ el parámetro arco), entonces la variación segunda del funcional longitud sobre dicha curva es:

$$
\begin{aligned}
\frac{d^{2} L}{d \xi^{2}}(0)= & \frac{1}{\|\dot{c}\|}\left(-\int_{t_{1}}^{t_{2}} d t\left\langle\frac{\mathrm{D}^{2} c^{\perp}}{\partial t^{2}}+R\left(\dot{c}, c^{\prime \perp}\right) \dot{c}, c^{\perp \perp}\right\rangle\right. \\
& \left.+\left.\left(\left\langle\frac{\mathrm{D} c^{\prime}}{\partial \xi}, \dot{c}\right\rangle+\left\langle\frac{\mathrm{D} c^{\prime \perp}}{\partial t}, c^{\prime \perp}\right\rangle\right)\right|_{t_{1}} ^{t_{2}}\right)
\end{aligned}
$$

donde $c^{\perp}$ es la componente de $c^{\prime}$ ortogonal a $\dot{c}$, es decir:

$$
c^{\perp}=c^{\prime}-\left\langle\frac{\dot{c}}{\|\dot{c}\|}, c^{\prime}\right\rangle \frac{\dot{c}}{\|\dot{c}\|}
$$

El operador $\frac{\mathrm{D}^{2}}{\partial t^{2}}+R(\dot{c}, \cdot) \dot{c}$ recibe el nombre de "operador de desviación geodésica". Los campos pertenecientes a su núcleo son llamados, a su vez, campos de Jacobi de la variedad.

\section{D.5 Teoría de Morse}

Finalizaremos este apéndice con una breve exposición de los aspectos fundamentales de la Teoría de Morse sobre espacios de curvas cerradas.

Sea $\Omega_{m_{0}}=\left\{\gamma: S^{1} \rightarrow M / \gamma(0)=\gamma(1)=m_{0}\right\}$ el espacio de caminos cerrados con un punto-base fijo $m_{0}$ en una variedad riemanniana $n$-dimensional $M$. La teoría de Morse establece una relación entre las propiedades topológicas del espacio $\Omega_{m_{0}}$ y las características de los puntos críticos del funcional de longitud $L=$ $\int\|\dot{\gamma}\| d t$ definido en $\Omega_{m_{0}}$, es decir, las curvas geodésicas cerradas $\gamma_{c}$ en $M$ (para detalles ver por ejemplo [70] y [74]). Plantearemos a continuación una versión de la teoría de Morse a la manera de Bott [14].

Sea

$$
P_{t}\left(\Omega_{m_{0}}\right)=\sum_{k=0}^{\infty} b_{k} t^{k}
$$


el polinomio de Poincaré de $\Omega_{m_{0}}$ en la incógnita $t$. Los coeficientes $b_{k}$ de dicho polinomio no son más que los números de Betti de $\Omega_{m_{0}}$, es decir, las dimensiones de los grupos de homología de dicho espacio:

$$
b_{k}=\operatorname{dim} H_{k}\left(\Omega_{m_{0}}, \mathbb{R}\right)
$$

y, por tanto, la topología de $\Omega_{m_{0}}$ está determinada por $P_{t}\left(\Omega_{m_{0}}\right)$.

Sea

$$
\mathcal{M}_{t}(L)=\sum_{N_{c}} P_{t}\left(N_{c}\right) t^{\mu\left(N_{c}\right)}
$$

el polinomio de Morse del funcional $L$. Por su parte $\mathcal{M}_{t}(L)$ codifica la estructura de las curvas críticas de $L$ puesto que la suma se lleva a cabo sobre las variedades críticas, $N_{c}$, formadas, según sean las características geométricas de la variedad $M$, bien por una geodésica aislada, bien por un conjunto continuo de geodésicas. $P_{t}\left(N_{c}\right)$ es el polinomio de Poincaré de $N_{c}$ y $\mu\left(N_{c}\right)$ es el índice de Morse de la variedad crítica $N_{c}$, que se define de la forma:

$\mu\left(N_{c}\right)$ es la dimensión del subespacio del espacio tangente $T_{\gamma_{c}} \Omega_{m_{0}}$ en el cual la forma cuadrática Hessiano de $L$ en $\gamma_{c} \in N_{c}$ es definida negativa en las direcciones ortogonales a $N_{c}$.

Las desigualdades de Morse

$$
\mathcal{M}_{t}(L) \geq P_{t}\left(\Omega_{m_{0}}\right)
$$

informan de que las características de la topología de $\Omega_{m_{0}}$ imponen la existencia de muchos de los puntos críticos de $L$.

El índice de Morse puede ser entendido, de manera equivalente, como el número de autovalores negativos del operador de desviación geodésica y, por tanto, $\mu\left(N_{c}\right)$ nos informa de la estabilidad o inestabilidad de una geodésica dada $\gamma_{c} \in N_{c}$. Por otra parte, este hecho nos permite evitar la resolución del problema espectral (en general difícil) calculando el índice, $\mu\left(N_{c}\right)$, por aplicación directa del Teorema del Índice de Morse:

Teorema del Índice de Morse. El índice de Morse de una geodésica $\gamma_{c} \in \Omega_{m_{0}}$ es igual al número de puntos conjugados al punto base $m_{0}$ por los que pasa $\gamma_{c}$, contados con su multiplicidad.

Recordemos entonces que se llama punto conjugado o focal de $m_{0}$ a todo punto de $M^{n}$ tal que existen infinitas geodésicas de $\Omega_{m_{0}}$ (y por tanto, que pasan por $m_{0}$ ) que pasan por el mismo. Este enunciado es equivalente a decir que existe un campo de Jacobi (es decir un campo solución de la Ecuación de Jacobi, y por 
tanto perteneciente al núcleo del operador de desviación geodésica) no trivial que se anula simultáneamente en $m_{0}$ y en el punto en cuestión. La multiplicidad del punto conjugado viene determinada por la dimensión de una sección transversal a la congruencia de geodésicas cerca del punto focal (ver [52], [67] y [68] para detalles). Equivalentemente, la multiplicidad será igual al número de parámetros independientes de que depende el conjunto infinito de geodésicas de la congruencia. 


\section{Apéndice E}

\section{Correcciones cuánticas a la masa de Kinks}

En este Apéndice calcularemos la energía del estado fundamental, en el sector topológico del Modelo Sigma $O(3)$ Lineal Deformado, al primer orden en la expansión semi-clásica. Como es bien sabido, los kinks estables, en modelos con un sólo campo escalar, dan lugar a un estado cuántico que es el fundamental en el sector topológico del sistema, y a una "torre" de estados excitados sobre él, con propiedades muy interesantes (ver [87] para detalles). La generalización a modelos con varios campos escalares no existe prácticamente en la literatura, para conseguirla, se han de salvar dos dificultades: a) Identificar los kinks estables, un problema mucho más difícil que en el caso $N=1$, y que ha sido discutido profusamente en la Memoria. b) Relacionado con él, manejar el espectro del operador de segunda variación (Hessiano) aunque sea mediante técnicas asintóticas.

El método que utilizaremos está desarrollado de forma completa en la Referencia [30] y sintetizado en [8]. Nos limitaremos a continuación a exponer la fórmula que proporciona la corrección cuántica y a calcular cada uno de sus términos. En cualquier caso, de cara a obtener un estado propio del Hamiltoniano del sistema cuántico a partir de una solución clásica, uno debe partir de un kink estable. De acuerdo con la elección que hemos realizado en la Memoria para los parámetros de deformación: $1>\sigma_{3}^{2}>\sigma_{2}^{2}$, el único candidato a dar lugar a un buen autoestado es, por tanto, el kink TK2 $\sigma_{2}$. Consideraremos, sin embargo, las siguientes situaciones: 1) $\sigma_{3}^{2}>\sigma_{2}^{2}>1$, en la que el kink TK1 es estable y no existen los kinks TK2 $\sigma_{2}$ y TK $\left.2 \sigma_{3} ; 2\right) \sigma_{3}^{2}>1>\sigma_{2}^{2}$, es decir, TK1 inestable, TK2 $\sigma_{2}$ estable, y no existe el TK2 $\sigma_{3}$; y 3) $1>\sigma_{3}^{2}>\sigma_{2}^{2}$, existen los tres, y sólo el kink TK $2 \sigma_{2}$ es estable. La consideración de estas tres posibilidares resultará, 
como veremos, muy iluminadora.

La aproximación semi-clásica a la energía del estado fundamental en el sector topológico se escribe en la forma (ver [87]):

$$
E_{q}\left[\vec{\phi}_{K}\right]=E_{c}\left[\vec{\phi}_{K}\right]+\frac{\hbar}{2}\left\{\varepsilon\left[\vec{\phi}_{K}\right]+\delta_{M} \varepsilon\left[\vec{\phi}_{K}\right]+\delta_{F} \varepsilon\left[\vec{\phi}_{K}\right]\right\}+\vartheta\left(\hbar^{2}\right)
$$

Donde

a)

$$
\varepsilon\left[\vec{\phi}_{K}\right]=\operatorname{Tr} \Delta^{\frac{1}{2}}\left[\vec{\phi}_{K}\right]-\operatorname{Tr} \Delta^{\frac{1}{2}}\left[\vec{\phi}_{V}\right]
$$

es la energía debida a la incertidumbre en los modos de fluctuación en torno al kink, medida respecto a la misma cantidad en el sector de vacío. $\Delta\left[\vec{\phi}_{K}\right]$ y $\Delta\left[\vec{\phi}_{V}\right]$ son pues los operadores segunda variación (Hessianos) en torno a las soluciones clásicas, $\vec{\phi}_{K}$ y $\vec{\phi}_{V}$. Si definimos la función zeta generalizada asociada a un operador $A$ que actúa en un espacio de Hilbert como la serie formal, [45]:

$$
\zeta_{A}(s)=\sum_{n} \frac{1}{\lambda_{n}^{s}}, \quad s \in \mathbb{C}
$$

donde $\lambda_{n}$ son sus autovalores, podemos escribir:

$$
\varepsilon\left[\vec{\phi}_{K}\right]=\zeta_{\Delta\left[\vec{\phi}_{K}\right]}\left(\frac{-1}{2}\right)-\zeta_{\Delta\left[\vec{\phi}_{V}\right]}\left(\frac{-1}{2}\right)
$$

b)

$$
\delta_{M} \varepsilon\left[\vec{\phi}_{K}\right]=-\frac{1}{4 \pi} \sum_{a=1}^{3} \zeta_{\Delta_{a}\left[\vec{\phi}_{v}\right]}\left(\frac{1}{2}\right) \int_{-\infty}^{\infty} d x \Lambda_{a}\left(\vec{\phi}_{K}\right)
$$

se debe a la contribución de los contra-términos, que han de ser introducidos de cara a renormalizar la masa, es decir:

$$
\Lambda_{a}\left(\vec{\phi}_{K}\right)=\frac{\delta^{2} U}{\delta \phi_{a} \delta \phi_{a}}\left(\vec{\phi}_{K}\right)-\frac{\delta^{2} U}{\delta \phi_{a} \delta \phi_{a}}\left(\vec{\phi}_{V}\right)
$$

y

$$
\Delta_{a}\left(\vec{\phi}_{K}\right)=-\frac{d^{2}}{d x^{2}}+\frac{\delta^{2} U}{\delta \phi_{a} \delta \phi_{a}}\left(\vec{\phi}_{V}\right)=-\frac{d^{2}}{d x^{2}}+\bar{\Lambda}_{a}\left(\vec{\phi}_{V}\right)
$$

c)

$$
\delta_{F} \varepsilon\left[\vec{\phi}_{K}\right]=\frac{1}{2 \pi} \int_{-\infty}^{\infty} d x \sum_{a=1}^{3} \Lambda_{a}\left(\vec{\phi}_{K}\right)
$$


compensa el hecho de que usualmente $\Delta\left(\vec{\phi}_{K}\right)$ tiene más autofunciones, modos de fluctuación, que $\Delta\left(\vec{\phi}_{V}\right)$, y las constantes cosmológicas en el sector topológico y en el vacío deben renormalizarse de distinta forma ${ }^{1}$.

El problema con el que nos encontramos al considerar la fórmula (E.1) es que las cantidades: $\zeta_{\Delta\left[\vec{\phi}_{K}\right]}\left(-\frac{1}{2}\right), \zeta_{\Delta\left[\vec{\phi}_{V}\right]}\left(-\frac{1}{2}\right)$ y $\zeta_{\Delta\left[\vec{\phi}_{V}\right]}\left(\frac{1}{2}\right)$, que entran en la definición de $E_{q}\left[\vec{\phi}_{K}\right]$, son estrictamente divergentes. En vez de utilizar el método convencional de imponer un corte ultravioleta, bien en la energía, bien en el número de modos, usaremos el método de la función zeta generalizada para regularizar las cantidades divergentes. La idea es tomar el valor de $\zeta_{\Delta}(s)$ como definición, donde $s$ es un punto del plano complejo que no es un polo de $\zeta_{\Delta}$, y, posteriormente, tomar el límite $s \rightarrow-\frac{1}{2}$ y $s \rightarrow \frac{1}{2}$ para obtener una respuesta finita, ver [30].

Para ello, es conveniente usar la definición de la función zeta como la transformada de Mellin de la función del calor

$$
\begin{aligned}
\zeta_{\Delta}(s) & =\frac{1}{\Gamma(s)} \int_{0}^{\infty} d \beta \beta^{s-1} \operatorname{Tr}\left(e^{-\beta \Delta}\right) \\
\zeta_{\Delta}^{*}(s) & =\frac{1}{\Gamma(s)} \int_{0}^{\infty} d \beta \beta^{s-1}\left[\operatorname{Tr}\left(e^{-\beta \Delta}\right)-m_{0}\right]
\end{aligned}
$$

En la segunda fórmula, la contribución de los $m_{0}$ modos cero ha sido sustraida. $\operatorname{Tr}\left(e^{-\beta \Delta}\right)$ es la traza del núcleo de la ecuación del calor. Además, $\Delta$ es de la forma:

$$
\Delta_{a b}=-\frac{d^{2}}{d x^{2}} \delta_{a b}+V_{a b}(x)
$$

$\mathrm{y}$, por ello, admite una expansión asintótica si $\beta<1$

$$
\operatorname{Tr}\left(e^{-\beta \Delta}\right)=\frac{1}{\sqrt{4 \pi}} \sum_{n<n_{0}} a_{n}(\Delta) \beta^{n-\frac{1}{2}}+\vartheta\left(\beta^{n_{0}-\frac{1}{2}}\right)
$$

a partir de la cual se determina la estructura de $\zeta_{\Delta}$ como función meromorfa de $s$. Los coeficientes $a_{n}(\Delta)$ son

$$
a_{n}(\Delta)=\sum_{a=1}^{3} \int d x a_{n}^{a a}(x, x)
$$

y se calculan de modo recurrente en términos de $V_{a b}$.

${ }^{1}$ En realidad, la expresión de $\delta_{F}$ es la siguiente:

$$
\delta_{F} \varepsilon\left[\vec{\phi}_{K}\right]=\frac{1}{2 \pi} \int_{-\infty}^{\infty} d x\left[\sum_{a, b=1}^{3}\left\{\frac{\delta^{2} U}{\delta \phi_{a} \delta \phi_{b}}\left(\vec{\phi}_{K}\right)-\frac{\delta^{2} U}{\delta \phi_{a} \delta \phi_{b}}\left(\vec{\phi}_{V}\right)\right\}\right]
$$

pero en todos los casos que consideraremos los términos no diagonales bien son nulos, bien proporcionan contribución nula a la integral. 


\section{E.1 Kinks cuánticos en el Modelo Sigma $O(3)$ Li- neal Deformado}

Si usamos variables sin dimensiones en el modo elegido en la Sección 2.2, el potencial de la teoría de campos es

$$
V\left(\phi_{1}, \phi_{2}, \phi_{3}\right)=\frac{1}{2}\left(\phi_{1}^{2}+\phi_{2}^{2}+\phi_{3}^{2}-1\right)^{2}+\frac{\sigma_{2}^{2}}{2} \phi_{2}^{2}+\frac{\sigma_{3}^{2}}{2} \phi_{3}^{2}
$$

En cualquiera de las configuraciones de vacío, $\vec{\phi}_{V}^{ \pm}= \pm \vec{e}_{1}$, el Hessiano $\Delta\left[\vec{\phi}_{V}\right]=$ $\Delta^{0}$ es:

$$
\Delta^{0} \equiv-\frac{d^{2}}{d x^{2}} \delta_{a b}+V_{a b}^{0}
$$

con:

$$
V_{11}^{0}=4, \quad V_{22}^{0}=\sigma_{2}^{2}, \quad V_{33}^{0}=\sigma_{3}^{2}
$$

y $V_{a b}^{0}=0$ si $a \neq b$.

Recuperar las dimensiones correctas requiere multiplicar $\zeta_{\Delta^{0}}\left(-\frac{1}{2}\right)$ por $\left(\frac{2}{m^{2}}\right)^{-\frac{1}{2}}$ y para regularizar por el método de la función zeta es necesario introducir un parámetro $\mu$ con dimensiones de masa. Así, debemos considerar $\mu\left(\frac{2 \mu^{2}}{m^{2}}\right)^{s} \zeta_{\Delta^{0}}(s)$. Si ponemos el sistema en un intervalo de longitud sin dimensiones $m L$, es fácil concluir que

$$
\operatorname{Tr}\left(e^{-\beta \Delta^{0}}\right)=\frac{m L}{\sqrt{8 \pi \beta}}\left(e^{-4 \beta}+e^{-\sigma_{2}^{2} \beta}+e^{-\sigma_{3}^{2} \beta}\right)
$$

y de ahí:

$$
\zeta_{\Delta^{0}}(s)=\frac{m L}{\sqrt{8 \pi}} \frac{\Gamma\left(s-\frac{1}{2}\right)}{\Gamma(s)}\left(2^{1-2 s}+\sigma_{2}^{1-2 s}+\sigma_{3}^{1-2 s}\right)
$$

\section{E.1.1 El Kink TK1}

Estudiemos, en primer lugar, el rango $\sigma_{3}^{2}>\sigma_{2}^{2}>1$ en que el único kink existente es el kink topológico de una componente, que es además estable. Si denotamos por $\Delta\left[\vec{\phi}_{\mathrm{TK} 1}\right]=\Delta^{1}$ el Hessiano evaluado en la configuración TK1, resulta que

$$
\Delta_{a b}^{1}=-\frac{d^{2}}{d x^{2}} \delta_{a b}+V_{a b}^{1}
$$

con

$$
V_{11}^{1}=V_{11}^{0}-6 \operatorname{sech}^{2} x, \quad V_{22}^{1}=V_{22}^{0}-2 \operatorname{sech}^{2} x, \quad V_{33}^{1}=V_{33}^{0}-2 \operatorname{sech}^{2} x
$$


$V_{a b}^{1}=0$ si $a \neq b$. Por tanto:

$$
\begin{aligned}
\operatorname{Tr}\left(e^{-\beta \Delta^{1}}\right)-\operatorname{Tr}\left(e^{-\beta \Delta^{0}}\right)-1 & =e^{-3 \beta}+e^{-\left(\sigma_{2}^{2}-1\right) \beta}+e^{-\left(\sigma_{3}^{2}-1\right) \beta}+\operatorname{Erfc}[2 \sqrt{\beta}]+ \\
& +\left(e^{-3 \beta}+e^{-\left(\sigma_{2}^{2}-1\right) \beta}+e^{-\left(\sigma_{3}^{2}-1\right) \beta}\right) \operatorname{Erfc}[\sqrt{\beta}]
\end{aligned}
$$

De ahí se obtiene

$$
\begin{aligned}
& \zeta_{\Delta^{1}}^{*}(s)-\zeta_{\Delta^{0}}(s)=\frac{1}{\left(\sigma_{2}^{2}-1\right)^{s}}+\frac{1}{\left(\sigma_{3}^{2}-1\right)^{s}} \\
& -\frac{1}{\sqrt{\pi}} \frac{\Gamma\left(s+\frac{1}{2}\right)}{s \Gamma(s)}\left({ }_{2} F_{1}\left(s, s+\frac{1}{2}, s+1 ; \bar{\sigma}_{2}^{2}\right)+{ }_{2} F_{1}\left(s, s+\frac{1}{2}, s+1 ; \bar{\sigma}_{3}^{2}\right)\right)+ \\
& +\frac{1}{\sqrt{\pi}} \frac{\Gamma\left(s+\frac{1}{2}\right)}{\Gamma(s)}\left(\frac{2}{3^{s+\frac{1}{2}}}{ }_{2} F_{1}\left(\frac{1}{2}, s+\frac{1}{2}, \frac{3}{2} ;-\frac{1}{3}\right)-\frac{1}{4^{s} s}\right)
\end{aligned}
$$

y, por ello:

$$
\varepsilon\left[\vec{\phi}_{\mathrm{TK} 1}\right]=\lim _{s \rightarrow-\frac{1}{2}} \mu\left(\frac{2 \mu^{2}}{m^{2}}\right)^{s}\left(\zeta_{\Delta^{1}}^{*}(s)-\zeta_{\Delta^{0}}(s)\right)
$$

La contribución que proviene de los contratérminos que renormalizan la masa es:

$$
\begin{aligned}
& \delta_{M} \varepsilon\left[\vec{\phi}_{\mathrm{TK} 1}\right]= \\
& =\lim _{L \rightarrow \infty} \lim _{s \rightarrow \frac{1}{2}} \frac{2^{s}}{L \sqrt{2}}\left(\frac{\mu^{2}}{m^{2}}\right)^{s-\frac{1}{2}} \int_{-\frac{m L}{2 \sqrt{2}}}^{\frac{m L}{2 \sqrt{2}}} \frac{d x}{\cosh ^{2} x}\left(6 \zeta_{\Delta_{1}}(s)+2 \zeta_{\Delta_{2}}(s)+2 \zeta_{\Delta_{3}}(s)\right)= \\
& =\frac{10 m}{\sqrt{2 \pi}} \lim _{s \rightarrow \frac{1}{2}} \frac{\Gamma\left(s-\frac{1}{2}\right)}{\Gamma(s)}
\end{aligned}
$$

toda vez que

$$
\begin{aligned}
\zeta_{\Delta_{1}}(s) & =\frac{m L}{\sqrt{8 \pi}} \frac{1}{4^{s-\frac{1}{2}}} \frac{\Gamma\left(s-\frac{1}{2}\right)}{\Gamma(s)} \\
\zeta_{\Delta_{2}}(s) & =\frac{m L}{\sqrt{8 \pi}} \frac{1}{\left(\sigma_{2}^{2}\right)^{s-\frac{1}{2}}} \frac{\Gamma\left(s-\frac{1}{2}\right)}{\Gamma(s)} \\
\zeta_{\Delta_{3}}(s) & =\frac{m L}{\sqrt{8 \pi}} \frac{1}{\left(\sigma_{3}^{2}\right)^{s-\frac{1}{2}}} \frac{\Gamma\left(s-\frac{1}{2}\right)}{\Gamma(s)}
\end{aligned}
$$

El término corrector

$$
\delta_{F} \varepsilon\left[\vec{\phi}_{\mathrm{TK} 1}\right]=-\frac{m}{2 \pi \sqrt{2}} \int_{-\infty}^{\infty} d x \frac{10}{\cosh ^{2} x}=-\frac{10 m}{\pi \sqrt{2}}
$$


completa la fórmula de la masa del kink TK1:

$$
\begin{aligned}
& \frac{\hbar}{2}\left[\varepsilon\left[\vec{\phi}_{\mathrm{TK} 1}\right]+\delta_{M} \varepsilon\left[\vec{\phi}_{\mathrm{TK} 1}\right]+\delta_{F} \varepsilon\left[\vec{\phi}_{\mathrm{TK} 1}\right]\right]= \\
& =\frac{\hbar m}{2 \sqrt{2}}\left[\sqrt{\sigma_{2}^{2}-1}+\sqrt{\sigma_{3}^{2}-1}-\frac{1}{\sqrt{\pi}} \lim _{s \rightarrow-\frac{1}{2}} \frac{\Gamma\left(s+\frac{1}{2}\right)}{s \Gamma(s)} .\right. \\
& \left.\cdot\left({ }_{2} F_{1}\left(s, s+\frac{1}{2}, s+1 ; \bar{\sigma}_{2}^{2}\right)+{ }_{2} F_{1}\left(s, s+\frac{1}{2}, s+1 ; \bar{\sigma}_{3}^{2}\right)\right)\right] \\
& +\frac{\hbar m}{2 \sqrt{2}}\left[\frac{1}{\sqrt{\pi}} \lim _{s \rightarrow-\frac{1}{2}} \frac{\Gamma\left(s+\frac{1}{2}\right)}{\Gamma(s)} .\right. \\
& \left.\cdot\left(2{ }_{2} F_{1}\left(\frac{1}{2}, s+\frac{1}{2}, \frac{3}{2} ;-\frac{1}{3}\right)-\frac{1}{s}\right)+\frac{10}{\sqrt{\pi}}\left(\lim _{s \rightarrow \frac{1}{2}} \frac{\Gamma\left(s-\frac{1}{2}\right)}{\Gamma(s)}-\frac{1}{\sqrt{\pi}}\right)\right]= \\
& =\hbar m\left[\frac{1}{6} \sqrt{\frac{3}{2}}-\frac{5}{\pi \sqrt{2}}+\frac{\sqrt{\sigma_{2}^{2}-1}}{\pi \sqrt{2}} \operatorname{arcsen} \frac{1}{\sigma_{2}}+\frac{\sqrt{\sigma_{3}^{2}-1}}{\pi \sqrt{2}} \operatorname{arcsen} \frac{1}{\sigma_{3}}\right]
\end{aligned}
$$

Como cabría esperar, si $\sigma_{2}^{2}$ y/ó $\sigma_{3}^{2}$ son menores que 1 , hay términos imaginarios que indican que el estado TK1, en ese régimen, es una resonancia.

\section{E.1.2 El Kink TK2 $\sigma_{2}$}

En los rangos $\sigma_{3}^{2}>1>\sigma_{2}^{2}$ y $1>\sigma_{3}^{2}>\sigma_{2}^{2}$, el kink TK2 $\sigma_{2}$ existe y es estable. El kink TK1 no da lugar a un estado estacionario, véase (E.2), donde es patente que su energía deviene imaginaria. Denotamos por $\Delta\left[\vec{\phi}_{\mathrm{TK} 2 \sigma_{2}}\right]=\Delta^{22}$ el Hessiano evaluado en la configuración TK2 $\sigma_{2}$ :

$$
\Delta_{a b}^{22}=-\frac{d^{2}}{d x^{2}} \delta_{a b}+V_{a b}^{22}(x)
$$

con:

$$
\begin{aligned}
& V_{11}^{22}(x)=V_{11}^{0}-\frac{2\left(2+\sigma_{2}^{2}\right)}{\cosh ^{2} \sigma_{2} x}, \quad V_{12}^{22}(x)=V_{21}^{22}(x)=4 \bar{\sigma}_{2} \frac{\tanh \sigma_{2} x}{\cosh \sigma_{2} x} \\
& V_{22}^{22}(x)=V_{22}^{0}-\frac{2\left(3 \sigma_{2}^{2}-2\right)}{\cosh ^{2} \sigma_{2} x}, \quad V_{13}^{22}(x)=V_{31}^{22}(x)=0, V_{33}^{22}(x)=V_{33}^{0}-\frac{2 \sigma_{2}^{2}}{\cosh ^{2} \sigma_{2} x}
\end{aligned}
$$

Introduciendo los operadores:

$$
\partial=\left(\begin{array}{ccc}
\Delta_{11}^{22} & \Delta_{12}^{22} & 0 \\
\Delta_{21}^{22} & \Delta_{22}^{22} & 0 \\
0 & 0 & 0
\end{array}\right), \quad d=\left(\begin{array}{ccc}
0 & 0 & 0 \\
0 & 0 & 0 \\
0 & 0 & \Delta_{33}^{22}
\end{array}\right)
$$


resulta que: $\Delta^{22}=\partial+d,[\partial, d]=0, \mathrm{y}$, por tanto,

$$
\begin{gathered}
\operatorname{Tr}\left(e^{-\beta \Delta^{22}}\right)=\operatorname{Tr}\left(e^{-\beta \partial}\right)+\operatorname{Tr}\left(e^{-\beta d}\right) \\
\zeta_{\Delta^{22}}(s)=\zeta_{\partial}(s)+\zeta_{d}(s)
\end{gathered}
$$

La función del calor y la función zeta generalizada del operador diagonal $d$ se pueden calcular exactamente:

$$
\begin{gathered}
\operatorname{Tr}\left(e^{-\beta d}\right)-\operatorname{Tr}\left(e^{-\beta \Delta_{3}}\right)=e^{-\beta\left(\sigma_{3}^{2}-\sigma_{2}^{2}\right)}\left(1-\operatorname{Erfc}\left[\sigma_{2} \sqrt{\beta}\right]\right) \\
\zeta_{d}(s)-\zeta_{\Delta_{3}}(s)=-\frac{1}{\sqrt{\pi}} \frac{1}{\sigma_{2}^{2 s}} \frac{\Gamma\left(s+\frac{1}{2}\right)}{\Gamma(s)}{ }_{2} F_{1}\left(s, s+\frac{1}{2}, s+1 ; 1-\frac{\sigma_{3}^{2}}{\sigma_{2}^{2}}\right)
\end{gathered}
$$

El operador $\partial$, sin embargo, no es diagonal, y su espectro es muy difícil de tratar. Su función del calor asociada se puede aproximar, sin embargo, si $\beta>1$, mediante la expansión asintótica:

$$
\begin{aligned}
& \operatorname{Tr}\left(e^{-\beta \partial}\right)-\operatorname{Tr}\left(e^{-\beta \partial_{0}}\right)-1=\operatorname{Tr}^{*}\left(e^{-\beta \partial}\right)-\operatorname{Tr}\left(e^{-\beta \partial_{0}}\right)= \\
& =-1+\frac{1}{\sqrt{4 \pi}}\left(\sum_{n=0}^{\infty}\left(e^{-4 \beta} a_{n}^{11}\left(\partial_{-}\right)+e^{-\sigma_{2}^{2} \beta} a_{n}^{22}\left(\partial_{-}\right)\right) \beta^{n-\frac{1}{2}}\right)
\end{aligned}
$$

donde:

$$
\partial_{-}=\partial-\left(\begin{array}{cc}
4 & 0 \\
0 & \sigma_{2}^{2}
\end{array}\right) ; \quad \partial_{0}=\left(\begin{array}{cc}
\Delta_{11}^{0} & \Delta_{12}^{0} \\
\Delta_{21}^{0} & \Delta_{22}^{0}
\end{array}\right)=\left(\begin{array}{cc}
\Delta_{1} & 0 \\
0 & \Delta_{2}
\end{array}\right)
$$

Los coeficientes de la expansión se definen como la integral:

$$
a_{n}^{i j}\left(\partial_{-}\right)=\int d x a_{n}^{i j}\left(x, x ; \partial_{-}\right), \quad i, j=1,2
$$

de los coeficientes de Seeley:

$$
\begin{gathered}
a_{0}^{i j}\left(x, x ; \partial_{-}\right)=1 \\
a_{1}^{11}\left(x, x ; \partial_{-}\right)=-V_{11}^{-}(x) \\
a_{1}^{12}\left(x, x ; \partial_{-}\right)=-V_{12}^{-}=a_{1}^{21}\left(x, x ; \partial_{-}\right) \\
a_{1}^{22}\left(x, x ; \partial_{-}\right)=-V_{22}^{-}(x) \\
a_{2}^{11}\left(x, x ; \partial_{-}\right)=-\frac{1}{6} \frac{\partial^{2} V_{11}^{-}}{\partial x^{2}}+\frac{1}{2} V_{11}^{-}(x) V_{11}^{-}(x)+\frac{1}{2} V_{12}^{-}(x) V_{12}^{-}(x) \\
a_{2}^{12}\left(x, x ; \partial_{-}\right)=-\frac{1}{6} \frac{\partial^{2} V_{12}^{-}}{\partial x^{2}}+\frac{1}{2} V_{12}^{-}(x)\left[V_{11}^{-}(x)+V_{22}^{-}(x)+4-\sigma_{2}^{2}\right] \\
a_{2}^{21}\left(x, x ; \partial_{-}\right)=-\frac{1}{6} \frac{\partial^{2} V_{12}^{-}}{\partial x^{2}}+\frac{1}{2} V_{12}^{-}(x)\left[V_{11}^{-}(x)+V_{22}^{-}(x)-4+\sigma_{2}^{2}\right] \\
a_{2}^{22}\left(x, x ; \partial_{-}\right)=-\frac{1}{6} \frac{\partial^{2} V_{22}^{-}}{\partial x^{2}}+\frac{1}{2} V_{22}^{-}(x) V_{22}^{-}(x)+\frac{1}{2} V_{12}^{-}(x) V_{12}^{-}(x)
\end{gathered}
$$




$$
\begin{aligned}
a_{3}^{11}\left(x, x ; \partial_{-}\right)= & -\frac{1}{60} \frac{\partial^{4} V_{11}^{-}}{\partial x^{4}}+\frac{1}{6} V_{11}^{-}(x) \frac{\partial^{2} V_{11}^{-}}{\partial x^{2}}+\frac{1}{6} V_{12}^{-}(x) \frac{\partial V_{12}^{-}}{\partial x^{2}}+\frac{1}{12} \frac{\partial V_{11}^{-}}{\partial x} \frac{\partial V_{11}^{-}}{\partial x}+ \\
& +\frac{1}{12} \frac{\partial V_{12}^{-}}{\partial x} \frac{\partial V_{12}^{-}}{\partial x}-\frac{1}{6}\left(V_{12}^{-}(x)\right)^{2}\left(V_{22}^{-}(x)-4+\sigma_{2}^{2}\right) \\
& -\frac{1}{6}\left(V_{11}^{-}(x)\right)^{3}-\frac{1}{3}\left(V_{12}^{-}(x)\right)^{2} V_{11}^{-}(x) \\
a_{3}^{22}\left(x, x ; \partial_{-}\right)= & -\frac{1}{60} \frac{\partial^{4} V_{22}^{-}}{\partial x^{4}}+\frac{1}{6} V_{22}^{-}(x) \frac{\partial^{2} V_{22}^{-}}{\partial x^{2}}+\frac{1}{6} V_{12}^{-}(x) \frac{\partial V_{12}^{-}}{\partial x^{2}}+\frac{1}{12} \frac{\partial V_{22}^{-}}{\partial x} \frac{\partial V_{22}^{-}}{\partial x}+ \\
& +\frac{1}{12} \frac{\partial V_{12}}{\partial x} \frac{\partial V_{12}^{-}}{\partial x}-\frac{1}{6}\left(V_{12}^{-}(x)\right)^{2}\left(V_{11}^{-}(x)+4-\sigma_{2}^{2}\right) \\
& -\frac{1}{6}\left(V_{22}^{-}(x)\right)^{3}-\frac{1}{3}\left(V_{12}^{-}(x)\right)^{2} V_{22}^{-}(x)
\end{aligned}
$$

donde $V^{-}=V^{22}-V^{0}$.

La función zeta generalizada es, por tanto

$$
\begin{gathered}
\zeta_{\partial}^{*}(s)-\zeta_{\partial^{0}}(s)=\frac{1}{\Gamma(s)}\left[\int _ { 0 } ^ { 1 } d \beta \left(-\beta^{s-1}+\right.\right. \\
\left.\left.+\frac{1}{\sqrt{4 \pi}} \sum_{i=1}^{2} \sum_{n=1}^{n_{0}-1} e^{-V_{i i}^{0} \beta} a_{n}^{i i}\left(\partial_{-}\right) \beta^{s+n-\frac{3}{2}}\right)+b_{n_{0}}(s)+B(s)\right] \\
b_{n_{0}}(s)=\int_{0}^{1} d \beta \frac{1}{\sqrt{4 \pi}} \sum_{i=1}^{2} \sum_{n=n_{0}}^{\infty} e^{-V_{i i}^{0} \beta} a_{n}^{i i}\left(\partial_{-}\right) \beta^{s+n-\frac{3}{2}} \\
B(s)=\int_{1}^{\infty} d \beta\left[\operatorname{Tr}^{*}\left(e^{-\beta \partial}\right)-\operatorname{Tr}\left(e^{-\beta \partial}\right)\right]
\end{gathered}
$$

Despreciar $b_{n_{0}}(s)$ y $B(s)$ da lugar a una muy buena aproximación, de modo que:

$$
\zeta_{\partial}^{*}(s)-\zeta_{\partial^{0}}(s) \cong \frac{1}{\Gamma(s)}\left[-\frac{1}{s}+\sum_{i=1}^{2} \sum_{n=1}^{n_{0}-1} \frac{a_{n}^{i i}}{\sqrt{4 \pi}} \gamma\left[s+n-\frac{1}{2} ; V_{i i}^{0}\right]\right]
$$

donde $\gamma[z ; c]$ es la función Gamma incompleta (ver [45]).

Las distintas contribuciones a la masa del kink TK2 $\sigma_{2}$, a orden un lazo son, por tanto:

$$
\begin{aligned}
& \text { - } \varepsilon\left[\vec{\phi}_{\mathrm{TK} 2 \sigma_{2}}\right]=\lim _{s \rightarrow-\frac{1}{2}} 2^{s} \frac{\mu^{2 s+1}}{m^{2 s}}\left[\zeta_{\partial}^{*}(s)-\zeta_{\partial^{0}}(s)+\zeta_{d}(s)-\zeta_{\Delta_{3}}(s)\right]= \\
& =\frac{m}{\sqrt{2}} \lim _{s \rightarrow-\frac{1}{2}}\left[-\frac{1}{s \Gamma(s)}+\sum_{i=1}^{2} \sum_{n=1}^{n_{0}-1} \frac{a_{n}^{i i}\left(\partial_{-}\right)}{2 \sqrt{\pi} \Gamma(s)} \gamma\left[s+n-\frac{1}{2}, V_{i i}^{0}\right]\right] \\
& -\frac{m}{\sqrt{2}} \frac{\sigma_{2}}{\sqrt{\pi}} \lim _{s \rightarrow-\frac{1}{2}}\left[\frac{\Gamma\left(s+\frac{1}{2}\right)}{s \Gamma(s)}{ }_{2} F_{1}\left[s, s+\frac{1}{2}, s+1 ; 1-\frac{\sigma_{3}^{2}}{\sigma_{2}^{2}}\right]\right]
\end{aligned}
$$




$$
\begin{aligned}
& \text { - } \delta_{M} \varepsilon\left[\vec{\phi}_{\mathrm{TK} 2 \sigma_{2}}\right]= \lim _{L \rightarrow \infty} \lim _{s \rightarrow \frac{1}{2}} \frac{2^{s}}{L \sqrt{2}}\left(\frac{\mu^{2}}{m^{2}}\right)^{s-\frac{1}{2}} \cdot \\
& \cdot\left(2\left(2+\sigma_{2}^{2}\right) \zeta_{\Delta_{1}}(s)+2\left(3 \sigma_{2}^{2}-2\right) \zeta_{\Delta_{2}}(s)+2 \sigma_{2}^{2} \zeta_{\Delta_{3}}(s)\right) . \\
& \cdot \int_{-\frac{m L}{2 \sqrt{2}}}^{\frac{m L}{2 \sqrt{2}}} \frac{d x}{\cosh ^{2} \sigma_{2} x} \\
& \delta_{M} \varepsilon\left[\vec{\phi}_{\mathrm{TK} 2 \sigma_{2}}\right]= \frac{m \sigma_{2}}{\sqrt{2 \pi}}(8+2) \lim _{s \rightarrow \frac{1}{2}} \frac{\Gamma\left(s-\frac{1}{2}\right)}{\Gamma(s)} \\
& \text { - } \delta_{F} \varepsilon\left[\vec{\phi}_{\mathrm{TK} 2 \sigma_{2}}\right]=-\frac{1}{2 \pi} \int_{-\infty}^{\infty} d x\left[\frac{2\left(2+\sigma_{2}^{2}\right)+2\left(3 \sigma_{2}^{2}-2\right)+2 \sigma_{2}^{2}}{\cosh ^{2} \sigma_{2} x}\right]=-\frac{10 \sigma_{2} m}{\pi \sqrt{2}}
\end{aligned}
$$

La primera corrección a la masa del kink TK2 $\sigma_{2}$ es pues:

$$
\begin{aligned}
& \frac{\hbar}{2}\left\{\varepsilon\left[\vec{\phi}_{\mathrm{TK} 2 \sigma_{2}}\right]+\delta_{M} \varepsilon\left[\vec{\phi}_{\mathrm{TK} 2 \sigma_{2}}\right]+\delta_{F} \varepsilon\left[\vec{\phi}_{\mathrm{TK} 2 \sigma_{2}}\right]\right\}= \\
& =\frac{\hbar m}{2 \sqrt{2}}\left[\lim _{s \rightarrow-\frac{1}{2}}\left(-\frac{1}{s \Gamma(s)}+\sum_{i=1}^{2} \sum_{n=1}^{n_{0}-1} \frac{a_{n}^{i i}\left(\partial_{-}\right)}{\sqrt{4 \pi} \Gamma(s)} \gamma\left[s+n-\frac{1}{2} ; V_{i i}^{0}\right]\right)+\right. \\
& \left.+\lim _{s \rightarrow \frac{1}{2}} \frac{8 \sigma_{2}}{\sqrt{\pi}} \frac{\left.\Gamma\left(s-\frac{1}{2}\right)\right]}{\Gamma(s)}\right]+\frac{\hbar m}{2 \sqrt{2}}\left[-\frac{\sigma_{2}}{\sqrt{\pi}} \lim _{s \rightarrow-\frac{1}{2}} \frac{\Gamma\left(s+\frac{1}{2}\right)}{\Gamma(s)}{ }_{2} F_{1}\left[s, s+\frac{1}{2}, s+1 ; 1-\frac{\sigma_{3}^{2}}{\sigma_{2}^{2}}\right]\right. \\
& \left.+\lim _{s \rightarrow \frac{1}{2}} \frac{2 \sigma_{2}}{\sqrt{\pi}} \frac{\Gamma\left(s-\frac{1}{2}\right)}{\Gamma(s)}\right]-\frac{\hbar m}{\pi \sqrt{2}} 5 \sigma_{2}= \\
& =\frac{\hbar m}{2 \sqrt{2}}\left[\lim _{s \rightarrow-\frac{1}{2}}\left(-\frac{1}{s \Gamma(s)}+\sum_{i=1}^{2} \sum_{n=1}^{n_{0}-1} \frac{a_{n}^{i i}\left(\partial_{-}\right)}{\sqrt{4 \pi} \Gamma(s)} \gamma\left[s+n-\frac{1}{2} ; V_{i i}^{0}\right]\right)+\right. \\
& \left.+\lim _{s \rightarrow \frac{1}{2}} \frac{8 \sigma_{2}}{\sqrt{\pi}} \frac{\Gamma\left(s-\frac{1}{2}\right)}{\Gamma(s)}-\frac{10 \sigma_{2}}{\pi}\right]+\frac{\hbar m}{2 \sqrt{2 \pi}}\left(-\frac{\sigma_{2}}{2}+\sqrt{\sigma_{3}^{2}-\sigma_{2}^{2}} \operatorname{arcsen} \frac{\sigma_{2}}{\sigma_{3}}\right)
\end{aligned}
$$

La fórmula (E.3) ha de entenderse en dos partes:

- I. La contribución del operador $\partial$ se ha computado asintóticamente. De un lado:

$$
\frac{\hbar}{2}\left[\lim _{s \rightarrow-\frac{1}{2}} \sum_{i=1}^{2} a_{1}^{i i}\left(\partial_{-}\right) \frac{\gamma\left[s+\frac{1}{2} ; V_{i i}^{0}\right]}{\Gamma(s)}+\lim _{s \rightarrow \frac{1}{2}} \frac{8 \sigma_{2} \Gamma\left(s-\frac{1}{2}\right)}{\sqrt{\pi} \Gamma(s)}\right]
$$

cancela exactamente con $-\frac{4 \sigma_{2}}{\pi} \hbar m$. 
De otro lado los coeficientes $a_{n}^{i i}$, de $n=2$ a $n=9$, se muestran en la siguiente Tabla para distintos valores de $\sigma_{2}{ }^{2}$

\begin{tabular}{|c|c|c|c|c|c|c|}
\hline & \multicolumn{2}{|c|}{$\sigma_{2}=0.5$} & \multicolumn{2}{c|}{$\sigma_{2}=0.7$} & \multicolumn{2}{c|}{$\sigma_{2}=0.8$} \\
\hline$i$ & $\tilde{a}_{i}^{11}$ & $\tilde{a}_{i}^{22}$ & $\tilde{a}_{i}^{11}$ & $\tilde{a}_{i}^{22}$ & $\tilde{a}_{i}^{11}$ & $\tilde{a}_{i}^{22}$ \\
\hline 1 & 17.9984 & -9.99909 & 14.2285 & -3.02857 & 13.2 & -0.4 \\
2 & 34.9975 & 16.3313 & 27.5051 & 4.95579 & 25.632 & 2.42133 \\
3 & 47.9636 & -16.8309 & 38.5365 & -3.88117 & 36.3858 & -1.46193 \\
4 & 49.4219 & 13.2491 & 40.9039 & 2.58248 & 33.7529 & -0.53935 \\
5 & 40.6415 & -8.41779 & 34.5847 & -1.43041 & 33.7529 & -0.53935 \\
6 & 27.7911 & 4.4983 & 24.2279 & 0.696572 & 23.9672 & 0.267282 \\
7 & 16.2632 & -2.08006 & 14.4768 & -0.30375 & 14.4819 & -0.117869 \\
8 & 8.31759 & -0.849979 & 7.53962 & 0.119982 & 7.61204 & 0.0464771 \\
9 & 3.77771 & -0.295903 & 3.47957 & -0.0425253 & 3.53989 & -0.0162638 \\
10 & 1.51992 & 0.0884623 & 1.44002 & 0.0132872 & 1.47558 & 0.00497705 \\
\hline
\end{tabular}

\begin{tabular}{|c|c|c|c|c|c|c|}
\hline & \multicolumn{2}{|c|}{$\sigma_{2}=0.9$} & \multicolumn{2}{c|}{$\sigma_{2}=0.95$} & \multicolumn{2}{c|}{$\sigma_{2} \geq 1$} \\
\hline$i$ & $\tilde{a}_{i}^{11}$ & $\tilde{a}_{i}^{22}$ & $\tilde{a}_{i}^{11}$ & $\tilde{a}_{i}^{22}$ & $\tilde{a}_{i}^{11}$ & $\tilde{a}_{i}^{22}$ \\
\hline 1 & 12.4889 & 1.91111 & 12.2211 & 2.97895 & 12.0 & 4.0 \\
2 & 24.5218 & 1.67378 & 24.1951 & 1.952444 & 24.0 & 2.66667 \\
3 & 35.3368 & -0.241656 & 35.16 .16 & 0.310882 & 35.2 & 1.066667 \\
4 & 38.8216 & 0.341514 & 38.9553 & 0.230323 & 39.3143 & 0.304762 \\
5 & 33.8715 & -0.16798 & 34.2227 & -0.053986 & 34.7429 & 0.0677249 \\
6 & 24.361 & 0.0863412 & 24.7475 & 0.0372813 & 25.2306 & 0.0123136 \\
7 & 14.8742 & -0.0370868 & 15.1758 & -0.0145382 & 15.5208 & 0.0018944 \\
8 & 7.88516 & 0.0140749 & 8.07351 & 0.00547229 & 8.27702 & 0.000252587 \\
9 & 3.69263 & -0.0046464 & 3.79191 & -0.00173035 & 3.89498 & $2.9713410^{-5}$ \\
10 & 1.54847 & 0.001313 & 1.594006 & 0.0003535 & 1.63998 & $3.1259110^{-6}$ \\
\hline
\end{tabular}

De ella se deduce la corrección cuántica a la masa del kink debida a la parte no diagonal del Hessiano $\Delta^{22}$, con un error debido a los términos $b_{11}\left(-\frac{1}{2}\right)$ y $B\left(-\frac{1}{2}\right)$, que se encuentra cuantificado en [8].

\begin{tabular}{|c|c|c|c|}
\hline$\sigma_{2}=0.5$ & $\sigma_{2}=0.7$ & $\sigma_{2}=0.8$ & $\sigma_{2}=0.9$ \\
\hline$-0.852622 \hbar m$ & $-0.583835 \hbar m$ & $-0.524363 \hbar m$ & $-0.505708 \hbar m$ \\
\hline \hline$\sigma_{2}=0.95$ & $\sigma_{2}=1.0$ & $\sigma_{2}=1.2$ & $\sigma_{2}=1.4$ \\
\hline$-0.511638 \hbar m$ & $-0.528311 \hbar m$ & $-0.518426 \hbar m$ & $-0.509645 \hbar m$ \\
\hline \hline$\sigma_{2}=1.6$ & $\sigma_{2}=1.8$ & $\sigma_{2}=2.0$ & $\sigma_{2}=2.5$ \\
\hline$-0.502291 \hbar m$ & $-0.496369 \hbar m$ & $-0.49172 \hbar m$ & $-0.484183 \hbar m$ \\
\hline \hline$\sigma_{2}=3.0$ & $\sigma_{2}=4.0$ & $\sigma_{2}=5.0$ & $\sigma_{2}=6.0$ \\
\hline$-0.480101 \hbar m$ & $-0.476181 \hbar m$ & & \\
\hline
\end{tabular}

\footnotetext{
${ }^{2}$ Se han incluido en la Tabla, para valores de $\sigma_{2}>1$, los coeficientes de la expansión asintótica para $\Delta\left[\vec{\phi}_{\mathrm{TK} 1}\right]=\Delta^{1}$ y la subsecuente aproximación a la corrección cuántica de la masa del kink TK1, que es tanto mejor cuanto más alejado del valor $\sigma_{2}=1$ se considere, toda vez que para ese valor existe un segundo autovalor cero.
} 


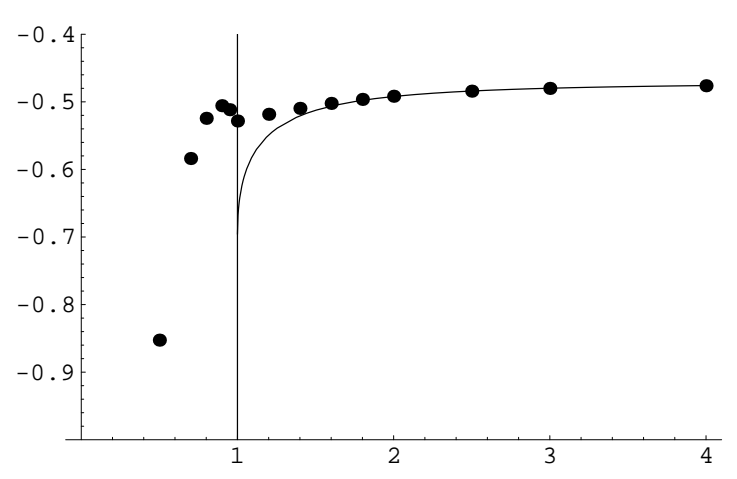

Figura E.1: Comparación de las contribuciones de $\partial$, como funciones de $\sigma$, calculadas mediante el desarrollo asintótico (puntos) y la respuesta exacta (tramo continuo), para los regímenes de estabilidad del TK1 $(\sigma>1)$, donde los dos son calculables, y TK2 $(\sigma \leq 1)$.

- II. Ello debe sumarse a la contribución del operador $d$, que se puede calcular exactamente y se encuentra recogida en la fórmula (E.3):

$$
\frac{\hbar m}{2 \sqrt{2 \pi}}\left[-\frac{\sigma_{2}}{2}+\sqrt{\sigma_{3}^{2}-\sigma_{2}^{2}} \operatorname{arcsen} \frac{\sigma_{2}}{\sigma_{3}}\right]-\frac{\hbar m \sigma_{2}}{\pi}
$$

En el rango $1>\sigma_{3}^{2}>\sigma_{2}^{2}$, el kink TK2 $\sigma_{3}$ también existe. El Hessiano en esta solución $\Delta\left[\vec{\phi}_{\mathrm{TK} 2 \sigma_{3}}\right]=\Delta^{23}$

$$
\Delta_{a b}^{23}=-\frac{d^{2}}{d x^{2}} \delta_{a b}+V_{a b}^{23}(x)
$$

es también no diagonal:

$$
\begin{aligned}
& V_{11}^{23}(x)=V_{11}^{0}-\frac{2\left(2+\sigma_{3}^{2}\right)}{\cosh ^{2} \sigma_{3} x}, \quad V_{13}^{23}(x)=V_{31}^{23}(x)=4 \bar{\sigma}_{3} \frac{\tanh \sigma_{3} x}{\cosh \sigma_{3} x} \\
& V_{22}^{23}(x)=V_{22}^{0}-\frac{2 \sigma_{3}^{2}}{\cosh ^{2} \sigma_{3} x}, \quad V_{33}^{23}(x)=V_{33}^{0}-\frac{2\left(3 \sigma_{3}^{2}-2\right)}{\cosh ^{2} \sigma_{3} x}, V_{12}^{23}(x)=V_{21}^{23}(x)=0
\end{aligned}
$$

y formalmente idéntico al $\Delta^{22}$. Hay, por tanto, un término en la fórmula de la masa del kink TK2 $\sigma_{3}$ cuántico de la forma

$$
\frac{\hbar m}{2 \sqrt{2 \pi}} \sqrt{\sigma_{2}^{2}-\sigma_{3}^{2}} \operatorname{arcsen} \frac{\sigma_{3}}{\sigma_{2}}
$$

proveniente de $\Delta_{22}^{23}$, que es imaginario puro e indica que el kink TK2 $\sigma_{3}$ es una resonancia. 


\section{Bibliografía}

[1] Ablowitz, M.J. y Clarkson, P.A., "Solitons, non-linear evolution equations and inverse scattering". Cambridge Univ. Press, Cambridge, 1991.

[2] Abramowitz, M. y Stegun, I.A., Eds., "Handbook of mathematical functions with formulas, graphs, and mathematical tables". Dover Publications, Inc., New York, 1992.

[3] Alonso Izquierdo, A., "Kinks, Sistemas Integrables y Supersimetría". Tesis Doctoral. Universidad de Salamanca, Salamanca, 2001.

[4] Alonso Izquierdo, A., González León, M.A. y Mateos Guilarte, J., Kink manifolds in (1+1)-dimensional scalar field theory, J. Phys. A: Math. Gen. 31 (1998), 209-229.

[5] Alonso Izquierdo, A., González León, M.A. y Mateos Guilarte, J., Kinks out of Geodesics. Topological Walls in the Linear Sigma Model, contenido en "Geometry and physics (Zaragoza, 1998)", An. Fís. Monogr., 5, 15-35. CIEMAT, Madrid, 1998.

[6] Alonso Izquierdo, A., González León, M.A. y Mateos Guilarte, J., $N=2$ supersymmetric kinks and real algebraic curves, Phys. Lett. B 480 (2000), 373-380.

[7] Alonso Izquierdo, A., González León, M.A. y Mateos Guilarte, J., Kinks from dynamical systems: domain walls in a deformed $O(N)$ linear sigma model, Nonlinearity 13 (2000), 1137-1169.

[8] Alonso Izquierdo, A., García Fuertes, W., González León, M.A. y Mateos Guilarte, J., Generalized Zeta Functions and One Loop Corrections to Quantum Kink Masses, Preprint USAL/UOV, 2001. 
[9] Arnold, V.I., "Mecánica Clásica: Métodos Matemáticos”. Ed. Paraninfo, Madrid, 1983.

[10] Atiyah, M. y Hitchin, N., "The geometry and dynamics of magnetic monopoles". Princeton Univ. Press, Princeton, 1988.

[11] Bazeia, D., Nascimento, J. R. S., Ribeiro, R. F. y Toledo, D., Soliton stability in systems of two real scalar fields, J. Phys. A: Math. Gen. 30 (1997), 81578166 .

[12] Birkhoff, G.D., “Dynamical Systems”. Amer. Math. Soc., Providence, 1927.

[13] Bogomol'nyi, E. B., The stability of classical solutions, Soviet J. Nuclear Phys. 24 (1976), 449-454.

[14] Bott, R., Lectures on Morse theory, old and new, Bull. Amer. Math. Soc. 7 (1982), 331-358.

[15] Bott, R., On Some Recent Developments in Yang-Mills Theory, contenido en: "R. Bott: Collected Papers", vol. 4, Birkhäuser Verlag, Boston, 1995.

[16] Boussinesq, J., Théorie de l'intumescence liquid appelée onde solitaire ou de la translation, se propageant dans un canal rectangulaire, Comptes Rendus Acad. Sci. Paris 72 (1871), 755-759.

[17] Boya, L.J., Cariñena, J.F. y Mateos Guilarte, J., Homotopy and Solitons, Fortschr. Phys. 26 (1978), 175-214.

[18] Boya, L.J. y Casahorrán, J., Normal-mode diagonalization for twocomponent topological kinks, Phys. Rev. A 39 (1989), 4298-4300. General scalar bidimensional models including kinks, Ann. Physics 196 (1989), 361385 .

[19] Cariñena, J.F., Canonical Group Actions, I.C.T.P. preprint IC/88/37 (1988).

[20] Cecotti, S. y Vafa, C., On classification of $N=2$ supersymmetric theories, Comm. Math. Phys. 158 (1993), 569-644.

[21] Christoffel, E.B., Über die Transformation der homogenen Differentialausdrücke zweiten Grades, J. Reine Angew. Math. 70 (1869), 46-70; ibid. 70 (1869), 241-245. 
[22] Coleman, S., Classical lumps and their quantum descendants (1975), contenido en "Aspects of Symmetry". Cambridge Univ. Press, Cambridge, 1990.

[23] Coleman, S., There are no Goldstone bosons in two dimensions, Comm. Math. Phys. 31 (1973), 259-264.

[24] Chang, S.J. y Friedberg, R., Elliptical billiards and Poncelet's theorem, J. Math. Phys. 29 (1988), 1537-1550.

[25] Da Rios, Sul moto d'un liquido indefinito con un filetto vorticoso di forma qualunque, Rend. Circ. Mat. Palermo 22 (1906), 117-135.

[26] Dashen, R.F., Hasslacher, B. y Neveu, A., Non-perturbative methods and extended hadron models in field theory: 1, semi-classical functional methods; 2, two-dimensional models and extended hadrons; 3, Four-dimensional nonAbelian models, Phys. Rev. D 10,(1974) 4114-4129; ibid. 10 (1974), 4130; ibid. 10, (1974), 4138-4142. Semiclassical bound states in an asymptotically free theory, Phys. Rev. D 12 (1975), 2443-2458.

[27] Davydov, A.S., "Solitons in Molecular Systems". Kluwer Academic Publ., Dordrecht, 1991.

[28] Drazin, P.G. y Johnson, R.S., "Solitons: an introduction". Cambridge Univ. Press, Cambridge, 1996.

[29] Dvali, G. y Shifman, M., Domain Walls in Strongly Coupled Theories, Phys. Lett. B 396 (1997), 64-69.

[30] Elizalde, E., Odintsov, S.D., Bytsenko, A.A. y Zerbini, S., "Zeta regularization techniques with applications". World Scientific, Singapur, 1994.

[31] Elsgoltz, L., "Ecuaciones Diferenciales y Cálculo Variacional". Ed. URSS, Moscú, 1994.

[32] Faddeev, L.D. y Korepin, V.E., Quantum theory of solitons, Phys. Rep. 42 (1978), 1-87.

[33] Faddeev, L.D. y Takhtajan, L.A., "Hamiltonian methods in the theory of solitons". Springer-Verlag, Berlín-New York, 1987.

[34] Fermi, E., Beweis dass ein mechanisches Normalsysteme im Allgemeinen quasi-ergodisch ist, Phys. Zeit. 24 (1923), 261-265. 
[35] Fermi, E., Pasta, J. y Ulam, S., Studies in Nonlinear Problems. I, contenido en "Nonlinear Wave Motion", Lectures in Applied Math., vol. 15, 142-155. Amer. Math. Soc., Providence, 1974.

[36] Freed, D. y Uhlenbeck, K., "Instantons and four manifolds". Springer Verlag, New York, 1984.

[37] Freund, P.G.O., "Introduction to supersymmetry". Cambridge Univ. Press, Cambridge-New York, 1986.

[38] Friedberg, R., Lee, T.D. y Sirlin, A., Class of scalar-field soliton solutions in three space dimensions, Phys. Rev. D 13 (1976), 2739-2761.

[39] García Fuertes, W., "Defectos Topológicos en Teoría de Campos. De kinks, Vórtices y Monopolos en Modelos Escalares y Teorías Gauge". Tesis Doctoral. Universidad de Oviedo, Oviedo, 1998.

[40] Gardner, C.S., Greene, J.M., Kruskal, M.D. y Miura, R.M., Method for solving the Korteweg-de Vries equation, Phys. Rev. Lett. 19 (1967), 10951097.

[41] Gardner, C. S. y Morikawa, G. K., The effect of temperature on the width of a small-amplitude, solitary wave in a collision-free plasma, Comm. Pure Appl. Math. 18 (1965), 35-49.

[42] Garnier, R., Sur une classe de Systèmes différentiels Abéliens deduits de la Théorie des équations linéaires, Rend. Circ. Mat. Palermo 43 (1919), 155191.

[43] Gell-Mann, M. y Lèvy, M., The axial vector current in beta decay, Nuovo Cimento 16 (1960), 705-726.

[44] Gibbons, G.W. y Townsend, P.K., A Bogomol'nyi equation for intersecting domain walls, Phys. Rev. Lett. 83 (1999), 1727-1730.

[45] Gilkey, P., "Invariance Theory, the Heat equation and the Atiyah-Singer Index Theorem". Publish or Perish Publ., Delaware, 1984.

[46] Goldstone, J. y Jackiw, R., Quantization of Nonlinear Waves, Phys. Rev. D 11 (1075), 1486-1498. 
[47] Grosse, H., Quasiclassical Estimates on Moments of the Energy Levels, Acta Physica Austriaca 52 (1980), 89-105.

[48] Ginzburg, V.L., Some remarks on phase transitions of the second kind and the microscopic theory of ferroelectric materials, Sov. Phys. Solid State 2 (1960), 1824.

[49] Hasimoto, H., Motion of a vortex filament and its relation to elastic, J. Phys. Soc. Japan 31 (1971), 293-295.

[50] Helgason, S., "Differential Geometry, Lie Groups, and Symmetric Spaces". Academic Press, Inc., New York, 1978.

[51] Hitchin, N., Integrable Systems in Riemannian Geometry, contenido en: "Integrable Systems", J. Differential Geometry Survey, vol. 4. International Press, Cambridge (MA), 1999.

[52] Ito, H. Kink energy sum rule in a two-component scalar field model of $1+1$ dimensions, Phys. Lett. A 112 (1985), 119-123.

[53] Ito, H y Tasaki, H., Stability theory for nonlinear Klein-Gordon kinks and Morse's index theorem, Phys. Lett. A 113 (1985), 179-182.

[54] Jacobi, C.G.J., "Vorlesungen über Dynamik". Editado por A. Clebsch. Reimer, Berlín, 1866.

[55] Jackiw, R., Quantum meaning of classical field theory, Rev. Modern Phys. 49 (1977), 681-706.

[56] Jackiw, R. y Rebbi, C., Solitons with fermion number 1/2, Phys. Rev. D (3) 13 (1976), 3398-3409.

[57] Jost, J., "Riemannian Geometry and Geometric Analysis". Springer-Verlag, Berlín-Heidelberg, 1995.

[58] Kirwan, F., "Complex Algebraic Curves". Cambridge Univ. Press, Cambridge, 1992.

[59] Kobayashi, S. y Nomizu, K., "Foundations of Differential Geometry". John Wiley \& Sons, New York, 1963. 
[60] Korteweg, D.J., de Vries, G., On the change of form of long waves advancing in a rectangular canal, and on a new type of long stacionary waves, Philos. Mag. Ser. 539 (1895), 422-443.

[61] Kozlov, V. V., "Symmetries, Topology and Resonances in Hamiltonian Mechanics”. Springer-Verlag, Berlín, 1996.

[62] Zabusky, N.J. y Kruskal, M.D., Interactions of solitons in a collisionless plasma and the recurrence of initial states, Phys. Rev. Lett. 15 (1965), 240243.

[63] Lee, T.D., "Particle Physics and Introduction to Field Theory". Harwood Academic Publishers, New York, 1990.

[64] Landau, L.D. y Lifshitz, E.M., "Mecánica”. Segunda edición. Ed. Reverté, Barcelona, 1985.

[65] Laugwitz, D. "Differential and Riemannian Geometry". Tercera edición. Academic Press, Inc., New York, 1970.

[66] Magyari, E. y Thomas, H., Solitary waves in a $1 D$ anharmonic lattice with two-component order parameter, Phys. Lett. A 100 (1984), 11-14.

[67] Mateos Guilarte, J., A note on Morse theory and one-dimensional solitons, Lett. Math. Phys. 14 (1987), 169-176.

[68] Mateos Guilarte, J., Stationary phase approximation and quantum soliton families, Ann. Physics 188 (1988), 307-346.

[69] Mateos Guilarte, J., Sphalerons and instantons in two-dimensional field theory, Ann. Physics 216 (1992), 122-151.

[70] Milnor, J., "Morse Theory". Princeton Univ. Press, Princeton, 1973.

[71] Montonen, C., On Solitons with an Abelian Charge in Scalar Field Theories: (I) Classical theory and Bohr-Sommerfeld quantization, Nucl. Phys. B 112 (1976), 349-357.

[72] Morris, J.R., Nested Domain Defects, Int. J. Mod. Phys. A 13 (1998), 11151128. 
[73] Morse, P.M. y Feshbach, H., "Methods of Theoretical Physics". McGraw-Hill Book Company, Inc., New York-Toronto-London, 1953.

[74] Morse, M., "Calculus of variations in the large". Amer. Math. Soc. Colloq. Publ., Providence, 1934.

[75] Deligne, P. y Mumford, D., The irreducibility of the space of curves of given genus, Inst. Hautes Études Sci. Publ. Math. No. 36 (1969), 75-109.

[76] Nielsen, H.B. y Olesen, P., Vortex-Line Models for Dual Strings, Nucl. Phys. B 61 (1973), 45-61.

[77] Novikov, S.P., Manakov, S.V., Pitaevskiü, L.P. y Zakharov, V.E., "Theory of solitons. The inverse scattering method". Plenum Press, New York-London, 1984.

[78] Novikov, S.P., "Solitons and geometry", Lezioni Fermiane, Scuola Normale Superiore, Pisa. Cambridge Univ. Press, Cambridge, 1994.

[79] Palais, R., The symmetries of solitons, Bull. Amer. Math. Soc. 34 (1997), 339-403.

[80] Perelomov, A., "Integrable Systems of Classical Mechanics and Lie Algebras". Birkhäuser Verlag, Basilea, 1990.

[81] Perelomov, A., "Lectures on Constrained Integrable Systems". Universidad de Salamanca, 1998.

[82] Petersen, P., Aspects of global Riemannian geometry, Bull. Amer. Math. Soc. 36 (1999), 297-344.

[83] Polyakov, A.M., Particle spectrum in quantum field theory, JETP Lett. 20 (1974), 194.

[84] Postnikov, M.M., "The Variational Theory of Geodesics". Dover Publications, Inc., New York, 1983.

[85] Rañada, M.F., Sistemas Sobreintegrables, Proceedings del VI Workshop de Otoño: Geometría y Física. Salamanca, 1997.

[86] Rajaraman, R. Solitons of Coupled Scalar Field Theories in Two Dimensions, Phys. Rev. Lett. 42 (1979), 200-204. 
[87] Rajaraman, R., "Solitons and instantons. An introduction to solitons and instantons in quantum field theory". North-Holland Publishing Co., Amsterdam-New York, 1982.

[88] Rayleigh, Lord, On waves, Phil. Mag. (5), 1 (1876), 257-279.

[89] Riemann, B., Pariserarbeit, Obras Completas (1876). Segunda Edición (1892), 391-404.

[90] Russell, J.S., Report on Waves, contenido en: 14th Mtg. of the British Assoc. for the Advance of Science, pp. 311-390. John Murray, Londres, 1844.

[91] Sarker, S., Trullinger, S.E. y Bishop, A.R., Solitary-wave solution for a complex one-dimensional field, Phys. Lett. A 59 (1976), 255-258.

[92] Saffin, P.M., Tiling with almost-BPS-invariant domain-wall junctions, Phys. Rev. Lett. 83 (1999), 4249-4252.

[93] Segev, M. y Stegeman, G., Self-Trapping of Optical Beams: Spatial Solitons, Phys. Today August 1998, 42-48.

[94] Shifman, M., Vainshtein, A. y Voloshin, M., Anomaly and quantum corrections to solitons in two-dimensional theories with minimal supersymmetry, Phys. Rev. D 59 (1999), 45016.

[95] Szydłowski, M., The generalized Maupertuis principle, Regul. Chaotic Dyn. 3 (1998), 10-19.

[96] Szydłowski, M., Heller, M. y Sasin, W., Geometry of spaces with the Jacobi metric, J. Math. Phys. 37 (1996), 346-360.

[97] 't Hooft, G., Magnetic monopoles in unified gauge theories, Nucl. Phys. B 79 (1974), 276-284.

[98] Terng, C.L., Soliton equations and differential geometry, J. Diff. Geom. 45 (1997), núm. 2, 407-445.

[99] Terng, C.L. y Uhlenbeck, K., Poisson Actions and Scattering Theory for Integrable Systems, contenido en: "Integrable Systems", J. Differential Geometry Survey, vol. 4. International Press, Cambridge, MA, 1999. 
[100] Terng, C.L. y Uhlenbeck, K., Geometry of solitons, Notices Amer. Math. Soc. 47 (2000), núm. 1, 17-25.

[101] Vanhaecke, P., "Integrable systems in the realm of algebraic geometry". Lecture Notes in Mathematics, 1638. Springer-Verlag, Berlín, 1996.

[102] Veltman, M.J.G., "Reflections on the Higgs system", CERN Yellow Report. CERN 97-05.

[103] Vilenkin, A., Cosmic strings and domain walls, Phys. Rep. 121 (1985), núm. 5, 263-315.

[104] Vilenkin, A. y Shellard, E.P.S., "Cosmic Strings and other Topological Defects". Cambridge University Press, Cambridge, 1994.

[105] Veerman, J.J.P., Bazeia, D. y Moraes, F., Soliton stability in a Z(2) field theory, J. Math. Phys. 40 (1999), 3925-3929.

[106] Weinberg, S., A Model of Leptons, Phys. Rev. Lett. 19 (1967), 1264-1266.

[107] Wess, J. y Bagger, J., "Supersymmetry and Supergravity". Segunda Edición. Princeton Univ. Press, Princeton, 1992.

[108] Wess, J. y Zumino, B., A Lagrangian Model Invariant under Supergauge Transformations, Phys. Lett. B 49 (1974), 52-54.

[109] Witten, E., Branes and the dynamics of QCD, Nucl. Phys. B (Proc. Suppl.) 68 (1998), 216-239.

[110] Willmore, T.J., "Total Curvature in Riemannian Geometry". Ellis Horwood Series in Mathematics and its Applications, Chichester, 1982.

[111] Wojciechowski, S., Integrability of one particle in a perturbed central quartic potential, Phys. Scripta 31 (1985), 433-438.

[112] Zamolodchikov, A.B., Integrable field theory from conformal field theory, contenido en: "Integrable systems in quantum field theory and statistical mechanics", Eds: M. Jimbo, T. Miwa y A. Tsuchiya, 641-674. Adv. Stud. Pure Math. 19, Academic Press, Boston, 1989. 